\title{
Volatilitätskontrollierte Fraktionierung refraktär-lithophiler Elemente in Meteoriten und der Erde
}

\section{Dissertation}

zur Erlangung des mathematisch-naturwissenschaftlichen Doktorgrades

„Doctor rerum naturalium“

der Georg-August-Universität Göttingen

im Promotionsprogramm Geowissenschaften

der Georg-August University School of Science (GAUSS)

vorgelegt von

Verena Bendel

aus Bremen

Göttingen 2013

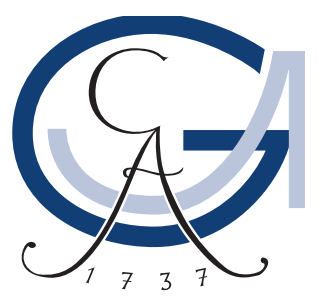


$\underline{\text { Betreuungsausschuss: }}$

Prof. Dr. Andreas Pack (GZG, Universität Göttingen)

Prof. Dr. Bent T. Hansen (GZG, Universität Göttingen)

$\underline{\text { Mitglieder der Prüfungskommission: }}$

Referent: Prof. Dr. Andreas Pack

Korreferent: Prof. Dr. Bent T. Hansen

weitere Mitglieder der Prüfungskommission:

Prof. Dr. Carsten Münker (Universität zu Köln)

Prof. Dr. Sharon Webb (GZG, Universität Göttingen)

Prof. Dr. Gerhard Wörner (GZG, Universität Göttingen)

PD Dr. Harald Krüger (MPI für Sonnensystemforschung)

Tag der mündlichen Prüfung: 24.01.2014 


\section{Inhaltsverzeichnis}

1. Einleitung 1

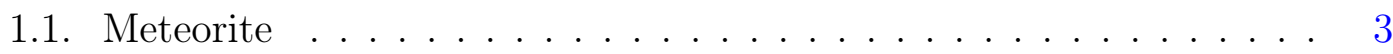

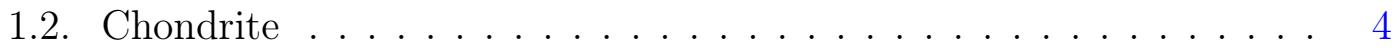

1.2.1. Komponenten von Chondriten . . . . . . . . . . . . . 4

1.2.2. Klassifikation der Chondrite . . . . . . . . . . . . . . 6

1.3. Kosmochemie der Seltenen Erden . . . . . . . . . . . . . . . . 8

1.3.1. Charakteristische REE-Muster in CAIs . . . . . . . . . . . 9

2. Probenmaterial und experimentelle Methoden 13

2.1. Probenmaterial . . . . . . . . . . . . . . . . . . 13

2.2. Probenpräparation . . . . . . . . . . . . . . . . . 20

2.3. Analytische Methoden . . . . . . . . . . . . . . . . . . 21

2.3.1. Elektronenstrahlmikrosonde . . . . . . . . . . . . . . . 21

2.3.2. Röntgenfluoreszenzanalyse . . . . . . . . . . . . . . . . 22

2.3.3. Massenspektrometrie mittels MC-ICPMS . . . . . . . . . . . 22

2.3.4. Massenspektrometrie mittels LA-ICPMS . . . . . . . . . . . 23

3. Ergebnisse 31

3.1. Komponenten von Leoville . . . . . . . . . . . . . . . . . . . 31

3.1.1. Seltene Erden . . . . . . . . . . . . . . . . . 31

3.1.2. $\mathrm{Y} / \mathrm{Ho}-, \mathrm{Zr} / \mathrm{Hf}-$ und $\mathrm{Nb} / \mathrm{Ta}-$ Verhältnisse . . . . . . . . . . . 37

3.2. Gesamtgesteinsanalysen an Chondriten, Achondriten und Planeten . 40

3.2.1. Quenchkugeln ... . . . . . . . . . . . . . . . 40

3.2.2. Volatilität von Haupt- und Spurenelementen während des

Aufschmelzprozesses . . . . . . . . . . . . . . 41

3.2.3. Röntgenfluoreszenzanalyse . . . . . . . . . . . . . . . . . 44

3.2.4. REE-Muster von Chondriten . . . . . . . . . . . . . 48

3.2.5. REE-Muster von Achondriten und terrestrischen Proben . . 56

3.2.6. HFSE-Elemente . . . . . . . . . . . . . . . . . 58

4. Diskussion $\quad \mathbf{6 1}$

4.1. Vergleich der LA-ICPMS-Daten mit Literaturdaten . . . . . . . . . . 61

4.2. Komponenten von Leoville . . . . . . . . . . . . . . . . . . . 64 
4.2.1. Refraktäre Einschlüsse . . . . . . . . . . . . . . . . 64

4.2.2. Chondren . . . . . . . . . . . . . . . . 66

4.2.3. Matrix . . . . . . . . . . . . . . . . . . . 69

4.2.4. Gesamt-Leoville . . . . . . . . . . . . . . . . 70

4.3. Bulk-Chondrite . . . . . . . . . . . . . . . . . . . . . . . . . . 73

4.3.1. Röntgenfluoreszenzanalysen . . . . . . . . . . . . . 73

4.3.2. Seltene Erden . . . . . . . . . . . . . . . . . 73

4.3.3. HFSE-Elemente . . . . . . . . . . . . . . . . . . . . 79

4.4. Tm-Anomalie im Sonnensystem . . . . . . . . . . . . . . 80

4.4.1. Berechnung der Tm-Anomalie. . . . . . . . . . . . . 80

4.4.2. Tm-Anomalien in Chondriten, Achondriten und der Erde . . 83

$\begin{array}{ll}\text { 5. Zusammenfassung und Ausblick } & 97\end{array}$

$\begin{array}{ll}\text { A. Anhang } & 101\end{array}$

A.1. Konzentrationsdaten . . . . . . . . . . . . . . . . . . . . 101

A.2. Übersicht der verwendeten Schliffpräparate . . . . . . . . . . . . 126

$\begin{array}{ll}\text { Literaturverzeichnis } & 133\end{array}$

$\begin{array}{lr}\text { Publikationen } & 147\end{array}$

$\begin{array}{lr}\text { Danksagung } & 149\end{array}$ 


\section{Abbildungsverzeichnis}

1.1. Verteilungskoeffizienten der Seltenen Erden zwischen fester und gasförmiger Phase. . . . . . . . . . . . . . . . . . 9 9

1.2. Fraktionierte Muster der Seltenen Erden. . . . . . . . . . . . . . . 10

2.1. Übersichtsbild des Dickschliffs von Leoville. . . . . . . . . . . . . . . . 14

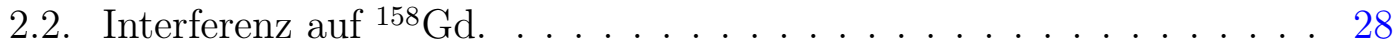

2.3. Eu/Tm vs. Tm-Anomalie. . . . . . . . . . . . . . . 30

3.1. REE-Muster in Chondren. . . . . . . . . . . . . . . . . . . 32

3.2. REE-Muster von Chondren und ihren Akkretionsrändern. . . . . . . 33

3.3. REE-Muster in refraktären Einschlüssen und der Matrix. . . . . . . . 35

3.4. Y/Ho-Verhältnisse in den Objekten von Leoville. . . . . . . . . . . . . 37

3.5. $\mathrm{Zr} / \mathrm{Hf}-$ und $\mathrm{Nb} / \mathrm{Ta}-$ Verhältnisse in den Objekten von Leoville. . . . . 38

3.6. Rückstreuelektronenbilder der Quenchkugeln. . . . . . . . . . . . . . . 40

3.7. Spurenelemente in den Quenchkugeln. . . . . . . . . . . . . . . . . . 42

3.8. Hauptelemente in den Quenchkugeln. . . . . . . . . . . . . . . . . 43

3.9. $\mathrm{Al} / \mathrm{Si}$ vs. $\mathrm{Ca} / \mathrm{Si}$ in Chondriten. . . . . . . . . . . . . . . . 44

3.10. Al/Si vs. Ti/Si in Chondriten. . . . . . . . . . . . . . . . . 45

3.11. $\mathrm{Al} / \mathrm{Si}$ vs. $\mathrm{Mg} / \mathrm{Si}$ in Chondriten. . . . . . . . . . . . . . . . 46

3.12. Seltene Erden in kohligen Chondriten (CI, CM, CV). . . . . . . . . . 49

3.13. Seltene Erden in kohligen Chondriten (CO, CK, CB) . . . . . . . . 50

3.14. Seltene Erden in gewöhnlichen Chondriten. . . . . . . . . . . . . . . 52

3.15. Seltene Erden in dem H-Chondrit Adrian. . . . . . . . . . . . . . . . . 53

3.16. REE-Muster mehrerer kleiner sowie jeweils einer größeren Probe von NWA 869 und Gao-Guenie. . . . . . . . . . . . . . . . . . . . . 54

3.17. Seltene Erden in Rumuruti- und Enstatit-Chondriten. . . . . . . . . . 55

3.18. REE-Muster von differenzierten Meteoriten. . . . . . . . . . . . 56

3.19. REE-Muster terrestrischer Proben. . . . . . . . . . . . . . . . 57

3.20. Zr/Hf- und Nb/Ta-Verhältnisse in Chondriten. . . . . . . . . . . . . 60

4.1. Literaturvergleich der LA-ICPMS-Daten für BCR-2G. . . . . . . . . 62

4.2. Literaturvergleich der LA-ICPMS-Daten für Orgueil und Allende. . 63

4.3. Anteil einer refraktären Komponente mit group-II-Muster in Alreichen Chondren. . . . . . . . . . . . . . . . . . 67 
4.4. Anteil fraktionierter Bestandteile in der Matrix von Leoville. . . . . 69

4.5. REE-Muster von den Komponenten Leovilles und des Gesamtchondrits. 71

4.6. Vergleich von Leoville mit anderen CV3-Chondriten. . . . . . . . . . 71

4.7. Mittlere Gehalte der Seltenen Erden in den Chondrit-Gruppen. . . . 74

4.8. Anteil einer group-II-Komponente in Allende. . . . . . . . . . . . . . . . 76

4.9. Anteil einer group-II-Komponente in Mighei. . . . . . . . . . . . . . 77

4.10. Anteil einer ultrarefraktären Komponente in North West Africa 3003. 78

4.11. Berechnung der Tm-Anomalie in chondritischen Proben. . . . . . . . 81

4.12. Berechnung der Tm-Anomalie in differenzierten Proben. . . . . . . . 82

4.13. Tm-Anomalien in Chondriten. . . . . . . . . . . . . . . . . 84

4.14. Tm-Anomalien in Achondriten und Planeten. . . . . . . . . . . . 87

4.15. Mittlere Tm-Anomalien von Chondriten, Achondriten und Planeten. 89

4.16. Anteil einer group-II-Komponente in Orgueil. . . . . . . . . . . . . . . 90

A.1. Schliffpräparate (1). . . . . . . . . . . . . . . . 126

A.2. Schliffpräparate (2). . . . . . . . . . . . . . . 127 


\section{Tabellenverzeichnis}

1.1. Modalbestände der Komponenten in den Chondritgruppen. . . . . . 4

2.1. Übersicht der Objekte in Leoville. . . . . . . . . . . . . . . . . . . . . . . . . 15

2.2. Probenliste Meteorite . . . . . . . . . . . . . . 16

2.3. Probenliste terrestrischer Gesteine. . . . . . . . . . . . . . . . 19

2.4. Übersicht der mittels LA-ICPMS analysierten Isotope. . . . . . . . . 24

3.1. REE-Konzentrationen der Objekte in Leoville. . . . . . . . . . . . . 36

3.2. Konzentrationen von $\mathrm{Zr}, \mathrm{Nb}, \mathrm{Hf}$ und $\mathrm{Ta}$ in den Objekten von Leoville. 39

3.3. Ergebnisse der RFA-Analysen. . . . . . . . . . . . . . . . . . 47

3.4. Ergebnisse der Isotopenverdünnungsanalysen. . . . . . . . . . . . . . . 59

4.1. Literaturvergleich der Seltenen Erden in BCR-2G. . . . . . . . . . . . 61

4.2. Literaturvergleich der Seltenen Erden in Orgueil und Allende. . . . . 63

4.3. Prozentuale Anteile der Komponenten in Leoville. . . . . . . . . . . . 70

4.4. Spurenelemente in Gesamt-Leoville und den Komponenten. . . . . . 72

4.5. Vergleich von Tm-Anomalien, berechnet aus Daten verschiedener Studien. . . . . . . . . . . . . . . . . . . 83

4.6. Tm-Anomalien von Chondriten, Achondriten und der Erde. . . . . . 85

4.7. REE/Er-Verhältnisse und REE-Konzentrationen von Orgueil und nichtkohligen Chondriten. . . . . . . . . . . . . . . . . 91

4.8. Tm-Anomalien der in dieser Arbeit analysierten Proben. . . . . . . . 92

4.9. Tm-Anomalien aus Literaturdaten von Chondriten und irdischen Gesteinen. . . . . . . . . . . . . . . . . . . . . 93

4.10. Tm-Anomalien von Ozeanbodenbasalten. . . . . . . . . . . . . . . . 94

A.1. CaO-Gehalte der Objekte in Leoville. . . . . . . . . . . . . . . . . . . . 101

A.2. RFA-Analysen an einer Basaltprobe. . . . . . . . . . . . . . . . 102

A.3. Ca-Konzentrationen der Chondritgruppen. . . . . . . . . . . . . . 103

A.4. Ergebnisse der LA-ICPMS-Messungen. . . . . . . . . . . . . . . . . . . 104

A.5. REE-Konzentrationen in den Quenchkugeln von Allende und Thuathe.114

A.6. Mittels EMS gemessene Zusammensetzung der Quenchkugeln und der natürlichen Gläser. . . . . . . . . . . . . . . . . . . . 116

A.7. Probenliste der Schliffpräparate. . . . . . . . . . . . . . . . . 128 



\section{Einleitung}

Unser Sonnensystem ist durch den gravitativen Kollaps einer großen Molekülwolke entstanden. Sein Entstehungsalter wurde anhand von Ca-Al-reichen Einschlüssen, die in primitiven Meteoriten vorkommen und die ersten Kondensate des Sonnensystems sind, auf 4,568 Milliarden Jahre datiert (Bouvier und Wadhwa, 2010). Nach dem Kollaps bildeten sich aus dem solaren Nebel eine Protosonne und eine darum rotierende Akkretionsscheibe aus Staub und Gas, in der mit zunehmender Verdichtung die Akkretion fester Materie begann. Zunächst hafteten kleine Staubteilchen und Aggregate aneinander, die durch weitere Kollisionen zu Planetisimalen und schließlich zu Planeten heranwuchsen (Chambers, 2004). Im Asteroidengürtel, der zwischen den Umlaufbahnen von Mars und Jupiter verläuft, ist kein Planet entstanden. Stattdessen bewegen sich dort zahlreiche Asteroiden auf ihren Umlaufbahnen um die Sonne. Möglicherweise waren in dieser Region Resonanzen des sich früh bildenden Jupiters für störende Einflüsse auf die Entstehung eines Planeten verantwortlich (Chambers, 2004).

Von besonderer wissenschaftlicher Bedeutung sind Elementfraktionierungen, die im solaren Nebel aufgrund von Volatilitätsunterschieden stattgefunden haben. Sie ermöglichen Rückschlüsse auf die Entstehungsbedingungen von Planeten und Asteroiden. Bei sehr hohen Temperaturen kondensierten zunächst die refraktären Elemente (das sind Elemente mit Kondensationstemperaturen von über $1400 \mathrm{~K}$ ) in Mineralphasen wie Korund, Hibonit, Grossit, Gehlenit, Perowskit und Ca-Titanat (Lodders, 2003). Mit der fortschreitenden Abkühlung des solaren Nebels gingen auch moderat-volatile und schließlich volatile Elemente in die Festphase über.

Der 1969 im mexikanischen Allende niedergegangene Meteorit (King et al., 1969), ermöglichte durch seine große Probenmenge zahlreiche chemische Analysen, die wertvolle Einblicke über Fraktionierungsprozesse im frühen Sonnensystem lieferten. Insbesondere die Beobachtung stark fraktionierter Seltener Erden in Ca-Al-reichen Einschlüssen (Martin und Mason, 1974; Grossman und Ganapathy, 1976b; Mason und Taylor, 1982), die im Meteorit Allende häufig vorkommen, führte zu neuen Erkenntnissen über ihre physikochemischen Entstehungsbedingungen.

Selbst auf einer größeren Skala von Asteroiden und Planeten treten REE- (rare earth elements) Fraktionierungen auf. Beispielsweise ist das Sm/Nd-Verhältnis in den terrestrischen Planeten Erde und Mars, sowie dem Mond etwa $5 \%$ höher als in den Chondriten (Caro et al., 2008). Verschiedene Chondritklassen sind ebenfalls gegeneinander fraktioniert. Nakamura (1974) beobachtete, dass sich die 
REE-Muster der gewöhnlichen Chondrite von CI- und anderen kohligen Chondriten unterscheiden. Er beschrieb insbesondere eine Anreicherung der leichten Seltenen Erden in kohligen Chondriten, die von Orgueil (CI1) über Murchison (CM2) bis hin zu Allende (CV3) zunehmen, sowie eine positive Yb-Anomalie kohliger Chondrite gegenüber gewöhnlichen Chondriten und brachte diese Beobachtungen mit Ybreichen Hochtemperaturkondensaten in kohligen Chondriten in Verbindung.

Auch die genaue chemische Zusammensetzung der Erde ist Gegenstand aktueller Forschung. Kosmochemische An- und Abreicherungstrends der Elemente zeigen, dass die Erde größere Ähnlichkeiten zu den kohligen Chondriten aufweist als zu den gewöhnlichen und den Enstatit-Chondriten (Allègre et al., 2001; Palme und O'Neill, 2003). Verhältnisse von stabilen Isotopen deuten dagegen an, dass sie aus demselben Material entstanden sein könnte wie die Enstatit-Chondrite (Javoy, 1995; Javoy et al., 2010).

Diese Arbeit leistet einen Beitrag zum Verständnis volatilitätskontrollierter Fraktionierungen refraktär-lithophiler Elemente in Chondritkomponenten sowie zwischen Bulk-Chondriten, Achondriten und Planeten. Schwerpunktmäßig wird dabei auf die Seltenen Erden eingegangen. Für die Untersuchungen der Chondritkomponenten wurde Leoville ausgewählt, der ein reduzierter CV3-Chondrit ist (McSween, 1977; Bonal et al., 2006). Da der Leoville-Mutterkörper nach seiner Entstehung nur geringfügige wässrige und thermische Alteration erfahren hat, ist er besonders gut für chemische Untersuchungen geeignet. Die Komponenten wurden in-situ als gesamte Objekte, also nicht ihre einzelnen Mineralphasen, analysiert. Die Ergebnisse ermöglichten wichtige Rückschlüsse auf die spezifischen Entstehungsbedingungen der jeweiligen Komponenten.

Für die Untersuchungen volatilitätskontrollierter Fraktionierungen auf einer Größsenskala von Asteroiden und Planeten wurde eine Auswahl an Meteoriten und terrestrischen Proben analysiert, die einen großen Bereich des inneren Sonnensystems von der Erde und dem Mond über den Mars bis hin zu differenzierten und undifferenzierten Meteoriten aus dem Asteroidengürtel umfasst. Ein Schwerpunkt dieser Arbeit liegt dabei auf der systematischen Gegenüberstellung verschiedener Chondritklassen und -gruppen. Die mittels LA-ICPMS (laser ablation inductively coupled mass spectrometry) durchgeführten Messungen umfassen, abgesehen von Cer, alle Seltenen Erden von Lanthan bis Lutetium, was in früheren vergleichenden Studien von Chondriten (Masuda et al., 1973; Nakamura und Masuda, 1973; Nakamura, 1974; Evensen et al., 1978) nicht gegeben war. Insbesondere die Messung des monoisotopischen Thuliums liefert wichtige Erkenntnisse über volatilitätskontrollierte Fraktionierungsprozesse im frühen Sonnensystem. Es wird gezeigt, dass sich die relativen Tm-Gehalte kohliger Chondrite deutlich von denen der anderen Objekte des inneren Sonnensystems unterscheiden und dass diese Unterschiede auf volatilitätskontrollierte Fraktionierungsprozesse zurückzuführen sind. 


\subsection{Meteorite}

Ein Meteorit ist ein Gesteinsbrocken extraterrestrischer Herkunft, der auf der Erdoberfläche aufgeschlagen ist, anstatt zuvor vollständig in der Erdatmosphäre verglüht zu sein. Meteorite stammen von Asteroiden, Planeten oder vom Mond und wurden bei Kollisionen mit anderen Körpern von diesen losgeschlagen. Die meisten Meteorite haben ihren Ursprung im Asteroidengürtel, der zwischen den Umlaufbahnen von Mars und Jupiter verläuft.

Nach gängigen Klassifikationsmodellen werden drei Arten von Meteoriten unterschieden: Undifferenzierte Meteorite (Chondrite), geringfügig differenzierte Meteorite (primitive Achondrite) und differenzierte Meteorite, die ihrerseits in Achondrite, Eisen- und Steineisenmeteorite unterteilt werden (Bischoff, 2001; Krot et al., 2003; Weisberg et al., 2006).

Chondrite sind primitive, das heißt undifferenzierte Meteorite. Ihr Name leitet sich davon ab, dass sie kleine silikatische Kügelchen - die Chondren - enthalten. Die Konzentrationsverhältnisse der nichtvolatilen Elemente in Chondriten entsprechen etwa denen des Sonnensystems. Nach ihrer Akkretion haben Chondrite nur geringfügige Veränderungen wie wässrige Alteration oder thermische Metamorphose auf dem Mutterkörper erfahren, sie wurden jedoch nicht aufgeschmolzen. Die verschiedenen Klassen der Chondrite sowie ihre Komponenten werden im Abschnitt 1.2 beschrieben.

Die primitiven Achondrite entsprechen in ihrer chemischen Zusammensetzung im Wesentlichen den Chondriten, haben im Gegensatz zu diesen jedoch eine partielle Aufschmelzung oder Rekristallisation auf dem Mutterkörper erfahren. Zu den primitiven Achondriten zählen die Gruppen der Acapulcoite, Lodranite und Winonaite (Bischoff, 2001).

Differenzierte Meteorite stammen von Mutterkörpern, die in ihrer Entstehungsphase aufgeschmolzen und differenziert wurden, so dass sich ein Eisenkern und ein silikatischer Mantel gebildet haben. Es wird zwischen Achondriten, Eisenund Steineisenmeteoriten unterschieden.

Achondrite sind silikatische Gesteine mit einem geringen Eisengehalt. Ihr Name leitet sich von der Tatsache ab, dass sie im Gegensatz zu den Chondriten keine Chondren enthalten. $\mathrm{Zu}$ den Achondriten zählen die Gruppen der Angrite, Aubrite, Brachinite, Ureilite, HED-Metoerite (Howardite, Diogenite und Eukrite), Mondmeteorite und Marsmeteorite (Krot et al., 2003). Es wird angenommen, dass die HED-Meteorite von einem gemeinsamen Mutterkörper, dem Asteroiden 4 Vesta stammen (Russell et al., 2012). Dementgegen stehen Hinweise auf einen Ursprung auf dem IIIAB-Mutterkörper (Wasson, 2013).

Eisenmeteorite bestehen aus Fe-Ni-Metall. Anhand der chemischen Zusammensetzung werden zwölf Gruppen von Eisenmeteoriten (IAB, IC, IIAB, IIC, IID, IIE, IIIAB, IIICD, IIIE, IIIF, IVA und IVB) unterschieden (Krot et al., 2003). 
Steineisenmeteorite bestehen hauptsächlich aus Olivin und einer metallischen Fe-Ni-Legierung. Es werden zwei Gruppen, die Pallasite und die Mesosiderite unterschieden (Krot et al., 2003).

\subsection{Chondrite}

\subsubsection{Komponenten von Chondriten}

Die Hauptbestandteile von Chondriten sind die Chondren und eine feinkörnige Matrix. In kohligen Chondriten spielen außerdem refraktäre Einschlüsse eine wichtige Rolle. Der Anteil der verschiedenen Komponenten kann sich je nach Chondritgruppe stark unterscheiden (Tabelle 1.1).

Tabelle 1.1: Modalbestände der Komponenten in den Chondritgruppen in Vol\%. Daten aus Weisberg et al. (2006).

\begin{tabular}{lcccc}
\hline & Chondren $^{\text {a }}$ & Matrix & CAIs \& AOAs & Metall \\
\hline CI & $\ll 1$ & $>99$ & $\ll 1$ & 0 \\
CM & $20^{b}$ & $70^{b}$ & 5 & 0,1 \\
CO & 48 & 34 & 13 & $1-5$ \\
CV & 45 & 40 & 10 & $0-5$ \\
CK & 45 & 40 & 10 & $0-5$ \\
CR & $50-60$ & $30-50$ & 0,5 & $5-8$ \\
CH & 70 & 5 & 0,1 & 20 \\
CB & $20-40$ & $\ll 1$ & $\ll 1$ & $60-80$ \\
H & $60-80$ & $10-15$ & $\ll 1$ & 8 \\
L & $60-80$ & $10-15$ & $\ll 1$ & 4 \\
LL & $60-80$ & $10-15$ & $\ll 1$ & 2 \\
EH & $60-80$ & $2-15^{c}$ & $\ll 1$ & 10 \\
EL & $60-80$ & $2-15^{c}$ & $\ll 1$ & 10 \\
R & $>40$ & 36 & 0 & 0,1 \\
K & 27 & 73 & $\ll 1$ & 7 \\
\hline B & 27 & & &
\end{tabular}

${ }^{a}$ Beinhaltet Mineralbruchstücke.

${ }^{b}$ Sehr variabel.

${ }^{c}$ Der Matrix-Anteil in E-Chondriten ist als Näherungswert zu betrachten.

Refraktäre Einschlüsse: Es gibt zwei Arten von refraktären Einschlüssen, die in Chondriten vorkommen: Ca-Al-reiche Einschlüsse (CAIs; Ca-Al-rich inclusions) und amöboide Olivinaggregate (AOAs). 
CAIs sind Submillimeter bis Zentimeter große Einschlüsse. Sie sind durch Kondensationsereignisse bei hohen Temperaturen im solaren Nebel enstanden und bestehen aus refraktären Ca-Al-Ti-reichen Mineralen wie Korund, Hibonit, Grossit, Perowskit, Mellilit, Spinell, Al-Ti-Diopsid, Anorthit und Forsterit (Scott und Krot, 2003). Einige der CAIs wurden nach ihrer Entstehung aufgeschmolzen.

CAIs sind die ältesten bekannten Objekte unseres Sonnensystems. Verschiedene Datierungsmethoden belegen, dass sie vor etwa 4567 bis 4568 Millionen Jahren entstanden (Burkhardt et al., 2008; Amelin et al., 2010; Bouvier und Wadhwa, 2010; Connelly et al., 2012). Sie haben sich wahrscheinlich innerhalb einer Zeitspanne von nur 20000 Jahren durch Kondensationsprozesse gebildet, anschließende Aufschmelzereignisse, die einige CAIs betreffen, fanden während einer etwas längeren Zeitspanne von ungefähr 0,7 Millionen Jahren nach der Entstehung der ersten CAIs statt (MacPherson et al., 2012).

Amöboide Olivinaggregate sind unregelmäßig geformte, Millimeter bis Zentimeter große Einschlüsse, die aus Hochtemperaturmineralen wie Forsterit, Fe-Ni-Metall und kleinen eingeschlossenen CAIs bestehen (Krot et al., 2009). In alterierten AOAs kommen auch sekundäre Phasen wie Nephelin und Sodalith vor (Scott und Krot, 2003).

Chondren: Chondren sind Submillimeter bis Millimeter große silikatische Kügelchen, die bis zu 80 Vol\% eines Chondrits ausmachen können (Tabelle 1.1). Sie bestehen vorwiegend aus den Mineralen Olivin, Pyroxen, Plagioklas, Spinell, Chromit, Troilit und Kamacit, welche in eine glasige oder mikrokristalline Mesostasis eingebettet sind (Jones et al., 2005). Das Ausgangsmaterial der Chondren wurde während der Entstehung aufgeschmolzen und erstarrte anschließend zu Schmelzkügelchen. Es gibt deutliche Hinweise darauf, dass es mehrere aufeinanderfolgende Aufschmelzereignisse gab, zum Beispiel das Vorkommen von Reliktkörnern einer früheren Chondrengeneration oder Akkretionsränder, die nachträglich aufgeschmolzen wurden (Jones et al., 2005; Krot et al., 2009).

Da die Gehalte an volatilen Elementen wie Natrium während der Entstehung von Chondren relativ konstant bleiben, erscheint es wahrscheinlich, dass sie sich in Regionen mit einem hohen Staubanteil gebildet haben (Alexander et al., 2008; Borisov et al., 2008). Anhand der Texturen von Chondren können die maximalen Temperaturen abgeschätzt werden, denen sie ausgesetzt waren; sie betrugen etwa 1400 bis $1850{ }^{\circ} \mathrm{C}$ (Hewins et al., 2005). Durch Experimente konnte abgeschätzt werden, dass die Abkühlungsraten zwischen 10 und $1000{ }^{\circ} \mathrm{C}$ pro Stunde betragen haben müssen (Hewins et al., 2005).

In der Literatur werden verschiedene Chondrenbildungsmodelle diskutiert, darunter Impaktmodelle, die auf Kollisionen von Planetisimalen beruhen (Asphaug et al., 2011; Sanders und Scott, 2012) und Schockwellenmodelle, bei denen angenommen 
wird, dass Schockwellen im solaren Nebel zur Aufschmelzung der Chondrenpräkursoren geführt haben (Desch und Connolly, 2002; Boss und Durisen, 2005; Hood et al., 2009). Datierungen belegen, dass die Chondrenbildung schon etwa zeitgleich mit der Entstehung von CAIs begonnen hat, sich aber über eine längere Zeitspanne von bis zu drei Millionen Jahren erstreckte (Bizzarro et al., 2004; Connelly et al., 2012).

Matrix: Chondritmatrix besteht aus feinkörnigem Material, mit Korngrößen, die typischerweise zwischen $5 \mathrm{~nm}$ und $10 \mu \mathrm{m}$ liegen (Scott und Krot, 2003). Sie dient als Grundmasse, in die Chondren und refraktäre Einschlüsse eingebettet sind. Feinkörnige Akkretionsränder um Chondren und CAIs werden auch als Matrix betrachtet.

Die häufigsten Matrixminerale sind Olivine, Pyroxene, Oxide, Sulfide und Fe-NiMetalle. In wässrig alterierten Chondriten finden sich häufig sekundäre Mineralphasen wie Phyllosilikate, Eisenoxide, Karbonate, Sulfate und Phosphate (Zolensky et al., 1993). Zu einem geringen Anteil können auch organisches Material (Busemann et al., 2006) und präsolare Körner, die älter als unser Sonnensystem sind (Nagashima et al., 2004), vorkommen.

\subsubsection{Klassifikation der Chondrite}

Chondrite sind chemisch primitives Material, das bezüglich der nichtvolatilen Elemente im Wesentlichen der Zusammensetzung des Sonnensystems entspricht. Es bestehen jedoch kleine systematische Unterschiede der chemischen Zusammensetzung zwischen verschiedenen Chondritklassen und -gruppen. Anhand der chemischen und petrologischen Eigenschaften werden vier Chondritklassen unterschieden: Kohlige Chondrite, gewöhnliche Chondrite, Enstatit-Chondrite und Rumuruti-Chondrite (Bischoff, 2001). Desweiteren gibt es unklassifizierte Chondrite, die keiner dieser Klassen zugeordnet werden können.

Kohlige Chondrite (CC; carbonaceous chondrites) unterscheiden sich von den anderen Chondritklassen dahingehend, dass sie refraktäre Einschlüsse enthalten, die in nichtkohligen Chondritklassen nur sehr sporadisch vorkommen (Tabelle 1.1). Die meisten kohligen Chondritgruppen haben gegenüber den CI-Chondriten eine relative Anreicherung an refraktär-lithophilen Elementen (Kallemeyn und Wasson, 1981). Darüberhinaus besitzen die meisten kohligen Chondrite einen hohen Matrixanteil und einen geringen Chondrenanteil. Es werden acht verschiedene Gruppen unterschieden (Krot et al., 2003):

- CI-Chondrite (Ivuna-Gruppe) bestehen zu über $99 \%$ aus Matrix und sind somit die einzige Chondritgruppe, die so gut wie keine Chondren und refraktären Einschlüsse enthält. Sie werden als die chemisch primitivsten Chondrite 
angesehen, da die Elementverhältnisse der nichtvolatilen Elemente dieser Chondritgruppe am besten mit spektroskopischen Daten der solaren Photosphäre übereinstimmen (Anders und Grevesse, 1989; Lodders et al., 2009). Der Mineralbestand der CI-Chondrite deutet allerdings an, dass sie starke sekundäre Veränderungen durch wässrige Alteration erfahren haben (Tomeoka und Buseck, 1988).

- CM-Chondrite (Mighei-Gruppe) sind im Vergleich zu CI-Chondriten an refraktär-lithophilen Elementen (RLE) angereichert: Die mittleren CInormierten RLE/Mg-Verhältnisse betragen 1,13 (Rubin, 2011). Sie enthalten kleine Chondren und refraktäre Einschlüsse. Klasten der CM-Chondrite sind häufig auch in anderen Meteoriten zu finden (Zolensky et al., 1996; Rubin und Bottke, 2009).

- Die Gruppe der CV-Chondrite (Vigarano-Gruppe) besitzt die höchsten Gehalte an refraktär-lithophilen Elementen. Die RLE/Mg-Verhältnisse haben einen durchschnittlichen Anreicherungsfaktor von 1,35 im Vergleich zu CIChondriten (Rubin, 2011). CV-Chondrite zeichnen sich durch im Durchschnitt fast einen Millimeter messende, also relativ große Chondren aus (Rubin, 2010), und enthalten mit $10 \%$ einen hohen Anteil an refraktären Einschlüssen (Tabelle 1.1).

- CO-Chondrite (Ornans-Gruppe) enthalten kleine Chondren von durchschnittlich $150 \mu \mathrm{m}$ (Rubin, 2010). Der Anteil der refraktären Einschlüsse ist in dieser Gruppe mit 13\% am höchsten (Tabelle 1.1). Der Anreicherungsfaktor der refraktär-lithophilen Elemente ist mit 1,11 ähnlich hoch wie bei den CM-Chondriten (Rubin, 2011).

- CK-Chondrite (Karoonda-Gruppe) sind, wie die CV-Chondrite, stark an refraktär-lithophilen Elementen angereichert, mit einem durchschnittlichen Anreicherungsfaktor von 1,24 (Rubin, 2011). Sie enthalten ebenfalls viele refraktäre Einschlüsse (10\%, Tabelle 1.1).

- CR-Chondrite (Renazzo-Gruppe) haben einen relativ großen Matrix-Anteil und nur wenige refraktäre Einschlüsse. Die Chondren sind mit durchschnittlich 0,7 mm (Rubin, 2010) relativ groß.

- CB-Chondrite (Bencubbin-Gruppe) bestehen fast ausschließlich aus Metall (60 bis $80 \%$ ) und Chondren (20 bis $40 \%$ ); im Gegensatz zu anderen kohligen Chondriten enthalten sie praktisch keine Matrix (Tabelle 1.1). Aufgrund ihrer chemischen und petrologischen Eigenschaften werden sie in zwei Untergruppen, die $\mathrm{CB}_{\mathrm{a}^{-}}$und $\mathrm{CB}_{\mathrm{b}}$-Chondrite, eingeteilt (Weisberg et al., 2006). 
- CH-Chondrite (metallreich) bestehen zu einem großen Anteil aus sehr kleinen, durchschnittlich $20 \mu \mathrm{m}$ messenden Chondren (Rubin, 2010). Sie enthalten nur wenig Matrix und fast keine refraktären Einschlüsse.

Gewöhnliche Chondrite (OC; ordinary chondrites) stellen die am häufigsten vorkommende Chondritgruppe dar. Charakteristisch für sie ist ein großer Anteil an Chondren (60 bis $80 \%$ ), die etwa 0,3 bis $0,6 \mathrm{~mm}$ groß sind (Rubin, 2010). Der Anteil an Matrix ist mit 10 bis $15 \%$ relativ gering und refraktäre Einschlüsse kommen mit weniger als $1 \%$ nur sehr selten vor (Tabelle 1.1). Im Gegensatz zu kohligen Chondriten sind gewöhnliche Chondrite an refraktären Elementen abgereichert: Die durchschnittlichen CI-normierten RLE/Mg-Verhältnisse betragen 0,89 bis 0,90 (Rubin, 2011).

Gewöhnliche Chondrite werden anhand der chemischen Zusammensetzung in drei Gruppen eingeteilt, die H- (high iron), L- (low iron) und LL- (low iron, low metal) Chondrite. Sie haben charakteristische $\mathrm{Fe} / \mathrm{SiO}_{2}$-Verhältnisse von durchschnittlich 0,77, 0,55 und 0,49 (Van Schmus und Wood, 1967).

Enstatit-Chondrite (EC) bestehen zu 60 bis $80 \%$ aus Chondren, die reich an Enstatit sind. Sie haben einen geringen Matrixanteil von 2 bis $15 \%$ und enthalten kaum refraktäre Einschlüsse (Tabelle 1.1). Der Mineralbestand der EnstatitChondrite weist auf reduzierende Bedingungen während ihrer Entstehung hin. Die Gruppe der Enstatit-Chondrite ist im Vergleich zu CI-Chondriten an refraktärlithophilen Elementen abgereichert: Die Mg- und CI-normierten RLE-Gehalte betragen 0,87 bis 0,88 (Rubin, 2011). Sie ist die einzige Chondritklasse, deren Sauerstoffisotopenzusammensetzung auf der terrestrischen Fraktionierungslinie liegt (Clayton et al., 1984). Enstatit-Chondrite werden - wie gewöhnliche Chondrite anhand ihres Eisengehaltes in EH- und EL-Chondrite unterteilt (Sears et al., 1982).

Rumuruti-Chondrite (RC) sind nach dem Fall von Rumuruti in Kenia benannt (Schulze et al., 1994). Sie bestehen fast ausschließlich aus Chondren und Matrix und enthalten kaum refraktäre Einschlüsse (Tabelle 1.1). Sie sind gegenüber den CI-Chondriten nur schwach an refraktär-lithophilen Elementen abgereichert und haben ein CI-normiertes RLE/Mg-Verhältnis von 0,97 (Rubin, 2011).

\subsection{Kosmochemie der Seltenen Erden}

Die Seltenen Erden gehören zu den refraktären Elementen. Sie kondensieren bei hohen Temperaturen in einem sich abkühlenden solaren Nebel in refraktäre Trägerphasen wie zum Beispiel Hibonit und Perowskit; ihre $50 \%$-Kondensationstemperaturen liegen bei einem Druck von $10^{-4}$ bar zwischen etwa $1660 \mathrm{~K}$ für die schweren Seltenen Erden und $1350 \mathrm{~K}$ für Europium (Lodders, 2003). Die Fraktionierung der Seltenen Erden während der Kondensation erfolgt nicht gleichmäßig als Funktion des 
Ionenradius, sondern diskontinuierlich in Abhängigkeit ihrer stark unterschiedlichen Volatilität (Boynton, 1975).

Die Volatilitätsunterschiede der Seltenen Erden beruhen auf ihrem Oxidationszustand in der Gasphase: In einem Gas solarer Zusammensetzung liegen die meisten Seltenen Erden überwiegend als Monoxid vor; Eu und Yb liegen dagegen überwiegend monoatomar vor und zeichnen sich daher durch eine höhere Volatilität aus (Boynton, 1989). Thulium, das etwa zur Hälfte monoatomar vorliegt, ist ebenfalls volatiler als die schweren Seltenen Erden Er und Lu. Die Verteilungskoeffizienten der Seltenen Erden zwischen der festen und der gasförmigen Phase nach den Berechnungen von Boynton (1989) sind in Abbildung 1.1 dargestellt.

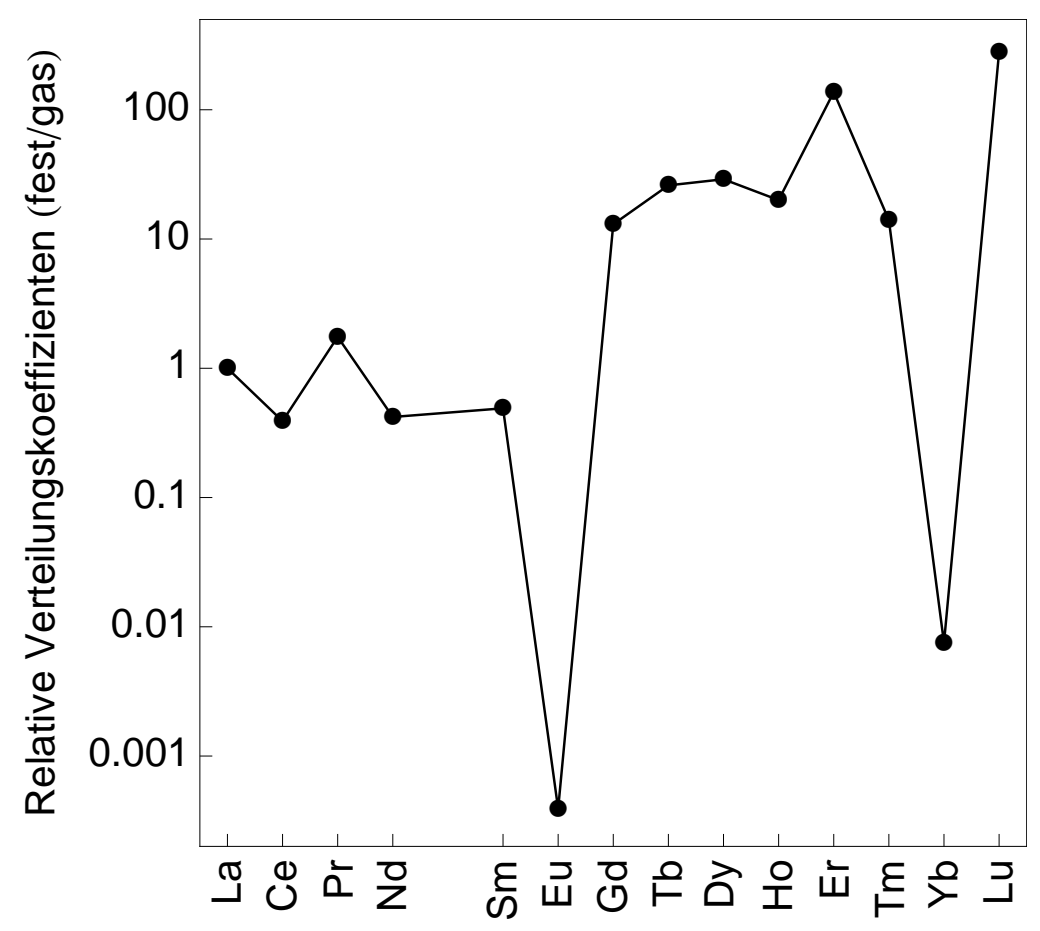

Abbildung 1.1: Relative Verteilungskoeffizienten der Seltenen Erden zwischen der festen und der gasförmigen Phase im solaren Nebel bei einer Temperatur von $1650 \mathrm{~K}$ mit Hibonit als Trägermineral. Nach Boynton (1989).

\subsubsection{Charakteristische REE-Muster in CAIs}

Ein Großteil der CAIs zeigt erhebliche REE-Fraktionierungen, die durch fraktionierte Gleichgewichtskondensation bei hohen Temperaturen zu erklären sind (Boynton, 1989). Nachfolgend werden die wichtigsten REE-Muster der CAIs kurz beschrieben. 
Ultrarefraktäres Muster: Ultrarefraktäre REE-Muster zeigen eine Anreicherung der schweren gegenüber den leichten Seltenen Erden, die beiden volatilsten $\mathrm{REE}-\mathrm{Eu}$ und $\mathrm{Yb}$ - sind extrem abgereichert und auch Tm ist gegenüber den anderen schweren Seltenen Erden leicht verarmt (Abbildung 1.2 a). CAIs mit solchen REE-Mustern stellen vermutlich die ersten Kondensate des solaren Nebels dar (Boynton, 1989).

Group-II-Muster: Das group-II-Muster ist, abgesehen von Eu und Yb, komplementär zum ultrarefraktären Muster; es ist an den leichten Seltenen Erden und Tm angereichert (Abbildung $1.2 \mathrm{~b}$ ). Die Entstehung von Kondensaten mit einem solchen Muster kann durch ein Zweistufenmodell erklärt werden: Nach der Kondensation und der Isolation einer ultrarefraktären Komponente ist das residuale Gas an den volatileren Seltenen Erden angereichert; in einem zweiten Kondensationsschritt entsteht nun eine refraktäre Komponente, die ein nahezu komplementäres REE-Muster - das group-II-Muster - hat, sie wird von der Gasphase isoliert, bevor die beiden volatilsten Seltenen Erden Eu und Yb vollständig auskondensiert sind (Boynton, 1989).
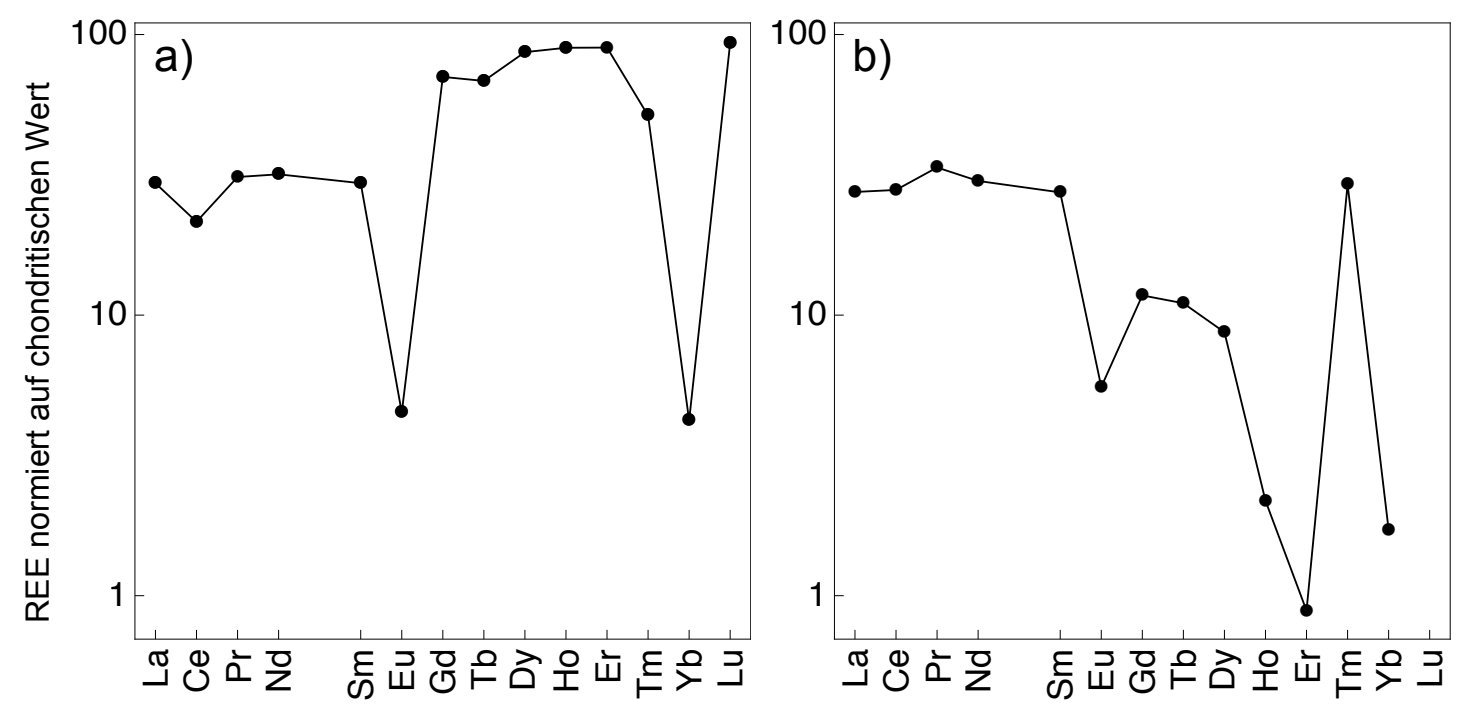

Abbildung 1.2: Fraktionierte Muster der Seltenen Erden. a) Ultrarefraktäres REE-Muster: Typisch sind starke Abreicherungen der volatilsten Seltenen Erden Eu und Yb; nach Boynton (1989). b) Group-II-Muster: Bis auf Eu und $\mathrm{Yb}$ ist es komplementär zum ultrarefraktären Muster; nach Mason und Taylor (1982). 
Andere Muster: Es gibt weitere charakteristische Muster der Seltenen Erden in CAIs, die abgesehen von Eu und Yb relativ unfraktioniert sind (Mason und Taylor, 1982):

- group-I: positive Eu-Anomalie, sonst unfraktioniert

- group-III: negative Eu- und Yb-Anomalien, sonst unfraktioniert

- group-V: unfraktioniert

- group-VI: positive Eu- und Yb-Anomalien, sonst unfraktioniert, komplementär zu group-III 
1. Einleitung 


\section{Probenmaterial und experimentelle Methoden}

\subsection{Probenmaterial}

Es wurden in-situ-Analysen der Komponenten des CV3-Chondrits Leoville an einem 2,2 $\mathrm{cm}^{2}$ großen polierten Dickschliff durchgeführt, der von J. Zipfel (Senckenbergmuseum, Frankfurt am Main) zur Verfügung gestellt wurde. Die Probe wurde bereits in einer vorhergehenden Studie von Patzer et al. (2012) petrographisch untersucht. Die Probe enthält zahlreiche Typ-1-Chondren, eine Typ-2-Chondre, drei Al-reiche Chondren, fünf CAIs, ein AOA und einige Klasten; der Matrix-Anteil beträgt ungefähr ein Drittel (Patzer et al., 2012). Es wurden 32 repräsentative Objekte ausgewählt und eingehender untersucht (siehe Abbildung 2.1 und Tabelle 2.1). Ausführliche Informationen dazu finden sich in Patzer et al. (2012).

Es wurden Gesamtgesteinsanalysen an 49 Chondriten und 15 Achondriten (Tabelle 2.2) sowie 46 terrestrischen Proben (Tabelle 2.3) durchgeführt. Die chondritischen Proben umfassen 14 kohlige Chondrite, 26 gewöhnliche Chondrite, acht EnstatitChondrite und einen Rumuruti-Chondrit. Die Auswahl der Achondrite besteht aus drei Mondmeteoriten, zwei Marsmeteoriten, acht HED-Meteoriten, einem Winonait und einem Aubrit. Die terrestrischen Proben stammen aus verschiedenen geologischen Reservoiren und schließen die Standardmaterialien BCR-2G, AGV-2, W-2a, BHVO-2, RGM-2, BIR-1, GSP-2, UB-N, NIM-P, NIM-D, DTS-1, JP-1 und PCC-1 ein.

Die Meteoritenproben wurden zur Verfügung gestellt von: A. Bischoff (Westfälische Wilhelms-Universität Münster), T. McCoy und L. Welzenbach (Smithsonian National Museum of Natural History, Washington), M. Shelley (Australian National University, Canberra), B. Zanda (Muséum National d'Histoire Naturelle, Paris), R. Schönberg (Leibniz Universität Hannover), A. Gehler (Private Sammlung), J. Zipfel (Senckenbergmuseum, Frankfurt am Main) und K. Bermingham (Westfälische Wilhelms-Universität Münster). Die terrestrischen Proben wurden bereit gestellt von: M. Reich, G. Hartmann, K. H. Wedepohl, B. Schmidt and W. Wegner (Georg-August-Universität Göttingen), H.-P. Schertl (Ruhr-Universität Bochum), H. Paulick und C. Münker (Universität zu Köln) und A. Gehler (Private Sammlung). 


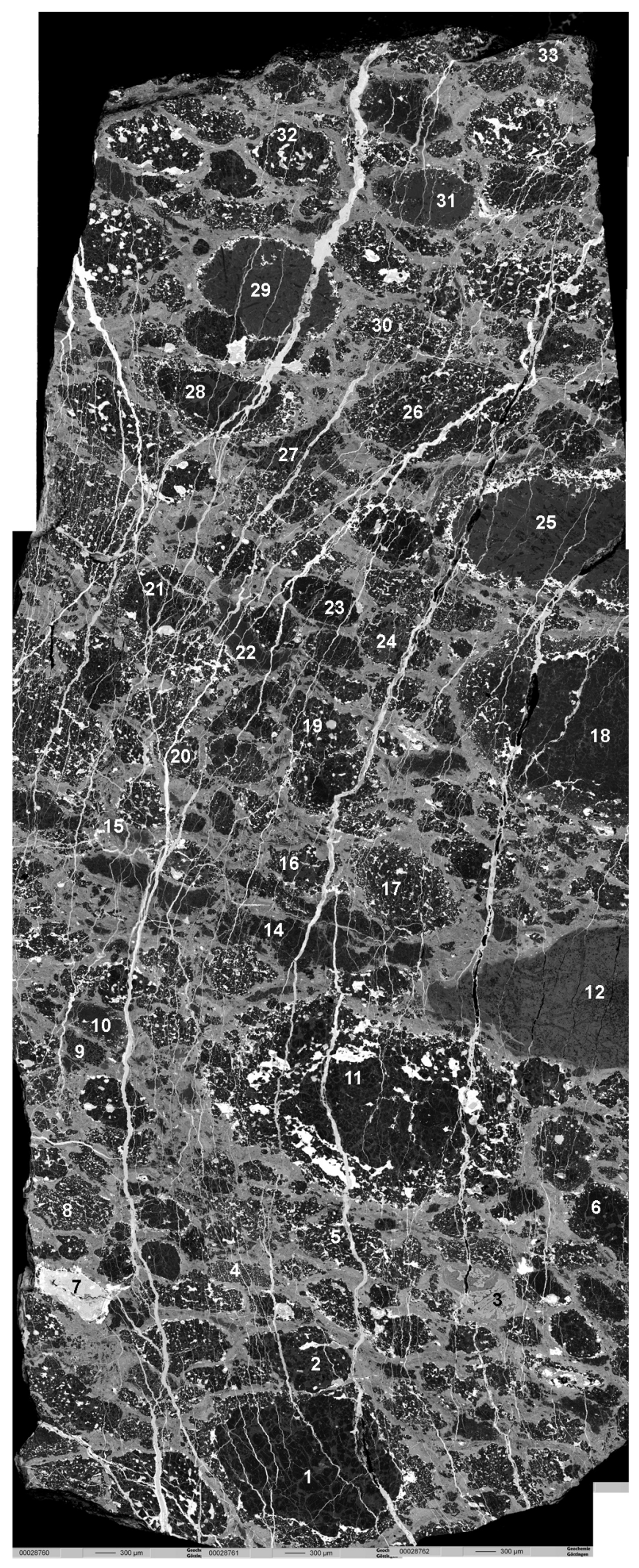

Abbildung 2.1: Übersichtsbild des Dickschliffs von Leoville. Die Zahlen kennzeichnen die analysierten Komponenten, Informationen dazu finden sich in Tabelle 2.1. Das Bild ist in anderer Form in Patzer et al. (2012) veröffentlicht. 
Tabelle 2.1: Übersicht der mittels LA-ICPMS analysierten Objekte. Daten aus Patzer et al. (2012). Die Größe der Objekte bezieht sich auf die jeweils längste Dimension. PO: porphyrischer Olivin; POP: porphyrischer Olivin + Pyroxen; PP: porphyrisches Pyroxen; G: granulär; C: kryptokristallin.

\begin{tabular}{|c|c|c|}
\hline Objekt & Typ & Größe $(\mathrm{mm})$ \\
\hline 1 & Typ-1-Chondre, PO, mit Akkretionsrand & 3,8 \\
\hline 2 & Typ-1-Chondre, PO & 1,6 \\
\hline 3 & Typ-2-Chondre, zusammengesetzt & 1,2 \\
\hline 4 & Typ-1-Chondre, G/PP & 0,9 \\
\hline 5 & Typ-1-Chondre, POP, teilweise mit Akkretionsrand & 1,3 \\
\hline 6 & Typ-1-Chondre, PO, mit Akkretionsrand & 1,4 \\
\hline 8 & Typ-1-Chondre, PP & 1,2 \\
\hline 9 & CAI, feinkörnig & 0,7 \\
\hline 10 & Typ-1-Chondre, C & 0,9 \\
\hline 11 & Typ-1-Chondre, PO, zusammengesetzt & 4,9 \\
\hline 12 & CAI, fein- bis grobkörnig & $>3,0$ \\
\hline 14 & Typ-1-Chondre, C/G & 1,9 \\
\hline 15 & CAI, sehr feinkörnig & 0,7 \\
\hline 16 & Typ-1-Chondre, POP, mit Akkretionsrand & 1,1 \\
\hline 17 & Typ-1-Chondre, PO, mit Akkretionsrand & 1,8 \\
\hline 18 & Typ-1-Chondre, PP, mit Akkretionsrand & $>3,0$ \\
\hline 19 & Typ-1-Chondre, POP, mit Akkretionsrand & 1,1 \\
\hline 20 & Typ-1-Chondre, PP & 0,7 \\
\hline 21 & Typ-1-Chondre, PO, teilweise mit Akkretionsrand & 1,4 \\
\hline 22 & CAI, sehr feinkörnig & 1,2 \\
\hline 23 & Typ-1-Chondre, PO & 1,2 \\
\hline 24 & Typ-1-Chondre, PP & 1,2 \\
\hline 25 & Al-reiche Chondre, mit Akkretionsrand & $>3,0$ \\
\hline 26 & Typ-1-Chondre, PO, mit Akkretionsrand & 2,7 \\
\hline 27 & AOA, feinkörnig & 1,2 \\
\hline 28 & Typ-1-Chondre, PP, mit Akkretionsrand & 2,5 \\
\hline 29 & Al-reiche Chondre & 2,2 \\
\hline 30 & Typ-1-Chondre, POP, mit Akkretionsrand & 1,8 \\
\hline 31 & Al-reiche Chondre & 1,5 \\
\hline 32 & Typ-1-Chondre, PO & 0,8 \\
\hline
\end{tabular}


Tabelle 2.2: Probenliste Meteorite.

\begin{tabular}{|c|c|c|c|c|c|c|c|}
\hline Probe & Gruppe & $\begin{array}{l}\text { Fund/ } \\
\text { Fall }\end{array}$ & $\begin{array}{l}\text { Masse } \\
(\mathrm{mg})\end{array}$ & $\begin{array}{l}\text { oxi- } \\
\text { diert }\end{array}$ & $\begin{array}{c}\# \\
\text { Kugeln }\end{array}$ & Quelle & $\begin{array}{l}\text { Katalog- } \\
\text { Nummer }\end{array}$ \\
\hline Alais & CI1 & Fall & 69 & nein & $2^{\mathrm{a}}$ & NMNH & USNM 949 \\
\hline Ivuna & CI1 & Fall & 205 & nein & $3^{\mathrm{a}}$ & NMNH & USNM 2478 \\
\hline Orgueil-A & CI1 & Fall & 432 & nein & $11^{\mathrm{b}}$ & MNHN & \\
\hline Orgueil-B & CI1 & Fall & 489 & nein & $8^{a}$ & MNHN & \\
\hline Orgueil-C & CI1 & Fall & 215 & nein & $3^{\mathrm{a}}$ & NMNH & USNM 6475 \\
\hline Orgueil-D & CI1 & Fall & 738 & nein & $1^{\mathrm{b}}$ & WWU & \\
\hline Orgueil-E & CI1 & Fall & 98 & nein & $4^{\mathrm{b}}$ & MNHN & \\
\hline Cold Bokkeveld & CM2 & Fall & 240 & ja & $3^{\mathrm{a}}$ & NMNH & USNM 1002 \\
\hline Mighei & CM2 & Fall & 190 & nein & $3^{\mathrm{a}}$ & NMNH & USNM 1081 \\
\hline Murchison-A & CM2 & Fall & 138 & nein & $2^{\mathrm{a}}$ & $\mathrm{ANU}$ & \\
\hline Murchison-B & CM2 & Fall & 116 & nein & $2^{\mathrm{a}}$ & ANU & \\
\hline Murchison-C & CM2 & Fall & 44 & nein & $2^{\mathrm{a}}$ & $\mathrm{ANU}$ & \\
\hline Murchison-D & CM2 & Fall & 135 & nein & $2^{\mathrm{b}}$ & ANU & \\
\hline Murchison-E & CM2 & Fall & 242 & nein & $3^{\mathrm{a}}$ & NMNH & USNM 6650-2 \\
\hline Murray & CM2 & Fall & 263 & nein & $3^{\mathrm{a}}$ & NMNH & USNM 1769-6 \\
\hline Allende-A & CV3 & Fall & 247 & nein & $5^{\mathrm{a}, \mathrm{b}}$ & ANU & \\
\hline Allende-B & CV3 & Fall & 225 & nein & $4^{\mathrm{a}, \mathrm{b}}$ & ANU & \\
\hline Allende-C & CV3 & Fall & 221 & nein & $2^{\mathrm{a}}$ & ANU & \\
\hline Allende-D & CV3 & Fall & 1500 & nein & $2^{\mathrm{b}}$ & LUH & \\
\hline Allende-E & CV3 & Fall & 16600 & nein & $3^{\mathrm{a}}$ & RFWU & \\
\hline Allende-M & CV3 & Fall & 5589 & nein & $2^{\mathrm{b}}$ & WWU & \\
\hline Allende-S & CV3 & Fall & 4000000 & nein & $4^{\mathrm{a}}$ & NMNH & USNM 3529 \\
\hline Axtell & CV3 & Fund & $100-200$ & nein & $2^{\mathrm{b}}$ & LUH & \\
\hline Mokoia & CV3 & Fall & 140 & nein & $4^{\mathrm{a}, \mathrm{b}}$ & $\mathrm{ANU}$ & \\
\hline Vigarano & CV3 & Fall & 1000 & nein & $3^{\mathrm{a}}$ & RFWU & \\
\hline NWA 3003 & $\mathrm{CO} 3$ & Fund & 100 & nein & $2^{\mathrm{b}}$ & GAUG & \\
\hline Karoonda-A & CK4 & Fall & 189 & nein & $4^{\mathrm{a}, \mathrm{b}}$ & $\mathrm{ANU}$ & \\
\hline Karoonda-B & CK4 & Fall & 257 & nein & $3^{\mathrm{a}}$ & ANU & \\
\hline Bencubbin & $\mathrm{CB}$ & Fund & 100 & nein & $2^{\mathrm{b}}$ & LUH & \\
\hline Chainpur-A & LL3 & Fall & 309 & nein & $5^{\mathrm{a}, \mathrm{b}}$ & ANU & \\
\hline Chainpur-B & LL3 & Fall & 328 & nein & $2^{\mathrm{a}}$ & ANU & \\
\hline Dar al Gani 298 & LL4 & Fund & 100 & nein & $2^{\mathrm{b}}$ & LUH & \\
\hline Dhurmsala & LL6 & Fall & 578 & ja & $2^{\mathrm{a}}$ & $\mathrm{PC}$ & \\
\hline Lake Labyrinth & LL6 & Fund & 1493 & ja & $2^{\mathrm{b}}$ & ANU & \\
\hline Saint-Séverin & LL6 & Fall & 82 & ja & $2^{\mathrm{a}}$ & WWU & \\
\hline Bruderheim & L6 & Fall & 49 & ja & $2^{\mathrm{a}}$ & WWU & \\
\hline Gashua & L6 & Fall & 155 & ja & $2^{\mathrm{a}}$ & $\mathrm{PC}$ & \\
\hline Homestead & L5 & Fall & 200 & nein & $2^{\mathrm{b}}$ & LUH & \\
\hline
\end{tabular}


Tabelle 2.2 (Fortsetzung)

\begin{tabular}{|c|c|c|c|c|c|c|c|}
\hline Probe & Gruppe & $\begin{array}{l}\text { Fund/ } \\
\text { Fall }\end{array}$ & $\begin{array}{c}\text { Masse } \\
(\mathrm{mg})\end{array}$ & $\begin{array}{l}\text { oxi- } \\
\text { diert }\end{array}$ & $\begin{array}{l}\# \\
\text { Kugeln }\end{array}$ & Quelle & $\begin{array}{l}\text { Katalog- } \\
\text { Nummer }\end{array}$ \\
\hline Mount Tazerzait & L5 & Fall & 100 & nein & $2^{\mathrm{b}}$ & $\overline{\mathrm{LUH}}$ & \\
\hline NWA 869 & L5 & Fund & 3157 & ja & $2^{\mathrm{a}}$ & $\mathrm{PC}$ & \\
\hline NWA 869-A & L5 & Fund & 26 & ja & $1^{\mathrm{a}}$ & $\mathrm{PC}$ & \\
\hline NWA 869-B & L5 & Fund & 24 & ja & $1^{\mathrm{a}}$ & $\mathrm{PC}$ & \\
\hline NWA 869-C & L5 & Fund & 23 & ja & $1^{\mathrm{a}}$ & $\mathrm{PC}$ & \\
\hline NWA 869-D & L5 & Fund & 22 & ja & $1^{\mathrm{a}}$ & $\mathrm{PC}$ & \\
\hline NWA $869-\mathrm{E}$ & L5 & Fund & 22 & ja & $1^{\mathrm{a}}$ & $\mathrm{PC}$ & \\
\hline NWA 869-F & L5 & Fund & 25 & ja & $1^{\mathrm{a}}$ & $\mathrm{PC}$ & \\
\hline NWA 869-G & L5 & Fund & 24 & ja & $1^{\mathrm{a}}$ & $\mathrm{PC}$ & \\
\hline NWA 869-H & L5 & Fund & 24 & ja & $1^{\mathrm{a}}$ & $\mathrm{PC}$ & \\
\hline NWA 869-I & L5 & Fund & 28 & ja & $1^{\mathrm{a}}$ & $\mathrm{PC}$ & \\
\hline NWA 869-J & L5 & Fund & 23 & ja & $1^{\mathrm{a}}$ & $\mathrm{PC}$ & \\
\hline NWA 869-K & L5 & Fund & 29 & ja & $1^{\mathrm{a}}$ & $\mathrm{PC}$ & \\
\hline SAU 001 & L4-5 & Fund & 1228 & $\mathrm{ja}$ & $2^{\mathrm{a}}$ & $\mathrm{PC}$ & \\
\hline SAU 001-B & L4-5 & Fund & 1181 & nein & - & $\mathrm{PC}$ & \\
\hline St. Michel & L6 & Fall & 329 & ja & $4^{\mathrm{a}, \mathrm{b}}$ & $\mathrm{ANU}$ & \\
\hline Zavid & L6 & Fall & 171 & ja & $4^{\mathrm{a}, \mathrm{b}}$ & ANU & \\
\hline Adrian & $\mathrm{H} 4$ & Fund & 612 & ja & $4^{\mathrm{a}, \mathrm{b}}$ & $\mathrm{ANU}$ & \\
\hline Dar al Gani 300 & H3-5 & Fund & 1000 & nein & $2^{\mathrm{b}}$ & LUH & \\
\hline Djoumine & $\mathrm{H} 5-6$ & Fund & $150-200$ & nein & $2^{\mathrm{b}}$ & LUH & \\
\hline El Hammami & H5 & Fund & 63 & ja & $2^{\mathrm{a}}$ & WWU & \\
\hline Forest City & H5 & Fall & 750 & ja & $4^{\mathrm{a}, \mathrm{b}}$ & $\mathrm{ANU}$ & \\
\hline Gao-Guenie & $\mathrm{H} 5$ & Fall & 915 & ja & $2^{\mathrm{a}}$ & $\mathrm{PC}$ & \\
\hline Gao-Guenie-A & H5 & Fall & $30-40$ & ja & $2^{\mathrm{a}}$ & $\mathrm{PC}$ & \\
\hline Gao-Guenie-B & H5 & Fall & $30-40$ & ja & $2^{\mathrm{a}}$ & $\mathrm{PC}$ & \\
\hline Gao-Guenie-C & H5 & Fall & $30-40$ & ja & $2^{\mathrm{a}}$ & $\mathrm{PC}$ & \\
\hline Gao-Guenie-D & H5 & Fall & $30-40$ & ja & $2^{\mathrm{a}}$ & $\mathrm{PC}$ & \\
\hline Gao-Guenie-E & H5 & Fall & $30-40$ & ja & $2^{\mathrm{a}}$ & $\mathrm{PC}$ & \\
\hline Gao-Guenie-F & H5 & Fall & $30-40$ & ja & $2^{\mathrm{a}}$ & $\mathrm{PC}$ & \\
\hline Hammond Downs & $\mathrm{H} 4$ & Fund & 248 & ja & $4^{\mathrm{a}, \mathrm{b}}$ & $\mathrm{ANU}$ & \\
\hline Ijopega & $\mathrm{H} 6$ & Fall & 383 & ja & $4^{\mathrm{a}, \mathrm{b}}$ & ANU & \\
\hline Jilin & $\mathrm{H} 5$ & Fall & 464 & nein & $2^{\mathrm{b}}$ & $\mathrm{SB}$ & \\
\hline Kunya-Urgench & $\mathrm{H} 5$ & Fall & 962 & ja & $2^{\mathrm{a}}$ & $\mathrm{PC}$ & \\
\hline Pultusk-A & $\mathrm{H} 5$ & Fall & 90 & ja & $4^{\mathrm{a}, \mathrm{b}}$ & $\mathrm{ANU}$ & \\
\hline Pultusk-B & $\mathrm{H} 5$ & Fall & 209 & ja & $2^{\mathrm{a}}$ & $\mathrm{ANU}$ & \\
\hline Richardton & $\mathrm{H} 5$ & Fall & 701 & ja & $4^{\mathrm{a}, \mathrm{b}}$ & $\mathrm{ANU}$ & \\
\hline Thuathe & $\mathrm{H} 4 / 5$ & Fall & 519 & $\mathrm{ja}$ & $6^{\mathrm{a}}$ & $\mathrm{PC}$ & \\
\hline Thuathe-B & $\mathrm{H} 4 / 5$ & Fall & 517 & nein & - & $\mathrm{PC}$ & \\
\hline
\end{tabular}


Tabelle 2.2 (Fortsetzung)

\begin{tabular}{lclccccc}
\hline Probe & Gruppe Fund/ & $\begin{array}{c}\text { Masse } \\
(\mathrm{mg})\end{array}$ & $\begin{array}{c}\text { oxi- } \\
\text { diert }\end{array}$ & $\begin{array}{c}\text { Kugeln } \\
\text { Kall }\end{array}$ & $\begin{array}{c}\text { Quelle } \\
\text { Katalog- } \\
\text { Numer }\end{array}$ \\
\hline NWA 753 & R3-6 & Fund & 206 & nein & $2^{\mathrm{a}}$ & WWU & \\
Atlanta & EL6 & Fund & $100-150$ & nein & $2^{\mathrm{b}}$ & LUH & \\
Eagle & EL6 & Fund & 50 & nein & $2^{\mathrm{b}}$ & LUH & \\
Hvittis & EL6 & Fall & 379 & ja & $2^{\mathrm{b}}$ & ANU & \\
Pillistfer & EL6 & Fall & 155 & ja & $4^{\mathrm{a}, \mathrm{b}}$ & ANU & \\
Abee & EH4 & Fall & 249 & ja & $2^{\mathrm{a}}$ & NMNH & USNM 2096 \\
Indarch & EH4 & Fall & 57 & ja & $2^{\mathrm{a}}$ & WWU & \\
Sahara 97072 & EH3 & Fund & 200 & nein & $1^{\mathrm{b}}$ & LUH & \\
St. Mark's & EH5 & Fall & 266 & ja & $2^{\mathrm{a}}$ & NMNH & USNM 486 \\
Bilanga & DIO & Fall & 346 & ja & $2^{\mathrm{a}}$ & PC & \\
Cachari & EUC & Fund & 86 & ja & $2^{\mathrm{a}}$ & PC & \\
Camel Donga & EUC & Fund & 50 & nein & $2^{\mathrm{b}}$ & LUH & \\
Dhofar 182 & EUC & Fund & 93 & nein & $2^{\mathrm{a}}$ & WWU & \\
Juvinas & EUC & Fall & 55 & nein & $2^{\mathrm{a}}$ & WWU & \\
Millbillillie-A & EUC & Fall & 100 & nein & $2^{\mathrm{b}}$ & LUH & \\
Millbillillie-B & EUC & Fall & 41 & nein & $2^{\mathrm{a}}$ & WWU & \\
NWA 3359 & EUC & Fund & 56 & nein & $2^{\mathrm{a}}$ & WWU & \\
Padvarninkai & EUC & Fall & 50 & nein & $2^{\mathrm{b}}$ & LUH & \\
NWA 1457 & WIN & Fund & 100 & nein & $2^{\mathrm{b}}$ & GAUG & \\
Mount Egerton & AUB & Fund & 100 & nein & $2^{\mathrm{b}}$ & GAUG & \\
NWA 4864 & Mars & Fund & 41 & nein & $2^{\mathrm{a}}$ & WWU & \\
SAU 051 & Mars & Fund & 28 & nein & $2^{\mathrm{a}}$ & WWU & \\
Dhofar 911 & Mond & Fund & 41 & nein & $2^{\mathrm{a}}$ & WWU & \\
Kalahari 008 & Mond & Fund & 31 & nein & $2^{\mathrm{a}}$ & WWU & \\
Kalahari 009 & Mond & Fund & 31 & nein & $2^{\mathrm{a}}$ & WWU & \\
\hline
\end{tabular}

a) eigene Präparation der Quenchkugeln; b) Präparierte Proben standen bereits zur Verfügung.

Probeninformationen: NWA: North West Africa; SAU: Sayh al Uhaymir; Bencubbin: Nur die Silikatphase wurde analysiert; Allende-S: Smithsonian Reference Powder (Jarosewich et al., 1987); Allende-M: Große Einschlüsse wurden entfernt (angegebene Probenmasse ist das Gesamtgewicht vor dem Entfernen der Einschlüsse), von K. Bermingham präpariert und zur Verfügung gestellt.

Quellen: ANU: Australian National University, Canberra; GAUG: Georg-AugustUniversität Göttingen; LUH: Leibniz Universität Hannover; MNHN: Muséum National d'Histoire Naturelle, Paris; NMNH: Smithsonian National Museum of Natural History; PC: Private Sammlung A. Gehler; RFWU: Rheinische Friedrich-Wilhelms-Universität Bonn; SB: Senckenbergmuseum, Frankfurt am Main; WWU: Westfälische WilhelmsUniversität Münster. 
Tabelle 2.3: Probenliste terrestrischer Gesteine.

\begin{tabular}{|c|c|c|c|c|}
\hline Probe & Gestein & Lokation & \# Kugeln & Quelle \\
\hline GZG.GSP.13450 & Lherzolith & Étang de Lers & $2^{\mathrm{a}}$ & SGAUG \\
\hline De-1 & Peridotit & Deudesfeld & $2^{\mathrm{a}}$ & GAUG \\
\hline De-2 & Peridotit & Deudesfeld & $2^{\mathrm{a}}$ & GAUG \\
\hline De-3 & Peridotit & Deudesfeld & $2^{\mathrm{a}}$ & GAUG \\
\hline $\mathrm{Zm}$ & Peridotit & Molines, France & $2^{\mathrm{a}}$ & GAUG \\
\hline SC-1 & Peridotit & San Carlos & $2^{\mathrm{a}}$ & GAUG \\
\hline SC-2 & Peridotit & San Carlos & $2^{\mathrm{a}}$ & GAUG \\
\hline Ва-1 & Peridotit & Balmuccia & $2^{\mathrm{a}}$ & GAUG \\
\hline Ba-2 & Peridotit & Balmuccia & $2^{\mathrm{a}}$ & GAUG \\
\hline $\mathrm{Za}-27$ & Peridotit & Barberton & $2^{\mathrm{a}}$ & UK \\
\hline $\mathrm{PCC}-1^{5}$ & Peridotit & Kalifornien & $2^{\mathrm{a}}$ & USGS \\
\hline $\mathrm{JP}-1^{5}$ & Peridotit & Japan & $2^{\mathrm{a}}$ & GSJ \\
\hline DTS- $1^{5}$ & Dunit & Washington & $2^{\mathrm{a}}$ & USGS \\
\hline NIM-D $^{5}$ & Dunit & Südafrika & $2^{\mathrm{a}}$ & MINTEK \\
\hline NIM-P ${ }^{5}$ & Pyroxenit & Südafrika & $2^{\mathrm{a}}$ & MINTEK \\
\hline UB-N ${ }^{5}$ & Serpentinit & Frankreich & $2^{\mathrm{a}}$ & CRPG \\
\hline El-1 & Serpentinit & Elba & $2^{\mathrm{a}}$ & GAUG \\
\hline 24803 & Komatiit & Zimbabwe & $2^{\mathrm{a}}$ & RUB \\
\hline 26575 & Komatiit & Komati River & $2^{\mathrm{a}}$ & RUB \\
\hline 26581 & Komatiit & Komati River & $2^{\mathrm{a}}$ & RUB \\
\hline$Z a-28 a^{2}$ & Komatiit & Barberton & $2^{\mathrm{a}}$ & UK \\
\hline$Z a-29 a^{2}$ & Komatiit & Barberton & $2^{\mathrm{a}}$ & UK \\
\hline MOL & Tektit & Europa & $2^{\mathrm{a}}$ & $\mathrm{PC}$ \\
\hline $\mathrm{KOH}$ & Tektit & Thailand & $2^{\mathrm{a}}$ & $\mathrm{PC}$ \\
\hline MUO & Tektit & Kambodscha & $2^{\mathrm{a}}$ & $\mathrm{PC}$ \\
\hline QUA & Tektit & China & $2^{\mathrm{a}}$ & $\mathrm{PC}$ \\
\hline El-2 & Radiolarit & Elba & $2^{\mathrm{a}}$ & GAUG \\
\hline $\mathrm{ES}^{1}$ & Tonschiefer & Europa & $2^{\mathrm{a}}$ & GAUG \\
\hline Pan-02-047 3 & Granit & Chagres, Panama & $2^{\mathrm{a}}$ & GAUG \\
\hline GZG.GSP.12569 & Obsidian & Island & - & SGAUG \\
\hline GSP-2 ${ }^{5}$ & Granodiorit & Colorado & $2^{\mathrm{b}}$ & USGS \\
\hline RGM- $2^{5}$ & Rhyolith & Kalifornien & $2^{\mathrm{b}}$ & USGS \\
\hline El-3 & Gabbro & Elba & $2^{\mathrm{a}}$ & GAUG \\
\hline LP1 & OIB & La Palma & $2^{\mathrm{a}}$ & UK \\
\hline LP4 & OIB & La Palma & $2^{\mathrm{a}}$ & UK \\
\hline $\mathrm{SC}-3$ & Basalt & San Carlos & $2^{\mathrm{a}}$ & GAUG \\
\hline 138 DS- $1^{4}$ & MORB & Süd-Mittelatl. R. & - & UK \\
\hline $155 \mathrm{DS}-1^{4}$ & MORB & Süd-Mittelatl. R. & - & UK \\
\hline $199 \mathrm{DS}-2^{4}$ & MORB & Süd-Mittelatl. R. & - & UK \\
\hline
\end{tabular}


Tabelle 2.3 (Fortsetzung)

\begin{tabular}{lllcc}
\hline Probe & Gestein & Lokation & \# Kugeln & Quelle \\
\hline 157 DS-3 & MORB & Süd-Mittelatl. R. & - & UK \\
137 DS-4 & MORB & Süd-Mittelatl. R. & - & UK \\
BIR-1 $^{5}$ & Basalt & Island & $2^{\mathrm{b}}$ & USGS \\
BHVO-2 $^{5}$ & Basalt & Hawaii & $2^{\mathrm{b}}$ & USGS \\
${\mathrm{W}-2 \mathrm{a}^{5}}^{5}$ & Diabas & Virginia & $2^{\mathrm{b}}$ & USGS \\
${\mathrm{AGV}-2^{5}}_{\text {BCR-2G }}^{5}$ & Andesit & Oregon & $2^{\mathrm{b}}$ & USGS \\
\hline
\end{tabular}

a) eigene Präparation der Quenchkugeln; b) Präparierte Proben standen bereits zur Verfügung.

Natürliche Gläser und der Standard BCR-2G wurden in-situ analysiert.

Probeninformationen: 1) ES: European shale composite, dieselbe Probe wie in Minami (1935); 2) dieselbe Probe wie in Münker et al. (2003); 3) dieselbe Probe wie in Wegner et al. (2011); 4) dieselbe Probe wie Paulick et al. (2010); 5) Geologischer Standard. Quellen: CRPG: Centre de Recherches Pétrographiques et Géochimiques, France; GAUG: Georg-August-Universität Göttingen; GSJ: Geologic Survey of Japan; MINTEK: Council for Mineral Technology, South Africa; PC: Private Sammlung A. Gehler; RFWU: Rheinische Friedrich-Wilhelms-Universität Bonn; RUB: Mineralogische Sammlung, Institut für Geologie, Mineralogie und Geophysik, Ruhr-Universität Bochum; SGAUG: Sammlungen des Geowissenschaftlichen Zentrums der GeorgAugust-Universität Göttingen; UK: Universität zu Köln; USGS: U.S. Geological Survey.

\subsection{Probenpräparation mittels Laserschmelzen unter aerodynamischer Levitation}

Aus den Gesamtgesteinsproben wurden durch den Einsatz einer aerodynamischen Levitationsanlage kleine Quenchkugeln nach der Methode von Pack et al. (2010) hergestellt. Dazu wurden diese zunächst je nach Probenmenge mit einem Achatmörser oder einer Schwingmühle (Retsch MM 301) zu sehr feinem Pulver gemörsert und homogenisiert. Einige metallhaltige Chondritproben (siehe Tabelle 2.2) wurden 24 Stunden in einem Muffelofen bei ca. $900^{\circ} \mathrm{C}$ an Luft oxidiert und anschließend erneut gemörsert, um ein feineres und homogeneres Pulver zu erhalten.

Die verwendete Levitationsanlage wird mit einem SYNRAD $50 \mathrm{~W} \mathrm{CO}_{2}$-Laser betrieben. Für die Präparation der Quenchkugeln ist nur eine geringe Probenmenge von 10 bis $20 \mathrm{mg}$ notwendig. Das Probenpulver wurde zunächst in einem Graphittiegel durch kurze Bestrahlung mit dem Laser zu einer Kugel geschmolzen. Um 
Kontaminationen zu vermeiden, wurde der Graphittiegel vor jedem Aufschmelzen gereinigt. Anschließend wurde die Probe auf eine Levitationsdüse aus Aluminium gesetzt und ein Gasstrom so eingestellt, dass sie frei im Gasstrom schwebte. Dort wurde sie ein oder zwei weitere Male aufgeschmolzen, was durch die gleichzeitige Bestrahlung mit zwei fokussierten Laserstrahlen von oben und unten erreicht wurde. Als Levitationsgase dienten Stickstoff (neutral), Luft (oxidierend) und Argon mit $2 \%$ Wasserstoffbeimischung (reduzierend). Die Aufschmelzdauer je Probe betrug nur wenige Sekunden. Anschließend wurden die Proben durch Ausschalten des Lasers abgeschreckt. Sie erstarrten je nach Zusammensetzung in eine glasige oder mikrokristalline Struktur. Von den meisten Proben wurden mindestens zwei Quenchkugeln präpariert. Diese wurden anschließend in Epoxidharz gegossen, angeschliffen und poliert. Etwa $30 \%$ der verwendeten Proben standen bereits im präparierten Zustand für die Analysen zur Verfügung (siehe Tabellen 2.2 und 2.3). Eine Übersicht der verwendeten Schliffpräparate befindet sich im Anhang (Abbildungen A.1 und A.2).

\subsection{Analytische Methoden}

\subsubsection{Elektronenstrahlmikrosonde}

An der Elektronenstrahlmikrosonde (EMS) des Typs JEOL JXA 8900 RL der Universität Göttingen wurden Rückstreuelektronenbilder (BSE; backscattered electrons) erstellt und quantitative wellenlängendispersive Röntgenmikroanalysen (WDX; wavelength dispersive X-ray spectroscopy) an den Quenchkugeln der Gesamtgesteinsproben durchgeführt. Zur Vermeidung elektrostatischer Aufladungseffekte wurden die Proben mit einer Kohlenstoffbedampfung versehen.

Die BSE-Bilder wurden zur Charakterisierung des Kristallgefüges der Quenchkugeln erstellt. Die verwendete Beschleunigungsspannung betrug dabei $15 \mathrm{kV}$ und die Strahlstromstärke etwa $15 \mathrm{nA}$.

Mittels WDX wurden die Gehalte der folgenden Elemente gemessen: Si, Na, K, Ca, Fe, Al, Mg, P, Ti, Mn, S, Cr und Ni. Dafür wurde ein defokussierter Elektronenstrahl von $30 \mu \mathrm{m}$ Durchmesser benutzt. Die Beschleunigungsspannung während der Messungen betrug entweder 15 oder $20 \mathrm{kV}$, die Strahlstromstärke wieder etwa $15 \mathrm{nA}$. Es wurden Messzeiten von 15 Sekunden für Si, Na, K, Ca, Fe, $\mathrm{Al}, \mathrm{Mg}$ und Ni sowie 30 Sekunden für die anderen Elemente gewählt. Der Untergrund wurde für jeweils 5 bzw. 15 Sekunden aufgezeichnet. Die Kalibration erfolgte durch die Standards Albit, Sanidin, Wollastonit, $\mathrm{TiO}_{2}, \mathrm{Cr}_{2} \mathrm{O}_{3}$, Rhodonit, Hämatit, NiO, Olivin, Anorthit, ZnS und Apatit. Es wurde eine Matrixkorrektur nach der $\Phi(\rho, z)$ Methode durchgeführt. Auf jeder Quenchkugel wurde an 5 bis 10 zufällig verteilten Punkten gemessen, die Elementkonzentrationen wurden anschließend gemittelt. 
Die Ergebnisse über die chemischen Zusammensetzungen einiger Kugeln wurden von A. Pack und A. Patzer zur Verfügung gestellt (siehe Tabelle A.6). Details zu den Messbedingungen sind in Patzer et al. (2010) zu finden.

\subsubsection{Röntgenfluoreszenzanalyse}

Die Röntgenfloureszenzanalysen (RFA) wurden an einem PANanlytical Axios Röntgenfloureszenzspektrometer der Universität Göttingen von G. Hartmann durchgeführt. Das Gerät ist mit einer Rhodium-Röntgenröhre des Typs SST-mAX als Anregungsquelle sowie einem wellenlängendispersiven Detektorsystem ausgestattet. Es wurden insgesamt 19 Meteoritenproben auf ihre Hauptelemente und einige Spurenelemente analysiert. Die Kalibrierung des Gerätes erfolgt über geeignete geologische Standards. Es ist mit der Auswertungssoftware Super Q4 ausgestattet.

Die Proben wurden eigenständig als Schmelztabletten präpariert. Dabei wurde im Wesentlichen die Methode von Wolf und Palme (2001) angewandt: Es wurde je $120 \mathrm{mg}$ Probenpulver mit $3600 \mathrm{mg}$ Schmelzmittel (66\% di-Lithiumtetraborat - 34\% Lithiummetaborat) vermischt und Ammoniumnitrat als Oxidationsmittel hinzugefügt. Das gut durchmischte Pulver wurde anschließend für ungefähr 9 Minuten bei etwa $1250^{\circ} \mathrm{C}$ in einem Platintiegel geschmolzen, danach in eine Platin-Abgießschale gegossen und innerhalb von 4 Minuten durch ein Gebläse auf Raumtemperatur abgekühlt.

Die Präzision der Messungen wurde an einem Basalt durch die Präparati-

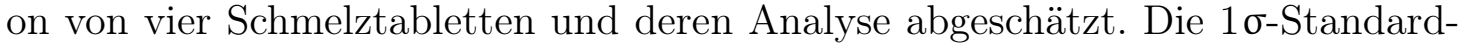
abweichungen der vier Messungen betragen für die Hauptelemente weniger als $2 \%$, lediglich für Na ergab sich eine größere Abweichung von 3,5\%. Die Standardabweichungen der meisten Spurenelemente liegen zwischen 1 und $10 \%$ (siehe Tabelle A.2). Höhere Abweichungen zeigten Nickel, Kupfer und Chlor mit 12, 25 und $72 \%$.

\subsubsection{Massenspektrometrie mittels MC-ICPMS}

An 22 Proben wurden die Seltenen Erden Sm, Nd und Lu sowie die high field strength elements (HFSE) Zr, Nb, Hf, Ta und W mittels Isotopenverdünnungs-MCICPMS (multicollector inductively coupled plasma mass spectrometry) analysiert. Die Messungen wurden von C. Münker an einem Thermo Scientific NeptuneMassenspektrometer an der Universität Bonn durchgeführt. Die Probenvorbereitung fand unter Anleitung von C. Münker statt und folgt den in Münker et al. (2001), Weyer et al. (2002), König et al. (2008) und Münker (2010) beschriebenen Vorgehensweisen.

Es wurde jeweils etwa $100 \mathrm{mg}$ Probenpulver eingewogen und mit kombinierten ${ }^{149} \mathrm{Sm}-{ }^{150} \mathrm{Nd}-$ und ${ }^{183} \mathrm{~W}-{ }^{180} \mathrm{Ta}-{ }^{180} \mathrm{Hf}-{ }^{176} \mathrm{Lu}-{ }^{94} \mathrm{Zr}-$ Spikes versehen, die mit $>99,9 \%$ reinen Metallstandards des Ames Laboratory kalibriert wurden. Anschließend 
wurden die Proben mit einer Mischung aus $\mathrm{HF}-\mathrm{HNO}_{3}$ aufgeschlossen. Etwa $15 \%$ des Aufschlusses wurde für die Separation von W unter Verwendung von BIORAD AG1-X8 Anionenaustauscherharzen benutzt (König et al., 2008). Zur Abtrennung der anderen Elemente wurde der verbleibende Anteil des Probenaufschlusses auf EICHROM Ln-Spec-Säulen geladen. Zuerst wurden die leichten Seltenen Erden zusammen mit der Matrix abgetrennt; diese Fraktion wurde später für die Separation von Sm und Nd mittels Kationenaustauschchromatographie und Eichrom Ln-SpecSäulen herangezogen (Pin und Zalduegui, 1997). Als nächstes wurde eine Lu-reiche HREE-Fraktion und anschließend eine HFSE-Fraktion gewonnen. Ein Teil dieser HFSE-Fraktion wurde für die Messungen von $\mathrm{Zr} / \mathrm{Nb}$ benutzt. Der andere Teil wurde unter Einsatz von BIORAD AG1-X8 Anionenaustauscherharzen für die Abtrennung von Ta und Hf verwendet; die Hf-Fraktion wurde anschließend unter Einsatz von Eichrom Ln-spec-Harzen weiter purifiziert (Münker et al., 2001).

Die Nb-Konzentrationen wurden über die $\mathrm{Zr} / \mathrm{Nb}$-Verhältnisse und die mittels Isotopenverdünnung gemessenen Zr-Konzentrationen berechnet (Weyer et al., 2002). Die externe Präzision der Messungen ist typischerweise besser als $\pm 2 \%$ für $\mathrm{Nb} / \mathrm{Ta}$, $\pm 0,3 \%$ für $\mathrm{Zr} / \mathrm{Hf}, \pm 0,1 \%$ für $\mathrm{Sm} / \mathrm{Nd}$ und $\pm 0,25 \%$ für $\mathrm{Hf} / \mathrm{W}(1 \sigma)$.

\subsubsection{Massenspektrometrie mittels LA-ICPMS}

Die Messungen mittels LA-ICPMS (laser ablation inductively coupled plasma mass spectrometry) wurden an der Australian National University (ANU) in Canberra an einem AGILENT 7500S Quadrupol-Massenspektrometer durchgeführt, das mit einem $193 \mathrm{~nm}$ Excimerlaser kombiniert ist. Das Massenspektrometer ist so kalibriert, dass die Oxidbildungsrate, gemessen als $\mathrm{ThO}^{+} / \mathrm{Th}^{+}$im synthetischen Glasstandard NIST SRM 612, kleiner als 0,5\% ist. Die Messungen der Gesamtgesteinsproben fanden an den gequenchten Kugeln bzw. für einige natürliche Gläser an Gesteinsbruchstücken statt. Sie wurden innerhalb von drei Messaufenthalten durchgeführt. Die jeweils analysierten Elemente sind in Tabelle 2.4 angegeben. Einige Messungen wurden von A. Pack (Messaufenthalt 1) bzw. A. Patzer und C. Allen (Messaufenthalt 2) durchgeführt. Alle Messungen erfolgten im Linienscan-Modus. Der Strahldurchmesser betrug dabei zwischen 81 und $112 \mu \mathrm{m}$, die Laserenergie betrug etwa $50 \mathrm{~mJ}$, die Wiederholfrequenz lag zwischen 5 und $10 \mathrm{~Hz}$ und die Ablationszeit variierte zwischen 100 und 200 Sekunden. Der Untergrund wurde jeweils 20 bis 30 Sekunden gemessen.

Die in-situ-Messungen an den Chondritkomponenten von Leoville wurden von A. Patzer vorbereitet und durchgeführt. Es wurden dabei die für Messaufenthalt 2 angegebenen Elemente analysiert. Der Laserstrahldurchmesser betrug $81 \mu \mathrm{m}$, die Frequenz lag bei $10 \mathrm{~Hz}$ und die Energiedichte variierte zwischen 6,5 und $7 \mathrm{~J} / \mathrm{cm}^{2}$. Die Messungen wurden ebenfalls im Linienscan-Modus mit einer Scangeschwindigkeit von $5 \mu \mathrm{m} / \mathrm{s}$ durchgeführt. Die Messdauer betrug je nach Größe des zu untersu- 
chenden Objektes zwischen 40 und 160 Sekunden. Vor jeder Messung wurde der Untergrund für 20 Sekunden aufgezeichnet.

\section{Reduktion der LA-ICPMS Daten}

Zur Auswertung der LA-ICPMS Daten wurden mit der Software Mathematica Skripte erstellt. Die Berechnung der Elementkonzentrationen in den Proben erfolgte wie im Folgenden beschrieben.

Jede Messung bestand aus mehreren hundert Zyklen $(n=200-400)$, in denen die Counts der Elemente $\left(\mathrm{E}^{+}\right)$bestimmt wurden. Nach einer Untergrundkorrektur wurden für jeden Messzyklus die gemessenen Counts der Elemente durch die

\begin{tabular}{lccc}
\hline Isotop & S1 & S2 & S3 \\
\hline${ }^{89} \mathrm{Y}$ & + & + & + \\
${ }^{90} \mathrm{Zr}$ & & + & + \\
${ }^{91} \mathrm{Zr}$ & & & + \\
${ }^{93} \mathrm{Nb}$ & & + & + \\
${ }^{139} \mathrm{La}$ & + & + & + \\
${ }^{140} \mathrm{Ce}$ & + & + & + \\
${ }^{141} \mathrm{Pr}$ & + & + & + \\
${ }^{146} \mathrm{Nd}$ & + & + & + \\
${ }^{147} \mathrm{Sm}$ & + & + & + \\
${ }^{153} \mathrm{Eu}$ & + & + & + \\
${ }^{157} \mathrm{Gd}$ & & + & + \\
${ }^{158} \mathrm{Gd}$ & + & & + \\
${ }^{159} \mathrm{~Tb}$ & + & + & + \\
${ }^{162} \mathrm{Dy}$ & + & & \\
${ }^{163} \mathrm{Dy}$ & + & + & + \\
${ }^{165} \mathrm{Ho}$ & + & + & + \\
${ }^{166} \mathrm{Er}$ & + & + & + \\
${ }^{169} \mathrm{Tm}$ & + & + & + \\
${ }^{172} \mathrm{Yb}$ & & + & + \\
${ }^{174} \mathrm{Yb}$ & + & & + \\
${ }^{175} \mathrm{Lu}$ & + & + & + \\
${ }^{178} \mathrm{Hf}$ & & + & + \\
${ }^{180} \mathrm{Hf}$ & & & + \\
${ }^{181} \mathrm{Ta}$ & & + & + \\
\hline
\end{tabular}

Tabelle 2.4: Mittels LA-ICPMS analysierte Isotope in den Gesamtgesteinsproben während der drei Messaufenthalte S1, S2 und S3. 
Intensitäten von ${ }^{43} \mathrm{Ca}$ geteilt. Anschließend wurde das arithmetische Mittel dieser Verhältnisse gebildet:

$$
\overline{\left(\frac{\mathrm{E}^{+}}{{ }^{43} \mathrm{Ca}^{+}}\right)}=\frac{1}{n} \sum_{i=1}^{n}\left(\frac{\mathrm{E}^{+}}{{ }^{43} \mathrm{Ca}^{+}}\right)_{i} .
$$

Die $\mathrm{E}^{+} /{ }^{43} \mathrm{Ca}^{+}$-Verhältnisse der einzelnen Messzyklen $i$ zeigen Normalverteilungen um die Mittelwerte. Die Standardfehler der Mittelwerte berechnen sich als:

$$
\sigma \overline{\left(\frac{\mathrm{E}^{+}}{{ }^{43} \mathrm{Ca}^{+}}\right)}=\frac{1}{n} \sqrt{\sum_{i=1}^{n}\left(\left(\frac{\mathrm{E}^{+}}{{ }^{43} \mathrm{Ca}^{+}}\right)-\overline{\left.\left(\frac{\mathrm{E}^{+}}{{ }^{43} \mathrm{Ca}^{+}}\right)\right)^{2}}\right.} .
$$

Alle $\mathrm{E}^{+} /{ }^{43} \mathrm{Ca}^{+}-$Verhältnisse, die um mehr als drei Standardabweichungen vom Mittelwert abwichen, wurden entfernt und in der weiteren Auswertung nicht berücksichtigt. Anschließend wurden die Elementkonzentrationen [E] der Proben berechnet:

$$
[\mathrm{E}]=\overline{\left(\frac{\mathrm{E}^{+}}{{ }^{43 \mathrm{Ca}^{+}}}\right)} \times \frac{1}{\mathrm{RSF}_{t}} \times[\mathrm{Ca}] .
$$

Dabei dienten die mittels EMS bestimmten Ca-Konzentrationen [Ca] der Quenchkugeln (Tabelle A.6) bzw. der Komponenten in Leoville (Tabelle A.1) als interner Standard. Als externer Standard wurde das synthetische Glas NIST SRM 612 verwendet, die Elementkonzentrationen dazu wurden aus der Studie von Jochum et al. (2011) entnommen.

Der Standard NIST SRM 612 wurde regelmäßig gemessen und relative Sensitivitätsfaktoren (RSF) für die $\mathrm{E}^{+} /{ }^{43} \mathrm{Ca}^{+}$-Verhältnisse berechnet. Da diese RSF-Werte einer geringen zeitlichen Gerätedrift unterlagen, wurden sie als zeitabhängige Funktion behandelt; zu einer bestimmten Zeit berechnen sie sich als:

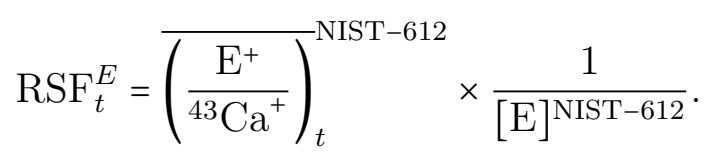

Zur Abschätzung der RSF-Werte zu einem beliebigen Zeitpunkt wurden die gemessenen RSF-Werte als Funktion der Zeit angefittet. Für die Messaufenthalte 1 und 2, in denen regelmäßige Messungen des Standards zwischen den Probenmessungen stattfanden, wurde ein Polynom dritter Ordnung gewählt. Beim Messaufenthalt 3 fanden die Messungen dagegen in kurzen Serien von typischerweise 12 Proben statt, wobei der Standard vor und nach der jeweiligen Serie gemessen wurde. Die zeitabhängigen RSF-Werte wurden daher in diesem Messaufenthalt für jede Serie durch eine lineare Interpolation berechnet. Diese zeitabhängigen RSF-Werte wurden 
anschließend für die Berechnung der Elementkonzentrationen in den Proben gemäß Gleichung 2.3 herangezogen.

Die Fehlerberechnung der ermittelten Elementkonzentrationen in den präparierten Quenchkugeln erfolgte anhand des Gauß'schen Fehlerfortpflanzungsgesetzes, wobei nur die Unsicherheiten $\sigma\left(\overline{E^{+} /{ }^{43} \mathrm{Ca}^{+}}\right)$und $\sigma(\mathrm{RSF})$ Berücksichtigung fanden. Die Messunsicherheiten bezüglich der mittels EMS bestimmten Ca-Konzentrationen wurden dagegen vernachlässigt, da absolute Konzentrationen in dieser Arbeit von geringerer Relevanz sind und aufgrund des Verlustes von volatilen Elementen während der Probenpräparation ohnehin nicht exakt bestimmt werden können. Stattdessen werden hauptsächlich Fraktionierungen der Seltenen Erden untereinander, sowie einiger anderer Elementverhältnisse diskutiert. Unsicherheiten der Ca-Konzentrationen wirken sich auf alle berechneten Elementkonzentrationen gleichermaßen aus, so dass sie sich bei dem Vergleich der Seltenen Erden untereinander oder bei der Betrachtung anderer Elementverhältnisse aufheben.

Da im Folgenden alle Verhältnisse der Seltenen Erden in Bezug auf den ebenfalls in dieser Arbeit gemessen CI-Chondrit Orgueil dargestellt werden, kürzen sich Unsicherheiten aufgrund der externen Kalibration mit dem Standard NIST-612 (Jochum et al., 2011), die in die Berechnung des RSF eingehen, ebenso heraus und wurden daher vernachlässigt. Der Fehler des RSF wurde für die Messaufenthalte 1 und 2 aus der Abweichung der Messwerte von der Fitkurve abgeschätzt. Beim Messaufenthalt 3 wurde als relativer Fehler des RSF für alle Elemente ein Wert von $0,25 \%$ angenommen, der aus den Standardfehlern der $\mathrm{E}^{+} /{ }^{43} \mathrm{Ca}^{+}-$Verhältnisse abgeschätzt wurde.

In einigen Messungen wurden entweder für Dysprosium oder für Gadolinium und Ytterbium je zwei verschiedene Isotope analysiert. Im Falle von Dy und $\mathrm{Yb}$ wurden Mittelwerte der beiden untersuchten Isotope verwendet. Beim Gd wurden dagegen für den ersten Messaufenthalt Daten des Isotops ${ }^{158} \mathrm{Gd}$ benutzt und für die Messaufenthalte 2 und 3 Daten des Isotops ${ }^{157} \mathrm{Gd}$ (siehe nächster Abschnitt). Auch für die Elemente Zr und Hf wurden in einigen Messungen zwei Isotope analysiert; für die weitere Auswertung wurden jedoch ausschließlich Daten der Isotope ${ }^{90} \mathrm{Zr}$ und ${ }^{178} \mathrm{Hf}$ verwendet.

Die aus der Zählstatistik ermittelte Präzision der Elementkonzentrationen einer

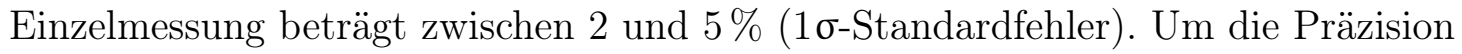
der Ergebnisse weiter zu erhöhen, wurden die Gesamtgesteinsproben jeweils mehrfach gemessen. Für die Konzentrationen der Seltenen Erden wurden Mittelwerte $\overline{[R E E]}$ berechnet. Da die ermittelten absoluten Konzentrationen der Seltenen Erden aufgrund der Präparationsmethode in den verschiedenen Quenchkugeln variieren, die Verhältnisse der Seltenen Erden untereinander aber stabil sind, wurden zunächst die REE-Konzentrationen aller Einzelmessungen an derselben Probe durch einen Faktor k auf ihren gemeinsamen Mittelwert normiert: 


$$
k \times\left(\sum[\mathrm{REE}]\right)_{\text {Einzelmessung }}=\left(\sum[\mathrm{REE}]\right)_{\text {Probenmittelwert }} .
$$

Anschließend wurde der Mittelwert dieser normierten REE-Konzentrationen $\left(\mathrm{REE}^{\mathrm{n}}\right)$ bestimmt und der Fehler folgendermaßen berechnet ( $\mathrm{N}$ ist die Anzahl der Messungen je Probe):

$$
\Delta \overline{\left[\mathrm{REE}^{\mathrm{n}}\right]}=\frac{1}{N} \sqrt{\sum_{i} \Delta\left[\mathrm{REE}^{\mathrm{n}}\right]_{i}^{2}}
$$

Für Elementverhältnisse wie $\mathrm{Y} / \mathrm{Ho}, \mathrm{Zr} / \mathrm{Hf}$ und Nb/Ta wurden ebenfalls Mittelwerte der Einzelmessungen jeder Probe gebildet und die Fehler entsprechend Gleichung 2.6 berechnet.

An den Einschlüssen von Leoville wurden je nach Größe ein bis fünf Linienscanmessungen mittels LA-ICPMS durchgeführt, die anschließend gemittelt wurden. Die Messungenauigkeit wurde anhand der Reproduzierbarkeit der Messungen abgeschätzt. Dazu wurde die relative Abweichung jeder Messung eines Objektes von dem gemeinsamen Mittelwert dieses Objektes berechnet. Anschließend wurde für alle Objekte gemeinsam die $1 \sigma$-Standardabweichung der einzelnen Abweichungen ermittelt und als relativer Fehler angenommen. Für die Seltenen Erden bezieht sich dieser Fehler nicht auf absolute Konzentrationen, sondern auf inter-REE Verhältnisse.

Korrektur der Zr/Hf- und Nb/Ta-Verhältnisse: Sowohl die Zr/Hf- als auch die $\mathrm{Nb} / \mathrm{Ta}$-Verhältnisse der LA-ICPMS-Messungen weichen von den mittels MCICPMS gemessenen Verhältnissen systematisch zu höheren Werten ab. Dieser Unterschied kann durch die externe Kalibration der LA-ICPMS-Daten mit dem Standard NIST SRM 612 begründet sein. Es wurde daher eine nachträgliche Korrektur der mit LA-ICPMS gemessenen $\mathrm{Zr} / \mathrm{Hf}$ - und Nb/Ta-Verhältnisse anhand des Basaltstandards BHVO-1 bzw. BHVO-2 vorgenommen. Dafür wurden durch MC-ICPMS gemessene Verhältnisse des BHVO-1 von Münker et al. (2003) herangezogen: Das Zr/Hf-Verhältnis beträgt 36,93 und das Nb/Ta-Verhältnis 15,4. Die am BHVO-2 durch LA-ICPMS ermittelten Werte betragen 37,7 und 16,4. Die Zr/Hf-Verhältnisse der LA-ICPMS-Daten wurden daher mit einem Faktor von 0,98 und die $\mathrm{Nb} / \mathrm{Ta}-$ Verhältnisse mit einem Faktor von 0,94 korrigiert.

\section{Interferenzen und Matrixeffekte der LA-ICPMS-Daten}

Das Massenspektrometer war so kalibriert, dass die Oxidbildungsrate von Thorium (gemessen als $\mathrm{ThO}^{+} / \mathrm{Th}^{+}$) weniger als $0,5 \%$ betrug. Es kann davon ausgegangen werden, dass die Oxidbildungsrate anderer Elemente - besonders der Seltenen Erden - noch geringer war, so dass Oxidinterferenzen in den Messungen vernachlässigbar klein sein sollten. Um zu überprüfen, ob dennoch polyatomare Interferenzen 

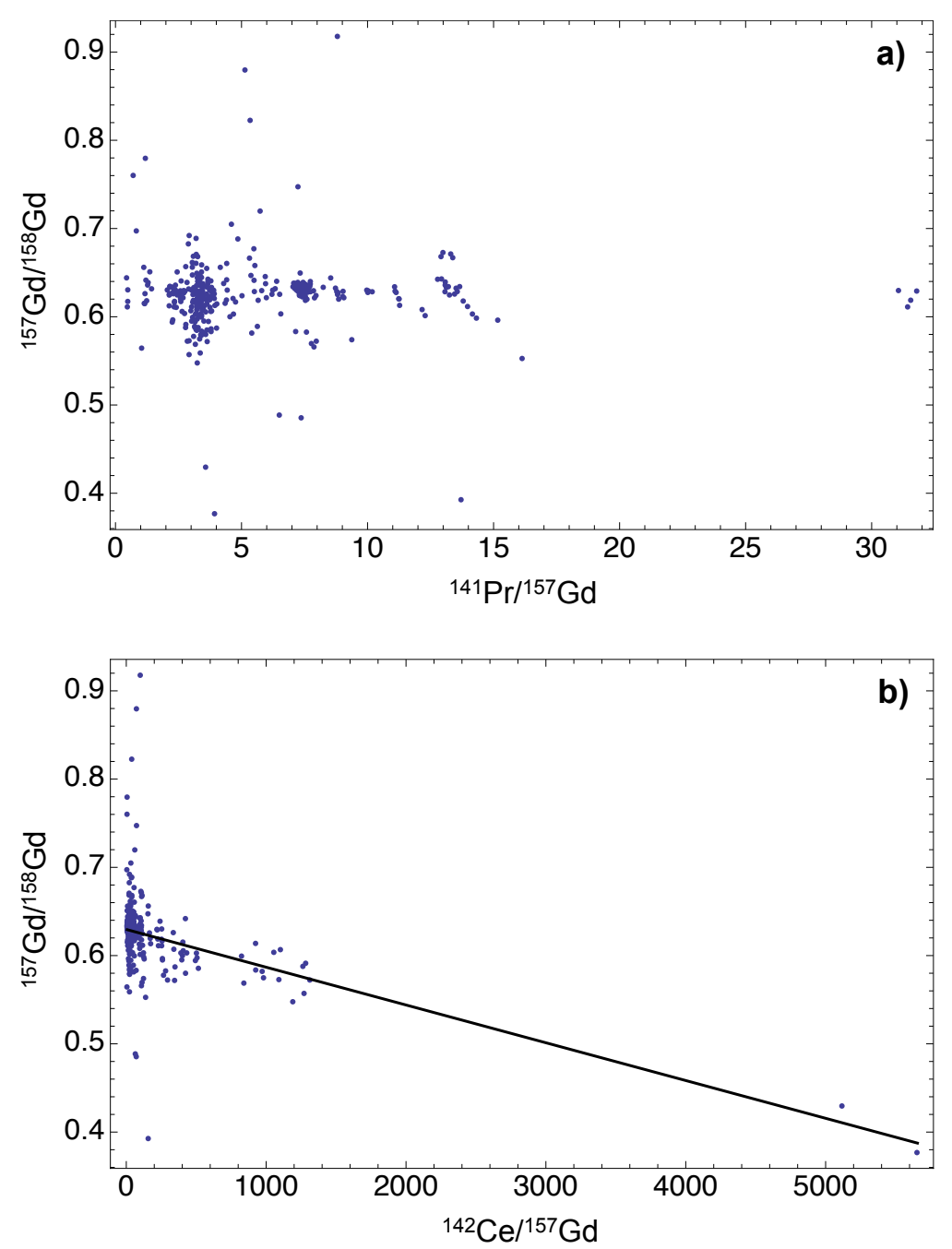

Abbildung 2.2: ${ }^{157} \mathrm{Gd} /{ }^{158} \mathrm{Gd}$ vs. ${ }^{141} \mathrm{Pr} /{ }^{157} \mathrm{Gd}$ und ${ }^{157} \mathrm{Gd} /{ }^{158} \mathrm{Gd}$ vs. ${ }^{142} \mathrm{Ce} /{ }^{157} \mathrm{Gd}$ : ${ }^{141} \mathrm{Pr}^{16} \mathrm{O}$ verursacht keine Interferenz auf ${ }^{157} \mathrm{Gd}$. Sehr hohe Gehalte an Ce verursachen dagegen eine Interferenz auf ${ }^{158} \mathrm{Gd}$.

vorliegen, wurden für drei Seltene Erden je zwei Isotope gemessen: ${ }^{157} \mathrm{Gd}-{ }^{158} \mathrm{Gd}$, ${ }^{162} \mathrm{Dy}-{ }^{163} \mathrm{Dy}$ und ${ }^{172} \mathrm{Yb}-{ }^{174} \mathrm{Yb}$.

Die mittleren gemessenen ${ }^{157} \mathrm{Gd} /{ }^{158} \mathrm{Gd}$-Verhältnisse der Standardmaterialien NIST SRM $612(0,634)$ und BCR-2G $(0,631)$ stimmen innerhalb von $1 \%$ mit dem natürlichen ${ }^{157} \mathrm{Gd} /{ }^{158} \mathrm{Gd}$-Verhältnis von 0,630 überein. Polyatomare Verbindungen, die Interferenzen auf einem der Gd-Isotope erzeugen können, sind beispielsweise ${ }^{141} \operatorname{Pr}^{16} \mathrm{O}$ auf ${ }^{157} \mathrm{Gd}$ bzw. ${ }^{142} \mathrm{Ce}^{16} \mathrm{O}$ auf ${ }^{158} \mathrm{Gd}$. Da keine Korrelation zwischen ${ }^{157} \mathrm{Gd} /{ }^{158} \mathrm{Gd}$ und ${ }^{141} \mathrm{Pr} /{ }^{157} \mathrm{Gd}$ in den gemessenen Proben besteht (Abbildung 2.2), 
wurde ausgeschlossen, dass ${ }^{141} \operatorname{Pr}^{16} \mathrm{O}$ Interferenzen verursacht. Dagegen wurde für einige Proben, die extrem hohe Gehalte an Ce aufweisen, eine geringe Interferenz von ${ }^{142} \mathrm{Ce}^{16} \mathrm{O}$ auf ${ }^{158} \mathrm{Gd}$ festgestellt. Deshalb wurden für die in den Messaufenthalten 2 und 3 gewonnenen Daten ausschließlich die Ergebnisse des Isotops ${ }^{157} \mathrm{Gd}$ benutzt. Während des Messaufenthaltes 1 wurde ausschließlich das Isotop ${ }^{158} \mathrm{Gd}$ gemessen. Alle in dieser Session untersuchten Proben haben jedoch so niedrige $\mathrm{Ce} / \mathrm{Gd}-$ Verhältnisse, dass Interferenzen, die durch ${ }^{142} \mathrm{Ce}^{16} \mathrm{O}$ verursacht würden, geringer als 0,3\% wären und somit innerhalb des Messfehlers lägen.

Die durchschnittlichen ${ }^{162}$ Dy/163 Dy-Verhältnisse der Standards NIST SRM 612 $(1,034)$ und BCR-2G (1,034) sowie der chondritischen Proben $(1,033)$ stimmen innerhalb von $1 \%$ mit dem natürlichen Isotopenverhältnis von 1,024 überein. Eine mögliche Interferenz von ${ }^{146} \mathrm{Nd}^{16} \mathrm{O}$ auf ${ }^{162} \mathrm{Dy}$ konnte nicht identifiziert werden.

Die in den Standards NIST SRM 612 und BCR-2G gemessenen durchschnittlichen ${ }^{172} \mathrm{Yb} /{ }^{174} \mathrm{Yb}-$ Verhältnisse sind ungefähr $5 \%$ niedriger als das natürliche Isotopenverhältnis von 0,689. Es konnte jedoch keine polyatomare Verbindung identifiziert werden, die derartige Interferenzen verursacht. Die ${ }^{172} \mathrm{Yb} /{ }^{174} \mathrm{Yb}$-Verhältnisse der untersuchten chondritischen und terrestrischen Proben stimmen im Mittel innerhalb von 1-2\% mit den gemessenen Standards überein. Mögliche Unsicherheiten der gemessenen Yb-Gehalte beeinflussen nicht die in Abschnitt 4.4 diskutierten Tm-Anomalien (relative Orgueil-normierte Tm-Gehalte gegenüber den anderen schweren Seltenen Erden, siehe Abschnitt 4.4.1), da die Yb-Konzentrationen nicht für die Berechnung herangezogen wurden.

Für die in Abschnitt 4.4 diskutierten Daten ist es außerdem wichtig, Interferenzen auf dem monoisotopischen Tm selbst auszuschließen. Die wahrscheinlichste Interferenz auf ${ }^{169} \mathrm{Tm}$ ist ${ }^{153} \mathrm{Eu}^{16} \mathrm{O}$. Bestünde eine solche Interferenz, würden Proben mit hohen Eu/Tm-Verhältnissen systematisch zu hohe gemessene Tm-Gehalte aufweisen, die in überschätzten Tm-Anomalien (relative Tm/HREE-Verhältnisse) resultierten. Es besteht jedoch keine Korrelation zwischen den $\mathrm{Eu} / \mathrm{Tm}-$ Verhältnissen und den berechneten Tm-Anomlien (Abbildung 2.3). Daher wurde eine durch ${ }^{153} \mathrm{Eu}^{16} \mathrm{O}$ verursachte Interferenz als Ursache für die Tm-Anomalien ausgeschlossen, gleiches gilt für andere polyatomare Verbindungen wie ${ }^{152} \mathrm{Sm}^{16} \mathrm{OH}$ und ${ }^{168} \mathrm{ErH}$.

Abgesehen von Interferenzen können auch Matrixeffekte zur systematischen Verfälschung der Messergebnisse führen (Sylvester, 2008). So können Unterschiede der chemischen Zusammensetzung oder der strukturellen Beschaffenheit in unterschiedlichen Ablationseigenschaften resultieren. Es ist unwahrscheinlich, dass nennenswerte Matrixeffekte zwischen verschiedenen Chondriten und ihren Komponenten auftreten, da sie ähnliche chemische Zusammensetzungen besitzen. Die terrestrischen Proben und die differenzierten Steinmeteorite unterscheiden sich in ihrer Zusammensetzung dagegen von den Chondriten, da sie stark an siderophilen Elementen abgereichert sind. Es konnte jedoch kein Zusammenhang zwischen der beobachteten 


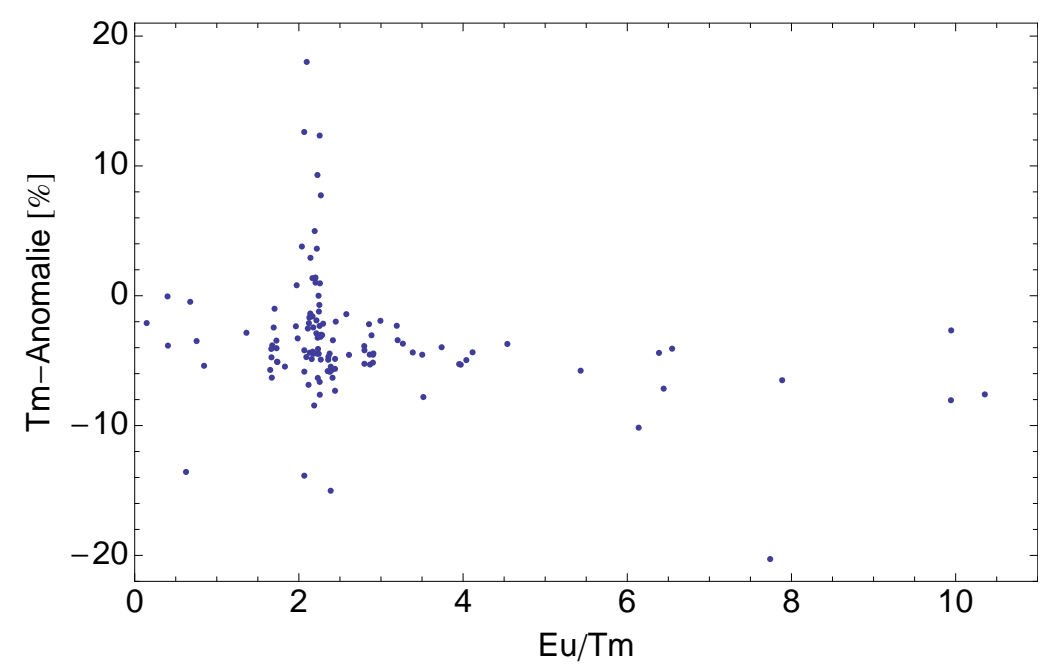

Abbildung 2.3: Eu/Tm vs. Tm-Anomalie. Für die Berechnung der Tm-Anomalie siehe Abschnitt 4.4. Da keine positive Korrelation zwischen der Tm-Anomalie und dem $\mathrm{Eu} / \mathrm{Tm}$-Verhältnis besteht, konnte eine Interferenz von ${ }^{153} \mathrm{Eu}^{16} \mathrm{O}$ auf ${ }^{169} \mathrm{Tm}$ ausgeschlossen werden.

Tm-Anomalie und der Hauptelementzusammensetzung der Proben festgestellt werden, der auf ein unterschiedliches Ablationsverhalten zwischen differenzierten und nichtdifferenzierten Proben hindeuten würde. Desweiteren konnte nachgewiesen werden, dass die erzielten Ergebnisse nicht durch die Probenpräparationsmethode beeinflusst werden, da in-situ untersuchte natürliche Gläser bezüglich der TmAnomalie gleiche Ergebnisse liefern wie experimentell hergestellte, abgeschreckte Proben. 


\section{Ergebnisse}

\subsection{Komponenten von Leoville}

Dieser Abschnitt behandelt Spurenelementfraktionierungen der Komponenten des CV3-Chondrits Leoville. Die hier präsentierten Daten wurden von A. Patzer gemessen und anschließend von der Verfasserin dieser Arbeit ausgewertet.

\subsubsection{Seltene Erden}

Zur graphischen Darstellung der Seltenen Erden wurden diese auf den CI-Chondrit Orgueil normiert. Es wurden hierfür gemittelte REE-Verhältnisse von fünf OrgueilProben (Abschnitt 3.2) herangezogen, die zuvor anhand der absoluten La-Konzentration von Orgueil von 0,235 ppm (Barrat et al., 2012) normiert wurden.

Die aus verschiedenen Messungen desselben Einschlusses bestimmten Anreicherungsfaktoren der Seltenen Erden unterscheiden sich um einen Faktor von bis zu drei voneinander. Diese großen Schwankungen der absoluten REE-Konzentrationen sind auf Probeninhomogenitäten zurückzuführen. Die REE-Verhältnisse verschiedener Messungen eines Einschlusses sind jedoch relativ konstant: Die Abweichungen der REE-Verhältnisse der Einzelmessungen voneinander betragen typischerweise unter $10 \%$, etwas größere Abweichungen von bis zu $20 \%$ ergaben sich für $\mathrm{Yb}$ und $\mathrm{Lu}$ und über $20 \%$ für $\mathrm{Eu}$.

Chondren: Es werden hier drei Untergruppen der Chondren getrennt betrachtet, die aufgrund ihrer chemischen Zusammensetzung und Petrologie unterschieden werden können: Die Typ-1-Chondren sind reich an $\mathrm{MgO}$ und reduzierter als die Typ-2-Chondren, welche einen höheren FeO-Gehalt aufweisen (McSween Jr., 1977). Al-reiche Chondren zeichnen sich dagegen durch hohe $\mathrm{Al}_{2} \mathrm{O}_{3}$-Gehalte von über 10 Gew.- $\%$ aus (Bischoff und Keil, 1984).

Die meisten Typ-1-Chondren haben relativ unfraktionierte REE-Muster, die gegenüber Orgueil 0,5 bis 10-fach angereichert sind (Abbildung $3.1 \mathrm{a}$ ). Viele der Chondren zeigen allerdings Eu-Anomalien, die für die Objekte 1 und 5 positiv und für die Objekte 6, 8, 10, 11, 14, 16, 17, 18 und 23 negativ ist. Abweichend davon besitzen vier Typ-1-Chondren (Objekte 2, 20, 24 und 26) fraktionierte group-II-Muster. 

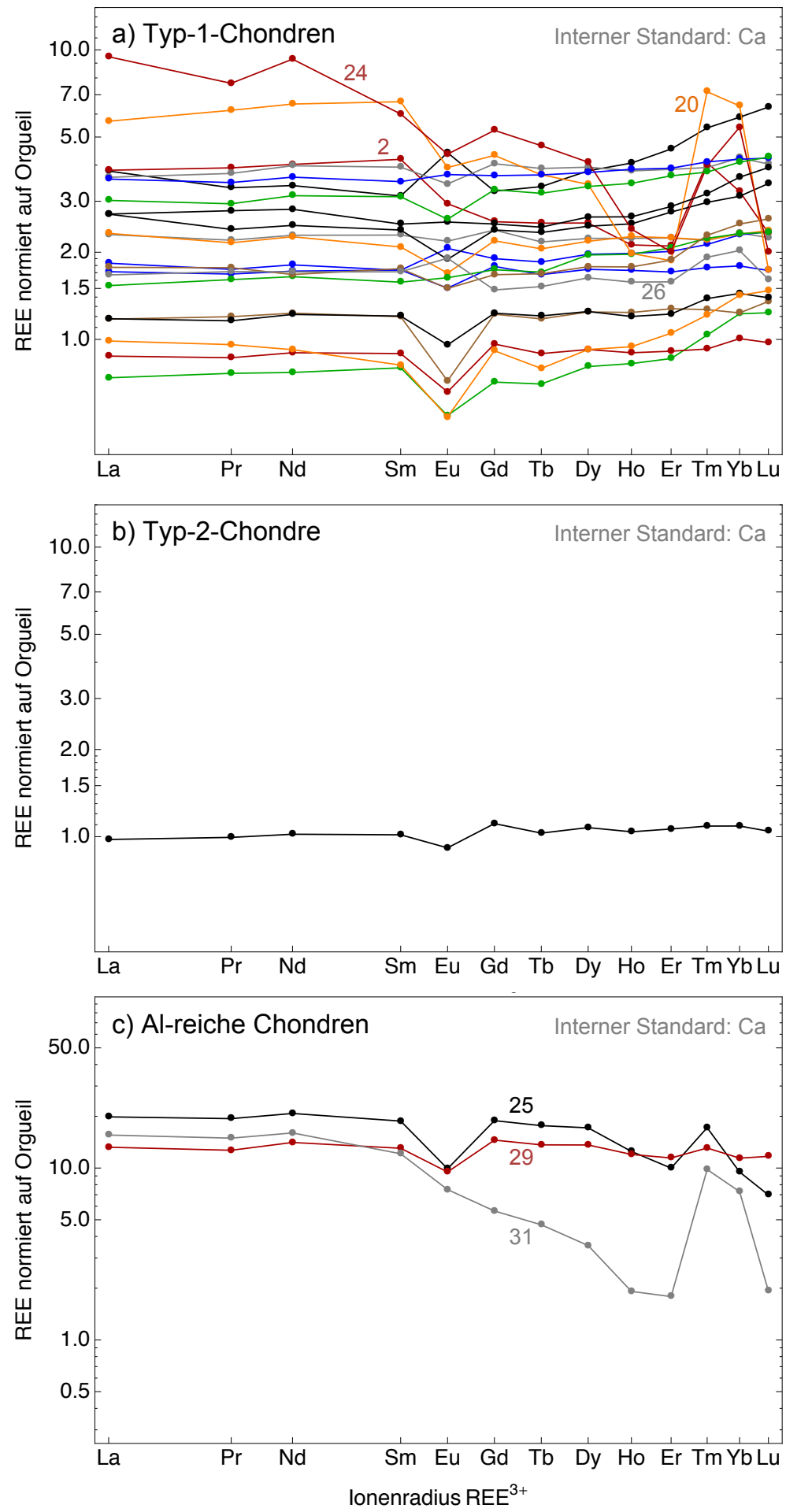

Abbildung 3.1: Orgueil-normierte REE-Muster in Typ-1-Chondren (a), einer Typ-2-Chondre (b) und Al-reichen Chondren (c). Für die Al-reichen Chondren und die Typ-1-Chondren mit einem group-II-Muster sind die Objektnummern angegeben. 
Die Typ-2-Chondre zeigt ebenfalls ein relativ unfraktioniertes REE-Muster mit einer kleinen negativen Eu-Anomalie (Abbildung 3.1b). Die Anreicherung der Seltenen Erden entspricht ungefähr der CI-Konzentration.

Im Gegensatz zu diesen beiden Chondrentypen sind die Seltenen Erden in den Al-reichen Chondren fraktioniert und in den leichten Seltenen Erden etwa 10 bis
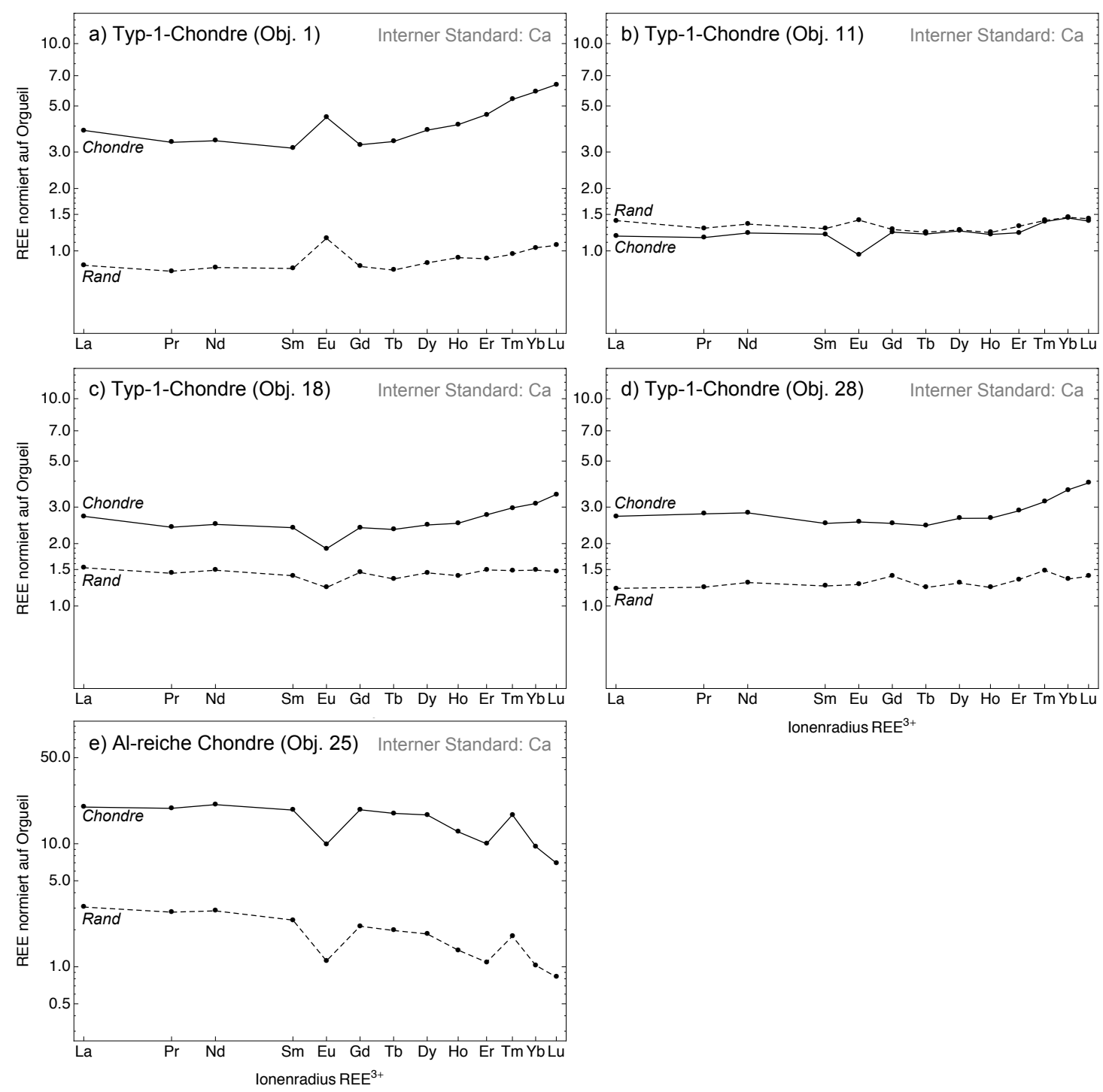

Abbildung 3.2: Orgueil-normierte REE-Muster einiger Chondren und ihrer Akkretionsränder. Es ist zu beachten, dass die absoluten Konzentrationen der Chondrenränder aufgrund der internen Kalibration mit Ca mit relativ großen Unsicherheiten behaftet sind. 
20-fach gegenüber Orgueil angereichert (Abbildung 3.1c). Die Einschlüsse 25 und 31 zeigen deutliche group-II-Muster mit Anreicherungen der leichten Seltenen Erden sowie Tm und Yb, während Objekt 29 nur schwach fraktioniert ist.

An fünf Chondren wurden zusätzlich Messungen ihrer Akkretionsränder durchgeführt, um diese mit dem Inneren der Chondren zu vergleichen. Die REE-Muster dieser Chondren und ihrer Ränder weisen große Ähnlichkeiten zueinander auf (Abbildung 3.2). Dies zeigt sich besonders deutlich bei der fraktionierten Al-reichen Chondre 25, deren Rand ein nahezu identisches REE-Muster besitzt. Der Rand der Typ-1-Chondre 11 zeigt dagegen mit einer geringen positiven Eu-Anomalie einen Unterschied zum Chondrenkern, der eine negative Eu-Anomalie aufweist. Die Chondrenränder haben, mit Ausnahme von Chondre 11, niedrigere Gehalte der Seltenen Erden als die Chondren selbst. Es ist dabei allerdings zu beachten, dass die berechneten absoluten REE-Konzentrationen in den Chondrenrändern aufgrund der internen Kalibration anhand der Ca-Konzentrationen mit relativ großen Unsicherheiten behaftet sind, da die Ca-Konzentrationen in den Rändern mit weniger als zwei Gewichtsprozent (siehe Tabelle A.1) gering sind.

Refraktäre Einschlüsse: Alle untersuchten CAIs haben fraktionierte Seltene Erden. Drei der vier CAIs (Einschlüsse 9, 15 und 22) zeigen deutliche group-IIMuster (Abbildung 3.3 a) mit Anreicherungsfaktoren der leichten Seltenen Erden zwischen 20 und 50 gegenüber Orgueil. Das REE-Muster des CAI 12 hat zwar Ähnlichkeiten zu einem typischen group-II-Muster, unterscheidet sich von diesem allerdings dahingehend, dass die leichten gegenüber den schweren Seltenen Erden nur gering angereichert sind, dass eine deutliche positive Eu-Anomalie besteht und dass Ytterbium etwas stärker angereichert ist als Thulium.

Das einzige in der Probe vorhandene amöboide Olivinaggregat besitzt ebenfalls fraktionierte Seltene Erden (group-II), wobei Yb, das typischerweise zusammen mit Tm angereichert ist, hier keine Anreicherung zeigt (Abbildung $3.3 \mathrm{~b}$ ).

Matrix: Die neun Matrix-Messungen zeigen, dass die Konzentrationen der Seltenen Erden in der Matrix von Leoville ungefähr mit der CI-Konzentration übereinstimmen, die Anreicherungsfaktoren betragen zwischen 0,5 und 1,5 (Abbildung $3.3 \mathrm{c}$ ). Abgesehen von einer ausgeprägten positiven Eu-Anomalie haben die Muster Ähnlichkeiten zu einem group-II-Muster: Die leichten Seltenen Erden sind schwach angereichert und unfraktioniert, während die schweren Seltenen Erden abgereichert sind und häufig kleine positive Anomalien in Tm und Yb haben. Im Gegensatz $\mathrm{zu}$ einem typischen group-II-Muster ist Yb in der Matrix im Durchschnitt stärker angereichert als Tm. 

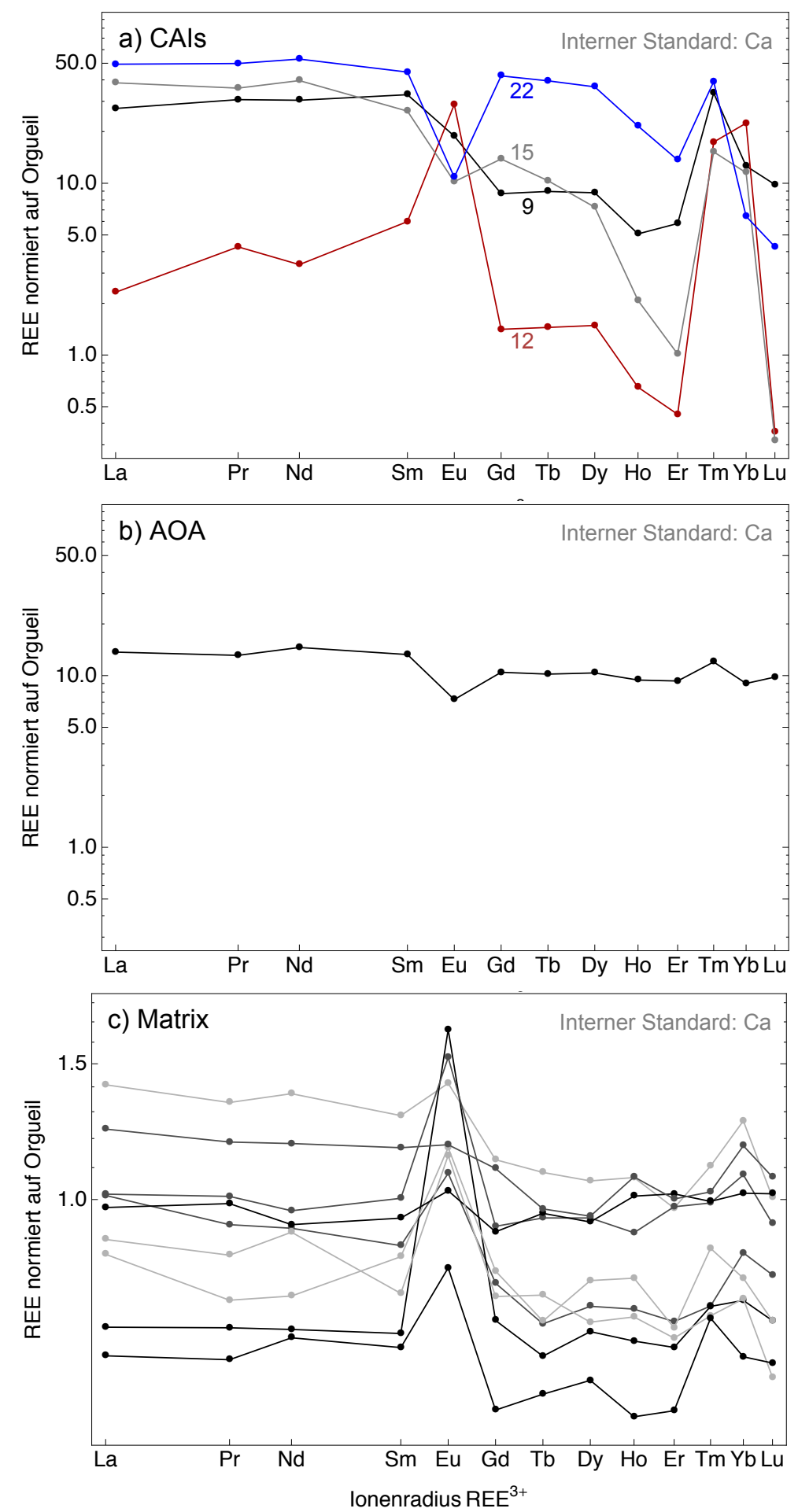

Abbildung 3.3: Orgueil-normierte REE-Muster in CAIs (a), einem AOA (b) und der Matrix (c). Als interner Standard für die in-situ-LA-ICPMS-Messungen dienten die Ca-Konzentrationen. Für die CAIs sind die Objektnummern angegeben. 


\begin{tabular}{|c|c|c|}
\hline 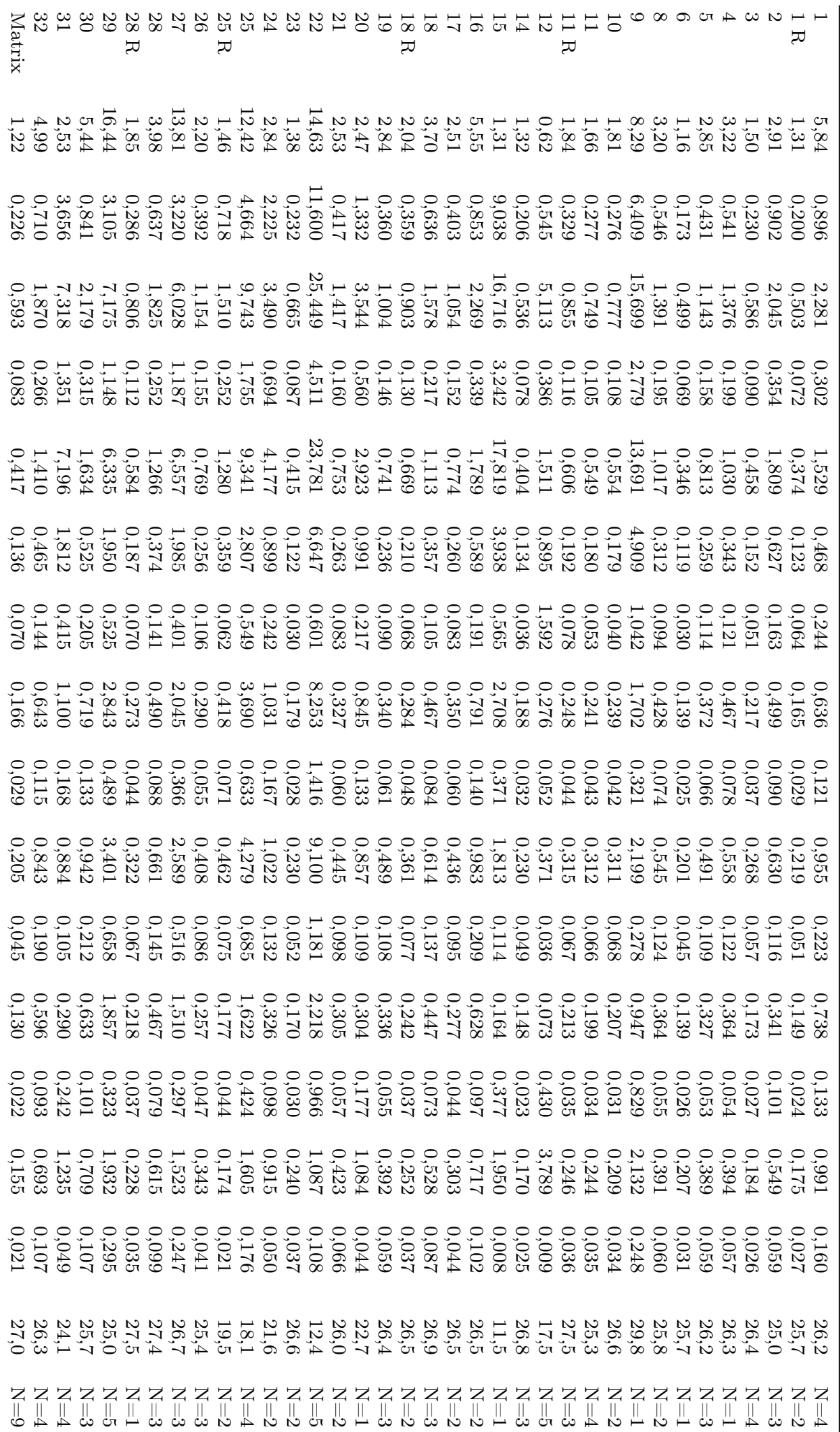 & $\mid \begin{array}{l}\forall \\
\ddot{\varphi} \\
\overline{0}\end{array}$ & 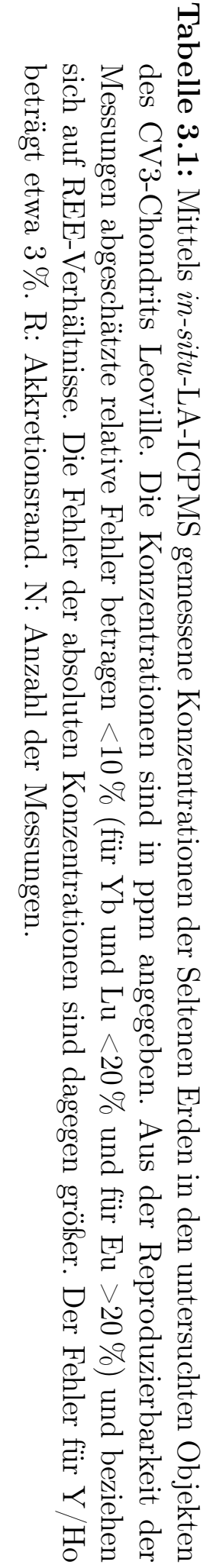 \\
\hline
\end{tabular}




\subsubsection{Y/Ho-, Zr/Hf- und Nb/Ta-Verhältnisse}

Die Y/Ho-Verhältnisse der Matrix sowie der Typ-1- und Typ-2-Chondren sind relativ konstant und stimmen, mit Ausnahme von zwei Typ-1-Chondren, innerhalb von $5 \%$ mit dem Y/Ho-Verhältnis kohliger Chondrite von 25,9 (Pack et al., 2007) überein (Abbildung 3.4). Die beiden abweichenden Chondren (Objekte 20 und 24), die sich außerdem durch fraktionierte REE group-II-Muster auszeichnen, haben subchondritische Y/Ho-Verhältnisse von 22,7 und 21,6.

Die Al-reichen Chondren haben ebenfalls subchondritische Y/Ho-Verhältnisse, die zwischen 18,1 und 25,0 liegen. Die Y/Ho-Verhältnisse der CAIs streuen sehr stark und reichen von deutlich subchondritischen Werten (11,5 für CAI 15) bis hin zu superchondritischen Verhältnissen (29,8 für CAI 9). Das Y/Ho-Verhältnis des AOA stimmt mit 26,7 ungefähr mit dem Wert kohliger Chondrite überein.

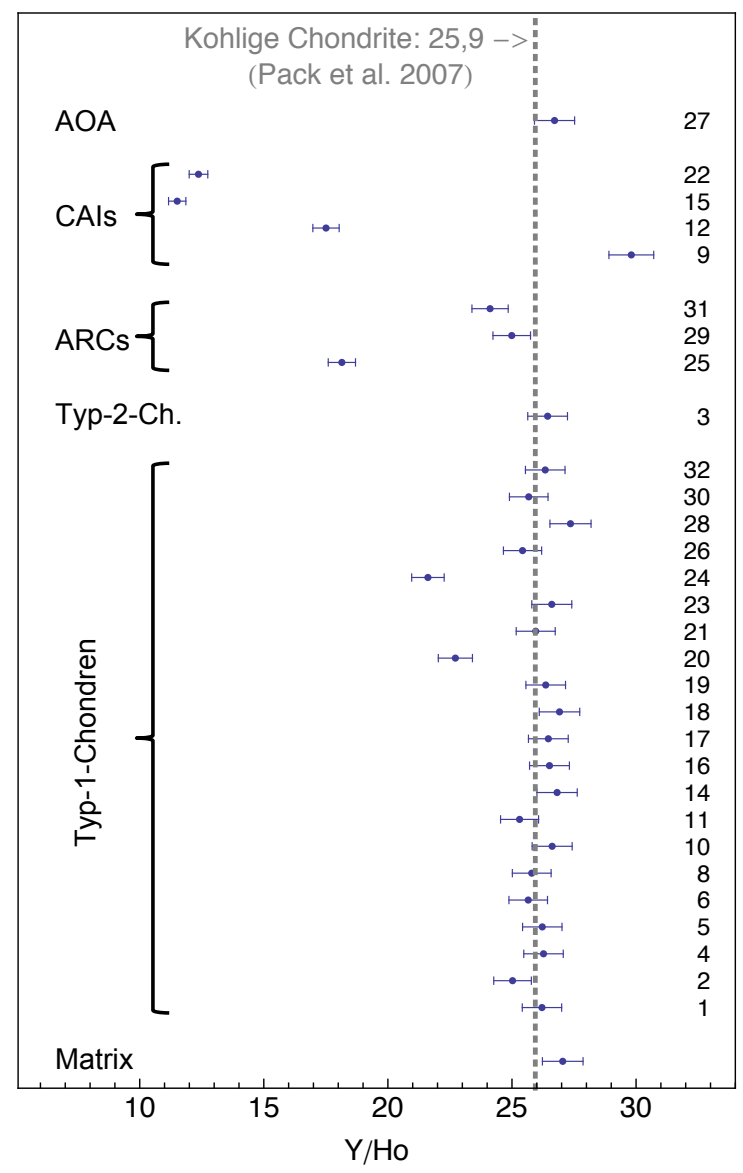

Abbildung 3.4: Y/Ho-Verhältnisse in den untersuchten Objekten. Als externer Standard diente NIST SRM 612. Die Matrix, die Typ-1- und Typ-2-Chondren haben chondritische $\mathrm{Y} / \mathrm{Ho}$-Verhältnisse mit Ausnahme von zwei Chondren (Objekte 20 und 24). Die Y/Ho-Verhältnisse der Al-reichen Chondren (ARCs) und der refraktären Einschlüsse variieren stark und sind im Durchschnitt niedriger. Es wurde für alle Objekte ein relativer Fehler von 3\% angenommen. 
Die $\mathrm{Zr} / \mathrm{Hf}-$ Verhältnisse der Komponenten von Leoville sind, mit Ausnahme von zwei CAIs, einheitlich und stimmen innerhalb von $10 \%$ mit dem chondritischen Wert von 34,3 (Münker et al., 2003) überein (Abbildung 3.5 a). Die davon abweichenden CAIs (Objekte 15 und 22) haben superchondritische Werte.

Die $\mathrm{Nb} / \mathrm{Ta}$-Verhältnisse der verschiedenen Objekte zeigen eine relativ große Streuung (Abbildung 3.5 b), wobei die Matrix sowie die Typ-1- und Typ-2-Chondren im Durchschnitt dem chondritischen Nb/Ta-Verhältnis von 19,9 (Münker et al., 2003) entsprechen. Die refraktären Einschlüsse und Al-reichen Chondren haben dagegen subchondritische Werte zwischen 1,2 und 14,0. Die Nb/Ta- sowie Zr/HfVerhältnisse aller Objekte sind in Tabelle 3.2 angegeben.
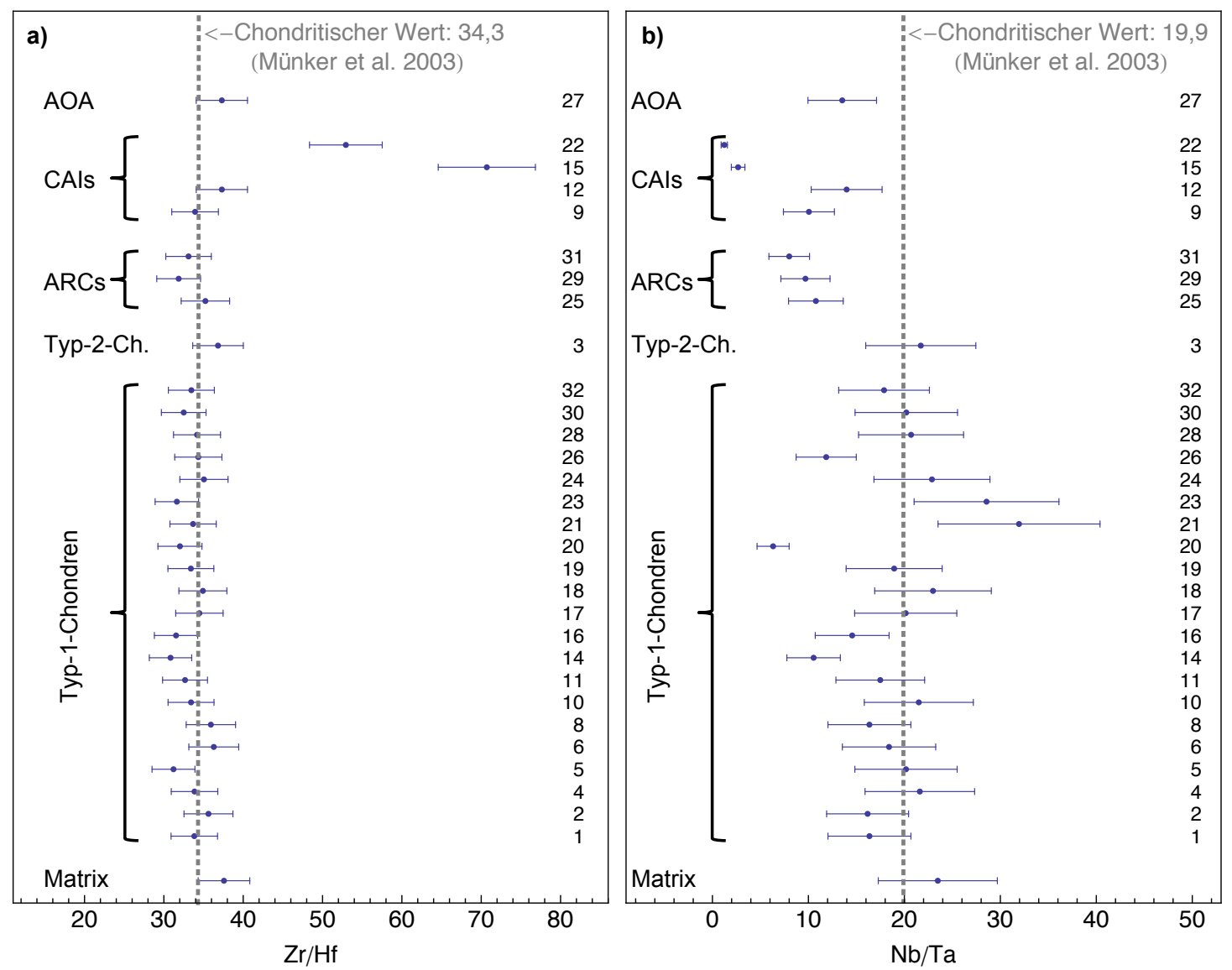

Abbildung 3.5: a) Die Zr/Hf-Verhältnisse sind, abgesehen von zwei CAIs, in den verschiedenen Komponenten chondritisch. b) Die Nb/Ta-Verhältnisse zeigen eine relativ große Streuung. Die Matrix sowie die Typ-1- und Typ-2-Chondren besitzen im Durchschnitt chondritische Werte, während die Al-reichen Chondren (ARCs) und die refraktären Einschlüsse subchondritische Verhältnisse haben. Es wurden für alle Objekte relative Fehler von $9 \%$ für $\mathrm{Zr} / \mathrm{Hf}$ und $27 \%$ für $\mathrm{Nb} / \mathrm{Ta}$ angenommen. Die externe Kalibration der Elementverhältnisse erfolgte mit dem Basaltstandard BHVO. 
Tabelle 3.2: Mittels in-situ-LA-ICPMS gemessene Konzentrationen (in ppm) an

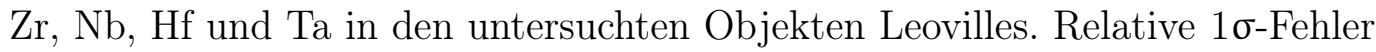
betragen ca. $20 \%$ für $\mathrm{Hf}, 25 \%$ für $\mathrm{Zr}$ sowie $30 \%$ für $\mathrm{Nb}$ und Ta, dabei sind die Unsicherheiten bezüglich der internen Kalibration mit Ca und der externen Kalibration mit NIST SRM 612 nicht miteinbezogen. Die mittleren Zr/Hf- und $\mathrm{Nb} / \mathrm{Ta}-$ Verhältnisse wurden aus den Elementverhältnissen der Einzelmessungen berechnet. Die Fehler für $\mathrm{Zr} / \mathrm{Hf}$ und Nb/Ta betragen etwa $9 \%$ und $27 \%$. Die mit * markierten Spalten enthalten mithilfe des Basaltstandards BHVO korrigierte $\mathrm{Zr} / \mathrm{Hf}-$ und Nb/Ta-Verhältnisse. $N$ : Anzahl der Messungen.

\begin{tabular}{|c|c|c|c|c|c|c|c|c|c|}
\hline Objekt & $\mathrm{Zr}$ & $\mathrm{Nb}$ & Hf & $\mathrm{Ta}$ & $\mathrm{Zr} / \mathrm{Hf}$ & $\mathrm{Nb} / \mathrm{Ta}$ & $\mathrm{Zr} / \mathrm{Hf}^{*}$ & $\mathrm{Nb} / \mathrm{Ta}^{*}$ & $N$ \\
\hline 1 & 12,47 & 1,00 & 0,356 & 0,057 & 34,5 & 17,4 & 33,8 & 16,4 & 4 \\
\hline 2 & 6,89 & 0,71 & 0,191 & 0,042 & 36,4 & 17,2 & 35,6 & 16,2 & 3 \\
\hline 3 & 4,34 & 0,33 & 0,113 & 0,015 & 37,6 & 23,1 & 36,8 & 21,7 & 4 \\
\hline 4 & 8,08 & 0,71 & 0,234 & 0,031 & 34,6 & 23,0 & 33,9 & 21,6 & 1 \\
\hline 5 & 6,25 & 0,55 & 0,196 & 0,026 & 31,9 & 21,5 & 31,2 & 20,2 & 3 \\
\hline 6 & 2,43 & 0,20 & 0,066 & 0,010 & 37,0 & 19,6 & 36,3 & 18,4 & 1 \\
\hline 8 & 7,96 & 0,56 & 0,217 & 0,033 & 36,7 & 17,4 & 35,9 & 16,4 & 2 \\
\hline 9 & 31,45 & 1,43 & 0,908 & 0,134 & 34,6 & 10,7 & 33,9 & 10,1 & 1 \\
\hline 10 & 4,43 & 0,38 & 0,130 & 0,017 & 34,1 & 22,9 & 33,4 & 21,5 & 2 \\
\hline 11 & 3,85 & 0,32 & 0,116 & 0,017 & 33,4 & 18,6 & 32,7 & 17,5 & 4 \\
\hline 12 & 1,18 & 10,51 & 0,035 & 0,660 & 38,1 & 14,9 & 37,3 & 14,0 & 5 \\
\hline 14 & 3,49 & 0,16 & 0,111 & 0,014 & 31,5 & 11,2 & 30,8 & 10,5 & 3 \\
\hline 15 & 1,66 & 1,06 & 0,023 & 0,370 & 72,2 & 2,9 & 70,7 & 2,7 & 1 \\
\hline 16 & 12,43 & 0,79 & 0,383 & 0,049 & 32,2 & 15,5 & 31,5 & 14,6 & 2 \\
\hline 17 & 5,82 & 0,54 & 0,165 & 0,025 & 35,2 & 21,4 & 34,5 & 20,1 & 2 \\
\hline 18 & 8,01 & 0,78 & 0,225 & 0,032 & 35,6 & 24,5 & 34,9 & 23,0 & 3 \\
\hline 19 & 6,03 & 0,48 & 0,176 & 0,024 & 34,1 & 20,2 & 33,4 & 18,9 & 3 \\
\hline 20 & 5,31 & 0,62 & 0,162 & 0,093 & 32,7 & 6,7 & 32,0 & 6,3 & 1 \\
\hline 21 & 5,51 & 0,68 & 0,160 & 0,021 & 34,4 & 34,0 & 33,7 & 31,9 & 2 \\
\hline 22 & 17,79 & 0,33 & 0,329 & 0,443 & 54,0 & 1,3 & 52,9 & 1,2 & 5 \\
\hline 23 & 2,60 & 0,34 & 0,080 & 0,013 & 32,3 & 30,4 & 31,6 & 28,6 & 2 \\
\hline 24 & 6,55 & 1,37 & 0,182 & 0,057 & 35,8 & 24,4 & 35,1 & 22,9 & 2 \\
\hline 25 & 21,26 & 2,29 & 0,599 & 0,204 & 36,0 & 11,5 & 35,2 & 10,8 & 4 \\
\hline 26 & 4,97 & 0,31 & 0,141 & 0,025 & 35,1 & 12,6 & 34,3 & 11,9 & 3 \\
\hline 27 & 31,59 & 1,28 & 0,814 & 0,092 & 38,1 & 14,4 & 37,3 & 13,5 & 3 \\
\hline 28 & 8,83 & 0,77 & 0,250 & 0,035 & 34,9 & 22,0 & 34,2 & 20,7 & 3 \\
\hline 29 & 34,05 & 1,43 & 1,048 & 0,139 & 32,5 & 10,3 & 31,9 & 9,7 & 5 \\
\hline 30 & 12,67 & 1,07 & 0,383 & 0,050 & 33,2 & 21,5 & 32,5 & 20,2 & 3 \\
\hline 31 & 6,03 & 1,43 & 0,178 & 0,168 & 33,8 & 8,5 & 33,1 & 8,0 & 4 \\
\hline 32 & 11,44 & 0,86 & 0,335 & 0,046 & 34,2 & 19,0 & 33,5 & 17,9 & 4 \\
\hline Matrix & 3,12 & 0,28 & 0,082 & 0,012 & 38,4 & 25,0 & 37,6 & 23,5 & 9 \\
\hline
\end{tabular}




\subsection{Gesamtgesteinsanalysen an Chondriten, Achondriten und Planeten}

\subsubsection{Quenchkugeln}

Die Quenchkugeln der Proben wurden rasterelektronenmikroskopisch charakterisiert. Das Gefüge der gequenchten Bulk-Chondritproben ist mikrokristallin mit länglichen Kristallen, die bis zu mehrere hundert Mikrometer messen können (Abbildung 3.6 a). Unter reduzierenden Bedingungen während der Levitation können sich auch kleine metallreiche und sulfidreiche Ausscheidungen bilden. Siliziumreiche Proben wie Achondrite und die meisten irdischen Proben erstarren in einem glasigen Zustand (Abbildung 3.6 b).
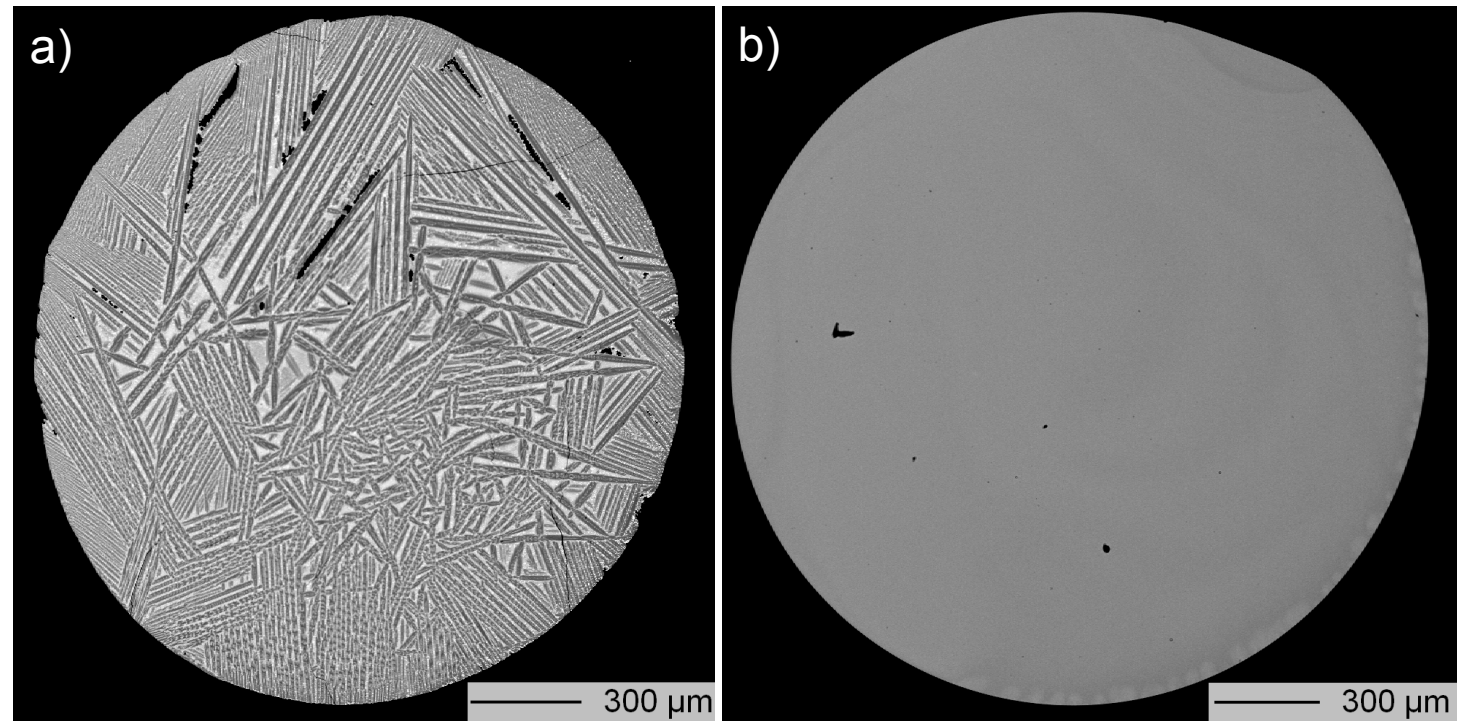

Abbildung 3.6: Rückstreuelektronenbilder der Quenchkugeln. a) Chondrit North West Africa 869 (L5) ist in einem mikrokristallinen Gefüge erstarrt, b) Achondrit Cachari (EUC) ist in einem glasigen Gefüge erstarrt.

Die Entstehung von kleinen Kristallen stellt für die Messungen mittels LAICPMS kein Problem dar, weil durch die gewählten Einstellungen während der Ablation (Strahldurchmesser 81 - $112 \mu \mathrm{m}$ und Linienscan von mehreren hundert Mikrometern) ein großer Bereich der Probenoberfläche ablatiert wird, so dass kleinskalige Inhomogenitäten nicht ins Gewicht fallen. Die erzielten Präzisionen für die Seltenen Erden lagen typischerweise im Bereich von zwei bis fünf Prozent.

Die Hauptelementmessungen mittels Elektronenstrahlmikrosonde sind dagegen mit Unsicherheiten behaftet, da Defokussierung des Elektronenstrahls auf $30 \mu \mathrm{m}$ beschränkt war und die Quenchkugeln auf dieser Größenskala aufgrund ihrer 
Kristallinität nicht homogen sind. Es wurden daher mindestens fünf Punkte pro Probe analysiert und anschließend gemittelt. Die relativen Standardabweichungen der Einzelmessungen betragen typischerweise bis zu $10 \%$ für Si, bis zu $20 \%$ für $\mathrm{Mg}$ und $\mathrm{Fe}$ und bis zu $30 \%$ für $\mathrm{Ca}$ und $\mathrm{Al}$.

\subsubsection{Volatilität von Haupt- und Spurenelementen während des Aufschmelzprozesses}

Um zu überprüfen, welche Elemente aufgrund ihrer Volatilität während der Probenpräparation teilweise oder vollständig entweichen, wurden Experimente an zwei Chondriten durchgeführt. Hierfür wurden ein kohliger Chondrit (Allende, CV3) und ein gewöhnlicher Chondrit (Thuathe, H4/5) ausgewählt. Von beiden Chondriten wurden je vier Quenchkugeln präpariert, die nach dem Vorschmelzen einbis viermal während der Levitation aufgeschmolzen wurden. Das Aufschmelzen dauerte normalerweise nur wenige Sekunden, der jeweils vierte Aufschmelzprozess wurde jedoch über eine deutlich längere Zeitspanne von ca. 30 Sekunden und bei maximaler Laserstärke durchgeführt.

In Abbildung 3.7 sind die refraktären Spurenelementverhältnisse REE/Er, Zr/Hf und $\mathrm{Nb} / \mathrm{Ta}$ der unterschiedlich häufig aufgeschmolzenen Kugeln gezeigt; die verschiedenen Messungen sind dabei auf ihren gemeinsamen Mittelwert normiert (Konzentrationsdaten sind in Tabelle A.5 zu finden). Innerhalb der Messungenauigkeit konnte, allein mit der Ausnahme von Ce, kein Verlust eines Seltenen Erdelements festgestellt werden. Die $\mathrm{Zr} / \mathrm{Hf}-$ und $\mathrm{Nb} / \mathrm{Ta}$-Verhältnisse sind nach mehreren Aufschmelzprozessen ebenfalls unverändert. Selbst ein Aufschmelzen von Probenmaterial über mehrere Stunden führt, abgesehen von Ce, zu keinem Verlust der Seltenen Erden (A. Pack, persönliche Kommunikation). Die gewählte Methode ist für die Analyse der Seltenen Erden und der HFSEs Zr, Nb, Hf und Ta folglich sehr gut geeignet. Cer evaporiert bei längerem Aufschmelzen (ca. 30 Sekunden) bis zu $20 \%$. Dies kann darauf zurückgeführt werden, dass es empfindlich auf die Sauerstofffugazität reagiert und unter oxidierenden Bedingungen besonders volatil ist (Boynton, 1984). Aus diesem Grunde werden für die Gesamtgesteinsanalysen keine Daten zu Ce präsentiert.

Abbildung 3.8 zeigt mittels EMS gemessene Hauptelemente (Tabelle A.6), die auf $\mathrm{Al}_{2} \mathrm{O}_{3}$ und die Ergebnisse der Röntgenfluoreszenzanalysen (Tabelle 3.3) normiert sind. Die Konzentrationen der meisten Hauptelemente nehmen in den Quenchkugeln bei mehrmaligem Aufschmelzen, insbesondere bei dem längeren vierten Aufschmelzprozess, deutlich ab. Schwefel, Natrium, Kalium, Phosphor und Nickel entweichen bei langem und starkem Aufschmelzen fast vollständig. Auch Silizium, Magnesium und Eisen evaporieren bei wiederholtem Schmelzen teilweise: Bei ein bis zwei kurzen Aufschmelzprozessen kann der Verlust bereits bis zu $20 \%$ betragen. 


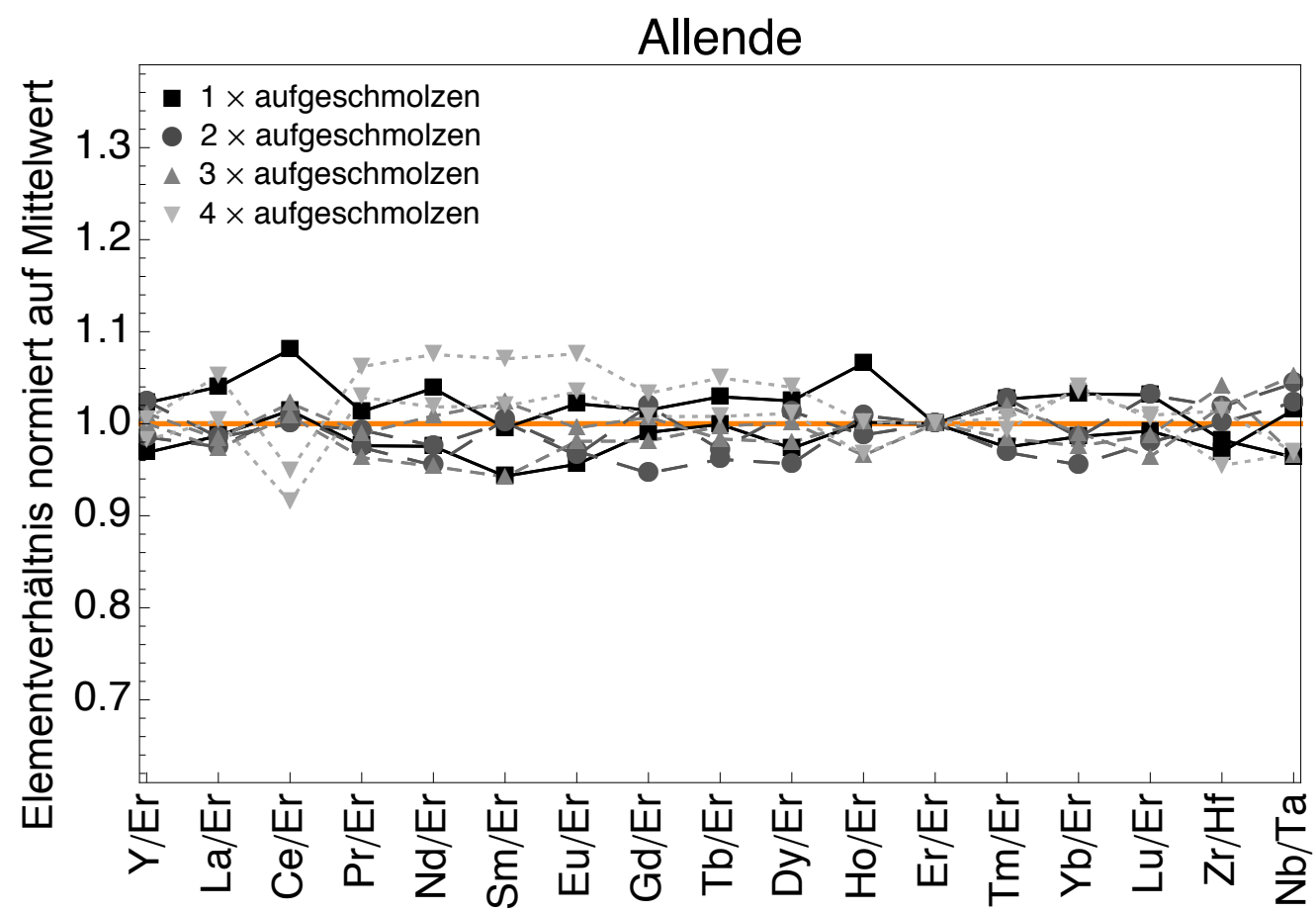

Thuathe

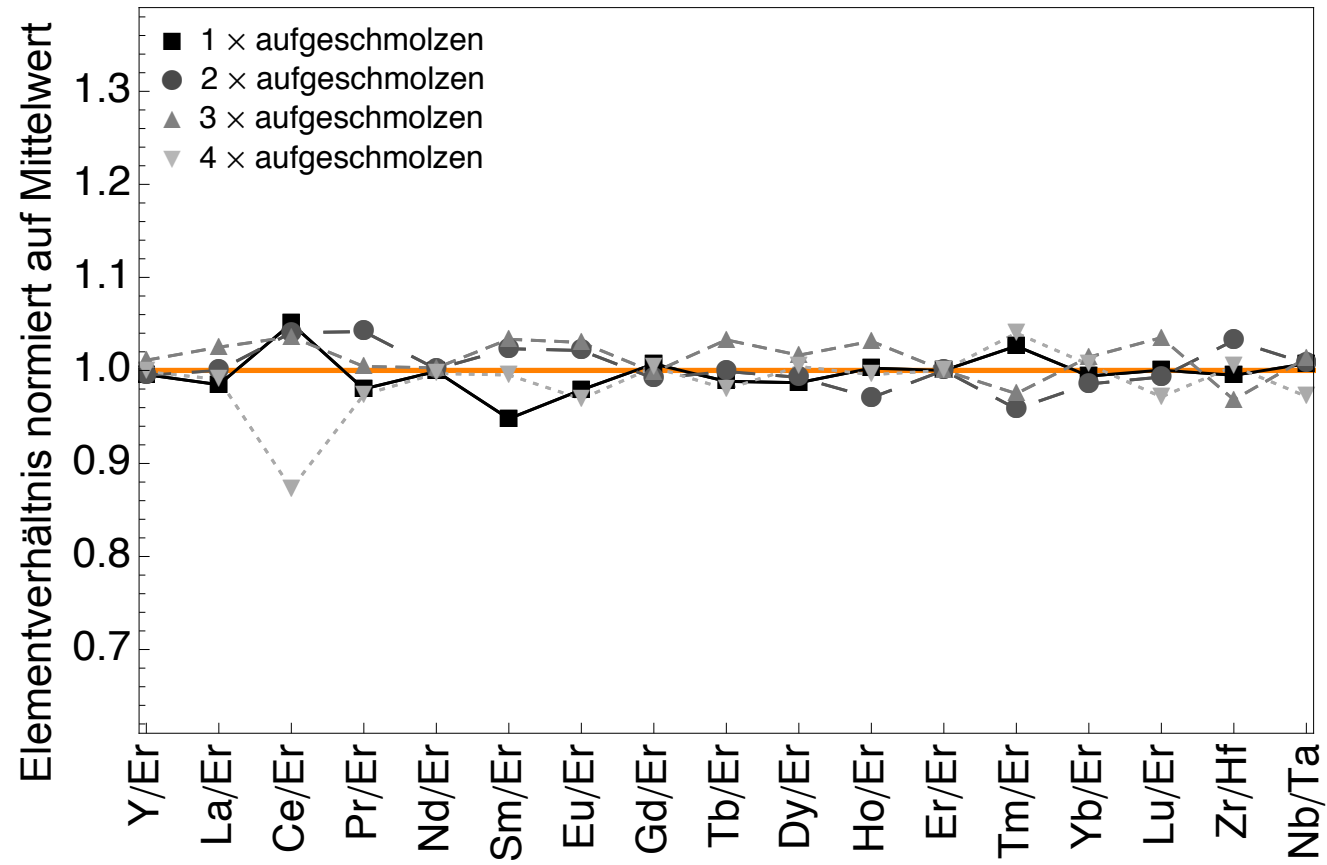

Abbildung 3.7: Mittels LA-ICPMS gemessene Spurenelemente in den Quenchkugeln (ein bis vier Mal aufgeschmolzen). Die Verhältnisse der Seltenen Erden (mit Ausnahme von $\mathrm{Ce}$ ) sowie $\mathrm{Zr} / \mathrm{Hf}$ und $\mathrm{Nb} / \mathrm{Ta}$ werden durch mehrfaches Aufschmelzen während der Probenpräparation nicht beeinflusst. 


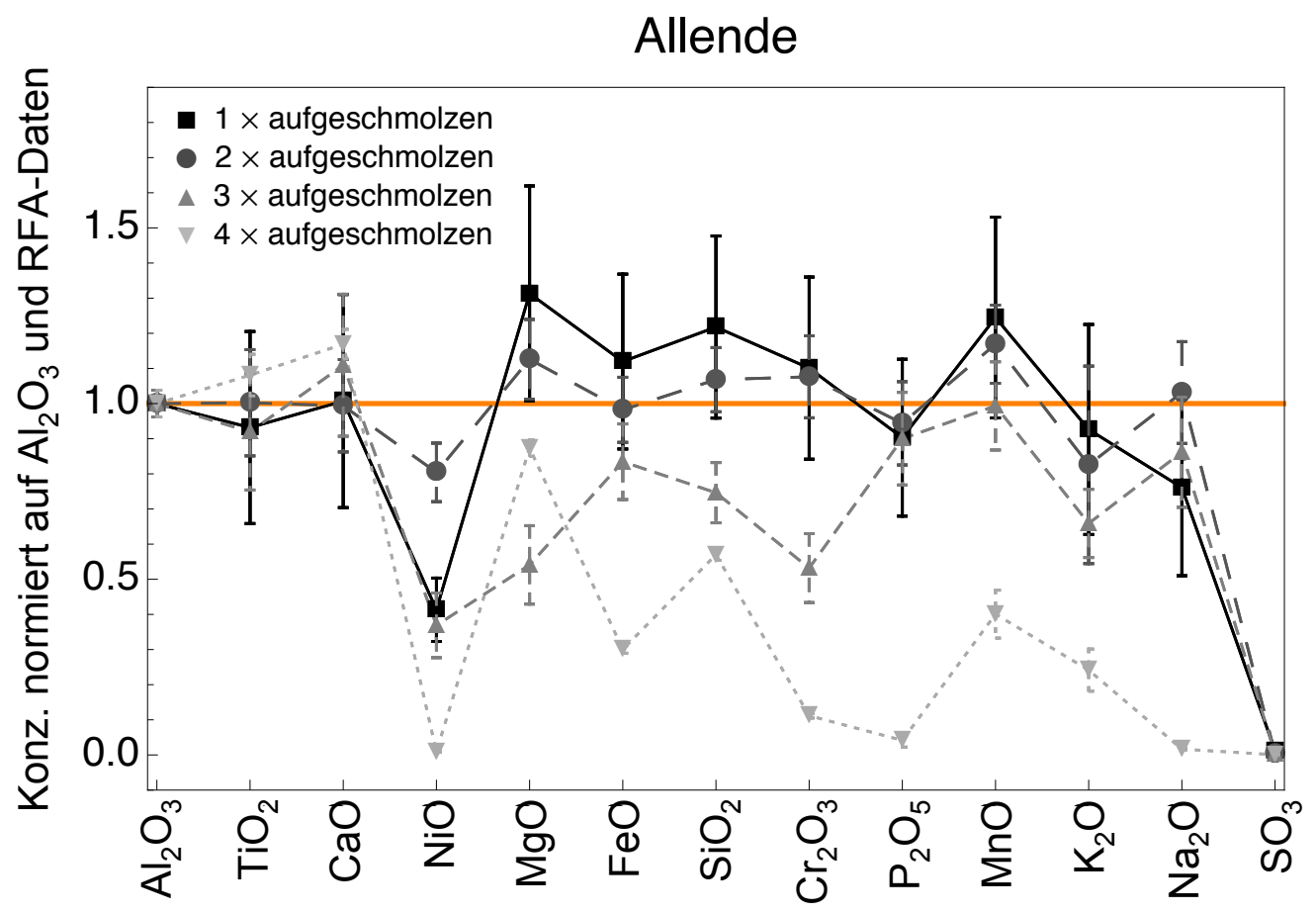

Thuathe

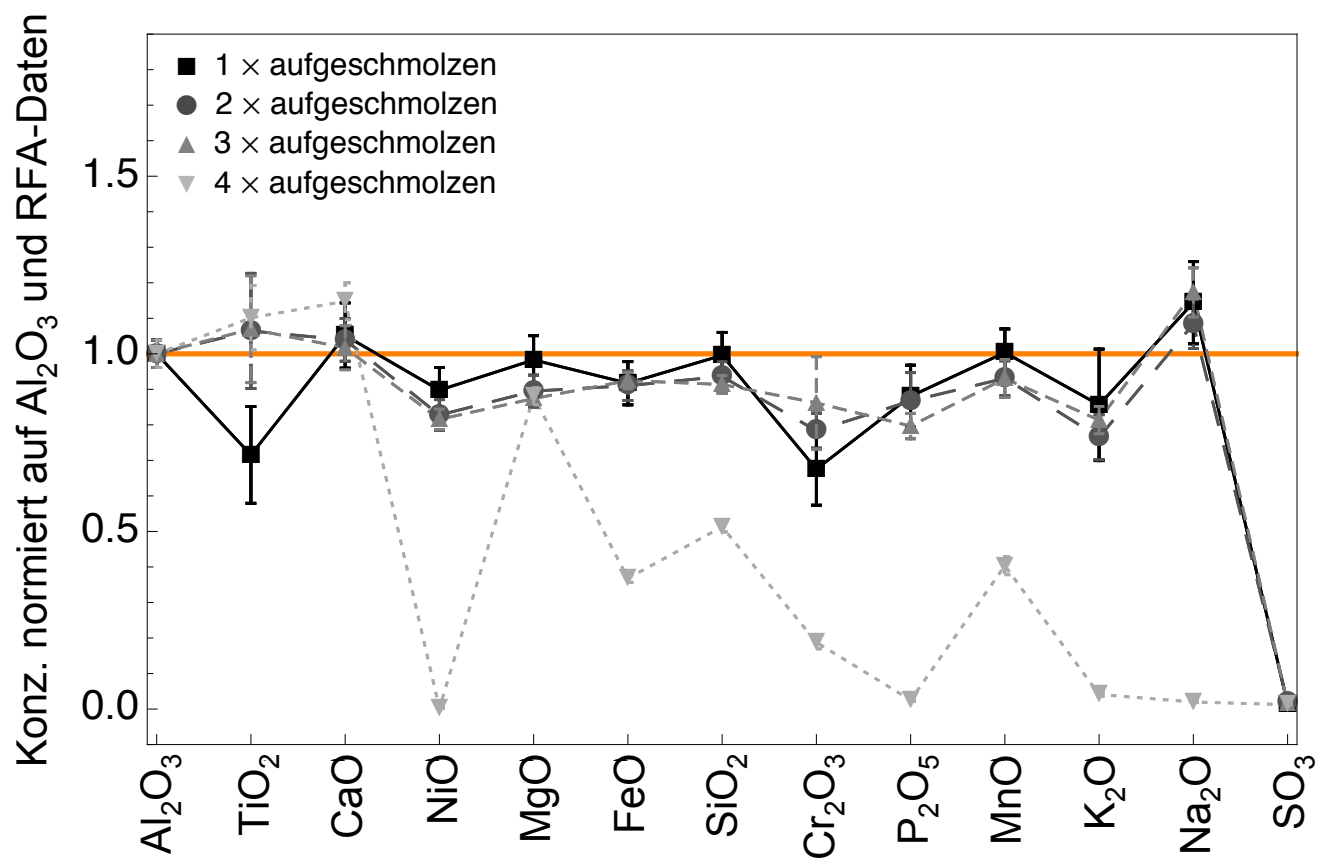

Abbildung 3.8: Mittels EMS gemessene Hauptelemente in den Quenchkugeln (ein bis vier Mal aufgeschmolzen). Beim Aufschmelzen gehen volatile und moderatvolatile Elemente partiell verloren. Die Fehlerbalken sind 1 $\sigma$-Standardfehler von je fünf Analysen. 
Die Verhältnisse der refraktärsten Hauptelemente Aluminium, Calcium und Titan werden durch mehrmaliges Aufschmelzen nicht beeinflusst und können unter Verwendung dieser Präparationsmethode zuverlässig bestimmt werden; für die anderen Hauptelemente ist die Methode jedoch nur bedingt geeignet.

\subsubsection{Röntgenfluoreszenzanalyse}

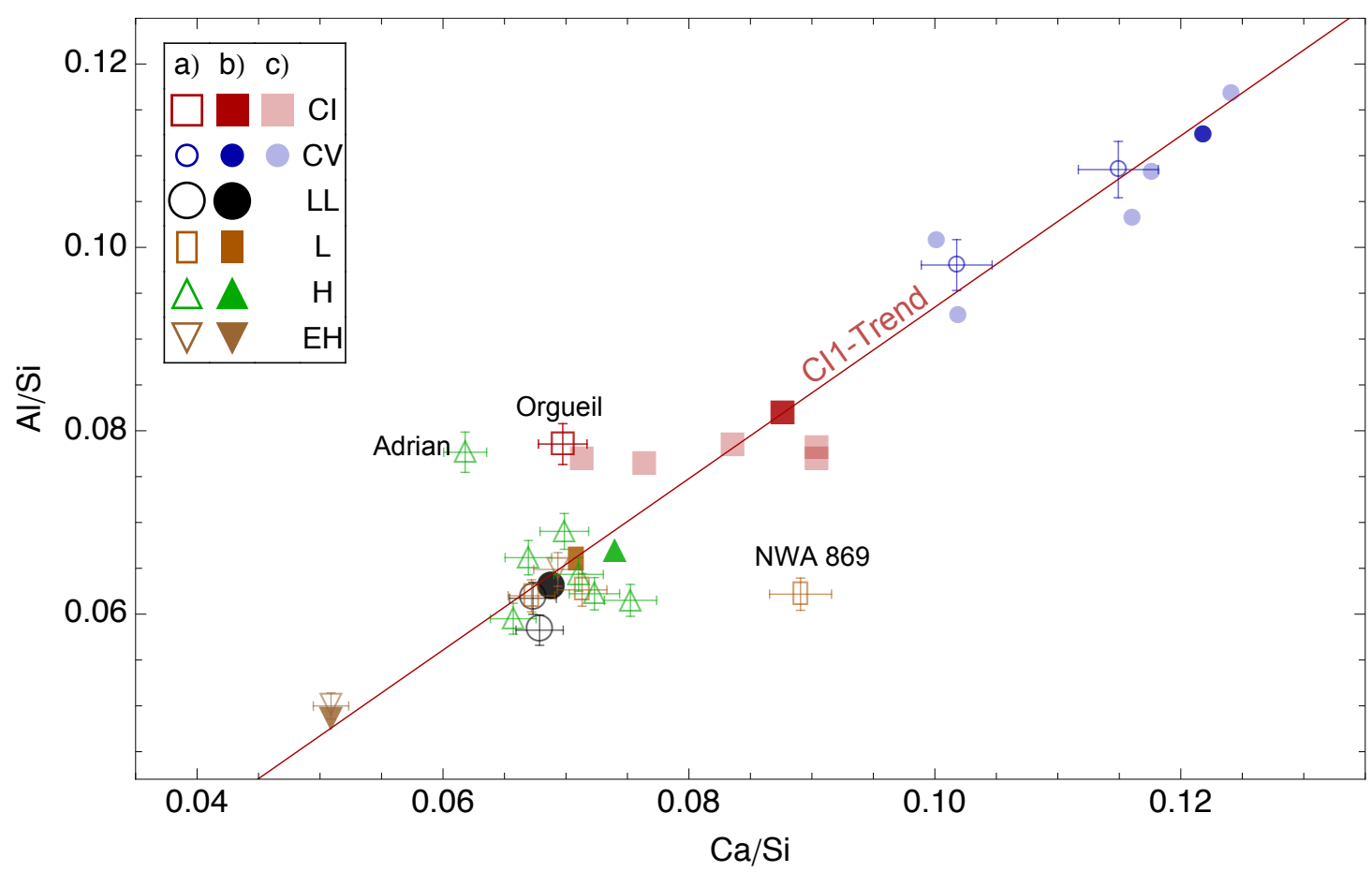

Abbildung 3.9: Gewichtsverhältnisse $\mathrm{Al} / \mathrm{Si}$ vs. $\mathrm{Ca} / \mathrm{Si}$ in Chondriten. Offene Symbole (a) sind RFA-Ergebnisse. Ausgefüllte Symbole (b) sind Gruppenmittelwerte aus Hutchison (2004). Blasse ausgefüllte Symbole (c) sind Daten aus Wolf und Palme (2001).

In den Abbildungen 3.9 bis 3.11 sind durch Röntgenfluoreszenzanlyse ermittelte Elementverhältnisse in Bulk-Chondriten gezeigt. Zum Vergleich sind Literaturdaten über die Mittelwerte der Chondritgruppen (Hutchison, 2004) dargestellt sowie RFA-Analysen für CI- und CV-Chondrite von Wolf und Palme (2001). Die Daten sind auch in guter Übereinstimmung mit anderen Literaturdaten und bestätigen bekannte Fraktionierungstrends von Chondriten (Larimer und Anders, 1970; Kallemeyn und Wasson, 1979; Jarosewich, 1990). 


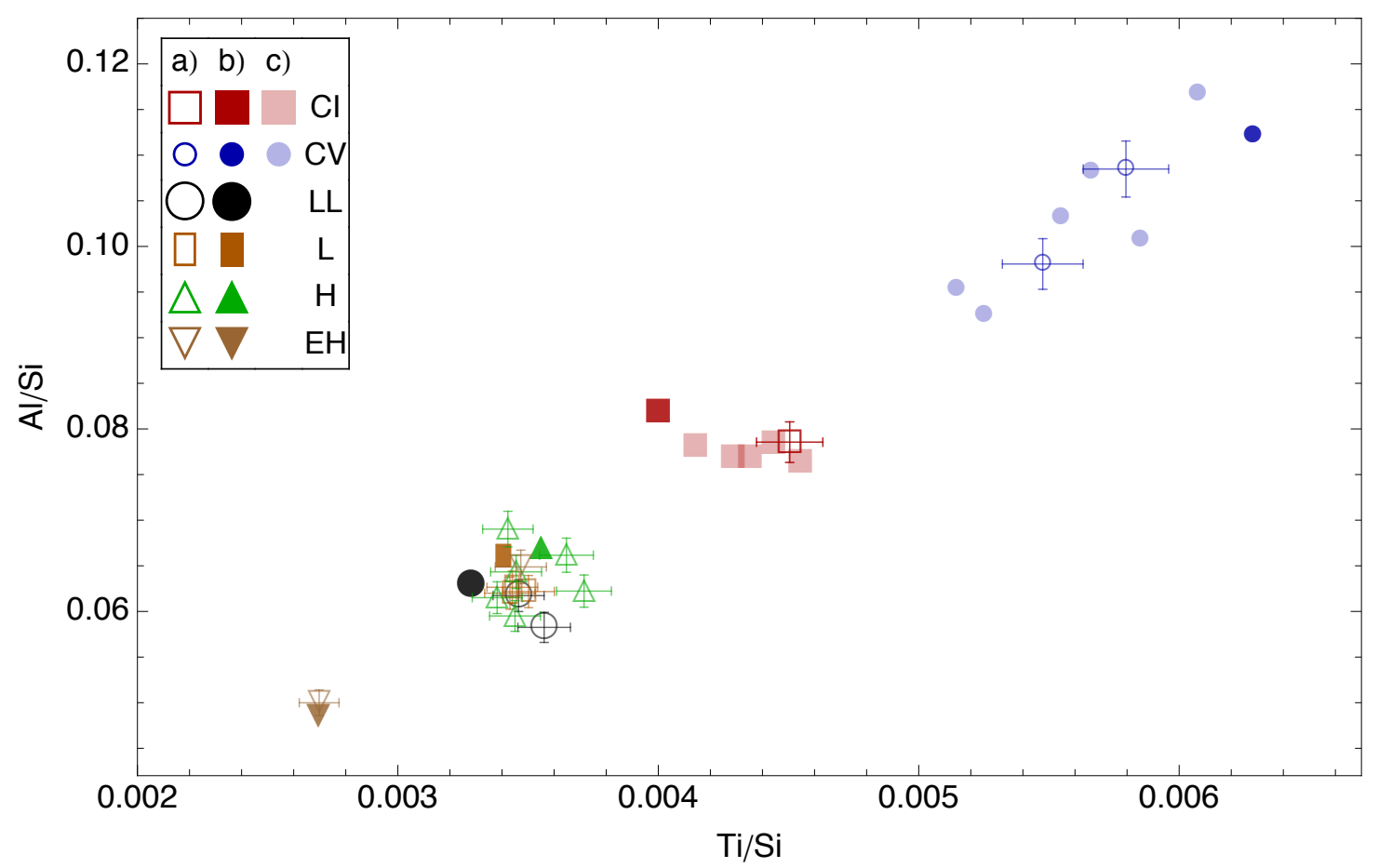

Abbildung 3.10: Gewichtsverhältnisse $\mathrm{Al} / \mathrm{Si}$ vs. $\mathrm{Ti} / \mathrm{Si}$ in Chondriten. Offene Symbole (a) sind RFA-Ergebnisse. Ausgefüllte Symbole (b) sind Gruppenmittelwerte aus Hutchison (2004). Blasse ausgefüllte Symbole (c) sind Daten aus Wolf und Palme (2001).

Abbildungen 3.9 und 3.10 zeigen, dass die refraktären Elemente Al, Ca und Ti gegenüber Si fraktioniert sind. Außerdem bestehen lineare Trends zwischen $\mathrm{Al} / \mathrm{Si}$ und $\mathrm{Ca} / \mathrm{Si}$ sowie zwischen $\mathrm{Al} / \mathrm{Si}$ und $\mathrm{Ti} / \mathrm{Si}$. Al, Ca und $\mathrm{Ti}$ sind folglich in den verschiedenen Chondritgruppen nicht gegeneinander fraktioniert. Verglichen mit CI-Chondriten haben CV-Chondrite etwa $30 \%$ höhere Verhältnisse von $\mathrm{Al} / \mathrm{Si}$, $\mathrm{Ca} / \mathrm{Si}$ und $\mathrm{Ti} / \mathrm{Si}$, sie sind also an den refraktären Elementen angereichert. Die gewöhnlichen und Enstatit-Chondrite sind dagegen gegenüber Orgueil an Al, Ca und Ti abgereichert.

Die in dieser Arbeit analysierte Orgueil-Probe weicht deutlich von dem linearen Trend zwischen $\mathrm{Al} / \mathrm{Si}$ und $\mathrm{Ca} / \mathrm{Si}$ ab (Abbildung 3.9). Literaturdaten von Wolf und Palme (2001) verdeutlichen, dass die Ca/Si-Verhältnisse in verschiedenen Proben der CI-Chondrite um bis zu $20 \%$ variieren.

Die Ti/Si-Verhältnisse in CI-Chondriten variieren ebenfalls (Abbildung 3.10), wobei der CI-Gruppenmittelwert (Hutchison, 2004) von dem linearen Trend zwischen $\mathrm{Al} / \mathrm{Si}$ und $\mathrm{Ti} / \mathrm{Si}$ der Chondrite abweicht. 


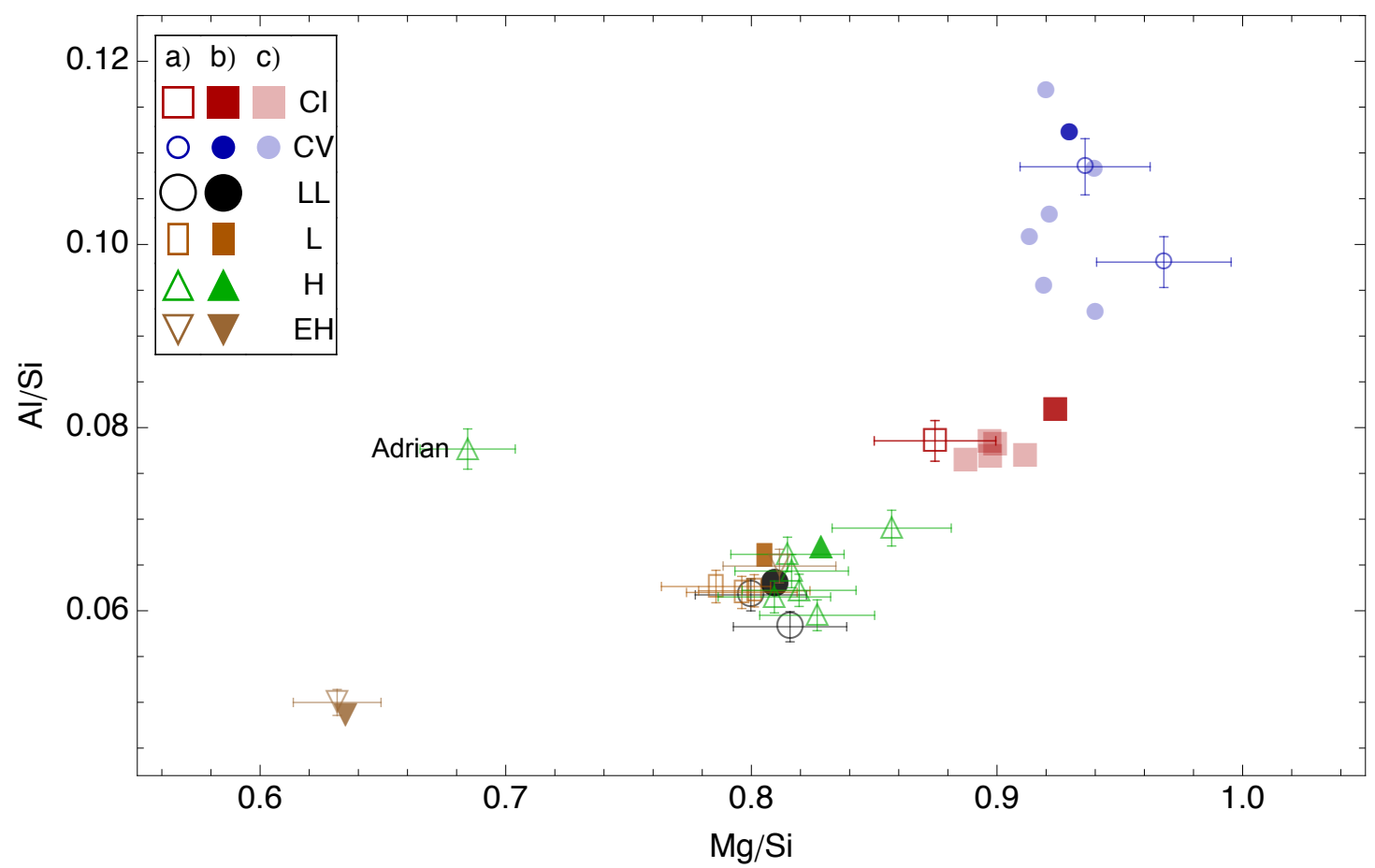

Abbildung 3.11: Gewichtsverhältnisse $\mathrm{Al} / \mathrm{Si}$ vs. $\mathrm{Mg} / \mathrm{Si}$ in Chondriten. Offene Symbole (a) sind RFA-Ergebnisse. Ausgefüllte Symbole (b) sind Gruppenmittelwerte aus Hutchison (2004). Blasse ausgefüllte Symbole (c) sind Daten aus Wolf und Palme (2001).

Magnesium ist ebenfalls zwischen verschiedenen Chondritgruppen gegenüber $\mathrm{Si}$ fraktioniert, allerdings in geringerem Maße als $\mathrm{Al}$ (Abbildung 3.11). Die $\mathrm{Mg} / \mathrm{Si}$ Verhältnisse sind in den CV-Chondriten am höchsten, gefolgt von CI-Chondriten, gewöhnlichen Chondriten und schließlich Enstatit-Chondriten. 


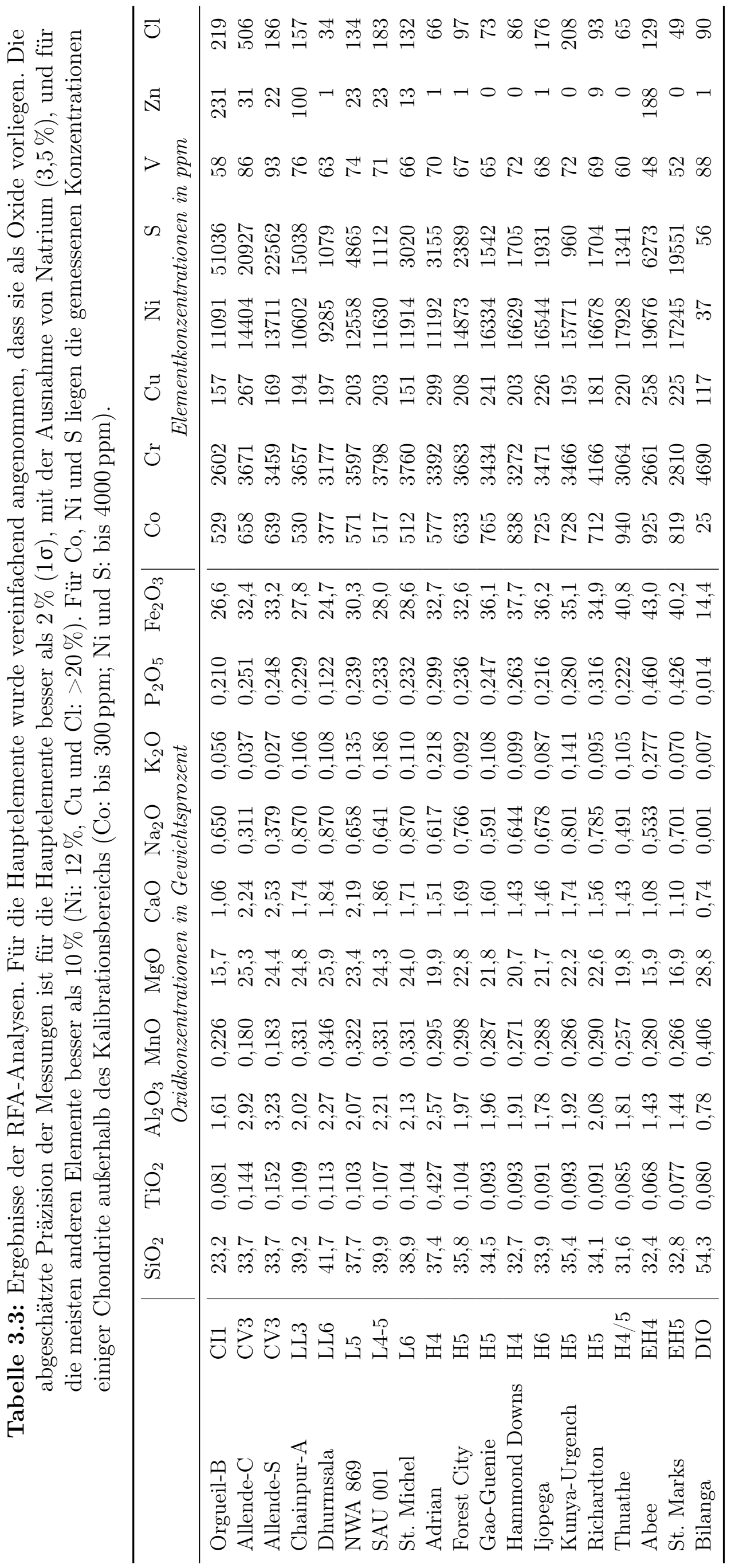




\subsubsection{REE-Muster von Chondriten}

Dieser Abschnitt behandelt REE-Muster von Bulk-Chondriten. Die Daten wurden dazu auf den Mittelwert der fünf analysierten Orgueil-Proben normiert. Da durch den Verlust der volatilen Elemente in den Schmelzkugeln die absoluten REEGehalte der Chondrite nicht ermittelt werden konnten, wurden die gemessenen REE-Konzentrationen anhand der mittleren Ca-Gehalte der Chondritgruppen kalibriert; Ca-Konzentrationen für CB-Chondrite wurden aus Kallemeyn et al. (1978) entnommen und für die übrigen Gruppen aus Hutchison (2004), siehe Tabelle A.3. Die REE-Konzentrationen in den graphischen Darstellungen sind daher nur Näherungswerte. Von großer Wichtigkeit sind in diesem Abschnitt die interREE-Fraktionierungen von Gesamtchondriten, für die absolute Konzentrationen unerheblich sind.

Kohlige Chondrite: Die REE-Muster der kohligen Chondrite sind in den Abbildungen 3.12 und 3.13 dargestellt. Die REE-Muster der fünf Proben von Orgueil zeigen nur geringe Variationen und sind innerhalb von $\pm 6 \%$ unfraktioniert, wenn sie auf ihren gemeinsamen Mittelwert normiert werden. Der scheinbare Unterschied der absoluten Konzentrationen der fünf Orgueil-Proben kann auf die interne Kalibration mit Ca zurückgeführt werden, da die Ca-Konzentrationen in CI-Chondriten um bis zu $20 \%$ voneinander abweichen können (siehe Abschnitt 3.2.3). Im Gegensatz zu Orgueil zeigen die REE-Muster von Ivuna und Alais starke Fraktionierungen von bis zu $30 \%$ mit einer Anreicherung der leichten Seltenen Erden gegenüber den schweren Seltenen Erden.

Die CM-Chondrite haben ebenfalls geringe Fraktionierungen, wobei die meisten Proben ein kleines Eu-Defizit aufweisen. Die REE-Muster von Mighei und Murray ähneln group-II-Mustern: Sie haben relative Anreicherungen an den leichten Seltenen Erden sowie an Tm und Yb, die etwa 5 bis $10 \%$ betragen. Die fünf Murchison-Proben und Murray zeigen keine charakteristischen volatilitätskontrollierten REE-Muster.

Alle untersuchten CV-Chondrite (Allende, Axtell, Mokoia und Vigarano) haben group-II-Muster. Die Probe Allende-S stammt von dem Smithsonian Reference Powder, das aus $4 \mathrm{~kg}$ Probenmenge hergestellt wurde (Jarosewich et al., 1987) und somit eine sehr repräsentative Probe für Allende darstellt. Diese Probe hat eine relative Anreicherung der leichten gegenüber den schweren Seltenen Erden von etwa $10 \%$. Die 5 kleineren Gesamtgesteinsproben von Allende (Allende-A bis Allende-E) zeigen - mit Ausnahme der Probe Allende-D - ebenfalls group-IIMuster mit unterschiedlich starken Fraktionierungen. Die stärkste Fraktionierung besitzt Allende-A: In dieser Probe sind die leichten Seltenen Erden und Tm um etwa $50 \%$ angereichert. Probe Allende-M, aus der vor dem Aufmörsern große 
a) $\mathrm{Cl}$-Chondrite

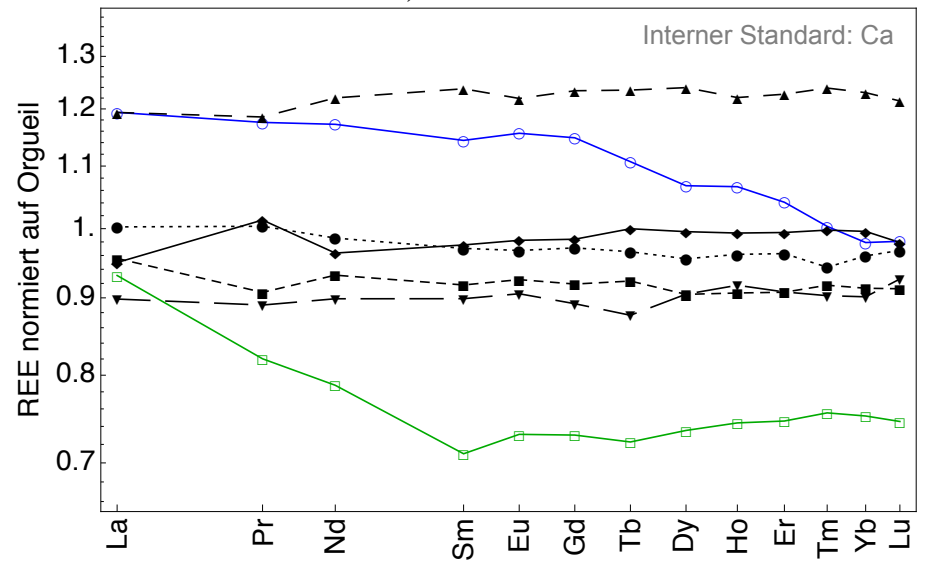

Alais $(69 \mathrm{mg})$ Ivuna (205 mg) Orgueil-A (432 mg)

- Orgueil-B (489 mg)

Orgueil-C (215 mg)

- Orgueil-D (738 mg)

$\leftarrow \quad$ Orgueil-E (98 mg)

b) $\mathrm{CM}-$ Chondrite

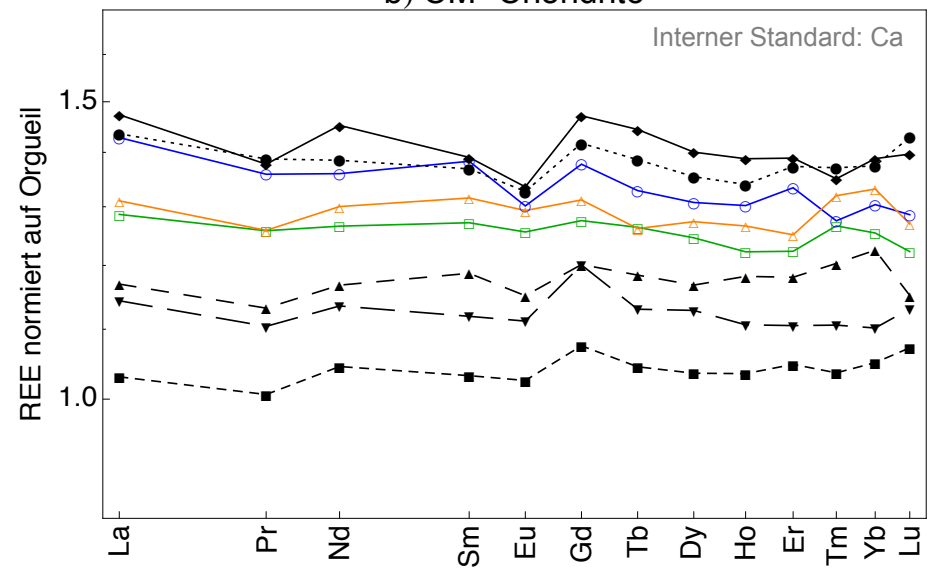

- Cold Bokkeveld (240 mg) Mighei (190 mg) Murchison-A (138 mg)

- Murchison-B (116 mg)

- Murchison-C (44 mg)

Murchison-D $(135 \mathrm{mg})$

Murchison-E (242 mg)

Murray (263 mg)

c) CV-Chondrite

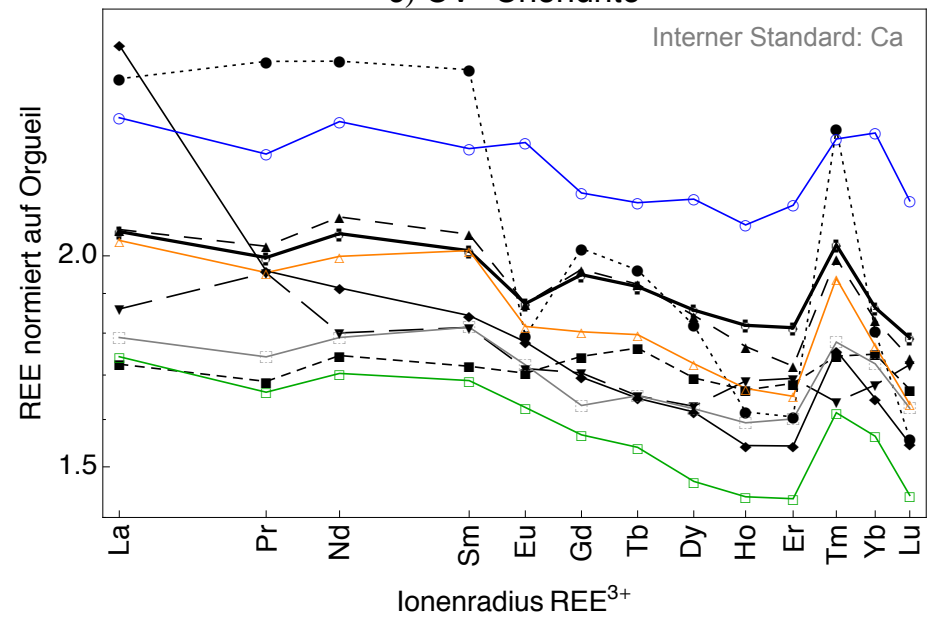

Allende-A (247 mg)

Allende-B $(225 \mathrm{mg})$

Allende-C $(221 \mathrm{mg})$

Allende-D $(1,5 \mathrm{~g})$

Allende-E $(16,6 \mathrm{~g})$

Allende-M $(5,5 \mathrm{~g})$

Allende-S (4 kg)

Axtell (ca. $150 \mathrm{mg}$ )

Mokoia $(140 \mathrm{mg})$

Vigarano (1 g)

Abbildung 3.12: LA-ICPMS-Gesamtgesteinsanalysen der Seltenen Erden in kohligen Chondriten (CI, CM, CV). Die absoluten Konzentrationen der Seltenen Erden sind Näherungswerte, da sie anhand der mittleren Ca-Gehalte der Chondritgruppen (Tabelle A.3) kalibriert wurden. 
a) CO-Chondrite

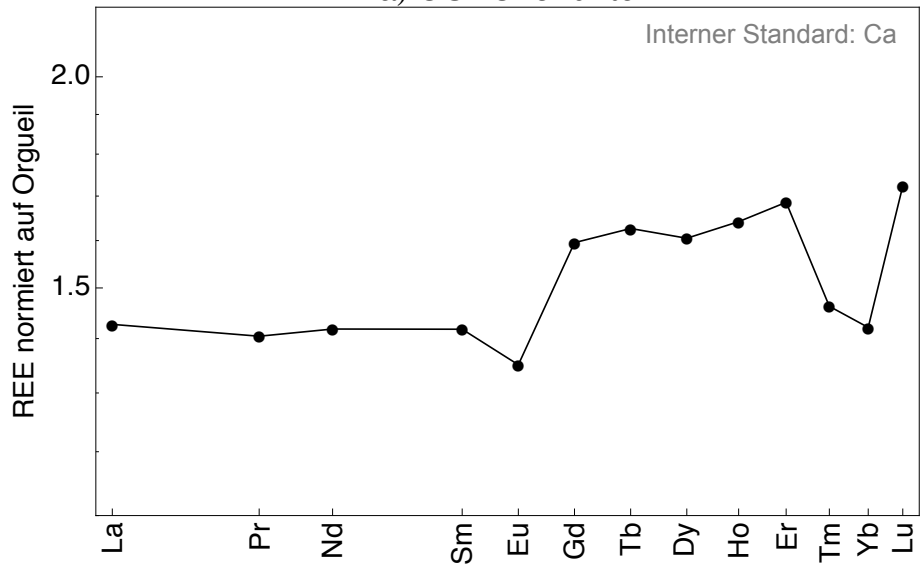

- $\quad$ NWA $3003(100 \mathrm{mg})$

b) CK-Chondrite

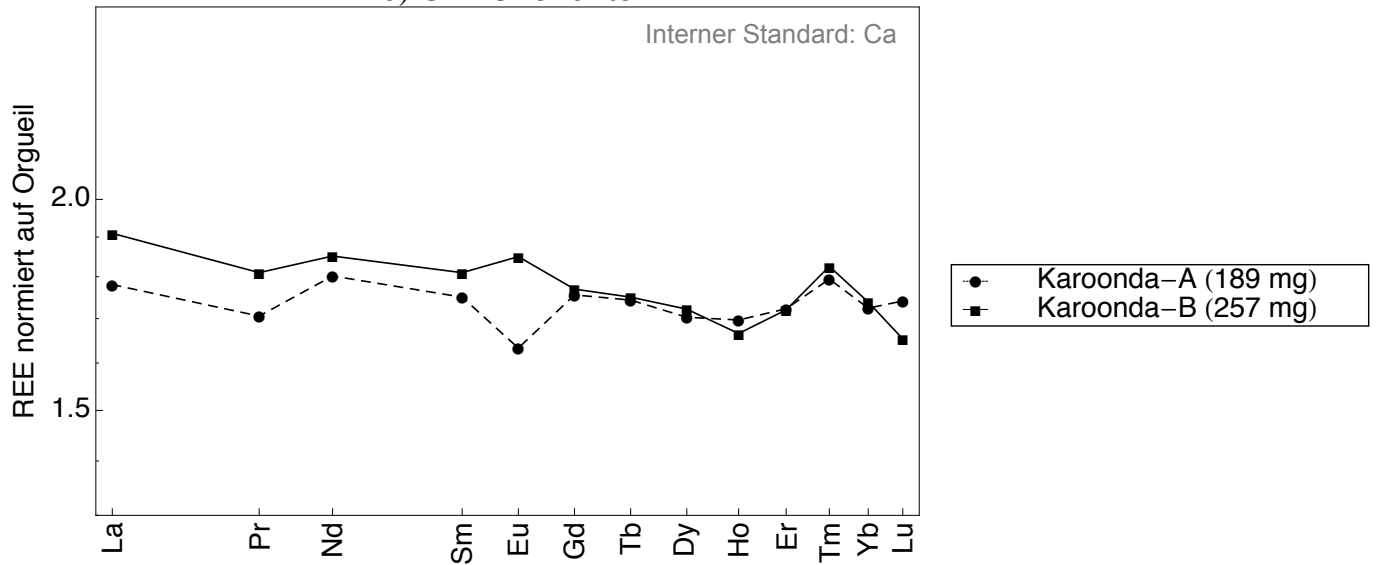

c) $\mathrm{CB}-$ Chondrite

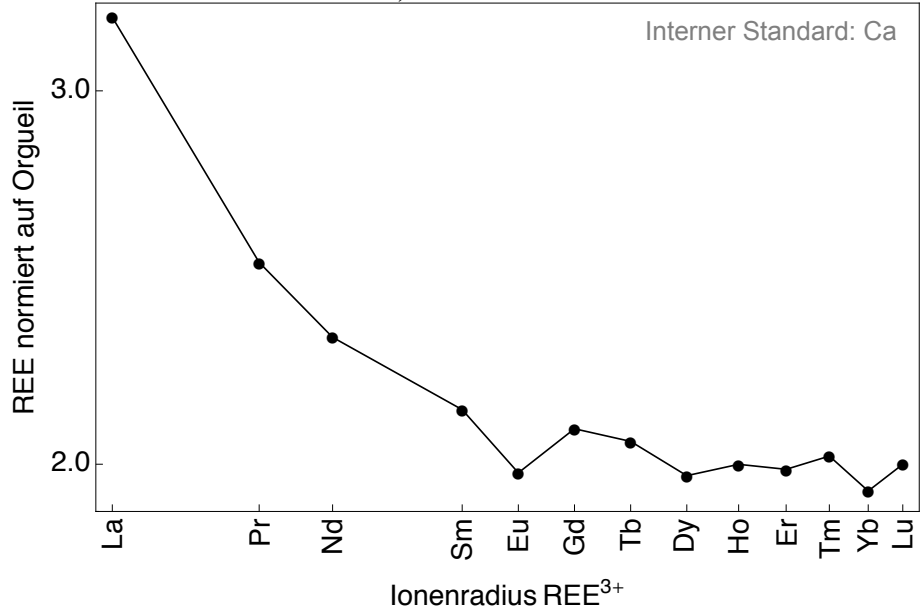

- $\quad$ Bencubbin (100 mg)

Abbildung 3.13: LA-ICPMS-Gesamtgesteinsanalysen der Seltenen Erden in kohligen Chondriten (CO, CK, CB). Die absoluten Konzentrationen der Seltenen Erden sind Näherungswerte, da sie anhand der mittleren Ca-Gehalte der Chondritgruppen (Tabelle A.3) kalibriert wurden. 
Chondren und CAIs entfernt wurden, hat eine ähnlich starke REE-Fraktionierung wie Referenzprobe von Allende.

Der CO-Chondrit North West Africa 3003 hat ein ultrarefraktäres Muster mit angereicherten schweren Seltenen Erden, aber einer signifikanten negativen Tmund Yb-Anomalie, die etwa $15 \%$ beträgt.

Die beiden von Karoonda (CK) analysierten Proben zeigen geringe Fraktionierungen mit schwach ausgeprägtem group-II-Muster. Beide Proben haben ähnliche Muster mit dem Unterschied, dass Karoonda-A eine negative und Karoonda-B eine positive Eu-Anomalie besitzen.

Von dem CB-Chondrit Bencubbin, der zu etwa $60 \%$ aus einer Metallphase und zu etwa $40 \%$ aus einer Silikatphase besteht (Kallemeyn et al., 1978), wurde nur der Silikatanteil analysiert. Er zeigt eine starke REE-Fraktionierung, wobei die leichten Seltenen Erden gegenüber den schweren Seltenen Erden angereichert sind.

Gewöhnliche Chondrite: Die gewöhnlichen Chondrite weisen nur geringe REEFraktionierungen auf (Abbildung 3.14). Es kommen allerdings häufig positive oder negative Eu-Anomalien vor. Bei den LL-Chondriten fallen Dhurmsala mit einer starken positiven und Saint-Séverin mit einer starken negativen Eu-Anomalie auf. Die L-Chondrite besitzen sehr ähnliche Muster, mit Ausnahme von Bruderheim, der eine Anreicherung der leichten Seltenen Erden sowie eine positive Eu-Anomalie hat. Die H-Chondrite zeigen ebenfalls ähnliche REE-Muster. Einige haben jedoch Anreicherungen der leichten Seltenen Erden. Insbesondere Adrian (H4) zeigt ein ungewöhnliches Muster mit einer sehr starken, etwa 30-fachen Anreicherung der leichten Seltenen Erden gegenüber Orgueil (Abbildung 3.15).

Alle gewöhnlichen Chondrite haben gemeinsam, dass sie gegenüber Orgueil eine geringe negative Anomalie an Tm von durchschnittlich etwa vier bis fünf Prozent besitzen. Viele der gewöhnlichen Chondrite haben zusätzlich ein Defizit an Yb, das jedoch noch kleiner ist als die Tm-Anomalie.

Um die Homogenität bezüglich der Seltenen Erden zu untersuchen, wurden an zwei gewöhnlichen Chondriten Messungen an kleinen Probenmengen durchgeführt (siehe Abbildung 3.16 und Tabelle A.4). Es wurden hierbei die inter-REEFraktionierungen, also nicht die absoluten Konzentrationen, untersucht. Von dem L-Chondrit North West Africa (NWA) 869 wurden 11 Proben mit 20 bis $30 \mathrm{mg}$ Probenmasse analysiert: Die REE-Verhältnisse stimmen in zehn der elf Proben innerhalb von etwa \pm 10 bis $\pm 20 \%$ überein. Die Abweichungen der elften Probe vom Mittelwert sind mit ca. $\pm 25 \%$ etwas größer. Der Vergleich des Mittelwertes der elf kleinen Proben (Gesamtmasse $270 \mathrm{mg}$ ) mit der größeren untersuchten Probe $(>3 \mathrm{~g})$ zeigt für alle Seltenen Erden nur geringe Abweichungen von wenigen Prozent $(<6 \%)$. 
a) LL-Chondrite

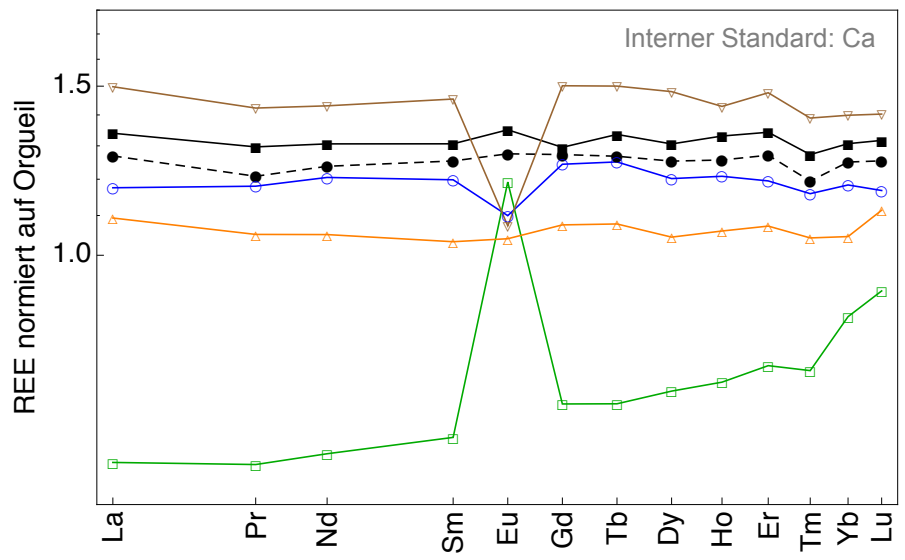

-. Chainpur-A (Fall)

- Chainpur-B (Fall)

- $\quad$ Dar al Gani 298 (Fund)

Dhurmsala (Fall)

Lake Labyrinth (Fund)

Saint-Séverin (Fall)

b) L-Chondrite

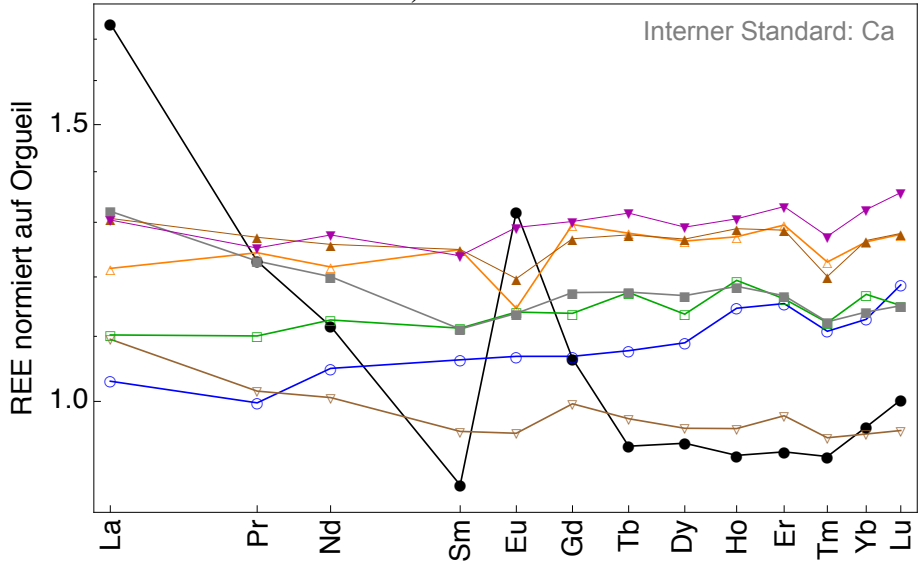

\begin{tabular}{|cl|}
\hline$\bullet$ & Bruderheim (Fall) \\
- & Gashua (Fall) \\
$\square$ & Homestead (Fall) \\
$\star$ & Mount Tazerzait (Fall) \\
- & NWA 869 (Fund) \\
- & SAU 001 (Fund) \\
- & St. Michel (Fall) \\
- & Zavid (Fall) \\
\hline
\end{tabular}

c) $\mathrm{H}$-Chondrite

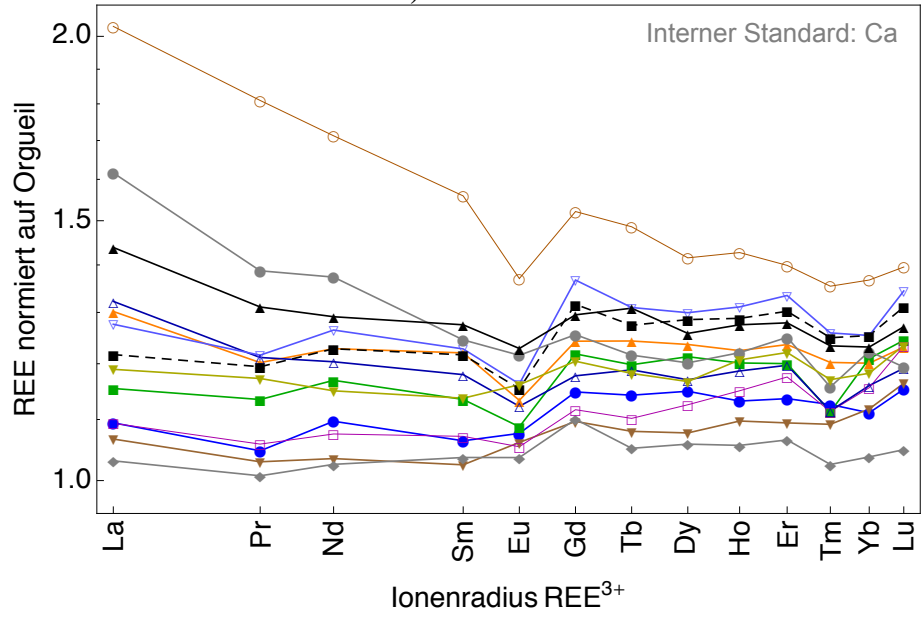

Dar al Gani 300 (Fund)

Djoumine (Fund)

El Hammami (Fund)

Forest City (Fall)

Gao-Guenie (Fall)

Hammond Downs (Fund)

ljopega (Fall)

Jilin (Fall)

Kunya-Urgench (Fall)

Pultusk-A (Fall)

Pultusk-B (Fall)

Richardton (Fall)

Thuathe (Fall)

Abbildung 3.14: LA-ICPMS-Gesamtgesteinsanalysen der Seltenen Erden in gewöhnlichen Chondriten (LL, L und H). Die absoluten Konzentrationen der Seltenen Erden sind Näherungswerte, da sie anhand der mittleren Ca-Gehalte der Chondritgruppen (Tabelle A.3) kalibriert wurden. 


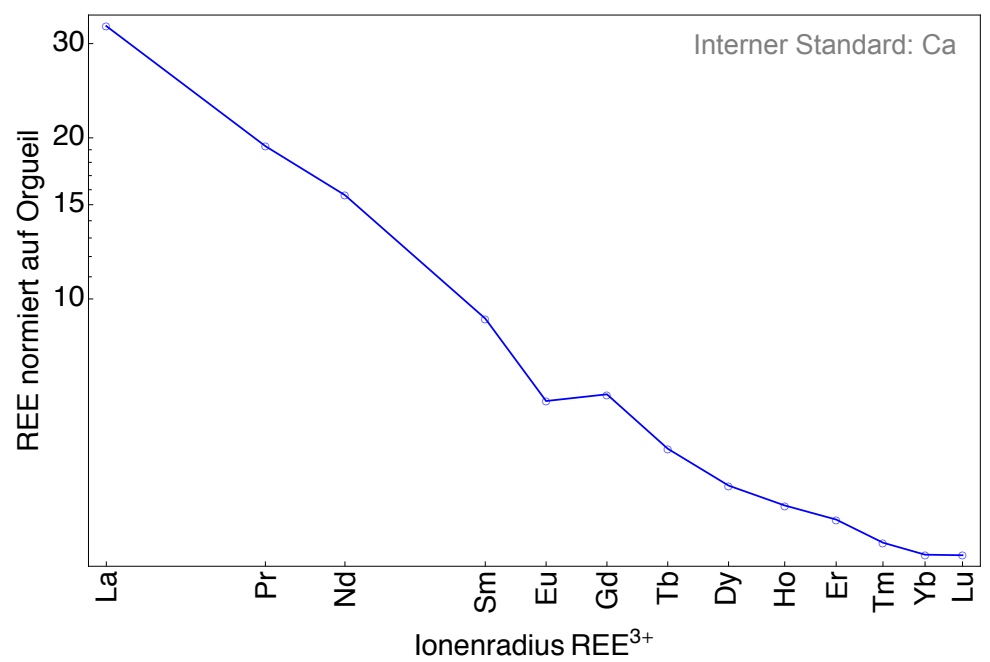

Abbildung 3.15: Seltene Erden in dem H-Chondrit Adrian. Die absoluten Konzentrationen der Seltenen Erden sind Näherungswerte, da sie anhand des mittleren Ca-Gehalte der H-Chondrite (Tabelle A.3) kalibriert wurden.

Zusätzlich wurden von dem H-Chondrit Gao-Guenie sechs Proben, die zwischen 30 und $40 \mathrm{mg}$ wogen, untersucht: Die REE-Verhältnisse stimmen hier ebenfalls innerhalb von etwa \pm 10 bis $\pm 20 \%$ überein, mit Ausnahme von Lanthan und Europium (bis zu $\pm 30 \%$ ). Der Mittelwert der sechs kleinen Proben (Gesamtmasse ca. $200 \mathrm{mg}$ ) stimmt für alle Seltenen Erden außer La innerhalb von $\pm 7 \%$ mit der größeren analysierten Probe (915 mg) überein. Für La beträgt die Abweichung etwa $30 \%$. Auf einer Gewichtsskala von einigen hundert Milligramm betragen die inter-REE-Fraktionierungen in gewöhnlichen Chondriten also typischerweise etwa \pm 5 bis $\pm 10 \%$.

Rumuruti-Chondrit: Der Rumuruti-Chondrit North West Africa 753 zeigt ebenfalls nur geringe Fraktionierungen. Er besitzt jedoch eine kleine positive EuAnomalie und weist, genau wie die gewöhnlichen Chondrite, eine etwa 5\%ige negative Tm-Anomalie auf (Abbildung $3.17 \mathrm{a}$ ).

Enstatit-Chondrite: Die EL-Chondrite haben ebenfalls eher geringe REEFraktionierungen, sie besitzen jedoch negative Eu-Anomalien (Abbildung $3.17 \mathrm{~b}$ ). Die EH-Chondrite haben unregelmäßige REE-Muster (Abbildung $3.17 \mathrm{c}$ ). Beide Gruppen der Enstatit-Chondrite zeigen, gleichermaßen wie gewöhnliche Chondrite, geringe Defizite an Tm und Yb. 

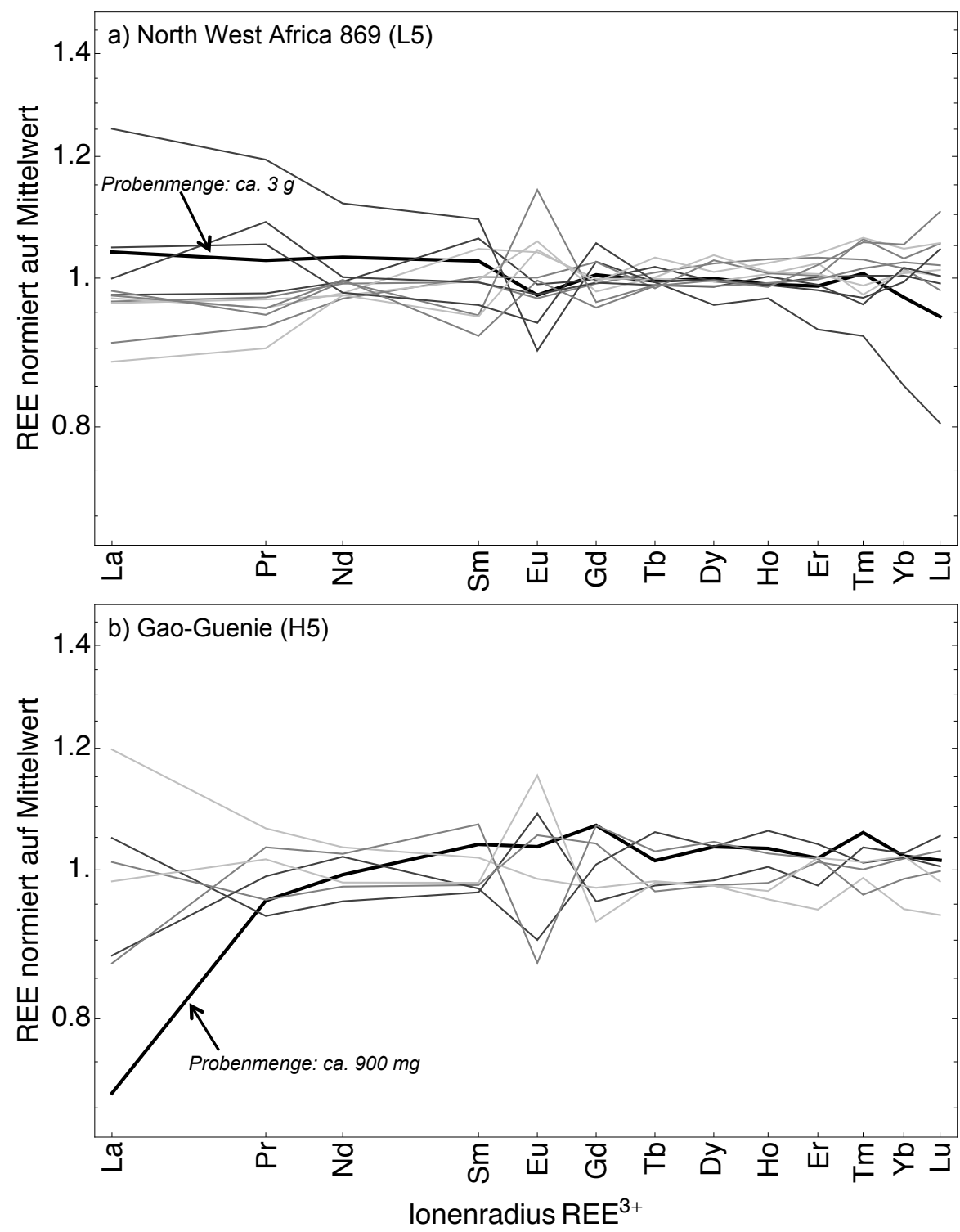

Abbildung 3.16: REE-Muster mehrerer kleiner sowie jeweils einer größeren Probe von a) North West Africa 869 und b) Gao-Guenie. 
a) R-Chondrite
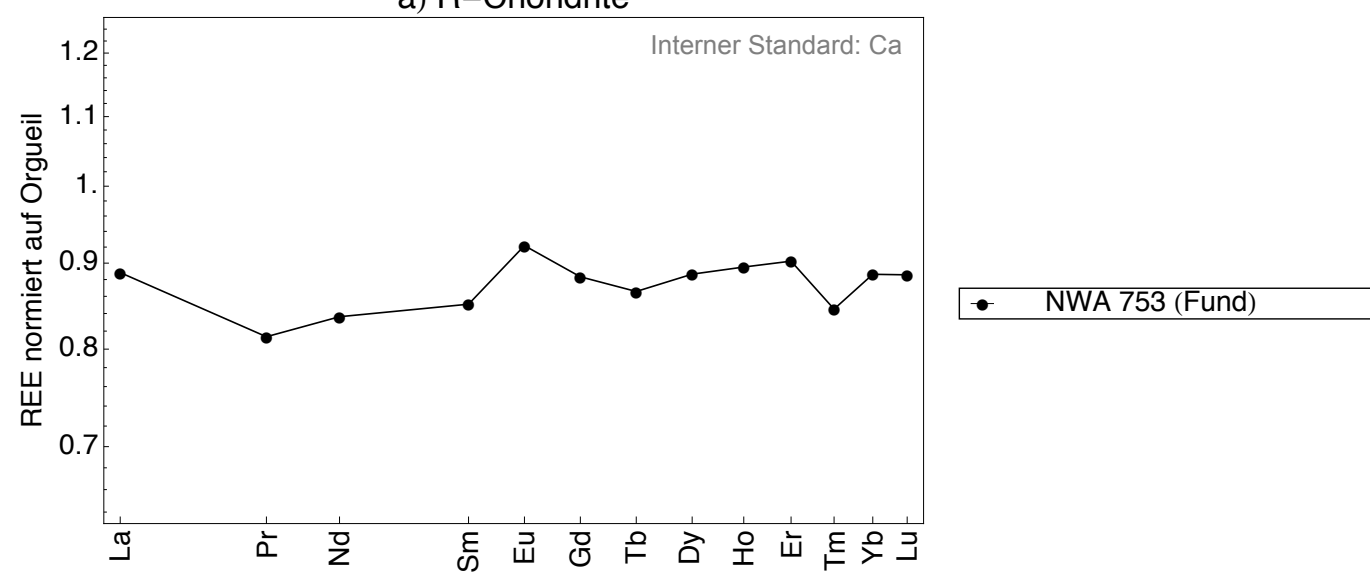

b) EL-Chondrite

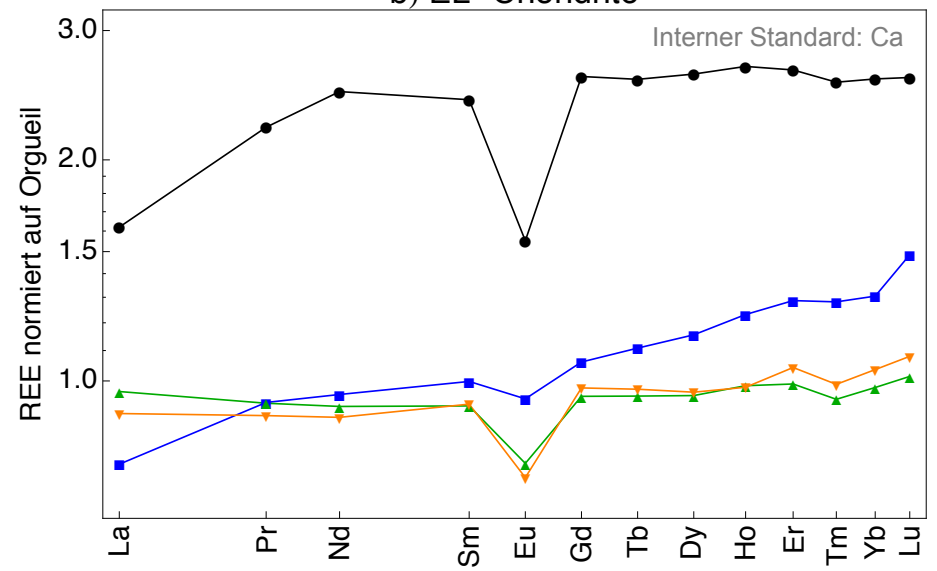

- $\quad$ Atlanta (Fund)

Eagle (Fund)

Hvittis (Fall)

Pillistfer (Fall)

c) EH-Chondrite

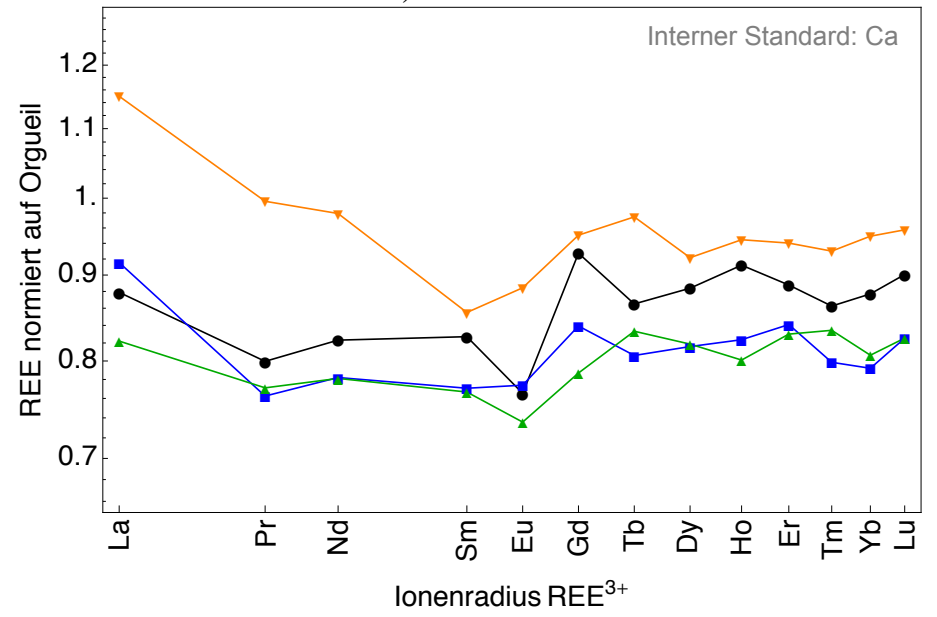

$\bullet \quad$ Abee (Fall)

Indarch (Fall)

Sahara 97072 (Fund)

St. Mark's (Fall)

Abbildung 3.17: LA-ICPMS-Gesamtgesteinsanalysen der Seltenen Erden in Rumuruti- und Enstatit-Chondriten (R, EL und EH). Die absoluten Konzentrationen der Seltenen Erden sind Näherungswerte, da sie anhand der mittleren Ca-Gehalte der Chondritgruppen (Tabelle A.3) kalibriert wurden. 


\subsubsection{REE-Muster von Achondriten und terrestrischen Proben}

In den Abbildungen 3.18 und 3.19 sind beispielhaft die REE-Muster einiger Achondrite inklusive der Mond- und Marsmeteorite sowie einiger terrestrischer Gesteinsarten dargestellt: Alle Proben weisen gleichmäßige REE-Fraktionierungen in Abhängigkeit des Ionenradius auf, die auf magmatische Prozesse zurückzuführen sind.

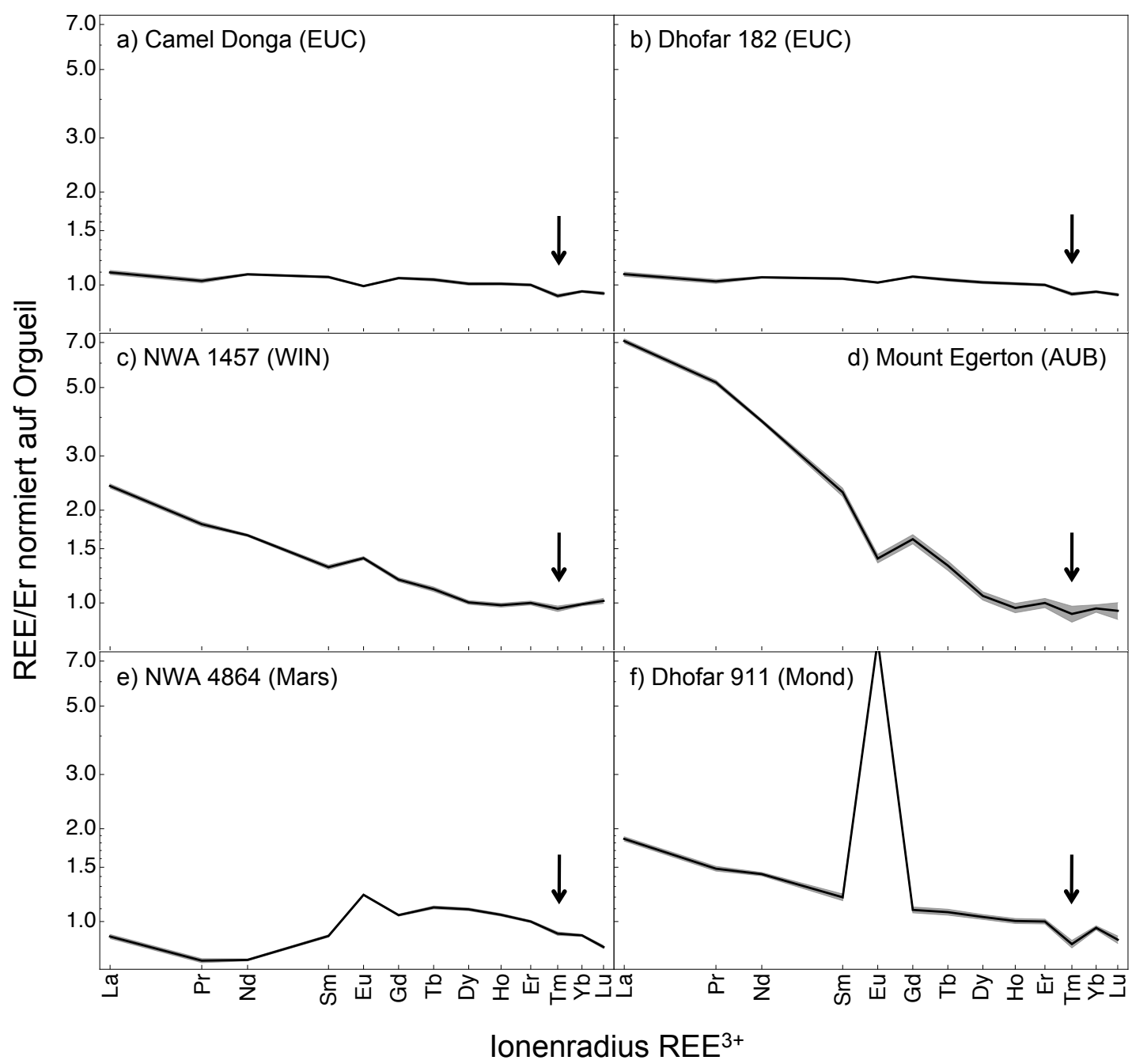

Abbildung 3.18: REE-Muster von differenzierten Meteoriten: a) und b) Eukrite, c) Winonait, d) Aubrit, e) Mars-Meteorit und f) Mond-Meteorit. Abgesehen von der gleichmäßigen Fraktionierung der Seltenen Erden gemäß ihrem Ionenradius sowie den zu erwartenden Eu-Anomalien fällt auf, dass alle Proben ein leichtes Tm-Defizit haben (Pfeile). 
Abweichend davon kommen positive und negative Eu-Anomalien vor, die ebenfalls durch magmatische Prozesse erklärt werden können, da Eu im Gegensatz zu den anderen Seltenen Erden zu einem großen Teil auch als zweiwertiges $\mathrm{Eu}^{2+}$ vorkommt, das aufgrund des größeren Ionenradius bevorzugt in andere Mineralphasen eingebaut wird als die dreiwertigen Seltenen Erden.

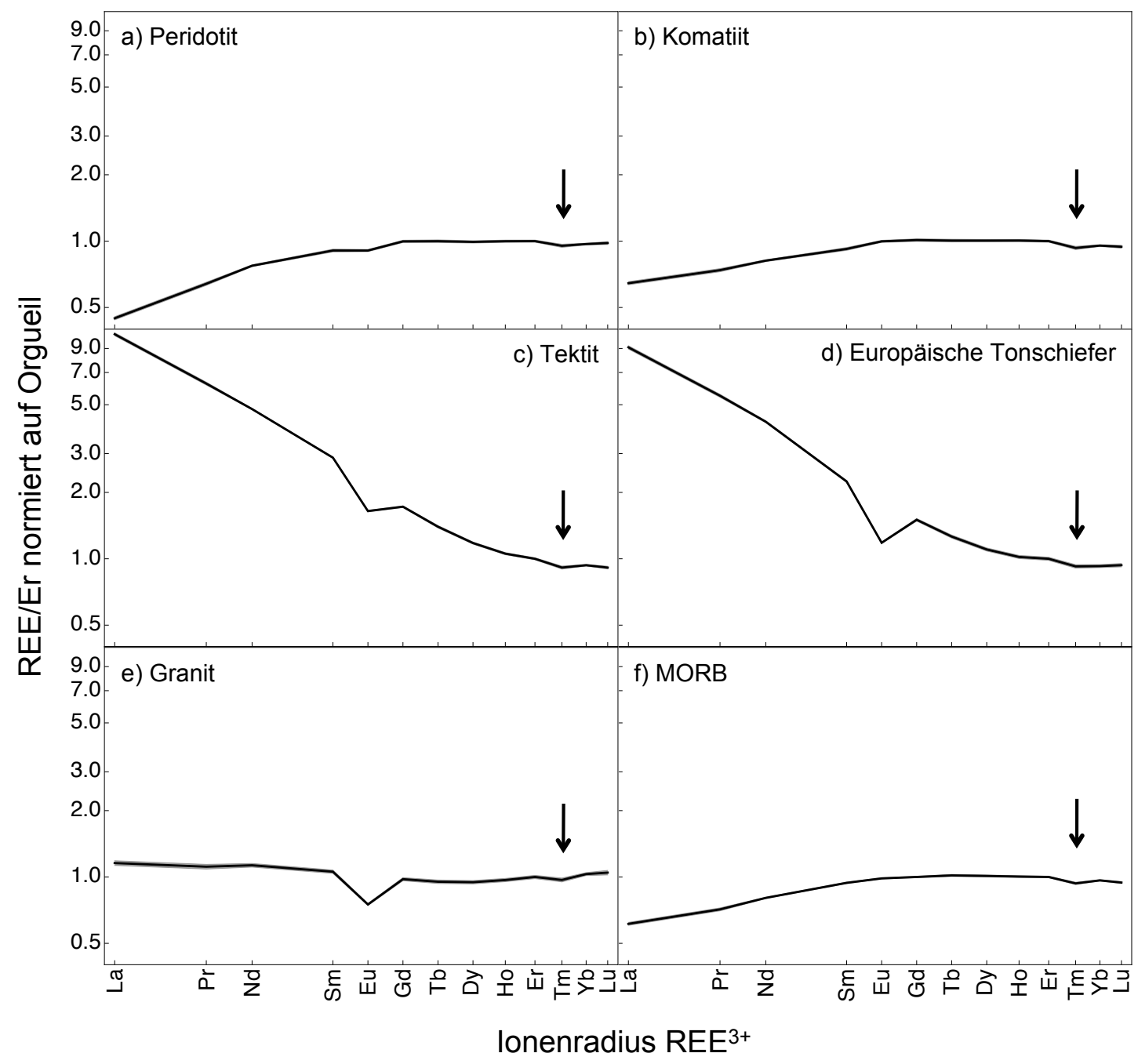

Abbildung 3.19: REE-Muster terrestrischer Proben aus unterschiedlichen geologischen Reservoiren: a) Peridotit (SC-1), b) Komatiit (24803), c) Tektit (MOL), d) Europäische Tonschiefer (ES), e) Granit (Pan-02-047) und f) MORB (137 DS-4). Abgesehen von der gleichmäßigen Fraktionierung der Seltenen Erden gemäß ihrem Ionenradius sowie den zu erwartenden Eu-Anomalien fällt auf, dass alle Proben ein leichtes Tm-Defizit haben (Pfeile). 
Auffallend ist, dass alle Proben im Vergleich zu Orgueil ein geringes Tm-Defizit von durchschnittlich etwa 5\% Prozent aufweisen, das nicht auf geochemische Prozesse zurückzuführen ist. Auf die REE-Muster der einzelnen Achondrite und terrestrischen Gesteine soll hier nicht ausführlich eingegangen werden; es sind für diese Arbeit lediglich die relativen Tm-Defizite, die durch volatilitätskontrollierte Fraktionierung entstanden sind, von Interesse. Es kann also festgehalten werden, dass die differenzierten Gesteinsproben der Erde und der Achondrite ein geringes relatives Tm-Defizit aufweisen, das mit ungefähr $5 \%$ etwa gleich groß ist wie das Tm-Defizit der nichtkohligen Chondrite (siehe Abschnitt 3.2.4).

\subsubsection{HFSE-Elemente}

Die mittels Isotopenverdünnungs-MC-ICPMS gemessenen Konzentrationen der HFSEs in Bulk-Chondriten sind in Tabelle 3.4 angegeben. In Abbildung 3.20 sind $\mathrm{Zr} / \mathrm{Hf}-$ und $\mathrm{Nb} / \mathrm{Ta}-$ Verhältnisse zusammen mit den Ergebnissen der LA-ICPMS dargestellt. Diese wurden anhand des Basaltstandards BHVO kalibriert (siehe Abschnitt 2.3.4) und sind in guter Übereinstimmung mit den Ergebnissen der MC-ICPMS. Es fällt allerdings auf, dass die Daten der LA-ICPMS stärker streuen als die der MC-ICPMS. Dies ist zum Teil darauf zurückzuführen, dass mittels LAICPMS eine größere Anzahl Proben gemessen wurde, darunter solche mit geringen Probenmengen unter $100 \mathrm{mg}$, sodass aufgrund der Probeninhomogenität eine größere Streuung der Daten entstanden ist. Beim Vergleich identischer Proben, die jeweils mit beiden Methoden gemessen wurden, zeigen die LA-ICPMS-Daten trotzdem eine etwas größere Streuung, die im Vergleich zu den Daten der MC-ICPMS für die $\mathrm{Zr} / \mathrm{Hf}$-Verhältnisse etwa doppelt so hoch und für die $\mathrm{Nb} / \mathrm{Ta}-$ Verhältnisse etwa dreimal so hoch ist.

Beide Methoden liefern jedoch übereinstimmende Ergebnisse. Die Zr/HfVerhältnisse aller Chondritgruppen ähneln dem von Münker et al. (2003) bestimmten chondritischen Mittelwert von 34,3. Dabei zeigen die kohligen Chondrite nur eine geringe Streuung und stimmen innerhalb von $\pm 5 \%$ mit diesem Verhältnis überein. Die CI-Chondrite entsprechen im Durchschnitt dem chondritischen Mittelwert von 34,3, während die anderen kohligen Chondrite mit einem durchschnittlichen Verhältnis von 33,5 um 2,5\% geringfügig niedriger liegen. Im Gegensatz zu den kohligen Chondriten zeigen die nichtkohligen Chondrite eine größere Streuung mit maximalen Abweichungen von dem chondritischen Mittelwert um bis zu etwa $\pm 15 \%$. Der durchschnittliche Wert der nichtkohligen Chondrite von $34,1 \pm 0,3(1 \sigma)$ stimmt aber mit dem chondritischen Mittelwert überein.

Viele der gewöhnlichen und Enstatit-Chondrite wurden während der Probenpräparation in einem Muffelofen oxidiert (siehe Tabelle 2.2). Wegen der eventuellen Kontamination durch Nb- oder Ta-haltige Heizdrähte sind die Daten dieser Proben nicht dargestellt. Die Nb/Ta-Verhältnisse der kohligen Chondrite zeigen eine relativ 


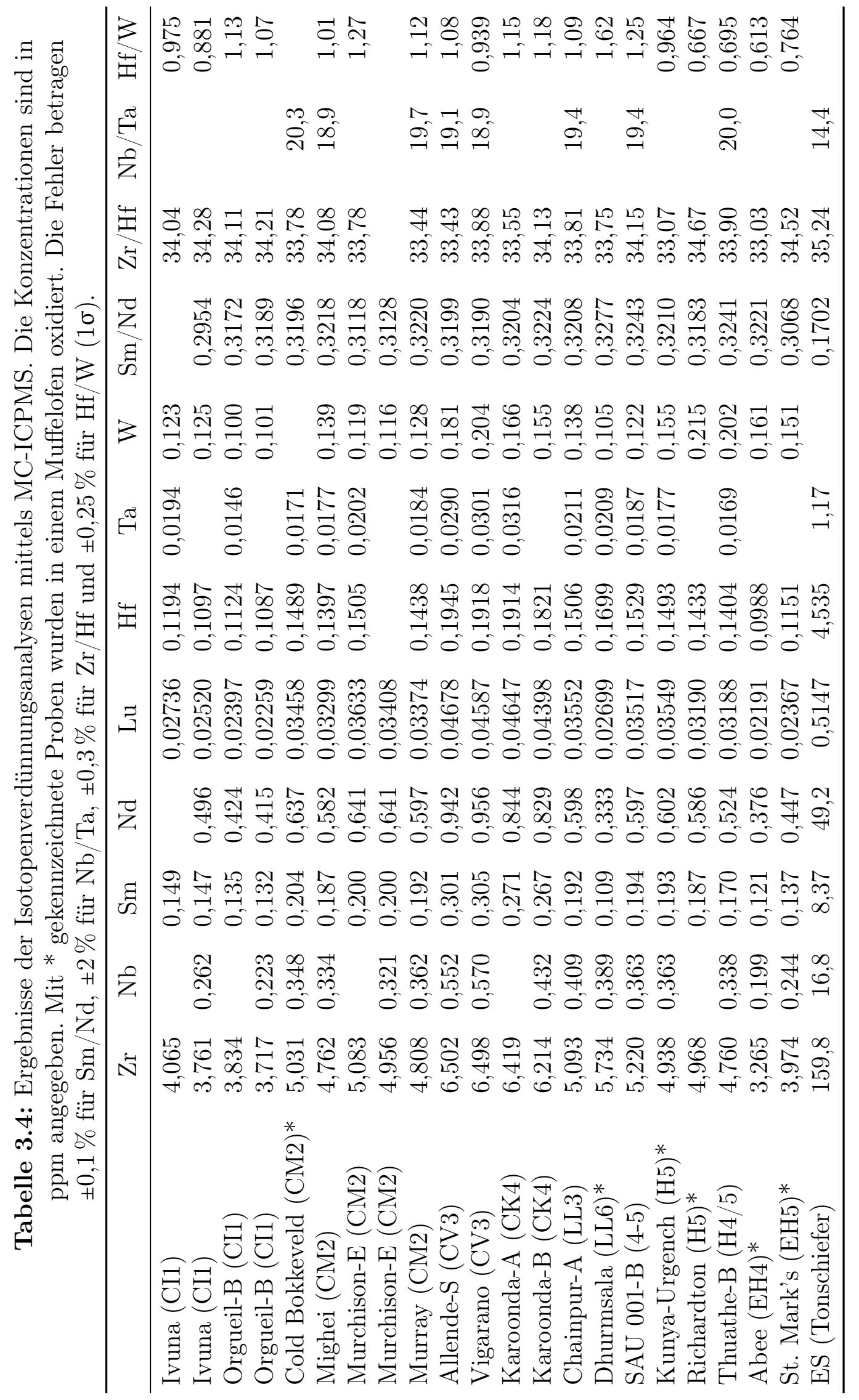


starke Streuung um den chondritischen Mittelwert von 19,9 (Münker et al., 2003), wobei die meisten kohligen Chondrite tendenziell etwas geringere Werte haben als die CI-Chondrite. Die Probe Allende-A, die außerdem ein stark fraktioniertes REE-Muster hat, fällt durch ein besonders niedriges Nb/Ta-Verhältnis von 11,0 auf. Bencubbin (CB) hat ebenfalls einen ungewöhnlich niedrigen Wert von 9,4. Im Gegensatz zu den kohligen Chondriten zeigen die Nb/Ta-Verhältnisse der gewöhnlichen Chondrite nur eine geringe Streuung, sie stimmen außerdem sehr gut mit dem chondritischen Mittelwert überein.
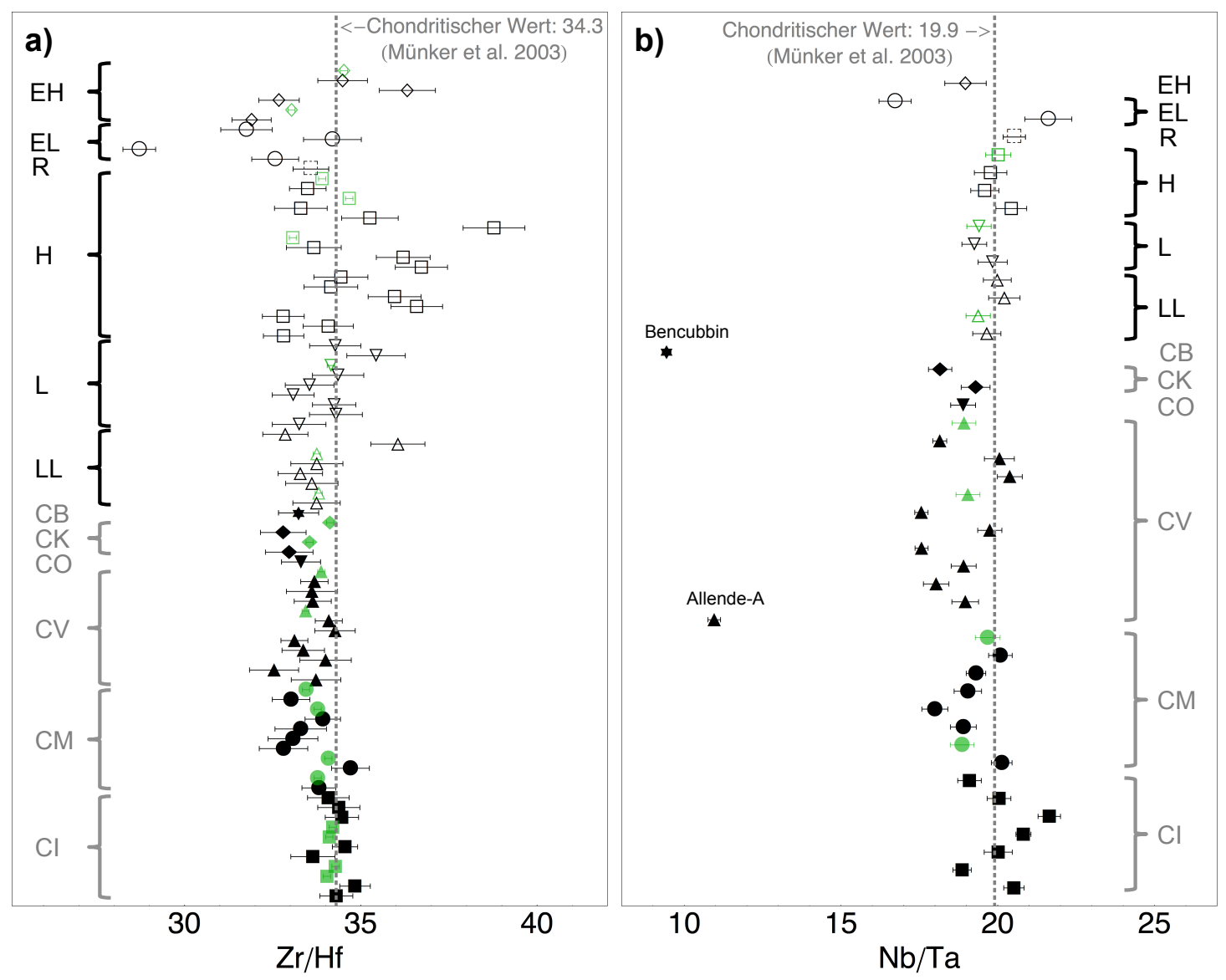

Abbildung 3.20: Zr/Hf-Verhältnisse (a) und Nb/Ta-Verhältnisse (b) in Chondri-

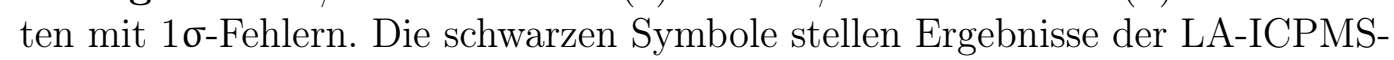
Analysen dar und wurden extern mit dem Basaltstandard BHVO kalibriert. Die grünen Symbole sind Ergebnisse der MC-ICPMS-Analysen. 


\section{Diskussion}

\subsection{Vergleich der LA-ICPMS-Daten mit Literaturdaten}

Der Basaltstandard BCR-2G wurde regelmäßig während aller Sessions gemessen. Er wurde dafür nicht als Quenchkugel präpariert. Die Ergebnisse wurden anschließend mit durch Lösungs- oder Laserablations-ICPMS gewonnenen Literaturdaten verglichen (siehe Tabelle 4.1). Gemittelte REE-Verhältnisse (REE/Er)

Tabelle 4.1: Literaturvergleich der Seltenen Erden in BCR-2G (*BCR-2).

\begin{tabular}{|c|c|c|c|c|c|c|c|c|c|c|}
\hline & \multirow{2}{*}{$\begin{array}{l}\text { diese } \\
\text { Arbeit }\end{array}$} & \multicolumn{9}{|c|}{ Literaturdaten } \\
\hline & & {$[1]$} & {$[2]$} & {$[3]$} & {$[4]$} & {$[5]$} & [6] & [7] & {$[8]^{*}$} & {$[9]^{*}$} \\
\hline La & 24,7 & 25,38 & 26,15 & 24,8 & 25 & 26,9 & 25,7 & 24,8 & 24,59 & 24,82 \\
\hline $\mathrm{Ce}$ & 51,9 & 52,82 & 57,03 & 52,5 & 52 & 53,1 & 53,9 & 52,1 & 53,3 & 53,48 \\
\hline $\operatorname{Pr}$ & 6,54 & 6,77 & 7,39 & 6,94 & 6,3 & 7,55 & 7,16 & 6,77 & 7,04 & 6,798 \\
\hline $\mathrm{Nd}$ & 28,1 & 28,71 & 30,26 & 28,2 & 27 & 30,6 & 31,1 & 28,5 & 28,5 & 28,9 \\
\hline $\mathrm{Sm}$ & 6,47 & 6,5 & 6,9 & 6,39 & 6,3 & 7,35 & 7,02 & 6,47 & 6,68 & 6,522 \\
\hline $\mathrm{Eu}$ & 1,94 & 1,87 & 2,04 & 1,98 & 1,91 & 2,13 & 2,17 & 1,97 & 1,99 & 1,964 \\
\hline $\mathrm{Gd}$ & 6,38 & 6,31 & 6,72 & 6,76 & 6,5 & 6,52 & 6,85 & 6,62 & 6,24 & 6,79 \\
\hline $\mathrm{Tb}$ & 0,99 & 0,95 & 1,04 & 1,05 & 0,95 & 1,07 & 1,05 & 1,1 & 1,04 & 1,046 \\
\hline Dy & 6,12 & 6,23 & 6,43 & 6,32 & 6 & 6,23 & 6,56 & 6,4 & 6,2 & 6,444 \\
\hline Ho & 1,25 & 1,32 & 1,36 & 1,3 & 1,2 & 1,33 & 1,34 & 1,28 & 1,33 & 1,284 \\
\hline Er & 3,51 & 3,65 & 3,6 & 3,64 & 3,3 & 3,68 & 3,55 & 3,69 & 3,48 & 3,642 \\
\hline $\mathrm{Tm}$ & 0,488 & 0,49 & 0,46 & 0,523 & 0,46 & 0,53 & 0,53 & 0,512 & 0,52 & 0,515 \\
\hline $\mathrm{Yb}$ & 3,36 & 3,33 & 3,49 & 3,52 & 3,2 & 3,41 & 3,53 & 3,43 & 3,35 & 3,347 \\
\hline $\mathrm{Lu}$ & 0,483 & 0,5 & 0,52 & 0,508 & 0,47 & 0,52 & 0,51 & 0,497 & 0,5 & 0,489 \\
\hline
\end{tabular}

[1] Lahaye et al. (1997), LA-HR-ICPMS; [2] Lahaye et al. (1997), Lösungs-HRICPMS; [3] Dulski (2001), ICPMS; [4] Gao et al. (2002), LA-ICPMS; [5] Strnad et al. (2005), Lösungs-ICPMS; [6] Strnad et al. (2005), LA-ICPMS; [7] Willbold und Jochum (2005), SF-ICPMS; [8] Huang et al. (2007), ICPMS; [9] Pourmand et al. (2012), MC-ICPMS. 
aus über 60 Messungen dieser Arbeit stimmen innerhalb von \pm 1 bis $\pm 3 \%$ mit dem Mittelwert der Literaturdaten überein (mit Ausnahme von $\operatorname{Pr}: \pm 4,4 \%$ ) und demonstrieren eine hohe Genauigkeit der gewählten Methode (Abbildung 4.1).

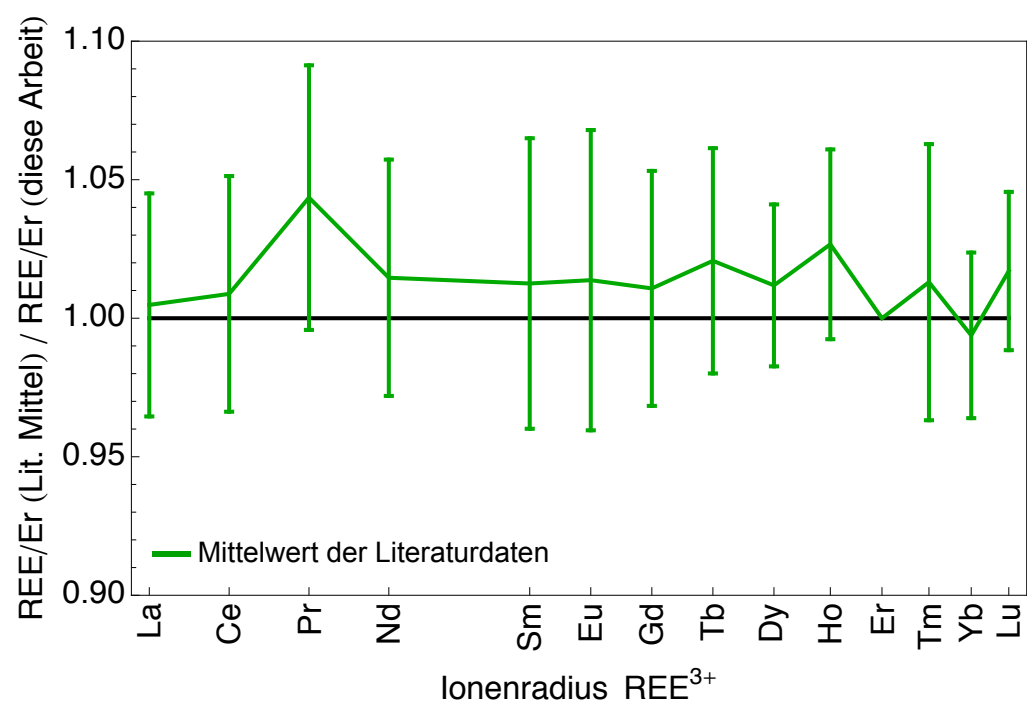

Abbildung 4.1: Vergleich der mittels LA-ICPMS gemessenen REE/ErVerhältnisse in BCR-2G mit dem Mittelwert aus neun Literaturdatensätzen

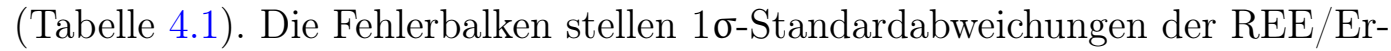
Verhältnisse der Literaturdaten dar.

Da in dieser Arbeit der CI-Chondrit Orgueil für die Normierung der Seltenen Erden der anderen Proben herangezogen wird, ist eine genaue Bestimmung der REE-Verhältnisse in Orgueil nötig. Dafür wurden insgesamt 46 Messungen an fünf verschiedenen Orgueil-Proben durchgeführt. Die 1 $\sigma$-Standardabweichungen der REE/Er-Verhältnisse der fünf Proben sind kleiner als $\pm 5 \%$ für die leichten REE und kleiner als $\pm 2 \%$ für die schweren REE. Die $1 \sigma$-Standardfehler der Mittelwerte betragen weniger als $\pm 2,5 \%$ für die leichten und $\pm 1 \%$ für die schweren Seltenen Erden.

Der Mittelwert von Orgueil wurde mit zwei Literaturdatensätzen (Barrat et al., 2012; Pourmand et al., 2012) verglichen, die mittels ICP-SFMS (ICP sector field mass spectrometry) bzw. MC-ICPMS gewonnen wurden und ebenfalls aus je fünf Proben gemittelt sind. Die REE/Er-Verhältnisse der drei Datensätze stimmen innerhalb von $\pm 5 \%$ überein (Abbildung $4.2 \mathrm{a}$ ). Es wurde ebenfalls ein Vergleich mit dem Referenzpulver des CV-Chondrits Allende durchgeführt: Die REE/ErVerhältnisse stimmen hier innerhalb von $\pm 10 \%$ überein (Abbildung $4.2 \mathrm{~b}$ ). Die Abweichungen der verschiedenen Methoden können auf Probeninhomogenitäten und systematischen Fehlern wie der Kalibration der LA-ICPMS-Daten mit dem externen Standard NIST SRM 612 beruhen. 


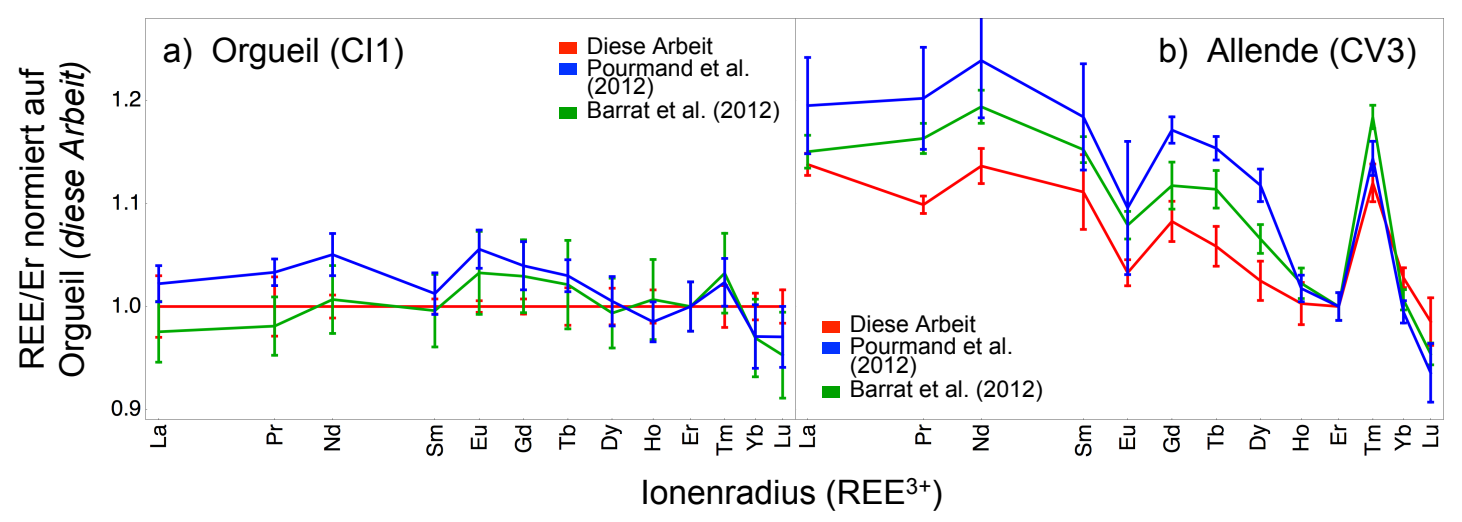

Abbildung 4.2: Vergleich der durch LA-ICPMS gemessenen REE/Er-Verhältnisse mit Literaturdaten (Tabelle 4.2) in Orgueil (a) und Allende (b). Die Fehlerbalken stellen 1 $\sigma$-Standardabweichungen dar.

Tabelle 4.2: Literaturvergleich der Seltenen Erden in Orgueil und Allende.

\begin{tabular}{l|c|cc|c|cc}
\hline & \multicolumn{3}{|c|}{ Orgueil } & \multicolumn{3}{|c}{ Allende } \\
& diese & \multicolumn{2}{|c|}{ Literaturdaten } & diese & \multicolumn{2}{c}{ Literaturdaten } \\
& Arbeit & {$[1]$} & {$[2]$} & Arbeit & {$[1]$} & {$[2]$} \\
\hline $\mathrm{La} / \mathrm{Er}$ & 1,45 & 1,41 & 1,48 & 1,65 & 1,67 & 1,73 \\
$\mathrm{Pr} / \mathrm{Er}$ & 0,558 & 0,547 & 0,576 & 0,613 & 0,649 & 0,670 \\
$\mathrm{Nd} / \mathrm{Er}$ & 2,77 & 2,79 & 2,91 & 3,15 & 3,31 & 3,43 \\
$\mathrm{Sm} / \mathrm{Er}$ & 0,922 & 0,918 & 0,934 & 1,024 & 1,062 & 1,092 \\
$\mathrm{Eu} / \mathrm{Er}$ & 0,341 & 0,352 & 0,360 & 0,352 & 0,368 & 0,374 \\
$\mathrm{Gd} / \mathrm{Er}$ & 1,20 & 1,24 & 1,25 & 1,30 & 1,35 & 1,41 \\
$\mathrm{~Tb} / \mathrm{Er}$ & 0,221 & 0,226 & 0,228 & 0,234 & 0,246 & 0,255 \\
$\mathrm{Dy} / \mathrm{Er}$ & 1,54 & 1,53 & 1,55 & 1,58 & 1,64 & 1,72 \\
$\mathrm{Ho} / \mathrm{Er}$ & 0,338 & 0,340 & 0,333 & 0,339 & 0,345 & 0,344 \\
$\mathrm{Tm} / \mathrm{Er}$ & 0,152 & 0,157 & 0,156 & 0,171 & 0,180 & 0,174 \\
$\mathrm{Yb} / \mathrm{Er}$ & 1,04 & 1,01 & 1,01 & 1,07 & 1,05 & 1,04 \\
$\mathrm{Lu} / \mathrm{Er}$ & 0,155 & 0,148 & 0,151 & 0,153 & 0,148 & 0,145 \\
\hline $\mathrm{Din} \mathrm{Ref}$ & & &
\end{tabular}

Die Referenzwerte der REE/Er-Verhältnisse sind berechnet aus Daten von [1] Barrat et al. (2012) und [2] Pourmand et al. (2012). 


\subsection{Komponenten von Leoville}

\subsubsection{Refraktäre Einschlüsse}

Die untersuchten refraktären Einschlüsse (vier CAIs und ein AOA) haben fraktionierte REE group-II-Muster (siehe Abbildung 3.3). Aus anderen Analysen refraktärer Einschlüsse Leovilles (Mao et al., 1990) sowie anderer CV-Chondrite (Allende, Kaba, Mokoia, Vigarano) ist bekannt, dass diese zwar häufig group-II-Muster besitzen, dass aber auch solche mit relativ unfraktionierten Seltenen Erden - abgesehen von Anomalien in Eu und Yb - vorkommen (Martin und Mason, 1974; Grossman und Ganapathy, 1976a; Grossman et al., 1979; Mason und Taylor, 1982; Liu und Schmitt, 1988; Mao et al., 1990; Sylvester et al., 1993; Huang et al., 2012). Refraktäre Einschlüsse mit solchen unfraktionierten REE-Mustern wurden in der hier verwendeten Probe nicht gefunden, was wahrscheinlich auf die geringe Anzahl der untersuchten Objekte zurückgeführt werden kann. Außerdem wurden auch keine ultrarefraktären REE-Muster identifiziert, die aus refraktären Einschlüssen in CM- und CO-Chondriten (Boynton et al., 1980; Palme et al., 1982; Davis, 1991; Simon et al., 1996) und vereinzelt sogar in CV-Chondriten (El Goresy et al., 2002) bekannt sind.

Typisch für das group-II-Muster ist, dass die leichten Seltenen Erden gegenüber den schweren Seltenen Erden angereichert sind (abgesehen von Tm und Yb, die ebenfalls angereichert sind); Europium ist dagegen abgereichert (Mason und Taylor, 1982). Die Entstehung dieser Fraktionierungen wird durch das Zweistufenmodell erklärt (Boynton, 1989), siehe Abschnitt 1.3. Das REE-Muster des CAI 12 ist dagegen ein modifiziertes group-II-Muster. Einschlüsse mit solchen REE-Mustern kommen eher selten vor, wurden aber bereits in Leoville (Mao et al., 1990) und anderen kohligen Chondriten (MacPherson et al., 1989) gefunden, und ihre Entstehung auf die Kondensation bei relativ geringen Temperaturen zurückgeführt. Die positive Eu-Anomalie in CAI 12, sowie die stärkere Anreicherung von Yb gegenüber Tm deuten darauf hin, dass dieser Einschluss bei deutlich geringerer Temperatur mit der Gasphase equilibriert ist als CAIs mit gewöhnlichen group-II-Mustern. Die abweichenden Temperaturbedingungen können ein Hinweis darauf sein, dass CAI $12 \mathrm{zu}$ einem späteren Zeitpunkt oder in einer anderen Region des Sonnensystems entstanden ist als die anderen CAIs.

Drei der untersuchten CAIs haben subchondritische Y/Ho-Verhältnisse. Berechnungen von Kornacki und Fegley (1986) zeigen, dass $\mathrm{Y}_{2} \mathrm{O}_{3}$ bei einem Druck von $10^{-3}$ bar eine etwa $15 \mathrm{~K}$ höhere $50 \%$-Kondensationstemperatur hat als $\mathrm{Ho}_{2} \mathrm{O}_{3}$. Es ist daher zu erwarten, dass die allerersten, ultrarefraktären Kondensate superchondritische Y/Ho-Verhältnisse besitzen. Kondensate, die sich anschließend aus dem komplementären Gas bildeten und ein REE group-II-Muster zeigen, haben dagegen niedrigere Y/Ho-Verhältnisse (Pack et al., 2007). Drei der vier analysierten 
CAIs mit group-II-Muster besitzen tatsächlich niedrige Y/Ho-Verhältnisse (siehe Abbildung 3.4). Der CAI 9 hat dagegen ein superchondritisches Y/Ho-Verhältnis; er zeigt außerdem nur eine geringe Anreicherung an $\mathrm{Yb}$, was auf eine höhere Entstehungstemperatur dieses CAI hindeutet.

Die Zr/Hf-Verhältnisse sind in den meisten Einschlüssen unfraktioniert. Zwei CAIs (Objekte 15 und 22) haben jedoch abweichend davon sehr hohe Zr/HfVerhältnisse (siehe Abbildung 3.5). Zirkonium und Hafnium sind refraktärer als die Seltenen Erden, wobei die 50\%-Kondensationstemperatur von Zr bei einem Druck von $10^{-4}$ bar um etwa $60 \mathrm{~K}$ höher liegt als die von Hf (Lodders, 2003). Folglich ist zu erwarten, dass die ersten Kondensate superchondritische $\mathrm{Zr} / \mathrm{Hf}$-Verhältnisse besitzen. Eine später aus einem Residuum kondensierte komplementäre Phase mit einem group-II-Muster hätte dagegen subchondritische $\mathrm{Zr}$ /Hf-Verhältnisse. Bei den CAIs 15 und 22, die beide ein group-II-Muster aufweisen, ist jedoch ein superchondritisches Zr/Hf-Verhältnis zu beobachten, das durch den Einbau einer ultrarefraktären Komponente mit superchondritischem Zr/Hf-Verhältnis erklärt werden kann.

Die variablen, subchondritischen $\mathrm{Nb} / \mathrm{Ta}-$ Verhältnisse der CAIs können auf fraktionierte Kondensation zurückgeführt werden. Die $50 \%$-Kondensationstemperatur von Ta beträgt bei einem Druck von $10^{-4}$ bar $1573 \mathrm{~K}$ und ist somit höher als die von $\mathrm{Nb}(1559 \mathrm{~K})$, aber niedriger als die der Seltenen Erden, außer Ce, Eu und Yb (Lodders, 2003). Die niedrigen Nb/Ta-Verhältnisse in den CAIs deuten darauf hin, dass diese in einem Temperaturbereich mit der Gasphase equilibriert sind, bei dem Ta bereits zu einem großen Teil in die Festphase übergegangen war (ebenso wie die meisten Seltenen Erden), Nb sich jedoch noch überwiegend in der Gasphase befand. Der CAI 12 zeigt neben dem oben erwähnten modifizierten group-II-Muster das höchste $\mathrm{Nb} / \mathrm{Ta}$-Verhältnis der vier CAIs. Dies ist zusätzlich zu dem ungewöhnlichen REE-Muster ein weiterer Hinweis darauf, dass er bei geringeren Temperaturen entstanden ist als die anderen CAIs, so dass bereits ein größerer Anteil des $\mathrm{Nb}$ auskondensiert war.

Alle untersuchten CAIs besitzen sowohl niedrige $\mathrm{Nb} / \mathrm{Ta}$-Verhältnisse als auch fraktionierte REE group-II-Muster. Es besteht jedoch keine Korrelation zwischen $\mathrm{Nb} / \mathrm{Ta}$ und $\mathrm{Tm} / \mathrm{Er}$, das bei einem stark fraktionierten group-II-Muster hoch ist. Eine Korrelation ist allerdings auch nicht unbedingt zu erwarten, da die Kondensationstemperaturen der meisten Seltenen Erden höher liegen als diejenigen von $\mathrm{Nb}$ und Ta (Lodders, 2003), so dass die Seltenen Erden während der Kondensation in einem anderen Temperaturintervall gegeneinander fraktionieren als Nb und Ta. 


\subsubsection{Chondren}

Die Anreicherungsfaktoren der Seltenen Erden in den Leoville-Chondren von etwa 0,5 bis 10-facher CI-Konzentration stimmen gut mit denen von Allende-Chondren überein, die im Bereich zwischen 0,15 und 10-facher CI-Konzentration liegen (Misawa und Nakamura, 1988a). Die relativ unfraktionierten REE-Muster (abgesehen von kleinen Eu-Anomalien) sowie unfraktionierte Y/Ho-, Nb/Ta- und Zr/Hf-Verhältnisse der meisten Typ-1-Chondren und der Typ-2-Chondre deuten darauf hin, dass sie aus einem unfraktionierten Gas entstanden sind. Es ist daher wahrscheinlich, dass sich die Chondren und refraktären Einschlüsse in unterschiedlichen Regionen des Sonnensystems oder zu unterschiedlichen Zeitpunkten gebildet haben. Die geringen, meist negativen Eu-Anomalien sind vermutlich durch fraktionierte Gleichgewichtskondensation entstanden, so dass die residuale Gasphase an Eu angereichert wurde. Die Präkursoren der Chondren mit positiver Eu-Anomalie hätten sich folglich aus dieser residualen Gasphase gebildet. Die gleichmäßige Anreicherung der schweren Seltenen Erden, die einige Chondren aufweisen, sind nicht durch volatilitätskontrollierte Fraktionierung zu erklären. Vielmehr entstehen solche Muster bei der Kristallisation von Chondren und sind typisch für Olivine und Enstatite (Jacquet et al., 2012).

Im Gegensatz zu den Typ-1- und Typ-2-Chondren enthalten die Al-reichen Chondren einen größeren Anteil refraktärer Komponenten (Misawa und Fujita, 1994; Russell et al., 2005; Scott, 2007). Dieses refraktäre Material kann Träger fraktionierter Seltener Erden sein, so dass Al-reiche Chondren häufig fraktionierte REE-Muster haben (Russell et al., 2005), es kommen aber auch solche vor, die keine volatilitätskontrollierte REE-Fraktionierung aufweisen (MacPherson und Huss, 2005). Die drei Al-reichen Chondren der hier untersuchten Probe zeigen fraktionierte REE group-II-Muster. Sie haben außerdem - wie die refraktären Einschlüsse - niedrige Nb/Ta-Verhältnisse. Daraus kann geschlossen werden, dass die Präkursoren der Al-reichen Chondren von Leoville refraktäres Material enthielten, das dem der CAIs ähnelt und unter vergleichbaren Bedingungen im solaren Nebel entstanden ist.

Um den Anteil einer solchen fraktionierten refraktären Komponente in den Al-reichen Chondren abzuschätzen, wurde eine auf den REE-Konzentrationen basierende Modellierung vorgenommen. Es wird dabei vereinfachend angenommen, dass die Präkursoren der Al-reichen Chondren aus einer Komponente mit unfraktionierten Seltenen Erden und variablem Anreicherungsfaktor gegenüber der CIKonzentration sowie einer zweiten fraktionierten Komponente bestehen (Abbildung 4.3). Als fraktionierte Komponente wurden die mittleren REE-Konzentrationen der vier analysierten CAIs in Leoville herangezogen. Die leichten Seltenen Erden sind dort im Durchschnitt etwa 30-fach gegenüber CI-Chondriten angereichert. In Allende-CAIs liegen die LREE-Anreicherungsfaktoren typischerweise zwischen 10 

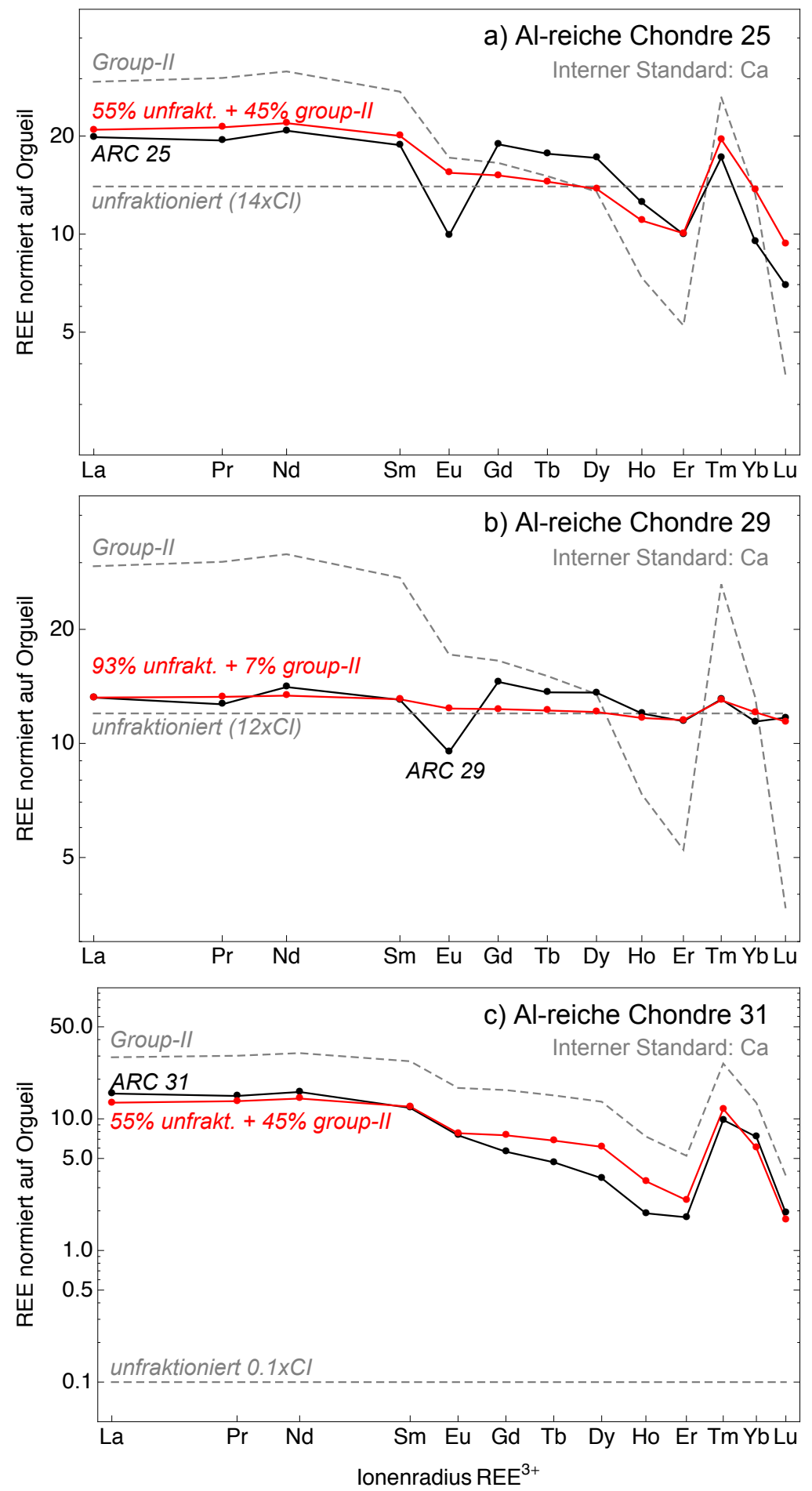

Abbildung 4.3: Anteil einer refraktären Komponente mit group-II-Muster in Al-reichen Chondren (ARC). 
und 50, so dass die durchschnittlichen Konzentrationen der vier CAIs aus Leoville einen realistischen Mittelwert für CAIs in CV-Chondriten darstellen (Mason und Taylor, 1982).

Das REE-Muster der Al-reichen Chondre 25 kann mithilfe dieses Modells durch eine Kombination aus 55 Gew.-\% einer unfraktionierten Komponente mit 14-facher Anreicherung der CI-Zusammensetzung und 45 Gew.- $\%$ der fraktionierten REE group-II-Komponente angenähert werden. Die Al-reiche Chondre 29 würde demnach zu etwa 93 Gew.-\% aus einer unfraktionierten Komponente, die 12-fach gegenüber CI-Chondriten angereichert ist, und nur zu etwa 7 Gew.- $\%$ aus der fraktionierten group-II-Komponente bestehen. Die Al-reiche Chondre 31 würde sich zu 55 Gew.-\% aus einer unfraktionierten, gegenüber CI-Chondriten abgereicherten $(0,1 \times \mathrm{CI})$ und zu 45 Gew.-\% aus der group-II-Komponente zusammensetzen. Die abgeschätzten Gewichtsanteile einer CAI-ähnlichen Komponente mit group-II-Muster in den drei Al-reichen Chondren liegen also zwischen etwa 7 und 45 Gew.-\%. Zusätzlich zu group-II-CAIs können aber auch CAIs mit unfraktionierten, angereicherten Seltenen Erden in den Präkursoren der Al-reichen Chondren vorkommen. Der Gesamt-CAIGehalt einer Al-reichen Chondre aus Mokoia wurde von Jones und Norman (2008) auf bis zu $30 \%$ geschätzt.

Ähnlich zu den Al-reichen Chondren haben auch vier der Typ-1-Chondren (Objekte 2, 20, 24, 26) fraktionierte Seltene Erden mit group-II-Muster (siehe Abbildung 3.1). Im Gegensatz zu den Al-reichen Chondren sind die Seltenen Erden in den gewöhnlichen Chondren jedoch weniger stark angereichert. Es erscheint daher plausibel, dass diese Chondren - wie die Al-reichen Chondren - ebenfalls durch den Einbau von refraktärem Material entstanden sind, mit dem Unterschied, dass der Anteil der refraktären Komponente geringer ist. Chondren mit fraktionierten Seltenen Erden wurden bereits in anderen kohligen und gewöhnlichen Chondriten beobachtet und diese Fraktionierungen wurden auf den Einbau refraktärer und ultrarefraktärer Präkursoren zurückgeführt (Misawa und Nakamura, 1988a,b; Pack et al., 2004; Wakaki et al., 2013). Durch Anwendung des einfachen Zweikomponentenmodells auf die vier Typ-1-Chondren ergeben sich Gewichtsanteile zwischen 2 und $20 \%$ für die refraktäre Komponente mit fraktioniertem group-II-Muster, während die Komponente mit unfraktionierten Seltenen Erden etwa 1 bis 2,5-fache CI-Konzentration aufweist.

Die Akkretionsränder der Chondren in Leoville sind aufgrund hoher Temperaturen rekristallisiert, jedoch nicht aufgeschmolzen worden (Patzer et al., 2012). Da die REE-Muster der Akkretionsränder und der Chondrenkerne sehr gut übereinstimmen, kann davon ausgegangen werden, dass sich die Chondren und ihre Akkretionsränder aus demselben Ausgangsmaterial gebildet haben. Insbesondere das group-II-Muster, das sowohl die Chondre 25 als auch ihr Akkretionsrand zeigen und nur durch volatilitätskontrollierte Fraktionierung erklärt werden kann, deutet an, dass dieses 
fraktionierte Material sowohl in den Präkursoren der Chondre selbst als auch in den Präkursoren des Chondrenrandes enthalten war.

\subsubsection{Matrix}

Chondritmatrix kann zusätzlich zu gewöhnlichen Matrixbestandteilen geringe Anteile refraktärer Komponenten aufweisen, die möglicherweise wie CAIs nahe der Sonne entstanden sind (Scott und Krot, 2005). Die identifizierte schwache Anreicherung der leichten Seltenen Erden sowie an $\mathrm{Tm}$ und $\mathrm{Yb}$ in der Matrix Leovilles deutet darauf hin, dass tatsächlich eine solche refraktäre Komponente enthalten ist. Aufgrund der guten Übereinstimmung des REE-Musters der Matrix mit einem group-II-Muster (abgesehen von $\mathrm{Eu}$ ) ist es wahrscheinlich, dass diese refraktäre Komponente in ihrer chemischen Zusammensetzung den CAIs ähnelt und sich in derselben Region des Sonnensystems gebildet hat. Ein Anteil einer group-II-Komponente von nur 0,6 Gew.-\% würde ausreichen, um die beobachteten Anreicherungen der leichten Seltenen Erden sowie von Tm und Yb zu erklären (Abbildung 4.4).

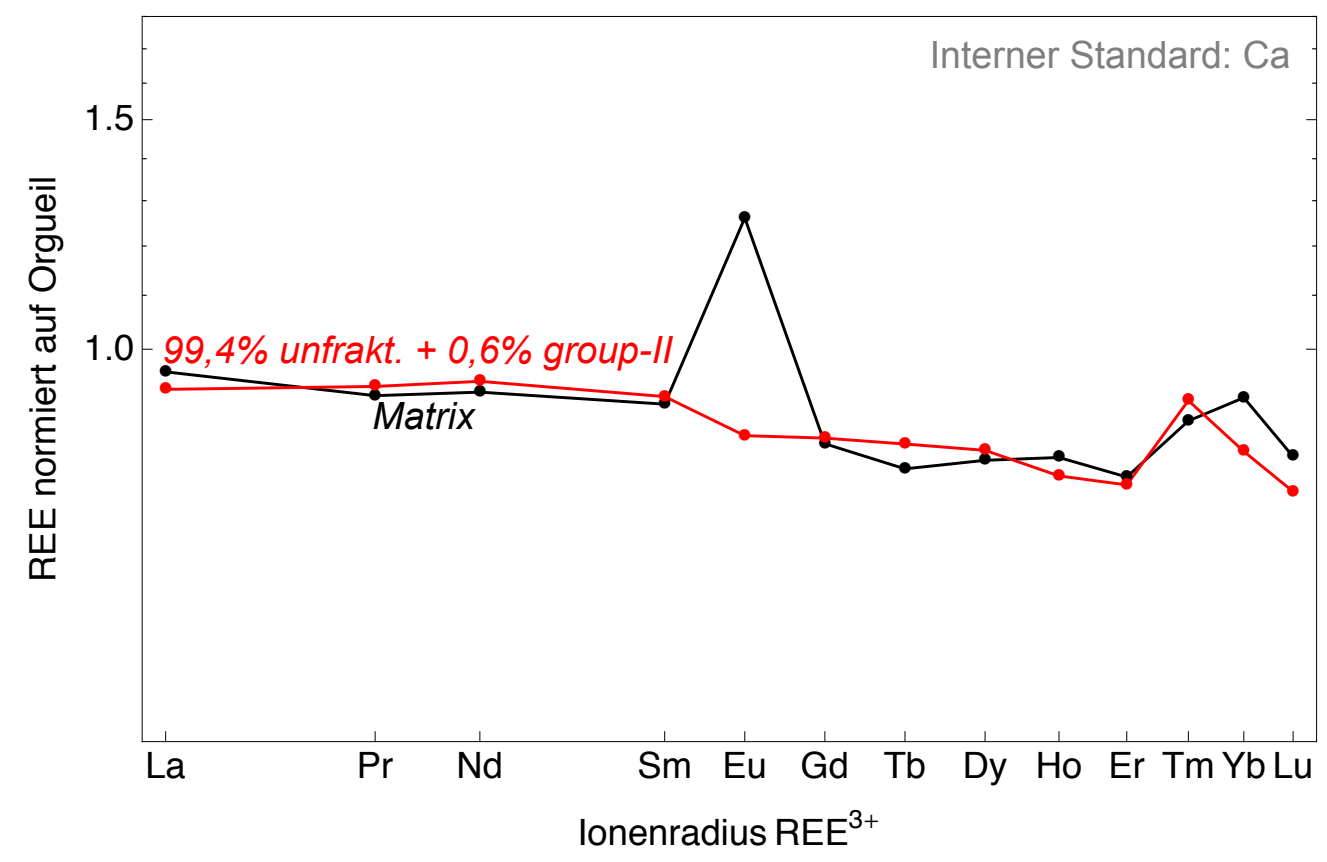

Abbildung 4.4: Das REE-Muster der Matrix von Leoville kann, abgesehen von der Eu-Anomalie, durch die Kombination aus 99,4\% unfraktionierten Bestandteilen und $0,6 \%$ einer refraktären Phase mit REE group-II-Muster erklärt werden.

Die Entstehung und Zusammensetzung von Chondritmatrix ist Gegenstand intensiver Diskussion. Es wird beispielsweise von Alexander (2005) angenommen, 
dass Chondritmatrix zu einem großen Teil aus primitivem Material besteht, dass in der chemischen Zusammensetzung dem der CI-Chondrite entspricht und unabhängig von den Chondren entstanden ist. Dementgegen steht die Annahme, dass sich Chondren und Matrix aufgrund ihrer chemischen Komplementarität in derselben Region des Sonnensystems gebildet haben müssen (Klerner und Palme, 1999; Bland et al., 2005; Hezel und Palme, 2008, 2010). Auch die REE-Muster der Matrix und der Chondren von Leoville sind in gewisser Weise komplementär zueinander, da die Matrix eine große positive Eu-Anomalie aufweist, die meisten Chondren besitzen jedoch eine negative Eu-Anomalie. Diese Komplementarität kann durch fraktionierte Kondensation in einer gemeinsamen Chondren-Matrix-Bildungsregion entstanden sein. Alternativ besteht die Möglichkeit, dass der Eu-Überschuss in der Matrix ebenfalls durch refraktäre Komponenten hervorgerufen wird. Hierfür kämen refraktäre Bestandteile mit REE group-I- und REE group-VI-Mustern (Mason und Taylor, 1982), die positive Eu-Anomalien haben, in Frage.

\subsubsection{Gesamt-Leoville}

Aus den mittleren REE-Konzentrationen der verschiedenen Komponenten und deren prozentualen Anteilen (Tabelle 4.3) wurden REE-Konzentrationen für den Gesamtchondrit Leoville berechnet (Tabelle 4.4, Abbildung 4.5). Das Muster für den Gesamtchondrit ist 2 bis 3,5-fach gegenüber Orgueil angereichert und zeigt ein deutliches group-II-Muster mit einer etwa 20 bis $30 \%$ igen Anreicherung der leichten Selten Erden gegenüber den schweren Seltenen Erden. Zu diesem Muster tragen im Wesentlichen die refraktären Einschlüsse bei, aber auch die Al-reichen Chondren, einige der Typ-1-Chondren sowie die Matrix. Das fraktionierte REEMuster ist in guter Übereinstimmung zu REE-Mustern anderer CV3-Chondrite (Abbildung 4.6). Es zeigt allerdings eine deutlich stärkere Fraktionierung. Dies

Tabelle 4.3: Prozentuale Anteile der hier diskutierten Komponenten in Leoville. Daten aus Patzer et al. (2012).

\begin{tabular}{lc}
\hline Komponenten & Flächen- $\%$ \\
\hline Typ-1-Chondren & 67 \\
Typ-2-Chondren & 0,5 \\
Al-reiche Chondren & 3 \\
CAIs & 3 \\
AOA & 0,5 \\
Matrix & 23 \\
\hline Summe & 97 \\
\hline
\end{tabular}




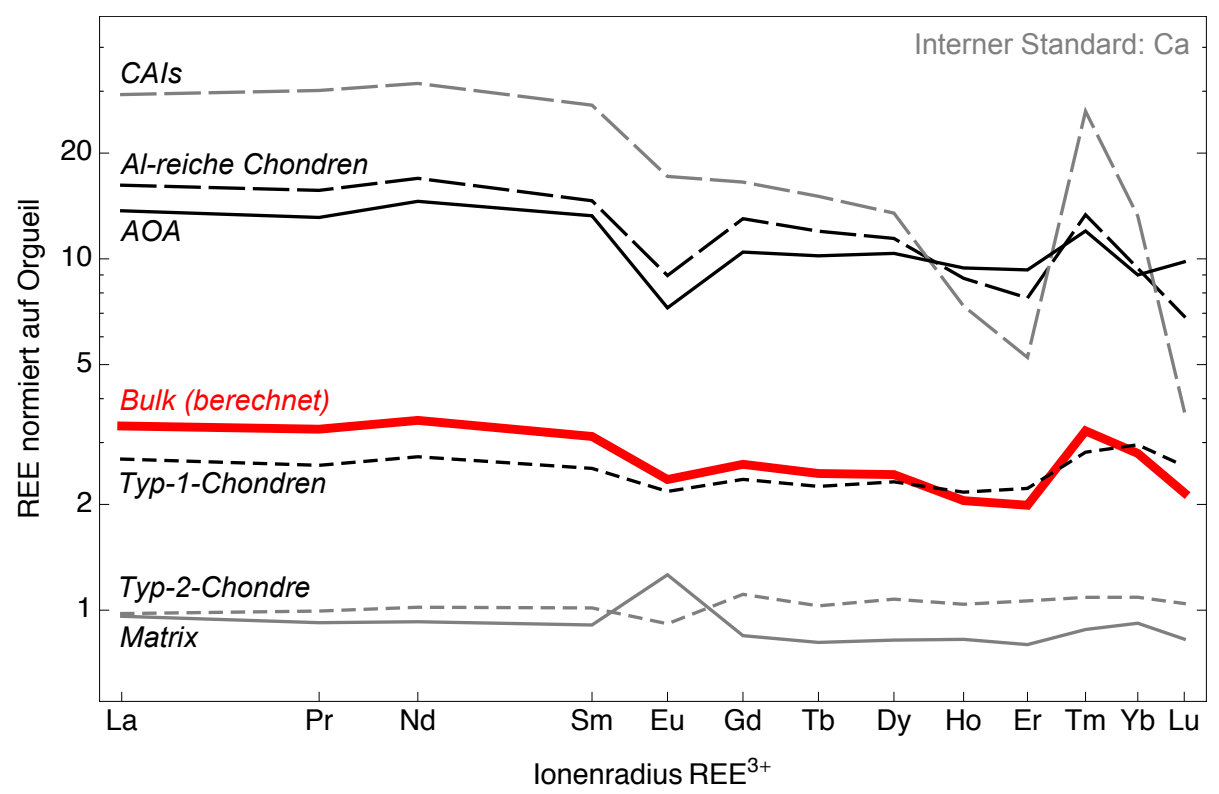

Abbildung 4.5: Mittelwerte der verschiedenen Komponenten Leovilles und das daraus berechnete REE-Muster für den Gesamtchondrit.

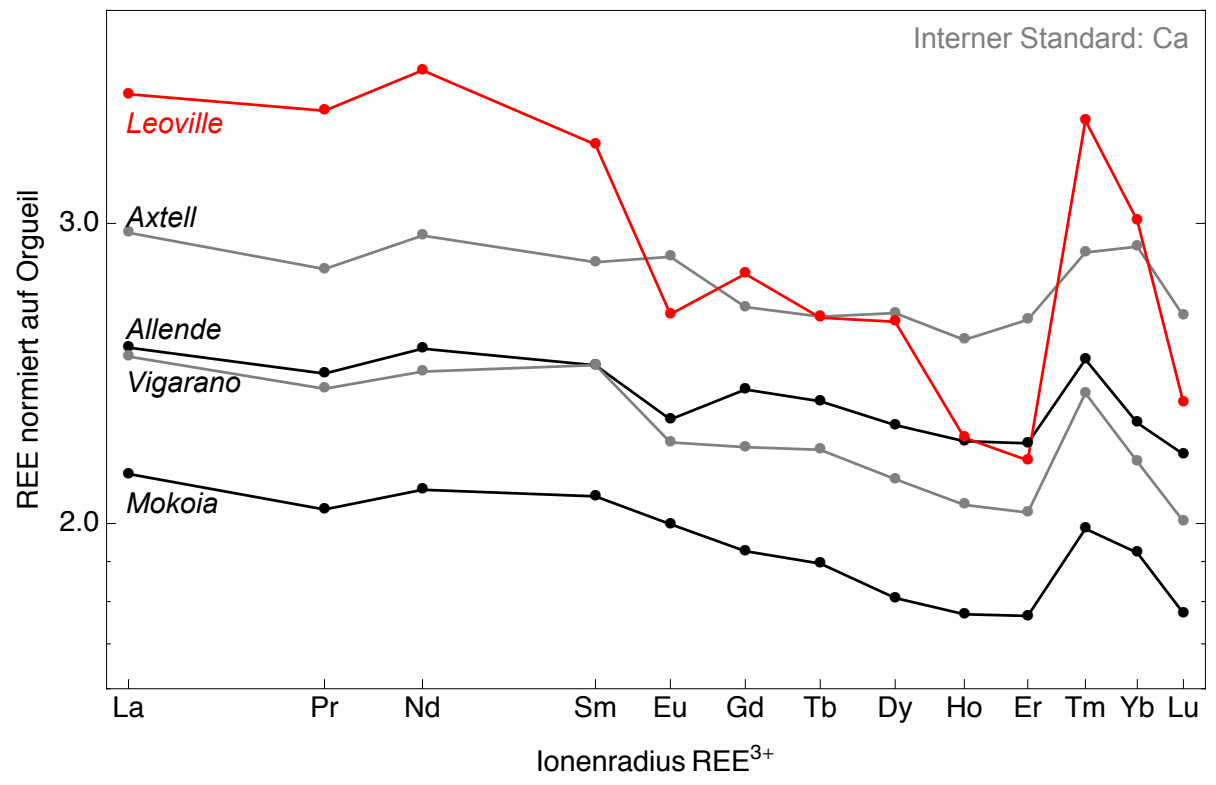

Abbildung 4.6: Vergleich von Leoville mit anderen CV3-Chondriten. Die absoluten REE-Konzentrationen der anderen CV3-Chondrite sind Näherungswerte, da sie anhand des mittleren Ca-Gehaltes der CV-Chondrite (Tabelle A.3) kalibriert wurden. 
kann unterschiedliche Gründe haben. Untersuchungen von Liu und Schmitt (1988) legen nahe, dass nur etwa $30 \%$ der CAIs in CV3-Chondriten fraktionierte REE group-II-Muster aufweisen. Es haben aber alle in dieser Leoville-Probe untersuchten refraktären Einschlüsse fraktionierte group-II-Muster. Daraus kann geschlossen werden, dass entweder der Anteil von group-II-CAIs in Leoville tatsächlich höher ist als in anderen CV3-Chondriten, oder dass die CAIs in dem untersuchten Dickschliff nicht repräsentativ für Leoville sind.

Die Berechnung der REE-Gehalte des Gesamtchondrits aus den durchschnittlichen REE-Gehalten der Komponenten kann außerdem zu einer Überschätzung der Fraktionierung führen, da die Größenverhältnisse der einzelnen Chondren und CAIs untereinander nicht berücksichtigt wurden. Zusätzlich können durch die interne Kalibration mit Ca weitere Unsicherheiten entstehen.

Tabelle 4.4: Mittlere Spurenelementkonzentrationen der Komponenten von Leoville und des Gesamtchondrits in ppm. $N$ : Anzahl der untersuchten Objekte.

\begin{tabular}{lrrrrrrr}
\hline & $\begin{array}{r}\text { Chondren } \\
\text { Typ-1 } \\
N=21\end{array}$ & $\begin{array}{r}\text { Chondre } \\
\text { Typ-2 } \\
N=1\end{array}$ & $\begin{array}{c}\text { Chondren } \\
\text { Al-reich }\end{array}$ & CAIs & AOA & Matrix & $\begin{array}{c}\text { Gesamt } \\
\text { berechnet }\end{array}$ \\
& 3,07 & 1,50 & 10,47 & 6,21 & 13,81 & 1,22 & 3,00 \\
\hline $\mathrm{Y}$ & 6,95 & 4,34 & 20,45 & 13,02 & 31,59 & 3,12 & 6,76 \\
$\mathrm{Zr}$ & 0,63 & 0,33 & 1,72 & 3,33 & 1,28 & 0,28 & 0,66 \\
$\mathrm{Nb}$ & 0,633 & 0,230 & 3,808 & 6,898 & 3,220 & 0,226 & 0,839 \\
$\mathrm{La}$ & 1,564 & 0,586 & 8,079 & 15,744 & 6,028 & 0,593 & 1,992 \\
$\mathrm{Ce}$ & 0,234 & 0,090 & 1,418 & 2,729 & 1,187 & 0,083 & 0,316 \\
$\mathrm{Pr}$ & 1,229 & 0,458 & 7,624 & 14,201 & 6,557 & 0,417 & 1,659 \\
$\mathrm{Nd}$ & 0,379 & 0,152 & 2,190 & 4,097 & 1,985 & 0,136 & 0,499 \\
$\mathrm{Sm}$ & 0,121 & 0,051 & 0,496 & 0,950 & 0,401 & 0,070 & 0,147 \\
$\mathrm{Eu}$ & 0,461 & 0,217 & 2,545 & 3,235 & 2,045 & 0,166 & 0,548 \\
$\mathrm{Gd}$ & 0,081 & 0,037 & 0,430 & 0,540 & 0,366 & 0,029 & 0,095 \\
$\mathrm{~Tb}$ & 0,579 & 0,268 & 2,855 & 3,371 & 2,589 & 0,205 & 0,656 \\
$\mathrm{Dy}$ & 0,119 & 0,057 & 0,483 & 0,402 & 0,516 & 0,045 & 0,123 \\
$\mathrm{Ho}$ & 0,361 & 0,173 & 1,256 & 0,851 & 1,510 & 0,130 & 0,354 \\
$\mathrm{Er}$ & 0,070 & 0,027 & 0,330 & 0,650 & 0,297 & 0,022 & 0,085 \\
$\mathrm{Tm}$ & 0,500 & 0,184 & 1,591 & 2,240 & 1,523 & 0,155 & 0,510 \\
$\mathrm{Yb}$ & 0,065 & 0,026 & 0,173 & 0,093 & 0,247 & 0,021 & 0,059 \\
$\mathrm{Lu}$ & 0,203 & 0,113 & 0,609 & 0,324 & 0,814 & 0,082 & 0,193 \\
$\mathrm{Hf}$ & 0,034 & 0,015 & 0,170 & 0,402 & 0,092 & 0,012 & 0,045 \\
$\mathrm{Ta}$ & & & & & & & \\
\hline & & & & & & & \\
\hline
\end{tabular}




\subsection{Bulk-Chondrite}

\subsubsection{Röntgenfluoreszenzanalysen}

Die beobachteten Hauptelementfraktionierungstrends der Chondrite zeigen eine gute Übereinstimmung mit Literaturdaten von Hutchison (2004) und Wolf und Palme (2001). Einige Elementkonzentrationen des H-Chondrits Adrian, besonders $\mathrm{Ti}, \mathrm{Al}$ und $\mathrm{Mg}$, weichen von den anderen gewöhnlichen Chondriten ab. Dieser Chondrit hat außerdem ein ungewöhnliches REE-Muster, das ein Hinweis auf terrestrische Kontamination ist (siehe Abschnitt 4.3.2).

Das Ca/Si-Verhältnis der untersuchten Orgueil-Probe (Orgueil-B) ist deutlich geringer als das Ca/Si-Verhältnis des CI-Gruppenmittelwerts (Hutchison, 2004). Dies beruht auf der geringen Ca-Konzentration von 0,76 Gew.-\% in der Probe. Die Daten mehrerer Orgueil-Proben von Wolf und Palme (2001), die aus jeweils etwa 1 Gramm Probe hergestellt worden sind, zeigen eine Variation der Ca-Konzentrationen zwischen 0,77 und 0,96 Gew.-\% und verdeutlichen, dass Ca auf dieser Gewichtsskala in Orgueil inhomogen verteilt ist. In Anbetracht der geringen Probenmasse von Orgueil-B (etwa $500 \mathrm{mg}$ ) ist der abweichende Ca-Gehalt nicht außergewöhnlich. Variationen des Ca-Gehalts in CI-Chondriten werden durch die inhomogene Verteilung von Mineralphasen wie Phosphaten, Karbonaten und Sulfaten, in denen Ca konzentriert ist, verursacht (Morlok et al., 2006).

\subsubsection{Seltene Erden}

Nichtkohlige Chondrite: Die Gruppen der nichtkohligen Chondrite (gewöhnliche, Enstatit- und Rumuruti-Chondrite) zeigen ähnliche REE-Muster mit nur geringen Fraktionierungen (Abbildung 4.7). Alle diese Gruppen haben jedoch im Vergleich zu Orgueil ein kleines Defizit an Tm und Yb. Solche Abreicherungen sind von ultrarefraktären REE-Mustern (Boynton et al., 1980) bekannt, die durch fraktionierte Kondensation bei hohen Temperaturen entstanden sind. Das Defizit an Tm und Yb könnte daher durch eine ultrarefraktäre Komponente in den nichtkohligen Chondriten erklärt werden, die in Orgueil nicht vorhanden ist. In diesem Falle wäre allerdings zu erwarten, dass Yb in den nichtkohligen Chondriten stärker abgereichert ist als Tm - tatsächlich zeigt aber Tm die größeren Anomalien. Es ist daher wahrscheinlicher, dass Orgueil eine refraktäre Komponente mit group-IIMuster enthält, die in den gewöhnlichen, Enstatit- und Rumuruti-Chondriten nicht vorhanden ist. In Abschnitt 4.4 wird ausführlicher auf dieses Thema eingegangen.

Die starke Anreicherung der leichten Seltenen Erden in dem H-Chondrit Adrian, der kein beobachteter Fall ist, ist ein Hinweis auf terrestrische Alteration (siehe auch Crozaz und Wadhwa (2001) und Crozaz et al. (2003)). Der ebenfalls an den leichten Seltenen Erden angereicherte Hammond Downs ist auch ein Fund 
und daher möglicherweise geringfügig terrestrisch alteriert. Andererseits besteht auch die Möglichkeit, dass die Anreicherungen der leichten Seltenen Erden auf Probeninhomogenitäten beruhen. Die Homogenitätsmessungen an den gewöhnlichen Chondriten North West Africa 869 und Gao-Guenie (Abbildung 3.16) belegen, dass Abweichungen der leichten Seltenen Erden, insbesondere von Lanthan, nicht ungewöhnlich sind.

Die beobachteten Eu-Anomalien der Chondrite können zwei Ursachen haben: Da Europium das volatilste Seltene Erdelement ist (Lodders, 2003), können die Eu-Anomalien durch volatilitätskontrollierte Fraktionierung im solaren Nebel entstanden sein. Bei geringen Probenmengen ist es auch möglich, dass die Anomalien durch Probeninhomogenitäten hervorgerufen werden.

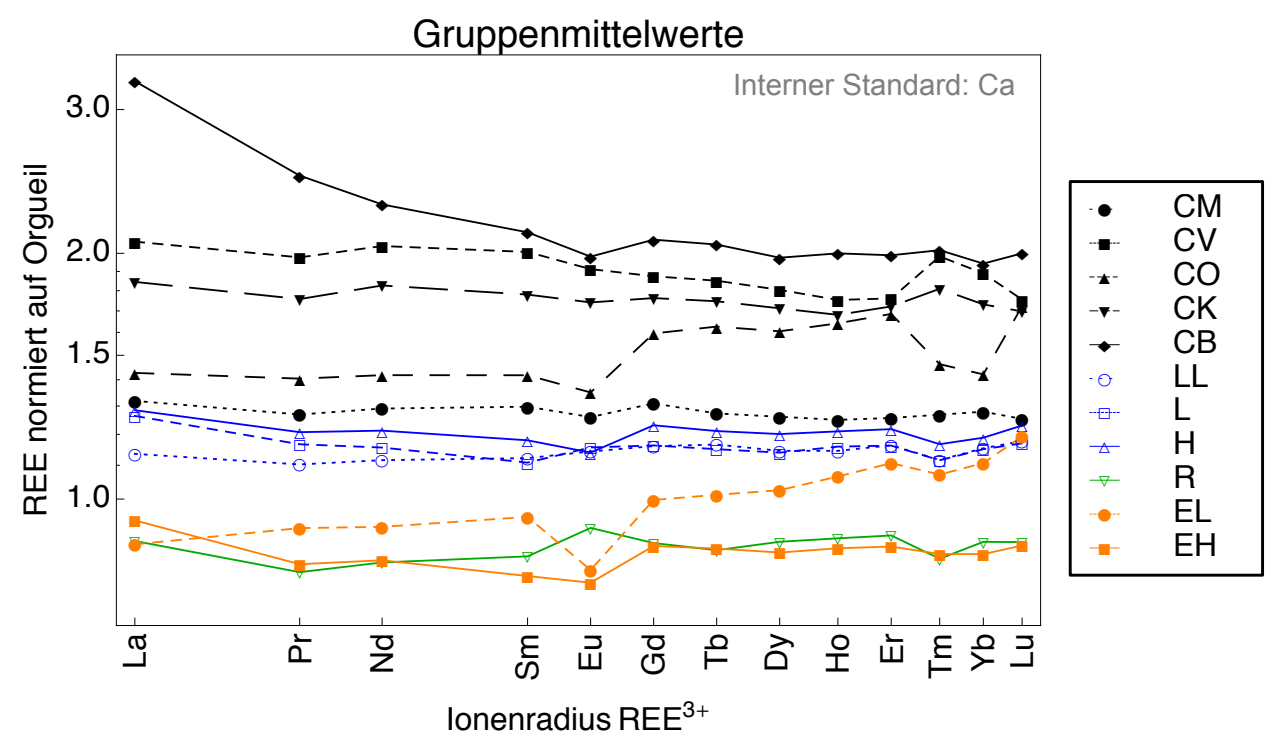

Abbildung 4.7: Mittlere Gehalte der Seltenen Erden in den Chondrit-Gruppen. Adrian (H) und Atlanta (EL) sind nicht in den Gruppenmittelwerten berücksichtigt. Die absoluten Konzentrationen sind Näherungswerte, da sie anhand der mittleren Ca-Gehalte der Chondritgruppen (Tabelle A.3) kalibriert wurden.

Kohlige Chondrite: Die meisten kohligen Chondrite zeigen REE-Fraktionierungen, die auf den Einbau refraktären Materials zurückgeführt werden können. Die beobachteten Fraktionierungen der beiden CI-Chondrite Alais und Ivuna, die an den leichten Seltenen Erden deutlich angereichert sind (Abbildung 3.12), stellen eine Ausnahme dar und sind nicht durch den Einbau von refraktärem Material zu erklären. Erwartungsgemäß sollten sowohl Alais als auch Ivuna im Vergleich 
zu Orgueil unfraktionierte REE-Muster haben (Barrat et al., 2012; Pourmand et al., 2012). Es gibt jedoch, abgesehen von Alais und Ivuna, auch Proben von Orgueil, die ebenfalls eine Anreicherung der leichten Seltenen Erden zeigen (Barrat et al., 2012; Pourmand et al., 2012). Ungewöhnliche REE-Muster können bei kleinen Probenmengen auftreten, da die Seltenen Erden in CI-Chondriten in einigen Mineralphasen (z.B. Phosphaten) stark angereichert sind und somit auf kleiner Größenskala nicht homogen im Chondrit verteilt sind (Morlok et al., 2006; Bouvier et al., 2008).

Die group-II-Muster der CV-Chondrite werden vermutlich durch das häufige Vorkommen von CAIs mit group-II-Muster (Mason und Taylor, 1982; Liu und Schmitt, 1988; Mao et al., 1990; Huang et al., 2012) verursacht, CAIs mit ultrarefraktären REE-Mustern treten dagegen in CV-Chondriten nur sporadisch auf (El Goresy et al., 2002). Das REE-Muster des Allende-Referenzpulvers kann als repräsentativ für den Gesamtchondrit angesehen werden. Die Fraktionierungen der fünf kleineren untersuchten Proben (Allende-A bis Allende-E mit Probenmengen zwischen $221 \mathrm{mg}$ und 16,6 g) belegen, dass die Seltenen Erden in Allende sehr inhomogen verteilt sind. Auch Untersuchungen von Stracke et al. (2012) an 39 Proben von Allende mit durchschnittlichen Probenmengen von über $600 \mathrm{mg}$, die ebenfalls Fraktionierungen aufweisen, bestätigen diese inhomogene Verteilung.

Analog zu den Al-reichen Chondren (Abschnitt 4.2.2) kann der Anteil einer fraktionierten group-II-Komponente in den Bulk-Chondriten abgeschätzt werden. Es wird dabei angenommen, dass sich die Gesamtchondrite aus einer im Vergleich zu CI-Chondriten unfraktionierten, aber variabel angereicherten Komponente und zusätzlichem refraktären Material mit group-II-Muster zusammensetzen. Als fraktionierte REE group-II-Komponente wurde wieder der Mittelwert der vier CAIs von Leoville herangezogen. Allende besteht demnach zu etwa 1,2 Gew.- \% aus einer refraktären Phase mit REE group-II-Muster (Abbildung 4.8). Die Gewichtsanteile der anderen CV-Chondrite wurden mit etwa 1\% für Mokoia, 1,5\% für Axtell und 1,7\% für Vigarano abgeschätzt. Da diese fraktionierte Komponente nicht ausschließlich in Form von CAIs, sondern auch als feiner Staub oder innerhalb von Chondren vorliegen kann, kann der Anteil an group-II-CAIs in den CV-Chondriten auf maximal 1 bis 1,7\% geschätzt werden. Dieser Anteil stimmt gut mit den Beobachtungen überein, dass die modale Häufigkeit von CAIs in CV-Chondriten etwa 3 bis $4 \%$ beträgt (Hezel et al., 2008; Stracke et al., 2012) und dass davon etwa $30 \%$ fraktionierte group-II-Muster aufweisen (Liu und Schmitt, 1988). Der Anteil an group-II-CAIs in der untersuchten Probe von Leoville ist im Gegensatz zu diesen vier CV-Chondriten mit etwa 3\% (Patzer et al., 2012) allerdings deutlich höher.

Das schwach ausgeprägte group-II-Muster von Karoonda (CK) wird wahrscheinlich ebenfalls durch das Vorkommen von refraktären Komponenten mit group-IIMuster verursacht. Ein Anteil einer solchen refraktären Komponente von etwa 0,4 


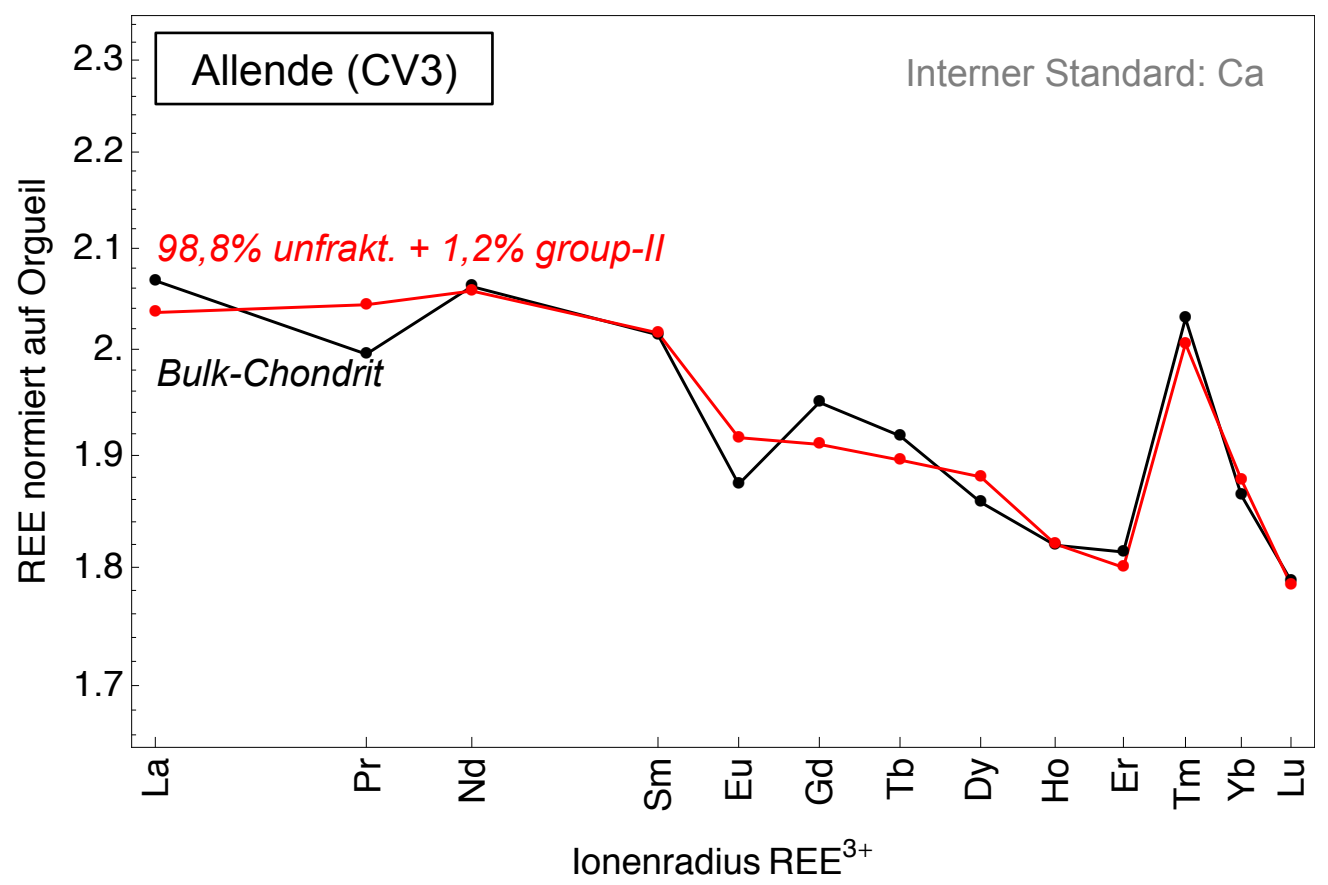

Abbildung 4.8: REE-Muster von Allende (Referenzpulver). Der Anteil einer group-II-Komponente wird auf 1,2 Gew.-\% geschätzt.

bis 0,8 Gew.-\% reicht aus, um die beobachtete Tm-Anomalie zu erklären und ist somit geringer als in den CV-Chondriten.

Die Ähnlichkeit der CV- und CK-Chondrite hinsichtlich ihrer Seltenen Erden stützt die Theorie, dass beide Gruppen aus demselben Ausgangsmaterial entstanden und von demselben Mutterkörper stammen könnten (Greenwood et al., 2010). Andere Übereinstimmungen der beiden Chondritgruppen sind beispielsweise die etwa gleich starke Anreicherung refraktär-lithophiler Elemente und vergleichbare Sauerstoffisotopenverhältnisse (Greenwood et al., 2010) sowie ähnliche Bestrahlungsalter (Scherer und Schultz, 2000).

Die REE-Muster der CM-Chondrite sind im Vergleich zu Orgueil leicht fraktioniert und einige entsprechen group-II-Mustern. In CM-Chondriten kommen sowohl refraktäre Einschlüsse mit relativ unfraktionierten als auch solche mit group-IIMustern (Ireland et al., 1988; Ireland, 1990; MacPherson und Davis, 1994) oder mit ultrarefraktären REE-Mustern (Boynton et al., 1980; Davis, 1991; Simon et al., 1996) vor. Die fünf analysierten Proben von Murchison haben unterschiedliche REE-Muster, können aber nicht eindeutig group-II-Mustern oder ultrarefraktären Mustern zugeordnet werden. Dagegen zeigen Mighei und Murray group-II-Muster, die wahrscheinlich, ähnlich wie bei den CV- und CK-Chondriten, durch das Vorhandensein von refraktärem Material verursacht werden. Die fraktionierten REE- 


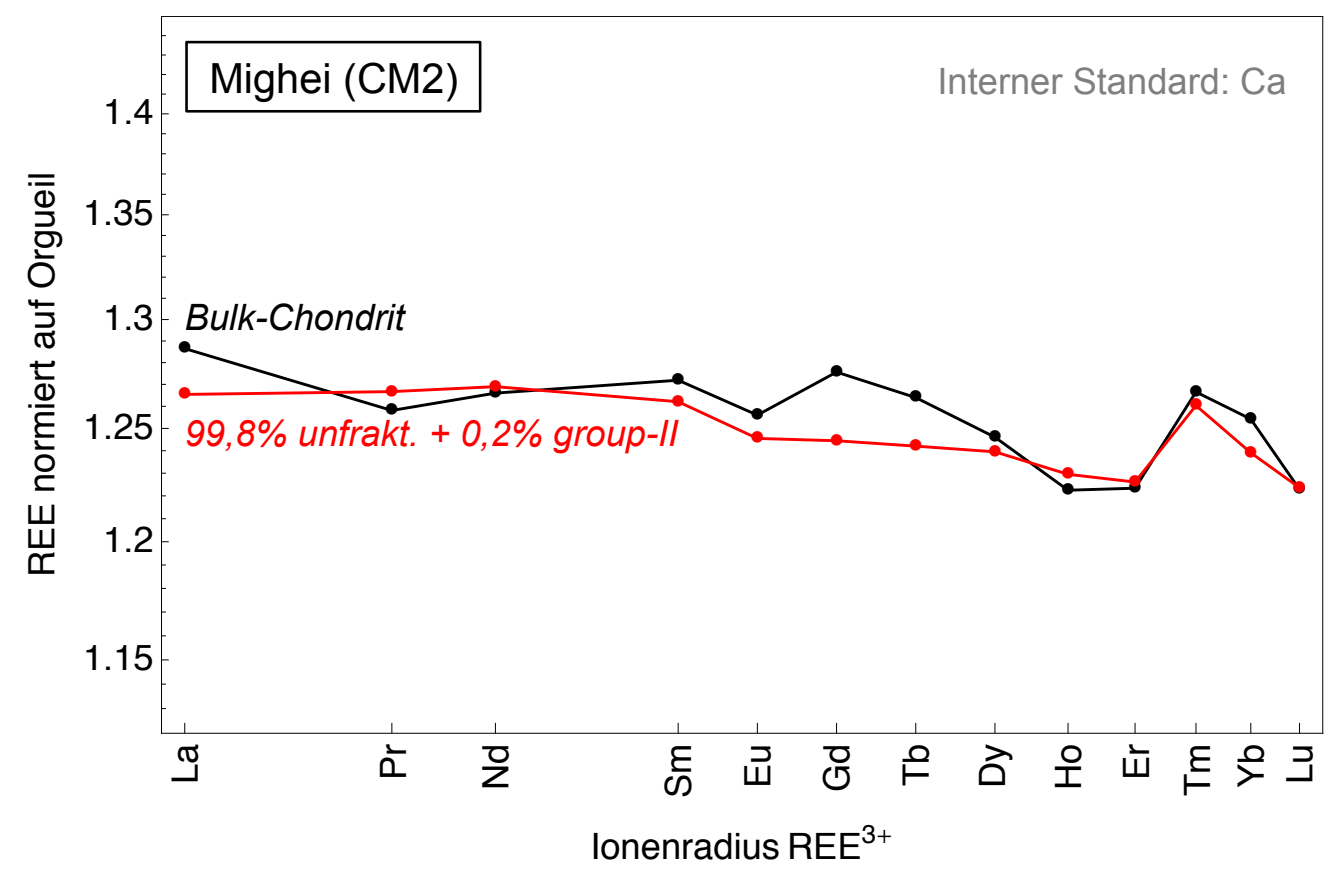

Abbildung 4.9: REE-Muster von Mighei. Der Anteil einer group-II-Komponente wird auf 0,2 Gew.- $\%$ geschätzt.

Muster von Mighei (Abbildung 4.9) und von Murray können erklärt werden, wenn sie zusätzlich zu einer gegenüber CI-Chondriten unfraktionierten Komponente eine group-II-Komponente mit einem Gewichtsanteil von 0,2\% beziehungsweise 0,4\% enthalten. Der Anteil der group-II-Komponente ist also in CM-Chondriten deutlich geringer als in CV-Chondriten.

Das ultrarefraktäre REE-Muster des CO-Chondrits North West Africa 3003 kann auf eine ultrarefraktäre Komponente in der Probe zurückgeführt werden. Einschlüsse mit ultrarefraktären REE-Mustern sind aus CO-Chondriten, z.B. Ornans (Palme et al., 1982; Davis, 1991) und Lancé (Fahey et al., 1994), bekannt, wobei ein Einschluss aus Zr-Y-Ti-Sc-Oxid innerhalb eines AOA in Ornans extrem hohe Anreicherungsfaktoren der refraktärsten Seltenen Erden von etwa hunderttausendfacher CI-Konzentration aufweist (Davis, 1991). Chondren in Felix weisen dagegen sowohl relativ unfraktionierte als auch group-II-Muster auf (Misawa und Nakamura, 1988b,c). Das REE-Muster von North West Africa 3003 kann durch die Kombination von 0,3\% einer ultrarefraktären Komponente, in der Lu etwa hundertfach gegenüber den CI-Chondriten angereichert ist (Boynton et al., 1980), und 99,7 \% einer unfraktionierten Komponente angenähert werden (Abbildung 4.10). Da die Anreicherungen der Seltenen Erden in den ultrarefraktären Einschlüssen in CO-Chondriten aber auch deutlich höher sein können, ist dies nur als grober 


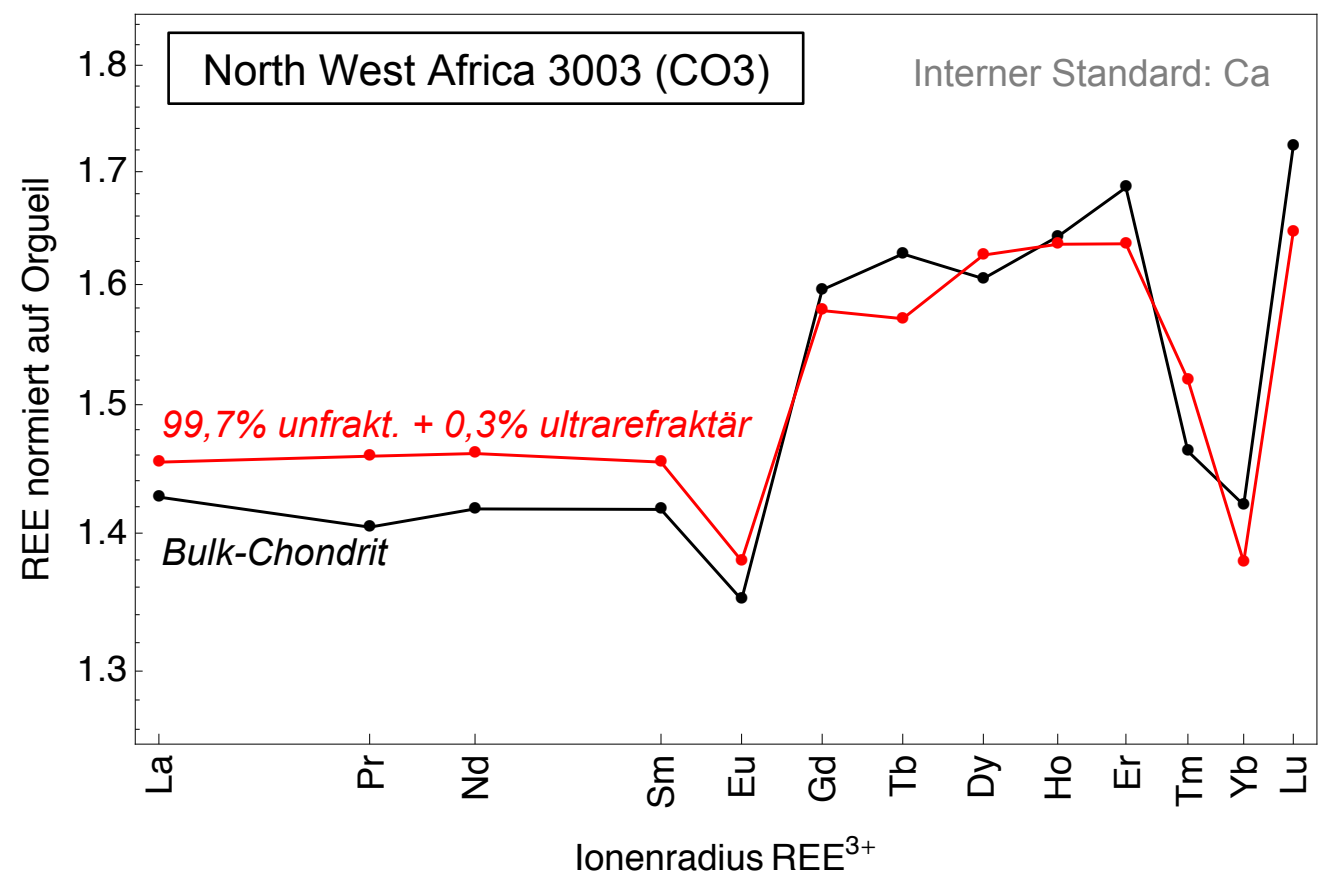

Abbildung 4.10: REE-Muster von North West Africa 3003. Der Anteil einer ultrarefraktären Komponente (Boynton et al., 1980) wird auf etwa 0,3 Gew.-\% geschätzt.

Schätzwert anzusehen. Hätte das ultrarefraktäre Material beispielsweise eine tausendfache Anreicherung an Lu gegenüber CI-Chondriten, betrüge der Anteil dieser ultrarefraktären Komponente in North West Africa 3003 nur 0,03 Gew.-\%.

Die starke Anreicherung der leichten Seltenen Erden in Bencubbin (CB) kann nicht auf volatilitätskontrollierte Fraktionierung zurückgeführt werden. Ein ähnliches an leichten Seltenen Erden angereichertes REE-Muster der Silikatphase von Bencubbin wurde ebenfalls von Kallemeyn et al. (1978) gemessen. Dieses REEMuster wurde von den Autoren auf die Fraktionierung während der Kristallisation nach einem Aufschmelzereignis zurückgeführt. Ein solches REE-Muster scheint allerdings für den Bulk-Chondrit Bencubbin nicht repräsentativ zu sein, da Kallemeyn et al. (1978) in zwei größeren Proben relativ unfraktionierte Seltene Erden fanden. Die in dieser Arbeit analysierte Probe hat eine Masse von nur $100 \mathrm{mg}$ und ist daher nicht repräsentativ für den Gesamtchondrit. 


\subsubsection{HFSE-Elemente}

Die Zr/Hf-Verhältnisse der kohligen Chondritgruppen CM, CV, CO, CK und CB liegen etwas niedriger als das der CI-Chondrite (Abbildung 3.20). Wie bereits in Abschnitt 4.2 erwähnt, sind Zr und Hf sehr refraktäre Elemente und fraktionieren während der Kondensation bei hohen Temperaturen. In frühen Kondensaten, wie zum Beispiel den CAIs, können die beiden Elemente daher gegeneinander fraktioniert sein. Die etwas niedrigeren Zr/Hf-Verhältnisse der meisten kohligen Chondrite können deshalb durch das Vorhandensein refraktärer Einschlüsse verursacht worden sein.

Die große Streuung in gewöhnlichen und Enstatit-Chondriten kann hingegen nicht auf das Vorkommen refraktärer Einschlüsse zurückgeführt werden, da diese dort nur sehr selten vorkommen. Im Gegensatz zu den Ergebnissen von Patzer et al. (2010) deuten die Ergebnisse dieser Arbeit darauf hin, dass bezüglich der $\mathrm{Zr} / \mathrm{Hf}-$ Verhältnisse kein systematischer Unterschied zwischen den verschiedenen Gruppen der gewöhnlichen und Enstatit-Chondrite besteht. Die durchschnittlichen $\mathrm{Zr} /$ Hf-Verhältnisse aller untersuchten Chondritklassen stimmen innerhalb von $\pm 5 \%$ mit dem chondritischen Mittelwert von Münker et al. (2003) überein. Es bestehen also nur geringfügige Fraktionierungen zwischen den Chondritklassen.

Die größere Streuung der Nb/Ta-Verhältnisse in den kohligen Chondriten im Vergleich zu den nichtkohligen Chondriten kann auf Probeninhomogenitäten beruhen, da kohlige Chondrite refraktäre Einschlüsse enthalten, die vom chondritischen Wert abweichende Nb/Ta-Verhältnisse aufweisen können (siehe Abschnitt 3.1). Die geringeren $\mathrm{Nb} / \mathrm{Ta}$-Verhältnisse der meisten kohligen Chondrite können ebenfalls auf den Einbau refraktären Materials zurückgeführt werden. Wie die Analyse refraktärer Einschlüsse Leovilles gezeigt hat, besitzen diese typischerweise subchondritische $\mathrm{Nb} / \mathrm{Ta}$-Verhältnisse (Abschnitt 3.1.2). Das Vorhandensein refraktärer Einschlüsse verringert so das $\mathrm{Nb} / \mathrm{Ta}-$ Verhältnis des Gesamtchondrits. Niedrige $\mathrm{Nb} / \mathrm{Ta}$-Verhältnisse wurden in CV-Chondriten ebenfalls von Münker et al. (2003) beobachtet. Auch Daten von Jochum et al. (2000) und Barrat et al. (2012) bestätigen, dass Allende im Gegensatz zu CI-Chondriten geringere Nb/Ta-Werte besitzen.

In kleinen Allende-Proben besteht ein Zusammenhang zwischen niedrigen $\mathrm{Nb} / \mathrm{Ta}-$ Verhältnissen und fraktionierten REE group-II-Mustern (Stracke et al., 2012; Münker et al., 2012): Es besteht eine negative Korrelation zwischen Nb/Ta und Tm/Er (das bei einem group-II-Muster superchondritisch ist). Eine solche Korrelation zwischen $\mathrm{Nb} / \mathrm{Ta}$ und den Seltenen Erden besteht für die hier untersuchten $\mathrm{CV}$ Chondrite und andere kohlige Chondrite nicht. Lediglich die Probe Allende-A, welche die stärkste REE-Fraktionierung aufweist, besitzt auch ein besonders niedriges $\mathrm{Nb} / \mathrm{Ta}$-Verhältnis. 
Das niedrige $\mathrm{Nb} / \mathrm{Ta}$-Verhältnis von Bencubbin ist möglicherweise auf seine spezielle Entstehungsgeschichte zurückzuführen. Da das Vorläufermaterial während der Entstehung durch ein Impaktereignis aufgeschmolzen wurde (Kallemeyn et al., 1978), kann die Fraktionierung von Nb und Ta im Zusammenhang mit der anschließenden Kristallisation stehen.

\subsection{Tm-Anomalie im Sonnensystem}

Die beobachteten Anomalien der relativen Tm-Gehalte in den chondritischen, achondritischen und planetaren Proben sollen in diesem Abschnitt näher untersucht werden. Dazu wird zunächst für alle Proben eine Tm-Anomalie quantitativ abgeschätzt. Ihre genaue Berechnung wird im Folgenden erläutert.

\subsubsection{Berechnung der Tm-Anomalie}

Für die Berechnung der Tm-Anomalie wurden nur die schweren Seltenen Erden von Gd bis Lu berücksichtigt. Da die absoluten Konzentrationen der Seltenen Erden für diese Fragestellung unerheblich sind, wurden HREE/Er-Verhältnisse benutzt. Um die Tm-Anomalie relativ zu Orgueil zu berechnen, wurden diese Verhältnisse wiederum auf Orgueil normiert:

$$
\mathrm{R}^{\mathrm{HREE}}=\frac{\mathrm{HREE}_{\text {Probe }} / \mathrm{Er}_{\text {Probe }}}{\mathrm{HREE}_{\text {Orgueil }} / \text { Er }_{\text {Orgueil }}} .
$$

Diese Orgueil- und Er-normierten Verhältnisse $\mathrm{R}^{\mathrm{HREE}}$ wurden gegen die Ionenradien bei achtfacher Koordinationsgeometrie (Shannon, 1976) geplottet. Für die Berechnung der Tm-Anomalie in Chondriten - die aus undifferenziertem Material bestehen - erfolgte zunächst eine lineare Regression zwischen Er und Lu (Abbildung 4.11, Tm selbst und $\mathrm{Yb}$ wurden dabei außer Acht gelassen). Das benachbarte $\mathrm{Yb}$ wurde nicht für die Berechnung der Tm-Anomalie herangezogen, da es selbst erheblich volatiler ist als die anderen schweren Seltenen Erden und in frühen Kondensaten des solaren Nebels variable relative Gehalte aufweist. Der Fehler in dem Bereich zwischen Er und Lu wurde ebenfalls als linear angenommen. Die Tm-Anomalie berechnet sich anschließend aus dem Er- und Orgueil-normierten Messwert für Tm $\mathrm{R}_{\text {gemessen }}^{\mathrm{Tm}}$ und dem erwarteten Wert aus der linearen Regression $\mathrm{R}_{\text {berechnet }}^{\mathrm{Tm}}$ :

$$
\text { Tm-Anomalie (\%) }=\left[\frac{\mathrm{R}_{\text {gemessen }}^{\text {Tm }}}{\mathrm{R}_{\text {berechnet }}^{T m}}-1\right] \times 100 .
$$

Der Fehler dieser Tm-Anomalie berechnet sich mit Hilfe des Gausschen Fehlerfortpflanzungsgesetzes. 


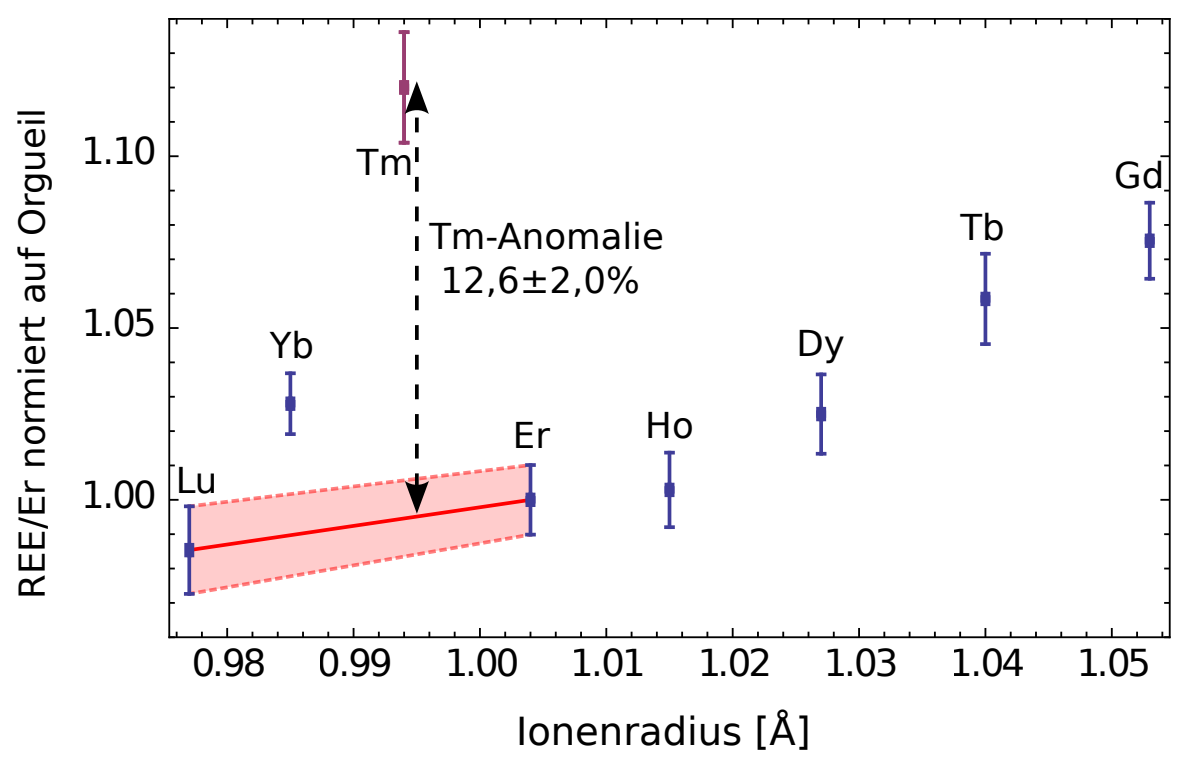

Abbildung 4.11: Berechnung der Tm-Anomalie in chondritischen Proben am Beispiel von Allende: Der gemessene Tm-Gehalt wird mit dem gemäß der linearen Regression erwarteten Wert verglichen.

Bei den Proben der differenzierten Objekte (Erde, Mond, Mars, HED-Mutterkörper und andere differenzierte Asteroiden) hat durch magmatische Prozesse auf dem Mutterkörper eine Fraktionierung der Seltenen Erden gemäß ihrem Ionenradius stattgefunden. Um diese Fraktionierungen zu berücksichtigen, wurden für die Berechnung der Tm-Anomalien der differenzierten Proben die Orgueil- und Er-normierten schweren Seltenen Erden von Gd bis Lu mit einem Polynom dritten Grades angefittet, ohne Einbeziehung von Tm und Yb (Abbildung 4.12). Diese Fitfunktion wurde ausgewählt, da die Messwerte auf diese Weise sehr gut angepasst werden konnten. Der Fehlerbereich des Fits wurde durch eine Monte Carlo Simulation abgeschätzt. Die Berechnung der Tm-Anomalie der differenzierten Proben erfolgte anschließend - in Analogie zu den Chondriten - aus dem Messwert für Tm und dem erwarteten Wert gemäß des Fits (Gleichung 4.2).

Zum Vergleich wurden aktuelle Literaturdaten von chondritischen sowie terrestrischen Proben für die Berechnung der Tm-Anomalien herangezogen. Diese Messdaten entstanden mittels ICP-SFMS (Barrat et al., 2012), MC-ICPMS (Pourmand et al., 2012) bzw. LA-ICPMS (Jenner und O'Neill, 2012a).

Die Studie von Pourmand et al. (2012) enthält Daten über fünf Chondrite (darunter Orgueil) sowie einige terrestrische Standardmaterialien und Post Archean Australian Shales (Post-archaische australische Tonschiefer, PAAS). Der Fehlerbereich der Chondrite wurde durch die für Orgueil angegebene relative Standardabweichung abgeschätzt. Für Orgueil selbst wurde ein Standardfehler der fünf 


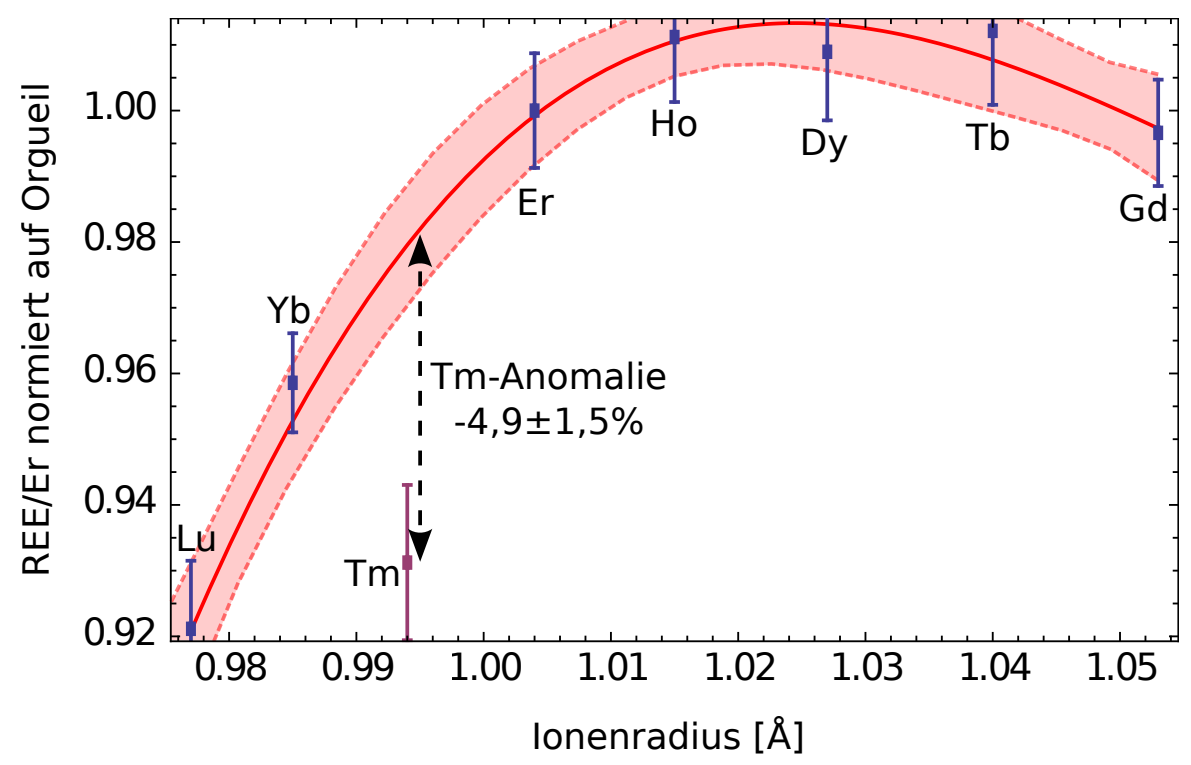

Abbildung 4.12: Berechnung der Tm-Anomalie in differenzierten Proben am Beispiel eines MORB (199 DS-24): Der gemessene Tm-Gehalt wird mit dem gemäß der kubischen Regression erwarteten Wert verglichen.

Messungen berechnet. Im Falle der terrestrischen Proben wurde ein Fehlerbereich entsprechend der angegebenen relativen Standardabweichungen der geologischen Standards G2 und G3 angenommen.

Die Studie von Barrat et al. (2012) liefert Daten über vier Chondrite (inklusive Orgueil). Als Fehler wurde für Ivuna und Alais die für Orgueil angegebene Standardabweichung benutzt. Im Falle der jeweils mehrfach gemessenen Chondrite Orgueil und Allende wurden die jeweiligen Mittelwerte mit Standardfehler herangezogen.

Die Arbeit von Jenner und O'Neill (2012a) enthält Daten über 616 Ozeanbodenbasalte (OFB; ocean floor basalt), welche mittels LA-ICPMS in-situ gemessen wurden. Die Analysen fanden an demselben Massenspektrometer statt wie die in dieser Arbeit durchgeführten Messungen. Der relative Fehler aller Seltenen Erden dieser Proben beträgt etwa $3 \%$ (Jenner und O'Neill, 2012b).

Da bei jeder Messmethode auch charakteristische systematische Fehler auftreten, wurden bei der Berechnung der Tm-Anomalie nur Daten derselben Methode miteinander verglichen, d.h. die Daten von Barrat et al. (2012) und Pourmand et al. (2012) wurden direkt auf die Orgueil-Konzentrationen der entsprechenden Studien normiert. Die OFB-Daten aus Jenner und O'Neill (2012a) wurden auf die Orgueil-Daten dieser Arbeit normiert, da sie an demselben Gerät gewonnen wurden. Der Vergleich der berechneten Tm-Anomalien der drei Chondrite Alais, Ivuna und Allende, die in dieser Arbeit sowie in den Arbeiten von Barrat et al. (2012) und Pourmand et al. (2012) gemessen wurden, zeigen eine gute Übereinstimmung. Die 
maximale absolute Abweichung beträgt weniger als drei Prozent und liegt somit innerhalb des Fehlerbereichs (Tabelle 4.5). Die berechneten Tm-Anomalien aller Proben sind in den Tabellen 4.8, 4.9 und 4.10 angegeben.

Tabelle 4.5: Tm-Anomalien in Prozent relativ zu Orgueil mit $1 \sigma$-Fehlern, berechnet aus Daten verschiedener Studien.

\begin{tabular}{lrrr}
\hline & Alais (CI) & Ivuna (CI) & Allende (CV) \\
\hline diese Arbeit & $-1,4 \pm 2,2$ & $1,4 \pm 2,1$ & $12,6 \pm 2,0$ \\
Pourmand et al. 2012 & $-1,6 \pm 3,8$ & $-0,8 \pm 3,8$ & $13,4 \pm 4,4$ \\
Barrat et al. 2012 & $-0,1 \pm 6,3$ & $1,6 \pm 6,5$ & $14,3 \pm 3,4$ \\
\hline
\end{tabular}

\subsubsection{Tm-Anomalien in Chondriten, Achondriten und der Erde}

Da der CI-Chondrit Orgueil für die Normierung der Seltenen Erden der anderen Proben benutzt wurde, sind die im Folgenden angegebenen Tm-Anomalien relativ zu Orgueil zu verstehen.

Wie bereits in Abschnitt 4.3.2 beschrieben, weisen die CI-Chondrite Ivuna und Alais ungewöhnliche Fraktionierungen der Seltenen Erden auf (Anreicherung der leichten Seltenen Erden gegenüber den schweren Seltenen Erden). Sie zeigen jedoch keine Anomalien, die auf Volatilitätsunterschiede der Seltenen Erden zurückzuführen sind. Insbesondere haben sie unter Berücksichtigung des Fehlerbereiches keine TmAnomalie.

Die anderen untersuchten kohligen Chondritgruppen besitzen, wie bereits in Abschnitt 4.3.2 diskutiert, volatilitätskontrollierte REE-Muster. Mit Ausnahme des CO-Chondrits NWA 3003, der ein deutliches ultrarefraktäres REE-Muster mit einer großen negativen Tm-Anomalie von $-13,9 \pm 2,0 \%$ besitzt, zeigen alle anderen kohligen Chondrite entweder keine oder positive Tm-Anomalien (Abbildung 4.13, Tabelle 4.6). So zeigen die Gruppe der CM-Chondrite mit $-0,1 \pm 0,9 \%$ und der CB-Chondrit Bencubbin mit 1,3 $\pm 2,4 \%$ im Rahmen des Fehlerbereiches keine TmAnomalien. Der CK-Chondrit Karoonda hat dagegen eine positive Tm-Anomalie von $5,2 \pm 1,7 \%$ und die CV-Chondrite haben im Mittel eine große positive TmAnomalie von $13,4 \pm 1,1 \%$.

Der Vergleich verschiedener CM- und CV-Chondrite untereinander verdeutlicht, dass die Anomalien innerhalb der Gruppen variabel sein können. Die Tm-Anomalien für CM-Chondrite reichen von -3,0\% für Cold Bokkeveld bis 5,0\% für Murray, die der CV-Chondrite von 9,3\% für Axtell bis 18,0\% für Vigarano (Tabelle 4.8). 


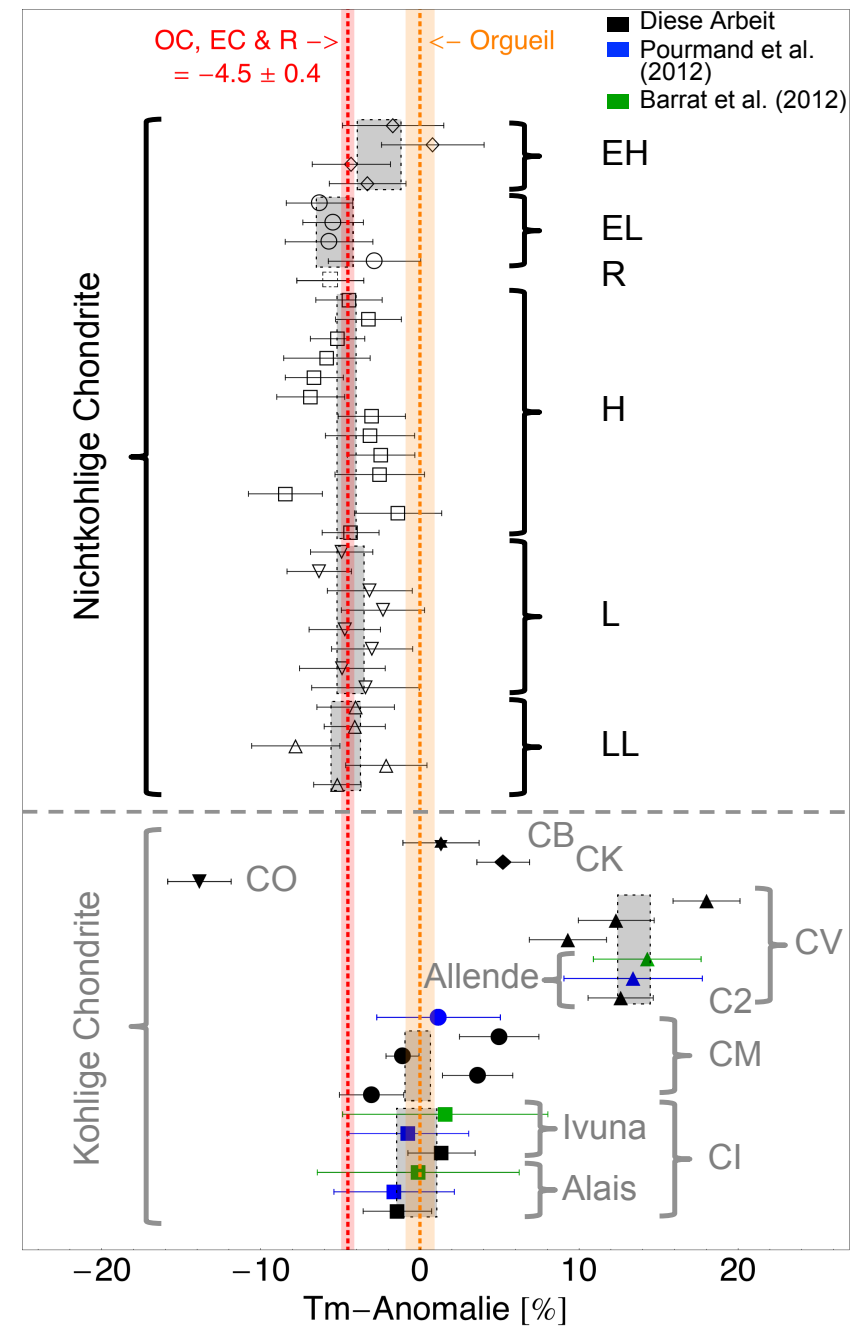

Abbildung 4.13: Tm-Anomalien in Chondriten relativ zu Orgueil. Die kohligen Chondrite haben (mit Ausnahme des CO-Chondrits) entweder keine oder positive Tm-Anomalien und zeigen eine starke Streuung. Die nichtkohligen Chondrite haben dagegen ähnliche negative Tm-Anomalien. Die grauen Rechtecke stellen gewichtete Gruppenmittelwerte mit Fehlerbereich dar. Der Fehler von Orgueil entspricht dem 1 $\sigma$-Standardfehler der fünf Orgueil-Proben. 
Tabelle 4.6: Gewichtete Mittelwerte der Tm-Anomalien der verschiedenen Chon-

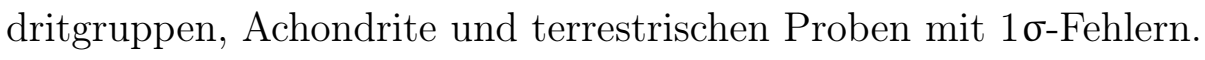

\begin{tabular}{|c|c|c|}
\hline \multicolumn{3}{|c|}{ Tm-Anomalie (\%) } \\
\hline \multicolumn{3}{|c|}{ Kohliges Chondrit-Reservoir } \\
\hline $\mathrm{CI}^{1}$ (Alais, Ivuna) & $-0,2 \pm 1,3$ & $(\mathrm{~N}=2)$ \\
\hline $\mathrm{CM}$ & $-0,1 \pm 0,9$ & $(\mathrm{~N}=4)$ \\
\hline $\mathrm{C} 2^{1}$ & $1,2 \pm 3,9$ & $(\mathrm{~N}=1)$ \\
\hline $\mathrm{CV}^{1}$ & $13,4 \pm 1,1$ & $(\mathrm{~N}=4)$ \\
\hline $\mathrm{CO}$ & $-13,9 \pm 2,0$ & $(\mathrm{~N}=1)$ \\
\hline CK & $5,2 \pm 1,7$ & $(\mathrm{~N}=1)$ \\
\hline $\mathrm{CB}^{2}$ & $1,3 \pm 2,4$ & $(\mathrm{~N}=1)$ \\
\hline \multicolumn{3}{|c|}{ Nichtkohliges Chondrit-Reservoir } \\
\hline LL & $-4,7 \pm 1,0$ & $(\mathrm{~N}=5)$ \\
\hline $\mathrm{L}$ & $-4,4 \pm 0,9$ & $(\mathrm{~N}=8)$ \\
\hline $\mathrm{H}$ & $-4,6 \pm 0,6$ & $(\mathrm{~N}=11)$ \\
\hline $\mathrm{R}$ & $-5,6 \pm 2,2$ & $(\mathrm{~N}=1)$ \\
\hline EL & $-5,4 \pm 1,2$ & $(\mathrm{~N}=4)$ \\
\hline $\mathrm{EH}$ & $-2,6 \pm 1,4$ & $(\mathrm{~N}=3)$ \\
\hline HED & $-4,1 \pm 0,6$ & $(\mathrm{~N}=8)$ \\
\hline WIN & $-3,6 \pm 2,5$ & $(\mathrm{~N}=1)$ \\
\hline AUB & $-4,1 \pm 7,0$ & $(\mathrm{~N}=1)$ \\
\hline Mars & $-3,0 \pm 1,2$ & $(\mathrm{~N}=2)$ \\
\hline Mond & $-4,5 \pm 1,1$ & $(\mathrm{~N}=3)$ \\
\hline Erde $^{1}$ & $-5,0 \pm 0,2$ & $(\mathrm{~N}=678)$ \\
\hline Peridotite ${ }^{1}$ & $-5,3 \pm 0,6$ & $(\mathrm{~N}=18)$ \\
\hline Komatiite & $-4,9 \pm 0,9$ & $(\mathrm{~N}=5)$ \\
\hline Tektite & $-3,6 \pm 0,9$ & $(\mathrm{~N}=4)$ \\
\hline Sedimente $^{1}$ & $-4,7 \pm 1,1$ & $(\mathrm{~N}=11)$ \\
\hline Felsische Gesteine ${ }^{1}$ & $-10,3 \pm 3,9$ & $(\mathrm{~N}=6)$ \\
\hline Mafische Gesteine ${ }^{1}$ & $-5,0 \pm 0,2$ & $(\mathrm{~N}=634)$ \\
\hline Mittelwert & $-4,8 \pm 0,2$ & \\
\hline
\end{tabular}

1 Mittelwert enthält Literaturdaten.

${ }^{2}$ Es wurde nur die Silikatphase untersucht.

N: Anzahl der untersuchten Proben. 
Alle Gruppen der gewöhnlichen, Enstatit- und Rumuruti-Chondrite zeigen im Vergleich zu Orgueil negative Tm-Anomalien zwischen -2,6\% für EH- und -5,6\% für R-Chondrite (Abbildung 4.13; Tabelle 4.6). Der gewichtete Mittelwert aller nichtkohligen Chondrite beträgt $-4,5 \pm 0,4 \%$.

Die berechneten Tm-Anomalien der differenzierten Proben liegen zwischen -3,0\% (Mars) und -5,0\% (Erde). Ihr gewichteter Mittelwert beträgt -4,9 $\pm 0,2 \%$ (Abbildung 4.14). Hierbei sind die terrestrischen Proben wegen ihrer hohen Anzahl sehr stark gewichtet. Dieser Mittelwert überlappt mit dem Fehlerbereich der nichtkohligen Chondrite von $-4,5 \pm 0,4 \%$. Es kann also festgehalten werden, dass die Proben der Erde, des Mond, des Mars und der anderen Achondrite eine ähnliche Tm-Anomalie aufweisen wie die nichtkohligen Chondrite.

Da die Tm-Anomalie in allen untersuchten terrestrischen Gesteinsarten existiert, kann ausgeschlossen werden, dass sie durch magmatische Fraktionierungsprozesse hervorgerufen wird. Außerdem wurden auch keine Hinweise auf Fraktionierungen aufgrund des Tetradeneffektes gefunden, der in aquatischen Systemen durch Komplexbildung auftreten kann (Bau, 1996; Monecke et al., 2002). Darüberhinaus ist es ebenfalls sehr unwahrscheinlich, dass die Tm-Anomalie der Erde auf moderat siderophiles Verhalten von Tm unter hohen Druck- und Temperaturbedingungen und den Einbau von Tm in den Erdkern zurückzuführen ist. In diesem Fall wäre eine kontinuierliche Abnahme der Tm-Anomalie, entsprechend der Größe der Objekte, von der Erde über den Mars bis hin zu den Achondriten zu erwarten. Dies ist jedoch nicht der Fall. Insbesondere die ähnliche Tm-Anomalie der Erde und der nichtkohligen Chondrite, die in ihrer Entstehungsgeschichte nicht aufgeschmolzen wurden, deutet auf einen kosmochemischen Ursprung der Tm-Anomalie hin.

Die Tatsache, dass die Tm-Anomalien der Erde, des Mondes und des Mars im Fehlerbereich mit denen der nichtkohligen Chondrite überlappen, sich jedoch signifikant von denen der kohligen Chondrite unterscheiden, spricht dafür, dass die Vorläufermaterialien der terrestrischen Planeten in ihrer chemischen Zusammensetzung eher den nichtkohligen als den kohligen Chondriten entsprachen.

Analog zu der Klassifikation von Warren (2011), die auf der Isotopenzusammensetzung von Sauerstoff $\left(\Delta^{17} \mathrm{O}\right)$, Chrom $\left(\epsilon^{54} \mathrm{Cr}\right)$, Titan $\left(\epsilon^{50} \mathrm{Ti}\right)$ und Nickel $\left(\epsilon^{62} \mathrm{Ni}\right)$ basiert, werden die Objekte des Sonnensystems in dieser Arbeit anhand ihrer Tm-Anomalien in zwei verschiedene Gruppen eingeteilt: Die kohligen Chondrite entstammen dem kohligen Chondrit-Reservoir, die gewöhnlichen, Enstatit- und Rumuruti-Chondrite, sowie die Achondrite und die terrestrischen Planeten entstammen dem nichtkohligen Chondrit-Reservoir. Die gewichtete mittlere Tm-Anomalie aller Objekte des nichtkohligen Chondrit-Reservoirs beträgt dann $-4,8 \pm 0,2 \%$ (Abbildung 4.15).

Es stellt sich nun die Frage, ob die durchschnittlichen HREE-Verhältnisse des gesamten Sonnensystems tatsächlich besser durch die CI-Chondrite (kohliges Chondrit- 

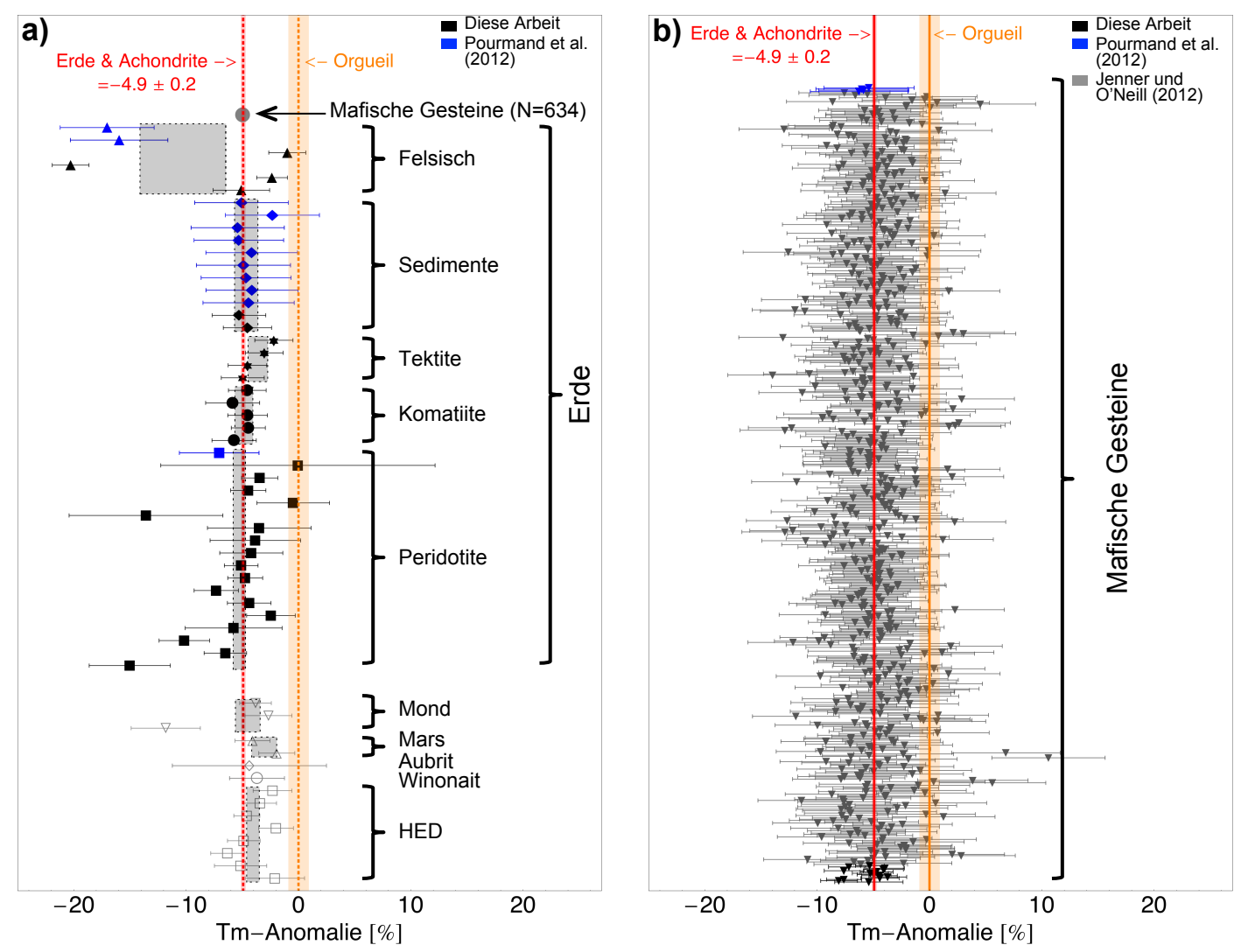

Abbildung 4.14: a) Tm-Anomalien in Achondriten und verschiedenen terrestrischen Gesteinstypen. Die mafischen Gesteine (634 Proben) werden durch ihren Mittelwert repräsentiert und sind in b) im Einzelnen gezeigt. Die Achondrite, inklusive der Mars- und Mondmeteorite sowie die irdischen Gesteine haben eine ähnliche negative Tm-Anomalie. Die grauen Rechtecke stellen gewichtete Gruppenmittelwerte mit Fehlerbereich dar (für die felsischen Gesteine: Mittelwert mit Standardfehler).

Reservoir) repräsentiert werden oder ob sie eher mit den Objekten des nichtkohligen Chondrit-Reservoirs übereinstimmen. Da die Sonne über $99 \%$ der Materie des Sonnensystems vereint, entspricht die solare Zusammensetzung im Wesentlichen auch der des gesamten Sonnensystems. Sie kann zwar durch spektroskopische Untersuchungen der Photosphäre (Lodders, 2003; Grevesse et al., 2007) oder durch Analysen des Sonnenwindes (Marty et al., 2011) gemessen werden. Da diese Untersuchungen jedoch mit relativ großen Ungenauigkeiten behaftet sind (Lodders, 2003; Grevesse et al., 2007), kann die Frage, ob CI-Chondrite hinsichtlich der Seltenen Erden tatsächlich der beste Proxy für das Sonnensystem sind, auf diese Weise nicht beantwortet werden. 
Die Objekte des nichtkohligen Chondrit-Reservoirs, die in dieser Arbeit beprobt wurden und alle eine ähnliche Tm-Anomalie aufweisen, entsprechen zusammen etwa $56 \%$ der Masse des inneren Sonnensystems. Dagegen ist die Masse der kohligen Chondrite und vor allem die der CI-Chondrite sehr klein. Es kommen zwei Interpretationsmöglichkeiten in Betracht:

1. Der CI-Chondrit Orgueil entspricht in den Tm/HREE-Verhältnissen dem Sonnensystem. In diesem Fall hätten alle Objekte des nichtkohligen ChondritReservoirs fraktionierte Seltene Erden relativ zum Sonnensystem. Negative Tm-Anomalien können beispielsweise durch die Zufuhr einer Komponente mit ultrarefraktären REE-Mustern oder durch das Entfernen einer refraktären Komponente mit group-II-Muster entstehen. Es wäre also ein Prozess in der frühen Phase des Sonnensystems nötig gewesen, der auf einer sehr großen Skala (von dem Enstehungsgebiet der Erde bis zum Asteroidengürtel) eine gleichmäßige Tm-Anomalie erzeugt hätte. Ein solcher Prozess ist bisher nicht bekannt und ist darüberhinaus sehr unwahrscheinlich.

2. Die Objekte des nichtkohligen Chondrit-Reservoirs entsprechen in ihren Tm/HREE-Verhältnissen dem Sonnensystem. In diesem Fall hätten die CIChondrite eine positive Tm-Anomalie im Vergleich zum Sonnensystem. Dies erscheint insbesondere deshalb plausibel, da auch die anderen kohligen Chondrite variable Tm-Anomalien aufweisen.

Da das zweite Szenario wahrscheinlicher erscheint als das erste, wird der Mittelwert des nichtkohligen Chondrit-Reservoirs im Folgenden als solarer Wert betrachtet. Alle kohligen Chondrite, mit Ausnahme des CO-Chondrits, haben folglich eine positive Tm-Anomalie im Vergleich zum Sonnensystem, die im Fall des CI-Chondrits Orgueil 4,8 $\pm 0,9 \%$ beträgt (Abbildung 4.15). Es ist wahrscheinlich, dass in der frühen Phase des Sonnensystems refraktärer Staub mit fraktionierten Seltenen Erden in die Bildungsregion der kohligen Chondrite transportiert wurde und den verschiedenen kohligen Chondritgruppen in unterschiedlichem Maße zugeführt wurde. Radialer Transport von Hochtemperaturmaterialien aus sonnennahen Regionen in die äußeren Gebiete des Sonnensystems wird anhand verschiedener Modelle diskutiert (Shu et al., 1996; Cuzzi et al., 2003; Ciesla, 2007; Wurm und Haack, 2009). Das Vorkommen von CAIs in dem Komet 81P/Wild2 (Zolensky et al., 2006) deutet die Durchmischung von Materialien des Sonnensystems auf einer sehr großen Skala an. Auch in CI-Chondriten kommen vereinzelt CAIs vor (Frank et al., 2011), so dass es plausibel erscheint, dass sie eine refraktäre Komponente mit REE group-II-Muster enthalten.

Bei der Normierung hochpräziser REE-Daten terrestrischer und extraterrestrischer Proben sollten diese Fraktionierungen der CI-Chondrite berücksichtigt 


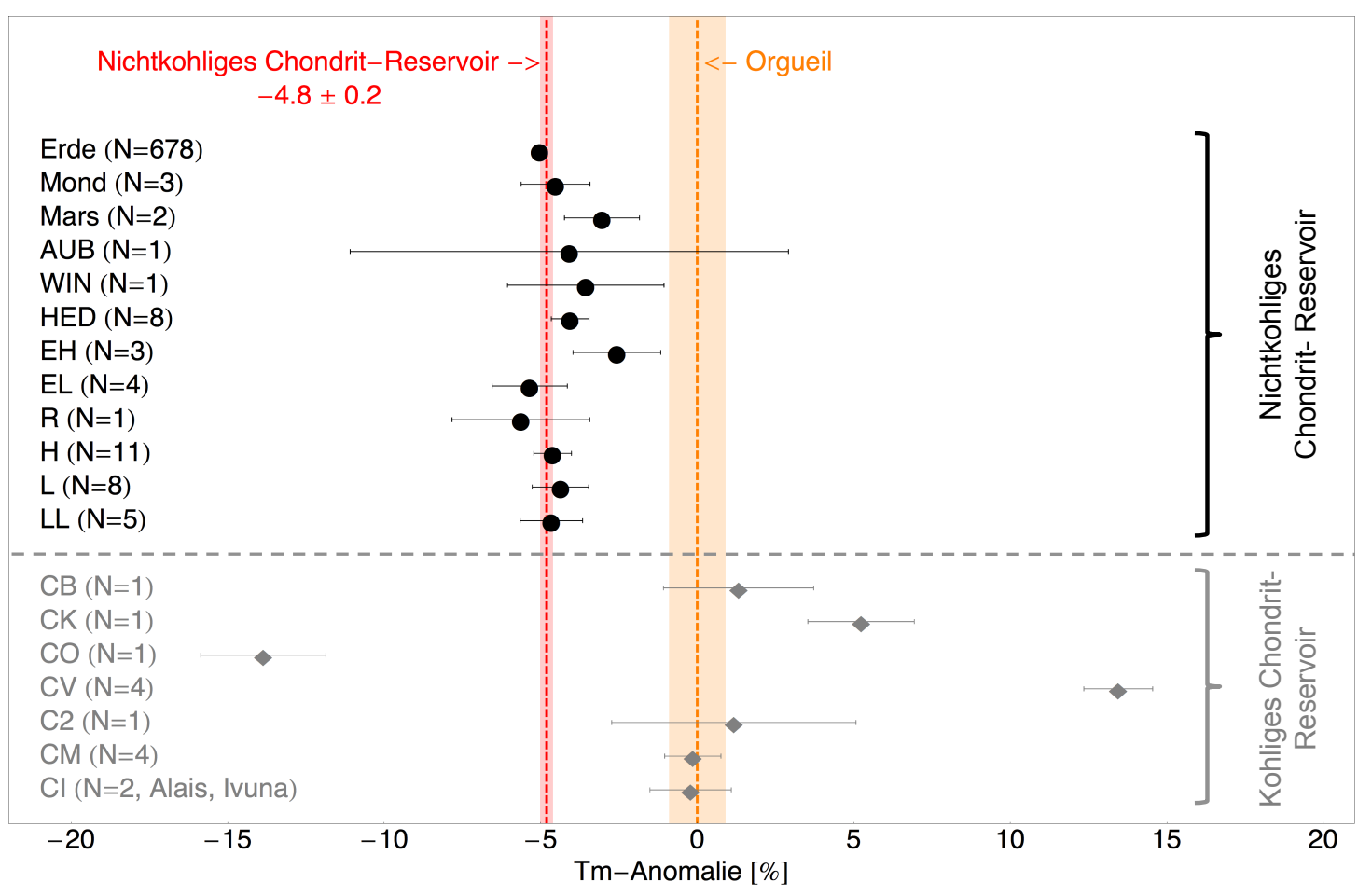

Abbildung 4.15: Mittlere Tm-Anomalien von Chondritgruppen, Achondritgruppen und Planeten. Die Objekte zeigen eine Zweiteilung bezüglich ihrer TmAnomalien. Es wird hier angenommen, dass das nichtkohlige Chondrit-Reservoir den solaren Wert widerspiegelt. Die Objekte des kohligen Chondrit-Reservoirs haben variable, meist positive Tm-Anomalien.

werden oder es sollte alternativ auf einen Mittelwert der nichtkohligen Chondrite normiert werden. In Tabelle 4.7 sind Konzentrationsdaten der Seltenen Erden von Orgueil und den nichtkohligen Chondriten angegeben. Diese wurden anhand der SmKonzentration von Orgueil von 0,149 ppm (Bouvier et al., 2008) bzw. der mittleren Sm-Konzentration der gewöhnlichen und Enstatit-Chondrite von 0,194 ppm, die sich ebenfalls aus den Daten von Bouvier et al. (2008) ergibt, berechnet.

Die positive Tm-Anomalie von Orgueil gegenüber den nichtkohligen Chondriten kann durch einen geringen Anteil einer refraktären Phase erklärt werden. Dabei werden die mittleren REE-Konzentrationen der nichtkohligen Chondrite als unfraktioniertes Ausgangsmaterial angenommen. Wenn der Abreicherungsfaktor der REE-Konzentrationen in Orgueil gegenüber den nichtkohligen Chondriten etwa 0,72 beträgt und zusätzlich ein geringer Anteil einer refraktären Phase mit groupII-Muster von 0,2\% in Orgueil vorhanden ist, stimmen die Fraktionierungen der schweren Seltenen Erden gut mit den tatsächlich gemessenen Konzentrationen überein (Abbildung 4.16). Für die leichten Seltenen Erden, insbesondere La, ergeben 


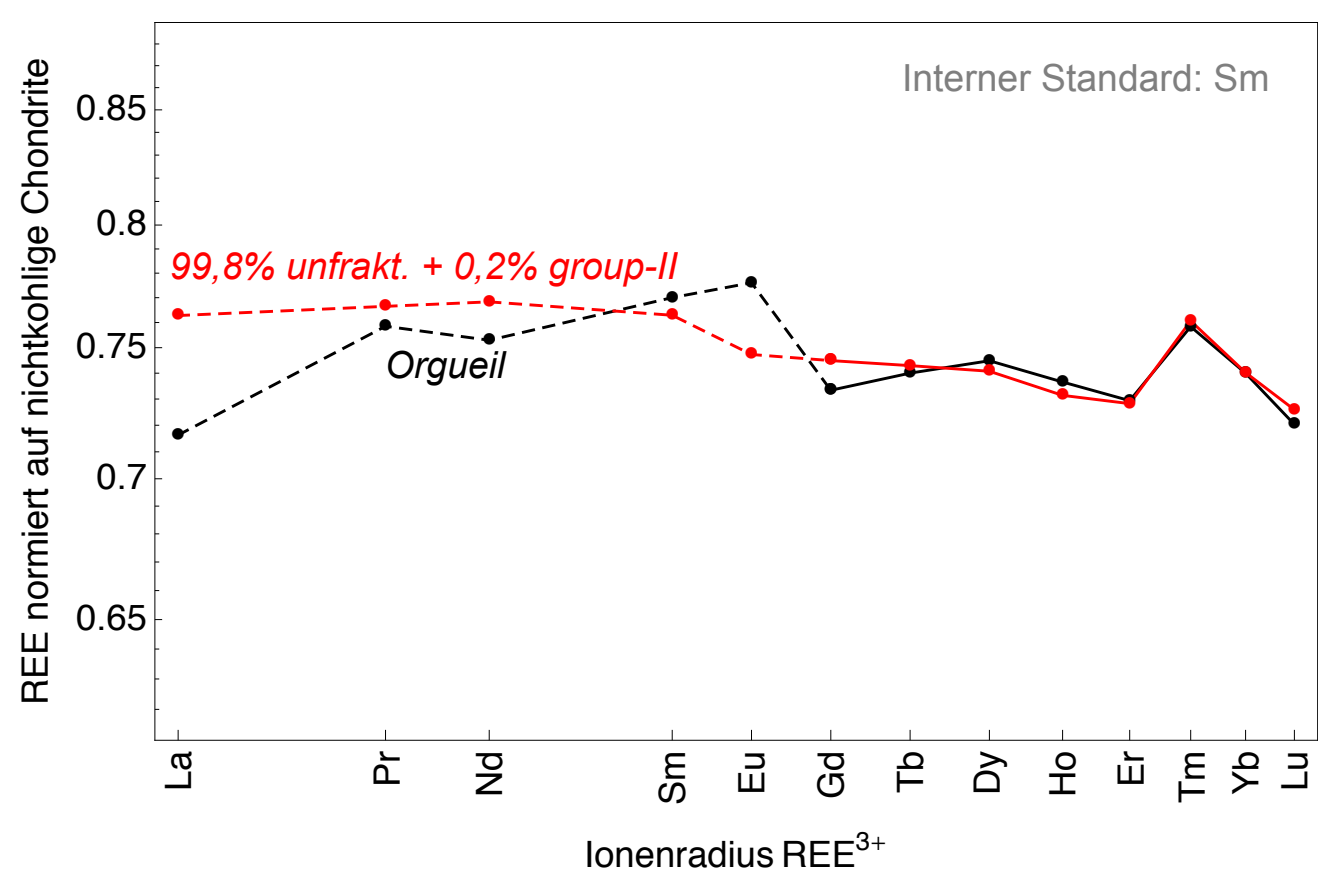

Abbildung 4.16: Refraktäre Komponente in Orgueil. Unter der Annahme, dass nichtkohlige Chondrite solare HREE-Verhältnisse widerspiegeln, wird der Anteil einer fraktionierten group-II-Komponente in Orgueil auf etwa 0,2 Gew.- \% geschätzt. Bei der Abschätzung wurden nur die schweren REE (durchgezogene Linien) berücksichtigt.

sich dagegen größere Abweichungen, die dadurch zustande kommen können, dass in einigen nichtkohligen Chondriten die leichten Seltenen Erden angereichert sind.

Die Bimodalität hinsichtlich der schweren Seltenen Erden zwischen dem kohligen und dem nichtkohligen Chondrit-Reservoir konnte für andere refraktäre Elementverhältnisse wie $\mathrm{Zr} / \mathrm{Hf}$ und $\mathrm{Nb} / \mathrm{Ta}$ nicht nachgewiesen werden (Abbildung 3.20). Diese Zweiteilung spiegelt sich jedoch in der Verteilung von stabilen Isotopen von $\epsilon^{54} \mathrm{Cr}$, $\epsilon^{50} \mathrm{Ti}$ und $\epsilon^{62} \mathrm{Ni}$ (Warren, 2011) wider. Es ist daher möglich, dass auch die Isotopenverhältnisse des kohligen Chondrit-Reservoirs, inklusive der CI-Chondrite, nicht der solaren Isotopenzusammensetzung entsprechen, sondern dass die Objekte des nichtkohligen Chondrit-Reservoirs die solare Zusammensetzung besser repräsentieren. Ein Eintrag von Material mit einer außergewöhnlichen Isotopenzusammensetzung bezüglich $\epsilon^{54} \mathrm{Cr}, \epsilon^{50} \mathrm{Ti}$ und $\epsilon^{62} \mathrm{Ni}$ würde die beobachteten Anomalien der kohligen Chondrite erklären. 
Tabelle 4.7: REE/Er-Verhältnisse der CI-Zusammensetzung (Orgueil) und der nichtkohligen Chondrite (Mittelwert aus gewöhnlichen, Enstatitund Rumuruti-Chondriten) sowie Konzentrationen, die mithilfe der Sm-Konzentration von Orgueil bzw. der mittleren Sm-Konzentration von gewöhnlichen und Enstatit-Chondriten (Bouvier et al., 2008) berechnet wurden. Die mittleren absoluten Konzentrationen der nichtkohligen Chondrite sind nur als Näherungswert zu betrachten. Der H-Chondrit Adrian, welcher eine starke REE-Fraktionierung aufweist, ist nicht im Mittelwert berücksichtigt.

\begin{tabular}{lcccc}
\hline & \multicolumn{2}{c}{ REE/Er-Verhältnisse } & \multicolumn{2}{c}{ REE-Konz. (ppm) } \\
& Orgueil & Mittelwert & Orgueil & Mittelwert \\
& $(\mathrm{CI})$ & OC, EC, RC & OC, EC, RC \\
\hline $\mathrm{La}$ & 1,45 & 1,47 & 0,234 & 0,327 \\
$\mathrm{Pr}$ & 0,558 & 0,536 & 0,090 & 0,119 \\
$\mathrm{Nd}$ & 2,77 & 2,68 & 0,448 & 0,595 \\
$\mathrm{Sm}$ & 0,922 & 0,873 & 0,149 & 0,194 \\
$\mathrm{Eu}$ & 0,341 & 0,320 & 0,055 & 0,071 \\
$\mathrm{Gd}$ & 1,20 & 1,20 & 0,195 & 0,266 \\
$\mathrm{~Tb}$ & 0,221 & 0,218 & 0,036 & 0,048 \\
$\mathrm{Dy}$ & 1,54 & 1,51 & 0,249 & 0,334 \\
$\mathrm{Ho}$ & 0,338 & 0,334 & 0,055 & 0,074 \\
$\mathrm{Er}$ & 1 & 1 & 0,162 & 0,222 \\
$\mathrm{Tm}$ & 0,152 & 0,146 & 0,025 & 0,032 \\
$\mathrm{Yb}$ & 1,04 & 1,03 & 0,169 & 0,228 \\
$\mathrm{Lu}$ & 0,155 & 0,157 & 0,025 & 0,035 \\
\hline
\end{tabular}


Tabelle 4.8: Tm-Anomalien der in dieser Arbeit analysierten meteoritischen und terrestrischen Proben mit 1 $\sigma$-Fehlern. Bei der Analyse mehrerer Proben desselben Meteorits wurden gewichtete Mittelwerte der Tm-Anomalien berechnet.

\begin{tabular}{|c|c|c|c|c|c|}
\hline Probe & \multirow[t]{2}{*}{$\begin{array}{c}\text { Tm- } \\
\text { Anomalie } \\
(\%)\end{array}$} & Probe & $\begin{array}{c}\text { Tm- } \\
\text { Anomalie } \\
(\%)\end{array}$ & Probe & $\begin{array}{c}\text { Tm- } \\
\text { Anomalie } \\
(\%)\end{array}$ \\
\hline Kohlige Chondrite & & Pultusk-A & $-5,8 \pm 2,2$ & \multicolumn{2}{|c|}{ Erde } \\
\hline Orgueil-A & $-2,2 \pm 2,0$ & Pultusk-B & $-4,2 \pm 2,7$ & \multicolumn{2}{|c|}{ Peridotite/Serpentinite } \\
\hline Orgueil-B & $1,0 \pm 1,8$ & Mittelwert & $-5,2 \pm 1,7$ & GZG.GSP.13450 & $-15,0 \pm 3,6$ \\
\hline Orgueil-C & $1,4 \pm 2,0$ & Richardton & $-3,2 \pm 2,1$ & De-1 & $-6,5 \pm 1,9$ \\
\hline Orgeuil-D & $-1,2 \pm 2,4$ & Thuathe & $-4,5 \pm 2,1$ & De-2 & $-10,2 \pm 2,2$ \\
\hline Orgeuil-E & $1,0 \pm 2,4$ & \multicolumn{2}{|c|}{ Rumuruti-Chondrite } & De-3 & $-5,8 \pm 4,3$ \\
\hline Mittelwert & $0,0 \pm 0,9$ & NWA 753 & $-5,6 \pm 2,1$ & $\mathrm{Zm}$ & $-2,4 \pm 2,2$ \\
\hline Alais & $-1,4 \pm 2,2$ & \multicolumn{2}{|c|}{ Enstatit-Chondrite } & $\mathrm{SC}-1$ & $-4,4 \pm 1,9$ \\
\hline Ivuna & $1,4 \pm 2,1$ & Atlanta & $-2,9 \pm 2,9$ & $\mathrm{SC}-2$ & $-7,3 \pm 2,0$ \\
\hline Cold Bokkeveld & $-3,0 \pm 2,0$ & Eagle & $-5,7 \pm 2,8$ & $\mathrm{Ba}-1$ & $-4,7 \pm 1,6$ \\
\hline Mighei & $3,6 \pm 2,2$ & Hvittis & $-5,5 \pm 1,9$ & $\mathrm{Ba}-2$ & $-5,1 \pm 1,5$ \\
\hline Murchison-A & $-1,6 \pm 2,6$ & Pillistfer & $-6,3 \pm 2,1$ & $\mathrm{Za}-27$ & $-4,2 \pm 2,8$ \\
\hline Murchison-B & $-1,9 \pm 2,7$ & Abee & $-3,3 \pm 2,4$ & PCC-15 & $-3,8 \pm 4,0$ \\
\hline Murchison-C & $2,9 \pm 2,9$ & Indarch & $-4,3 \pm 2,5$ & JP-15 & $-3,5 \pm 4,6$ \\
\hline Murchison-D & $-0,7 \pm 1,8$ & Sahara 97072 & $0,8 \pm 3,2$ & DTS-15 & $-13,6 \pm 6,8$ \\
\hline Murchison-E & $-2,9 \pm 2,1$ & St. Mark's & $-1,7 \pm 3,2$ & NIM-D5 & $-0,5 \pm 3,2$ \\
\hline Mittelwert & $-1,1 \pm 1,0$ & HED-Meteor & & NIM-P5 & $-4,5 \pm 1,6$ \\
\hline Murray & $5,0 \pm 2,5$ & Bilanga & $-2,1 \pm 2,6$ & UB-N5 & $-3,5 \pm 1,6$ \\
\hline Allende-S & $12,6 \pm 2,0$ & Cachari & $-5,2 \pm 2,3$ & El-1 & $-0,1 \pm 12,2$ \\
\hline Axtell & $9,3 \pm 2,4$ & Camel Donga & $-6,3 \pm 1,5$ & \multicolumn{2}{|l|}{ Komatiite } \\
\hline Mokoia & $12,3 \pm 2,4$ & Dhofar 182 & $-4,9 \pm 1,5$ & 24803 & $-5,7 \pm 2,0$ \\
\hline Vigarano & $18,0 \pm 2,1$ & Juvinas & $-2,0 \pm 1,5$ & 26575 & $-4,5 \pm 1,5$ \\
\hline NWA 3003 & $-13,9 \pm 2,0$ & Millbillillie-A & $-3,9 \pm 1,7$ & 26581 & $-4,5 \pm 1,8$ \\
\hline Karoonda-A & $3,8 \pm 2,1$ & Millbillillie-B & $-5,2 \pm 1,6$ & $\mathrm{Za}-28 \mathrm{a} 2$ & $-5,9 \pm 2,4$ \\
\hline Karoonda-B & $7,7 \pm 2,7$ & Mittelwert & $-4,6 \pm 1,2$ & Za-29a2 & $-4,6 \pm 1,7$ \\
\hline Mittelwert & $5,2 \pm 1,7$ & NWA 3359 & $-3,4 \pm 1,5$ & \multicolumn{2}{|l|}{ Tektite } \\
\hline Bencubbin & $1,3 \pm 2,4$ & Padvarninkai & $-2,3 \pm 1,7$ & MOL & $-5,0 \pm 1,9$ \\
\hline \multicolumn{2}{|c|}{ Gewöhnliche Chondrite } & \multicolumn{2}{|l|}{ Winonait } & $\mathrm{KOH}$ & $-4,5 \pm 1,7$ \\
\hline Chainpur-A & $-5,5 \pm 1,8$ & NWA 1457 & $-3,7 \pm 2,4$ & MUO & $-3,0 \pm 1,7$ \\
\hline Chainpur-B & $-4,5 \pm 2,8$ & Aubrit & & QUA & $-2,2 \pm 1,7$ \\
\hline Mittelwert & $-5,2 \pm 1,5$ & Mount Egertor & $-4,4 \pm 6,9$ & \multicolumn{2}{|l|}{ Sedimente } \\
\hline Dar al Gani 298 & $-2,1 \pm 2,6$ & Mars-Meteo & & El-2 & $-4,5 \pm 2,1$ \\
\hline Dhurmsala & $-7,8 \pm 2,8$ & NWA 4864 & $-1,9 \pm 1,6$ & ES1 & $-5,3 \pm 2,4$ \\
\hline Lake Labyrinth & $-4,1 \pm 1,9$ & SAU 051 & $-4,1 \pm 1,6$ & \multicolumn{2}{|c|}{ Felsische Gesteine } \\
\hline Saint-Séverin & $-4,0 \pm 2,4$ & \multicolumn{2}{|c|}{ Mond-Meteorite } & Pan-02-0473 & $-5,1 \pm 2,5$ \\
\hline Bruderheim & $-3,4 \pm 3,4$ & Dhofar 911 & $-11,8 \pm 3,1$ & GZG.GSP.12569 & $-2,4 \pm 1,4$ \\
\hline Gashua & $-4,9 \pm 2,7$ & Kalahari 008 & $-2,7 \pm 2,1$ & GSP-25 & $-20,3 \pm 1,6$ \\
\hline Homestead & $-3,0 \pm 2,5$ & Kalahari 009 & $-3,8 \pm 1,4$ & RGM-25 & $-1,0 \pm 1,6$ \\
\hline Mount Tazerzait & $-4,7 \pm 2,2$ & & & \multicolumn{2}{|c|}{ Mafische Gesteine } \\
\hline NWA 869 & $-2,3 \pm 2,6$ & & & El-3 & $-5,4 \pm 3,0$ \\
\hline SAU 001 & $-3,2 \pm 2,7$ & & & LP1 & $-8,0 \pm 1,6$ \\
\hline St. Michel & $-6,3 \pm 2,0$ & & & LP4 & $-7,6 \pm 1,6$ \\
\hline Zavid & $-4,9 \pm 2,0$ & & & $\mathrm{SC}-3$ & $-4,4 \pm 1,7$ \\
\hline Adrian & $-4,4 \pm 1,8$ & & & 138 DS-14 & $-3,7 \pm 1,7$ \\
\hline Dar al Gani 300 & $-1,4 \pm 2,7$ & & & 155 DS-14 & $-4,4 \pm 1,6$ \\
\hline Djoumine & $-8,5 \pm 2,3$ & & & 199 DS-24 & $-4,9 \pm 1,5$ \\
\hline El Hammami & $-2,5 \pm 2,8$ & & & 157 DS-34 & $-5,3 \pm 1,6$ \\
\hline Forest City & $-2,4 \pm 2,1$ & & & 137 DS-44 & $-4,7 \pm 1,5$ \\
\hline Gao-Guenie & $-3,1 \pm 2,8$ & & & BIR-15 & $-7,6 \pm 1,7$ \\
\hline Hammond Downs & $-3,0 \pm 2,1$ & & & BHVO-25 & $-4,1 \pm 1,8$ \\
\hline Ijopega & $-6,9 \pm 2,1$ & & & W-2a5 & $-4,0 \pm 1,7$ \\
\hline Jilin & $-6,6 \pm 1,8$ & & & AGV-25 & $-7,2 \pm 1,8$ \\
\hline Kunya-Urgench & $-5,8 \pm 2,7$ & & & BCR-2G5 & $-5,3 \pm 1,3$ \\
\hline
\end{tabular}

NWA: North West Africa.

SAU: Sayh al Uhaymir. 
Tabelle 4.9: Tm-Anomalien, berechnet aus Literaturdaten von Chondriten und irdischen Gesteinen.

\begin{tabular}{llrc}
\hline Probe & Gesteinsart & Tm-Anomalie $(\%)$ & Referenz \\
\hline Alais & CI1 & $-1,6 \pm 3,8$ & $\mathrm{P}$ \\
Alais & CI1 & $-0,1 \pm 6,3$ & $\mathrm{~B}$ \\
Ivuna & CI1 & $-0,8 \pm 3,8$ & $\mathrm{P}$ \\
Ivuna & CI1 & $1,6 \pm 6,5$ & $\mathrm{~B}$ \\
Tagish Lake & C2 & $1,2 \pm 3,9$ & $\mathrm{P}$ \\
Allende & CV3 & $13,4 \pm 4,4$ & $\mathrm{P}$ \\
Allende & CV3 & $14,3 \pm 3,4$ & $\mathrm{~B}$ \\
PCC-1 & Peridotit & $-7,1 \pm 3,5$ & $\mathrm{P}$ \\
BHVO-1 & Basalt & $-6,3 \pm 4,3$ & $\mathrm{P}$ \\
BIR-1 & Basalt & $-5,8 \pm 4,0$ & $\mathrm{P}$ \\
BCR-2 & Basalt & $-6,0 \pm 4,1$ & $\mathrm{P}$ \\
W-2 & Diabas & $-5,4 \pm 4,0$ & $\mathrm{P}$ \\
G-2 & Granit & $-16,0 \pm 4,3$ & $\mathrm{P}$ \\
G-3 & Granit & $-17,0 \pm 4,2$ & $\mathrm{P}$ \\
AO-6 & PAAS & $-4,4 \pm 4,1$ & $\mathrm{P}$ \\
AO-7 & PAAS & $-4,1 \pm 4,1$ & $\mathrm{P}$ \\
AO-9 & PAAS & $-4,7 \pm 4,0$ & $\mathrm{P}$ \\
AO-10 & PAAS & $-4,9 \pm 4,2$ & $\mathrm{P}$ \\
AO-12 & PAAS & $-4,2 \pm 4,1$ & $\mathrm{P}$ \\
SC-7 & PAAS & $-5,3 \pm 4,0$ & $\mathrm{P}$ \\
SC-8 & PAAS & $-5,4 \pm 4,1$ & $\mathrm{P}$ \\
PL-1 & PAAS & $-2,3 \pm 4,2$ & $\mathrm{P}$ \\
PW-5 & PAAS & $-5,1 \pm 4,2$ & $\mathrm{P}$ \\
\hline B Barat & $(2012)$ & &
\end{tabular}

B: Barrat et al. (2012).

P: Pourmand et al. (2012).

PAAS: Post Archean Australian Shales. 
Tabelle 4.10: Tm-Anomalien der Ozeanbodenbasalte berechnet anhand der Messwerte aus der Studie von Jenner und O'Neill (2012a).

\begin{tabular}{|c|c|}
\hline $\begin{array}{r}\text { Probe } \\
\text { Nr. }\end{array}$ & $\begin{array}{c}\text { Tm- } \\
\text { Anomalie } \\
(\%)\end{array}$ \\
\hline 175 & $-6,2 \pm 4,1$ \\
\hline 176 & $-2,4 \pm 4,5$ \\
\hline 177 & $-6,8 \pm 4,2$ \\
\hline 178 & $-5,5 \pm 4,3$ \\
\hline 179 & $-10,8 \pm 4,0$ \\
\hline 180 & $-5,1 \pm 4,1$ \\
\hline 183 & $-4,2 \pm 4,2$ \\
\hline 184 & $3,1 \pm 4,6$ \\
\hline 185 & $2,3 \pm 4,6$ \\
\hline 186 & $-4,7 \pm 4,3$ \\
\hline 187 & $-4,1 \pm 4,3$ \\
\hline 188 & $-5,4 \pm 4,2$ \\
\hline 189 & $-2,2 \pm 4,6$ \\
\hline 191 & $-2,1 \pm 4,4$ \\
\hline 192 & $-3,0 \pm 4,5$ \\
\hline 193 & $-2,1 \pm 4,3$ \\
\hline 194 & $-4,7 \pm 4,3$ \\
\hline 195 & $-7,4 \pm 4,0$ \\
\hline 196 & $-1,5 \pm 4,4$ \\
\hline 197 & $-4,1 \pm 4,2$ \\
\hline 198 & $-0,2 \pm 4,5$ \\
\hline 199 & $-4,5 \pm 4,4$ \\
\hline 200 & $-9,3 \pm 4,2$ \\
\hline 201 & $-6,0 \pm 4,3$ \\
\hline 202 & $-7,4 \pm 4,2$ \\
\hline 203 & $-6,5 \pm 4,2$ \\
\hline 204 & $-3,4 \pm 4,4$ \\
\hline 285 & $-6,4 \pm 4,3$ \\
\hline 286 & $-3,9 \pm 4,4$ \\
\hline 287 & $-8,1 \pm 4,1$ \\
\hline 289 & $-3,1 \pm 4,4$ \\
\hline 290 & $-3,7 \pm 4,4$ \\
\hline 291 & $-2,5 \pm 4,4$ \\
\hline 293 & $-9,6 \pm 4,0$ \\
\hline 294 & $-5,0 \pm 4,2$ \\
\hline 304 & $-8,6 \pm 3,9$ \\
\hline 305 & $-7,3 \pm 4,1$ \\
\hline 307 & $-6,3 \pm 4,1$ \\
\hline 308 & $1,4 \pm 4,6$ \\
\hline 309 & $-7,8 \pm 4,3$ \\
\hline 310 & $-0,4 \pm 4,5$ \\
\hline 311 & $-6,9 \pm 4,2$ \\
\hline 312 & $-3,9 \pm 4,2$ \\
\hline 313 & $-7,0 \pm 4,3$ \\
\hline 314 & $-7,7 \pm 4,1$ \\
\hline 315 & $-2,6 \pm 4,5$ \\
\hline 316 & $-7,3 \pm 4,1$ \\
\hline 317 & $-2,4 \pm 4,5$ \\
\hline 318 & $-6,8 \pm 4,3$ \\
\hline 319 & $0,6 \pm 4,4$ \\
\hline 320 & $-7,8 \pm 4,3$ \\
\hline 321 & $-11,0 \pm 3,9$ \\
\hline 322 & $-7,8 \pm 4,3$ \\
\hline 323 & $-6,0 \pm 4,3$ \\
\hline 332 & $-3,7 \pm 4,2$ \\
\hline 333 & $-3,8 \pm 4,4$ \\
\hline 334 & $-8,9 \pm 4,2$ \\
\hline 335 & $-0,5 \pm 4,3$ \\
\hline 336 & $-5,7 \pm 4,2$ \\
\hline 337 & $-1,6 \pm 4,5$ \\
\hline 338 & $-4,4 \pm 4,3$ \\
\hline 339 & $-3,6 \pm 4,3$ \\
\hline
\end{tabular}

\begin{tabular}{|c|c|}
\hline $\begin{array}{r}\text { Probe } \\
\text { Nr. }\end{array}$ & $\begin{array}{c}\text { Tm- } \\
\text { Anomalie } \\
(\%)\end{array}$ \\
\hline 340 & $-5,7 \pm 4,3$ \\
\hline 341 & $-1,1 \pm 4,6$ \\
\hline 372 & $-5,4 \pm 4,2$ \\
\hline 373 & $5,4 \pm 4,6$ \\
\hline 374 & $0,4 \pm 4,7$ \\
\hline 375 & $4,2 \pm 4,7$ \\
\hline 376 & $-7,5 \pm 4,3$ \\
\hline 377 & $-6,0 \pm 4,0$ \\
\hline 378 & $-7,6 \pm 4,2$ \\
\hline 380 & $-6,6 \pm 4,3$ \\
\hline 381 & $-1,8 \pm 4,4$ \\
\hline 382 & $-5,8 \pm 4,1$ \\
\hline 383 & $-4,8 \pm 4,3$ \\
\hline 384 & $-0,6 \pm 4,5$ \\
\hline 385 & $-7,9 \pm 4,2$ \\
\hline 386 & $-5,6 \pm 4,2$ \\
\hline 387 & $-2,4 \pm 4,5$ \\
\hline 388 & $-4,4 \pm 4,2$ \\
\hline 389 & $-4,5 \pm 4,4$ \\
\hline 390 & $-7,2 \pm 4,2$ \\
\hline 391 & $-9,1 \pm 4,1$ \\
\hline 612 & $-3,6 \pm 4,2$ \\
\hline 614 & $-2,1 \pm 4,4$ \\
\hline 617 & $10,8 \pm 4,9$ \\
\hline 618 & $-7,0 \pm 4,3$ \\
\hline 619 & $-5,4 \pm 4,1$ \\
\hline 620 & $-2,4 \pm 4,3$ \\
\hline 621 & $6,6 \pm 4,7$ \\
\hline 622 & $-9,7 \pm 3,9$ \\
\hline 623 & $-5,8 \pm 4,1$ \\
\hline 624 & $-4,0 \pm 4,2$ \\
\hline 625 & $-4,7 \pm 4,2$ \\
\hline 626 & $-5,7 \pm 4,1$ \\
\hline 627 & $-2,5 \pm 4,3$ \\
\hline 628 & $-6,9 \pm 4,0$ \\
\hline 629 & $-3,2 \pm 4,2$ \\
\hline 631 & $-9,5 \pm 3,9$ \\
\hline 662 & $-3,3 \pm 4,5$ \\
\hline 663 & $-6,9 \pm 4,1$ \\
\hline 664 & $-6,8 \pm 4,2$ \\
\hline 665 & $-4,3 \pm 4,2$ \\
\hline 666 & $-6,0 \pm 4,1$ \\
\hline 667 & $-4,1 \pm 4,1$ \\
\hline 670 & $0,5 \pm 4,5$ \\
\hline 671 & $-4,8 \pm 4,1$ \\
\hline 672 & $-3,4 \pm 4,3$ \\
\hline 673 & $-4,5 \pm 4,3$ \\
\hline 674 & $-3,3 \pm 4,3$ \\
\hline 678 & $-5,5 \pm 4,2$ \\
\hline 679 & $-10,0 \pm 3,9$ \\
\hline 680 & $-5,9 \pm 4,0$ \\
\hline 681 & $-6,9 \pm 4,0$ \\
\hline 682 & $0,7 \pm 4,3$ \\
\hline 684 & $-0,4 \pm 4,3$ \\
\hline 685 & $-0,9 \pm 4,4$ \\
\hline 686 & $-1,7 \pm 4,3$ \\
\hline 687 & $-11,7 \pm 3,9$ \\
\hline 688 & $0,3 \pm 4,4$ \\
\hline 689 & $-4,4 \pm 4,1$ \\
\hline 690 & $-5,0 \pm 4,2$ \\
\hline 691 & $-8,0 \pm 4,0$ \\
\hline 722 & $-6,1 \pm 4,2$ \\
\hline
\end{tabular}

\begin{tabular}{|c|c|}
\hline $\begin{array}{r}\text { Probe } \\
\mathrm{Nr} .\end{array}$ & $\begin{array}{c}\text { Tm- } \\
\text { Anomalie } \\
(\%)\end{array}$ \\
\hline 723 & $-6,3 \pm 4,2$ \\
\hline 724 & $-4,4 \pm 4,2$ \\
\hline 725 & $-5,8 \pm 4,2$ \\
\hline 726 & $-6,3 \pm 4,2$ \\
\hline 727 & $-1,8 \pm 4,4$ \\
\hline 752 & $-7,9 \pm 4,1$ \\
\hline 753 & $-3,7 \pm 4,4$ \\
\hline 754 & $-2,8 \pm 4,5$ \\
\hline 755 & $-7,3 \pm 4,0$ \\
\hline 756 & $-4,0 \pm 4,2$ \\
\hline 757 & $-1,9 \pm 4,4$ \\
\hline 758 & $-4,8 \pm 4,2$ \\
\hline 759 & $-2,2 \pm 4,3$ \\
\hline 760 & $-1,8 \pm 4,6$ \\
\hline 761 & $-2,2 \pm 4,4$ \\
\hline 782 & $-1,0 \pm 4,5$ \\
\hline 783 & $-0,6 \pm 4,5$ \\
\hline 784 & $-7,9 \pm 4,2$ \\
\hline 785 & $-2,5 \pm 4,4$ \\
\hline 786 & $-5,4 \pm 4,3$ \\
\hline 788 & $0,3 \pm 4,6$ \\
\hline 789 & $-2,0 \pm 4,4$ \\
\hline 832 & $-4,6 \pm 4,1$ \\
\hline 833 & $-1,8 \pm 4,3$ \\
\hline 834 & $-2,9 \pm 4,4$ \\
\hline 835 & $-4,5 \pm 4,2$ \\
\hline 836 & $-10,0 \pm 4,0$ \\
\hline 838 & $-3,9 \pm 4,2$ \\
\hline 839 & $1,6 \pm 4,5$ \\
\hline 840 & $-6,1 \pm 4,2$ \\
\hline 841 & $-5,3 \pm 4,2$ \\
\hline 882 & $-3,6 \pm 4,2$ \\
\hline 883 & $0,5 \pm 4,6$ \\
\hline 884 & $-2,6 \pm 4,4$ \\
\hline 885 & $-4,5 \pm 4,1$ \\
\hline 886 & $-3,3 \pm 4,4$ \\
\hline 887 & $-4,8 \pm 4,4$ \\
\hline 888 & $-6,2 \pm 4,1$ \\
\hline 889 & $-6,0 \pm 4,1$ \\
\hline 890 & $-5,7 \pm 4,3$ \\
\hline 891 & $-5,0 \pm 4,1$ \\
\hline 922 & $-5,7 \pm 4,2$ \\
\hline 923 & $-6,7 \pm 4,1$ \\
\hline 929 & $-2,4 \pm 4,5$ \\
\hline 930 & $-0,4 \pm 4,5$ \\
\hline 931 & $0,9 \pm 4,4$ \\
\hline 952 & $-6,5 \pm 4,2$ \\
\hline 954 & $-2,5 \pm 4,2$ \\
\hline 955 & $-2,1 \pm 4,5$ \\
\hline 956 & $-7,0 \pm 4,1$ \\
\hline 957 & $2,4 \pm 4,7$ \\
\hline 958 & $-9,0 \pm 4,0$ \\
\hline 959 & $-1,8 \pm 4,5$ \\
\hline 960 & $-12,2 \pm 3,9$ \\
\hline 990 & $-9,9 \pm 4,1$ \\
\hline 1307 & $-4,3 \pm 4,2$ \\
\hline 1308 & $-6,9 \pm 4,1$ \\
\hline 1309 & $-9,2 \pm 4,0$ \\
\hline 1310 & $-4,4 \pm 4,3$ \\
\hline 1311 & $-5,6 \pm 4,1$ \\
\hline 1470 & $-4,5 \pm 4,2$ \\
\hline 1471 & $-5,9 \pm 4,2$ \\
\hline
\end{tabular}

\begin{tabular}{|c|c|}
\hline $\begin{array}{r}\text { Probe } \\
\mathrm{Nr} .\end{array}$ & $\begin{array}{c}\text { Tm- } \\
\text { Anomalie } \\
(\%)\end{array}$ \\
\hline 1472 & $-4,5 \pm 4,3$ \\
\hline 1473 & $-3,5 \pm 4,3$ \\
\hline 1474 & $-3,8 \pm 4,3$ \\
\hline 1475 & $-3,0 \pm 4,3$ \\
\hline 1476 & $-3,8 \pm 4,3$ \\
\hline 1477 & $-5,5 \pm 4,3$ \\
\hline 1478 & $-3,4 \pm 4,3$ \\
\hline 1479 & $-7,0 \pm 4,2$ \\
\hline 1570 & $-5,4 \pm 4,2$ \\
\hline 1571 & $-5,7 \pm 4,3$ \\
\hline 1572 & $-3,5 \pm 4,3$ \\
\hline 1573 & $-5,1 \pm 4,2$ \\
\hline 1672 & $-4,7 \pm 4,3$ \\
\hline 1673 & $-9,2 \pm 4,1$ \\
\hline 1674 & $-5,1 \pm 4,3$ \\
\hline 1676 & $-3,7 \pm 4,1$ \\
\hline 1677 & $-3,3 \pm 4,4$ \\
\hline 1678 & $2,1 \pm 4,5$ \\
\hline 1679 & $-5,0 \pm 4,1$ \\
\hline 1693 & $-6,2 \pm 4,2$ \\
\hline 1694 & $-6,1 \pm 4,2$ \\
\hline 1695 & $-7,5 \pm 4,1$ \\
\hline 1696 & $-8,3 \pm 4,0$ \\
\hline 1698 & $-5,9 \pm 4,1$ \\
\hline 1699 & $-4,6 \pm 4,2$ \\
\hline 1715 & $-6,7 \pm 4,1$ \\
\hline 1716 & $-6,9 \pm 4,3$ \\
\hline 1718 & $-4,5 \pm 4,3$ \\
\hline 1719 & $-6,6 \pm 4,0$ \\
\hline 1960 & $-6,3 \pm 4,1$ \\
\hline 1961 & $-6,2 \pm 4,1$ \\
\hline 1962 & $-3,9 \pm 4,2$ \\
\hline 1963 & $-6,0 \pm 4,2$ \\
\hline 1966 & $-3,4 \pm 4,3$ \\
\hline 1969 & $-4,6 \pm 4,3$ \\
\hline 2110 & $-3,9 \pm 4,4$ \\
\hline 2111 & $-3,0 \pm 4,3$ \\
\hline 2112 & $-2,9 \pm 4,2$ \\
\hline 2113 & $-5,1 \pm 4,1$ \\
\hline 2114 & $-4,3 \pm 4,2$ \\
\hline 2115 & $-5,2 \pm 4,2$ \\
\hline 2116 & $-4,7 \pm 4,1$ \\
\hline 2117 & $-5,6 \pm 4,1$ \\
\hline 2118 & $-5,0 \pm 4,1$ \\
\hline 2119 & $-3,3 \pm 4,3$ \\
\hline 2250 & $-4,7 \pm 4,1$ \\
\hline 2251 & $-4,4 \pm 4,3$ \\
\hline 2252 & $-3,5 \pm 4,3$ \\
\hline 2253 & $-6,0 \pm 4,3$ \\
\hline 2254 & $-5,3 \pm 4,2$ \\
\hline 2255 & $-4,4 \pm 4,3$ \\
\hline 2256 & $-4,6 \pm 4,2$ \\
\hline 2257 & $-7,4 \pm 4,2$ \\
\hline 2258 & $-4,5 \pm 4,3$ \\
\hline 2259 & $-4,2 \pm 4,2$ \\
\hline 2290 & $-6,7 \pm 4,0$ \\
\hline 2291 & $-5,9 \pm 4,2$ \\
\hline 2292 & $-6,4 \pm 4,1$ \\
\hline 2293 & $-5,2 \pm 4,1$ \\
\hline 2294 & $-3,3 \pm 4,3$ \\
\hline 2295 & $-2,6 \pm 4,3$ \\
\hline 2296 & $-2,2 \pm 4,2$ \\
\hline
\end{tabular}

\begin{tabular}{|c|c|}
\hline $\begin{array}{r}\text { Probe } \\
\text { Nr. }\end{array}$ & $\begin{array}{c}\text { Tm- } \\
\text { Anomalie } \\
(\%)\end{array}$ \\
\hline 2297 & $-4,5 \pm 4,1$ \\
\hline 2298 & $-4,7 \pm 4,2$ \\
\hline 2299 & $-4,2 \pm 4,2$ \\
\hline 2340 & $-3,9 \pm 4,1$ \\
\hline 2341 & $-4,1 \pm 4,1$ \\
\hline 2342 & $-7,6 \pm 3,9$ \\
\hline 2343 & $-6,9 \pm 4,1$ \\
\hline 2344 & $-9,3 \pm 3,9$ \\
\hline 2345 & $-4,5 \pm 4,1$ \\
\hline 2346 & $-5,9 \pm 4,0$ \\
\hline 2970 & $-11,1 \pm 3,9$ \\
\hline 2971 & $1,2 \pm 4,5$ \\
\hline 2972 & $-8,8 \pm 3,9$ \\
\hline 2973 & $-2,5 \pm 4,4$ \\
\hline 2975 & $-3,9 \pm 4,3$ \\
\hline 2976 & $-8,6 \pm 4,0$ \\
\hline 2977 & $-7,5 \pm 4,2$ \\
\hline 2978 & $-12,9 \pm 3,9$ \\
\hline 2979 & $-12,1 \pm 3,9$ \\
\hline 3050 & $-5,3 \pm 4,2$ \\
\hline 3052 & $-4,5 \pm 4,1$ \\
\hline 3056 & $-9,5 \pm 4,0$ \\
\hline 3059 & $-7,7 \pm 4,2$ \\
\hline 3070 & $-8,1 \pm 4,0$ \\
\hline 3071 & $-4,1 \pm 4,1$ \\
\hline 3072 & $2,2 \pm 4,5$ \\
\hline 3073 & $-12,7 \pm 3,7$ \\
\hline 3074 & $-0,9 \pm 4,3$ \\
\hline 3075 & $-2,1 \pm 4,4$ \\
\hline 3076 & $-8,4 \pm 3,8$ \\
\hline 3077 & $-2,5 \pm 4,2$ \\
\hline 3078 & $-6,6 \pm 3,9$ \\
\hline 3079 & $-2,8 \pm 4,2$ \\
\hline 3090 & $-10,5 \pm 3,9$ \\
\hline 3091 & $-7,4 \pm 4,1$ \\
\hline 3092 & $-3,6 \pm 4,2$ \\
\hline 3093 & $-6,9 \pm 4,2$ \\
\hline 3094 & $-3,8 \pm 4,3$ \\
\hline 3095 & $-7,6 \pm 4,1$ \\
\hline 3096 & $-3,9 \pm 4,3$ \\
\hline 3097 & $-3,4 \pm 4,4$ \\
\hline 3098 & $-4,1 \pm 4,2$ \\
\hline 3099 & $-8,8 \pm 4,0$ \\
\hline 3220 & $-5,0 \pm 4,3$ \\
\hline 3221 & $-6,9 \pm 4,1$ \\
\hline 3222 & $-4,7 \pm 4,1$ \\
\hline 3223 & $-2,7 \pm 4,3$ \\
\hline 3224 & $-3,7 \pm 4,2$ \\
\hline 3225 & $-5,3 \pm 4,4$ \\
\hline 3226 & $-1,8 \pm 4,6$ \\
\hline 3228 & $-3,9 \pm 4,4$ \\
\hline 3330 & $-2,9 \pm 4,3$ \\
\hline 3331 & $-5,7 \pm 4,1$ \\
\hline 3334 & $-3,4 \pm 4,2$ \\
\hline 3335 & $-4,0 \pm 4,3$ \\
\hline 3336 & $-1,3 \pm 4,4$ \\
\hline 3450 & $-4,3 \pm 4,6$ \\
\hline 3451 & $-11,8 \pm 4,1$ \\
\hline 3452 & $-3,1 \pm 4,5$ \\
\hline 3456 & $-1,2 \pm 4,3$ \\
\hline 3458 & $-3,7 \pm 4,2$ \\
\hline 3570 & $1,8 \pm 4,6$ \\
\hline
\end{tabular}


Tabelle 4.10 (Fortsetzung)

\begin{tabular}{|c|c|}
\hline $\begin{array}{r}\text { Probe } \\
\text { Nr. }\end{array}$ & $\begin{array}{c}\text { Tm- } \\
\text { Anomalie } \\
(\%)\end{array}$ \\
\hline 3571 & $-4,6 \pm 4,2$ \\
\hline 3572 & $-8,8 \pm 4,0$ \\
\hline 3573 & $-6,3 \pm 4,2$ \\
\hline 3576 & $-7,1 \pm 4,3$ \\
\hline 3577 & $-1,0 \pm 4,5$ \\
\hline 3578 & $1,6 \pm 4,6$ \\
\hline 3579 & $-3,0 \pm 4,4$ \\
\hline 3590 & $-5,4 \pm 4,2$ \\
\hline 3591 & $-7,4 \pm 4,1$ \\
\hline 3592 & $-7,4 \pm 4,1$ \\
\hline 3593 & $-5,5 \pm 4,2$ \\
\hline 3594 & $-7,6 \pm 4,2$ \\
\hline 3595 & $-5,5 \pm 4,3$ \\
\hline 3596 & $-4,6 \pm 4,3$ \\
\hline 3610 & $-5,5 \pm 4,2$ \\
\hline 3611 & $-7,4 \pm 4,1$ \\
\hline 3612 & $-5,2 \pm 4,3$ \\
\hline 3613 & $-6,8 \pm 4,2$ \\
\hline 3614 & $-5,6 \pm 4,1$ \\
\hline 3615 & $-6,0 \pm 4,1$ \\
\hline 3616 & $-7,0 \pm 4,0$ \\
\hline 3617 & $-3,5 \pm 4,2$ \\
\hline 3618 & $-4,3 \pm 4,3$ \\
\hline 3619 & $-9,4 \pm 4,0$ \\
\hline 3620 & $-4,0 \pm 4,2$ \\
\hline 3621 & $-5,1 \pm 4,2$ \\
\hline 3622 & $-4,5 \pm 4,2$ \\
\hline 3623 & $-5,5 \pm 4,3$ \\
\hline 3624 & $-6,2 \pm 4,2$ \\
\hline 3625 & $-3,9 \pm 4,3$ \\
\hline 3626 & $-5,4 \pm 4,3$ \\
\hline 3627 & $-7,1 \pm 4,2$ \\
\hline 3628 & $-4,0 \pm 4,2$ \\
\hline 3629 & $-6,7 \pm 4,1$ \\
\hline 3760 & $-2,3 \pm 4,2$ \\
\hline 3761 & $-7,4 \pm 4,2$ \\
\hline 3763 & $-12,9 \pm 3,9$ \\
\hline 3764 & $-4,5 \pm 4,3$ \\
\hline 3765 & $-12,4 \pm 3,8$ \\
\hline 3766 & $1,8 \pm 4,6$ \\
\hline 3767 & $2,1 \pm 4,6$ \\
\hline 3768 & $-2,4 \pm 4,5$ \\
\hline 3769 & $2,3 \pm 4,6$ \\
\hline 3790 & $-5,5 \pm 4,2$ \\
\hline 3791 & $-4,2 \pm 4,2$ \\
\hline 3792 & $-8,4 \pm 4,1$ \\
\hline 3793 & $-9,5 \pm 4,1$ \\
\hline 3794 & $-4,8 \pm 4,1$ \\
\hline 3795 & $-0,7 \pm 4,3$ \\
\hline 3796 & $-9,0 \pm 4,1$ \\
\hline 3797 & $-6,8 \pm 4,1$ \\
\hline 3798 & $0,0 \pm 4,3$ \\
\hline 3799 & $-2,9 \pm 4,3$ \\
\hline 3830 & $2,3 \pm 4,7$ \\
\hline 3831 & $-4,9 \pm 4,2$ \\
\hline 3832 & $-4,2 \pm 4,4$ \\
\hline 3833 & $-4,4 \pm 4,3$ \\
\hline 3834 & $-3,3 \pm 4,3$ \\
\hline 3835 & $-2,8 \pm 4,4$ \\
\hline 3838 & $-2,9 \pm 4,4$ \\
\hline 3839 & $-7,3 \pm 4,1$ \\
\hline 3920 & $3,0 \pm 4,7$ \\
\hline
\end{tabular}

\begin{tabular}{|c|c|}
\hline $\begin{array}{r}\text { Probe } \\
\text { Nr. }\end{array}$ & $\begin{array}{c}\text { Tm- } \\
\text { Anomalie } \\
(\%)\end{array}$ \\
\hline 3921 & $-5,9 \pm 4,1$ \\
\hline 3922 & $-6,0 \pm 4,2$ \\
\hline 3923 & $-5,1 \pm 4,3$ \\
\hline 3924 & $-2,8 \pm 4,3$ \\
\hline 3925 & $-11,1 \pm 4,0$ \\
\hline 3926 & $-7,6 \pm 4,0$ \\
\hline 3927 & $-6,0 \pm 4,2$ \\
\hline 3928 & $-1,3 \pm 4,4$ \\
\hline 3960 & $-3,8 \pm 4,2$ \\
\hline 3961 & $-10,2 \pm 3,9$ \\
\hline 3962 & $-3,8 \pm 4,1$ \\
\hline 3963 & $-5,8 \pm 4,0$ \\
\hline 3964 & $-6,3 \pm 4,2$ \\
\hline 3965 & $-5,9 \pm 4,1$ \\
\hline 3966 & $-6,2 \pm 4,2$ \\
\hline 3967 & $-4,8 \pm 4,2$ \\
\hline 3968 & $-5,8 \pm 4,1$ \\
\hline 3969 & $-1,7 \pm 4,3$ \\
\hline 4080 & $-14,1 \pm 3,9$ \\
\hline 4081 & $-10,7 \pm 4,2$ \\
\hline 4082 & $-7,4 \pm 4,4$ \\
\hline 4083 & $-6,9 \pm 4,2$ \\
\hline 4084 & $-5,4 \pm 4,3$ \\
\hline 4085 & $-2,7 \pm 4,6$ \\
\hline 4087 & $-1,4 \pm 4,3$ \\
\hline 4088 & $-4,8 \pm 4,0$ \\
\hline 4089 & $-2,0 \pm 4,2$ \\
\hline 4250 & $-3,9 \pm 4,3$ \\
\hline 4251 & $-6,0 \pm 4,2$ \\
\hline 4252 & $-7,1 \pm 4,2$ \\
\hline 4253 & $-7,2 \pm 4,2$ \\
\hline 4254 & $-1,9 \pm 4,2$ \\
\hline 4255 & $-6,8 \pm 4,3$ \\
\hline 4256 & $-7,5 \pm 4,3$ \\
\hline 4257 & $-5,9 \pm 4,4$ \\
\hline 4258 & $-4,4 \pm 4,4$ \\
\hline 4259 & $-4,8 \pm 4,3$ \\
\hline 4370 & $-5,3 \pm 4,1$ \\
\hline 4371 & $-6,8 \pm 4,2$ \\
\hline 4372 & $-4,8 \pm 4,3$ \\
\hline 4373 & $-6,1 \pm 4,2$ \\
\hline 4374 & $-3,3 \pm 4,4$ \\
\hline 4375 & $-8,4 \pm 4,1$ \\
\hline 4376 & $-0,7 \pm 4,4$ \\
\hline 4377 & $-6,2 \pm 4,2$ \\
\hline 4378 & $-3,6 \pm 4,4$ \\
\hline 4379 & $-4,8 \pm 4,3$ \\
\hline 4420 & $-5,5 \pm 4,2$ \\
\hline 4421 & $-13,1 \pm 3,8$ \\
\hline 4425 & $1,1 \pm 4,4$ \\
\hline 4426 & $-1,4 \pm 4,6$ \\
\hline 4427 & $3,0 \pm 4,9$ \\
\hline 4428 & $2,1 \pm 4,7$ \\
\hline 4510 & $-6,2 \pm 4,1$ \\
\hline 4512 & $-6,2 \pm 4,1$ \\
\hline 4513 & $-5,2 \pm 4,2$ \\
\hline 4514 & $-6,7 \pm 4,0$ \\
\hline 4520 & $-7,1 \pm 4,2$ \\
\hline 4521 & $-2,8 \pm 4,3$ \\
\hline 4522 & $-6,7 \pm 4,0$ \\
\hline 4523 & $-3,2 \pm 4,2$ \\
\hline 4524 & $-2,7 \pm 4,2$ \\
\hline
\end{tabular}

\begin{tabular}{|c|c|}
\hline $\begin{array}{r}\text { Probe } \\
\text { Nr. }\end{array}$ & $\begin{array}{c}\text { Tm- } \\
\text { Anomalie } \\
(\%)\end{array}$ \\
\hline 4525 & $-5,0 \pm 4,2$ \\
\hline 4526 & $-3,2 \pm 4,3$ \\
\hline 4527 & $-2,0 \pm 4,3$ \\
\hline 4528 & $-5,1 \pm 4,2$ \\
\hline 4529 & $-8,1 \pm 4,1$ \\
\hline 4560 & $-2,6 \pm 4,4$ \\
\hline 4561 & $-2,5 \pm 4,3$ \\
\hline 4562 & $-10,9 \pm 3,9$ \\
\hline 4563 & $-12,2 \pm 3,9$ \\
\hline 4620 & $-4,6 \pm 4,2$ \\
\hline 4621 & $-3,9 \pm 4,6$ \\
\hline 4622 & $-5,1 \pm 4,4$ \\
\hline 4623 & $-7,4 \pm 4,2$ \\
\hline 4624 & $-5,4 \pm 4,2$ \\
\hline 4625 & $-3,9 \pm 4,4$ \\
\hline 4626 & $-6,4 \pm 4,2$ \\
\hline 4627 & $-11,1 \pm 4,0$ \\
\hline 4628 & $-5,3 \pm 4,2$ \\
\hline 4629 & $-6,3 \pm 4,0$ \\
\hline 4700 & $-7,6 \pm 3,9$ \\
\hline 4701 & $-3,4 \pm 4,2$ \\
\hline 4702 & $-3,6 \pm 4,2$ \\
\hline 4703 & $-4,5 \pm 4,2$ \\
\hline 4704 & $1,4 \pm 4,6$ \\
\hline 4705 & $-2,4 \pm 4,3$ \\
\hline 4708 & $-2,5 \pm 4,3$ \\
\hline 4709 & $-5,6 \pm 4,1$ \\
\hline 4750 & $-4,9 \pm 4,4$ \\
\hline 4751 & $-4,4 \pm 4,2$ \\
\hline 4754 & $-6,1 \pm 4,3$ \\
\hline 4755 & $-4,5 \pm 4,4$ \\
\hline 4780 & $-5,3 \pm 4,2$ \\
\hline 4781 & $-3,1 \pm 4,1$ \\
\hline 4782 & $-4,2 \pm 4,2$ \\
\hline 4783 & $-2,6 \pm 4,3$ \\
\hline 4784 & $-4,0 \pm 4,2$ \\
\hline 4785 & $-6,7 \pm 3,9$ \\
\hline 4786 & $-6,7 \pm 4,0$ \\
\hline 4787 & $-1,6 \pm 4,4$ \\
\hline 4788 & $-5,3 \pm 4,3$ \\
\hline 4789 & $-2,5 \pm 4,2$ \\
\hline 4970 & $-0,9 \pm 4,4$ \\
\hline 4971 & $-6,8 \pm 4,1$ \\
\hline 4972 & $-4,7 \pm 4,2$ \\
\hline 4973 & $-4,5 \pm 4,1$ \\
\hline 4974 & $-7,4 \pm 4,1$ \\
\hline 4975 & $-5,3 \pm 4,2$ \\
\hline 4976 & $-4,2 \pm 4,2$ \\
\hline 4977 & $-5,6 \pm 4,1$ \\
\hline 4978 & $-5,7 \pm 4,1$ \\
\hline 4979 & $-6,4 \pm 4,0$ \\
\hline 5350 & $-0,2 \pm 4,5$ \\
\hline 5351 & $-8,8 \pm 4,2$ \\
\hline 5352 & $-8,0 \pm 4,2$ \\
\hline 5353 & $-12,3 \pm 3,9$ \\
\hline 5354 & $-0,5 \pm 4,7$ \\
\hline 5355 & $-5,2 \pm 4,4$ \\
\hline 5356 & $-5,5 \pm 4,4$ \\
\hline 5357 & $-4,7 \pm 4,4$ \\
\hline 5358 & $-7,5 \pm 4,3$ \\
\hline 5359 & $-3,5 \pm 4,5$ \\
\hline 5370 & $-1,9 \pm 4,3$ \\
\hline
\end{tabular}

\begin{tabular}{|c|c|}
\hline $\begin{array}{r}\text { Probe } \\
\text { Nr. }\end{array}$ & $\begin{array}{c}\text { Tm- } \\
\text { Anomalie } \\
(\%)\end{array}$ \\
\hline 5371 & $-6,5 \pm 4,2$ \\
\hline 5372 & $-5,7 \pm 4,3$ \\
\hline 5373 & $-5,1 \pm 4,2$ \\
\hline 5374 & $-7,9 \pm 4,1$ \\
\hline 5375 & $-3,6 \pm 4,4$ \\
\hline 5376 & $0,1 \pm 4,5$ \\
\hline 5377 & $-3,7 \pm 4,4$ \\
\hline 5378 & $-8,5 \pm 3,9$ \\
\hline 5380 & $-3,6 \pm 4,3$ \\
\hline 5382 & $-3,9 \pm 4,3$ \\
\hline 5383 & $-4,2 \pm 4,3$ \\
\hline 5384 & $-1,6 \pm 4,4$ \\
\hline 5385 & $-3,0 \pm 4,3$ \\
\hline 5386 & $-5,0 \pm 4,3$ \\
\hline 5387 & $-9,3 \pm 3,9$ \\
\hline 5388 & $-2,5 \pm 4,2$ \\
\hline 5389 & $-4,4 \pm 4,3$ \\
\hline 5690 & $-1,5 \pm 4,4$ \\
\hline 5691 & $-5,7 \pm 4,2$ \\
\hline 5693 & $-8,1 \pm 4,0$ \\
\hline 5696 & $-5,3 \pm 4,1$ \\
\hline 5697 & $-7,0 \pm 4,1$ \\
\hline 5699 & $-5,8 \pm 4,2$ \\
\hline 5760 & $-6,5 \pm 4,2$ \\
\hline 5761 & $-2,8 \pm 4,4$ \\
\hline 5762 & $-5,4 \pm 4,2$ \\
\hline 5763 & $-5,0 \pm 4,3$ \\
\hline 5764 & $-4,1 \pm 4,2$ \\
\hline 5765 & $-4,4 \pm 4,3$ \\
\hline 5767 & $-3,3 \pm 4,4$ \\
\hline 5768 & $-5,1 \pm 4,2$ \\
\hline 5769 & $-2,4 \pm 4,3$ \\
\hline 5780 & $-4,7 \pm 4,2$ \\
\hline 5781 & $-3,2 \pm 4,2$ \\
\hline 5783 & $-2,7 \pm 4,4$ \\
\hline 5784 & $-1,0 \pm 4,5$ \\
\hline 5789 & $-4,6 \pm 4,2$ \\
\hline 6820 & $-1,7 \pm 4,3$ \\
\hline 6821 & $-4,2 \pm 4,2$ \\
\hline 6822 & $1,5 \pm 4,5$ \\
\hline 6823 & $-5,2 \pm 4,0$ \\
\hline 6824 & $-7,5 \pm 4,0$ \\
\hline 6825 & $-3,1 \pm 4,2$ \\
\hline 6826 & $-4,5 \pm 4,4$ \\
\hline 6829 & $-2,7 \pm 4,4$ \\
\hline 7200 & $-5,8 \pm 4,2$ \\
\hline 7201 & $-7,1 \pm 4,3$ \\
\hline 7202 & $-4,1 \pm 4,1$ \\
\hline 7203 & $-5,4 \pm 4,1$ \\
\hline 7204 & $-0,1 \pm 4,5$ \\
\hline 7205 & $-5,1 \pm 4,2$ \\
\hline 7206 & $-4,9 \pm 4,3$ \\
\hline 7207 & $-5,1 \pm 4,2$ \\
\hline 7208 & $-3,1 \pm 4,3$ \\
\hline 7209 & $-3,6 \pm 4,3$ \\
\hline 7220 & $-3,1 \pm 4,3$ \\
\hline 7222 & $-2,8 \pm 4,4$ \\
\hline 7224 & $-5,0 \pm 4,2$ \\
\hline 7225 & $-4,5 \pm 4,2$ \\
\hline 7226 & $-7,8 \pm 4,1$ \\
\hline 7227 & $-3,9 \pm 4,4$ \\
\hline 7228 & $-4,3 \pm 4,1$ \\
\hline
\end{tabular}

\begin{tabular}{|c|c|}
\hline $\begin{array}{r}\text { Probe } \\
\mathrm{Nr} .\end{array}$ & $\begin{array}{c}\text { Tm- } \\
\text { Anomalie } \\
(\%)\end{array}$ \\
\hline 7229 & $-7,6 \pm 4,1$ \\
\hline 7232 & $-1,2 \pm 4,3$ \\
\hline 7233 & $-4,2 \pm 4,3$ \\
\hline 7234 & $-1,7 \pm 4,4$ \\
\hline 7235 & $-3,5 \pm 4,1$ \\
\hline 7260 & $-3,7 \pm 4,3$ \\
\hline 7262 & $-4,7 \pm 4,2$ \\
\hline 7263 & $-4,8 \pm 4,3$ \\
\hline 7264 & $-5,4 \pm 4,2$ \\
\hline 7265 & $-7,0 \pm 4,2$ \\
\hline 7266 & $-4,0 \pm 4,3$ \\
\hline 7267 & $-2,1 \pm 4,4$ \\
\hline 7268 & $-2,7 \pm 4,5$ \\
\hline 7269 & $-8,1 \pm 4,1$ \\
\hline 8510 & $-5,6 \pm 4,3$ \\
\hline 8511 & $-9,3 \pm 3,9$ \\
\hline 8512 & $-3,7 \pm 4,3$ \\
\hline 8513 & $-2,2 \pm 4,5$ \\
\hline 8514 & $-2,7 \pm 4,3$ \\
\hline 8515 & $-2,0 \pm 4,4$ \\
\hline 8516 & $-2,1 \pm 4,4$ \\
\hline 8517 & $-3,9 \pm 4,2$ \\
\hline 8518 & $-6,8 \pm 4,1$ \\
\hline 8519 & $-7,2 \pm 4,2$ \\
\hline 8550 & $-8,3 \pm 4,3$ \\
\hline 8551 & $-2,4 \pm 4,6$ \\
\hline 8553 & $-3,7 \pm 4,4$ \\
\hline 8554 & $0,5 \pm 4,7$ \\
\hline 8555 & $-12,9 \pm 3,9$ \\
\hline 8556 & $-8,6 \pm 4,1$ \\
\hline 8557 & $-7,7 \pm 4,2$ \\
\hline 8558 & $-8,5 \pm 4,2$ \\
\hline 8559 & $-0,9 \pm 4,4$ \\
\hline 8590 & $-2,0 \pm 4,3$ \\
\hline 8591 & $-4,0 \pm 4,2$ \\
\hline 8592 & $-5,4 \pm 4,2$ \\
\hline 8593 & $-2,9 \pm 4,3$ \\
\hline 8594 & $-6,2 \pm 4,2$ \\
\hline 8595 & $-4,0 \pm 4,3$ \\
\hline 8596 & $-4,9 \pm 4,2$ \\
\hline 8597 & $-5,2 \pm 4,2$ \\
\hline 8598 & $-3,4 \pm 4,3$ \\
\hline 8599 & $-4,4 \pm 4,4$ \\
\hline 11240 & $-5,9 \pm 4,1$ \\
\hline 11241 & $-4,6 \pm 4,4$ \\
\hline 11242 & $-0,1 \pm 4,4$ \\
\hline 11243 & $-2,1 \pm 4,3$ \\
\hline 11244 & $-2,3 \pm 4,5$ \\
\hline 11245 & $4,6 \pm 4,8$ \\
\hline 11246 & $0,7 \pm 4,6$ \\
\hline 11247 & $-8,3 \pm 4,0$ \\
\hline 11248 & $-1,5 \pm 4,5$ \\
\hline 11249 & $-0,5 \pm 4,4$ \\
\hline 11250 & $-3,8 \pm 4,3$ \\
\hline 11253 & $-0,4 \pm 4,4$ \\
\hline 11254 & $-6,6 \pm 4,2$ \\
\hline 11255 & $-5,4 \pm 4,1$ \\
\hline 11256 & $-7,6 \pm 4,1$ \\
\hline
\end{tabular}


4. Diskussion 


\section{Zusammenfassung und Ausblick}

In dieser Arbeit wurden volatilitätskontrollierte Fraktionierungen refraktär-lithophiler Elemente in Chondritkomponenten sowie in Bulk-Chondriten, Achondriten und Planeten analysiert. Die wichtigsten Ergebnisse und Schlussfolgerungen sind nachfolgend zusammengefasst.

Es wurden Gesamtgesteinsuntersuchungen durch LA-ICPMS durchgeführt; zur Probenpräparation diente Laserschmelzen unter aerodynamischer Levitation. Dieses Verfahren erfordert nur geringe Probenmengen (ca. $10 \mathrm{mg}$ ), was besonders für Untersuchungen an Meteoriten von großem Vorteil ist. Die Methode erwies sich als sehr geeignet für die Analyse refraktär-lithophiler Elemente. Für Konzentrationsmessungen von Hauptelementen wie $\mathrm{Si}, \mathrm{Mg}$ und $\mathrm{Fe}$ in Chondriten ist die Präparationstechnik dagegen weniger gut anwendbar, da diese beim Aufschmelzen teilweise entweichen.

Es wurde eine vergleichende Untersuchung der Seltenen Erden und HFSEs an den verschiedenen Bestandteilen des CV-Chondrits Leoville durchgeführt. Dafür wurde ein $2,2 \mathrm{~cm}^{2}$-großer Dickschliff verwendet, der in-situ mittels LA-ICPMS analysiert wurde. Alle refraktären Einschlüsse - vier CAIs und ein AOA - haben volatilitätskontrollierte fraktionierte REE group-II-Muster. Sie sind aus einem residualen Gas entstanden, von dem zuvor eine ultrarefraktäre Komponente isoliert worden war. Typisch für die refraktären Einschlüsse sind auch subchondritische $\mathrm{Nb} / \mathrm{Ta}$-Verhältnisse.

Im Gegensatz zu den refraktären Einschlüssen haben Chondren zumeist relativ unfraktionierte REE-Muster sowie unfraktionierte Zr/Hf- und Nb/Ta-Verhältnisse. Einige Typ-1-Chondren und die Al-reichen Chondren weisen jedoch fraktionierte REE-Muster auf, die darauf hindeuten, dass die Präkursoren dieser Chondren Beimengungen refraktären Materials mit group-II-Muster enthielten. Der Anteil einer solchen fraktionierten Komponente in den Al-reichen Chondren wurde auf bis zu 45 Gew.-\% abgeschätzt.

Die Matrix von Leoville weist ebenfalls REE-Fraktionierungen auf, die - abgesehen von einer positiven Eu-Anomalie - einem group-II-Muster entsprechen. Dies deutet darauf hin, dass auch die Matrix refraktäre Bestandteile enthält. Unter der Annahme einer zum größten Teil aus CI-ähnlichem Material bestehenden Matrix, kann der Anteil fraktionierter Bestandteile mit group-II-Muster auf etwa 0,6 Gew.-\% abgeschätzt werden. 
Diese Beobachtungen lassen darauf schließen, dass sowohl die refraktären Einschlüsse als auch die refraktären Präkursoren der Al-reichen sowie einiger Typ1-Chondren und der in die Matrix eingebaute refraktäre Staub einen ähnlichen Ursprung haben: Sie sind wahrscheinlich unter hohen Temperaturen durch fraktionierte Kondensation aus einem residualen Gas entstanden.

Die LA-ICPMS-Untersuchungen an Bulk-Chondriten ergaben, dass die kohligen Chondrite im Vergleich zu Orgueil charakteristische volatilitätskontrollierte REEMuster haben, was auf den Einbau refraktärer Komponenten mit fraktionierten Seltenen Erden zurückgeführt wurde. Alle analysierten CV- und CK-Chondrite weisen group-II-Muster auf, die durch den Einbau einer fraktionierten Komponente von etwa 1 bis 1,7 Gew.-\% für CV-Chondrite und etwa 0,4 bis 0,8 Gew.-\% für Karoonda (CK) erklärt wurden. Unter den CM-Chondriten kommen sowohl groupII-Muster als auch relativ unfraktionierte REE-Muster vor. Die fraktionierten REE-Muster von Mighei und Murray wurden durch einen Anteil von 0,2 bis 0,4 Gew.-\% einer fraktionierten Komponente erklärt. Der CO-Chondrit NWA 3003 hat dagegen ein deutliches ultrarefraktäres REE-Muster, was auf den Einbau von etwa 0,3 Gew.-\% ultrarefraktären Materials zurückgeführt wurde. Die Mehrheit der gewöhnlichen, Rumuruti- und Enstatit-Chondrite hat relativ unfraktionierte REE-Muster. Charakteristisch ist allerdings ein kleines Defizit an Tm und Yb im Vergleich zu Orgueil.

Die mittleren Zr/Hf-Verhältnisse der verschiedenen Chondritgruppen stimmen innerhalb von $\pm 5 \%$ überein, wobei die kohligen Chondrite (außer CI) etwas geringere Werte besitzen. Die Nb/Ta-Verhältnisse sind ebenfalls in den kohligen Chondriten (außer CI) niedriger als in den gewöhnlichen Chondriten, was vermutlich auf dem Vorkommen refraktärer Einschlüsse mit abweichenden $\mathrm{Nb} / \mathrm{Ta}-$ Verhältnissen in kohligen Chondriten beruht.

Sowohl gewöhnliche, Enstatit- und Rumuruti-Chondrite als auch Proben der Achondrite, des Mars, der Erde und des Mondes weisen geringe negative TmAnomalien gegenüber Orgueil auf. Die Objekte des inneren Sonnensystems wurden daher anhand ihrer relativen HREE-Gehalte in zwei Reservoire eingeteilt: ein kohliges und ein nichtkohliges Chondrit-Reservoir. Dem nichtkohligen ChondritReservoir gehören auch die Achondrite, der Mars, die Erde und der Mond an. Im Gegensatz zu den Seltenen Erden zeigen die refraktären Elementverhältnisse Zr/Hf und $\mathrm{Nb} / \mathrm{Ta}$ keine solche Zweiteilung zwischen kohligen und nichtkohligen Chondriten. Aufgrund der Größe des nichtkohligen Chondrit-Reservoirs wurde angenommen, dass die Objekte dieses Reservoirs die HREE-Verhältnisse des Sonnensystems repräsentieren. Die kohligen Chondrite haben dagegen variable Tm-Anomalien, welche durch den Eintrag fraktionierter refraktärer Komponenten in die Entstehungsregion der kohligen Chondrite zu erklären sind. 
Die CI-Chondrite, welche allgemein als die chemisch primitivste Chondritgruppe angesehen werden, hätten in diesem Fall eine positive Tm-Anomalie von 4,8 \pm 0,9\% und stimmten somit chemisch nicht mit dem Sonnensystem überein. Ein Gewichtsanteil von nur 0,2 \% einer refraktären Komponente mit REE group-II-Muster würde ausreichen, um diese Tm-Anomalie zu verursachen. CI-Chondrite wären somit für die Normierung hochpräziser REE-Daten kosmo- und geochemischer Analysen nur bedingt geeignet. Es wurde außerdem gefolgert, dass die Präkursoren der Erde und des Mars bezüglich der Seltenen Erden eher den nichtkohligen als den kohligen Chondriten entsprechen. Dies gilt möglicherweise auch für die anderen terrestrischen Planeten Merkur und Venus.

Für weiterführende Studien empfiehlt es sich, die beobachtete Tm-Anomalie zwischen dem kohligen und dem nichtkohligen Reservoir durch eine andere analytische Methode zu bestätigen, beispielsweise mittels Lösungs-ICPMS nach vorheriger chromatographischer Abtrennung der Seltenen Erden. Darüberhinaus wäre es hilfreich, weitere CK-Chondrite zu analysieren, um die Ähnlichkeit ihrer REEMuster zu denen der CV-Chondrite zu überprüfen. Dies würde zur Klärung der Fragestellung, ob diese beiden Chondritgruppen aus demselben Ausgangsmaterial entstanden sind, beitragen. Ebenso vielversprechend erscheint die Untersuchung weiterer CO-Chondrite. Der in dieser Arbeit untersuchte North West Africa 3003 weicht hinsichtlich seines relativen Tm-Gehaltes als Einziger deutlich von den anderen kohligen Chondriten ab und hat im Gegensatz zu diesen eine große negative Tm-Anomalie. Die Analyse weiterer CO-Chondrite würde Aufschluss darüber geben, ob solche ultrarefraktären REE-Muster typisch für diese Chondritgruppe sind. Dies würde implizieren, dass in der frühen Phase des Sonnensystems größere Mengen an Material mit ultrarefraktären REE-Mustern in die Bildungsregion der CO-Chondrite eingetragen wurde, während das in die Bildungsregion der CV- und CK-Chondrite transportierte refraktäre Material überwiegend group-II-Muster oder unfraktionierte REE-Muster aufwies. 
5. Zusammenfassung und Ausblick 


\section{A. Anhang}

\section{A.1. Konzentrationsdaten}

Tabelle A.1: Mittels EMS bestimmte mittlere CaO-Gehalte der Objekte in Leoville in Gewichtsprozent. Die CaO-Gehalte der Einschlüsse wurden über Phasenkarten und der CaO-Gehalt der Matrix mithilfe von Punktmessungen mit defokussiertem Elektronenstrahl ermittelt. Unveröffentlichte Daten von A. Patzer.

\begin{tabular}{|c|c|c|c|}
\hline Objek & $\mathrm{CaO}$ & Objekt & $\mathrm{CaO}$ \\
\hline 1 & 4,5 & $18 \mathrm{R}$ & 1,6 \\
\hline $1 \mathrm{R}$ & 1 & 19 & 2,3 \\
\hline 2 & 3,4 & 20 & 8,4 \\
\hline 3 & 1,2 & 21 & 2,1 \\
\hline 4 & 2,5 & 22 & 15,5 \\
\hline 5 & 2,1 & 23 & 0,9 \\
\hline 6 & 1,0 & 24 & 7,2 \\
\hline 8 & 1,9 & 25 & 15,0 \\
\hline 9 & 26,2 & $25 \mathrm{R}$ & 1 \\
\hline 10 & 1,2 & 26 & 1,8 \\
\hline 11 & 1,2 & 27 & 8,8 \\
\hline $11 R$ & 1,2 & 28 & 3,6 \\
\hline 12 & 27,0 & $28 \mathrm{R}$ & 1,4 \\
\hline 14 & 1,0 & 29 & 14,1 \\
\hline 15 & 19,5 & 30 & 3,8 \\
\hline 16 & 4,6 & 31 & 11 \\
\hline 17 & 1,6 & 32 & 3,2 \\
\hline 18 & 2,6 & Matrix & 1 \\
\hline
\end{tabular}


Tabelle A.2: RFA-Analysen an einer Basaltprobe, die viermal präpariert und analysiert wurde. Die Präzision der Messungen wurde anhand der absoluten und relativen 1 $\sigma$-Standardabweichung (SD bzw. RSD) abgeschätzt.

\begin{tabular}{llrrrrrc}
\hline & & $\mathbf{1}$ & $\mathbf{2}$ & $\mathbf{3}$ & $\mathbf{4}$ & SD & RSD (\%) \\
\hline $\mathrm{SiO}_{2}$ & $(\%)$ & 45,2 & 45,4 & 45,6 & 46,0 & 0,3 & 0,7 \\
$\mathrm{TiO}_{2}$ & $(\%)$ & 2,74 & 2,75 & 2,76 & 2,78 & 0,02 & 0,7 \\
$\mathrm{Al}_{2} \mathrm{O}_{3}$ & $(\%)$ & 14,8 & 14,9 & 14,9 & 15,1 & 0,1 & 0,7 \\
$\mathrm{MnO}$ & $(\%)$ & 0,185 & 0,186 & 0,187 & 0,190 & 0,002 & 1,1 \\
$\mathrm{MgO}$ & $(\%)$ & 9,0 & 9,0 & 9,0 & 9,2 & 0,1 & 1,1 \\
$\mathrm{CaO}$ & $(\%)$ & 9,4 & 9,4 & 9,5 & 9,6 & 0,1 & 0,9 \\
$\mathrm{Na}_{2} \mathrm{O}$ & $(\%)$ & 3,66 & 3,44 & 3,42 & 3,38 & 0,12 & 3,5 \\
$\mathrm{~K}_{2} \mathrm{O}$ & $(\%)$ & 1,98 & 1,99 & 1,99 & 2,01 & 0,01 & 0,6 \\
$\mathrm{P}_{2} \mathrm{O}_{5}$ & $(\%)$ & 0,747 & 0,748 & 0,749 & 0,751 & 0,002 & 0,2 \\
$\mathrm{Fe}_{2} \mathrm{O}_{3}$ & $(\%)$ & 11,6 & 11,7 & 11,7 & 11,8 & 0,1 & 0,9 \\
\hline $\mathrm{Co}$ & $(\mathrm{ppm})$ & 48 & 47 & 44 & 48 & 2 & 4 \\
$\mathrm{Cr}$ & $(\mathrm{ppm})$ & 220 & 219 & 222 & 231 & 6 & 2 \\
$\mathrm{Cu}$ & $(\mathrm{ppm})$ & 78 & 106 & 125 & 143 & 28 & 25 \\
$\mathrm{Ni}$ & $(\mathrm{ppm})$ & 183 & 183 & 181 & 229 & 23 & 12 \\
$\mathrm{~S}$ & $(\mathrm{ppm})$ & 169 & 145 & 143 & 145 & 13 & 8 \\
$\mathrm{~V}$ & $(\mathrm{ppm})$ & 222 & 231 & 229 & 233 & 5 & 2 \\
$\mathrm{Zn}$ & $(\mathrm{ppm})$ & 103 & 101 & 102 & 102 & 1 & 1 \\
$\mathrm{Cl}$ & $(\mathrm{ppm})$ & 1293 & 444 & 402 & 351 & 449 & 72 \\
\hline
\end{tabular}


Tabelle A.3: Ca-Konzentrationen der Chondritgruppen. Die für die CB-Chondrite angegebene Konzentration ist ein Mittelwert aus drei Analysen der silikatischen Phase von Bencubbin (Kallemeyn et al., 1978). Die anderen Konzentrationen sind Mittelwerte der Chondritgruppen aus Hutchison (2004).

\begin{tabular}{cc}
\hline Gruppe & Ca (Gew.-\%) \\
\hline CI & 0,92 \\
CM & 1,27 \\
CV & 1,90 \\
CO & 1,58 \\
CK & 1,72 \\
CB & 2,1 \\
LL & 1,30 \\
L & 1,31 \\
H & 1,25 \\
R & 1,20 \\
EL & 1,01 \\
EH & 0,85 \\
\hline
\end{tabular}


Tabelle A.4: Ergebnisse der LA-ICPMS-Messungen. Die Konzentrationen sind in ppm angegeben und beziehen sich auf die Gehalte in den Quenchkugeln. Sie wurden intern mit den Ca-Konzentrationen der Kugeln kalibriert.

\begin{tabular}{|c|c|c|c|c|c|c|c|c|}
\hline & $\mathrm{Y}$ & $\mathrm{La}$ & $\mathrm{Pr}$ & $\mathrm{Nd}$ & $\mathrm{Sm}$ & $\mathrm{Eu}$ & Gd & $\mathrm{Tb}$ \\
\hline \multicolumn{9}{|l|}{ Chondrite } \\
\hline \multirow[t]{2}{*}{ Alais (CI1) } & 2,31 & 0,442 & 0,168 & 0,832 & 0,27 & 0,101 & 0,355 & 0,0627 \\
\hline & $\pm 0,01$ & $\pm 0,002$ & $\pm 0,002$ & $\pm 0,006$ & $\pm 0,004$ & $\pm 0,002$ & $\pm 0,005$ & $\pm 0,0007$ \\
\hline \multirow[t]{2}{*}{ Ivuna (CI1) } & 2,92 & 0,511 & 0,174 & 0,829 & 0,249 & 0,0947 & 0,334 & 0,0607 \\
\hline & $\pm 0,01$ & $\pm 0,003$ & $\pm 0,002$ & $\pm 0,006$ & $\pm 0,004$ & $\pm 0,0012$ & $\pm 0,005$ & $\pm 0,0007$ \\
\hline \multirow{2}{*}{$\begin{array}{l}\text { Orgueil-A } \\
\text { (CI1) }\end{array}$} & 2,49 & 0,433 & 0,167 & 0,816 & 0,267 & 0,0985 & 0,35 & 0,0638 \\
\hline & $\pm 0,02$ & $\pm 0,004$ & $\pm 0,002$ & $\pm 0,009$ & $\pm 0,003$ & $\pm 0,0011$ & $\pm 0,004$ & $\pm 0,0007$ \\
\hline \multirow{2}{*}{$\begin{array}{l}\text { Orgueil-B } \\
\text { (CI1) }\end{array}$} & 2,07 & 0,36 & 0,132 & 0,672 & 0,22 & 0,0821 & 0,288 & 0,0531 \\
\hline & $\pm 0,01$ & $\pm 0,002$ & $\pm 0,001$ & $\pm 0,004$ & $\pm 0,003$ & $\pm 0,0007$ & $\pm 0,003$ & $\pm 0,0005$ \\
\hline \multirow{2}{*}{$\begin{array}{l}\text { Orgueil-C } \\
\text { (CI1) }\end{array}$} & 2,92 & 0,468 & 0,179 & 0,917 & 0,31 & 0,113 & 0,404 & 0,0741 \\
\hline & $\pm 0,01$ & $\pm 0,002$ & $\pm 0,002$ & $\pm 0,006$ & $\pm 0,004$ & $\pm 0,002$ & $\pm 0,004$ & $\pm 0,0007$ \\
\hline \multirow{2}{*}{$\begin{array}{l}\text { Orgueil-D } \\
\text { (CI1) }\end{array}$} & 2,11 & 0,35 & 0,134 & 0,671 & 0,223 & 0,0832 & 0,29 & 0,0522 \\
\hline & $\pm 0,01$ & $\pm 0,003$ & $\pm 0,002$ & $\pm 0,007$ & $\pm 0,004$ & $\pm 0,0012$ & $\pm 0,005$ & $\pm 0,0008$ \\
\hline \multirow{2}{*}{$\begin{array}{l}\text { Orgueil-E } \\
\text { (CI1) }\end{array}$} & 2,33 & 0,38 & 0,156 & 0,739 & 0,249 & 0,0928 & 0,329 & 0,0612 \\
\hline & $\pm 0,02$ & $\pm 0,004$ & $\pm 0,002$ & $\pm 0,01$ & $\pm 0,004$ & $\pm 0,0013$ & $\pm 0,005$ & $\pm 0,0009$ \\
\hline \multirow{2}{*}{$\begin{array}{l}\text { Cold Bokke- } \\
\text { veld (CM2) }\end{array}$} & 2,39 & 0,434 & 0,159 & 0,792 & 0,268 & 0,0932 & 0,349 & 0,0617 \\
\hline & $\pm 0,01$ & $\pm 0,003$ & $\pm 0,002$ & $\pm 0,006$ & $\pm 0,004$ & $\pm 0,0011$ & $\pm 0,005$ & $\pm 0,0007$ \\
\hline \multirow[t]{2}{*}{ Mighei (CM2) } & 2,91 & 0,521 & 0,196 & 0,982 & 0,329 & 0,12 & 0,431 & 0,0783 \\
\hline & $\pm 0,01$ & $\pm 0,003$ & $\pm 0,002$ & $\pm 0,008$ & $\pm 0,005$ & $\pm 0,002$ & $\pm 0,006$ & $\pm 0,001$ \\
\hline \multirow{2}{*}{$\begin{array}{l}\text { Murchison-A } \\
\text { (CM2) }\end{array}$} & 3,46 & 0,593 & 0,221 & 1,1 & 0,361 & 0,129 & 0,488 & 0,0875 \\
\hline & $\pm 0,04$ & $\pm 0,006$ & $\pm 0,002$ & $\pm 0,02$ & $\pm 0,005$ & $\pm 0,002$ & $\pm 0,008$ & $\pm 0,0013$ \\
\hline \multirow{2}{*}{$\begin{array}{l}\text { Murchison-B } \\
\text { (CM2) }\end{array}$} & 3,37 & 0,553 & 0,208 & 1,08 & 0,354 & 0,13 & 0,482 & 0,0858 \\
\hline & $\pm 0,04$ & $\pm 0,005$ & $\pm 0,002$ & $\pm 0,02$ & $\pm 0,006$ & $\pm 0,002$ & $\pm 0,008$ & $\pm 0,0014$ \\
\hline \multirow{2}{*}{$\begin{array}{l}\text { Murchison-C } \\
\text { (CM2) }\end{array}$} & 2,75 & 0,458 & 0,171 & 0,876 & 0,296 & 0,106 & 0,392 & 0,0709 \\
\hline & $\pm 0,03$ & $\pm 0,004$ & $\pm 0,002$ & $\pm 0,009$ & $\pm 0,005$ & $\pm 0,002$ & $\pm 0,007$ & $\pm 0,0011$ \\
\hline \multirow{2}{*}{$\begin{array}{l}\text { Murchison-D } \\
\text { (CM2) }\end{array}$} & 2,67 & 0,456 & 0,17 & 0,868 & 0,285 & 0,105 & 0,399 & 0,069 \\
\hline & $\pm 0,01$ & $\pm 0,003$ & $\pm 0,002$ & $\pm 0,007$ & $\pm 0,003$ & $\pm 0,002$ & $\pm 0,004$ & $\pm 0,0009$ \\
\hline \multirow{2}{*}{$\begin{array}{l}\text { Murchison-E } \\
\text { (CM2) }\end{array}$} & 2,93 & 0,532 & 0,192 & 1 & 0,32 & 0,114 & 0,443 & 0,0798 \\
\hline & $\pm 0,01$ & $\pm 0,003$ & $\pm 0,002$ & $\pm 0,01$ & $\pm 0,005$ & $\pm 0,002$ & $\pm 0,006$ & $\pm 0,0009$ \\
\hline \multirow[t]{2}{*}{ Murray (CM2) } & 2,71 & 0,478 & 0,177 & 0,909 & 0,306 & 0,111 & 0,399 & 0,0704 \\
\hline & $\pm 0,02$ & $\pm 0,003$ & $\pm 0,002$ & $\pm 0,008$ & $\pm 0,006$ & $\pm 0,002$ & $\pm 0,006$ & $\pm 0,001$ \\
\hline \multirow{2}{*}{$\begin{array}{l}\text { Allende-A } \\
\text { (CV3) }\end{array}$} & 2,97 & 0,786 & 0,31 & 1,54 & 0,508 & 0,13 & 0,52 & 0,0926 \\
\hline & $\pm 0,02$ & $\pm 0,004$ & $\pm 0,002$ & $\pm 0,01$ & $\pm 0,004$ & $\pm 0,001$ & $\pm 0,005$ & $\pm 0,0009$ \\
\hline Allende-B & 2,9 & 0,495 & 0,186 & 0,96 & 0,315 & 0,115 & 0,417 & 0,0774 \\
\hline (CV3) & $\pm 0,03$ & $\pm 0,003$ & $\pm 0,001$ & $\pm 0,007$ & $\pm 0,004$ & $\pm 0,001$ & $\pm 0,005$ & $\pm 0,0009$ \\
\hline Allende-C & 3,23 & 0,634 & 0,239 & 1,24 & 0,402 & 0,135 & 0,501 & 0,09 \\
\hline (CV3) & $\pm 0,04$ & $\pm 0,005$ & $\pm 0,002$ & $\pm 0,02$ & $\pm 0,006$ & $\pm 0,002$ & $\pm 0,008$ & $\pm 0,0014$ \\
\hline Allende-D & 2,96 & 0,549 & 0,222 & 1,02 & 0,342 & 0,119 & 0,42 & 0,0746 \\
\hline (CV3) & $\pm 0,01$ & $\pm 0,003$ & $\pm 0,002$ & $\pm 0,01$ & $\pm 0,006$ & $\pm 0,002$ & $\pm 0,006$ & $\pm 0,001$ \\
\hline Allende-E & 2,91 & 0,847 & 0,24 & 1,17 & 0,374 & 0,133 & 0,449 & 0,0801 \\
\hline (CV3) & $\pm 0,01$ & $\pm 0,003$ & $\pm 0,002$ & $\pm 0,01$ & $\pm 0,004$ & $\pm 0,002$ & $\pm 0,005$ & $\pm 0,0007$ \\
\hline Allende-M & 2,52 & 0,477 & 0,179 & 0,914 & 0,309 & 0,108 & 0,363 & 0,0674 \\
\hline (CV3) & $\pm 0,01$ & $\pm 0,003$ & $\pm 0,002$ & $\pm 0,008$ & $\pm 0,005$ & $\pm 0,002$ & $\pm 0,006$ & $\pm 0,0009$ \\
\hline Allende-S & 3,11 & 0,603 & 0,224 & 1,15 & 0,375 & 0,129 & 0,475 & 0,0856 \\
\hline (CV3) & $\pm 0,01$ & $\pm 0,003$ & $\pm 0,002$ & $\pm 0,01$ & $\pm 0,004$ & $\pm 0,002$ & $\pm 0,005$ & $\pm 0,0008$ \\
\hline Axtell (CV3) & 3,11 & 0,61 & 0,224 & 1,16 & 0,373 & 0,139 & 0,459 & 0,0831 \\
\hline & $\pm 0,01$ & $\pm 0,004$ & $\pm 0,002$ & $\pm 0,01$ & $\pm 0,005$ & $\pm 0,002$ & $\pm 0,006$ & $\pm 0,001$ \\
\hline Mokoia (CV3) & 2,86 & 0,569 & 0,209 & 1,07 & 0,351 & 0,125 & 0,426 & 0,0769 \\
\hline & $\pm 0,03$ & $\pm 0,004$ & $\pm 0,002$ & $\pm 0,01$ & $\pm 0,004$ & $\pm 0,002$ & $\pm 0,005$ & $\pm 0,0009$ \\
\hline Vigarano & 3,05 & 0,643 & 0,237 & 1,21 & 0,405 & 0,135 & 0,474 & 0,0865 \\
\hline (CV3) & $\pm 0,01$ & $\pm 0,003$ & $\pm 0,002$ & $\pm 0,01$ & $\pm 0,004$ & $\pm 0,002$ & $\pm 0,005$ & $\pm 0,0008$ \\
\hline NWA 3003 & 3,37 & 0,484 & 0,184 & 0,922 & 0,307 & 0,108 & 0,451 & 0,0844 \\
\hline$(\mathrm{CO} 3)$ & $\pm 0,02$ & $\pm 0,003$ & $\pm 0,002$ & $\pm 0,008$ & $\pm 0,005$ & $\pm 0,002$ & $\pm 0,007$ & $\pm 0,001$ \\
\hline Karoonda-A & 3,41 & 0,596 & 0,22 & 1,15 & 0,374 & 0,129 & 0,49 & 0,0892 \\
\hline (CK4) & $\pm 0,03$ & $\pm 0,004$ & $\pm 0,002$ & $\pm 0,01$ & $\pm 0,004$ & $\pm 0,002$ & $\pm 0,005$ & $\pm 0,001$ \\
\hline
\end{tabular}




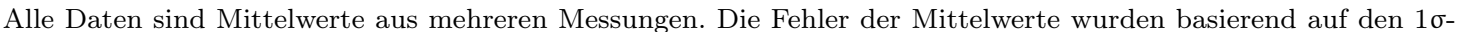
Standardfehlern der Einzelmessungen berechnet. Die Messungen wurden von V. Bendel («), A. Pack ()), A. Patzer $(\diamond)$ und C. Allen $(\Delta)$ durchgeführt. Daten zu Y und Ho der von A. Pack durchgeführten Einzelmessungen sind in Pack et al. (2007) veröffentlicht. N: Anzahl der Messungen.

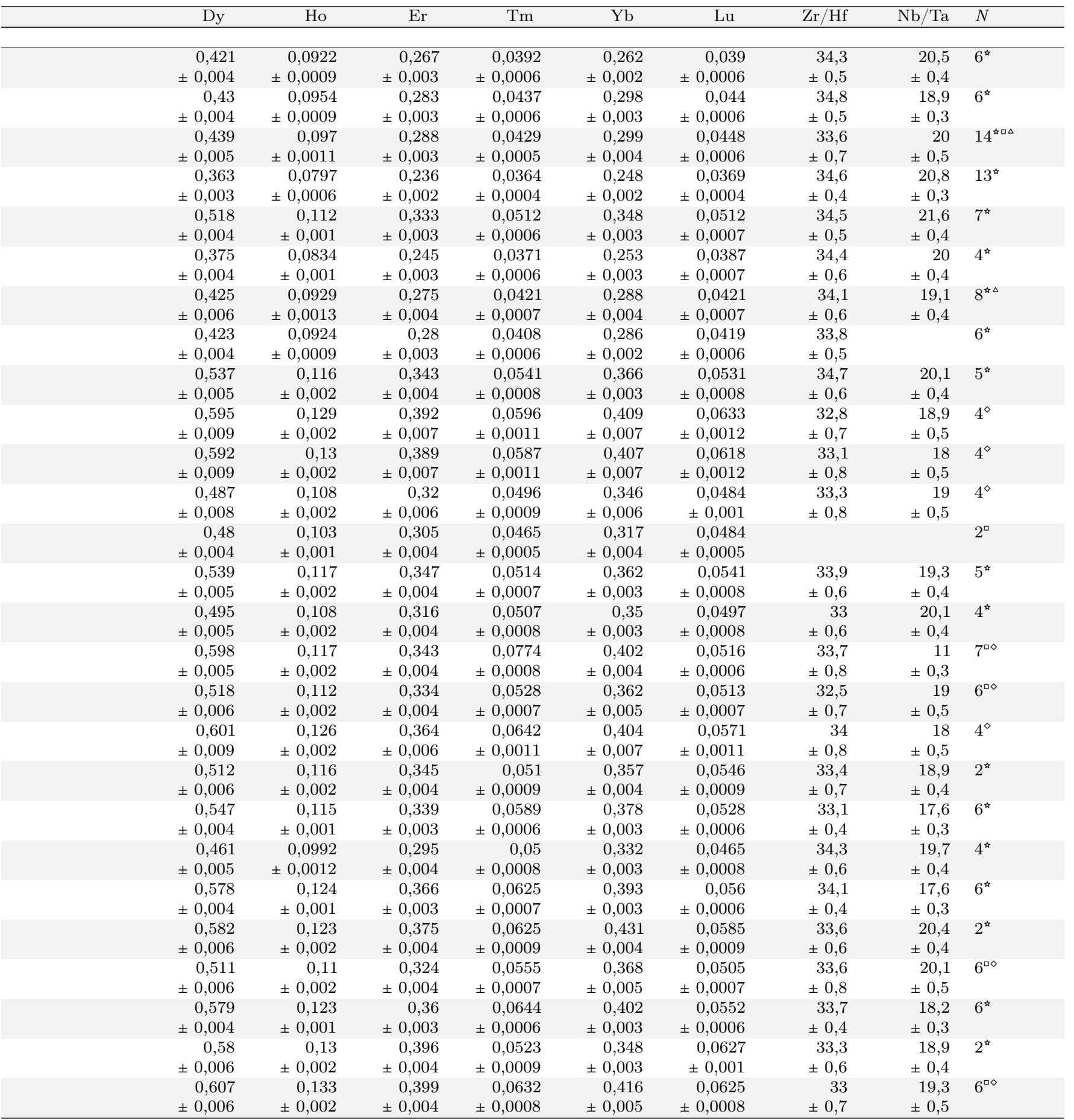


Tabelle A.4 (Fortsetzung)

\begin{tabular}{|c|c|c|c|c|c|c|c|c|}
\hline & $\mathrm{Y}$ & $\mathrm{La}$ & $\mathrm{Pr}$ & $\mathrm{Nd}$ & $\mathrm{Sm}$ & $\mathrm{Eu}$ & $\mathrm{Gd}$ & $\mathrm{Tb}$ \\
\hline Karoonda-B & 3,14 & 0,602 & 0,22 & 1,12 & 0,364 & 0,138 & 0,465 & 0,0844 \\
\hline$(\mathrm{CK} 4)$ & $\pm 0,02$ & $\pm 0,004$ & $\pm 0,002$ & $\pm 0,02$ & $\pm 0,007$ & $\pm 0,003$ & $\pm 0,008$ & $\pm 0,0013$ \\
\hline Bencubbin & 3,18 & 0,851 & 0,251 & 1,15 & 0,355 & 0,122 & 0,454 & 0,0822 \\
\hline$(\mathrm{CB})$ & $\pm 0,01$ & $\pm 0,004$ & $\pm 0,002$ & $\pm 0,01$ & $\pm 0,006$ & $\pm 0,002$ & $\pm 0,006$ & $\pm 0,0011$ \\
\hline Chainpur-A & 2,56 & 0,427 & 0,157 & 0,797 & 0,269 & 0,101 & 0,357 & 0,0652 \\
\hline (LL3) & $\pm 0,02$ & $\pm 0,003$ & $\pm 0,001$ & $\pm 0,005$ & $\pm 0,003$ & $\pm 0,001$ & $\pm 0,004$ & $\pm 0,0006$ \\
\hline Chainpur-B & 2,58 & 0,443 & 0,165 & 0,828 & 0,276 & 0,105 & 0,358 & 0,0676 \\
\hline (LL3) & $\pm 0,02$ & $\pm 0,004$ & $\pm 0,002$ & $\pm 0,01$ & $\pm 0,007$ & $\pm 0,002$ & $\pm 0,007$ & $\pm 0,0012$ \\
\hline Dar al Gani & 2,41 & 0,389 & 0,151 & 0,765 & 0,253 & 0,0859 & 0,344 & 0,0634 \\
\hline 298 (LL4) & $\pm 0,01$ & $\pm 0,003$ & $\pm 0,002$ & $\pm 0,007$ & $\pm 0,005$ & $\pm 0,0013$ & $\pm 0,006$ & $\pm 0,0009$ \\
\hline Dhurmsala & 1,73 & 0,239 & 0,0916 & 0,468 & 0,162 & 0,11 & 0,229 & 0,0421 \\
\hline$(\mathrm{LL} 6)$ & $\pm 0,02$ & $\pm 0,003$ & $\pm 0,001$ & $\pm 0,006$ & $\pm 0,004$ & $\pm 0,002$ & $\pm 0,005$ & $\pm 0,0008$ \\
\hline Lake Labyrinth & 2,25 & 0,382 & 0,141 & 0,703 & 0,23 & 0,0856 & 0,313 & 0,0575 \\
\hline$(\mathrm{LL} 6)$ & $\pm 0,01$ & $\pm 0,002$ & $\pm 0,001$ & $\pm 0,005$ & $\pm 0,003$ & $\pm 0,0009$ & $\pm 0,004$ & $\pm 0,0006$ \\
\hline Saint-Séverin & 2,83 & 0,477 & 0,174 & 0,872 & 0,295 & 0,0805 & 0,398 & 0,073 \\
\hline (LL6) & $\pm 0,02$ & $\pm 0,004$ & $\pm 0,002$ & $\pm 0,01$ & $\pm 0,006$ & $\pm 0,0014$ & $\pm 0,007$ & $\pm 0,0011$ \\
\hline Bruderheim & 1,6 & 0,508 & 0,138 & 0,625 & 0,165 & 0,091 & 0,259 & 0,0419 \\
\hline (L6) & $\pm 0,01$ & $\pm 0,004$ & $\pm 0,002$ & $\pm 0,008$ & $\pm 0,005$ & $\pm 0,0018$ & $\pm 0,006$ & $\pm 0,0009$ \\
\hline \multirow[t]{2}{*}{ Gashua (L6) } & 2,57 & 0,384 & 0,143 & 0,75 & 0,253 & 0,0939 & 0,332 & 0,0614 \\
\hline & $\pm 0,03$ & $\pm 0,004$ & $\pm 0,002$ & $\pm 0,008$ & $\pm 0,005$ & $\pm 0,0014$ & $\pm 0,006$ & $\pm 0,0011$ \\
\hline Homestead & 2,29 & 0,352 & 0,135 & 0,69 & 0,227 & 0,0858 & 0,303 & 0,0573 \\
\hline (L5) & $\pm 0,01$ & $\pm 0,003$ & $\pm 0,002$ & $\pm 0,007$ & $\pm 0,004$ & $\pm 0,0013$ & $\pm 0,005$ & $\pm 0,0008$ \\
\hline Mount & 2,19 & 0,35 & 0,138 & 0,672 & 0,229 & 0,0778 & 0,311 & 0,0563 \\
\hline Tazerzait (L5) & $\pm 0,01$ & $\pm 0,002$ & $\pm 0,002$ & $\pm 0,006$ & $\pm 0,004$ & $\pm 0,0011$ & $\pm 0,005$ & $\pm 0,0008$ \\
\hline \multirow[t]{2}{*}{ NWA 869 (L5) } & 2,51 & 0,46 & 0,164 & 0,81 & 0,257 & 0,0946 & 0,349 & 0,0627 \\
\hline & $\pm 0,03$ & $\pm 0,005$ & $\pm 0,002$ & $\pm 0,008$ & $\pm 0,004$ & $\pm 0,001$ & $\pm 0,006$ & $\pm 0,001$ \\
\hline NWA 869-A & 2,73 & 0,475 & 0,172 & 0,86 & 0,292 & 0,106 & 0,382 & 0,0705 \\
\hline (L5) & $\pm 0,05$ & $\pm 0,005$ & $\pm 0,002$ & $\pm 0,011$ & $\pm 0,006$ & $\pm 0,002$ & $\pm 0,008$ & $\pm 0,0014$ \\
\hline NWA 869-B & 2,96 & 0,504 & 0,183 & 0,917 & 0,293 & 0,111 & 0,406 & 0,0748 \\
\hline$(\mathrm{L} 5)$ & $\pm 0,05$ & $\pm 0,006$ & $\pm 0,003$ & $\pm 0,012$ & $\pm 0,006$ & $\pm 0,002$ & $\pm 0,008$ & $\pm 0,0015$ \\
\hline NWA 869-C & 2,59 & 0,448 & 0,16 & 0,801 & 0,273 & 0,105 & 0,362 & 0,0657 \\
\hline (L5) & $\pm 0,04$ & $\pm 0,005$ & $\pm 0,002$ & $\pm 0,01$ & $\pm 0,006$ & $\pm 0,002$ & $\pm 0,008$ & $\pm 0,0013$ \\
\hline NWA 869-D & 2,67 & 0,634 & 0,219 & 1,01 & 0,313 & 0,0998 & 0,408 & 0,0717 \\
\hline$(\mathrm{L} 5)$ & $\pm 0,04$ & $\pm 0,007$ & $\pm 0,003$ & $\pm 0,02$ & $\pm 0,007$ & $\pm 0,0017$ & $\pm 0,008$ & $\pm 0,0014$ \\
\hline NWA 869-E & 2,1 & 0,338 & 0,125 & 0,64 & 0,211 & 0,0816 & 0,299 & 0,0521 \\
\hline (L5) & $\pm 0,04$ & $\pm 0,004$ & $\pm 0,002$ & $\pm 0,009$ & $\pm 0,005$ & $\pm 0,0015$ & $\pm 0,007$ & $\pm 0,0011$ \\
\hline NWA 869-F & 2,08 & 0,346 & 0,126 & 0,621 & 0,203 & 0,0833 & 0,277 & 0,0512 \\
\hline$(\mathrm{L} 5)$ & $\pm 0,04$ & $\pm 0,004$ & $\pm 0,002$ & $\pm 0,009$ & $\pm 0,005$ & $\pm 0,0017$ & $\pm 0,007$ & $\pm 0,0012$ \\
\hline NWA 869-G & 2,12 & 0,374 & 0,147 & 0,665 & 0,21 & 0,0801 & 0,292 & 0,0527 \\
\hline (L5) & $\pm 0,04$ & $\pm 0,004$ & $\pm 0,002$ & $\pm 0,009$ & $\pm 0,005$ & $\pm 0,0014$ & $\pm 0,006$ & $\pm 0,001$ \\
\hline NWA $869-\mathrm{H}$ & 2,37 & 0,421 & 0,147 & 0,758 & 0,23 & 0,108 & 0,325 & 0,0605 \\
\hline$(\mathrm{L} 5)$ & $\pm 0,04$ & $\pm 0,005$ & $\pm 0,003$ & $\pm 0,011$ & $\pm 0,006$ & $\pm 0,002$ & $\pm 0,008$ & $\pm 0,0013$ \\
\hline NWA 869-I & 2,01 & 0,31 & 0,114 & 0,607 & 0,188 & 0,0804 & 0,274 & 0,0515 \\
\hline (L5) & $\pm 0,03$ & $\pm 0,004$ & $\pm 0,002$ & $\pm 0,009$ & $\pm 0,005$ & $\pm 0,0016$ & $\pm 0,007$ & $\pm 0,0012$ \\
\hline NWA 869-J & 2,72 & 0,516 & 0,188 & 0,855 & 0,268 & 0,101 & 0,408 & 0,0698 \\
\hline$(\mathrm{L} 5)$ & $\pm 0,05$ & $\pm 0,006$ & $\pm 0,003$ & $\pm 0,011$ & $\pm 0,006$ & $\pm 0,002$ & $\pm 0,009$ & $\pm 0,0015$ \\
\hline NWA 869-K & 1,96 & 0,34 & 0,121 & 0,618 & 0,181 & 0,0763 & 0,262 & 0,0491 \\
\hline (L5) & $\pm 0,03$ & $\pm 0,004$ & $\pm 0,002$ & $\pm 0,009$ & $\pm 0,005$ & $\pm 0,0015$ & $\pm 0,007$ & $\pm 0,0011$ \\
\hline SAU 001 & 2,56 & 0,474 & 0,17 & 0,825 & 0,255 & 0,0962 & 0,351 & 0,0644 \\
\hline$(\mathrm{L} 4-5)$ & $\pm 0,03$ & $\pm 0,004$ & $\pm 0,002$ & $\pm 0,008$ & $\pm 0,004$ & $\pm 0,0011$ & $\pm 0,006$ & $\pm 0,001$ \\
\hline \multirow[t]{2}{*}{ St. Michel (L6) } & 2,34 & 0,393 & 0,147 & 0,724 & 0,239 & 0,0848 & 0,318 & 0,0586 \\
\hline & $\pm 0,02$ & $\pm 0,003$ & $\pm 0,001$ & $\pm 0,006$ & $\pm 0,003$ & $\pm 0,0009$ & $\pm 0,004$ & $\pm 0,0007$ \\
\hline \multirow[t]{2}{*}{ Zavid (L6) } & 2,44 & 0,402 & 0,149 & 0,754 & 0,244 & 0,0939 & 0,335 & 0,0622 \\
\hline & $\pm 0,02$ & $\pm 0,003$ & $\pm 0,001$ & $\pm 0,006$ & $\pm 0,003$ & $\pm 0,0009$ & $\pm 0,004$ & $\pm 0,0007$ \\
\hline \multirow[t]{2}{*}{ Adrian (H4) } & 7,79 & 9,09 & 2,09 & 8,42 & 1,65 & 0,427 & 1,56 & 0,226 \\
\hline & $\pm 0,06$ & $\pm 0,04$ & $\pm 0,01$ & $\pm 0,04$ & $\pm 0,02$ & $\pm 0,003$ & $\pm 0,02$ & $\pm 0,002$ \\
\hline Dar al Gani & 2,16 & 0,341 & 0,126 & 0,655 & 0,212 & 0,0791 & 0,298 & 0,0545 \\
\hline 300 (H3-5) & $\pm 0,01$ & $\pm 0,003$ & $\pm 0,002$ & $\pm 0,007$ & $\pm 0,004$ & $\pm 0,0012$ & $\pm 0,005$ & $\pm 0,0009$ \\
\hline Djoumine & 2,39 & 0,39 & 0,148 & 0,757 & 0,245 & 0,0867 & 0,343 & 0,0619 \\
\hline$(\mathrm{H} 5-6)$ & $\pm 0,01$ & $\pm 0,003$ & $\pm 0,002$ & $\pm 0,007$ & $\pm 0,005$ & $\pm 0,0013$ & $\pm 0,005$ & $\pm 0,0009$ \\
\hline
\end{tabular}




\begin{tabular}{|c|c|c|c|c|c|c|c|c|}
\hline Dy & Ho & Er & Tm & $\mathrm{Yb}$ & $\mathrm{Lu}$ & $\mathrm{Zr} / \mathrm{Hf}$ & $\mathrm{Nb} / \mathrm{Ta}$ & $N$ \\
\hline 0,578 & 0,123 & 0,375 & 0,0607 & 0,396 & 0,056 & 32,8 & 18,2 & $3^{4}$ \\
\hline $\pm 0,007$ & $\pm 0,002$ & $\pm 0,005$ & $\pm 0,0011$ & $\pm 0,004$ & $\pm 0,001$ & $\pm 0,7$ & $\pm 0,4$ & \\
\hline 0,551 & 0,122 & 0,361 & 0,0557 & 0,367 & 0,0563 & 33,2 & 9,44 & $2^{\text {से }}$ \\
\hline 0,449 & 0,0987 & 0,296 & 0,0423 & 0,303 & 0,0452 & 33,7 & 19,7 & $7^{\square \diamond}$ \\
\hline $\pm 0,004$ & $\pm 0,0008$ & $\pm 0,003$ & $\pm 0,0005$ & $\pm 0,003$ & $\pm 0,0005$ & $\pm 0,7$ & $\pm 0,5$ & \\
\hline 0,46 & 0,103 & 0,308 & 0,0444 & 0,312 & 0,0468 & 33,6 & 20,2 & $2^{\hbar}$ \\
\hline $\pm 0,007$ & $\pm 0,002$ & $\pm 0,005$ & $\pm 0,001$ & $\pm 0,004$ & $\pm 0,001$ & $\pm 0,8$ & $\pm 0,6$ & \\
\hline 0,424 & 0,0935 & 0,274 & 0,0405 & 0,283 & 0,0415 & 33,3 & 20 & $2^{\text {मे }}$ \\
\hline $\pm 0,005$ & $\pm 0,0011$ & $\pm 0,004$ & $\pm 0,0008$ & $\pm 0,003$ & $\pm 0,0008$ & $\pm 0,7$ & $\pm 0,5$ & \\
\hline 0,302 & 0,0677 & 0,209 & 0,0314 & 0,245 & 0,0387 & 33,8 & & $4^{\diamond}$ \\
\hline $\pm 0,005$ & $\pm 0,0012$ & $\pm 0,004$ & $\pm 0,0007$ & $\pm 0,005$ & $\pm 0,0008$ & $\pm 0,8$ & & \\
\hline 0,388 & 0,0864 & 0,259 & 0,0383 & 0,263 & 0,0417 & 36,1 & & $4^{\text {मेᄆ }}$ \\
\hline $\pm 0,003$ & $\pm 0,0006$ & $\pm 0,003$ & $\pm 0,0005$ & $\pm 0,003$ & $\pm 0,0005$ & $\pm 0,8$ & & \\
\hline 0,502 & 0,106 & 0,325 & 0,0466 & 0,321 & 0,0479 & 32,9 & & $2^{\star \Delta}$ \\
\hline $\pm 0,006$ & $\pm 0,002$ & $\pm 0,005$ & $\pm 0,0008$ & $\pm 0,004$ & $\pm 0,0008$ & $\pm 0,7$ & & \\
\hline 0,433 & 0,0998 & 0,298 & 0,0435 & 0,303 & 0,0475 & 34,3 & & $4^{\diamond}$ \\
\hline $\pm 0,007$ & $\pm 0,0016$ & $\pm 0,005$ & $\pm 0,0009$ & $\pm 0,006$ & $\pm 0,001$ & $\pm 0,8$ & & \\
\hline 0,387 & 0,0891 & 0,257 & 0,0378 & 0,27 & 0,0395 & 34,2 & 19,8 & $2^{\text {मे }}$ \\
\hline $\pm 0,005$ & $\pm 0,0011$ & $\pm 0,004$ & $\pm 0,0007$ & $\pm 0,003$ & $\pm 0,0008$ & $\pm 0,7$ & $\pm 0,5$ & \\
\hline 0,388 & 0,0856 & 0,258 & 0,0372 & 0,262 & 0,0395 & 33,1 & 19,3 & $2^{\text {मे }}$ \\
\hline $\pm 0,004$ & $\pm 0,001$ & $\pm 0,003$ & $\pm 0,0006$ & $\pm 0,003$ & $\pm 0,0007$ & $\pm 0,6$ & $\pm 0,4$ & \\
\hline 0,431 & 0,0944 & 0,285 & 0,042 & 0,289 & 0,0433 & 33,5 & & $4^{\diamond}$ \\
\hline $\pm 0,007$ & $\pm 0,0015$ & $\pm 0,005$ & $\pm 0,0008$ & $\pm 0,005$ & $\pm 0,0008$ & $\pm 0,7$ & & \\
\hline 0,472 & 0,104 & 0,318 & 0,0461 & 0,329 & 0,05 & 36 & & $2^{\diamond}$ \\
\hline $\pm 0,009$ & $\pm 0,002$ & $\pm 0,007$ & $\pm 0,0011$ & $\pm 0,007$ & $\pm 0,0012$ & \pm 1 & & \\
\hline 0,519 & 0,115 & 0,35 & 0,0505 & 0,356 & 0,053 & 33,2 & & $2^{\diamond}$ \\
\hline $\pm 0,01$ & $\pm 0,003$ & $\pm 0,007$ & $\pm 0,0011$ & $\pm 0,008$ & $\pm 0,0012$ & \pm 1 & & \\
\hline 0,447 & 0,1 & 0,308 & 0,0425 & 0,314 & 0,048 & 34 & & $2^{\diamond}$ \\
\hline $\pm 0,008$ & $\pm 0,002$ & $\pm 0,006$ & $\pm 0,001$ & $\pm 0,007$ & $\pm 0,0011$ & \pm 1 & & \\
\hline 0,474 & 0,106 & 0,306 & 0,0438 & 0,29 & 0,0422 & 32,5 & & $2^{\diamond}$ \\
\hline $\pm 0,009$ & $\pm 0,002$ & $\pm 0,006$ & $\pm 0,001$ & $\pm 0,006$ & $\pm 0,0011$ & $\pm 0,9$ & & \\
\hline 0,362 & 0,078 & 0,235 & 0,0335 & 0,243 & 0,0376 & 32 & & $2^{\diamond}$ \\
\hline $\pm 0,008$ & $\pm 0,0016$ & $\pm 0,005$ & $\pm 0,0009$ & $\pm 0,006$ & $\pm 0,001$ & $\pm 1,1$ & & \\
\hline 0,359 & 0,0807 & 0,241 & 0,0339 & 0,256 & 0,0388 & 35,9 & & $2^{\diamond}$ \\
\hline $\pm 0,007$ & $\pm 0,0015$ & $\pm 0,005$ & $\pm 0,0008$ & $\pm 0,006$ & $\pm 0,001$ & \pm 1 & & \\
\hline 0,413 & 0,0918 & 0,279 & 0,0411 & 0,296 & 0,0453 & 33,1 & & $2^{\diamond}$ \\
\hline $\pm 0,008$ & $\pm 0,0019$ & $\pm 0,006$ & $\pm 0,0011$ & $\pm 0,007$ & $\pm 0,0013$ & \pm 1 & & \\
\hline 0,345 & 0,0773 & 0,237 & 0,0352 & 0,247 & 0,0383 & 33,9 & & $2^{\diamond}$ \\
\hline $\pm 0,007$ & $\pm 0,0016$ & $\pm 0,005$ & $\pm 0,001$ & $\pm 0,006$ & $\pm 0,001$ & $\pm 1,1$ & & \\
\hline 0,478 & 0,105 & 0,315 & 0,0451 & 0,329 & 0,0533 & 34,4 & & $2^{\diamond}$ \\
\hline $\pm 0,009$ & $\pm 0,002$ & $\pm 0,007$ & $\pm 0,0011$ & $\pm 0,007$ & $\pm 0,0012$ & \pm 1 & & \\
\hline 0,339 & 0,0741 & 0,232 & 0,0347 & 0,247 & 0,0399 & 34,2 & & $2^{\diamond}$ \\
\hline $\pm 0,007$ & $\pm 0,0016$ & $\pm 0,005$ & $\pm 0,0009$ & $\pm 0,006$ & $\pm 0,0011$ & $\pm 1,1$ & & \\
\hline 0,446 & 0,0992 & 0,29 & 0,0425 & 0,295 & 0,0443 & 34,4 & & $4^{\diamond}$ \\
\hline $\pm 0,007$ & $\pm 0,0016$ & $\pm 0,005$ & $\pm 0,0008$ & $\pm 0,005$ & $\pm 0,0009$ & $\pm 0,8$ & & \\
\hline 0,406 & 0,0903 & 0,267 & 0,038 & 0,274 & 0,0412 & 35,4 & & $6^{\square \diamond}$ \\
\hline $\pm 0,005$ & $\pm 0,001$ & $\pm 0,003$ & $\pm 0,0005$ & $\pm 0,004$ & $\pm 0,0006$ & $\pm 0,9$ & & \\
\hline 0,424 & 0,0941 & 0,284 & 0,0414 & 0,295 & 0,045 & 34,3 & & $6^{\square \diamond}$ \\
\hline $\pm 0,004$ & $\pm 0,0009$ & $\pm 0,003$ & $\pm 0,0005$ & $\pm 0,004$ & $\pm 0,0006$ & $\pm 0,8$ & & \\
\hline 1,34 & 0,27 & 0,753 & 0,104 & 0,675 & 0,1 & 32,8 & & $6^{\square \diamond}$ \\
\hline $\pm 0,02$ & $\pm 0,003$ & $\pm 0,007$ & $\pm 0,002$ & $\pm 0,007$ & $\pm 0,002$ & $\pm 0,6$ & & \\
\hline 0,382 & 0,0824 & 0,245 & 0,037 & 0,25 & 0,0386 & 34,1 & 20,4 & $2^{\text {해 }}$ \\
\hline $\pm 0,005$ & $\pm 0,0011$ & $\pm 0,004$ & $\pm 0,0008$ & $\pm 0,003$ & $\pm 0,0008$ & $\pm 0,8$ & $\pm 0,5$ & \\
\hline 0,437 & 0,0948 & 0,281 & 0,0397 & 0,295 & 0,0452 & 32,8 & 19,6 & $2^{\text {औ }}$ \\
\hline $\pm 0,005$ & $\pm 0,0011$ & $\pm 0,004$ & $\pm 0,0007$ & $\pm 0,003$ & $\pm 0,0009$ & $\pm 0,6$ & $\pm 0,5$ & \\
\hline
\end{tabular}


Tabelle A.4 (Fortsetzung)

\begin{tabular}{|c|c|c|c|c|c|c|c|c|}
\hline & $\mathrm{Y}$ & $\mathrm{La}$ & $\operatorname{Pr}$ & $\mathrm{Nd}$ & $\mathrm{Sm}$ & $\mathrm{Eu}$ & $\mathrm{Gd}$ & $\mathrm{Tb}$ \\
\hline El Hammami & 2,09 & 0,363 & 0,129 & 0,657 & 0,217 & 0,0746 & 0,289 & 0,0531 \\
\hline (H5) & $\pm 0,01$ & $\pm 0,003$ & $\pm 0,002$ & $\pm 0,009$ & $\pm 0,005$ & $\pm 0,0014$ & $\pm 0,006$ & $\pm 0,001$ \\
\hline Forest City & 2,23 & 0,36 & 0,134 & 0,669 & 0,221 & 0,0844 & 0,309 & 0,0557 \\
\hline (H5) & $\pm 0,02$ & $\pm 0,003$ & $\pm 0,001$ & $\pm 0,005$ & $\pm 0,003$ & $\pm 0,0009$ & $\pm 0,004$ & $\pm 0,0007$ \\
\hline Gao-Guenie & 2,15 & 0,337 & 0,127 & 0,643 & 0,216 & 0,08 & 0,3 & 0,0526 \\
\hline (H5) & $\pm 0,03$ & $\pm 0,003$ & $\pm 0,001$ & $\pm 0,007$ & $\pm 0,004$ & $\pm 0,001$ & $\pm 0,005$ & $\pm 0,0009$ \\
\hline Gao-Guenie-A & 1,82 & 0,457 & 0,115 & 0,571 & 0,186 & 0,0776 & 0,247 & 0,0468 \\
\hline (H5) & $\pm 0,02$ & $\pm 0,005$ & $\pm 0,002$ & $\pm 0,01$ & $\pm 0,006$ & $\pm 0,0019$ & $\pm 0,008$ & $\pm 0,0012$ \\
\hline Gao-Guenie-B & 2,21 & 0,522 & 0,139 & 0,69 & 0,223 & 0,0889 & 0,319 & 0,0549 \\
\hline (H5) & $\pm 0,02$ & $\pm 0,005$ & $\pm 0,002$ & $\pm 0,011$ & $\pm 0,008$ & $\pm 0,002$ & $\pm 0,009$ & $\pm 0,0014$ \\
\hline Gao-Guenie-C & 2,1 & 0,612 & 0,153 & 0,726 & 0,23 & 0,0826 & 0,296 & 0,0553 \\
\hline (H5) & $\pm 0,02$ & $\pm 0,005$ & $\pm 0,003$ & $\pm 0,011$ & $\pm 0,007$ & $\pm 0,002$ & $\pm 0,008$ & $\pm 0,0014$ \\
\hline Gao-Guenie-D & 2,06 & 0,41 & 0,13 & 0,653 & 0,2 & 0,0687 & 0,28 & 0,0543 \\
\hline (H5) & $\pm 0,02$ & $\pm 0,005$ & $\pm 0,002$ & $\pm 0,011$ & $\pm 0,007$ & $\pm 0,0019$ & $\pm 0,008$ & $\pm 0,0014$ \\
\hline Gao-Guenie-E & 2,28 & 0,442 & 0,148 & 0,716 & 0,241 & 0,0725 & 0,324 & 0,0576 \\
\hline (H5) & $\pm 0,02$ & $\pm 0,005$ & $\pm 0,002$ & $\pm 0,01$ & $\pm 0,007$ & $\pm 0,0018$ & $\pm 0,008$ & $\pm 0,0013$ \\
\hline Gao-Guenie-F & 1,51 & 0,346 & 0,101 & 0,474 & 0,152 & 0,0664 & 0,194 & 0,038 \\
\hline (H5) & $\pm 0,02$ & $\pm 0,004$ & $\pm 0,002$ & $\pm 0,01$ & $\pm 0,006$ & $\pm 0,0019$ & $\pm 0,007$ & $\pm 0,001$ \\
\hline Hammond & 2,56 & 0,611 & 0,21 & 0,988 & 0,299 & 0,0973 & 0,382 & 0,0684 \\
\hline Downs (H4) & $\pm 0,02$ & $\pm 0,004$ & $\pm 0,002$ & $\pm 0,007$ & $\pm 0,004$ & $\pm 0,001$ & $\pm 0,005$ & $\pm 0,0008$ \\
\hline \multirow[t]{2}{*}{ Ijopega (H6) } & 1,94 & 0,305 & 0,114 & 0,575 & 0,191 & 0,0693 & 0,26 & 0,047 \\
\hline & $\pm 0,02$ & $\pm 0,002$ & $\pm 0,001$ & $\pm 0,005$ & $\pm 0,003$ & $\pm 0,0009$ & $\pm 0,004$ & $\pm 0,0007$ \\
\hline \multirow[t]{2}{*}{ Jilin (H5) } & 2,21 & 0,409 & 0,144 & 0,714 & 0,233 & 0,0819 & 0,304 & 0,0563 \\
\hline & $\pm 0,01$ & $\pm 0,002$ & $\pm 0,001$ & $\pm 0,005$ & $\pm 0,003$ & $\pm 0,0009$ & $\pm 0,003$ & $\pm 0,0006$ \\
\hline Kunya-Ur- & 2,72 & 0,432 & 0,159 & 0,82 & 0,265 & 0,0928 & 0,386 & 0,0678 \\
\hline gench (H5) & $\pm 0,03$ & $\pm 0,004$ & $\pm 0,002$ & $\pm 0,009$ & $\pm 0,005$ & $\pm 0,0014$ & $\pm 0,007$ & $\pm 0,0011$ \\
\hline Pultusk-A & 2,01 & 0,429 & 0,142 & 0,698 & 0,211 & 0,0761 & 0,278 & 0,0494 \\
\hline (H5) & $\pm 0,02$ & $\pm 0,003$ & $\pm 0,001$ & $\pm 0,006$ & $\pm 0,003$ & $\pm 0,0009$ & $\pm 0,004$ & $\pm 0,0007$ \\
\hline Pultusk-B & 2,41 & 0,367 & 0,139 & 0,709 & 0,234 & 0,082 & 0,331 & 0,0587 \\
\hline (H5) & $\pm 0,03$ & $\pm 0,003$ & $\pm 0,002$ & $\pm 0,008$ & $\pm 0,004$ & $\pm 0,0011$ & $\pm 0,006$ & $\pm 0,001$ \\
\hline Richardton & 2,42 & 0,448 & 0,157 & 0,77 & 0,253 & 0,0902 & 0,336 & 0,0623 \\
\hline (H5) & $\pm 0,02$ & $\pm 0,003$ & $\pm 0,002$ & $\pm 0,006$ & $\pm 0,003$ & $\pm 0,001$ & $\pm 0,004$ & $\pm 0,0007$ \\
\hline Thuathe & 2,09 & 0,344 & 0,131 & 0,637 & 0,21 & 0,0792 & 0,29 & 0,0523 \\
\hline$(\mathrm{H} 4 / 5)$ & $\pm 0,02$ & $\pm 0,002$ & $\pm 0,001$ & $\pm 0,005$ & $\pm 0,003$ & $\pm 0,0009$ & $\pm 0,004$ & $\pm 0,0006$ \\
\hline NWA 753 & 2,25 & 0,381 & 0,134 & 0,687 & 0,233 & 0,0932 & 0,316 & 0,0568 \\
\hline (R3-6) & $\pm 0,01$ & $\pm 0,003$ & $\pm 0,002$ & $\pm 0,006$ & $\pm 0,004$ & $\pm 0,0012$ & $\pm 0,005$ & $\pm 0,0007$ \\
\hline \multirow[t]{2}{*}{ Atlanta (EL6) } & 1,77 & 0,172 & 0,0906 & 0,504 & 0,164 & 0,0388 & 0,23 & 0,0418 \\
\hline & $\pm 0,01$ & $\pm 0,002$ & $\pm 0,001$ & $\pm 0,006$ & $\pm 0,004$ & $\pm 0,0009$ & $\pm 0,005$ & $\pm 0,0007$ \\
\hline \multirow[t]{2}{*}{ Eagle (EL6) } & 1,58 & 0,16 & 0,075 & 0,382 & 0,133 & 0,0464 & 0,184 & 0,0353 \\
\hline & $\pm 0,01$ & $\pm 0,002$ & $\pm 0,0009$ & $\pm 0,006$ & $\pm 0,004$ & $\pm 0,001$ & $\pm 0,004$ & $\pm 0,0007$ \\
\hline \multirow[t]{2}{*}{ Hvittis (EL6) } & 1,86 & 0,294 & 0,109 & 0,537 & 0,179 & 0,0553 & 0,241 & 0,0443 \\
\hline & $\pm 0,01$ & $\pm 0,002$ & $\pm 0,001$ & $\pm 0,004$ & $\pm 0,002$ & $\pm 0,0007$ & $\pm 0,003$ & $\pm 0,0005$ \\
\hline \multirow[t]{2}{*}{ Pillistfer (EL6) } & 1,81 & 0,265 & 0,101 & 0,502 & 0,174 & 0,0511 & 0,239 & 0,0437 \\
\hline & $\pm 0,02$ & $\pm 0,002$ & $\pm 0,001$ & $\pm 0,005$ & $\pm 0,003$ & $\pm 0,0007$ & $\pm 0,004$ & $\pm 0,0006$ \\
\hline \multirow[t]{2}{*}{ Abee (EH4) } & 1,57 & 0,261 & 0,0917 & 0,469 & 0,157 & 0,0537 & 0,23 & 0,0394 \\
\hline & $\pm 0,01$ & $\pm 0,002$ & $\pm 0,0009$ & $\pm 0,005$ & $\pm 0,003$ & $\pm 0,0009$ & $\pm 0,004$ & $\pm 0,0006$ \\
\hline \multirow[t]{2}{*}{ Indarch (EH4) } & 1,51 & 0,277 & 0,0888 & 0,453 & 0,149 & 0,0552 & 0,212 & 0,0373 \\
\hline & $\pm 0,01$ & $\pm 0,002$ & $\pm 0,0009$ & $\pm 0,005$ & $\pm 0,004$ & $\pm 0,001$ & $\pm 0,004$ & $\pm 0,0006$ \\
\hline Sahara 97072 & 1,59 & 0,254 & 0,0919 & 0,463 & 0,151 & 0,0537 & 0,203 & 0,0394 \\
\hline (EH3) & $\pm 0,01$ & $\pm 0,002$ & $\pm 0,001$ & $\pm 0,006$ & $\pm 0,004$ & $\pm 0,001$ & $\pm 0,005$ & $\pm 0,0007$ \\
\hline St. Mark's & 1,71 & 0,349 & 0,116 & 0,57 & 0,166 & 0,0633 & 0,241 & 0,0453 \\
\hline (EH5) & $\pm 0,01$ & $\pm 0,003$ & $\pm 0,002$ & $\pm 0,008$ & $\pm 0,005$ & $\pm 0,0014$ & $\pm 0,005$ & $\pm 0,0009$ \\
\hline \multicolumn{9}{|l|}{ Achondrite } \\
\hline \multirow[t]{2}{*}{ Bilanga (DIO) } & 1,02 & 0,0286 & 0,0228 & 0,0716 & 0,0432 & 0,00302 & 0,0846 & 0,0199 \\
\hline & $\pm 0,02$ & $\pm 0,0005$ & $\pm 0,0004$ & $\pm 0,0014$ & $\pm 0,0013$ & $\pm 0,00018$ & $\pm 0,002$ & $\pm 0,0004$ \\
\hline \multirow[t]{2}{*}{ Cachari (EUC) } & 15,9 & 2,97 & 1,14 & 5,66 & 1,69 & 0,734 & 2,32 & 0,434 \\
\hline & $\pm 0,2$ & $\pm 0,03$ & $\pm 0,01$ & $\pm 0,05$ & $\pm 0,03$ & $\pm 0,008$ & $\pm 0,04$ & $\pm 0,006$ \\
\hline Camel Donga & 18,1 & 3,29 & 1,19 & 6,21 & 2,03 & 0,7 & 2,63 & 0,476 \\
\hline$(\mathrm{EUC})$ & $\pm 0,1$ & $\pm 0,02$ & $\pm 0,01$ & $\pm 0,03$ & $\pm 0,02$ & $\pm 0,005$ & $\pm 0,02$ & $\pm 0,003$ \\
\hline
\end{tabular}




\begin{tabular}{|c|c|c|c|c|c|c|c|c|}
\hline Dy & Ho & Er & $\mathrm{Tm}$ & $\mathrm{Yb}$ & $\mathrm{Lu}$ & $\mathrm{Zr} / \mathrm{Hf}$ & $\mathrm{Nb} / \mathrm{Ta}$ & $N$ \\
\hline 0,368 & 0,0799 & 0,239 & 0,0354 & 0,242 & 0,0369 & 36,6 & & $2^{\not h}$ \\
\hline $\pm 0,005$ & $\pm 0,0011$ & $\pm 0,004$ & $\pm 0,0008$ & $\pm 0,003$ & $\pm 0,0008$ & $\pm 0,8$ & & \\
\hline 0,387 & 0,0865 & 0,255 & 0,0388 & 0,272 & 0,0422 & 36 & & $6^{\square \diamond}$ \\
\hline $\pm 0,004$ & $\pm 0,0009$ & $\pm 0,003$ & $\pm 0,0006$ & $\pm 0,004$ & $\pm 0,0006$ & $\pm 0,8$ & & \\
\hline 0,369 & 0,0807 & 0,241 & 0,0354 & 0,245 & 0,0369 & 34,2 & & $4^{\diamond}$ \\
\hline $\pm 0,006$ & $\pm 0,0013$ & $\pm 0,005$ & $\pm 0,0007$ & $\pm 0,005$ & $\pm 0,0008$ & $\pm 0,8$ & & \\
\hline 0,324 & 0,0725 & 0,214 & 0,032 & 0,227 & 0,0353 & 33,4 & & $2^{\text {मे }}$ \\
\hline $\pm 0,007$ & $\pm 0,0015$ & $\pm 0,005$ & $\pm 0,001$ & $\pm 0,004$ & $\pm 0,001$ & \pm 1 & & \\
\hline 0,381 & 0,0838 & 0,262 & 0,0366 & 0,267 & 0,0409 & 33,6 & & $2^{\hat{\psi}}$ \\
\hline $\pm 0,007$ & $\pm 0,0016$ & $\pm 0,005$ & $\pm 0,0011$ & $\pm 0,004$ & $\pm 0,0011$ & \pm 1 & & \\
\hline 0,377 & 0,081 & 0,242 & 0,0358 & 0,245 & 0,0368 & 35,3 & & $2^{\text {म }}$ \\
\hline $\pm 0,007$ & $\pm 0,0017$ & $\pm 0,005$ & $\pm 0,0011$ & $\pm 0,004$ & $\pm 0,0012$ & $\pm 1,1$ & & \\
\hline 0,365 & 0,0819 & 0,243 & 0,0334 & 0,242 & 0,0361 & 34,3 & & $2^{\text {मे }}$ \\
\hline $\pm 0,007$ & $\pm 0,0016$ & $\pm 0,005$ & $\pm 0,001$ & $\pm 0,004$ & $\pm 0,0011$ & $\pm 1,1$ & & \\
\hline 0,401 & 0,0864 & 0,26 & 0,0348 & 0,255 & 0,0391 & 35 & & $2^{\hat{h}}$ \\
\hline $\pm 0,007$ & $\pm 0,0017$ & $\pm 0,005$ & $\pm 0,001$ & $\pm 0,004$ & $\pm 0,0011$ & $\pm 1,2$ & & \\
\hline 0,26 & 0,0565 & 0,18 & 0,0252 & 0,183 & 0,0266 & 35,9 & & $2^{\hat{h}}$ \\
\hline $\pm 0,006$ & $\pm 0,0013$ & $\pm 0,004$ & $\pm 0,0009$ & $\pm 0,004$ & $\pm 0,0009$ & $\pm 1,1$ & & \\
\hline 0,454 & 0,1 & 0,291 & 0,043 & 0,297 & 0,0451 & 34,4 & & $6^{\square \diamond}$ \\
\hline $\pm 0,005$ & $\pm 0,001$ & $\pm 0,004$ & $\pm 0,0006$ & $\pm 0,004$ & $\pm 0,0007$ & $\pm 0,8$ & & \\
\hline 0,334 & 0,0749 & 0,227 & 0,0327 & 0,233 & 0,0369 & 36,7 & & $6^{\square \diamond}$ \\
\hline $\pm 0,004$ & $\pm 0,0008$ & $\pm 0,003$ & $\pm 0,0005$ & $\pm 0,003$ & $\pm 0,0006$ & $\pm 0,8$ & & \\
\hline 0,386 & 0,0858 & 0,256 & 0,0364 & 0,259 & 0,0396 & 36,2 & 19,8 & $4^{\text {मे }}$ \\
\hline $\pm 0,003$ & $\pm 0,0006$ & $\pm 0,003$ & $\pm 0,0005$ & $\pm 0,002$ & $\pm 0,0005$ & $\pm 0,8$ & $\pm 0,6$ & \\
\hline 0,468 & 0,104 & 0,312 & 0,0449 & 0,306 & 0,0488 & 33,7 & & $4^{\diamond}$ \\
\hline $\pm 0,007$ & $\pm 0,002$ & $\pm 0,006$ & $\pm 0,0009$ & $\pm 0,006$ & $\pm 0,001$ & $\pm 0,8$ & & \\
\hline 0,34 & 0,0757 & 0,23 & 0,0324 & 0,234 & 0,034 & 38,8 & & $6^{\square \diamond}$ \\
\hline $\pm 0,004$ & $\pm 0,0009$ & $\pm 0,003$ & $\pm 0,0005$ & $\pm 0,003$ & $\pm 0,0006$ & $\pm 0,9$ & & \\
\hline 0,413 & 0,0906 & 0,271 & 0,0397 & 0,273 & 0,0424 & 35,3 & & $4^{\diamond}$ \\
\hline $\pm 0,006$ & $\pm 0,0014$ & $\pm 0,005$ & $\pm 0,0008$ & $\pm 0,005$ & $\pm 0,0009$ & $\pm 0,9$ & & \\
\hline 0,417 & 0,0927 & 0,275 & 0,0405 & 0,277 & 0,0425 & 33,3 & & $6^{\square \diamond}$ \\
\hline $\pm 0,005$ & $\pm 0,001$ & $\pm 0,003$ & $\pm 0,0006$ & $\pm 0,004$ & $\pm 0,0006$ & $\pm 0,8$ & & \\
\hline 0,359 & 0,0815 & 0,244 & 0,0357 & 0,247 & 0,0383 & 33,5 & & 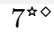 \\
\hline $\pm 0,004$ & $\pm 0,0009$ & $\pm 0,003$ & $\pm 0,0005$ & $\pm 0,003$ & $\pm 0,0006$ & $\pm 0,6$ & & \\
\hline 0,405 & 0,0897 & 0,268 & 0,0382 & 0,274 & 0,0408 & 33,6 & 20,5 & $4^{\text {मे }}$ \\
\hline $\pm 0,004$ & $\pm 0,0009$ & $\pm 0,003$ & $\pm 0,0006$ & $\pm 0,003$ & $\pm 0,0006$ & $\pm 0,6$ & $\pm 0,4$ & \\
\hline 0,296 & 0,0664 & 0,195 & 0,0285 & 0,197 & 0,0295 & 32,6 & 21,6 & $2^{\hat{\psi h}}$ \\
\hline $\pm 0,004$ & $\pm 0,0009$ & $\pm 0,003$ & $\pm 0,0007$ & $\pm 0,003$ & $\pm 0,0007$ & $\pm 0,7$ & $\pm 0,8$ & \\
\hline 0,256 & 0,0599 & 0,185 & 0,0281 & 0,196 & 0,0332 & 28,7 & 16,7 & $2^{\star \Downarrow}$ \\
\hline $\pm 0,004$ & $\pm 0,001$ & $\pm 0,003$ & $\pm 0,0006$ & $\pm 0,003$ & $\pm 0,0008$ & $\pm 0,5$ & $\pm 0,6$ & \\
\hline 0,309 & 0,0699 & 0,208 & 0,0302 & 0,215 & 0,0331 & 34,2 & & $4^{\text {म力口 }}$ \\
\hline $\pm 0,003$ & $\pm 0,0006$ & $\pm 0,002$ & $\pm 0,0004$ & $\pm 0,002$ & $\pm 0,0004$ & $\pm 0,9$ & & \\
\hline 0,302 & 0,0672 & 0,212 & 0,0306 & 0,219 & 0,034 & 31,8 & & $6^{\square \diamond}$ \\
\hline $\pm 0,004$ & $\pm 0,0008$ & $\pm 0,003$ & $\pm 0,0005$ & $\pm 0,003$ & $\pm 0,0005$ & $\pm 0,8$ & & \\
\hline 0,28 & 0,0634 & 0,183 & 0,0271 & 0,188 & 0,0288 & 31,9 & & $4^{*}$ \\
\hline $\pm 0,004$ & $\pm 0,0008$ & $\pm 0,003$ & $\pm 0,0005$ & $\pm 0,002$ & $\pm 0,0005$ & $\pm 0,6$ & & \\
\hline 0,263 & 0,0582 & 0,176 & 0,0254 & 0,173 & 0,0268 & 32,7 & & $4^{\text {मे }}$ \\
\hline $\pm 0,003$ & $\pm 0,0008$ & $\pm 0,003$ & $\pm 0,0005$ & $\pm 0,002$ & $\pm 0,0005$ & $\pm 0,6$ & & \\
\hline 0,27 & 0,0579 & 0,178 & 0,0272 & 0,18 & 0,0274 & 36,3 & 19 & $2^{\hat{\lambda}}$ \\
\hline $\pm 0,004$ & $\pm 0,0009$ & $\pm 0,003$ & $\pm 0,0007$ & $\pm 0,003$ & $\pm 0,0007$ & $\pm 0,8$ & $\pm 0,7$ & \\
\hline 0,298 & 0,067 & 0,198 & 0,0298 & 0,208 & 0,0312 & 34,5 & & $4^{\star}$ \\
\hline $\pm 0,005$ & $\pm 0,001$ & $\pm 0,003$ & $\pm 0,0008$ & $\pm 0,003$ & $\pm 0,0007$ & $\pm 0,8$ & & \\
\hline 0,153 & 0,0381 & 0,128 & 0,0207 & 0,153 & 0,0254 & 36,6 & & $4^{\diamond}$ \\
\hline $\pm 0,003$ & $\pm 0,0007$ & $\pm 0,003$ & $\pm 0,0005$ & $\pm 0,003$ & $\pm 0,0006$ & $\pm 1,1$ & & \\
\hline 2,81 & 0,604 & 1,8 & 0,253 & 1,82 & 0,274 & 34,7 & & $4^{\diamond}$ \\
\hline $\pm 0,04$ & $\pm 0,009$ & $\pm 0,03$ & $\pm 0,004$ & $\pm 0,03$ & $\pm 0,005$ & $\pm 0,7$ & & \\
\hline 3,21 & 0,705 & 2,07 & 0,29 & 2,06 & 0,302 & 34,3 & 18,3 & $2^{\text {औ }}$ \\
\hline $\pm 0,02$ & $\pm 0,004$ & $\pm 0,02$ & $\pm 0,003$ & $\pm 0,01$ & $\pm 0,003$ & $\pm 0,3$ & $\pm 0,2$ & \\
\hline
\end{tabular}


Tabelle A.4 (Fortsetzung)

\begin{tabular}{|c|c|c|c|c|c|c|c|c|}
\hline & $\mathrm{Y}$ & $\mathrm{La}$ & $\mathrm{Pr}$ & $\mathrm{Nd}$ & $\mathrm{Sm}$ & $\mathrm{Eu}$ & Gd & $\mathrm{Tb}$ \\
\hline Dhofar 182 & 15,8 & 2,91 & 1,06 & 5,44 & 1,79 & 0,644 & 2,38 & 0,426 \\
\hline$(\mathrm{EUC})$ & $\pm 0,1$ & $\pm 0,01$ & $\pm 0,01$ & $\pm 0,03$ & $\pm 0,02$ & $\pm 0,004$ & $\pm 0,02$ & $\pm 0,003$ \\
\hline \multirow[t]{2}{*}{ Juvinas (EUC) } & 13,1 & 2,12 & 0,785 & 4,04 & 1,34 & 0,565 & 1,83 & 0,338 \\
\hline & $\pm 0,1$ & $\pm 0,01$ & $\pm 0,004$ & $\pm 0,02$ & $\pm 0,02$ & $\pm 0,004$ & $\pm 0,02$ & $\pm 0,003$ \\
\hline Millbillillie-A & 11,1 & 1,8 & 0,671 & 3,42 & 1,15 & 0,528 & 1,53 & 0,283 \\
\hline$(\mathrm{EUC})$ & $\pm 0,1$ & $\pm 0,01$ & $\pm 0,004$ & $\pm 0,02$ & $\pm 0,02$ & $\pm 0,004$ & $\pm 0,02$ & $\pm 0,003$ \\
\hline Millbillillie-B & 12,6 & 2,79 & 0,99 & 4,95 & 1,53 & 0,545 & 1,9 & 0,34 \\
\hline (EUC) & $\pm 0,1$ & $\pm 0,01$ & $\pm 0,004$ & $\pm 0,03$ & $\pm 0,02$ & $\pm 0,004$ & $\pm 0,02$ & $\pm 0,003$ \\
\hline NWA 3359 & 16,2 & 2,9 & 1,06 & 5,38 & 1,82 & 0,676 & 2,39 & 0,443 \\
\hline (EUC) & $\pm 0,1$ & $\pm 0,01$ & $\pm 0,01$ & $\pm 0,03$ & $\pm 0,02$ & $\pm 0,004$ & $\pm 0,02$ & $\pm 0,003$ \\
\hline Padvarninkai & 11,9 & 1,94 & 0,681 & 3,5 & 1,18 & 0,647 & 1,55 & 0,296 \\
\hline (EUC) & $\pm 0,1$ & $\pm 0,01$ & $\pm 0,004$ & $\pm 0,02$ & $\pm 0,02$ & $\pm 0,005$ & $\pm 0,02$ & $\pm 0,003$ \\
\hline NWA 1457 & 2,31 & 0,906 & 0,262 & 1,2 & 0,315 & 0,124 & 0,375 & 0,0639 \\
\hline$(\mathrm{WIN})$ & $\pm 0,01$ & $\pm 0,005$ & $\pm 0,002$ & $\pm 0,01$ & $\pm 0,005$ & $\pm 0,002$ & $\pm 0,006$ & $\pm 0,0009$ \\
\hline Mount Egerton & 0,365 & 0,445 & 0,126 & 0,468 & 0,0916 & 0,0206 & 0,0843 & 0,0127 \\
\hline$(\mathrm{AUB})$ & $\pm 0,003$ & $\pm 0,003$ & $\pm 0,002$ & $\pm 0,006$ & $\pm 0,0029$ & $\pm 0,0007$ & $\pm 0,0029$ & $\pm 0,0005$ \\
\hline NWA 4864 & 12,1 & 1,81 & 0,581 & 2,9 & 1,15 & 0,581 & 1,76 & 0,343 \\
\hline (Mars) & $\pm 0,1$ & $\pm 0,01$ & $\pm 0,003$ & $\pm 0,02$ & $\pm 0,01$ & $\pm 0,004$ & $\pm 0,02$ & $\pm 0,003$ \\
\hline SAU 051 & 6,15 & 0,0999 & 0,0461 & 0,344 & 0,295 & 0,169 & 0,698 & 0,147 \\
\hline (Mars) & $\pm 0,02$ & $\pm 0,001$ & $\pm 0,0006$ & $\pm 0,004$ & $\pm 0,005$ & $\pm 0,002$ & $\pm 0,006$ & $\pm 0,002$ \\
\hline Dhofar 911 & 2,35 & 0,749 & 0,231 & 1,1 & 0,308 & 0,777 & 0,367 & 0,0661 \\
\hline (Mond) & $\pm 0,02$ & $\pm 0,005$ & $\pm 0,003$ & $\pm 0,02$ & $\pm 0,008$ & $\pm 0,007$ & $\pm 0,008$ & $\pm 0,0014$ \\
\hline Kalahari 008 & 7,67 & 2,32 & 0,671 & 3,11 & 0,903 & 1,27 & 1,11 & 0,201 \\
\hline (Mond) & $\pm 0,03$ & $\pm 0,01$ & $\pm 0,005$ & $\pm 0,03$ & $\pm 0,013$ & $\pm 0,01$ & $\pm 0,02$ & $\pm 0,003$ \\
\hline Kalahari 009 & 12,8 & 1,2 & 0,325 & 1,64 & 0,643 & 0,483 & 1,08 & 0,235 \\
\hline (Mond) & $\pm 0,1$ & $\pm 0,01$ & $\pm 0,003$ & $\pm 0,02$ & $\pm 0,008$ & $\pm 0,004$ & $\pm 0,01$ & $\pm 0,002$ \\
\hline \multicolumn{9}{|l|}{ Erde } \\
\hline GZG.GSP.13450 & 0,294 & 0,0743 & 0,0302 & 0,144 & 0,0377 & 0,0133 & 0,0439 & 0,00708 \\
\hline (Lherzolith) & $\pm 0,003$ & $\pm 0,0008$ & $\pm 0,0005$ & $\pm 0,003$ & $\pm 0,0013$ & $\pm 0,0004$ & $\pm 0,0014$ & $\pm 0,00022$ \\
\hline De-1 & 4,66 & 2,33 & 1,04 & 5,21 & 1,41 & 0,462 & 1,38 & 0,196 \\
\hline (Peridotit) & $\pm 0,01$ & $\pm 0,01$ & $\pm 0,01$ & $\pm 0,02$ & $\pm 0,01$ & $\pm 0,003$ & $\pm 0,01$ & $\pm 0,002$ \\
\hline De-2 & 1,32 & 2,24 & 0,635 & 2,46 & 0,426 & 0,125 & 0,349 & 0,0458 \\
\hline (Peridotit) & $\pm 0,01$ & $\pm 0,01$ & $\pm 0,003$ & $\pm 0,02$ & $\pm 0,005$ & $\pm 0,002$ & $\pm 0,004$ & $\pm 0,0006$ \\
\hline De-3 & 0,348 & 0,864 & 0,168 & 0,623 & 0,105 & 0,0298 & 0,0849 & 0,0123 \\
\hline (Peridotit) & $\pm 0,003$ & $\pm 0,004$ & $\pm 0,002$ & $\pm 0,005$ & $\pm 0,003$ & $\pm 0,0006$ & $\pm 0,0019$ & $\pm 0,0003$ \\
\hline \multirow[t]{2}{*}{ Zm (Peridotit) } & 1,27 & 0,292 & 0,0625 & 0,311 & 0,0984 & 0,0393 & 0,152 & 0,0295 \\
\hline & $\pm 0,01$ & $\pm 0,002$ & $\pm 0,0006$ & $\pm 0,004$ & $\pm 0,002$ & $\pm 0,0007$ & $\pm 0,003$ & $\pm 0,0005$ \\
\hline $\mathrm{SC}-1$ & 2,06 & 0,154 & 0,0848 & 0,509 & 0,198 & 0,0733 & 0,285 & 0,0524 \\
\hline (Peridotit) & $\pm 0,01$ & $\pm 0,002$ & $\pm 0,0007$ & $\pm 0,005$ & $\pm 0,003$ & $\pm 0,0009$ & $\pm 0,004$ & $\pm 0,0006$ \\
\hline $\mathrm{SC}-2$ & 1,16 & 0,332 & 0,0892 & 0,409 & 0,133 & 0,0475 & 0,177 & 0,0319 \\
\hline (Peridotit) & $\pm 0,01$ & $\pm 0,002$ & $\pm 0,0006$ & $\pm 0,004$ & $\pm 0,002$ & $\pm 0,0006$ & $\pm 0,003$ & $\pm 0,0004$ \\
\hline $\mathrm{Ba}-1$ & 3,02 & 0,0751 & 0,065 & 0,446 & 0,224 & 0,0888 & 0,37 & 0,0716 \\
\hline (Peridotit) & $\pm 0,01$ & $\pm 0,0007$ & $\pm 0,0005$ & $\pm 0,004$ & $\pm 0,003$ & $\pm 0,0008$ & $\pm 0,004$ & $\pm 0,0006$ \\
\hline $\mathrm{Ba}-2$ & 3,21 & 0,063 & 0,0631 & 0,459 & 0,237 & 0,0961 & 0,392 & 0,0762 \\
\hline (Peridotit) & $\pm 0,01$ & $\pm 0,0006$ & $\pm 0,0005$ & $\pm 0,004$ & $\pm 0,003$ & $\pm 0,0008$ & $\pm 0,004$ & $\pm 0,0006$ \\
\hline $\mathrm{Za}-27$ & 2,88 & 0,392 & 0,169 & 0,877 & 0,284 & 0,134 & 0,398 & 0,075 \\
\hline (Peridotit) & $\pm 0,02$ & $\pm 0,004$ & $\pm 0,002$ & $\pm 0,011$ & $\pm 0,007$ & $\pm 0,003$ & $\pm 0,008$ & $\pm 0,0013$ \\
\hline PCC-1 & 0,0978 & 0,0333 & 0,00793 & 0,0306 & 0,00547 & 0,00129 & 0,00735 & 0,00139 \\
\hline (Peridotit) & $\pm 0,001$ & $\pm 0,0004$ & $\pm 0,00017$ & $\pm 0,0009$ & $\pm 0,00043$ & $\pm 0,00011$ & $\pm 0,00048$ & $\pm 0,00008$ \\
\hline JP-1 & 0,109 & 0,0321 & 0,00886 & 0,0366 & 0,00972 & 0,00193 & 0,00993 & 0,00213 \\
\hline (Peridotit) & $\pm 0,001$ & $\pm 0,0004$ & $\pm 0,00019$ & $\pm 0,001$ & $\pm 0,00053$ & $\pm 0,00013$ & $\pm 0,00059$ & $\pm 0,0001$ \\
\hline \multirow[t]{2}{*}{ DTS-1 (Dunit) } & 0,0535 & 0,0373 & 0,00916 & 0,0335 & 0,00588 & 0,00087 & 0,00671 & 0,00139 \\
\hline & $\pm 0,0009$ & $\pm 0,0006$ & $\pm 0,00024$ & $\pm 0,0011$ & $\pm 0,0006$ & $\pm 0,00013$ & $\pm 0,00058$ & $\pm 0,0001$ \\
\hline NIM-D & 0,211 & 0,0715 & 0,0178 & 0,0721 & 0,0166 & 0,00449 & 0,0211 & 0,00369 \\
\hline (Dunit) & $\pm 0,002$ & $\pm 0,0007$ & $\pm 0,0003$ & $\pm 0,0014$ & $\pm 0,0008$ & $\pm 0,00019$ & $\pm 0,0009$ & $\pm 0,00013$ \\
\hline NIM-P & 3,16 & 1,96 & 0,481 & 1,98 & 0,446 & 0,133 & 0,477 & 0,0809 \\
\hline (Pyroxenit) & $\pm 0,01$ & $\pm 0,01$ & $\pm 0,002$ & $\pm 0,01$ & $\pm 0,004$ & $\pm 0,001$ & $\pm 0,004$ & $\pm 0,0007$ \\
\hline UB-N & 3,16 & 0,384 & 0,142 & 0,735 & 0,268 & 0,0955 & 0,398 & 0,0765 \\
\hline (Serpentinit) & $\pm 0,01$ & $\pm 0,002$ & $\pm 0,001$ & $\pm 0,005$ & $\pm 0,003$ & $\pm 0,0009$ & $\pm 0,004$ & $\pm 0,0006$ \\
\hline
\end{tabular}




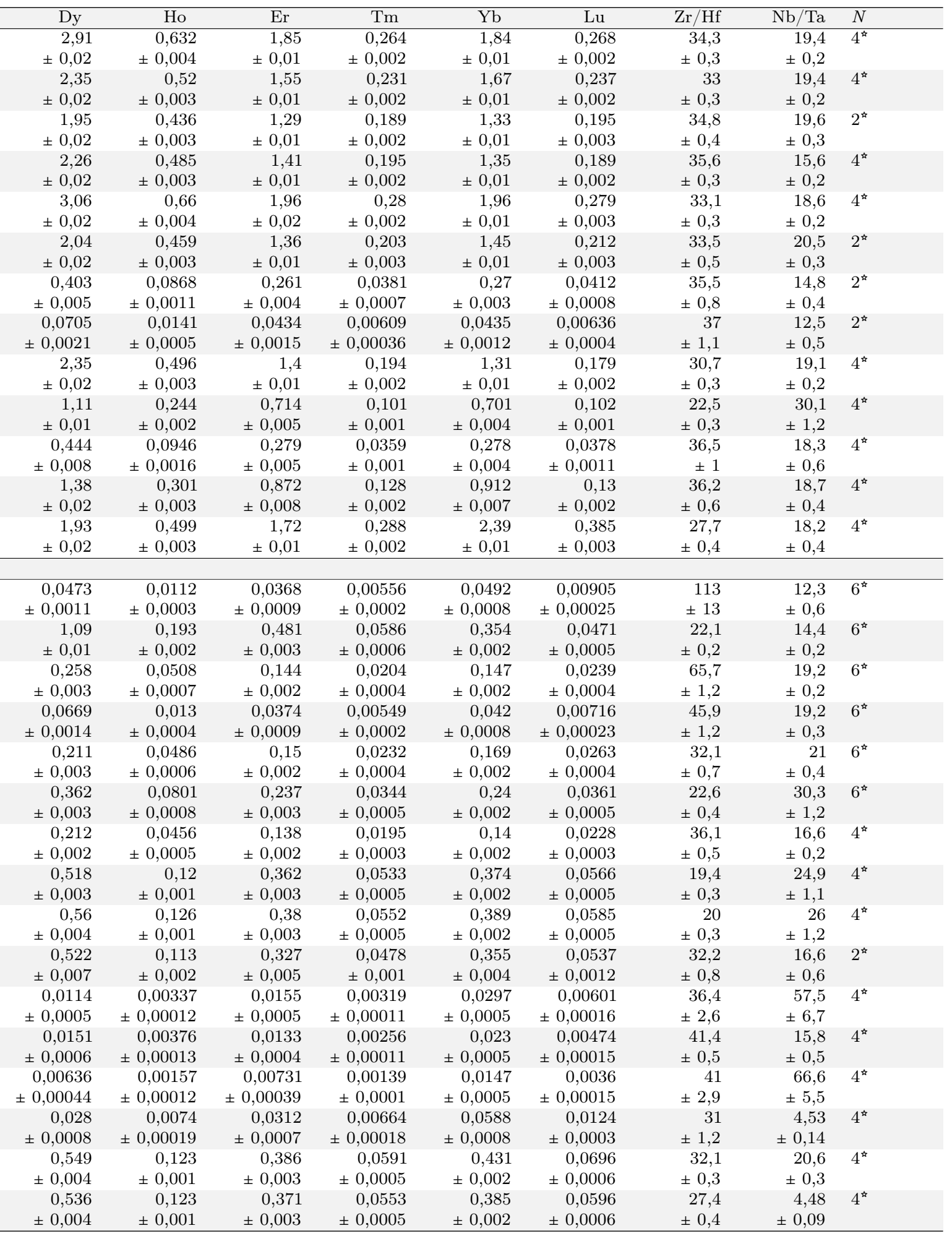


Tabelle A.4 (Fortsetzung)

\begin{tabular}{|c|c|c|c|c|c|c|c|c|}
\hline & $\mathrm{Y}$ & $\mathrm{La}$ & $\mathrm{Pr}$ & $\mathrm{Nd}$ & $\mathrm{Sm}$ & $\mathrm{Eu}$ & $\mathrm{Gd}$ & $\mathrm{Tb}$ \\
\hline El-1 & 1,38 & 0,0328 & 0,014 & 0,0746 & 0,0341 & 0,0119 & 0,11 & 0,0248 \\
\hline (Serpentinit) & $\pm 0,09$ & $\pm 0,0025$ & $\pm 0,0013$ & $\pm 0,0084$ & $\pm 0,0065$ & $\pm 0,0019$ & $\pm 0,01$ & $\pm 0,003$ \\
\hline 24803 & 10,4 & 1,12 & 0,493 & 2,71 & 1,02 & 0,408 & 1,46 & 0,266 \\
\hline (Komatiit) & $\pm 0,1$ & $\pm 0,01$ & $\pm 0,004$ & $\pm 0,03$ & $\pm 0,02$ & $\pm 0,005$ & $\pm 0,02$ & $\pm 0,003$ \\
\hline 26575 & 13,5 & 1,53 & 0,652 & 3,38 & 1,19 & 0,481 & 1,75 & 0,339 \\
\hline (Komatiit) & $\pm 0,1$ & $\pm 0,01$ & $\pm 0,004$ & $\pm 0,02$ & $\pm 0,02$ & $\pm 0,004$ & $\pm 0,02$ & $\pm 0,003$ \\
\hline 26581 & 5,27 & 0,937 & 0,366 & 1,82 & 0,588 & 0,252 & 0,771 & 0,14 \\
\hline (Komatiit) & $\pm 0,02$ & $\pm 0,004$ & $\pm 0,002$ & $\pm 0,02$ & $\pm 0,007$ & $\pm 0,003$ & $\pm 0,008$ & $\pm 0,002$ \\
\hline $\mathrm{Za}-28 \mathrm{a}$ & 3,92 & 0,697 & 0,27 & 1,35 & 0,439 & 0,155 & 0,581 & 0,105 \\
\hline (Komatiit) & $\pm 0,02$ & $\pm 0,005$ & $\pm 0,003$ & $\pm 0,02$ & $\pm 0,008$ & $\pm 0,003$ & $\pm 0,01$ & $\pm 0,002$ \\
\hline Za-29a & 21,2 & 5,93 & 2,09 & 9,9 & 2,86 & 0,908 & 3,31 & 0,584 \\
\hline (Komatiit) & $\pm 0,1$ & $\pm 0,03$ & $\pm 0,01$ & $\pm 0,05$ & $\pm 0,03$ & $\pm 0,008$ & $\pm 0,03$ & $\pm 0,005$ \\
\hline \multirow[t]{2}{*}{ MOL (Tektit) } & 15,2 & 24,5 & 5,63 & 21,4 & 4,29 & 0,909 & 3,36 & 0,5 \\
\hline & $\pm 0,1$ & $\pm 0,1$ & $\pm 0,03$ & $\pm 0,1$ & $\pm 0,04$ & $\pm 0,008$ & $\pm 0,03$ & $\pm 0,005$ \\
\hline \multirow[t]{2}{*}{ KOH (Tektit) } & 27,2 & 38,7 & 8,55 & 32,7 & 6,45 & 1,24 & 5,59 & 0,853 \\
\hline & $\pm 0,1$ & $\pm 0,2$ & $\pm 0,04$ & $\pm 0,2$ & $\pm 0,05$ & $\pm 0,01$ & $\pm 0,04$ & $\pm 0,006$ \\
\hline \multirow[t]{2}{*}{ MUO (Tektit) } & 26 & 36,3 & 8,05 & 30,8 & 6,12 & 1,19 & 5,22 & 0,795 \\
\hline & $\pm 0,1$ & $\pm 0,2$ & $\pm 0,03$ & $\pm 0,2$ & $\pm 0,04$ & $\pm 0,01$ & $\pm 0,04$ & $\pm 0,006$ \\
\hline \multirow[t]{2}{*}{ QUA (Tektit) } & 28,3 & 40,4 & 8,91 & 34 & 6,73 & 1,3 & 5,77 & 0,895 \\
\hline & $\pm 0,1$ & $\pm 0,2$ & $\pm 0,04$ & $\pm 0,2$ & $\pm 0,05$ & $\pm 0,01$ & $\pm 0,04$ & $\pm 0,007$ \\
\hline $\mathrm{El}-2$ & 14,6 & 17 & 4,3 & 17,5 & 3,85 & 0,904 & 3,57 & 0,562 \\
\hline (Radiolarit) & $\pm 0,2$ & $\pm 0,2$ & $\pm 0,04$ & $\pm 0,2$ & $\pm 0,05$ & $\pm 0,011$ & $\pm 0,04$ & $\pm 0,006$ \\
\hline $\mathrm{ES}$ & 54,6 & 73,5 & 17,1 & 64,7 & 11,5 & 2,25 & 10,1 & 1,55 \\
\hline (Tonschiefer) & $\pm 0,7$ & $\pm 0,6$ & $\pm 0,2$ & $\pm 0,6$ & $\pm 0,2$ & $\pm 0,02$ & $\pm 0,2$ & $\pm 0,03$ \\
\hline Pan-02-047 & 63,4 & 12,2 & 4,53 & 22,9 & 7,11 & 1,87 & 8,6 & 1,53 \\
\hline (Granit) & \pm 1 & $\pm 0,3$ & $\pm 0,09$ & $\pm 0,5$ & $\pm 0,13$ & $\pm 0,02$ & $\pm 0,14$ & $\pm 0,03$ \\
\hline GZG.GSP.12569 & 88,2 & 76,8 & 18,7 & 73,6 & 15,9 & 2,77 & 15,1 & 2,51 \\
\hline (Obsidian) & $\pm 0,3$ & $\pm 0,3$ & $\pm 0,1$ & $\pm 0,3$ & $\pm 0,1$ & $\pm 0,02$ & $\pm 0,1$ & $\pm 0,02$ \\
\hline GSP-2 & 28,8 & 216 & 62,3 & 238 & 30,3 & 2,59 & 14,2 & 1,45 \\
\hline (Granodiorit) & $\pm 0,2$ & \pm 1 & $\pm 0,2$ & \pm 1 & $\pm 0,2$ & $\pm 0,02$ & $\pm 0,1$ & $\pm 0,01$ \\
\hline RGM-2 & 24,8 & 26,3 & 5,79 & 21,7 & 4,53 & 0,704 & 4,09 & 0,668 \\
\hline (Rhyolith) & $\pm 0,1$ & $\pm 0,1$ & $\pm 0,03$ & $\pm 0,1$ & $\pm 0,04$ & $\pm 0,006$ & $\pm 0,03$ & $\pm 0,005$ \\
\hline \multirow[t]{2}{*}{ El-3 (Gabbro) } & 1,82 & 0,0986 & 0,0202 & 0,116 & 0,0606 & 0,0302 & 0,161 & 0,0346 \\
\hline & $\pm 0,02$ & $\pm 0,0016$ & $\pm 0,0007$ & $\pm 0,004$ & $\pm 0,0031$ & $\pm 0,0011$ & $\pm 0,005$ & $\pm 0,001$ \\
\hline \multirow[t]{2}{*}{ LP1 (OIB) } & 33,4 & 85,8 & 19,3 & 76,3 & 13,8 & 4,1 & 10,7 & 1,42 \\
\hline & $\pm 0,1$ & $\pm 0,2$ & $\pm 0,1$ & $\pm 0,2$ & $\pm 0,1$ & $\pm 0,02$ & $\pm 0,1$ & $\pm 0,01$ \\
\hline \multirow[t]{2}{*}{ LP4 (OIB) } & 31 & 91,6 & 19,2 & 73,9 & 13,2 & 3,96 & 10,2 & 1,34 \\
\hline & $\pm 0,1$ & $\pm 0,2$ & $\pm 0,1$ & $\pm 0,2$ & $\pm 0,1$ & $\pm 0,02$ & $\pm 0,1$ & $\pm 0,01$ \\
\hline \multirow[t]{2}{*}{ SC-3 (Basalt) } & 25,5 & 40,2 & 8,72 & 35,3 & 7,14 & 2,33 & 6,32 & 0,918 \\
\hline & $\pm 0,1$ & $\pm 0,2$ & $\pm 0,03$ & $\pm 0,2$ & $\pm 0,05$ & $\pm 0,02$ & $\pm 0,04$ & $\pm 0,006$ \\
\hline 138 DS-1 & 21,8 & 14 & 3,85 & 17,2 & 4,24 & 1,49 & 4,36 & 0,703 \\
\hline (MORB) & $\pm 0,1$ & $\pm 0,1$ & $\pm 0,02$ & $\pm 0,1$ & $\pm 0,03$ & $\pm 0,01$ & $\pm 0,03$ & $\pm 0,005$ \\
\hline 155 DS-1 & 17,6 & 2,25 & 0,945 & 5,18 & 1,91 & 0,824 & 2,61 & 0,48 \\
\hline (MORB) & $\pm 0,1$ & $\pm 0,01$ & $\pm 0,005$ & $\pm 0,03$ & $\pm 0,02$ & $\pm 0,006$ & $\pm 0,02$ & $\pm 0,004$ \\
\hline 199 DS-2 & 21,4 & 1,75 & 0,911 & 5,35 & 2,07 & 0,817 & 2,94 & 0,546 \\
\hline (MORB) & $\pm 0,1$ & $\pm 0,01$ & $\pm 0,005$ & $\pm 0,03$ & $\pm 0,02$ & $\pm 0,006$ & $\pm 0,03$ & $\pm 0,004$ \\
\hline 157 DS-3 & 18,6 & 4,74 & 1,77 & 9,08 & 2,89 & 1,11 & 3,37 & 0,564 \\
\hline (MORB) & $\pm 0,1$ & $\pm 0,02$ & $\pm 0,01$ & $\pm 0,04$ & $\pm 0,03$ & $\pm 0,01$ & $\pm 0,03$ & $\pm 0,004$ \\
\hline 137 DS-4 & 23,5 & 2,4 & 1,08 & 6,04 & 2,35 & 0,911 & 3,27 & 0,609 \\
\hline (MORB) & $\pm 0,1$ & $\pm 0,01$ & $\pm 0,01$ & $\pm 0,03$ & $\pm 0,02$ & $\pm 0,006$ & $\pm 0,03$ & $\pm 0,004$ \\
\hline \multirow[t]{2}{*}{ BIR-1 (Basalt) } & 13,9 & 0,614 & 0,358 & 2,37 & 1,12 & 0,526 & 1,83 & 0,348 \\
\hline & $\pm 0,1$ & $\pm 0,006$ & $\pm 0,004$ & $\pm 0,03$ & $\pm 0,02$ & $\pm 0,006$ & $\pm 0,02$ & $\pm 0,004$ \\
\hline BHVO-2 & 23,4 & 15,3 & 5,17 & 24,2 & 6,01 & 2,05 & 5,9 & 0,883 \\
\hline (Basalt) & $\pm 0,1$ & $\pm 0,1$ & $\pm 0,02$ & $\pm 0,1$ & $\pm 0,04$ & $\pm 0,02$ & $\pm 0,04$ & $\pm 0,006$ \\
\hline \multirow[t]{2}{*}{ W-2a (Diabas) } & 19,7 & 10,9 & 3,03 & 13,4 & 3,39 & 1,13 & 3,6 & 0,597 \\
\hline & $\pm 0,1$ & $\pm 0,1$ & $\pm 0,02$ & $\pm 0,1$ & $\pm 0,03$ & $\pm 0,01$ & $\pm 0,03$ & $\pm 0,005$ \\
\hline AGV-2 & 18 & 39 & 8,18 & 31 & 5,64 & 1,59 & 4,53 & 0,624 \\
\hline (Andesit) & $\pm 0,1$ & $\pm 0,2$ & $\pm 0,03$ & $\pm 0,2$ & $\pm 0,04$ & $\pm 0,01$ & $\pm 0,04$ & $\pm 0,005$ \\
\hline BCR-2G & 32,3 & 24,7 & 6,54 & 28,1 & 6,47 & 1,94 & 6,38 & 0,991 \\
\hline (Basalt) & $\pm 0,1$ & $\pm 0,1$ & $\pm 0,01$ & $\pm 0,1$ & $\pm 0,02$ & $\pm 0,01$ & $\pm 0,02$ & $\pm 0,003$ \\
\hline
\end{tabular}




\begin{tabular}{|c|c|c|c|c|c|c|c|c|}
\hline Dy & Ho & Er & Tm & $\mathrm{Yb}$ & $\mathrm{Lu}$ & $\mathrm{Zr} / \mathrm{Hf}$ & $\mathrm{Nb} / \mathrm{Ta}$ & $N$ \\
\hline 0,181 & 0,0512 & 0,173 & 0,0298 & 0,226 & 0,0364 & 20,6 & & $2^{4}$ \\
\hline $\pm 0,023$ & $\pm 0,0047$ & $\pm 0,017$ & $\pm 0,0028$ & $\pm 0,013$ & $\pm 0,0032$ & $\pm 6,2$ & & \\
\hline 1,85 & 0,407 & 1,2 & 0,17 & 1,19 & 0,175 & 31,1 & 15,7 & $2^{\text {म }}$ \\
\hline 2,39 & 0,529 & 1,57 & 0,221 & 1,51 & 0,216 & 33,3 & 15,3 & $4^{\star}$ \\
\hline $\pm 0,02$ & $\pm 0,003$ & $\pm 0,01$ & $\pm 0,002$ & $\pm 0,01$ & $\pm 0,002$ & $\pm 0,3$ & $\pm 0,2$ & \\
\hline 0,949 & 0,207 & 0,618 & 0,088 & 0,607 & 0,0889 & 33,2 & 14 & $4^{\hbar}$ \\
\hline $\pm 0,007$ & $\pm 0,002$ & $\pm 0,005$ & $\pm 0,001$ & $\pm 0,004$ & $\pm 0,001$ & $\pm 0,4$ & $\pm 0,2$ & \\
\hline 0,739 & 0,157 & 0,461 & 0,065 & 0,471 & 0,0678 & 33,6 & 14,8 & $2^{\star \pi}$ \\
\hline $\pm 0,008$ & $\pm 0,002$ & $\pm 0,006$ & $\pm 0,0012$ & $\pm 0,005$ & $\pm 0,0012$ & $\pm 0,7$ & $\pm 0,4$ & \\
\hline 3,89 & 0,846 & 2,45 & 0,348 & 2,43 & 0,35 & 35,6 & 15,3 & $2^{\hbar}$ \\
\hline $\pm 0,03$ & $\pm 0,006$ & $\pm 0,02$ & $\pm 0,004$ & $\pm 0,02$ & $\pm 0,004$ & $\pm 0,4$ & $\pm 0,2$ & \\
\hline 2,94 & 0,577 & 1,62 & 0,225 & 1,58 & 0,229 & 34,8 & 11,4 & $2^{\text {多 }}$ \\
\hline $\pm 0,02$ & $\pm 0,005$ & $\pm 0,02$ & $\pm 0,003$ & $\pm 0,01$ & $\pm 0,003$ & $\pm 0,3$ & $\pm 0,1$ & \\
\hline 5,18 & 1,05 & 3,03 & 0,427 & 2,97 & 0,427 & 35,6 & 12,4 & $2^{\hbar}$ \\
\hline $\pm 0,04$ & $\pm 0,01$ & $\pm 0,03$ & $\pm 0,004$ & $\pm 0,02$ & $\pm 0,004$ & $\pm 0,3$ & $\pm 0,1$ & \\
\hline $\pm 0,03$ & $\pm 0,007$ & $\pm 0,02$ & $\pm 0,004$ & $\pm 0,02$ & $\pm 0,004$ & $\pm 0,3$ & $\pm 0,1$ & \\
\hline 5,48 & 1,1 & 3,15 & 0,454 & 3,16 & 0,456 & 35,2 & 12,2 & $2^{\text {म }}$ \\
\hline $\pm 0,04$ & $\pm 0,01$ & $\pm 0,02$ & $\pm 0,004$ & $\pm 0,02$ & $\pm 0,004$ & $\pm 0,3$ & $\pm 0,1$ & \\
\hline 3,36 & 0,665 & 1,89 & 0,258 & 1,73 & 0,255 & 38,2 & 14,5 & $2^{\hbar}$ \\
\hline $\pm 0,04$ & $\pm 0,008$ & $\pm 0,03$ & $\pm 0,004$ & $\pm 0,02$ & $\pm 0,004$ & $\pm 0,6$ & $\pm 0,3$ & \\
\hline 9,46 & 1,92 & 5,58 & 0,785 & 5,39 & 0,81 & 36,7 & 12,5 & $4^{\diamond}$ \\
\hline $\pm 0,13$ & $\pm 0,03$ & $\pm 0,09$ & $\pm 0,012$ & $\pm 0,08$ & $\pm 0,013$ & $\pm 0,8$ & $\pm 0,2$ & \\
\hline 10,6 & 2,39 & 7,3 & 1,08 & 7,84 & 1,19 & 26,4 & 13,1 & $2^{\text {मे }}$ \\
\hline $\pm 0,2$ & $\pm 0,04$ & $\pm 0,12$ & $\pm 0,02$ & $\pm 0,11$ & $\pm 0,03$ & \pm 1 & $\pm 0,5$ & \\
\hline 16,2 & 3,38 & 9,82 & 1,41 & 9,69 & 1,37 & 31 & 16,2 & $4^{\text {मे }}$ \\
\hline $\pm 0,1$ & $\pm 0,02$ & $\pm 0,04$ & $\pm 0,01$ & $\pm 0,03$ & $\pm 0,01$ & $\pm 0,2$ & $\pm 0,1$ & \\
\hline 6,75 & 1,13 & 2,77 & 0,335 & 2,05 & 0,271 & 36,8 & 25,6 & $2^{\hat{\psi}}$ \\
\hline $\pm 0,04$ & $\pm 0,01$ & $\pm 0,02$ & $\pm 0,004$ & $\pm 0,02$ & $\pm 0,004$ & $\pm 0,3$ & $\pm 0,2$ & \\
\hline 4,26 & 0,894 & 2,71 & 0,413 & 3,02 & 0,456 & 35,4 & 8,14 & $2^{\text {से }}$ \\
\hline $\pm 0,03$ & $\pm 0,006$ & $\pm 0,02$ & $\pm 0,004$ & $\pm 0,02$ & $\pm 0,004$ & $\pm 0,3$ & $\pm 0,06$ & \\
\hline 0,281 & 0,0724 & 0,229 & 0,0357 & 0,265 & 0,041 & 6,02 & & $2^{\text {多 }}$ \\
\hline $\pm 0,006$ & $\pm 0,0013$ & $\pm 0,004$ & $\pm 0,001$ & $\pm 0,004$ & $\pm 0,001$ & $\pm 0,36$ & & \\
\hline 7,09 & 1,25 & 3,07 & 0,382 & 2,43 & 0,316 & 42,1 & 17,6 & $4^{\text {th }}$ \\
\hline $\pm 0,03$ & $\pm 0,01$ & $\pm 0,02$ & $\pm 0,003$ & $\pm 0,01$ & $\pm 0,003$ & $\pm 0,2$ & $\pm 0,1$ & \\
\hline 5,26 & 1,01 & 2,69 & 0,365 & 2,43 & 0,344 & 42,9 & 15,8 & $2^{\text {म }}$ \\
\hline $\pm 0,03$ & $\pm 0,01$ & $\pm 0,02$ & $\pm 0,004$ & $\pm 0,02$ & $\pm 0,003$ & $\pm 0,3$ & $\pm 0,1$ & \\
\hline 4,31 & 0,876 & 2,39 & 0,329 & 2,22 & 0,309 & 39 & 16,3 & $4^{\star 2}$ \\
\hline $\pm 0,03$ & $\pm 0,005$ & $\pm 0,02$ & $\pm 0,003$ & $\pm 0,02$ & $\pm 0,003$ & $\pm 0,3$ & $\pm 0,1$ & \\
\hline 3,24 & 0,697 & 2,02 & 0,283 & 1,98 & 0,282 & 32,9 & 16,6 & $4^{\star}$ \\
\hline $\pm 0,02$ & $\pm 0,005$ & $\pm 0,02$ & $\pm 0,003$ & $\pm 0,01$ & $\pm 0,003$ & $\pm 0,3$ & $\pm 0,2$ & \\
\hline 3,79 & 0,835 & 2,44 & 0,347 & 2,44 & 0,35 & 31,7 & 15,7 & $4^{\text {म }}$ \\
\hline $\pm 0,02$ & $\pm 0,005$ & $\pm 0,02$ & $\pm 0,003$ & $\pm 0,02$ & $\pm 0,003$ & $\pm 0,3$ & $\pm 0,3$ & \\
\hline 3,64 & 0,74 & 2,06 & 0,28 & 1,93 & 0,27 & 36,1 & 16,7 & $4^{\star}$ \\
\hline $\pm 0,02$ & $\pm 0,005$ & $\pm 0,02$ & $\pm 0,003$ & $\pm 0,01$ & $\pm 0,003$ & $\pm 0,3$ & $\pm 0,2$ & \\
\hline 4,22 & 0,919 & 2,71 & 0,386 & 2,73 & 0,397 & 30,1 & 16,8 & $4^{\text {और }}$ \\
\hline $\pm 0,02$ & $\pm 0,005$ & $\pm 0,02$ & $\pm 0,003$ & $\pm 0,02$ & $\pm 0,003$ & $\pm 0,3$ & $\pm 0,2$ & \\
\hline 2,5 & 0,56 & 1,67 & 0,233 & 1,69 & 0,247 & 23,8 & 13,8 & $2^{\text {मे }}$ \\
\hline $\pm 0,02$ & $\pm 0,005$ & $\pm 0,02$ & $\pm 0,003$ & $\pm 0,02$ & $\pm 0,003$ & $\pm 0,4$ & $\pm 0,4$ & \\
\hline 5,08 & 0,95 & 2,43 & 0,313 & 1,99 & 0,26 & 36,9 & 15,4 & $2^{\text {मे }}$ \\
\hline $\pm 0,03$ & $\pm 0,006$ & $\pm 0,02$ & $\pm 0,003$ & $\pm 0,02$ & $\pm 0,003$ & $\pm 0,3$ & $\pm 0,1$ & \\
\hline 3,78 & 0,776 & 2,18 & 0,303 & 2,08 & 0,293 & 36 & 15,8 & $2^{\text {से }}$ \\
\hline $\pm 0,03$ & $\pm 0,006$ & $\pm 0,02$ & $\pm 0,003$ & $\pm 0,02$ & $\pm 0,003$ & $\pm 0,3$ & $\pm 0,2$ & \\
\hline 3,48 & 0,673 & 1,8 & 0,246 & 1,7 & 0,247 & 41,5 & 16 & $2^{\text {और }}$ \\
\hline $\pm 0,03$ & $\pm 0,006$ & $\pm 0,02$ & $\pm 0,003$ & $\pm 0,01$ & $\pm 0,003$ & $\pm 0,3$ & $\pm 0,2$ & \\
\hline 6,12 & 1,25 & 3,51 & 0,488 & 3,36 & 0,483 & 36 & 15,6 & $64^{\text {↔ } \square \diamond \Delta}$ \\
\hline $\pm 0,02$ & $\pm 0,01$ & $\pm 0,01$ & $\pm 0,002$ & $\pm 0,01$ & $\pm 0,002$ & $\pm 0,2$ & $\pm 0,1$ & \\
\hline
\end{tabular}




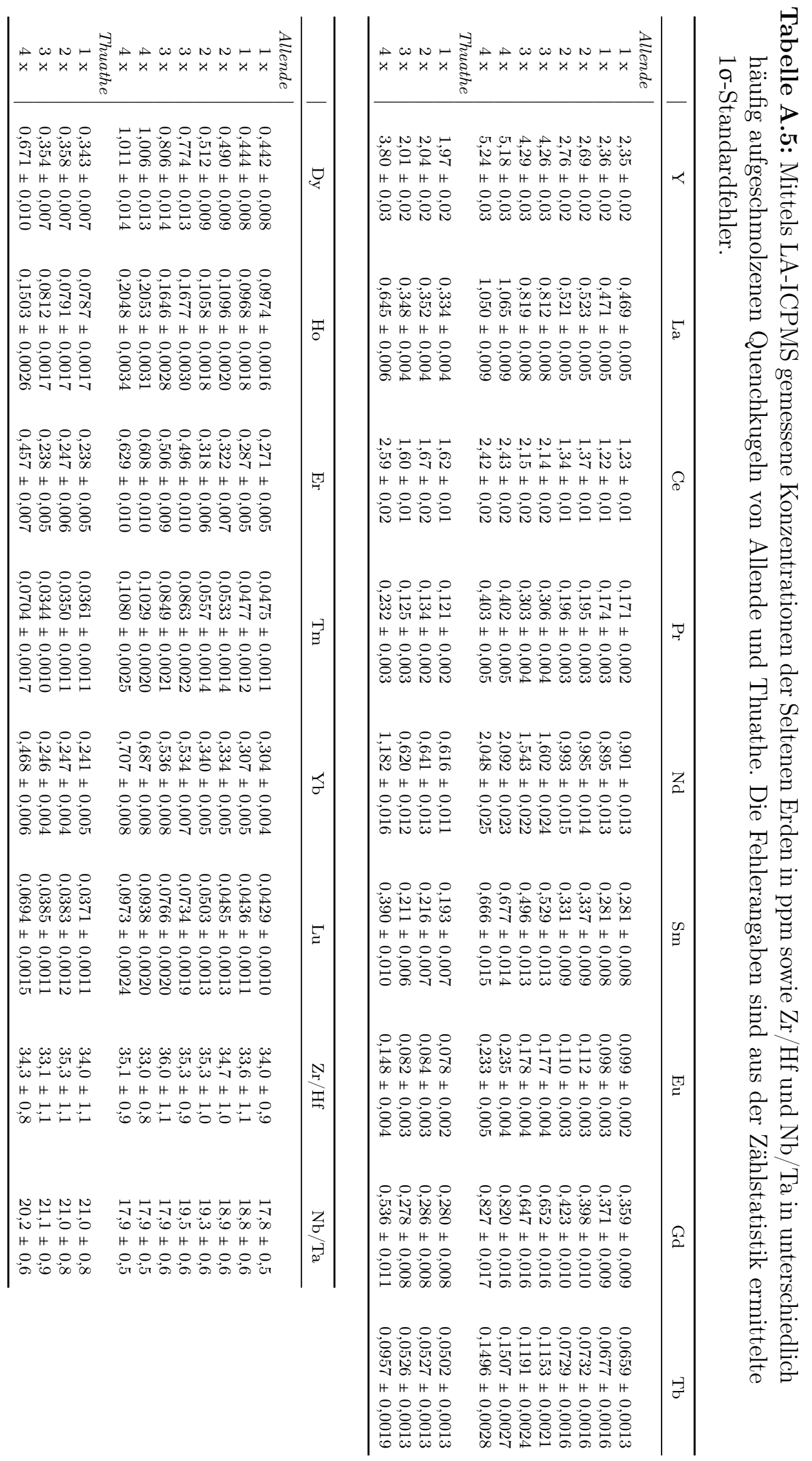


Tabelle A.6: Mittels EMS gemessene Konzentrationen in Gewichtsprozent der Quenchkugeln und der natürlichen Gläser.

\begin{tabular}{|c|c|c|c|c|c|c|}
\hline Probe & Kugel & $\mathrm{SiO}_{2}$ & $\mathrm{Na}_{2} \mathrm{O}$ & $\mathrm{K}_{2} \mathrm{O}$ & $\mathrm{CaO}$ & $\mathrm{FeO}$ \\
\hline \multicolumn{7}{|l|}{ Chondrite } \\
\hline Alais (CI1) & $3-1$ & $36,31 \pm 0,09$ & $0,74 \pm 0,03$ & $0,08 \pm 0,01$ & $1,72 \pm 0,06$ & $35,08 \pm 0,42$ \\
\hline Alais (CI1) & $3-2$ & $36,60 \pm 0,10$ & $0,36 \pm 0,02$ & $0,03 \pm 0,01$ & $1,60 \pm 0,05$ & $34,60 \pm 0,16$ \\
\hline Ivuna (CI1) & $3-3$ & $36,05 \pm 0,11$ & $0,38 \pm 0,03$ & $0,03 \pm 0,01$ & $2,48 \pm 0,07$ & $33,79 \pm 0,26$ \\
\hline Ivuna (CI1) & $3-4$ & $35,33 \pm 0,14$ & $0,39 \pm 0,04$ & $0,02 \pm 0,01$ & $2,36 \pm 0,11$ & $34,54 \pm 0,60$ \\
\hline Ivuna (CI1) & $3-5$ & $35,44 \pm 0,22$ & $0,18 \pm 0,03$ & $0,02 \pm 0,01$ & $2,79 \pm 0,38$ & $35,76 \pm 1,06$ \\
\hline Orgueil-A (CI1) ${ }^{\square}$ & $\mathrm{A}-32$ & $36,07 \pm 0,23$ & $0,16 \pm 0,02$ & u.NG. & $1,93 \pm 0,05$ & $35,37 \pm 0,25$ \\
\hline Orgueil-A (CI1) ${ }^{\square}$ & B-23 & $35,81 \pm 0,32$ & $0,47 \pm 0,07$ & $0,03 \pm 0,01$ & $1,81 \pm 0,07$ & $34,55 \pm 0,52$ \\
\hline Orgueil-A (CI1) ${ }^{\square}$ & B-24 & $35,98 \pm 0,24$ & $0,68 \pm 0,08$ & $0,04 \pm 0,01$ & $1,98 \pm 0,19$ & $35,96 \pm 1,13$ \\
\hline Orgueil-A (CI1) & B-25 & $35,65 \pm 0,18$ & $0,29 \pm 0,01$ & $0,01 \pm 0,01$ & $1,91 \pm 0,03$ & $35,50 \pm 0,45$ \\
\hline Orgueil-A (CI1) ${ }^{\diamond}$ & $\mathrm{C}-32$ & $34,47 \pm 0,87$ & $0,75 \pm 0,04$ & $0,04 \pm 0,01$ & $2,02 \pm 0,14$ & $35,05 \pm 1,48$ \\
\hline Orgueil-A (CI1) ${ }^{\diamond}$ & $\mathrm{C}-33$ & $36,11 \pm 0,59$ & $0,65 \pm 0,04$ & $0,03 \pm 0,01$ & $2,25 \pm 0,16$ & $34,06 \pm 0,53$ \\
\hline Orgueil-A (CI1) & $\mathrm{D}-19$ & $36,28 \pm 0,16$ & $0,42 \pm 0,04$ & $0,04 \pm 0,01$ & $1,91 \pm 0,10$ & $33,50 \pm 0,57$ \\
\hline Orgueil-A (CI1) & D-20 & $35,81 \pm 0,18$ & $0,65 \pm 0,09$ & $0,04 \pm 0,01$ & $2,22 \pm 0,25$ & $35,77 \pm 1,29$ \\
\hline Orgueil-A (CI1) & $\mathrm{D}-21$ & $36,31 \pm 0,23$ & $0,21 \pm 0,02$ & $0,02 \pm 0,01$ & $1,93 \pm 0,05$ & $34,07 \pm 0,32$ \\
\hline Orgueil-A (CI1) & $\mathrm{D}-22$ & $35,29 \pm 0,53$ & $0,92 \pm 0,07$ & $0,06 \pm 0,01$ & $2,38 \pm 0,12$ & $37,42 \pm 1,03$ \\
\hline Orgueil-A (CI1) & D-23 & $35,23 \pm 0,18$ & $0,81 \pm 0,04$ & $0,07 \pm 0,01$ & $2,03 \pm 0,09$ & $35,89 \pm 0,53$ \\
\hline Orgueil-B (CI1) & $3-6$ & $35,27 \pm 0,27$ & $0,91 \pm 0,04$ & $0,06 \pm 0,01$ & $1,60 \pm 0,09$ & $34,74 \pm 0,84$ \\
\hline Orgueil-B (CI1) & $3-7$ & $34,73 \pm 0,30$ & $0,61 \pm 0,10$ & $0,04 \pm 0,01$ & $1,50 \pm 0,20$ & $35,08 \pm 1,06$ \\
\hline Orgueil-B (CI1) & $4-31$ & $36,45 \pm 0,47$ & $0,85 \pm 0,10$ & $0,06 \pm 0,02$ & $1,66 \pm 0,21$ & $31,14 \pm 1,78$ \\
\hline Orgueil-B (CI1) & $4-32$ & $35,79 \pm 0,26$ & $0,27 \pm 0,10$ & $0,02 \pm 0,01$ & $1,51 \pm 0,49$ & $34,03 \pm 2,81$ \\
\hline Orgueil-B (CI1) & $5-39$ & $35,14 \pm 0,40$ & $0,53 \pm 0,03$ & $0,03 \pm 0,01$ & $1,44 \pm 0,10$ & $35,16 \pm 0,64$ \\
\hline Orgueil-B (CI1) & $6-37$ & $35,96 \pm 0,24$ & $0,06 \pm 0,01$ & u.NG. & $1,73 \pm 0,04$ & $34,89 \pm 0,16$ \\
\hline Orgueil-B (CI1) & $7-15$ & $35,99 \pm 0,37$ & $0,48 \pm 0,03$ & $0,03 \pm 0,01$ & $1,72 \pm 0,09$ & $35,12 \pm 0,36$ \\
\hline Orgueil-B (CI1) & $8-40$ & $35,89 \pm 0,48$ & $1,07 \pm 0,17$ & $0,07 \pm 0,02$ & $2,30 \pm 0,31$ & $37,24 \pm 1,97$ \\
\hline Orgueil-C (CI1) & $3-8$ & $35,20 \pm 0,12$ & $0,89 \pm 0,05$ & $0,06 \pm 0,01$ & $1,75 \pm 0,06$ & $35,65 \pm 0,28$ \\
\hline Orgueil-C (CI1) & $3-9$ & $35,07 \pm 0,14$ & $0,74 \pm 0,03$ & $0,04 \pm 0,01$ & $1,78 \pm 0,11$ & $35,88 \pm 0,34$ \\
\hline Orgueil-C (CI1) & $3-10$ & $34,57 \pm 0,50$ & $0,82 \pm 0,03$ & $0,05 \pm 0,01$ & $1,74 \pm 0,06$ & $35,56 \pm 0,77$ \\
\hline Orgueil-D (CI1) & $3-11$ & $35,15 \pm 0,36$ & $0,75 \pm 0,02$ & $0,04 \pm 0,01$ & $1,74 \pm 0,08$ & $34,81 \pm 0,36$ \\
\hline Orgueil-E (CI1) ${ }^{\diamond}$ & $\mathrm{C}-28$ & $36,44 \pm 1,06$ & $0,35 \pm 0,05$ & $0,02 \pm 0,01$ & $1,72 \pm 0,25$ & $31,50 \pm 0,79$ \\
\hline Orgueil-E (CI1) ${ }^{\diamond}$ & $\mathrm{C}-29$ & $35,86 \pm 0,80$ & $0,68 \pm 0,04$ & $0,03 \pm 0,01$ & $1,75 \pm 0,10$ & $31,75 \pm 1,29$ \\
\hline Orgueil-E (CI1) ${ }^{\diamond}$ & $\mathrm{C}-30$ & $34,53 \pm 0,68$ & $0,94 \pm 0,08$ & $0,06 \pm 0,01$ & $1,90 \pm 0,16$ & $33,60 \pm 0,77$ \\
\hline Orgueil-E (CI1) ${ }^{\diamond}$ & $\mathrm{C}-31$ & $34,67 \pm 0,53$ & $0,83 \pm 0,08$ & $0,05 \pm 0,01$ & $1,66 \pm 0,15$ & $32,23 \pm 1,13$ \\
\hline Cold Bokkeveld (CM2) & $3-12$ & $34,16 \pm 0,25$ & $0,62 \pm 0,05$ & $0,16 \pm 0,01$ & $2,12 \pm 0,11$ & $37,34 \pm 1,04$ \\
\hline Cold Bokkeveld (CM2) & $3-13$ & $34,68 \pm 0,40$ & $0,60 \pm 0,03$ & $0,18 \pm 0,02$ & $1,78 \pm 0,07$ & $35,59 \pm 0,50$ \\
\hline Cold Bokkeveld (CM2) & $3-14$ & $34,53 \pm 0,55$ & $0,66 \pm 0,04$ & $0,19 \pm 0,01$ & $1,86 \pm 0,10$ & $35,34 \pm 0,32$ \\
\hline Mighei (CM2) & $3-15$ & $37,54 \pm 0,13$ & $0,24 \pm 0,01$ & $0,02 \pm 0,01$ & $2,48 \pm 0,07$ & $30,63 \pm 0,23$ \\
\hline Mighei (CM2) & $3-16$ & $37,71 \pm 0,19$ & $0,50 \pm 0,01$ & $0,05 \pm 0,01$ & $2,50 \pm 0,05$ & $30,29 \pm 0,20$ \\
\hline Mighei (CM2) & $3-17$ & $37,67 \pm 0,18$ & $0,48 \pm 0,10$ & $0,03 \pm 0,01$ & $4,08 \pm 0,81$ & $35,07 \pm 1,99$ \\
\hline Murchison-A (CM2) & $2-15$ & $38,34 \pm 0,12$ & $0,03 \pm 0,01$ & $0,02 \pm 0,01$ & $3,02 \pm 0,49$ & $28,17 \pm 1,33$ \\
\hline Murchison-A (CM2) & $2-16$ & $37,49 \pm 0,14$ & $0,05 \pm 0,04$ & $0,01 \pm 0,01$ & $2,51 \pm 0,14$ & $28,32 \pm 0,58$ \\
\hline Murchison-B (CM2) & $2-17$ & $36,98 \pm 0,79$ & $0,25 \pm 0,05$ & $0,01 \pm 0,01$ & $3,28 \pm 0,58$ & $30,38 \pm 2,25$ \\
\hline Murchison-B (CM2) & $2-18$ & $37,73 \pm 0,06$ & $0,04 \pm 0,01$ & $0,01 \pm 0,01$ & $3,31 \pm 0,16$ & $27,68 \pm 0,37$ \\
\hline Murchison-C (CM2) & $2-19$ & $37,28 \pm 0,12$ & $0,10 \pm 0,02$ & $0,02 \pm 0,01$ & $2,41 \pm 0,15$ & $29,15 \pm 0,55$ \\
\hline Murchison-D (CM2) ${ }^{\square}$ & $\mathrm{B}-16$ & $36,52 \pm 0,28$ & $0,35 \pm 0,01$ & $0,02 \pm 0,01$ & $2,36 \pm 0,06$ & $32,49 \pm 0,52$ \\
\hline Murchison-D (CM2) ${ }^{\square}$ & $\mathrm{B}-17$ & $36,42 \pm 0,36$ & $0,12 \pm 0,01$ & u.NG. & $2,49 \pm 0,04$ & $32,60 \pm 0,73$ \\
\hline Murchsion-E (CM2) & $3-18$ & $36,96 \pm 0,07$ & $0,69 \pm 0,03$ & $0,05 \pm 0,01$ & $2,11 \pm 0,08$ & $31,40 \pm 0,25$ \\
\hline Murchsion-E (CM2) & $3-19$ & $35,74 \pm 0,20$ & $0,85 \pm 0,08$ & $0,07 \pm 0,01$ & $2,38 \pm 0,17$ & $33,86 \pm 0,91$ \\
\hline Murchsion-E (CM2) & $3-20$ & $36,37 \pm 0,10$ & $0,74 \pm 0,02$ & $0,05 \pm 0,01$ & $2,27 \pm 0,05$ & $31,87 \pm 0,25$ \\
\hline Murray (CM2) & $3-21$ & $35,67 \pm 0,07$ & $0,10 \pm 0,02$ & $0,02 \pm 0,01$ & $2,24 \pm 0,08$ & $33,81 \pm 0,18$ \\
\hline Murray (CM2) & $3-22$ & $35,91 \pm 0,40$ & $0,33 \pm 0,13$ & $0,03 \pm 0,01$ & $2,48 \pm 0,61$ & $34,22 \pm 2,48$ \\
\hline Murray (CM2) & $3-23$ & $35,76 \pm 0,10$ & $0,19 \pm 0,02$ & $0,02 \pm 0,01$ & $2,25 \pm 0,13$ & $33,65 \pm 0,45$ \\
\hline Allende-A $(\mathrm{CV} 3)^{\square}$ & $\mathrm{A}-15$ & $36,52 \pm 0,40$ & $0,09 \pm 0,01$ & u.NG. & $2,79 \pm 0,06$ & $30,08 \pm 0,57$ \\
\hline Allende-A (CV3) ${ }^{\square}$ & $\mathrm{A}-16$ & $37,91 \pm 0,43$ & $0,29 \pm 0,02$ & $0,02 \pm 0,01$ & $2,80 \pm 0,15$ & $28,76 \pm 0,85$ \\
\hline Allende-A $(\mathrm{CV} 3)^{\square}$ & $\mathrm{A}-17$ & $36,16 \pm 0,37$ & $0,31 \pm 0,03$ & $0,02 \pm 0,01$ & $2,83 \pm 0,23$ & $30,18 \pm 0,38$ \\
\hline Allende-A (CV3) & $2-21$ & $36,39 \pm 0,17$ & $0,05 \pm 0,01$ & $0,02 \pm 0,01$ & $3,24 \pm 0,18$ & $27,97 \pm 0,65$ \\
\hline Allende-A (CV3) & $2-22$ & $37,42 \pm 0,15$ & $0,11 \pm 0,02$ & $0,01 \pm 0,01$ & $3,22 \pm 0,26$ & $28,42 \pm 0,81$ \\
\hline Allende-B $(\mathrm{CV} 3)^{\square}$ & $B-20$ & $36,94 \pm 0,23$ & $0,29 \pm 0,03$ & u.NG. & $2,60 \pm 0,08$ & $31,74 \pm 0,61$ \\
\hline Allende-B $(\mathrm{CV} 3)^{\square}$ & $\mathrm{B}-21$ & $36,84 \pm 0,08$ & $0,19 \pm 0,02$ & u.NG. & $2,46 \pm 0,10$ & $31,75 \pm 0,47$ \\
\hline Allende-B (CV3) & $2-23$ & $36,89 \pm 0,15$ & $0,02 \pm 0,01$ & $0,02 \pm 0,01$ & $2,58 \pm 0,20$ & $29,17 \pm 0,55$ \\
\hline Allende-B (CV3) & $2-24$ & $36,73 \pm 0,09$ & $0,04 \pm 0,01$ & $0,01 \pm 0,01$ & $2,75 \pm 0,06$ & $29,69 \pm 0,16$ \\
\hline Allende-C (CV3) & $2-25$ & $36,40 \pm 0,42$ & $0,13 \pm 0,03$ & $0,02 \pm 0,01$ & $2,85 \pm 0,36$ & $27,49 \pm 1,77$ \\
\hline Allende-C (CV3) & $2-26$ & $37,05 \pm 0,12$ & $0,02 \pm 0,01$ & $0,01 \pm 0,01$ & $2,82 \pm 0,13$ & $26,60 \pm 0,39$ \\
\hline
\end{tabular}




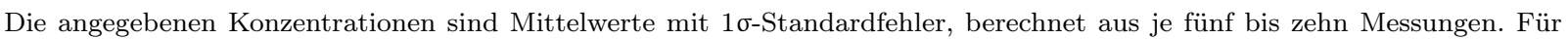
die mit $\square$ und $\diamond$ markierten Proben wurden die Messergebnisse von A. Pack und A. Patzer zur Verfügung gestellt und sind in Patzer et al. (2010) veröffentlicht. Die mit * markierten Quenchkugeln von Allende und Thuathe wurden je ein bis vier Mal aufgeschmolzen und für die Volatilitätsexperimente benutzt. u.NG.: unterhalb der Nachweisgrenze.

\begin{tabular}{|c|c|c|c|c|c|c|c|}
\hline $\mathrm{Al}_{2} \mathrm{O}_{3}$ & $\mathrm{MgO}$ & $\mathrm{P}_{2} \mathrm{O}_{5}$ & $\mathrm{TiO}_{2}$ & $\mathrm{MnO}$ & $\mathrm{SO}_{3}$ & $\mathrm{Cr}_{2} \mathrm{O}_{3}$ & $\mathrm{NiO}$ \\
\hline $2,77 \pm 0,10$ & $23,40 \pm 0,40$ & $0,19 \pm 0,02$ & $0,10 \pm 0,01$ & $0,38 \pm 0,01$ & $0,17 \pm 0,01$ & $0,56 \pm 0,05$ & $0,44 \pm 0,02$ \\
\hline $2,44 \pm 0,05$ & $24,30 \pm 0,23$ & $0,13 \pm 0,02$ & $0,12 \pm 0,01$ & $0,37 \pm 0,02$ & $0,06 \pm 0,01$ & $0,58 \pm 0,04$ & $0,49 \pm 0,02$ \\
\hline $2,62 \pm 0,10$ & $24,61 \pm 0,40$ & $0,24 \pm 0,01$ & $0,14 \pm 0,02$ & $0,43 \pm 0,02$ & $0,20 \pm 0,02$ & $0,53 \pm 0,07$ & $0,32 \pm 0,02$ \\
\hline $2,51 \pm 0,12$ & $24,46 \pm 0,67$ & $0,23 \pm 0,01$ & $0,12 \pm 0,02$ & $0,41 \pm 0,02$ & $0,06 \pm 0,01$ & $0,65 \pm 0,08$ & $0,65 \pm 0,02$ \\
\hline $2,88 \pm 0,39$ & $22,87 \pm 1,69$ & $0,19 \pm 0,02$ & $0,15 \pm 0,02$ & $0,44 \pm 0,02$ & $0,19 \pm 0,03$ & $0,59 \pm 0,05$ & $0,32 \pm 0,02$ \\
\hline $2,59 \pm 0,06$ & $24,66 \pm 0,12$ & $0,13 \pm 0,02$ & $0,12 \pm 0,01$ & $0,37 \pm 0,01$ & $0,01 \pm 0,01$ & $0,45 \pm 0,02$ & $1,23 \pm 0,03$ \\
\hline $2,47 \pm 0,09$ & $26,00 \pm 0,60$ & $0,22 \pm 0,04$ & $0,11 \pm 0,01$ & $0,38 \pm 0,02$ & $0,02 \pm 0,01$ & $0,49 \pm 0,02$ & $1,59 \pm 0,11$ \\
\hline $2,60 \pm 0,19$ & $23,59 \pm 1,49$ & $0,26 \pm 0,03$ & $0,12 \pm 0,01$ & $0,38 \pm 0,03$ & u.NG. & $0,48 \pm 0,03$ & $1,59 \pm 0,13$ \\
\hline $2,59 \pm 0,03$ & $24,67 \pm 0,11$ & $0,16 \pm 0,02$ & $0,12 \pm 0,01$ & $0,36 \pm 0,02$ & $0,01 \pm 0,01$ & $0,50 \pm 0,02$ & $1,42 \pm 0,03$ \\
\hline $2,56 \pm 0,10$ & $21,62 \pm 1,31$ & & $0,12 \pm 0,01$ & $0,40 \pm 0,01$ & & $0,42 \pm 0,03$ & $1,68 \pm 0,09$ \\
\hline $2,78 \pm 0,14$ & $21,33 \pm 1,01$ & & $0,12 \pm 0,01$ & $0,40 \pm 0,02$ & & $0,38 \pm 0,02$ & $1,50 \pm 0,10$ \\
\hline $2,64 \pm 0,12$ & $24,67 \pm 0,84$ & $0,21 \pm 0,01$ & $0,12 \pm 0,01$ & $0,38 \pm 0,01$ & $0,46 \pm 0,12$ & $0,58 \pm 0,03$ & $0,26 \pm 0,02$ \\
\hline $3,00 \pm 0,25$ & $21,96 \pm 2,05$ & $0,24 \pm 0,01$ & $0,13 \pm 0,02$ & $0,41 \pm 0,02$ & $0,69 \pm 0,15$ & $0,56 \pm 0,04$ & $0,34 \pm 0,04$ \\
\hline $2,69 \pm 0,04$ & $24,45 \pm 0,26$ & $0,14 \pm 0,01$ & $0,12 \pm 0,01$ & $0,38 \pm 0,01$ & $0,16 \pm 0,03$ & $0,51 \pm 0,03$ & $0,40 \pm 0,01$ \\
\hline $3,37 \pm 0,14$ & $18,58 \pm 1,00$ & $0,29 \pm 0,02$ & $0,15 \pm 0,01$ & $0,40 \pm 0,01$ & $0,29 \pm 0,19$ & $0,45 \pm 0,07$ & $0,55 \pm 0,08$ \\
\hline $2,74 \pm 0,11$ & $22,26 \pm 0,65$ & $0,30 \pm 0,02$ & $0,12 \pm 0,01$ & $0,40 \pm 0,01$ & $0,31 \pm 0,06$ & $0,55 \pm 0,02$ & $0,67 \pm 0,03$ \\
\hline $2,41 \pm 0,09$ & $24,46 \pm 0,87$ & $0,25 \pm 0,02$ & $0,09 \pm 0,01$ & $0,36 \pm 0,02$ & $0,07 \pm 0,02$ & $0,52 \pm 0,05$ & $1,18 \pm 0,04$ \\
\hline $2,32 \pm 0,30$ & $24,92 \pm 1,40$ & $0,21 \pm 0,03$ & $0,11 \pm 0,02$ & $0,35 \pm 0,02$ & $0,03 \pm 0,01$ & $0,54 \pm 0,04$ & $1,10 \pm 0,07$ \\
\hline $2,26 \pm 0,22$ & $26,94 \pm 2,23$ & $0,25 \pm 0,03$ & $0,10 \pm 0,02$ & $0,36 \pm 0,03$ & $0,02 \pm 0,01$ & $0,57 \pm 0,13$ & $1,49 \pm 0,13$ \\
\hline $2,10 \pm 0,55$ & $26,30 \pm 3,91$ & $0,15 \pm 0,03$ & $0,11 \pm 0,03$ & $0,34 \pm 0,03$ & $0,02 \pm 0,01$ & $0,55 \pm 0,06$ & $0,74 \pm 0,11$ \\
\hline $2,21 \pm 0,12$ & $24,35 \pm 0,53$ & $0,14 \pm 0,02$ & $0,08 \pm 0,02$ & $0,32 \pm 0,01$ & u.NG. & $0,61 \pm 0,07$ & $1,93 \pm 0,04$ \\
\hline $2,52 \pm 0,06$ & $24,04 \pm 0,21$ & $0,10 \pm 0,02$ & $0,12 \pm 0,01$ & $0,36 \pm 0,02$ & u.NG. & $0,42 \pm 0,05$ & $0,81 \pm 0,02$ \\
\hline $2,54 \pm 0,09$ & $24,24 \pm 0,44$ & $0,21 \pm 0,01$ & $0,11 \pm 0,01$ & $0,34 \pm 0,01$ & $0,02 \pm 0,01$ & $0,56 \pm 0,04$ & $0,76 \pm 0,03$ \\
\hline $3,23 \pm 0,38$ & $20,06 \pm 2,28$ & $0,25 \pm 0,03$ & $0,12 \pm 0,02$ & $0,38 \pm 0,02$ & $0,01 \pm 0,01$ & $0,46 \pm 0,06$ & $1,38 \pm 0,16$ \\
\hline $2,66 \pm 0,07$ & $24,07 \pm 0,49$ & $0,36 \pm 0,02$ & $0,13 \pm 0,02$ & $0,36 \pm 0,03$ & $0,35 \pm 0,05$ & $0,59 \pm 0,06$ & $0,44 \pm 0,10$ \\
\hline $2,55 \pm 0,07$ & $23,25 \pm 0,59$ & $0,27 \pm 0,01$ & $0,11 \pm 0,02$ & $0,37 \pm 0,02$ & $0,02 \pm 0,01$ & $0,47 \pm 0,04$ & $1,13 \pm 0,07$ \\
\hline $2,42 \pm 0,03$ & $22,90 \pm 0,30$ & $0,33 \pm 0,02$ & $0,11 \pm 0,02$ & $0,41 \pm 0,01$ & $0,01 \pm 0,01$ & $0,49 \pm 0,04$ & $1,62 \pm 0,02$ \\
\hline $2,38 \pm 0,06$ & $23,59 \pm 0,15$ & $0,21 \pm 0,01$ & $0,12 \pm 0,01$ & $0,36 \pm 0,02$ & $0,01 \pm 0,01$ & $0,55 \pm 0,03$ & $1,83 \pm 0,02$ \\
\hline $2,40 \pm 0,22$ & $25,68 \pm 1,65$ & & $0,10 \pm 0,02$ & $0,38 \pm 0,02$ & & $0,32 \pm 0,04$ & $1,61 \pm 0,19$ \\
\hline $2,44 \pm 0,09$ & $24,82 \pm 0,84$ & & $0,10 \pm 0,01$ & $0,35 \pm 0,01$ & & $0,33 \pm 0,03$ & $1,89 \pm 0,07$ \\
\hline $2,45 \pm 0,14$ & $23,28 \pm 0,95$ & & $0,11 \pm 0,01$ & $0,36 \pm 0,01$ & & $0,46 \pm 0,03$ & $1,80 \pm 0,10$ \\
\hline $2,28 \pm 0,17$ & $25,31 \pm 1,58$ & & $0,11 \pm 0,01$ & $0,35 \pm 0,01$ & & $0,43 \pm 0,02$ & $1,95 \pm 0,13$ \\
\hline $2,64 \pm 0,15$ & $21,44 \pm 0,96$ & $0,25 \pm 0,01$ & $0,14 \pm 0,02$ & $0,31 \pm 0,02$ & u.NG. & $0,49 \pm 0,03$ & $1,90 \pm 0,10$ \\
\hline $2,31 \pm 0,07$ & $23,49 \pm 0,37$ & $0,26 \pm 0,02$ & $0,11 \pm 0,01$ & $0,29 \pm 0,01$ & $0,01 \pm 0,01$ & $0,49 \pm 0,04$ & $2,09 \pm 0,03$ \\
\hline $2,42 \pm 0,07$ & $22,93 \pm 0,35$ & $0,25 \pm 0,02$ & $0,11 \pm 0,01$ & $0,28 \pm 0,02$ & $0,01 \pm 0,01$ & $0,51 \pm 0,06$ & $2,04 \pm 0,04$ \\
\hline $3,03 \pm 0,09$ & $25,83 \pm 0,31$ & $0,19 \pm 0,02$ & $0,13 \pm 0,02$ & $0,28 \pm 0,02$ & $0,46 \pm 0,03$ & $0,58 \pm 0,02$ & $0,11 \pm 0,02$ \\
\hline $3,09 \pm 0,07$ & $25,70 \pm 0,16$ & $0,26 \pm 0,01$ & $0,14 \pm 0,02$ & $0,30 \pm 0,02$ & $0,58 \pm 0,06$ & $0,57 \pm 0,03$ & $0,19 \pm 0,04$ \\
\hline $4,88 \pm 0,89$ & $18,15 \pm 3,94$ & $0,25 \pm 0,04$ & $0,22 \pm 0,05$ & $0,34 \pm 0,03$ & $0,66 \pm 0,16$ & $0,55 \pm 0,04$ & $0,12 \pm 0,02$ \\
\hline $3,72 \pm 0,56$ & $26,50 \pm 2,41$ & $0,04 \pm 0,01$ & $0,18 \pm 0,03$ & $0,27 \pm 0,02$ & $0,24 \pm 0,04$ & $0,55 \pm 0,02$ & $0,06 \pm 0,01$ \\
\hline $3,14 \pm 0,18$ & $28,60 \pm 0,85$ & $0,04 \pm 0,01$ & $0,15 \pm 0,02$ & $0,29 \pm 0,01$ & $0,14 \pm 0,08$ & $0,58 \pm 0,02$ & $0,06 \pm 0,01$ \\
\hline $3,68 \pm 0,54$ & $24,83 \pm 2,86$ & $0,12 \pm 0,02$ & $0,15 \pm 0,03$ & $0,29 \pm 0,02$ & $1,66 \pm 0,45$ & $0,61 \pm 0,05$ & $0,06 \pm 0,02$ \\
\hline $3,72 \pm 0,18$ & $27,79 \pm 0,66$ & $0,04 \pm 0,01$ & $0,15 \pm 0,02$ & $0,28 \pm 0,01$ & $0,08 \pm 0,01$ & $0,56 \pm 0,03$ & $0,04 \pm 0,01$ \\
\hline $3,18 \pm 0,20$ & $27,91 \pm 0,76$ & $0,07 \pm 0,01$ & $0,15 \pm 0,02$ & $0,30 \pm 0,01$ & $0,84 \pm 0,06$ & $0,57 \pm 0,03$ & $0,10 \pm 0,02$ \\
\hline $2,91 \pm 0,07$ & $26,50 \pm 0,32$ & $0,19 \pm 0,02$ & $0,13 \pm 0,01$ & $0,28 \pm 0,02$ & u.NG. & $0,48 \pm 0,03$ & $1,47 \pm 0,02$ \\
\hline $2,92 \pm 0,05$ & $26,16 \pm 0,16$ & $0,14 \pm 0,02$ & $0,13 \pm 0,01$ & $0,31 \pm 0,02$ & $0,02 \pm 0,01$ & $0,40 \pm 0,02$ & $1,10 \pm 0,03$ \\
\hline $2,95 \pm 0,10$ & $25,66 \pm 0,46$ & $0,23 \pm 0,02$ & $0,14 \pm 0,02$ & $0,29 \pm 0,02$ & $0,44 \pm 0,03$ & $0,58 \pm 0,05$ & $0,12 \pm 0,02$ \\
\hline $3,32 \pm 0,25$ & $23,83 \pm 1,23$ & $0,26 \pm 0,03$ & $0,18 \pm 0,02$ & $0,29 \pm 0,03$ & $0,58 \pm 0,09$ & $0,61 \pm 0,06$ & $0,25 \pm 0,06$ \\
\hline $3,14 \pm 0,07$ & $25,50 \pm 0,26$ & $0,23 \pm 0,01$ & $0,17 \pm 0,02$ & $0,27 \pm 0,02$ & $0,39 \pm 0,05$ & $0,54 \pm 0,03$ & $0,26 \pm 0,03$ \\
\hline $2,86 \pm 0,11$ & $25,24 \pm 0,35$ & $0,20 \pm 0,02$ & $0,12 \pm 0,01$ & $0,28 \pm 0,02$ & $0,15 \pm 0,01$ & $0,54 \pm 0,06$ & $0,38 \pm 0,01$ \\
\hline $3,38 \pm 0,95$ & $23,00 \pm 4,10$ & $0,29 \pm 0,06$ & $0,13 \pm 0,04$ & $0,30 \pm 0,03$ & $0,36 \pm 0,09$ & $0,64 \pm 0,07$ & $0,32 \pm 0,03$ \\
\hline $2,89 \pm 0,14$ & $25,12 \pm 0,44$ & $0,27 \pm 0,02$ & $0,13 \pm 0,02$ & $0,29 \pm 0,01$ & $0,19 \pm 0,02$ & $0,55 \pm 0,04$ & $0,47 \pm 0,02$ \\
\hline $3,91 \pm 0,06$ & $27,13 \pm 0,11$ & $0,12 \pm 0,02$ & $0,19 \pm 0,02$ & $0,21 \pm 0,01$ & $0,01 \pm 0,01$ & $0,40 \pm 0,02$ & $1,08 \pm 0,04$ \\
\hline $3,92 \pm 0,18$ & $27,81 \pm 1,05$ & $0,15 \pm 0,02$ & $0,17 \pm 0,01$ & $0,19 \pm 0,02$ & u.NG. & $0,39 \pm 0,03$ & $1,19 \pm 0,06$ \\
\hline $3,85 \pm 0,21$ & $26,50 \pm 1,17$ & $0,19 \pm 0,02$ & $0,18 \pm 0,01$ & $0,21 \pm 0,03$ & $0,02 \pm 0,01$ & $0,43 \pm 0,02$ & $1,28 \pm 0,09$ \\
\hline $4,46 \pm 0,24$ & $28,05 \pm 0,96$ & $0,03 \pm 0,01$ & $0,19 \pm 0,02$ & $0,20 \pm 0,01$ & $0,40 \pm 0,03$ & $0,55 \pm 0,02$ & $0,08 \pm 0,01$ \\
\hline $4,46 \pm 0,36$ & $26,46 \pm 1,38$ & $0,06 \pm 0,01$ & $0,20 \pm 0,02$ & $0,21 \pm 0,01$ & $0,46 \pm 0,04$ & $0,56 \pm 0,02$ & $0,07 \pm 0,01$ \\
\hline $3,09 \pm 0,11$ & $26,73 \pm 0,91$ & $0,31 \pm 0,02$ & $0,14 \pm 0,01$ & $0,21 \pm 0,02$ & $0,03 \pm 0,01$ & $0,48 \pm 0,02$ & $1,40 \pm 0,07$ \\
\hline $2,90 \pm 0,11$ & $26,95 \pm 0,81$ & $0,21 \pm 0,02$ & $0,13 \pm 0,01$ & $0,21 \pm 0,02$ & $0,03 \pm 0,01$ & $0,46 \pm 0,02$ & $1,45 \pm 0,06$ \\
\hline $3,16 \pm 0,28$ & $27,84 \pm 1,01$ & $0,07 \pm 0,01$ & $0,15 \pm 0,01$ & $0,20 \pm 0,01$ & $0,42 \pm 0,05$ & $0,59 \pm 0,03$ & $0,07 \pm 0,01$ \\
\hline $3,25 \pm 0,08$ & $27,44 \pm 0,18$ & $0,07 \pm 0,01$ & $0,15 \pm 0,01$ & $0,21 \pm 0,01$ & $0,60 \pm 0,02$ & $0,57 \pm 0,03$ & $0,08 \pm 0,02$ \\
\hline $3,82 \pm 0,46$ & $29,12 \pm 2,25$ & $0,13 \pm 0,02$ & $0,16 \pm 0,02$ & $0,21 \pm 0,02$ & $0,94 \pm 0,15$ & $0,56 \pm 0,06$ & $0,11 \pm 0,02$ \\
\hline $3,66 \pm 0,21$ & $30,33 \pm 0,69$ & $0,04 \pm 0,01$ & $0,17 \pm 0,02$ & $0,19 \pm 0,01$ & $0,39 \pm 0,03$ & $0,54 \pm 0,04$ & $0,04 \pm 0,01$ \\
\hline
\end{tabular}


Tabelle A.6 (Fortsetzung)

\begin{tabular}{|c|c|c|c|c|c|c|}
\hline Probe & Kugel & $\mathrm{SiO}_{2}$ & $\mathrm{Na}_{2} \mathrm{O}$ & $\mathrm{K}_{2} \mathrm{O}$ & $\mathrm{CaO}$ & $\mathrm{FeO}$ \\
\hline Allende-D (CV3) & $\mathrm{C}-38$ & $36,92 \pm 1,06$ & $0,17 \pm 0,03$ & u.NG. & $2,54 \pm 0,41$ & $28,97 \pm 1,91$ \\
\hline Allende-D (CV3) & $\mathrm{C}-39$ & $36,07 \pm 0,88$ & $0,01 \pm 0,01$ & $0,01 \pm 0,01$ & $2,91 \pm 0,20$ & $28,92 \pm 1,16$ \\
\hline Allende-E (CV3) & $3-24$ & $35,62 \pm 0,23$ & $0,32 \pm 0,02$ & $0,03 \pm 0,01$ & $2,95 \pm 0,16$ & $31,35 \pm 0,40$ \\
\hline Allende-E (CV3) & $3-25$ & $35,15 \pm 0,22$ & $0,35 \pm 0,02$ & $0,03 \pm 0,01$ & $3,20 \pm 0,09$ & $32,59 \pm 0,30$ \\
\hline Allende-E (CV3) & $3-26$ & $35,45 \pm 0,25$ & $0,23 \pm 0,02$ & $0,02 \pm 0,01$ & $2,91 \pm 0,16$ & $31,03 \pm 0,72$ \\
\hline Allende-M (CV3) & $3-27$ & $36,11 \pm 0,71$ & $0,27 \pm 0,04$ & $0,03 \pm 0,01$ & $2,30 \pm 0,23$ & $29,08 \pm 1,32$ \\
\hline Allende-M (CV3) & $3-28$ & $36,79 \pm 0,59$ & $0,32 \pm 0,02$ & $0,02 \pm 0,01$ & $2,51 \pm 0,14$ & $30,19 \pm 1,62$ \\
\hline Allende-S (CV3) *1 & $3-29$ & $36,49 \pm 0,41$ & $0,26 \pm 0,07$ & $0,02 \pm 0,01$ & $2,27 \pm 0,49$ & $29,74 \pm 1,94$ \\
\hline Allende-S (CV3) *2 & $3-30$ & $36,32 \pm 0,28$ & $0,39 \pm 0,05$ & $0,02 \pm 0,01$ & $2,54 \pm 0,26$ & $29,61 \pm 1,20$ \\
\hline Allende-S (CV3) *3 & $3-31$ & $35,99 \pm 0,22$ & $0,47 \pm 0,07$ & $0,03 \pm 0,01$ & $4,02 \pm 0,57$ & $35,67 \pm 2,10$ \\
\hline Allende-S (CV3) *4 & $3-32$ & $34,71 \pm 0,33$ & $0,01 \pm 0,01$ & $0,01 \pm 0,01$ & $5,35 \pm 0,14$ & $16,21 \pm 0,38$ \\
\hline Axtell (CV3) & $\mathrm{C}-3$ & $38,46 \pm 0,55$ & $0,05 \pm 0,02$ & u.NG. & $2,31 \pm 0,17$ & $28,12 \pm 1,16$ \\
\hline Axtell (CV3) $)^{\diamond}$ & $\mathrm{C}-4$ & $37,02 \pm 0,73$ & $0,03 \pm 0,01$ & u.NG. & $2,28 \pm 0,17$ & $30,79 \pm 0,91$ \\
\hline Mokoia $(\mathrm{CV} 3)^{\square}$ & B-8 & $36,23 \pm 0,70$ & $0,28 \pm 0,03$ & $0,01 \pm 0,01$ & $2,54 \pm 0,14$ & $31,67 \pm 0,82$ \\
\hline Mokoia $(\mathrm{CV} 3)^{\square}$ & B-9 & $35,93 \pm 0,31$ & $0,14 \pm 0,01$ & u.NG. & $2,75 \pm 0,04$ & $33,96 \pm 0,48$ \\
\hline Mokoia (CV3) & $2-33$ & $37,38 \pm 0,10$ & $0,40 \pm 0,03$ & $0,03 \pm 0,01$ & $3,04 \pm 0,16$ & $29,65 \pm 0,70$ \\
\hline Mokoia (CV3) & $2-34$ & $35,57 \pm 0,12$ & $0,04 \pm 0,01$ & $0,01 \pm 0,01$ & $3,27 \pm 0,04$ & $29,73 \pm 0,19$ \\
\hline Vigarano (CV3) & $3-33$ & $36,81 \pm 0,25$ & $0,18 \pm 0,02$ & $0,02 \pm 0,01$ & $2,67 \pm 0,18$ & $30,22 \pm 0,49$ \\
\hline Vigarano (CV3) & $3-34$ & $35,94 \pm 0,17$ & $0,11 \pm 0,02$ & $0,02 \pm 0,01$ & $3,07 \pm 0,16$ & $31,78 \pm 0,68$ \\
\hline Vigarano (CV3) & $3-35$ & $35,86 \pm 0,21$ & $0,15 \pm 0,03$ & $0,03 \pm 0,01$ & $3,24 \pm 0,66$ & $31,71 \pm 1,18$ \\
\hline NWA $3003(\mathrm{CO} 3)^{\diamond}$ & $\mathrm{C}-9$ & $35,81 \pm 1,10$ & $0,06 \pm 0,01$ & u.NG. & $2,60 \pm 0,31$ & $33,59 \pm 2,14$ \\
\hline NWA $3003(\mathrm{CO} 3)^{\diamond}$ & $\mathrm{C}-10$ & $36,29 \pm 0,65$ & $0,06 \pm 0,01$ & u.NG. & $2,52 \pm 0,23$ & $32,10 \pm 0,50$ \\
\hline Karoonda-A (CK4) ${ }^{\square}$ & B-14 & $37,69 \pm 0,22$ & $0,25 \pm 0,03$ & $0,01 \pm 0,01$ & $2,79 \pm 0,13$ & $30,61 \pm 0,72$ \\
\hline Karoonda-A (CK4) ${ }^{\square}$ & B-15 & $36,78 \pm 0,25$ & $0,17 \pm 0,02$ & u.NG. & $2,85 \pm 0,13$ & $30,96 \pm 1,08$ \\
\hline Karoonda-A (CK4) & $1-14$ & $36,47 \pm 0,14$ & $0,08 \pm 0,02$ & $0,02 \pm 0,01$ & $2,62 \pm 0,20$ & $29,43 \pm 0,80$ \\
\hline Karoonda-A (CK4) & $1-15$ & $35,90 \pm 0,30$ & $0,11 \pm 0,02$ & $0,01 \pm 0,01$ & $3,05 \pm 0,49$ & $30,56 \pm 1,50$ \\
\hline Karoonda-B (CK4) & $3-36$ & $36,73 \pm 0,65$ & $0,43 \pm 0,03$ & $0,03 \pm 0,01$ & $2,62 \pm 0,12$ & $29,90 \pm 0,88$ \\
\hline Karoonda-B (CK4) & $3-37$ & $35,68 \pm 0,45$ & $0,33 \pm 0,08$ & $0,03 \pm 0,01$ & $2,09 \pm 0,37$ & $29,68 \pm 2,20$ \\
\hline Karoonda-B (CK4) & $3-38$ & $35,84 \pm 0,96$ & $0,52 \pm 0,06$ & $0,03 \pm 0,01$ & $3,37 \pm 0,39$ & $34,06 \pm 1,74$ \\
\hline Bencubbin $(\mathrm{CB})^{\diamond}$ & $\mathrm{C}-17$ & $36,40 \pm 1,94$ & $0,07 \pm 0,01$ & u.NG. & $2,72 \pm 0,50$ & $29,79 \pm 2,59$ \\
\hline Bencubbin $(\mathrm{CB})^{\diamond}$ & $\mathrm{C}-18$ & $36,03 \pm 0,49$ & $0,08 \pm 0,02$ & u.NG. & $2,54 \pm 0,24$ & $31,03 \pm 1,60$ \\
\hline Chainpur-A (LL3) ${ }^{\square}$ & B-3 & $42,39 \pm 0,36$ & $0,51 \pm 0,03$ & $0,04 \pm 0,01$ & $2,05 \pm 0,08$ & $25,72 \pm 0,57$ \\
\hline Chainpur-A (LL3) ${ }^{\square}$ & B-4 & $41,87 \pm 0,36$ & $0,39 \pm 0,08$ & $0,03 \pm 0,01$ & $2,08 \pm 0,03$ & $27,20 \pm 0,32$ \\
\hline Chainpur-A (LL3) ${ }^{\square}$ & $\mathrm{B}-5$ & $41,89 \pm 0,25$ & $0,25 \pm 0,03$ & $0,02 \pm 0,01$ & $2,11 \pm 0,10$ & $27,15 \pm 0,77$ \\
\hline Chainpur-A (LL3) & $1-12$ & $45,43 \pm 0,17$ & $0,35 \pm 0,01$ & $0,03 \pm 0,01$ & $2,27 \pm 0,08$ & $18,22 \pm 0,18$ \\
\hline Chainpur-A (LL3) & $1-13$ & $45,61 \pm 0,19$ & $0,09 \pm 0,01$ & $0,03 \pm 0,01$ & $2,40 \pm 0,09$ & $18,46 \pm 0,21$ \\
\hline Chainpur-B (LL3) & $5-1$ & $43,45 \pm 0,12$ & $0,85 \pm 0,03$ & $0,08 \pm 0,01$ & $2,08 \pm 0,05$ & $24,49 \pm 0,06$ \\
\hline Chainpur-B (LL3) & $5-2$ & $43,80 \pm 0,87$ & $0,86 \pm 0,15$ & $0,09 \pm 0,02$ & $2,44 \pm 0,36$ & $26,90 \pm 1,24$ \\
\hline Dar al Gani $298(\text { LL4) })^{\diamond}$ & $\mathrm{C}-21$ & $42,98 \pm 0,10$ & $0,62 \pm 0,02$ & $0,05 \pm 0,01$ & $2,04 \pm 0,04$ & $22,32 \pm 0,11$ \\
\hline Dar al Gani 298 (LL4) $)^{\diamond}$ & $\mathrm{C}-22$ & $43,48 \pm 0,07$ & $0,06 \pm 0,01$ & $0,01 \pm 0,01$ & $2,07 \pm 0,02$ & $22,25 \pm 0,05$ \\
\hline Dhurmsala (LL6) & $2-3$ & $41,63 \pm 0,12$ & $0,14 \pm 0,01$ & $0,02 \pm 0,01$ & $2,46 \pm 0,10$ & $23,78 \pm 0,29$ \\
\hline Dhurmsala (LL6) & $2-4$ & $41,75 \pm 0,18$ & $0,10 \pm 0,01$ & $0,02 \pm 0,01$ & $2,55 \pm 0,21$ & $24,31 \pm 0,92$ \\
\hline Lake Labyrinth (LL6) ${ }^{口}$ & $\mathrm{~B}-18$ & $42,22 \pm 0,23$ & $0,10 \pm 0,02$ & $0,01 \pm 0,01$ & $2,17 \pm 0,06$ & $25,11 \pm 1,05$ \\
\hline Lake Labyrinth (LL6) ${ }^{\square}$ & B-19 & $42,79 \pm 0,16$ & $0,56 \pm 0,05$ & $0,14 \pm 0,02$ & $2,28 \pm 0,09$ & $25,63 \pm 0,65$ \\
\hline Saint-Séverin (LL6) & $5-3$ & $41,16 \pm 0,34$ & $0,92 \pm 0,08$ & $0,11 \pm 0,02$ & $2,00 \pm 0,13$ & $25,39 \pm 0,43$ \\
\hline Saint-Séverin (LL6) & $5-4$ & $41,62 \pm 0,56$ & $0,88 \pm 0,11$ & $0,11 \pm 0,02$ & $2,03 \pm 0,27$ & $25,42 \pm 0,72$ \\
\hline Bruderheim (L6) & $5-5$ & $35,83 \pm 0,85$ & $0,81 \pm 0,14$ & $0,11 \pm 0,02$ & $1,70 \pm 0,23$ & $34,96 \pm 1,91$ \\
\hline Bruderheim (L6) & $5-6$ & $37,77 \pm 0,58$ & $0,97 \pm 0,13$ & $0,13 \pm 0,02$ & $2,19 \pm 0,33$ & $34,28 \pm 2,19$ \\
\hline Gashua (L6) & $1-34$ & $41,60 \pm 0,28$ & $0,50 \pm 0,07$ & $0,08 \pm 0,01$ & $2,29 \pm 0,25$ & $27,98 \pm 1,04$ \\
\hline Gashua (L6) & $1-35$ & $41,62 \pm 0,44$ & $0,05 \pm 0,01$ & $0,02 \pm 0,01$ & $2,48 \pm 0,30$ & $28,73 \pm 0,94$ \\
\hline Homestead (L5) ${ }^{\diamond}$ & $\mathrm{C}-25$ & $40,94 \pm 0,48$ & $0,54 \pm 0,04$ & $0,03 \pm 0,01$ & $1,98 \pm 0,13$ & $26,33 \pm 1,37$ \\
\hline Homestead (L5) $\diamond$ & $\mathrm{C}-26$ & $41,67 \pm 0,20$ & $0,35 \pm 0,02$ & $0,02 \pm 0,01$ & $2,01 \pm 0,05$ & $25,98 \pm 0,45$ \\
\hline Mount Tazerzait (L5) & $\mathrm{C}-1$ & $38,49 \pm 0,51$ & $0,26 \pm 0,02$ & $0,03 \pm 0,01$ & $1,74 \pm 0,11$ & $29,68 \pm 1,18$ \\
\hline Mount Tazerzait (L5) ${ }^{\diamond}$ & $\mathrm{C}-2$ & $39,32 \pm 0,30$ & $0,02 \pm 0,01$ & $0,01 \pm 0,01$ & $1,87 \pm 0,08$ & $27,69 \pm 0,55$ \\
\hline NWA 869 (L5) & $2-11$ & $40,75 \pm 0,17$ & $0,36 \pm 0,02$ & $0,04 \pm 0,01$ & $2,67 \pm 0,16$ & $26,85 \pm 0,39$ \\
\hline NWA 869 (L5) & $2-12$ & $40,88 \pm 0,34$ & $0,16 \pm 0,03$ & $0,02 \pm 0,01$ & $2,68 \pm 0,31$ & $27,45 \pm 0,77$ \\
\hline NWA 869-A (L5) & $1-1$ & $40,71 \pm 0,26$ & $0,16 \pm 0,03$ & $0,03 \pm 0,01$ & $2,67 \pm 0,18$ & $26,35 \pm 0,82$ \\
\hline NWA 869-B (L5) & $1-2$ & $41,05 \pm 0,20$ & $0,30 \pm 0,02$ & $0,05 \pm 0,01$ & $3,24 \pm 0,24$ & $27,60 \pm 0,56$ \\
\hline NWA 869-C (L5) & $1-3$ & $40,00 \pm 0,18$ & $0,09 \pm 0,02$ & $0,02 \pm 0,01$ & $2,72 \pm 0,29$ & $26,98 \pm 0,81$ \\
\hline NWA 869-D (L5) & $1-4$ & $39,99 \pm 0,19$ & $0,39 \pm 0,04$ & $0,05 \pm 0,01$ & $2,32 \pm 0,11$ & $27,41 \pm 0,25$ \\
\hline NWA 869-E (L5) & $1-5$ & $39,46 \pm 0,17$ & $0,12 \pm 0,02$ & $0,03 \pm 0,01$ & $2,21 \pm 0,23$ & $27,24 \pm 0,69$ \\
\hline NWA 869-F (L5) & $1-6$ & $39,38 \pm 0,09$ & $0,04 \pm 0,01$ & $0,01 \pm 0,01$ & $2,18 \pm 0,11$ & $29,05 \pm 0,58$ \\
\hline NWA 869-G (L5) & $1-7$ & $40,26 \pm 0,37$ & $0,23 \pm 0,05$ & $0,03 \pm 0,01$ & $2,41 \pm 0,26$ & $24,88 \pm 0,74$ \\
\hline NWA 869-H (L5) & $1-8$ & $39,18 \pm 0,25$ & $0,42 \pm 0,06$ & $0,07 \pm 0,01$ & $2,59 \pm 0,34$ & $29,07 \pm 1,02$ \\
\hline NWA 869-I (L5) & $1-9$ & $38,87 \pm 0,13$ & $0,21 \pm 0,02$ & $0,03 \pm 0,01$ & $2,16 \pm 0,16$ & $29,66 \pm 0,59$ \\
\hline NWA 869-J (L5) & $1-10$ & $39,47 \pm 0,11$ & $0,05 \pm 0,01$ & $0,01 \pm 0,01$ & $2,86 \pm 0,21$ & $29,17 \pm 0,91$ \\
\hline NWA 869-K (L5) & $1-11$ & $40,40 \pm 0,12$ & $0,17 \pm 0,02$ & $0,02 \pm 0,01$ & $2,74 \pm 0,09$ & $29,09 \pm 0,31$ \\
\hline SAU 001 (L4-5) & $2-13$ & $41,75 \pm 0,24$ & $0,14 \pm 0,02$ & $0,03 \pm 0,01$ & $2,36 \pm 0,14$ & $27,73 \pm 0,37$ \\
\hline SAU 001 (L4-5) & $2-14$ & $39,82 \pm 0,27$ & $0,19 \pm 0,03$ & $0,04 \pm 0,01$ & $2,03 \pm 0,25$ & $26,11 \pm 0,52$ \\
\hline
\end{tabular}




\begin{tabular}{|c|c|c|c|c|c|c|c|}
\hline $\mathrm{Al}_{2} \mathrm{O}_{3}$ & $\mathrm{MgO}$ & $\mathrm{P}_{2} \mathrm{O}_{5}$ & $\mathrm{TiO}_{2}$ & $\mathrm{MnO}$ & $\mathrm{SO}_{3}$ & $\mathrm{Cr}_{2} \mathrm{O}_{3}$ & $\mathrm{NiO}$ \\
\hline $2,97 \pm 0,36$ & $26,75 \pm 2,51$ & & $0,12 \pm 0,02$ & $0,22 \pm 0,02$ & & $0,34 \pm 0,05$ & $1,41 \pm 0,18$ \\
\hline $3,30 \pm 0,13$ & $28,18 \pm 0,59$ & & $0,14 \pm 0,01$ & $0,21 \pm 0,01$ & & $0,18 \pm 0,02$ & $0,46 \pm 0,02$ \\
\hline $3,61 \pm 0,14$ & $26,21 \pm 0,65$ & $0,25 \pm 0,02$ & $0,15 \pm 0,01$ & $0,20 \pm 0,02$ & $0,08 \pm 0,01$ & $0,52 \pm 0,04$ & $0,65 \pm 0,02$ \\
\hline $4,03 \pm 0,10$ & $24,74 \pm 0,27$ & $0,27 \pm 0,01$ & $0,18 \pm 0,01$ & $0,20 \pm 0,01$ & $0,15 \pm 0,01$ & $0,65 \pm 0,07$ & $0,48 \pm 0,02$ \\
\hline $3,38 \pm 0,17$ & $26,20 \pm 0,90$ & $0,22 \pm 0,01$ & $0,14 \pm 0,02$ & $0,19 \pm 0,02$ & $0,02 \pm 0,01$ & $0,52 \pm 0,07$ & $1,02 \pm 0,05$ \\
\hline $2,82 \pm 0,24$ & $28,03 \pm 1,43$ & $0,20 \pm 0,03$ & $0,12 \pm 0,02$ & $0,19 \pm 0,01$ & u.NG. & $0,52 \pm 0,05$ & $1,80 \pm 0,10$ \\
\hline $3,22 \pm 0,22$ & $26,17 \pm 1,45$ & $0,19 \pm 0,02$ & $0,14 \pm 0,02$ & $0,20 \pm 0,02$ & u.NG. & $0,53 \pm 0,06$ & $1,72 \pm 0,08$ \\
\hline $2,87 \pm 0,61$ & $28,54 \pm 2,75$ & $0,20 \pm 0,03$ & $0,13 \pm 0,03$ & $0,20 \pm 0,02$ & $0,06 \pm 0,02$ & $0,49 \pm 0,06$ & $0,64 \pm 0,04$ \\
\hline $3,26 \pm 0,28$ & $27,78 \pm 1,49$ & $0,24 \pm 0,03$ & $0,15 \pm 0,02$ & $0,22 \pm 0,01$ & $0,02 \pm 0,01$ & $0,55 \pm 0,04$ & $1,42 \pm 0,09$ \\
\hline $4,62 \pm 0,54$ & $18,92 \pm 3,25$ & $0,32 \pm 0,03$ & $0,20 \pm 0,03$ & $0,26 \pm 0,02$ & $0,03 \pm 0,02$ & $0,38 \pm 0,06$ & $0,92 \pm 0,21$ \\
\hline $5,83 \pm 0,16$ & $38,59 \pm 0,19$ & $0,02 \pm 0,01$ & $0,30 \pm 0,02$ & $0,13 \pm 0,03$ & u.NG. & $0,10 \pm 0,01$ & $0,03 \pm 0,01$ \\
\hline $3,75 \pm 0,23$ & $26,55 \pm 1,47$ & & $0,16 \pm 0,01$ & $0,19 \pm 0,01$ & & $0,34 \pm 0,02$ & $0,45 \pm 0,04$ \\
\hline $3,79 \pm 0,19$ & $25,60 \pm 1,16$ & & $0,18 \pm 0,02$ & $0,20 \pm 0,01$ & & $0,37 \pm 0,03$ & $0,42 \pm 0,04$ \\
\hline $3,08 \pm 0,07$ & $27,61 \pm 0,59$ & $0,22 \pm 0,03$ & $0,14 \pm 0,01$ & $0,18 \pm 0,02$ & $0,02 \pm 0,02$ & $0,55 \pm 0,16$ & $1,54 \pm 0,09$ \\
\hline $3,18 \pm 0,06$ & $25,52 \pm 0,19$ & $0,18 \pm 0,03$ & $0,15 \pm 0,02$ & $0,20 \pm 0,02$ & $0,03 \pm 0,01$ & $0,43 \pm 0,01$ & $1,41 \pm 0,03$ \\
\hline $3,70 \pm 0,18$ & $25,43 \pm 0,97$ & $0,23 \pm 0,02$ & $0,16 \pm 0,01$ & $0,22 \pm 0,01$ & $1,36 \pm 0,10$ & $0,57 \pm 0,02$ & $0,04 \pm 0,02$ \\
\hline $3,86 \pm 0,05$ & $27,28 \pm 0,14$ & $0,03 \pm 0,01$ & $0,17 \pm 0,01$ & $0,22 \pm 0,01$ & $0,80 \pm 0,04$ & $0,52 \pm 0,03$ & $0,06 \pm 0,02$ \\
\hline $3,53 \pm 0,28$ & $27,42 \pm 0,79$ & $0,24 \pm 0,03$ & $0,17 \pm 0,02$ & $0,22 \pm 0,01$ & $0,23 \pm 0,02$ & $0,54 \pm 0,05$ & $0,27 \pm 0,02$ \\
\hline $3,94 \pm 0,16$ & $25,08 \pm 0,84$ & $0,23 \pm 0,01$ & $0,18 \pm 0,02$ & $0,19 \pm 0,01$ & $0,05 \pm 0,01$ & $0,51 \pm 0,06$ & $0,62 \pm 0,02$ \\
\hline $4,15 \pm 0,74$ & $24,92 \pm 2,61$ & $0,26 \pm 0,04$ & $0,17 \pm 0,03$ & $0,20 \pm 0,01$ & $0,11 \pm 0,03$ & $0,62 \pm 0,04$ & $0,51 \pm 0,04$ \\
\hline $3,21 \pm 0,29$ & $23,27 \pm 2,12$ & & $0,14 \pm 0,02$ & $0,21 \pm 0,01$ & & $0,32 \pm 0,05$ & $1,07 \pm 0,11$ \\
\hline $3,07 \pm 0,21$ & $24,20 \pm 1,37$ & & $0,14 \pm 0,01$ & $0,22 \pm 0,01$ & & $0,29 \pm 0,02$ & $1,16 \pm 0,08$ \\
\hline $3,17 \pm 0,11$ & $26,94 \pm 0,98$ & $0,26 \pm 0,02$ & $0,34 \pm 0,02$ & $0,21 \pm 0,02$ & u.NG. & $0,40 \pm 0,03$ & $1,33 \pm 0,08$ \\
\hline $3,31 \pm 0,15$ & $26,69 \pm 0,81$ & $0,22 \pm 0,02$ & $0,34 \pm 0,02$ & $0,22 \pm 0,01$ & $0,01 \pm 0,01$ & $0,41 \pm 0,02$ & $1,22 \pm 0,05$ \\
\hline $3,33 \pm 0,24$ & $28,08 \pm 1,09$ & $0,09 \pm 0,02$ & $0,32 \pm 0,03$ & $0,19 \pm 0,01$ & $0,57 \pm 0,09$ & $0,52 \pm 0,05$ & $0,08 \pm 0,02$ \\
\hline $3,70 \pm 0,55$ & $26,56 \pm 2,19$ & $0,10 \pm 0,02$ & $0,37 \pm 0,06$ & $0,20 \pm 0,01$ & $0,84 \pm 0,15$ & $0,49 \pm 0,04$ & $0,11 \pm 0,02$ \\
\hline $3,09 \pm 0,08$ & $26,16 \pm 0,40$ & $0,27 \pm 0,02$ & $0,13 \pm 0,01$ & $0,21 \pm 0,02$ & $0,01 \pm 0,01$ & $0,47 \pm 0,08$ & $1,80 \pm 0,04$ \\
\hline $2,72 \pm 0,44$ & $28,13 \pm 2,48$ & $0,21 \pm 0,02$ & $0,14 \pm 0,02$ & $0,20 \pm 0,03$ & u.NG. & $0,58 \pm 0,07$ & $1,94 \pm 0,18$ \\
\hline $3,92 \pm 0,40$ & $20,80 \pm 2,31$ & $0,27 \pm 0,03$ & $0,16 \pm 0,04$ & $0,22 \pm 0,03$ & u.NG. & $0,45 \pm 0,08$ & $1,36 \pm 0,18$ \\
\hline $3,30 \pm 0,39$ & $25,43 \pm 2,48$ & & $0,15 \pm 0,02$ & $0,18 \pm 0,02$ & & $0,37 \pm 0,08$ & $1,61 \pm 0,23$ \\
\hline $3,14 \pm 0,25$ & $25,40 \pm 1,88$ & & $0,14 \pm 0,02$ & $0,17 \pm 0,01$ & & $0,36 \pm 0,03$ & $1,68 \pm 0,13$ \\
\hline $2,42 \pm 0,08$ & $26,99 \pm 0,79$ & $0,17 \pm 0,02$ & $0,13 \pm 0,01$ & $0,35 \pm 0,01$ & $0,01 \pm 0,02$ & $0,46 \pm 0,02$ & $0,96 \pm 0,03$ \\
\hline $2,63 \pm 0,04$ & $27,32 \pm 0,61$ & $0,12 \pm 0,02$ & $0,13 \pm 0,01$ & $0,35 \pm 0,02$ & $0,01 \pm 0,01$ & $0,47 \pm 0,03$ & $0,86 \pm 0,10$ \\
\hline $2,50 \pm 0,11$ & $27,61 \pm 0,97$ & $0,11 \pm 0,02$ & $0,13 \pm 0,01$ & $0,36 \pm 0,03$ & $0,01 \pm 0,01$ & $0,46 \pm 0,05$ & $0,71 \pm 0,13$ \\
\hline $2,76 \pm 0,08$ & $29,70 \pm 0,50$ & $0,03 \pm 0,01$ & $0,13 \pm 0,01$ & $0,40 \pm 0,01$ & $0,27 \pm 0,02$ & $0,61 \pm 0,01$ & $0,03 \pm 0,01$ \\
\hline $2,93 \pm 0,09$ & $29,73 \pm 0,58$ & $0,02 \pm 0,01$ & $0,14 \pm 0,01$ & $0,41 \pm 0,01$ & $0,06 \pm 0,01$ & $0,63 \pm 0,01$ & $0,02 \pm 0,01$ \\
\hline $2,51 \pm 0,06$ & $26,51 \pm 0,21$ & $0,18 \pm 0,02$ & $0,11 \pm 0,01$ & $0,37 \pm 0,01$ & $0,11 \pm 0,01$ & $0,57 \pm 0,01$ & $0,17 \pm 0,01$ \\
\hline $2,89 \pm 0,43$ & $22,88 \pm 2,94$ & $0,18 \pm 0,02$ & $0,16 \pm 0,03$ & $0,39 \pm 0,03$ & $0,04 \pm 0,01$ & $0,60 \pm 0,05$ & $0,28 \pm 0,06$ \\
\hline $2,28 \pm 0,03$ & $27,71 \pm 0,22$ & & $0,11 \pm 0,01$ & $0,37 \pm 0,01$ & & $0,47 \pm 0,01$ & $0,90 \pm 0,02$ \\
\hline $2,32 \pm 0,02$ & $27,66 \pm 0,07$ & & $0,11 \pm 0,01$ & $0,36 \pm 0,01$ & & $0,10 \pm 0,01$ & $0,59 \pm 0,01$ \\
\hline $3,27 \pm 0,14$ & $28,27 \pm 0,55$ & $0,02 \pm 0,01$ & $0,14 \pm 0,01$ & $0,39 \pm 0,01$ & $0,02 \pm 0,01$ & $0,44 \pm 0,01$ & $0,44 \pm 0,03$ \\
\hline $3,25 \pm 0,28$ & $27,44 \pm 1,59$ & $0,03 \pm 0,01$ & $0,14 \pm 0,02$ & $0,39 \pm 0,02$ & $0,02 \pm 0,01$ & $0,46 \pm 0,02$ & $0,32 \pm 0,03$ \\
\hline $2,48 \pm 0,09$ & $26,58 \pm 0,38$ & $0,06 \pm 0,02$ & $0,16 \pm 0,01$ & $0,35 \pm 0,02$ & $0,01 \pm 0,01$ & $0,48 \pm 0,02$ & $0,65 \pm 0,03$ \\
\hline $2,41 \pm 0,06$ & $26,44 \pm 0,93$ & $0,19 \pm 0,03$ & $0,16 \pm 0,01$ & $0,37 \pm 0,02$ & u.NG. & $0,52 \pm 0,03$ & $0,90 \pm 0,06$ \\
\hline $2,13 \pm 0,11$ & $26,64 \pm 0,96$ & $0,25 \pm 0,02$ & $0,11 \pm 0,02$ & $0,34 \pm 0,02$ & u.NG. & $0,55 \pm 0,02$ & $2,04 \pm 0,14$ \\
\hline $2,16 \pm 0,23$ & $26,39 \pm 1,63$ & $0,24 \pm 0,03$ & $0,14 \pm 0,03$ & $0,37 \pm 0,03$ & u.NG. & $0,60 \pm 0,05$ & $1,86 \pm 0,17$ \\
\hline $2,00 \pm 0,22$ & $23,00 \pm 1,88$ & $0,31 \pm 0,04$ & $0,11 \pm 0,02$ & $0,32 \pm 0,03$ & $0,01 \pm 0,01$ & $0,40 \pm 0,04$ & $2,29 \pm 0,22$ \\
\hline $2,29 \pm 0,28$ & $20,92 \pm 2,86$ & $0,37 \pm 0,04$ & $0,11 \pm 0,02$ & $0,35 \pm 0,02$ & $0,01 \pm 0,01$ & $0,26 \pm 0,05$ & $1,80 \pm 0,33$ \\
\hline $2,50 \pm 0,25$ & $23,44 \pm 1,95$ & $0,10 \pm 0,02$ & $0,14 \pm 0,02$ & $0,37 \pm 0,02$ & $0,01 \pm 0,01$ & $0,54 \pm 0,03$ & $0,93 \pm 0,11$ \\
\hline $2,71 \pm 0,32$ & $23,60 \pm 2,03$ & $0,03 \pm 0,01$ & $0,16 \pm 0,02$ & $0,38 \pm 0,02$ & $0,02 \pm 0,01$ & $0,56 \pm 0,03$ & $0,45 \pm 0,08$ \\
\hline $2,35 \pm 0,12$ & $25,91 \pm 1,38$ & & $0,11 \pm 0,01$ & $0,35 \pm 0,01$ & & $0,38 \pm 0,03$ & $1,15 \pm 0,07$ \\
\hline $2,45 \pm 0,05$ & $26,06 \pm 0,39$ & & $0,12 \pm 0,01$ & $0,36 \pm 0,01$ & & $0,30 \pm 0,02$ & $0,88 \pm 0,02$ \\
\hline $2,16 \pm 0,12$ & $26,50 \pm 1,20$ & & $0,10 \pm 0,01$ & $0,34 \pm 0,01$ & & $0,44 \pm 0,04$ & $1,18 \pm 0,08$ \\
\hline $2,17 \pm 0,06$ & $27,87 \pm 0,73$ & & $0,10 \pm 0,01$ & $0,35 \pm 0,01$ & & $0,15 \pm 0,01$ & $0,61 \pm 0,03$ \\
\hline $2,61 \pm 0,14$ & $25,21 \pm 0,78$ & $0,10 \pm 0,01$ & $0,12 \pm 0,02$ & $0,37 \pm 0,01$ & $0,02 \pm 0,01$ & $0,57 \pm 0,02$ & $0,77 \pm 0,04$ \\
\hline $2,55 \pm 0,30$ & $25,22 \pm 1,71$ & $0,05 \pm 0,01$ & $0,12 \pm 0,02$ & $0,37 \pm 0,01$ & $0,02 \pm 0,01$ & $0,61 \pm 0,03$ & $0,38 \pm 0,04$ \\
\hline $2,90 \pm 0,19$ & $26,61 \pm 0,92$ & $0,05 \pm 0,01$ & $0,13 \pm 0,01$ & $0,37 \pm 0,01$ & $0,02 \pm 0,01$ & $0,54 \pm 0,02$ & $0,22 \pm 0,03$ \\
\hline $3,13 \pm 0,21$ & $23,62 \pm 1,27$ & $0,09 \pm 0,01$ & $0,14 \pm 0,02$ & $0,39 \pm 0,01$ & $0,02 \pm 0,01$ & $0,62 \pm 0,03$ & $0,46 \pm 0,04$ \\
\hline $2,89 \pm 0,30$ & $26,45 \pm 1,66$ & $0,04 \pm 0,01$ & $0,14 \pm 0,02$ & $0,37 \pm 0,02$ & $0,02 \pm 0,01$ & $0,59 \pm 0,02$ & $0,15 \pm 0,04$ \\
\hline $2,32 \pm 0,10$ & $25,83 \pm 0,63$ & $0,26 \pm 0,03$ & $0,11 \pm 0,01$ & $0,34 \pm 0,01$ & $0,02 \pm 0,01$ & $0,60 \pm 0,02$ & $0,40 \pm 0,08$ \\
\hline $2,31 \pm 0,25$ & $27,47 \pm 1,28$ & $0,05 \pm 0,01$ & $0,10 \pm 0,02$ & $0,34 \pm 0,01$ & $0,01 \pm 0,01$ & $0,52 \pm 0,02$ & $0,64 \pm 0,04$ \\
\hline $2,41 \pm 0,12$ & $26,06 \pm 0,86$ & $0,04 \pm 0,01$ & $0,12 \pm 0,01$ & $0,33 \pm 0,01$ & $0,02 \pm 0,01$ & $0,56 \pm 0,02$ & $0,62 \pm 0,02$ \\
\hline $2,45 \pm 0,25$ & $29,02 \pm 1,47$ & $0,07 \pm 0,02$ & $0,10 \pm 0,02$ & $0,34 \pm 0,02$ & $0,01 \pm 0,01$ & $0,50 \pm 0,02$ & $0,37 \pm 0,04$ \\
\hline $3,00 \pm 0,35$ & $23,78 \pm 1,81$ & $0,11 \pm 0,02$ & $0,13 \pm 0,02$ & $0,34 \pm 0,02$ & $0,02 \pm 0,01$ & $0,57 \pm 0,05$ & $1,29 \pm 0,11$ \\
\hline $2,23 \pm 0,15$ & $25,62 \pm 0,91$ & $0,07 \pm 0,01$ & $0,10 \pm 0,01$ & $0,33 \pm 0,01$ & $0,01 \pm 0,01$ & $0,45 \pm 0,02$ & $0,96 \pm 0,05$ \\
\hline $2,75 \pm 0,20$ & $24,87 \pm 1,39$ & $0,03 \pm 0,01$ & $0,14 \pm 0,02$ & $0,37 \pm 0,02$ & $0,02 \pm 0,01$ & $0,52 \pm 0,02$ & $0,30 \pm 0,03$ \\
\hline $2,21 \pm 0,06$ & $24,00 \pm 0,47$ & $0,03 \pm 0,01$ & $0,12 \pm 0,01$ & $0,36 \pm 0,01$ & $0,02 \pm 0,01$ & $0,48 \pm 0,01$ & $0,81 \pm 0,03$ \\
\hline $2,93 \pm 0,15$ & $24,15 \pm 0,78$ & $0,05 \pm 0,01$ & $0,13 \pm 0,01$ & $0,37 \pm 0,02$ & $0,01 \pm 0,01$ & $0,57 \pm 0,02$ & $0,65 \pm 0,03$ \\
\hline $2,47 \pm 0,32$ & $28,25 \pm 1,28$ & $0,05 \pm 0,01$ & $0,13 \pm 0,01$ & $0,34 \pm 0,01$ & u.NG. & $0,58 \pm 0,03$ & $0,73 \pm 0,05$ \\
\hline
\end{tabular}


Tabelle A.6 (Fortsetzung)

\begin{tabular}{|c|c|c|c|c|c|c|}
\hline Probe & Kugel & $\mathrm{SiO}_{2}$ & $\mathrm{Na}_{2} \mathrm{O}$ & $\mathrm{K}_{2} \mathrm{O}$ & $\mathrm{CaO}$ & $\mathrm{FeO}$ \\
\hline St. Michel (L6) & $\mathrm{B}-12$ & $41,81 \pm 0,27$ & $0,47 \pm 0,04$ & $0,04 \pm 0,01$ & $1,75 \pm 0,14$ & $26,36 \pm 0,83$ \\
\hline St. Michel (L6) ${ }^{\square}$ & B-13 & $41,64 \pm 0,19$ & $0,13 \pm 0,01$ & $0,01 \pm 0,01$ & $1,96 \pm 0,04$ & $27,49 \pm 0,12$ \\
\hline St. Michel (L6) & $1-30$ & $40,93 \pm 0,09$ & $0,53 \pm 0,02$ & $0,05 \pm 0,01$ & $1,89 \pm 0,03$ & $27,28 \pm 0,04$ \\
\hline St. Michel (L6) & $1-31$ & $41,50 \pm 0,14$ & $0,33 \pm 0,04$ & $0,03 \pm 0,01$ & $1,90 \pm 0,08$ & $26,95 \pm 0,21$ \\
\hline Zavid (L6) ${ }^{\square}$ & $\mathrm{A}-30$ & $41,45 \pm 0,22$ & $0,24 \pm 0,02$ & $0,02 \pm 0,01$ & $1,95 \pm 0,08$ & $27,83 \pm 0,57$ \\
\hline Zavid (L6) ${ }^{\square}$ & $\mathrm{A}-31$ & $40,88 \pm 0,34$ & $0,06 \pm 0,01$ & u.NG. & $1,96 \pm 0,03$ & $26,71 \pm 0,40$ \\
\hline Zavid (L6) & $1-24$ & $39,87 \pm 0,11$ & $0,07 \pm 0,01$ & $0,02 \pm 0,01$ & $1,94 \pm 0,12$ & $26,57 \pm 0,38$ \\
\hline Zavid (L6) & $1-25$ & $40,90 \pm 0,19$ & $0,36 \pm 0,03$ & $0,03 \pm 0,01$ & $2,19 \pm 0,16$ & $28,09 \pm 0,70$ \\
\hline Adrian $(\mathrm{H} 4)^{\square}$ & B-6 & $40,28 \pm 0,13$ & $0,24 \pm 0,02$ & $0,04 \pm 0,01$ & $1,81 \pm 0,06$ & $31,99 \pm 0,76$ \\
\hline Adrian $(\mathrm{H} 4)^{\square}$ & $\mathrm{B}-7$ & $39,30 \pm 0,17$ & $0,12 \pm 0,02$ & $0,01 \pm 0,01$ & $1,69 \pm 0,02$ & $32,69 \pm 0,24$ \\
\hline Adrian (H4) & $1-22$ & $37,76 \pm 0,15$ & $0,08 \pm 0,01$ & $0,02 \pm 0,01$ & $2,08 \pm 0,09$ & $32,19 \pm 0,31$ \\
\hline Adrian (H4) & $1-23$ & $38,94 \pm 0,12$ & $0,25 \pm 0,03$ & $0,05 \pm 0,01$ & $1,95 \pm 0,16$ & $32,22 \pm 0,84$ \\
\hline Dar al Gani $300(\mathrm{H} 3-5)^{\diamond}$ & $\mathrm{C}-13$ & $34,46 \pm 1,07$ & $0,41 \pm 0,06$ & $0,03 \pm 0,01$ & $1,59 \pm 0,20$ & $36,48 \pm 1,64$ \\
\hline Dar al Gani $300(\mathrm{H} 3-5)^{\diamond}$ & $\mathrm{C}-14$ & $39,11 \pm 1,40$ & $0,34 \pm 0,04$ & $0,02 \pm 0,01$ & $2,12 \pm 0,20$ & $32,65 \pm 1,74$ \\
\hline Djoumine $(\mathrm{H} 5-6)^{\diamond}$ & $\mathrm{C}-5$ & $37,78 \pm 0,44$ & $0,25 \pm 0,02$ & $0,02 \pm 0,01$ & $1,83 \pm 0,13$ & $30,08 \pm 1,29$ \\
\hline Djoumine $(\mathrm{H} 5-6)^{\diamond}$ & $\mathrm{C}-6$ & $40,83 \pm 0,75$ & $0,13 \pm 0,02$ & u.NG. & $2,21 \pm 0,34$ & $28,52 \pm 1,82$ \\
\hline El Hammami (H5) & $5-7$ & $36,94 \pm 0,31$ & $0,67 \pm 0,03$ & $0,07 \pm 0,01$ & $1,70 \pm 0,04$ & $34,00 \pm 0,50$ \\
\hline El Hammami (H5) & $5-8$ & $37,82 \pm 0,41$ & $0,67 \pm 0,10$ & $0,08 \pm 0,01$ & $1,65 \pm 0,16$ & $31,73 \pm 0,81$ \\
\hline Forest City (H5) ${ }^{\square}$ & $\mathrm{A}-22$ & $39,62 \pm 0,46$ & $0,54 \pm 0,04$ & $0,04 \pm 0,01$ & $1,85 \pm 0,10$ & $29,57 \pm 0,66$ \\
\hline Forest City (H5) & $\mathrm{A}-23$ & $40,55 \pm 0,31$ & $0,67 \pm 0,05$ & $0,06 \pm 0,01$ & $2,09 \pm 0,08$ & $29,88 \pm 0,78$ \\
\hline Forest City (H5) & $1-26$ & $38,15 \pm 0,09$ & $0,22 \pm 0,02$ & $0,02 \pm 0,01$ & $2,13 \pm 0,07$ & $31,47 \pm 0,12$ \\
\hline Forest City (H5) & $1-27$ & $38,23 \pm 0,08$ & $0,29 \pm 0,02$ & $0,02 \pm 0,01$ & $2,00 \pm 0,09$ & $31,18 \pm 0,31$ \\
\hline Gao-Guenie (H5) & $2-7$ & $36,11 \pm 0,33$ & $0,28 \pm 0,02$ & $0,03 \pm 0,01$ & $2,03 \pm 0,12$ & $35,85 \pm 0,89$ \\
\hline Gao-Guenie (H5) & $2-8$ & $36,27 \pm 0,21$ & $0,32 \pm 0,06$ & $0,04 \pm 0,01$ & $1,69 \pm 0,30$ & $33,45 \pm 1,65$ \\
\hline Gao-Guenie-A (H5) & $8-28$ & $38,32 \pm 0,16$ & $0,84 \pm 0,05$ & $0,12 \pm 0,01$ & $1,89 \pm 0,09$ & $32,86 \pm 0,58$ \\
\hline Gao-Guenie-A (H5) & $8-29$ & $37,41 \pm 0,25$ & $0,72 \pm 0,03$ & $0,09 \pm 0,01$ & $1,62 \pm 0,08$ & $32,80 \pm 0,18$ \\
\hline Gao-Guenie-B (H5) & $8-30$ & $38,82 \pm 0,49$ & $0,78 \pm 0,06$ & $0,10 \pm 0,01$ & $1,96 \pm 0,15$ & $32,17 \pm 0,32$ \\
\hline Gao-Guenie-B (H5) & $8-31$ & $37,94 \pm 0,36$ & $0,72 \pm 0,05$ & $0,08 \pm 0,01$ & $1,93 \pm 0,12$ & $33,73 \pm 0,34$ \\
\hline Gao-Guenie-C (H5) & $8-32$ & $34,77 \pm 0,24$ & $0,79 \pm 0,12$ & $0,10 \pm 0,02$ & $1,86 \pm 0,22$ & $38,24 \pm 1,29$ \\
\hline Gao-Guenie-C (H5) & $8-33$ & $35,21 \pm 0,62$ & $0,36 \pm 0,05$ & $0,02 \pm 0,01$ & $1,75 \pm 0,24$ & $37,75 \pm 0,84$ \\
\hline Gao-Guenie-D (H5) & $8-34$ & $33,45 \pm 0,74$ & $0,62 \pm 0,04$ & $0,08 \pm 0,01$ & $1,56 \pm 0,09$ & $39,47 \pm 0,93$ \\
\hline Gao-Guenie-D (H5) & $8-35$ & $34,20 \pm 0,64$ & $0,51 \pm 0,04$ & $0,06 \pm 0,01$ & $1,71 \pm 0,10$ & $39,35 \pm 0,57$ \\
\hline Gao-Guenie-E (H5) & $8-36$ & $35,99 \pm 0,35$ & $0,55 \pm 0,03$ & $0,06 \pm 0,01$ & $1,42 \pm 0,03$ & $36,34 \pm 0,40$ \\
\hline Gao-Guenie-E (H5) & $8-37$ & $35,69 \pm 0,24$ & $0,78 \pm 0,03$ & $0,10 \pm 0,01$ & $1,52 \pm 0,04$ & $36,94 \pm 0,44$ \\
\hline Gao-Guenie-F (H5) & $8-38$ & $36,29 \pm 0,48$ & $0,55 \pm 0,12$ & $0,07 \pm 0,02$ & $1,22 \pm 0,25$ & $33,05 \pm 2,35$ \\
\hline Gao-Guenie-F (H5) & $8-39$ & $35,51 \pm 0,19$ & $0,82 \pm 0,03$ & $0,09 \pm 0,01$ & $1,62 \pm 0,04$ & $37,95 \pm 0,67$ \\
\hline Hammond Downs $(\mathrm{H} 4)^{\square}$ & $\mathrm{A}-18$ & $37,51 \pm 0,59$ & $0,26 \pm 0,02$ & $0,02 \pm 0,01$ & $1,93 \pm 0,03$ & $34,08 \pm 0,75$ \\
\hline Hammond Downs (H4) & $A-19$ & $37,12 \pm 0,23$ & $0,23 \pm 0,03$ & $0,02 \pm 0,01$ & $1,81 \pm 0,07$ & $35,39 \pm 0,62$ \\
\hline Hammond Downs (H4) & $1-18$ & $34,52 \pm 0,08$ & $0,32 \pm 0,02$ & $0,03 \pm 0,01$ & $1,75 \pm 0,04$ & $37,04 \pm 0,24$ \\
\hline Hammond Downs (H4) & $1-19$ & $35,76 \pm 0,08$ & $0,08 \pm 0,01$ & $0,01 \pm 0,01$ & $1,63 \pm 0,05$ & $36,22 \pm 0,21$ \\
\hline Ijopega $(\mathrm{H} 6)^{\square}$ & B-1 & $37,16 \pm 0,58$ & $0,38 \pm 0,02$ & $0,02 \pm 0,01$ & $1,79 \pm 0,08$ & $35,73 \pm 0,69$ \\
\hline Ijopega $(\mathrm{H} 6)^{\square}$ & B-2 & $37,00 \pm 0,41$ & $0,09 \pm 0,01$ & u.NG. & $1,68 \pm 0,03$ & $35,34 \pm 0,47$ \\
\hline Ijopega (H6) & $1-28$ & $35,93 \pm 0,32$ & $0,26 \pm 0,04$ & $0,03 \pm 0,01$ & $1,50 \pm 0,23$ & $34,61 \pm 1,91$ \\
\hline Ijopega (H6) & $1-29$ & $37,13 \pm 0,12$ & $0,09 \pm 0,02$ & $0,02 \pm 0,01$ & $1,71 \pm 0,16$ & $33,06 \pm 0,75$ \\
\hline Jilin $(\mathrm{H} 5)^{\square}$ & $\mathrm{A}-26$ & $39,09 \pm 0,14$ & $0,33 \pm 0,01$ & $0,02 \pm 0,01$ & $1,90 \pm 0,03$ & $30,77 \pm 0,44$ \\
\hline Jilin $(\mathrm{H} 5)^{\square}$ & $\mathrm{A}-27$ & $37,68 \pm 0,28$ & $0,29 \pm 0,02$ & $0,02 \pm 0,01$ & $1,83 \pm 0,05$ & $31,20 \pm 0,32$ \\
\hline Kunya-Urgench (H5) & $2-1$ & $35,32 \pm 0,49$ & $0,03 \pm 0,01$ & $0,02 \pm 0,01$ & $2,60 \pm 0,48$ & $35,23 \pm 2,31$ \\
\hline Kunya-Urgench (H5) & $2-2$ & $36,88 \pm 0,12$ & $0,15 \pm 0,01$ & $0,02 \pm 0,01$ & $2,04 \pm 0,09$ & $32,48 \pm 0,34$ \\
\hline Pultusk-A (H5) & $\mathrm{A}-24$ & $35,21 \pm 1,26$ & $0,75 \pm 0,03$ & $0,05 \pm 0,01$ & $1,49 \pm 0,08$ & $38,16 \pm 1,28$ \\
\hline Pultusk-A (H5) ${ }^{\square}$ & $\mathrm{A}-25$ & $35,04 \pm 0,45$ & $0,24 \pm 0,01$ & $0,02 \pm 0,01$ & $1,62 \pm 0,06$ & $37,23 \pm 0,54$ \\
\hline Pultusk-A (H5) & $2-29$ & $33,86 \pm 0,21$ & $0,14 \pm 0,02$ & $0,02 \pm 0,01$ & $1,62 \pm 0,11$ & $39,52 \pm 0,76$ \\
\hline Pultusk-A (H5) & $2-30$ & $33,36 \pm 0,09$ & $0,44 \pm 0,02$ & $0,04 \pm 0,01$ & $1,63 \pm 0,04$ & $39,29 \pm 0,13$ \\
\hline Pultusk-B (H5) & $2-31$ & $35,26 \pm 0,11$ & $0,21 \pm 0,01$ & $0,03 \pm 0,01$ & $1,83 \pm 0,05$ & $37,26 \pm 0,12$ \\
\hline Pultusk-B (H5) & $2-32$ & $35,67 \pm 0,21$ & $0,23 \pm 0,04$ & $0,03 \pm 0,01$ & $1,83 \pm 0,22$ & $36,20 \pm 1,07$ \\
\hline Richardton $(\mathrm{H} 5)^{\square}$ & $\mathrm{A}-20$ & $37,84 \pm 0,24$ & $0,51 \pm 0,11$ & $0,04 \pm 0,02$ & $1,97 \pm 0,12$ & $33,11 \pm 1,26$ \\
\hline Richardton (H5) & $\mathrm{A}-21$ & $37,68 \pm 0,13$ & $0,01 \pm 0,01$ & u.NG. & $1,88 \pm 0,04$ & $32,03 \pm 0,33$ \\
\hline Richardton (H5) & $1-20$ & $36,59 \pm 0,25$ & $0,16 \pm 0,02$ & $0,02 \pm 0,01$ & $1,80 \pm 0,13$ & $33,46 \pm 1,27$ \\
\hline Richardton (H5) & $1-21$ & $36,16 \pm 0,13$ & $0,27 \pm 0,02$ & $0,03 \pm 0,01$ & $1,92 \pm 0,08$ & $33,74 \pm 0,47$ \\
\hline Thuathe (H4/5) & $1-32$ & $34,99 \pm 0,36$ & $0,09 \pm 0,02$ & $0,02 \pm 0,01$ & $1,74 \pm 0,18$ & $38,62 \pm 0,98$ \\
\hline Thuathe $(\mathrm{H} 4 / 5)$ & $1-33$ & $33,77 \pm 0,23$ & $0,16 \pm 0,02$ & $0,02 \pm 0,01$ & $1,76 \pm 0,13$ & $39,35 \pm 0,46$ \\
\hline Thuathe $(\mathrm{H} 4 / 5) * 1$ & $5-9$ & $34,91 \pm 0,41$ & $0,62 \pm 0,05$ & $0,10 \pm 0,02$ & $1,67 \pm 0,10$ & $37,45 \pm 0,67$ \\
\hline Thuathe $(\mathrm{H} 4 / 5) *_{2}$ & $5-10$ & $34,41 \pm 0,28$ & $0,62 \pm 0,03$ & $0,09 \pm 0,01$ & $1,73 \pm 0,07$ & $38,97 \pm 0,49$ \\
\hline Thuathe $(\mathrm{H} 4 / 5) * 3$ & $5-11$ & $33,87 \pm 0,87$ & $0,68 \pm 0,04$ & $0,10 \pm 0,01$ & $1,71 \pm 0,11$ & $39,91 \pm 0,93$ \\
\hline Thuathe $(\mathrm{H} 4 / 5) * 4$ & $5-12$ & $32,69 \pm 0,22$ & $0,02 \pm 0,01$ & $0,01 \pm 0,01$ & $3,32 \pm 0,12$ & $27,29 \pm 0,38$ \\
\hline NWA 753 (R3-6) & $5-19$ & $38,59 \pm 0,31$ & $0,67 \pm 0,04$ & $0,08 \pm 0,01$ & $2,44 \pm 0,06$ & $34,41 \pm 0,72$ \\
\hline NWA 753 (R3-6) & $5-20$ & $38,79 \pm 0,12$ & $0,64 \pm 0,04$ & $0,08 \pm 0,02$ & $2,59 \pm 0,08$ & $34,91 \pm 0,39$ \\
\hline Atlanta $(\text { EL6) })^{\diamond}$ & $\mathrm{C}-23$ & $40,73 \pm 0,38$ & $0,37 \pm 0,02$ & $0,02 \pm 0,01$ & $0,56 \pm 0,03$ & $29,95 \pm 0,41$ \\
\hline Atlanta $(\text { EL6) })^{\diamond}$ & $\mathrm{C}-24$ & $37,14 \pm 0,34$ & $0,12 \pm 0,01$ & $0,01 \pm 0,01$ & $0,47 \pm 0,04$ & $35,21 \pm 0,70$ \\
\hline Eagle $(\text { EL } 6)^{\diamond}$ & $\mathrm{C}-7$ & $40,71 \pm 0,56$ & $0,57 \pm 0,04$ & $0,05 \pm 0,01$ & $0,90 \pm 0,06$ & $32,01 \pm 0,83$ \\
\hline
\end{tabular}




\begin{tabular}{|c|c|c|c|c|c|c|c|}
\hline $\mathrm{Al}_{2} \mathrm{O}_{3}$ & $\mathrm{MgO}$ & $\mathrm{P}_{2} \mathrm{O}_{5}$ & $\mathrm{TiO}_{2}$ & $\mathrm{MnO}$ & $\mathrm{SO}_{3}$ & $\mathrm{Cr}_{2} \mathrm{O}_{3}$ & $\mathrm{NiO}$ \\
\hline $2,29 \pm 0,14$ & $28,19 \pm 1,29$ & $0,12 \pm 0,02$ & $0,11 \pm 0,02$ & $0,36 \pm 0,02$ & $0,01 \pm 0,01$ & $0,53 \pm 0,02$ & $1,20 \pm 0,12$ \\
\hline $2,51 \pm 0,06$ & $26,74 \pm 0,15$ & $0,05 \pm 0,03$ & $0,12 \pm 0,01$ & $0,36 \pm 0,03$ & u.NG. & $0,43 \pm 0,03$ & $0,77 \pm 0,02$ \\
\hline $2,41 \pm 0,03$ & $25,09 \pm 0,13$ & $0,09 \pm 0,01$ & $0,12 \pm 0,01$ & $0,36 \pm 0,01$ & $0,01 \pm 0,01$ & $0,60 \pm 0,03$ & $0,71 \pm 0,03$ \\
\hline $2,43 \pm 0,09$ & $25,70 \pm 0,48$ & $0,08 \pm 0,01$ & $0,11 \pm 0,01$ & $0,36 \pm 0,01$ & $0,01 \pm 0,01$ & $0,56 \pm 0,02$ & $0,91 \pm 0,05$ \\
\hline $2,55 \pm 0,08$ & $27,99 \pm 0,61$ & $0,08 \pm 0,02$ & $0,12 \pm 0,01$ & $0,38 \pm 0,02$ & $0,01 \pm 0,01$ & $0,45 \pm 0,02$ & $0,90 \pm 0,06$ \\
\hline $2,51 \pm 0,06$ & $28,57 \pm 0,26$ & $0,04 \pm 0,02$ & $0,13 \pm 0,01$ & $0,38 \pm 0,03$ & $0,01 \pm 0,01$ & $0,42 \pm 0,01$ & $0,56 \pm 0,02$ \\
\hline $2,55 \pm 0,14$ & $28,14 \pm 0,66$ & $0,02 \pm 0,01$ & $0,11 \pm 0,01$ & $0,35 \pm 0,01$ & u.NG. & $0,59 \pm 0,03$ & $0,42 \pm 0,01$ \\
\hline $2,94 \pm 0,20$ & $24,01 \pm 1,20$ & $0,08 \pm 0,01$ & $0,13 \pm 0,02$ & $0,38 \pm 0,01$ & $0,01 \pm 0,01$ & $0,62 \pm 0,03$ & $0,71 \pm 0,05$ \\
\hline $3,07 \pm 0,12$ & $22,95 \pm 0,89$ & $0,11 \pm 0,02$ & $0,15 \pm 0,01$ & $0,36 \pm 0,03$ & $0,02 \pm 0,01$ & $0,40 \pm 0,02$ & $0,83 \pm 0,08$ \\
\hline $2,85 \pm 0,04$ & $21,94 \pm 0,10$ & $0,06 \pm 0,02$ & $0,16 \pm 0,01$ & $0,34 \pm 0,03$ & $0,01 \pm 0,01$ & $0,45 \pm 0,02$ & $0,82 \pm 0,03$ \\
\hline $3,62 \pm 0,14$ & $21,87 \pm 0,60$ & $0,04 \pm 0,01$ & $0,20 \pm 0,01$ & $0,33 \pm 0,01$ & $0,01 \pm 0,01$ & $0,52 \pm 0,03$ & $0,43 \pm 0,02$ \\
\hline $3,53 \pm 0,27$ & $20,11 \pm 1,55$ & $0,07 \pm 0,01$ & $0,19 \pm 0,02$ & $0,33 \pm 0,02$ & $0,02 \pm 0,01$ & $0,51 \pm 0,03$ & $0,72 \pm 0,06$ \\
\hline $1,99 \pm 0,17$ & $22,35 \pm 1,72$ & & $0,09 \pm 0,01$ & $0,29 \pm 0,02$ & & $0,38 \pm 0,03$ & $2,15 \pm 0,13$ \\
\hline $2,54 \pm 0,19$ & $22,23 \pm 1,62$ & & $0,12 \pm 0,01$ & $0,34 \pm 0,02$ & & $0,33 \pm 0,06$ & $1,14 \pm 0,15$ \\
\hline $2,15 \pm 0,12$ & $26,04 \pm 1,55$ & & $0,11 \pm 0,02$ & $0,33 \pm 0,01$ & & $0,37 \pm 0,02$ & $1,36 \pm 0,10$ \\
\hline $2,44 \pm 0,24$ & $25,03 \pm 2,30$ & & $0,11 \pm 0,02$ & $0,35 \pm 0,02$ & & $0,22 \pm 0,03$ & $0,96 \pm 0,12$ \\
\hline $2,12 \pm 0,05$ & $23,55 \pm 0,22$ & $0,21 \pm 0,01$ & $0,15 \pm 0,02$ & $0,34 \pm 0,02$ & u.NG. & $0,49 \pm 0,06$ & $2,04 \pm 0,03$ \\
\hline $2,06 \pm 0,21$ & $24,89 \pm 1,24$ & $0,22 \pm 0,02$ & $0,15 \pm 0,03$ & $0,30 \pm 0,01$ & u.NG. & $0,41 \pm 0,03$ & $2,08 \pm 0,12$ \\
\hline $2,29 \pm 0,10$ & $25,81 \pm 1,02$ & $0,14 \pm 0,02$ & $0,11 \pm 0,01$ & $0,28 \pm 0,03$ & u.NG. & $0,48 \pm 0,02$ & $1,50 \pm 0,09$ \\
\hline $2,44 \pm 0,04$ & $25,02 \pm 0,82$ & $0,24 \pm 0,02$ & $0,12 \pm 0,01$ & $0,30 \pm 0,02$ & $0,01 \pm 0,01$ & $0,46 \pm 0,03$ & $1,46 \pm 0,07$ \\
\hline $2,60 \pm 0,10$ & $24,07 \pm 0,21$ & $0,07 \pm 0,01$ & $0,11 \pm 0,01$ & $0,32 \pm 0,01$ & $0,01 \pm 0,01$ & $0,59 \pm 0,04$ & $0,60 \pm 0,05$ \\
\hline $2,52 \pm 0,11$ & $24,57 \pm 0,51$ & $0,07 \pm 0,01$ & $0,11 \pm 0,01$ & $0,33 \pm 0,01$ & $0,02 \pm 0,01$ & $0,57 \pm 0,03$ & $0,97 \pm 0,03$ \\
\hline $2,37 \pm 0,12$ & $22,11 \pm 0,93$ & $0,10 \pm 0,01$ & $0,11 \pm 0,01$ & $0,32 \pm 0,01$ & $0,02 \pm 0,01$ & $0,48 \pm 0,06$ & $1,37 \pm 0,06$ \\
\hline $2,09 \pm 0,35$ & $24,59 \pm 2,04$ & $0,10 \pm 0,02$ & $0,10 \pm 0,02$ & $0,30 \pm 0,02$ & $0,02 \pm 0,01$ & $0,52 \pm 0,04$ & $1,60 \pm 0,15$ \\
\hline $2,31 \pm 0,07$ & $22,77 \pm 0,65$ & $0,18 \pm 0,01$ & $0,13 \pm 0,02$ & $0,31 \pm 0,02$ & $0,01 \pm 0,01$ & $0,37 \pm 0,05$ & $2,02 \pm 0,07$ \\
\hline $2,11 \pm 0,06$ & $23,95 \pm 0,24$ & $0,15 \pm 0,02$ & $0,10 \pm 0,02$ & $0,32 \pm 0,02$ & u.NG. & $0,41 \pm 0,04$ & $2,19 \pm 0,05$ \\
\hline $2,30 \pm 0,15$ & $22,41 \pm 0,73$ & $0,25 \pm 0,02$ & $0,09 \pm 0,01$ & $0,34 \pm 0,02$ & u.NG. & $0,39 \pm 0,05$ & $1,91 \pm 0,09$ \\
\hline $2,34 \pm 0,14$ & $22,14 \pm 0,72$ & $0,20 \pm 0,02$ & $0,11 \pm 0,01$ & $0,33 \pm 0,01$ & u.NG. & $0,50 \pm 0,05$ & $1,85 \pm 0,08$ \\
\hline $2,09 \pm 0,24$ & $20,57 \pm 1,65$ & $0,25 \pm 0,03$ & $0,11 \pm 0,02$ & $0,29 \pm 0,02$ & u.NG. & $0,59 \pm 0,06$ & $2,33 \pm 0,20$ \\
\hline $1,99 \pm 0,20$ & $21,43 \pm 1,23$ & $0,13 \pm 0,01$ & $0,11 \pm 0,01$ & $0,31 \pm 0,03$ & $0,01 \pm 0,01$ & $0,55 \pm 0,10$ & $2,09 \pm 0,15$ \\
\hline $1,80 \pm 0,04$ & $21,91 \pm 0,41$ & $0,20 \pm 0,02$ & $0,10 \pm 0,01$ & $0,27 \pm 0,02$ & $0,01 \pm 0,01$ & $0,44 \pm 0,06$ & $2,39 \pm 0,03$ \\
\hline $2,01 \pm 0,07$ & $20,81 \pm 0,31$ & $0,17 \pm 0,01$ & $0,08 \pm 0,01$ & $0,30 \pm 0,02$ & u.NG. & $0,40 \pm 0,05$ & $2,10 \pm 0,04$ \\
\hline $1,96 \pm 0,07$ & $22,51 \pm 0,17$ & $0,17 \pm 0,03$ & $0,10 \pm 0,02$ & $0,28 \pm 0,01$ & $0,01 \pm 0,01$ & $0,46 \pm 0,08$ & $2,13 \pm 0,03$ \\
\hline $2,07 \pm 0,07$ & $21,52 \pm 0,29$ & $0,24 \pm 0,02$ & $0,11 \pm 0,02$ & $0,31 \pm 0,01$ & u.NG. & $0,49 \pm 0,05$ & $2,02 \pm 0,03$ \\
\hline $1,75 \pm 0,30$ & $25,61 \pm 2,44$ & $0,15 \pm 0,02$ & $0,08 \pm 0,01$ & $0,30 \pm 0,03$ & u.NG. & $0,50 \pm 0,05$ & $2,59 \pm 0,27$ \\
\hline $2,29 \pm 0,08$ & $20,49 \pm 0,71$ & $0,16 \pm 0,01$ & $0,13 \pm 0,02$ & $0,30 \pm 0,02$ & u.NG. & $0,48 \pm 0,03$ & $1,99 \pm 0,10$ \\
\hline $2,54 \pm 0,05$ & $24,50 \pm 0,45$ & $0,10 \pm 0,02$ & $0,12 \pm 0,01$ & $0,29 \pm 0,02$ & u.NG. & $0,31 \pm 0,02$ & $1,02 \pm 0,03$ \\
\hline $2,32 \pm 0,07$ & $24,49 \pm 0,89$ & $0,11 \pm 0,01$ & $0,10 \pm 0,01$ & $0,34 \pm 0,02$ & $0,01 \pm 0,01$ & $0,36 \pm 0,03$ & $1,32 \pm 0,04$ \\
\hline $2,32 \pm 0,04$ & $22,78 \pm 0,17$ & $0,11 \pm 0,01$ & $0,11 \pm 0,01$ & $0,30 \pm 0,01$ & $0,01 \pm 0,01$ & $0,49 \pm 0,03$ & $1,18 \pm 0,11$ \\
\hline $2,17 \pm 0,08$ & $23,16 \pm 0,30$ & $0,05 \pm 0,01$ & $0,10 \pm 0,01$ & $0,30 \pm 0,01$ & $0,02 \pm 0,01$ & $0,52 \pm 0,02$ & $0,37 \pm 0,03$ \\
\hline $2,07 \pm 0,06$ & $24,28 \pm 0,34$ & $0,08 \pm 0,02$ & $0,10 \pm 0,01$ & $0,33 \pm 0,03$ & $0,01 \pm 0,01$ & $0,41 \pm 0,05$ & $1,33 \pm 0,10$ \\
\hline $2,07 \pm 0,05$ & $24,44 \pm 0,19$ & $0,07 \pm 0,02$ & $0,09 \pm 0,01$ & $0,34 \pm 0,02$ & u.NG. & $0,40 \pm 0,01$ & $1,16 \pm 0,03$ \\
\hline $1,92 \pm 0,30$ & $24,41 \pm 2,04$ & $0,08 \pm 0,02$ & $0,09 \pm 0,02$ & $0,31 \pm 0,02$ & $0,01 \pm 0,01$ & $0,56 \pm 0,04$ & $1,37 \pm 0,11$ \\
\hline $2,12 \pm 0,19$ & $25,52 \pm 1,04$ & $0,03 \pm 0,01$ & $0,10 \pm 0,02$ & $0,30 \pm 0,01$ & $0,01 \pm 0,01$ & $0,49 \pm 0,02$ & $0,60 \pm 0,03$ \\
\hline $2,31 \pm 0,04$ & $26,35 \pm 0,32$ & $0,13 \pm 0,02$ & $0,11 \pm 0,01$ & $0,35 \pm 0,02$ & $0,02 \pm 0,01$ & $0,41 \pm 0,01$ & $1,13 \pm 0,03$ \\
\hline $2,25 \pm 0,09$ & $25,46 \pm 0,45$ & $0,10 \pm 0,02$ & $0,11 \pm 0,01$ & $0,32 \pm 0,02$ & u.NG. & $0,44 \pm 0,02$ & $1,20 \pm 0,03$ \\
\hline $2,80 \pm 0,49$ & $23,21 \pm 2,85$ & $0,03 \pm 0,01$ & $0,13 \pm 0,03$ & $0,33 \pm 0,02$ & $0,01 \pm 0,01$ & $0,51 \pm 0,05$ & $0,64 \pm 0,09$ \\
\hline $2,29 \pm 0,09$ & $25,50 \pm 0,47$ & $0,05 \pm 0,01$ & $0,10 \pm 0,01$ & $0,31 \pm 0,01$ & u.NG. & $0,44 \pm 0,03$ & $1,06 \pm 0,03$ \\
\hline $2,09 \pm 0,06$ & $22,28 \pm 0,54$ & $0,24 \pm 0,03$ & $0,09 \pm 0,01$ & $0,28 \pm 0,03$ & $0,02 \pm 0,01$ & $0,52 \pm 0,20$ & $2,18 \pm 0,15$ \\
\hline $2,19 \pm 0,05$ & $22,40 \pm 0,30$ & $0,09 \pm 0,01$ & $0,10 \pm 0,02$ & $0,29 \pm 0,02$ & $0,01 \pm 0,01$ & $0,39 \pm 0,02$ & $1,56 \pm 0,02$ \\
\hline $2,13 \pm 0,15$ & $21,83 \pm 0,77$ & $0,07 \pm 0,01$ & $0,10 \pm 0,01$ & $0,28 \pm 0,01$ & $0,02 \pm 0,01$ & $0,53 \pm 0,04$ & $0,59 \pm 0,05$ \\
\hline $2,05 \pm 0,05$ & $21,01 \pm 0,11$ & $0,13 \pm 0,01$ & $0,09 \pm 0,01$ & $0,27 \pm 0,01$ & $0,02 \pm 0,01$ & $0,47 \pm 0,02$ & $1,78 \pm 0,05$ \\
\hline $2,25 \pm 0,04$ & $22,12 \pm 0,13$ & $0,09 \pm 0,01$ & $0,10 \pm 0,01$ & $0,29 \pm 0,01$ & $0,02 \pm 0,01$ & $0,49 \pm 0,05$ & $0,79 \pm 0,03$ \\
\hline $2,31 \pm 0,25$ & $23,42 \pm 1,35$ & $0,09 \pm 0,02$ & $0,10 \pm 0,02$ & $0,29 \pm 0,01$ & $0,03 \pm 0,01$ & $0,49 \pm 0,04$ & $0,44 \pm 0,04$ \\
\hline $2,57 \pm 0,14$ & $24,60 \pm 1,55$ & $0,25 \pm 0,04$ & $0,10 \pm 0,01$ & $0,31 \pm 0,02$ & $0,02 \pm 0,02$ & $0,54 \pm 0,03$ & $1,38 \pm 0,21$ \\
\hline $2,48 \pm 0,05$ & $26,65 \pm 0,21$ & $0,08 \pm 0,02$ & $0,10 \pm 0,01$ & $0,32 \pm 0,02$ & $0,01 \pm 0,01$ & $0,44 \pm 0,02$ & $0,73 \pm 0,02$ \\
\hline $2,50 \pm 0,14$ & $24,78 \pm 1,35$ & $0,07 \pm 0,01$ & $0,10 \pm 0,01$ & $0,32 \pm 0,02$ & $0,02 \pm 0,01$ & $0,63 \pm 0,05$ & $0,45 \pm 0,04$ \\
\hline $2,68 \pm 0,12$ & $24,12 \pm 0,50$ & $0,12 \pm 0,01$ & $0,11 \pm 0,01$ & $0,31 \pm 0,01$ & $0,02 \pm 0,02$ & $0,65 \pm 0,06$ & $0,79 \pm 0,08$ \\
\hline $2,11 \pm 0,21$ & $22,03 \pm 1,11$ & $0,07 \pm 0,01$ & $0,09 \pm 0,01$ & $0,30 \pm 0,01$ & $0,02 \pm 0,01$ & $0,38 \pm 0,04$ & $0,94 \pm 0,06$ \\
\hline $2,18 \pm 0,14$ & $21,53 \pm 0,54$ & $0,08 \pm 0,01$ & $0,09 \pm 0,01$ & $0,29 \pm 0,01$ & $0,02 \pm 0,01$ & $0,47 \pm 0,06$ & $1,33 \pm 0,08$ \\
\hline $2,01 \pm 0,13$ & $21,68 \pm 0,60$ & $0,22 \pm 0,02$ & $0,07 \pm 0,02$ & $0,29 \pm 0,01$ & u.NG. & $0,34 \pm 0,05$ & $2,28 \pm 0,08$ \\
\hline $2,11 \pm 0,10$ & $20,69 \pm 0,51$ & $0,22 \pm 0,02$ & $0,11 \pm 0,02$ & $0,28 \pm 0,01$ & u.NG. & $0,41 \pm 0,02$ & $2,20 \pm 0,07$ \\
\hline $2,12 \pm 0,03$ & $20,38 \pm 0,33$ & $0,21 \pm 0,01$ & $0,11 \pm 0,02$ & $0,28 \pm 0,02$ & u.NG. & $0,45 \pm 0,07$ & $2,19 \pm 0,08$ \\
\hline $3,65 \pm 0,10$ & $35,28 \pm 0,21$ & $0,01 \pm 0,01$ & $0,19 \pm 0,02$ & $0,21 \pm 0,02$ & u.NG. & $0,17 \pm 0,02$ & $0,01 \pm 0,01$ \\
\hline $2,32 \pm 0,06$ & $21,17 \pm 0,58$ & $0,24 \pm 0,02$ & $0,11 \pm 0,01$ & $0,29 \pm 0,01$ & u.NG. & $0,51 \pm 0,03$ & $1,28 \pm 0,05$ \\
\hline $2,46 \pm 0,10$ & $20,24 \pm 0,61$ & $0,25 \pm 0,02$ & $0,12 \pm 0,02$ & $0,30 \pm 0,02$ & $0,01 \pm 0,01$ & $0,51 \pm 0,02$ & $1,16 \pm 0,07$ \\
\hline $1,95 \pm 0,07$ & $23,90 \pm 0,47$ & & $0,08 \pm 0,01$ & $0,23 \pm 0,01$ & & $0,40 \pm 0,03$ & $1,61 \pm 0,07$ \\
\hline $1,65 \pm 0,10$ & $22,62 \pm 1,09$ & & $0,07 \pm 0,01$ & $0,21 \pm 0,01$ & & $0,23 \pm 0,02$ & $1,82 \pm 0,12$ \\
\hline $1,98 \pm 0,10$ & $21,47 \pm 1,11$ & & $0,06 \pm 0,01$ & $0,32 \pm 0,02$ & & $0,27 \pm 0,02$ & $1,66 \pm 0,14$ \\
\hline
\end{tabular}


Tabelle A.6 (Fortsetzung)

\begin{tabular}{|c|c|c|c|c|c|c|}
\hline Probe & Kugel & $\mathrm{SiO}_{2}$ & $\mathrm{Na}_{2} \mathrm{O}$ & $\mathrm{K}_{2} \mathrm{O}$ & $\mathrm{CaO}$ & $\mathrm{FeO}$ \\
\hline Eagle (EL6) ${ }^{\diamond}$ & $\mathrm{C}-8$ & $41,74 \pm 0,53$ & $0,09 \pm 0,01$ & $0,01 \pm 0,01$ & $1,11 \pm 0,07$ & $30,89 \pm 0,93$ \\
\hline Hvittis (EL6) ${ }^{\circ}$ & $\mathrm{A}-28$ & $40,63 \pm 0,23$ & $0,20 \pm 0,03$ & $0,02 \pm 0,01$ & $1,56 \pm 0,04$ & $32,83 \pm 0,50$ \\
\hline Hvittis (EL6) ${ }^{\square}$ & $\mathrm{A}-29$ & $40,61 \pm 0,39$ & $0,15 \pm 0,01$ & $0,01 \pm 0,01$ & $1,48 \pm 0,02$ & $32,62 \pm 0,45$ \\
\hline Pillistfer (EL6) ${ }^{\square}$ & $B-10$ & $39,51 \pm 0,27$ & $0,31 \pm 0,04$ & $0,02 \pm 0,01$ & $1,34 \pm 0,13$ & $34,05 \pm 1,75$ \\
\hline Pillistfer (EL6) ${ }^{\square}$ & $\mathrm{B}-11$ & $39,71 \pm 0,18$ & $0,17 \pm 0,01$ & $0,01 \pm 0,01$ & $1,43 \pm 0,02$ & $34,65 \pm 0,36$ \\
\hline Pillistfer (EL6) & $1-16$ & $39,02 \pm 0,04$ & $0,28 \pm 0,02$ & $0,03 \pm 0,01$ & $1,46 \pm 0,03$ & $33,70 \pm 0,14$ \\
\hline Pillistfer (EL6) & $1-17$ & $36,90 \pm 0,17$ & $0,04 \pm 0,01$ & $0,01 \pm 0,01$ & $1,65 \pm 0,13$ & $33,79 \pm 0,88$ \\
\hline Abee (EH4) & $5-13$ & $35,40 \pm 0,11$ & $0,65 \pm 0,02$ & $0,21 \pm 0,01$ & $1,23 \pm 0,02$ & $42,09 \pm 0,13$ \\
\hline Abee (EH4) & $5-14$ & $35,74 \pm 0,31$ & $0,66 \pm 0,05$ & $0,23 \pm 0,02$ & $1,25 \pm 0,06$ & $41,71 \pm 0,26$ \\
\hline Indarch (EH4) & $5-15$ & $37,45 \pm 0,46$ & $0,88 \pm 0,06$ & $0,15 \pm 0,01$ & $1,28 \pm 0,09$ & $39,43 \pm 0,34$ \\
\hline Indarch (EH4) & $5-16$ & $37,41 \pm 0,22$ & $0,72 \pm 0,01$ & $0,13 \pm 0,01$ & $1,24 \pm 0,03$ & $39,17 \pm 0,39$ \\
\hline Sahara $97072(\mathrm{EH} 3)^{\diamond}$ & $\mathrm{C}-27$ & $36,42 \pm 0,33$ & $0,46 \pm 0,03$ & $0,04 \pm 0,01$ & $1,28 \pm 0,07$ & $37,53 \pm 0,46$ \\
\hline St. Mark's (EH5) & $5-17$ & $37,55 \pm 0,21$ & $0,73 \pm 0,05$ & $0,06 \pm 0,01$ & $1,30 \pm 0,04$ & $39,30 \pm 0,81$ \\
\hline St. Mark's (EH5) & $5-18$ & $36,85 \pm 0,41$ & $0,68 \pm 0,05$ & $0,06 \pm 0,01$ & $1,14 \pm 0,06$ & $38,21 \pm 1,13$ \\
\hline \multicolumn{7}{|l|}{ Achondrite } \\
\hline Bilanga (DIO) & $2-27$ & $52,46 \pm 0,15$ & $0,02 \pm 0,01$ & $0,01 \pm 0,01$ & $0,79 \pm 0,02$ & $13,45 \pm 0,04$ \\
\hline Bilanga (DIO) & $2-28$ & $50,86 \pm 0,20$ & $0,03 \pm 0,01$ & $0,02 \pm 0,01$ & $0,85 \pm 0,02$ & $13,54 \pm 0,05$ \\
\hline Cachari (EUC) & $2-5$ & $45,25 \pm 0,16$ & $0,21 \pm 0,02$ & $0,04 \pm 0,01$ & $10,56 \pm 0,04$ & $19,57 \pm 0,07$ \\
\hline Cachari (EUC) & $2-6$ & $44,00 \pm 0,29$ & $0,17 \pm 0,02$ & $0,03 \pm 0,01$ & $10,79 \pm 0,07$ & $19,75 \pm 0,08$ \\
\hline Camel Donga (EUC) $)^{\diamond}$ & $\mathrm{C}-11$ & $39,65 \pm 0,09$ & $0,05 \pm 0,01$ & u.NG. & $13,07 \pm 0,03$ & $19,77 \pm 0,06$ \\
\hline Camel Donga (EUC) ${ }^{\diamond}$ & $\mathrm{C}-12$ & $44,61 \pm 0,11$ & $0,14 \pm 0,01$ & u.NG. & $11,40 \pm 0,02$ & $19,51 \pm 0,12$ \\
\hline Dhofar 182 (EUC) & $5-21$ & $50,61 \pm 0,04$ & $0,44 \pm 0,02$ & $0,05 \pm 0,01$ & $10,19 \pm 0,04$ & $17,94 \pm 0,06$ \\
\hline Dhofar 182 (EUC) & $5-22$ & $50,61 \pm 0,08$ & $0,40 \pm 0,02$ & $0,03 \pm 0,01$ & $10,18 \pm 0,03$ & $17,73 \pm 0,06$ \\
\hline Juvinas (EUC) & $5-23$ & $49,23 \pm 0,07$ & $0,33 \pm 0,01$ & $0,03 \pm 0,01$ & $10,26 \pm 0,04$ & $19,45 \pm 0,07$ \\
\hline Juvinas (EUC) & $5-24$ & $49,58 \pm 0,04$ & $0,25 \pm 0,02$ & $0,03 \pm 0,01$ & $10,35 \pm 0,04$ & $19,41 \pm 0,10$ \\
\hline Millbillilie-A (EUC) ${ }^{\diamond}$ & $\mathrm{C}-19$ & $47,55 \pm 0,05$ & $0,35 \pm 0,01$ & $0,03 \pm 0,01$ & $10,07 \pm 0,04$ & $18,97 \pm 0,06$ \\
\hline Millbillilie-A (EUC) ${ }^{\diamond}$ & $\mathrm{C}-20$ & $47,63 \pm 0,08$ & $0,33 \pm 0,01$ & $0,03 \pm 0,01$ & $10,02 \pm 0,03$ & $18,76 \pm 0,05$ \\
\hline Millbillillie-B (EUC) & $5-25$ & $48,11 \pm 0,14$ & $0,30 \pm 0,02$ & $0,02 \pm 0,01$ & $9,53 \pm 0,05$ & $20,22 \pm 0,05$ \\
\hline Millbillillie-B (EUC) & $5-26$ & $48,12 \pm 0,04$ & $0,36 \pm 0,01$ & $0,03 \pm 0,01$ & $9,40 \pm 0,04$ & $20,07 \pm 0,07$ \\
\hline NWA 3359 (EUC) & $5-27$ & $50,44 \pm 0,14$ & $0,39 \pm 0,03$ & $0,06 \pm 0,01$ & $10,17 \pm 0,06$ & $17,94 \pm 0,15$ \\
\hline NWA 3359 (EUC) & $5-28$ & $50,94 \pm 0,06$ & $0,36 \pm 0,03$ & $0,05 \pm 0,01$ & $10,27 \pm 0,05$ & $17,85 \pm 0,06$ \\
\hline Padvarninkai (EUC) $)^{\diamond}$ & $\mathrm{C}-15$ & $47,74 \pm 0,10$ & $0,33 \pm 0,01$ & $0,02 \pm 0,01$ & $10,63 \pm 0,03$ & $17,93 \pm 0,08$ \\
\hline Padvarninkai (EUC) $)^{\diamond}$ & $\mathrm{C}-16$ & $47,70 \pm 0,08$ & $0,38 \pm 0,01$ & $0,02 \pm 0,01$ & $10,54 \pm 0,05$ & $17,96 \pm 0,06$ \\
\hline NWA 1457 (WIN) ${ }^{\diamond}$ & $\mathrm{C}-34$ & $42,89 \pm 0,06$ & $0,92 \pm 0,02$ & $0,09 \pm 0,01$ & $1,78 \pm 0,02$ & $23,11 \pm 0,06$ \\
\hline NWA $1457(\text { WIN })^{\diamond}$ & $\mathrm{C}-35$ & $41,48 \pm 0,11$ & $0,56 \pm 0,01$ & $0,05 \pm 0,01$ & $1,87 \pm 0,01$ & $25,51 \pm 0,11$ \\
\hline Mount Egerton (AUB) $)^{\diamond}$ & $\mathrm{C}-36$ & $58,99 \pm 0,05$ & $0,01 \pm 0,01$ & $0,01 \pm 0,01$ & $0,47 \pm 0,01$ & $1,28 \pm 0,02$ \\
\hline Mount Egerton (AUB) ${ }^{\diamond}$ & $\mathrm{C}-37$ & $58,62 \pm 0,06$ & $0,02 \pm 0,01$ & $0,02 \pm 0,01$ & $0,48 \pm 0,01$ & $1,19 \pm 0,02$ \\
\hline NWA 4864 (Mars) & $5-35$ & $52,39 \pm 0,08$ & $1,64 \pm 0,05$ & $0,12 \pm 0,01$ & $10,81 \pm 0,03$ & $15,62 \pm 0,04$ \\
\hline NWA 4864 (Mars) & $5-36$ & $52,47 \pm 0,10$ & $1,71 \pm 0,03$ & $0,14 \pm 0,02$ & $10,90 \pm 0,05$ & $15,48 \pm 0,05$ \\
\hline SAU 051 (Mars) & $5-37$ & $47,66 \pm 0,08$ & $0,49 \pm 0,02$ & $0,02 \pm 0,01$ & $5,57 \pm 0,03$ & $18,77 \pm 0,04$ \\
\hline SAU 051 (Mars) & $5-38$ & $47,80 \pm 0,10$ & $0,25 \pm 0,02$ & $0,01 \pm 0,01$ & $5,59 \pm 0,02$ & $18,76 \pm 0,06$ \\
\hline Dhofar 911 (Mond) & $5-29$ & $43,05 \pm 0,14$ & $0,18 \pm 0,02$ & $0,01 \pm 0,01$ & $17,62 \pm 0,03$ & $3,16 \pm 0,04$ \\
\hline Dhofar 911 (Mond) & $5-30$ & $43,43 \pm 0,10$ & $0,12 \pm 0,01$ & $0,01 \pm 0,01$ & $17,69 \pm 0,06$ & $3,13 \pm 0,02$ \\
\hline Kalahari 008 (Mond) & $5-31$ & $45,55 \pm 0,11$ & $0,31 \pm 0,02$ & $0,11 \pm 0,01$ & $15,05 \pm 0,06$ & $5,31 \pm 0,04$ \\
\hline Kalahari 008 (Mond) & $5-32$ & $46,26 \pm 0,12$ & $0,42 \pm 0,03$ & $0,16 \pm 0,01$ & $15,04 \pm 0,09$ & $5,31 \pm 0,03$ \\
\hline Kalahari 009 (Mond) & $5-33$ & $47,07 \pm 0,08$ & $0,23 \pm 0,01$ & $0,07 \pm 0,01$ & $10,71 \pm 0,07$ & $19,76 \pm 0,09$ \\
\hline Kalahari 009 (Mond) & $5-34$ & $47,71 \pm 0,05$ & $0,24 \pm 0,02$ & $0,07 \pm 0,01$ & $10,79 \pm 0,05$ & $19,85 \pm 0,06$ \\
\hline \multicolumn{7}{|l|}{ Erde } \\
\hline GZG.GSP.13450 (Lherzolith) & $4-1$ & $44,30 \pm 0,17$ & $0,01 \pm 0,01$ & $0,02 \pm 0,01$ & $0,64 \pm 0,01$ & $8,13 \pm 0,12$ \\
\hline GZG.GSP.13450 (Lherzolith) & $4-2$ & $44,53 \pm 0,21$ & $0,03 \pm 0,01$ & $0,01 \pm 0,01$ & $0,64 \pm 0,01$ & $8,17 \pm 0,11$ \\
\hline De-1 (Peridotit) & $4-3$ & $47,82 \pm 0,17$ & $0,14 \pm 0,01$ & $0,01 \pm 0,01$ & $13,21 \pm 0,05$ & $6,58 \pm 0,04$ \\
\hline De-1 (Peridotit) & $4-4$ & $47,94 \pm 0,18$ & $0,10 \pm 0,01$ & $0,01 \pm 0,01$ & $13,35 \pm 0,06$ & $6,54 \pm 0,06$ \\
\hline De-2 (Peridotit) & $4-5$ & $45,63 \pm 0,20$ & $0,16 \pm 0,02$ & $0,05 \pm 0,01$ & $1,61 \pm 0,11$ & $9,22 \pm 0,38$ \\
\hline De-2 (Peridotit) & $4-6$ & $45,25 \pm 0,11$ & $0,09 \pm 0,01$ & $0,02 \pm 0,01$ & $1,49 \pm 0,02$ & $8,03 \pm 0,04$ \\
\hline De-3 (Peridotit) & $4-7$ & $43,85 \pm 0,08$ & $0,03 \pm 0,01$ & $0,01 \pm 0,01$ & $0,51 \pm 0,01$ & $8,46 \pm 0,07$ \\
\hline De-3 (Peridotit) & $4-8$ & $43,77 \pm 0,05$ & $0,02 \pm 0,01$ & $0,01 \pm 0,01$ & $0,51 \pm 0,02$ & $8,50 \pm 0,10$ \\
\hline $\mathrm{Zm}$ (Peridotit) & $4-9$ & $44,49 \pm 0,21$ & $0,08 \pm 0,01$ & $0,01 \pm 0,01$ & $1,43 \pm 0,04$ & $8,25 \pm 0,03$ \\
\hline Zm (Peridotit) & $4-10$ & $44,69 \pm 0,18$ & $0,03 \pm 0,01$ & $0,02 \pm 0,01$ & $1,43 \pm 0,04$ & $8,07 \pm 0,05$ \\
\hline SC-1 (Peridotit) & $4-11$ & $44,92 \pm 0,11$ & $0,07 \pm 0,01$ & $0,02 \pm 0,01$ & $3,08 \pm 0,04$ & $8,92 \pm 0,04$ \\
\hline SC-1 (Peridotit) & $4-12$ & $44,99 \pm 0,17$ & $0,06 \pm 0,01$ & $0,01 \pm 0,01$ & $3,07 \pm 0,05$ & $8,98 \pm 0,03$ \\
\hline SC-2 (Peridotit) & $4-13$ & $44,17 \pm 0,64$ & $0,10 \pm 0,04$ & $0,02 \pm 0,01$ & $1,55 \pm 0,32$ & $9,18 \pm 1,03$ \\
\hline SC-2 (Peridotit) & $4-14$ & $44,08 \pm 0,13$ & u.NG. & $0,01 \pm 0,01$ & $1,28 \pm 0,03$ & $8,06 \pm 0,03$ \\
\hline Ba-1 (Peridotit) & $4-15$ & $45,41 \pm 0,14$ & $0,12 \pm 0,01$ & u.NG. & $3,44 \pm 0,05$ & $8,59 \pm 0,03$ \\
\hline Ba-1 (Peridotit) & $4-16$ & $44,96 \pm 0,07$ & $0,05 \pm 0,01$ & u.NG. & $3,36 \pm 0,05$ & $8,56 \pm 0,06$ \\
\hline Ba-2 (Peridotit) & $4-17$ & $45,21 \pm 0,12$ & $0,16 \pm 0,01$ & u.NG. & $3,53 \pm 0,05$ & $8,49 \pm 0,04$ \\
\hline Ba-2 (Peridotit) & $4-18$ & $44,93 \pm 0,64$ & $0,08 \pm 0,02$ & $0,01 \pm 0,01$ & $3,09 \pm 0,61$ & $7,90 \pm 0,52$ \\
\hline Za-27 (Peridotit) & $6-15$ & $46,19 \pm 0,14$ & $0,03 \pm 0,01$ & $0,02 \pm 0,01$ & $2,41 \pm 0,06$ & $9,04 \pm 0,04$ \\
\hline Za-27 (Peridotit) & $6-16$ & $46,53 \pm 0,11$ & $0,02 \pm 0,01$ & $0,01 \pm 0,01$ & $2,61 \pm 0,02$ & $9,03 \pm 0,05$ \\
\hline PCC-1 (Peridotit) & $4-19$ & $44,56 \pm 0,16$ & $0,02 \pm 0,01$ & $0,02 \pm 0,01$ & $0,61 \pm 0,02$ & $8,07 \pm 0,05$ \\
\hline
\end{tabular}




\begin{tabular}{|c|c|c|c|c|c|c|c|}
\hline $\mathrm{Al}_{2} \mathrm{O}_{3}$ & $\mathrm{MgO}$ & $\mathrm{P}_{2} \mathrm{O}_{5}$ & $\mathrm{TiO}_{2}$ & $\mathrm{MnO}$ & $\mathrm{SO}_{3}$ & $\mathrm{Cr}_{2} \mathrm{O}_{3}$ & $\mathrm{NiO}$ \\
\hline $2,47 \pm 0,12$ & $22,56 \pm 0,76$ & & $0,08 \pm 0,01$ & $0,35 \pm 0,02$ & & $0,20 \pm 0,02$ & $0,90 \pm 0,05$ \\
\hline $2,13 \pm 0,04$ & $24,99 \pm 0,49$ & $0,08 \pm 0,02$ & $0,09 \pm 0,01$ & $0,19 \pm 0,02$ & $0,01 \pm 0,01$ & $0,38 \pm 0,03$ & $0,93 \pm 0,10$ \\
\hline $2,01 \pm 0,03$ & $24,02 \pm 0,27$ & $0,06 \pm 0,04$ & $0,10 \pm 0,01$ & $0,20 \pm 0,02$ & $0,02 \pm 0,01$ & $0,33 \pm 0,03$ & $1,01 \pm 0,05$ \\
\hline $1,90 \pm 0,15$ & $23,56 \pm 1,70$ & $0,17 \pm 0,02$ & $0,08 \pm 0,01$ & $0,20 \pm 0,02$ & $0,01 \pm 0,01$ & $0,31 \pm 0,02$ & $1,46 \pm 0,19$ \\
\hline $1,96 \pm 0,04$ & $22,87 \pm 0,15$ & $0,11 \pm 0,02$ & $0,08 \pm 0,01$ & $0,20 \pm 0,02$ & u.NG. & $0,26 \pm 0,02$ & $1,27 \pm 0,01$ \\
\hline $2,14 \pm 0,05$ & $22,04 \pm 0,21$ & $0,11 \pm 0,01$ & $0,08 \pm 0,01$ & $0,20 \pm 0,01$ & $0,02 \pm 0,01$ & $0,38 \pm 0,02$ & $0,92 \pm 0,07$ \\
\hline $2,43 \pm 0,19$ & $25,27 \pm 1,05$ & $0,03 \pm 0,01$ & $0,09 \pm 0,01$ & $0,21 \pm 0,01$ & $0,02 \pm 0,01$ & $0,35 \pm 0,02$ & $0,47 \pm 0,02$ \\
\hline $1,63 \pm 0,03$ & $16,73 \pm 0,11$ & $0,36 \pm 0,02$ & $0,06 \pm 0,01$ & $0,30 \pm 0,02$ & u.NG. & $0,42 \pm 0,02$ & $2,52 \pm 0,02$ \\
\hline $1,58 \pm 0,07$ & $16,39 \pm 0,40$ & $0,35 \pm 0,03$ & $0,06 \pm 0,02$ & $0,30 \pm 0,02$ & u.NG. & $0,40 \pm 0,02$ & $2,50 \pm 0,07$ \\
\hline $1,64 \pm 0,09$ & $17,36 \pm 0,45$ & $0,39 \pm 0,03$ & $0,09 \pm 0,03$ & $0,32 \pm 0,02$ & $0,01 \pm 0,01$ & $0,41 \pm 0,02$ & $2,22 \pm 0,11$ \\
\hline $1,61 \pm 0,05$ & $18,06 \pm 0,34$ & $0,31 \pm 0,02$ & $0,06 \pm 0,01$ & $0,30 \pm 0,02$ & u.NG. & $0,41 \pm 0,02$ & $2,30 \pm 0,04$ \\
\hline $1,57 \pm 0,05$ & $18,83 \pm 0,55$ & & $0,07 \pm 0,01$ & $0,28 \pm 0,01$ & & $0,35 \pm 0,01$ & $2,23 \pm 0,10$ \\
\hline $1,82 \pm 0,12$ & $17,97 \pm 0,80$ & $0,31 \pm 0,02$ & $0,09 \pm 0,01$ & $0,33 \pm 0,02$ & u.NG. & $0,43 \pm 0,04$ & $2,17 \pm 0,15$ \\
\hline $1,51 \pm 0,10$ & $19,80 \pm 0,91$ & $0,35 \pm 0,02$ & $0,07 \pm 0,02$ & $0,30 \pm 0,02$ & $0,01 \pm 0,01$ & $0,46 \pm 0,05$ & $2,48 \pm 0,14$ \\
\hline $0,89 \pm 0,01$ & $30,71 \pm 0,09$ & $0,02 \pm 0,01$ & $0,07 \pm 0,01$ & $0,43 \pm 0,01$ & $0,02 \pm 0,01$ & $0,70 \pm 0,01$ & $0,01 \pm 0,01$ \\
\hline $0,92 \pm 0,01$ & $32,21 \pm 0,16$ & u.NG. & $0,08 \pm 0,01$ & $0,43 \pm 0,01$ & $0,02 \pm 0,01$ & $0,66 \pm 0,01$ & $0,03 \pm 0,01$ \\
\hline $14,77 \pm 0,08$ & $7,11 \pm 0,04$ & $0,01 \pm 0,01$ & $0,55 \pm 0,02$ & $0,63 \pm 0,01$ & $0,01 \pm 0,01$ & $0,31 \pm 0,01$ & $0,02 \pm 0,01$ \\
\hline $15,17 \pm 0,16$ & $7,28 \pm 0,05$ & $0,01 \pm 0,01$ & $0,56 \pm 0,01$ & $0,63 \pm 0,01$ & $0,01 \pm 0,01$ & $0,30 \pm 0,01$ & $0,02 \pm 0,02$ \\
\hline $15,95 \pm 0,03$ & $8,60 \pm 0,03$ & & $0,83 \pm 0,01$ & $0,65 \pm 0,01$ & & $0,18 \pm 0,01$ & u.NG. \\
\hline $13,92 \pm 0,04$ & $7,58 \pm 0,04$ & & $0,72 \pm 0,01$ & $0,63 \pm 0,01$ & & $0,22 \pm 0,01$ & u.NG. \\
\hline $12,83 \pm 0,04$ & $6,54 \pm 0,04$ & $0,05 \pm 0,02$ & $0,67 \pm 0,02$ & $0,54 \pm 0,02$ & $0,01 \pm 0,01$ & $0,30 \pm 0,01$ & $0,02 \pm 0,02$ \\
\hline $12,71 \pm 0,04$ & $6,51 \pm 0,04$ & $0,04 \pm 0,02$ & $0,66 \pm 0,03$ & $0,55 \pm 0,03$ & $0,01 \pm 0,01$ & $0,30 \pm 0,01$ & u.NG. \\
\hline $11,57 \pm 0,05$ & $7,12 \pm 0,04$ & $0,02 \pm 0,01$ & $0,52 \pm 0,02$ & $0,60 \pm 0,02$ & $0,01 \pm 0,01$ & $0,33 \pm 0,02$ & $0,02 \pm 0,01$ \\
\hline $11,64 \pm 0,04$ & $7,22 \pm 0,04$ & $0,02 \pm 0,01$ & $0,53 \pm 0,01$ & $0,61 \pm 0,03$ & u.NG. & $0,31 \pm 0,02$ & u.NG. \\
\hline $13,12 \pm 0,05$ & $7,20 \pm 0,02$ & & $0,54 \pm 0,01$ & $0,57 \pm 0,01$ & & $0,28 \pm 0,01$ & u.NG. \\
\hline $13,14 \pm 0,04$ & $7,20 \pm 0,03$ & & $0,54 \pm 0,01$ & $0,58 \pm 0,01$ & & $0,16 \pm 0,01$ & u.NG. \\
\hline $12,57 \pm 0,05$ & $7,43 \pm 0,04$ & $0,04 \pm 0,02$ & $0,46 \pm 0,02$ & $0,63 \pm 0,01$ & $0,01 \pm 0,01$ & $0,31 \pm 0,01$ & $0,01 \pm 0,01$ \\
\hline $12,63 \pm 0,04$ & $7,42 \pm 0,03$ & $0,03 \pm 0,01$ & $0,45 \pm 0,01$ & $0,61 \pm 0,01$ & u.NG. & $0,30 \pm 0,01$ & $0,02 \pm 0,01$ \\
\hline $12,32 \pm 0,04$ & $6,88 \pm 0,02$ & $0,03 \pm 0,01$ & $0,75 \pm 0,01$ & $0,55 \pm 0,02$ & $0,02 \pm 0,01$ & $0,35 \pm 0,01$ & $0,01 \pm 0,01$ \\
\hline $12,44 \pm 0,04$ & $6,87 \pm 0,04$ & $0,03 \pm 0,01$ & $0,78 \pm 0,02$ & $0,53 \pm 0,01$ & $0,01 \pm 0,01$ & $0,34 \pm 0,02$ & $0,03 \pm 0,02$ \\
\hline $14,05 \pm 0,05$ & $6,50 \pm 0,02$ & & $0,52 \pm 0,01$ & $0,54 \pm 0,01$ & & $0,23 \pm 0,01$ & u.NG. \\
\hline $14,07 \pm 0,04$ & $6,53 \pm 0,02$ & & $0,54 \pm 0,01$ & $0,54 \pm 0,01$ & & $0,23 \pm 0,01$ & u.NG. \\
\hline $2,70 \pm 0,02$ & $24,90 \pm 0,05$ & & $0,13 \pm 0,01$ & $0,30 \pm 0,01$ & & $0,37 \pm 0,01$ & $1,06 \pm 0,01$ \\
\hline $2,39 \pm 0,02$ & $24,97 \pm 0,06$ & & $0,12 \pm 0,01$ & $0,29 \pm 0,01$ & & $0,33 \pm 0,01$ & $1,14 \pm 0,02$ \\
\hline $0,20 \pm 0,01$ & $39,03 \pm 0,06$ & & $0,02 \pm 0,01$ & $0,03 \pm 0,01$ & & $0,06 \pm 0,01$ & $0,04 \pm 0,01$ \\
\hline $0,20 \pm 0,01$ & $39,13 \pm 0,05$ & & $0,02 \pm 0,01$ & $0,03 \pm 0,01$ & & $0,04 \pm 0,01$ & $0,05 \pm 0,01$ \\
\hline $10,76 \pm 0,05$ & $6,94 \pm 0,04$ & $0,23 \pm 0,04$ & $0,56 \pm 0,03$ & $0,43 \pm 0,02$ & u.NG. & $0,10 \pm 0,01$ & u.NG. \\
\hline $10,77 \pm 0,07$ & $6,89 \pm 0,02$ & $0,31 \pm 0,04$ & $0,58 \pm 0,01$ & $0,43 \pm 0,02$ & $0,01 \pm 0,01$ & $0,08 \pm 0,01$ & $0,02 \pm 0,01$ \\
\hline $3,65 \pm 0,03$ & $22,06 \pm 0,03$ & $0,19 \pm 0,01$ & $0,33 \pm 0,02$ & $0,48 \pm 0,01$ & u.NG. & $0,90 \pm 0,01$ & $0,04 \pm 0,01$ \\
\hline $3,73 \pm 0,03$ & $22,20 \pm 0,04$ & $0,10 \pm 0,01$ & $0,34 \pm 0,02$ & $0,50 \pm 0,02$ & $0,01 \pm 0,01$ & $0,92 \pm 0,01$ & $0,03 \pm 0,01$ \\
\hline $27,87 \pm 0,12$ & $6,50 \pm 0,03$ & $0,02 \pm 0,01$ & $0,11 \pm 0,02$ & $0,05 \pm 0,02$ & u.NG. & $0,06 \pm 0,01$ & $0,01 \pm 0,01$ \\
\hline $28,21 \pm 0,12$ & $6,42 \pm 0,04$ & $0,01 \pm 0,01$ & $0,13 \pm 0,01$ & $0,07 \pm 0,01$ & $0,01 \pm 0,01$ & $0,07 \pm 0,01$ & $0,01 \pm 0,01$ \\
\hline $27,55 \pm 0,16$ & $4,40 \pm 0,04$ & $0,02 \pm 0,01$ & $0,42 \pm 0,02$ & $0,09 \pm 0,01$ & u.NG. & $0,11 \pm 0,02$ & $0,03 \pm 0,01$ \\
\hline $27,55 \pm 0,09$ & $4,35 \pm 0,01$ & $0,03 \pm 0,01$ & $0,40 \pm 0,02$ & $0,09 \pm 0,01$ & $0,01 \pm 0,01$ & $0,13 \pm 0,01$ & $0,02 \pm 0,01$ \\
\hline $11,60 \pm 0,05$ & $8,06 \pm 0,03$ & $0,16 \pm 0,01$ & $0,59 \pm 0,02$ & $0,34 \pm 0,02$ & $0,01 \pm 0,01$ & $0,34 \pm 0,02$ & $0,02 \pm 0,01$ \\
\hline $11,92 \pm 0,05$ & $8,04 \pm 0,03$ & $0,12 \pm 0,01$ & $0,58 \pm 0,03$ & $0,32 \pm 0,02$ & $0,01 \pm 0,01$ & $0,33 \pm 0,02$ & $0,02 \pm 0,01$ \\
\hline $0,85 \pm 0,04$ & $45,33 \pm 0,19$ & u.NG. & $0,04 \pm 0,01$ & $0,13 \pm 0,01$ & u.NG. & $0,30 \pm 0,01$ & $0,22 \pm 0,02$ \\
\hline $0,89 \pm 0,03$ & $45,33 \pm 0,11$ & u.NG. & $0,06 \pm 0,01$ & $0,13 \pm 0,01$ & u.NG. & $0,29 \pm 0,01$ & $0,25 \pm 0,01$ \\
\hline $3,45 \pm 0,02$ & $26,87 \pm 0,08$ & $0,02 \pm 0,01$ & $0,47 \pm 0,02$ & $0,13 \pm 0,01$ & u.NG. & $0,69 \pm 0,01$ & $0,07 \pm 0,01$ \\
\hline $3,52 \pm 0,04$ & $26,88 \pm 0,03$ & $0,01 \pm 0,01$ & $0,48 \pm 0,02$ & $0,13 \pm 0,01$ & u.NG. & $0,70 \pm 0,02$ & $0,05 \pm 0,02$ \\
\hline $1,66 \pm 0,10$ & $42,12 \pm 0,84$ & $0,03 \pm 0,01$ & $0,04 \pm 0,01$ & $0,14 \pm 0,01$ & u.NG. & $0,44 \pm 0,03$ & $0,28 \pm 0,02$ \\
\hline $1,49 \pm 0,04$ & $43,37 \pm 0,07$ & $0,04 \pm 0,02$ & $0,03 \pm 0,02$ & $0,14 \pm 0,01$ & u.NG. & $0,42 \pm 0,02$ & $0,22 \pm 0,01$ \\
\hline $0,80 \pm 0,02$ & $46,60 \pm 0,16$ & $0,02 \pm 0,01$ & $0,02 \pm 0,01$ & $0,12 \pm 0,01$ & u.NG. & $0,32 \pm 0,01$ & $0,24 \pm 0,02$ \\
\hline $0,83 \pm 0,02$ & $46,40 \pm 0,16$ & u.NG. & $0,04 \pm 0,02$ & $0,11 \pm 0,02$ & u.NG. & $0,34 \pm 0,02$ & $0,23 \pm 0,02$ \\
\hline $2,00 \pm 0,06$ & $43,36 \pm 0,15$ & $0,02 \pm 0,01$ & $0,07 \pm 0,01$ & $0,12 \pm 0,02$ & u.NG. & $0,40 \pm 0,02$ & $0,25 \pm 0,01$ \\
\hline $2,05 \pm 0,07$ & $43,64 \pm 0,21$ & $0,02 \pm 0,01$ & $0,03 \pm 0,01$ & $0,16 \pm 0,02$ & u.NG. & $0,39 \pm 0,01$ & $0,15 \pm 0,01$ \\
\hline $2,88 \pm 0,05$ & $39,31 \pm 0,07$ & $0,01 \pm 0,01$ & $0,12 \pm 0,02$ & $0,15 \pm 0,02$ & u.NG. & $0,44 \pm 0,01$ & $0,17 \pm 0,01$ \\
\hline $2,84 \pm 0,02$ & $39,35 \pm 0,17$ & $0,02 \pm 0,01$ & $0,13 \pm 0,01$ & $0,14 \pm 0,01$ & u.NG. & $0,45 \pm 0,01$ & $0,18 \pm 0,01$ \\
\hline $1,77 \pm 0,36$ & $43,34 \pm 2,28$ & $0,02 \pm 0,01$ & $0,07 \pm 0,02$ & $0,15 \pm 0,02$ & u.NG. & $0,38 \pm 0,04$ & $0,29 \pm 0,02$ \\
\hline $1,42 \pm 0,03$ & $44,99 \pm 0,11$ & u.NG. & $0,04 \pm 0,02$ & $0,13 \pm 0,02$ & u.NG. & $0,32 \pm 0,01$ & $0,10 \pm 0,02$ \\
\hline $3,82 \pm 0,06$ & $38,72 \pm 0,14$ & u.NG. & $0,12 \pm 0,01$ & $0,14 \pm 0,02$ & u.NG. & $0,36 \pm 0,01$ & $0,24 \pm 0,01$ \\
\hline $3,88 \pm 0,06$ & $39,21 \pm 0,11$ & $0,01 \pm 0,01$ & $0,13 \pm 0,02$ & $0,13 \pm 0,01$ & $0,01 \pm 0,01$ & $0,35 \pm 0,01$ & $0,15 \pm 0,01$ \\
\hline $3,77 \pm 0,07$ & $38,59 \pm 0,09$ & $0,01 \pm 0,01$ & $0,12 \pm 0,02$ & $0,14 \pm 0,02$ & $0,01 \pm 0,01$ & $0,38 \pm 0,02$ & $0,24 \pm 0,01$ \\
\hline $3,59 \pm 0,69$ & $41,16 \pm 2,36$ & u.NG. & $0,11 \pm 0,03$ & $0,14 \pm 0,02$ & u.NG. & $0,37 \pm 0,03$ & $0,20 \pm 0,03$ \\
\hline $2,93 \pm 0,06$ & $38,91 \pm 0,22$ & $0,02 \pm 0,01$ & $0,11 \pm 0,01$ & $0,17 \pm 0,02$ & u.NG. & $0,53 \pm 0,01$ & $0,24 \pm 0,02$ \\
\hline $2,92 \pm 0,04$ & $38,75 \pm 0,09$ & $0,02 \pm 0,01$ & $0,12 \pm 0,02$ & $0,16 \pm 0,01$ & u.NG. & $0,47 \pm 0,01$ & $0,22 \pm 0,02$ \\
\hline $0,72 \pm 0,04$ & $45,85 \pm 0,11$ & u.NG. & $0,01 \pm 0,01$ & $0,12 \pm 0,02$ & $0,01 \pm 0,01$ & $0,43 \pm 0,02$ & $0,27 \pm 0,01$ \\
\hline
\end{tabular}


Tabelle A.6 (Fortsetzung)

\begin{tabular}{|c|c|c|c|c|c|c|}
\hline Probe & Kugel & $\mathrm{SiO}_{2}$ & $\mathrm{Na}_{2} \mathrm{O}$ & $\mathrm{K}_{2} \mathrm{O}$ & $\mathrm{CaO}$ & $\mathrm{FeO}$ \\
\hline PCC-1 (Peridotit) & $4-20$ & $44,45 \pm 0,15$ & u.NG. & $0,01 \pm 0,01$ & $0,62 \pm 0,02$ & $8,03 \pm 0,08$ \\
\hline JP-1 (Peridotit) & $4-21$ & $43,55 \pm 0,24$ & $0,02 \pm 0,01$ & $0,01 \pm 0,01$ & $0,54 \pm 0,03$ & $7,84 \pm 0,12$ \\
\hline JP-1 (Peridotit) & $4-22$ & $44,11 \pm 0,07$ & $0,03 \pm 0,02$ & $0,01 \pm 0,01$ & $0,59 \pm 0,03$ & $7,97 \pm 0,12$ \\
\hline DTS-1 (Dunit) & $4-23$ & $40,81 \pm 0,12$ & u.NG. & u.NG. & $0,19 \pm 0,02$ & $8,76 \pm 0,47$ \\
\hline DTS-1 (Dunit) & $4-24$ & $40,67 \pm 0,19$ & $0,02 \pm 0,01$ & u.NG. & $0,15 \pm 0,02$ & $8,75 \pm 0,48$ \\
\hline NIM-D (Dunit) & $4-25$ & $39,41 \pm 0,39$ & $0,02 \pm 0,01$ & $0,01 \pm 0,01$ & $0,35 \pm 0,05$ & $17,62 \pm 1,32$ \\
\hline NIM-D (Dunit) & $4-26$ & $39,59 \pm 0,13$ & $0,03 \pm 0,01$ & $0,01 \pm 0,01$ & $0,27 \pm 0,02$ & $15,13 \pm 0,33$ \\
\hline NIM-P (Pyroxenit) & $4-27$ & $51,88 \pm 0,16$ & $0,34 \pm 0,02$ & $0,08 \pm 0,01$ & $2,70 \pm 0,04$ & $11,85 \pm 0,06$ \\
\hline NIM-P (Pyroxenit) & $4-28$ & $51,53 \pm 0,09$ & $0,26 \pm 0,02$ & $0,06 \pm 0,01$ & $2,63 \pm 0,01$ & $11,61 \pm 0,10$ \\
\hline UB-N (Serpentinit) & $4-29$ & $44,38 \pm 0,08$ & u.NG. & $0,02 \pm 0,01$ & $1,48 \pm 0,01$ & $7,74 \pm 0,04$ \\
\hline UB-N (Serpentinit) & $4-30$ & $45,21 \pm 0,25$ & $0,03 \pm 0,01$ & u.NG. & $1,45 \pm 0,02$ & $8,46 \pm 0,09$ \\
\hline El-1 (Serpentinit) & $6-1$ & $47,35 \pm 0,15$ & $0,02 \pm 0,01$ & u.NG. & $0,03 \pm 0,01$ & $8,50 \pm 0,08$ \\
\hline El-1 (Serpentinit) & $6-2$ & $47,33 \pm 0,12$ & $0,01 \pm 0,01$ & $0,02 \pm 0,01$ & $0,03 \pm 0,01$ & $8,63 \pm 0,06$ \\
\hline 24803 (Komatiit) & $6-5$ & $51,58 \pm 0,05$ & $0,68 \pm 0,02$ & $0,03 \pm 0,01$ & $9,26 \pm 0,04$ & $11,94 \pm 0,06$ \\
\hline 24803 (Komatiit) & $6-6$ & $52,03 \pm 0,07$ & $0,84 \pm 0,02$ & $0,03 \pm 0,01$ & $9,01 \pm 0,03$ & $11,95 \pm 0,05$ \\
\hline 26575 (Komatiit) & $6-7$ & $53,66 \pm 0,19$ & $2,55 \pm 0,03$ & $0,09 \pm 0,01$ & $10,80 \pm 0,02$ & $10,03 \pm 0,05$ \\
\hline 26575 (Komatiit) & $6-8$ & $53,92 \pm 0,06$ & $1,79 \pm 0,06$ & $0,05 \pm 0,01$ & $10,99 \pm 0,05$ & $10,15 \pm 0,05$ \\
\hline 26581 (Komatiit) & $6-9$ & $48,45 \pm 0,05$ & $0,59 \pm 0,02$ & $0,08 \pm 0,01$ & $6,63 \pm 0,02$ & $12,31 \pm 0,07$ \\
\hline 26581 (Komatiit) & $6-10$ & $48,71 \pm 0,02$ & $0,32 \pm 0,02$ & $0,05 \pm 0,01$ & $6,81 \pm 0,04$ & $12,31 \pm 0,03$ \\
\hline Za-28a (Komatiit) & $6-11$ & $46,77 \pm 0,04$ & $0,20 \pm 0,01$ & $0,09 \pm 0,01$ & $4,30 \pm 0,04$ & $10,94 \pm 0,04$ \\
\hline Za-28a (Komatiit) & $6-12$ & $46,93 \pm 0,12$ & $0,12 \pm 0,01$ & $0,04 \pm 0,01$ & $4,40 \pm 0,03$ & $10,89 \pm 0,06$ \\
\hline Za-29a (Komatiit) & $6-13$ & $54,24 \pm 0,12$ & $3,11 \pm 0,05$ & $0,63 \pm 0,01$ & $9,87 \pm 0,04$ & $11,64 \pm 0,07$ \\
\hline Za-29a (Komatiit) & $6-14$ & $54,53 \pm 0,12$ & $2,94 \pm 0,05$ & $0,60 \pm 0,02$ & $9,86 \pm 0,03$ & $11,39 \pm 0,07$ \\
\hline MOL (Tektit) & $6-25$ & $79,80 \pm 0,14$ & $0,47 \pm 0,01$ & $3,89 \pm 0,04$ & $3,68 \pm 0,02$ & $1,45 \pm 0,02$ \\
\hline MOL (Tektit) & $6-26$ & $80,07 \pm 0,12$ & $0,43 \pm 0,03$ & $3,77 \pm 0,11$ & $3,71 \pm 0,03$ & $1,47 \pm 0,05$ \\
\hline $\mathrm{KOH}$ (Tektit) & $6-27$ & $73,76 \pm 0,10$ & $1,37 \pm 0,04$ & $2,51 \pm 0,03$ & $2,37 \pm 0,02$ & $4,55 \pm 0,05$ \\
\hline $\mathrm{KOH}$ (Tektit) & $6-28$ & $73,40 \pm 0,05$ & $1,36 \pm 0,03$ & $2,53 \pm 0,02$ & $2,35 \pm 0,01$ & $4,53 \pm 0,07$ \\
\hline MUO (Tektit) & $6-29$ & $75,11 \pm 0,36$ & $1,62 \pm 0,03$ & $2,55 \pm 0,03$ & $2,07 \pm 0,08$ & $4,20 \pm 0,11$ \\
\hline MUO (Tektit) & $6-30$ & $75,81 \pm 0,33$ & $1,64 \pm 0,03$ & $2,59 \pm 0,04$ & $1,94 \pm 0,08$ & $4,06 \pm 0,08$ \\
\hline QUA (Tektit) & $6-31$ & $72,96 \pm 0,14$ & $1,38 \pm 0,03$ & $2,42 \pm 0,02$ & $2,36 \pm 0,02$ & $4,59 \pm 0,05$ \\
\hline QUA (Tektit) & $6-32$ & $73,44 \pm 0,08$ & $1,38 \pm 0,02$ & $2,42 \pm 0,03$ & $2,34 \pm 0,02$ & $4,62 \pm 0,04$ \\
\hline El-2 (Radiolarit) & $6-33$ & $77,19 \pm 0,07$ & $0,05 \pm 0,01$ & $2,98 \pm 0,05$ & $0,14 \pm 0,01$ & $9,09 \pm 0,03$ \\
\hline El-2 (Radiolarit) & $6-34$ & $76,55 \pm 0,19$ & $0,05 \pm 0,02$ & $2,94 \pm 0,06$ & $0,13 \pm 0,01$ & $9,00 \pm 0,10$ \\
\hline ES (Tonschiefer) & $2-9$ & $55,01 \pm 1,57$ & $0,46 \pm 0,06$ & $3,08 \pm 0,28$ & $0,38 \pm 0,02$ & $6,62 \pm 0,32$ \\
\hline ES (Tonschiefer) & $2-10$ & $40,65 \pm 0,63$ & $0,21 \pm 0,02$ & $1,28 \pm 0,07$ & $0,52 \pm 0,01$ & $8,89 \pm 0,07$ \\
\hline Pan-02-047 (Granit) & $6-23$ & $82,46 \pm 3,87$ & $4,07 \pm 0,66$ & $0,69 \pm 0,11$ & $1,25 \pm 0,29$ & $2,00 \pm 0,41$ \\
\hline Pan-02-047 (Granit) & $6-24$ & $77,42 \pm 2,18$ & $3,78 \pm 0,33$ & $0,73 \pm 0,05$ & $1,90 \pm 0,30$ & $2,57 \pm 0,33$ \\
\hline GZG.GSP.12569 (Obsidian) & $7-11$ & $75,38 \pm 0,14$ & $4,69 \pm 0,02$ & $3,46 \pm 0,02$ & $1,07 \pm 0,02$ & $2,31 \pm 0,02$ \\
\hline GZG.GSP.12569 (Obsidian) & $7-12$ & $75,35 \pm 0,15$ & $4,68 \pm 0,03$ & $3,49 \pm 0,03$ & $1,06 \pm 0,02$ & $2,34 \pm 0,03$ \\
\hline GSP-2 (Granodiorit) & $\mathrm{S}-6$ & $65,75 \pm 1,18$ & $1,67 \pm 0,10$ & $3,87 \pm 0,18$ & $2,43 \pm 0,14$ & $4,57 \pm 0,16$ \\
\hline GSP-2 (Granodiorit) & $\mathrm{S}-7$ & $66,93 \pm 0,59$ & $1,80 \pm 0,16$ & $3,86 \pm 0,21$ & $2,43 \pm 0,09$ & $4,56 \pm 0,08$ \\
\hline RGM-2 (Rhyolith) & $\mathrm{S}-18$ & $74,32 \pm 0,66$ & $2,91 \pm 0,22$ & $3,59 \pm 0,19$ & $1,36 \pm 0,06$ & $1,64 \pm 0,05$ \\
\hline RGM-2 (Rhyolith) & $\mathrm{S}-19$ & $72,97 \pm 0,98$ & $2,99 \pm 0,25$ & $3,68 \pm 0,21$ & $1,46 \pm 0,10$ & $1,79 \pm 0,06$ \\
\hline El-3 (Gabbro) & $6-3$ & $46,77 \pm 0,15$ & $0,01 \pm 0,01$ & $0,01 \pm 0,01$ & $2,28 \pm 0,05$ & $8,12 \pm 0,08$ \\
\hline El-3 (Gabbro) & $6-4$ & $46,51 \pm 0,13$ & $0,02 \pm 0,01$ & u.NG. & $2,26 \pm 0,02$ & $7,91 \pm 0,10$ \\
\hline LP1 (OIB) & $6-17$ & $44,96 \pm 0,11$ & $3,70 \pm 0,05$ & $1,47 \pm 0,04$ & $10,77 \pm 0,05$ & $11,96 \pm 0,04$ \\
\hline LP1 (OIB) & $6-18$ & $45,03 \pm 0,19$ & $3,97 \pm 0,08$ & $1,59 \pm 0,06$ & $10,73 \pm 0,05$ & $11,91 \pm 0,05$ \\
\hline LP4 (OIB) & $6-19$ & $44,84 \pm 0,05$ & $3,47 \pm 0,03$ & $1,42 \pm 0,03$ & $11,36 \pm 0,03$ & $12,10 \pm 0,04$ \\
\hline LP4 (OIB) & $6-20$ & $45,17 \pm 0,13$ & $3,37 \pm 0,08$ & $1,33 \pm 0,03$ & $11,45 \pm 0,05$ & $12,06 \pm 0,07$ \\
\hline SC-3 (Basalt) & $6-21$ & $45,82 \pm 0,25$ & $4,38 \pm 0,10$ & $2,56 \pm 0,06$ & $7,44 \pm 0,07$ & $11,71 \pm 0,26$ \\
\hline SC-3 (Basalt) & $6-22$ & $46,46 \pm 0,09$ & $3,48 \pm 0,05$ & $2,09 \pm 0,03$ & $7,56 \pm 0,06$ & $11,99 \pm 0,04$ \\
\hline 138 DS-1 (MORB) & $7-1$ & $48,17 \pm 0,25$ & $2,42 \pm 0,15$ & $0,28 \pm 0,05$ & $12,67 \pm 0,66$ & $7,70 \pm 1,11$ \\
\hline 138 DS-1 (MORB) & $7-2$ & $48,22 \pm 0,20$ & $2,70 \pm 0,04$ & $0,29 \pm 0,04$ & $12,14 \pm 0,49$ & $8,38 \pm 0,95$ \\
\hline 155 DS-1 (MORB) & $7-3$ & $50,39 \pm 0,12$ & $2,58 \pm 0,02$ & $0,10 \pm 0,01$ & $11,01 \pm 0,03$ & $10,11 \pm 0,04$ \\
\hline 155 DS-1 (MORB) & $7-4$ & $50,43 \pm 0,12$ & $2,54 \pm 0,02$ & $0,10 \pm 0,01$ & $11,07 \pm 0,03$ & $10,07 \pm 0,03$ \\
\hline 199 DS-2 (MORB) & $7-5$ & $50,18 \pm 0,15$ & $2,21 \pm 0,02$ & $0,05 \pm 0,01$ & $12,31 \pm 0,07$ & $9,07 \pm 0,08$ \\
\hline 199 DS-2 (MORB) & $7-6$ & $50,33 \pm 0,11$ & $2,21 \pm 0,02$ & $0,05 \pm 0,01$ & $12,26 \pm 0,03$ & $9,18 \pm 0,04$ \\
\hline 157 DS-3 (MORB) & $7-7$ & $50,78 \pm 0,08$ & $2,43 \pm 0,03$ & $0,23 \pm 0,01$ & $11,54 \pm 0,04$ & $8,93 \pm 0,05$ \\
\hline 157 DS-3 (MORB) & $7-8$ & $50,62 \pm 0,10$ & $2,42 \pm 0,02$ & $0,23 \pm 0,01$ & $11,55 \pm 0,03$ & $8,94 \pm 0,03$ \\
\hline 137 DS-4 (MORB) & $7-9$ & $50,22 \pm 0,19$ & $2,25 \pm 0,04$ & $0,07 \pm 0,01$ & $11,80 \pm 0,05$ & $9,72 \pm 0,03$ \\
\hline 137 DS-4 (MORB) & $7-10$ & $50,52 \pm 0,10$ & $2,28 \pm 0,05$ & $0,07 \pm 0,01$ & $11,78 \pm 0,03$ & $9,85 \pm 0,05$ \\
\hline BIR-1 (Basalt) & $\mathrm{S}-14$ & $47,82 \pm 0,06$ & $1,42 \pm 0,03$ & $0,02 \pm 0,01$ & $13,04 \pm 0,03$ & $10,15 \pm 0,04$ \\
\hline BIR-1 (Basalt) & $\mathrm{S}-15$ & $48,43 \pm 0,04$ & $1,08 \pm 0,04$ & $0,02 \pm 0,01$ & $13,29 \pm 0,04$ & $10,14 \pm 0,03$ \\
\hline BHVO-2 (Basalt) & $\mathrm{S}-22$ & $49,99 \pm 0,08$ & $1,70 \pm 0,06$ & $0,39 \pm 0,02$ & $11,27 \pm 0,03$ & $11,13 \pm 0,05$ \\
\hline BHVO-2 (Basalt) & $\mathrm{S}-23$ & $50,46 \pm 0,07$ & $1,30 \pm 0,02$ & $0,29 \pm 0,01$ & $11,85 \pm 0,03$ & $11,14 \pm 0,04$ \\
\hline W-2a (Diabas) & S-26 & $52,79 \pm 0,19$ & $1,72 \pm 0,15$ & $0,45 \pm 0,04$ & $11,33 \pm 0,15$ & $9,81 \pm 0,24$ \\
\hline W-2a (Diabas) & $\mathrm{S}-27$ & $53,18 \pm 0,16$ & $1,56 \pm 0,08$ & $0,46 \pm 0,03$ & $11,17 \pm 0,06$ & $9,74 \pm 0,06$ \\
\hline AGV-2 (Andesit) & $\mathrm{S}-34$ & $61,42 \pm 0,19$ & $3,33 \pm 0,05$ & $2,47 \pm 0,05$ & $5,41 \pm 0,05$ & $6,20 \pm 0,05$ \\
\hline AGV-2 (Andesit) & $\mathrm{S}-35$ & $61,30 \pm 0,15$ & $3,07 \pm 0,17$ & $2,20 \pm 0,13$ & $5,51 \pm 0,08$ & $6,19 \pm 0,06$ \\
\hline
\end{tabular}




\begin{tabular}{|c|c|c|c|c|c|c|c|}
\hline $\mathrm{Al}_{2} \mathrm{O}_{3}$ & $\mathrm{MgO}$ & $\mathrm{P}_{2} \mathrm{O}_{5}$ & $\mathrm{TiO}_{2}$ & $\mathrm{MnO}$ & $\mathrm{SO}_{3}$ & $\mathrm{Cr}_{2} \mathrm{O}_{3}$ & $\mathrm{NiO}$ \\
\hline $0,71 \pm 0,02$ & $45,90 \pm 0,11$ & u.NG. & $0,01 \pm 0,01$ & $0,12 \pm 0,01$ & u.NG. & $0,43 \pm 0,01$ & $0,15 \pm 0,02$ \\
\hline $0,68 \pm 0,04$ & $46,68 \pm 0,35$ & $0,01 \pm 0,01$ & $0,01 \pm 0,01$ & $0,12 \pm 0,01$ & $0,01 \pm 0,01$ & $0,47 \pm 0,02$ & $0,28 \pm 0,02$ \\
\hline $0,77 \pm 0,03$ & $46,10 \pm 0,16$ & $0,02 \pm 0,01$ & u.NG. & $0,15 \pm 0,01$ & $0,01 \pm 0,01$ & $0,43 \pm 0,01$ & $0,27 \pm 0,02$ \\
\hline $0,25 \pm 0,02$ & $49,99 \pm 0,37$ & $0,02 \pm 0,01$ & $0,04 \pm 0,01$ & $0,14 \pm 0,01$ & $0,01 \pm 0,01$ & $0,47 \pm 0,06$ & $0,26 \pm 0,01$ \\
\hline $0,29 \pm 0,04$ & $49,86 \pm 0,35$ & $0,02 \pm 0,01$ & $0,01 \pm 0,01$ & $0,15 \pm 0,01$ & u.NG. & $0,68 \pm 0,06$ & $0,26 \pm 0,01$ \\
\hline $0,26 \pm 0,02$ & $43,86 \pm 1,04$ & $0,01 \pm 0,01$ & $0,03 \pm 0,01$ & $0,26 \pm 0,03$ & u.NG. & $0,41 \pm 0,08$ & $0,25 \pm 0,02$ \\
\hline $0,22 \pm 0,03$ & $45,12 \pm 0,41$ & u.NG. & $0,03 \pm 0,01$ & $0,21 \pm 0,02$ & u.NG. & $0,34 \pm 0,04$ & $0,21 \pm 0,02$ \\
\hline $4,18 \pm 0,05$ & $25,45 \pm 0,14$ & $0,02 \pm 0,01$ & $0,19 \pm 0,02$ & $0,22 \pm 0,02$ & u.NG. & $3,53 \pm 0,08$ & $0,07 \pm 0,02$ \\
\hline $4,16 \pm 0,02$ & $25,67 \pm 0,07$ & $0,02 \pm 0,01$ & $0,20 \pm 0,02$ & $0,23 \pm 0,02$ & $0,01 \pm 0,01$ & $3,49 \pm 0,05$ & $0,06 \pm 0,02$ \\
\hline $3,44 \pm 0,02$ & $42,24 \pm 0,06$ & $0,01 \pm 0,01$ & $0,12 \pm 0,01$ & $0,15 \pm 0,01$ & u.NG. & $0,23 \pm 0,01$ & u.NG. \\
\hline $3,33 \pm 0,06$ & $40,62 \pm 0,07$ & $0,02 \pm 0,01$ & $0,12 \pm 0,01$ & $0,12 \pm 0,01$ & u.NG. & $0,35 \pm 0,02$ & $0,11 \pm 0,01$ \\
\hline $1,63 \pm 0,03$ & $42,06 \pm 0,19$ & u.NG. & $0,04 \pm 0,01$ & $0,13 \pm 0,02$ & $0,01 \pm 0,01$ & $0,43 \pm 0,01$ & $0,22 \pm 0,01$ \\
\hline $1,50 \pm 0,03$ & $42,58 \pm 0,11$ & u.NG. & $0,03 \pm 0,01$ & $0,14 \pm 0,02$ & u.NG. & $0,41 \pm 0,01$ & $0,15 \pm 0,02$ \\
\hline $9,88 \pm 0,05$ & $15,63 \pm 0,05$ & u.NG. & $0,49 \pm 0,02$ & $0,20 \pm 0,02$ & $0,01 \pm 0,01$ & $0,26 \pm 0,01$ & $0,05 \pm 0,02$ \\
\hline $9,58 \pm 0,06$ & $15,76 \pm 0,05$ & $0,02 \pm 0,01$ & $0,46 \pm 0,02$ & $0,23 \pm 0,02$ & u.NG. & $0,30 \pm 0,01$ & $0,05 \pm 0,01$ \\
\hline $10,31 \pm 0,06$ & $11,51 \pm 0,05$ & $0,04 \pm 0,01$ & $0,64 \pm 0,01$ & $0,14 \pm 0,01$ & $0,01 \pm 0,01$ & $0,11 \pm 0,01$ & $0,02 \pm 0,01$ \\
\hline $10,51 \pm 0,03$ & $11,65 \pm 0,04$ & $0,01 \pm 0,01$ & $0,66 \pm 0,01$ & $0,14 \pm 0,02$ & u.NG. & $0,11 \pm 0,01$ & u.NG. \\
\hline $4,45 \pm 0,02$ & $26,54 \pm 0,05$ & $0,02 \pm 0,01$ & $0,25 \pm 0,02$ & $0,22 \pm 0,02$ & u.NG. & $0,49 \pm 0,02$ & $0,17 \pm 0,02$ \\
\hline $4,56 \pm 0,02$ & $26,68 \pm 0,06$ & $0,02 \pm 0,01$ & $0,26 \pm 0,02$ & $0,23 \pm 0,02$ & $0,01 \pm 0,01$ & $0,49 \pm 0,02$ & $0,15 \pm 0,01$ \\
\hline $3,45 \pm 0,03$ & $33,44 \pm 0,09$ & $0,03 \pm 0,01$ & $0,20 \pm 0,02$ & $0,16 \pm 0,02$ & u.NG. & $0,43 \pm 0,02$ & $0,26 \pm 0,01$ \\
\hline $3,43 \pm 0,04$ & $33,58 \pm 0,05$ & $0,03 \pm 0,01$ & $0,17 \pm 0,02$ & $0,16 \pm 0,03$ & $0,01 \pm 0,01$ & $0,39 \pm 0,01$ & $0,19 \pm 0,01$ \\
\hline $10,33 \pm 0,08$ & $8,97 \pm 0,06$ & $0,07 \pm 0,01$ & $0,79 \pm 0,02$ & $0,21 \pm 0,02$ & $0,01 \pm 0,01$ & $0,09 \pm 0,01$ & $0,03 \pm 0,01$ \\
\hline $10,65 \pm 0,05$ & $8,74 \pm 0,03$ & $0,05 \pm 0,01$ & $0,80 \pm 0,02$ & $0,23 \pm 0,01$ & u.NG. & $0,07 \pm 0,01$ & u.NG. \\
\hline $8,88 \pm 0,06$ & $1,84 \pm 0,03$ & $0,06 \pm 0,01$ & $0,26 \pm 0,01$ & $0,10 \pm 0,01$ & u.NG. & $0,02 \pm 0,02$ & u.NG. \\
\hline $9,10 \pm 0,05$ & $1,85 \pm 0,03$ & $0,04 \pm 0,01$ & $0,26 \pm 0,02$ & $0,08 \pm 0,01$ & u.NG. & $0,02 \pm 0,01$ & $0,01 \pm 0,01$ \\
\hline $12,82 \pm 0,02$ & $2,03 \pm 0,02$ & $0,05 \pm 0,01$ & $0,79 \pm 0,02$ & $0,09 \pm 0,02$ & u.NG. & $0,03 \pm 0,02$ & $0,06 \pm 0,03$ \\
\hline $12,59 \pm 0,04$ & $2,01 \pm 0,03$ & $0,05 \pm 0,01$ & $0,76 \pm 0,02$ & $0,10 \pm 0,02$ & u.NG. & $0,02 \pm 0,01$ & $0,03 \pm 0,02$ \\
\hline $11,88 \pm 0,18$ & $1,87 \pm 0,05$ & $0,11 \pm 0,02$ & $0,72 \pm 0,02$ & $0,07 \pm 0,01$ & u.NG. & $0,02 \pm 0,01$ & $0,02 \pm 0,01$ \\
\hline $11,68 \pm 0,11$ & $1,79 \pm 0,07$ & $0,11 \pm 0,01$ & $0,72 \pm 0,01$ & $0,09 \pm 0,01$ & u.NG. & $0,02 \pm 0,01$ & $0,02 \pm 0,01$ \\
\hline $12,71 \pm 0,08$ & $2,07 \pm 0,03$ & $0,07 \pm 0,01$ & $0,80 \pm 0,02$ & $0,10 \pm 0,02$ & $0,01 \pm 0,01$ & $0,01 \pm 0,01$ & $0,02 \pm 0,01$ \\
\hline $12,88 \pm 0,03$ & $2,06 \pm 0,03$ & $0,06 \pm 0,01$ & $0,81 \pm 0,02$ & $0,09 \pm 0,01$ & $0,01 \pm 0,01$ & $0,03 \pm 0,01$ & $0,01 \pm 0,01$ \\
\hline $8,20 \pm 0,06$ & $1,72 \pm 0,02$ & $0,06 \pm 0,01$ & $0,28 \pm 0,02$ & $0,04 \pm 0,03$ & u.NG. & $0,02 \pm 0,01$ & $0,04 \pm 0,02$ \\
\hline $8,19 \pm 0,09$ & $1,74 \pm 0,02$ & $0,05 \pm 0,01$ & $0,31 \pm 0,01$ & $0,04 \pm 0,02$ & $0,01 \pm 0,01$ & $0,02 \pm 0,01$ & $0,02 \pm 0,01$ \\
\hline $28,38 \pm 1,49$ & $3,05 \pm 0,16$ & $0,03 \pm 0,01$ & $1,30 \pm 0,07$ & $0,11 \pm 0,01$ & $0,05 \pm 0,02$ & $0,03 \pm 0,01$ & $0,02 \pm 0,01$ \\
\hline $39,87 \pm 0,55$ & $4,29 \pm 0,06$ & $0,02 \pm 0,01$ & $1,80 \pm 0,03$ & $0,15 \pm 0,01$ & $0,01 \pm 0,01$ & $0,02 \pm 0,01$ & $0,02 \pm 0,01$ \\
\hline $10,28 \pm 2,03$ & $0,18 \pm 0,05$ & $0,03 \pm 0,01$ & $0,16 \pm 0,05$ & $0,10 \pm 0,02$ & u.NG. & $0,01 \pm 0,01$ & $0,02 \pm 0,01$ \\
\hline $13,51 \pm 1,23$ & $0,25 \pm 0,04$ & $0,04 \pm 0,01$ & $0,21 \pm 0,02$ & $0,17 \pm 0,03$ & u.NG. & u.NG. & $0,01 \pm 0,01$ \\
\hline $12,36 \pm 0,04$ & $0,14 \pm 0,01$ & $0,03 \pm 0,01$ & $0,22 \pm 0,01$ & $0,08 \pm 0,01$ & u.NG. & $0,02 \pm 0,01$ & $0,02 \pm 0,01$ \\
\hline $12,34 \pm 0,04$ & $0,12 \pm 0,01$ & $0,02 \pm 0,01$ & $0,21 \pm 0,01$ & $0,07 \pm 0,02$ & $0,01 \pm 0,01$ & $0,02 \pm 0,01$ & $0,01 \pm 0,01$ \\
\hline $18,58 \pm 0,96$ & $1,13 \pm 0,07$ & $0,09 \pm 0,02$ & $0,80 \pm 0,05$ & $0,05 \pm 0,01$ & $0,01 \pm 0,01$ & $0,02 \pm 0,01$ & $0,02 \pm 0,01$ \\
\hline $17,86 \pm 0,65$ & $1,14 \pm 0,04$ & $0,07 \pm 0,02$ & $0,81 \pm 0,04$ & $0,04 \pm 0,01$ & $0,01 \pm 0,01$ & $0,02 \pm 0,01$ & $0,02 \pm 0,01$ \\
\hline $15,72 \pm 0,76$ & $0,31 \pm 0,02$ & $0,04 \pm 0,01$ & $0,29 \pm 0,02$ & $0,03 \pm 0,01$ & u.NG. & u.NG. & $0,02 \pm 0,01$ \\
\hline $16,78 \pm 1,07$ & $0,33 \pm 0,02$ & $0,03 \pm 0,01$ & $0,32 \pm 0,02$ & $0,05 \pm 0,01$ & $0,01 \pm 0,01$ & $0,02 \pm 0,01$ & $0,01 \pm 0,01$ \\
\hline $2,32 \pm 0,05$ & $40,36 \pm 0,20$ & $0,02 \pm 0,02$ & $0,05 \pm 0,02$ & $0,15 \pm 0,02$ & u.NG. & $0,42 \pm 0,01$ & $0,25 \pm 0,01$ \\
\hline $2,24 \pm 0,02$ & $41,01 \pm 0,08$ & u.NG. & $0,06 \pm 0,01$ & $0,18 \pm 0,02$ & $0,01 \pm 0,01$ & $0,43 \pm 0,01$ & $0,17 \pm 0,01$ \\
\hline $13,92 \pm 0,05$ & $7,40 \pm 0,02$ & $0,40 \pm 0,02$ & $3,86 \pm 0,05$ & $0,19 \pm 0,02$ & u.NG. & $0,05 \pm 0,01$ & $0,02 \pm 0,01$ \\
\hline $14,10 \pm 0,07$ & $7,31 \pm 0,03$ & $0,51 \pm 0,08$ & $3,88 \pm 0,03$ & $0,21 \pm 0,02$ & u.NG. & $0,04 \pm 0,01$ & $0,02 \pm 0,01$ \\
\hline $13,48 \pm 0,05$ & $8,35 \pm 0,04$ & $0,48 \pm 0,04$ & $3,74 \pm 0,03$ & $0,22 \pm 0,02$ & u.NG. & $0,04 \pm 0,01$ & $0,04 \pm 0,01$ \\
\hline $13,55 \pm 0,03$ & $8,43 \pm 0,04$ & $0,36 \pm 0,05$ & $3,75 \pm 0,04$ & $0,22 \pm 0,02$ & u.NG. & $0,05 \pm 0,01$ & $0,04 \pm 0,01$ \\
\hline $14,55 \pm 0,29$ & $9,03 \pm 0,32$ & $0,55 \pm 0,04$ & $2,71 \pm 0,04$ & $0,19 \pm 0,01$ & u.NG. & $0,04 \pm 0,01$ & $0,04 \pm 0,01$ \\
\hline $14,81 \pm 0,06$ & $9,24 \pm 0,04$ & $0,25 \pm 0,02$ & $2,85 \pm 0,04$ & $0,19 \pm 0,01$ & u.NG. & $0,04 \pm 0,01$ & $0,03 \pm 0,02$ \\
\hline $19,53 \pm 2,13$ & $6,73 \pm 1,00$ & $0,27 \pm 0,03$ & $1,65 \pm 0,29$ & $0,16 \pm 0,02$ & $0,09 \pm 0,01$ & $0,03 \pm 0,01$ & $0,02 \pm 0,01$ \\
\hline $18,75 \pm 1,58$ & $6,71 \pm 0,84$ & $0,22 \pm 0,03$ & $1,76 \pm 0,20$ & $0,13 \pm 0,02$ & $0,10 \pm 0,02$ & $0,03 \pm 0,01$ & $0,02 \pm 0,01$ \\
\hline $15,43 \pm 0,05$ & $8,08 \pm 0,03$ & $0,08 \pm 0,01$ & $1,07 \pm 0,02$ & $0,17 \pm 0,01$ & $0,08 \pm 0,01$ & $0,05 \pm 0,01$ & $0,02 \pm 0,01$ \\
\hline $15,45 \pm 0,03$ & $8,08 \pm 0,02$ & $0,08 \pm 0,01$ & $1,08 \pm 0,02$ & $0,18 \pm 0,02$ & $0,09 \pm 0,01$ & $0,04 \pm 0,01$ & $0,02 \pm 0,01$ \\
\hline $15,02 \pm 0,16$ & $8,45 \pm 0,08$ & $0,09 \pm 0,01$ & $0,98 \pm 0,02$ & $0,16 \pm 0,02$ & $0,09 \pm 0,01$ & $0,07 \pm 0,01$ & $0,03 \pm 0,01$ \\
\hline $14,90 \pm 0,06$ & $8,47 \pm 0,03$ & $0,08 \pm 0,01$ & $1,02 \pm 0,01$ & $0,16 \pm 0,01$ & $0,10 \pm 0,01$ & $0,05 \pm 0,01$ & $0,02 \pm 0,01$ \\
\hline $14,98 \pm 0,03$ & $8,05 \pm 0,04$ & $0,14 \pm 0,01$ & $1,49 \pm 0,02$ & $0,16 \pm 0,01$ & $0,09 \pm 0,01$ & $0,06 \pm 0,01$ & $0,02 \pm 0,01$ \\
\hline $14,88 \pm 0,05$ & $8,01 \pm 0,03$ & $0,14 \pm 0,01$ & $1,50 \pm 0,02$ & $0,16 \pm 0,01$ & $0,09 \pm 0,01$ & $0,05 \pm 0,01$ & $0,03 \pm 0,01$ \\
\hline $14,57 \pm 0,07$ & $8,40 \pm 0,03$ & $0,08 \pm 0,02$ & $1,12 \pm 0,01$ & $0,18 \pm 0,01$ & $0,10 \pm 0,01$ & $0,04 \pm 0,01$ & $0,01 \pm 0,01$ \\
\hline $14,66 \pm 0,04$ & $8,36 \pm 0,04$ & $0,07 \pm 0,02$ & $1,14 \pm 0,02$ & $0,17 \pm 0,02$ & $0,11 \pm 0,01$ & $0,05 \pm 0,01$ & $0,03 \pm 0,01$ \\
\hline $15,85 \pm 0,04$ & $9,55 \pm 0,03$ & $0,03 \pm 0,01$ & $0,97 \pm 0,01$ & $0,18 \pm 0,01$ & $0,01 \pm 0,01$ & $0,05 \pm 0,01$ & $0,02 \pm 0,01$ \\
\hline $15,50 \pm 0,05$ & $9,74 \pm 0,02$ & $0,02 \pm 0,01$ & $0,97 \pm 0,02$ & $0,17 \pm 0,01$ & u.NG. & $0,05 \pm 0,01$ & $0,02 \pm 0,01$ \\
\hline $13,92 \pm 0,04$ & $7,22 \pm 0,03$ & $0,11 \pm 0,02$ & $2,79 \pm 0,03$ & $0,17 \pm 0,01$ & $0,01 \pm 0,01$ & $0,04 \pm 0,01$ & $0,03 \pm 0,01$ \\
\hline $14,06 \pm 0,05$ & $7,49 \pm 0,03$ & $0,05 \pm 0,01$ & $2,84 \pm 0,02$ & $0,17 \pm 0,02$ & $0,01 \pm 0,01$ & $0,03 \pm 0,01$ & $0,02 \pm 0,01$ \\
\hline $15,92 \pm 0,39$ & $6,60 \pm 0,14$ & $0,05 \pm 0,02$ & $1,06 \pm 0,06$ & $0,18 \pm 0,01$ & $0,01 \pm 0,01$ & $0,02 \pm 0,01$ & $0,02 \pm 0,01$ \\
\hline $15,85 \pm 0,14$ & $6,56 \pm 0,04$ & $0,05 \pm 0,01$ & $1,11 \pm 0,03$ & $0,18 \pm 0,01$ & u.NG. & $0,02 \pm 0,01$ & $0,01 \pm 0,01$ \\
\hline $17,83 \pm 0,19$ & $1,85 \pm 0,02$ & $0,23 \pm 0,03$ & $1,11 \pm 0,02$ & $0,12 \pm 0,01$ & $0,01 \pm 0,01$ & $0,01 \pm 0,01$ & $0,02 \pm 0,01$ \\
\hline $18,24 \pm 0,34$ & $1,90 \pm 0,03$ & $0,20 \pm 0,04$ & $1,13 \pm 0,02$ & $0,10 \pm 0,01$ & u.NG. & $0,01 \pm 0,01$ & $0,02 \pm 0,01$ \\
\hline
\end{tabular}




\section{A.2. Übersicht der verwendeten Schliffpräparate}

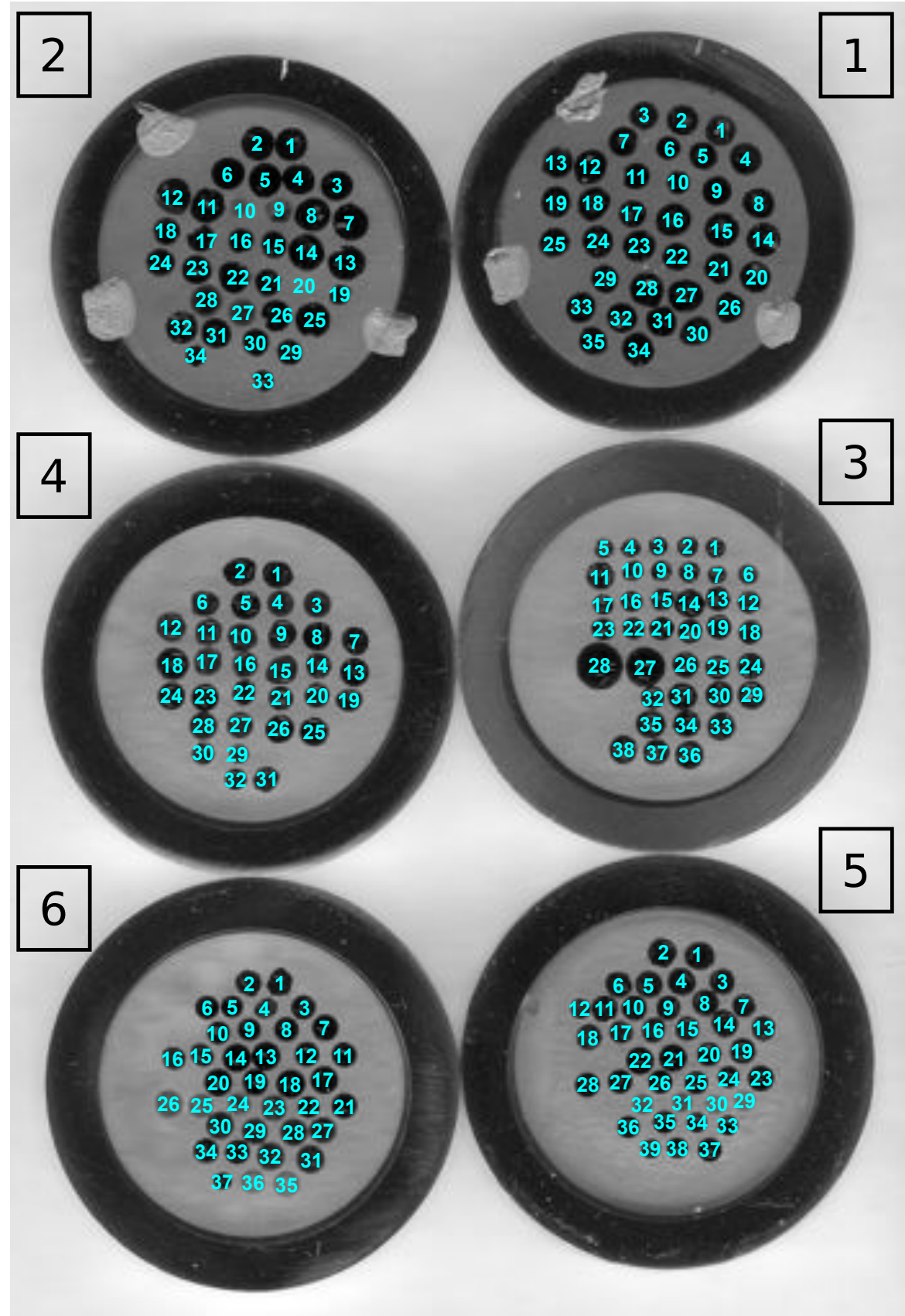

Abbildung A.1: Verwendete Schliffpräparate (1), vgl. Tabelle A.7. 


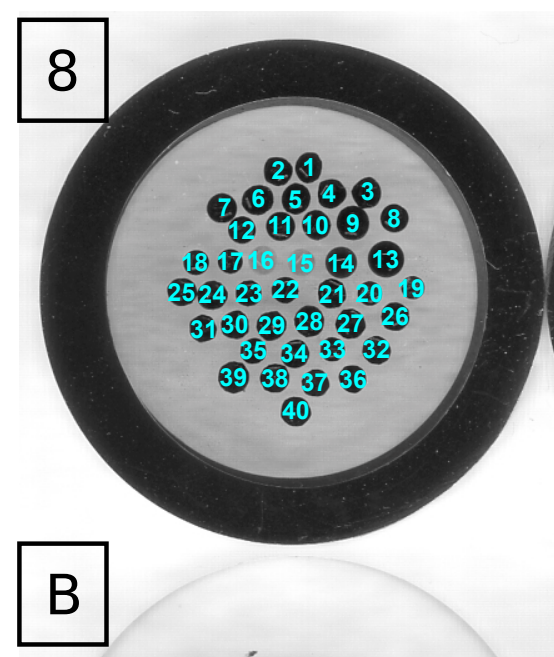

654321

(12)11 10987

1716151413

21201918

252423
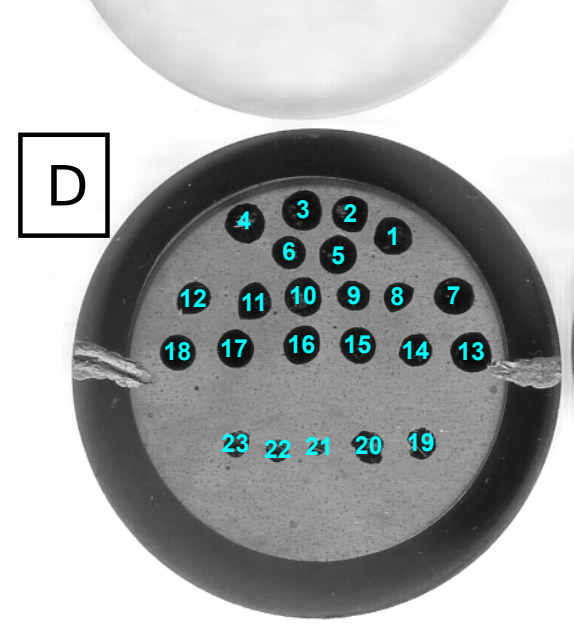

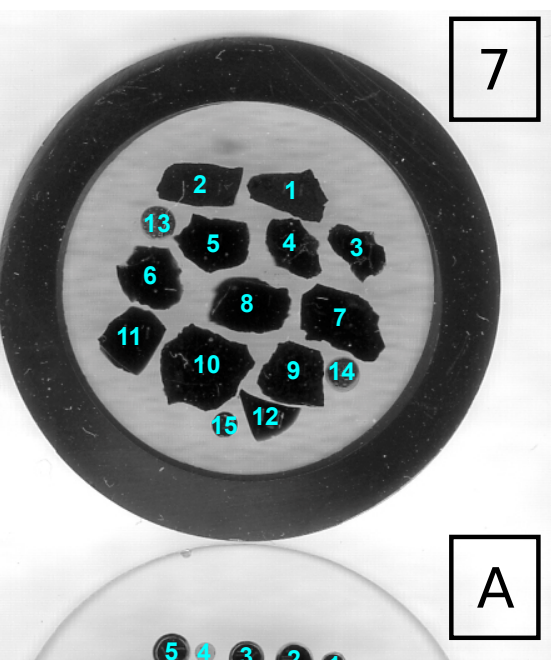

(5) (9) 8 (3) 8 (15) (14) 13 (12) 11 2019) (19) 17 (16) (26) 2524232221 (3i) (30) 292827

A
C

C

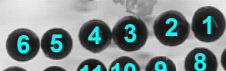

(13) (12)10987

2019) 1817161514

$2726252423 \div 21$

333231 (30) 28

$39{ }^{3}(3)$

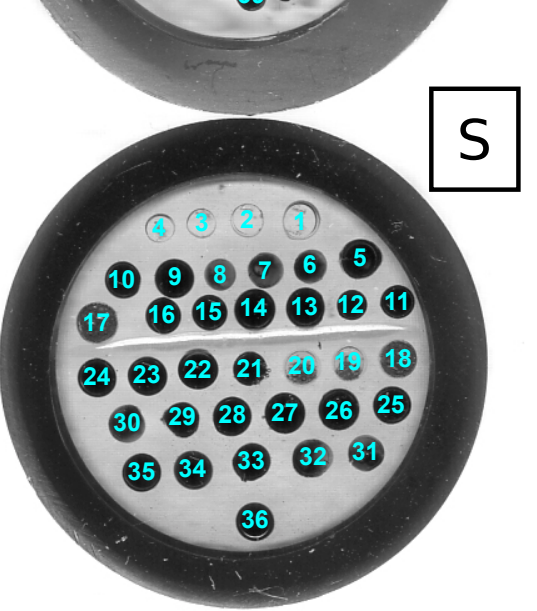

Abbildung A.2: Verwendete Schliffpräparate (2), vgl. Tabelle A.7. 
Tabelle A.7: Probenliste der verwendeten Schliffpräparate mit Angabe, wie häufig die Proben während der Levitation aufgeschmolzen wurden.

\begin{tabular}{|c|c|}
\hline \multicolumn{2}{|c|}{$\begin{array}{ll}\text { Nr. } & \text { Probe }\end{array}$} \\
\hline \multicolumn{2}{|c|}{ Schliff 1} \\
\hline 1 & NWA 869-A (L5), 1x \\
\hline 2 & NWA 869-B (L5), 1x \\
\hline 3 & NWA $869-\mathrm{C}$ (L5), 1x \\
\hline 4 & NWA $869-\mathrm{D}$ (L5), 1x \\
\hline 5 & NWA 869-E (L5), 1x \\
\hline 6 & NWA $869-\mathrm{F}$ (L5), 1x \\
\hline 7 & NWA 869-G (L5), 1x \\
\hline 8 & NWA $869-\mathrm{H}(\mathrm{L} 5), 1 \mathrm{x}$ \\
\hline 9 & NWA 869-I (L5), 1x \\
\hline 10 & NWA 869-J (L5), 1x \\
\hline 11 & NWA 869-K (L5), 1x \\
\hline 12 & Chainpur-A (LL3), 1x \\
\hline 13 & Chainpur-A (LL3), 1x \\
\hline 14 & Karoonda-A (CK4), 1x \\
\hline 15 & Karoonda-A (CK4), 1x \\
\hline 16 & Pillistfer (EL6), 1x \\
\hline 17 & Pillistfer (EL6), 1x \\
\hline 18 & Hammond Downs (H4), 1x \\
\hline 19 & Hammond Downs (H4), 1x \\
\hline 20 & Richardton (H5), 1x \\
\hline 21 & Richardton (H5), 1x \\
\hline 22 & Adrian (H4), 1x \\
\hline 23 & Adrian (H4), 1x \\
\hline 24 & Zavid (L6), 1x \\
\hline 25 & Zavid (L6), 1x \\
\hline 26 & Forest City (H5), 1x \\
\hline 27 & Forest City (H5), 1x \\
\hline 28 & Ijopega $(\mathrm{H} 6), 1 \mathrm{x}$ \\
\hline 29 & Ijopega $(\mathrm{H} 6), 1 \mathrm{x}$ \\
\hline 30 & St. Michel (L6), 1x \\
\hline 31 & St. Michel (L6), 1x \\
\hline 32 & Thuathe $(\mathrm{H} 4 / 5), 1 \mathrm{x}$ \\
\hline 33 & Thuathe $(\mathrm{H} 4 / 5), 1 \mathrm{x}$ \\
\hline 34 & Gashua (L6), 1x \\
\hline 35 & Gashua (L6), $1 \mathrm{x}$ \\
\hline \multicolumn{2}{|c|}{ Schliff 2} \\
\hline 1 & Kunya-Urgench (H5), 1x \\
\hline 2 & Kunya-Urgench (H5), 1x \\
\hline 3 & Dhurmsala (LL6), 1x \\
\hline 4 & Dhurmsala (LL6), 1x \\
\hline 5 & Cachari (EUC), 1x \\
\hline 6 & Cachari (EUC), 1x \\
\hline 7 & Gao-Guenie (H5), 1x \\
\hline 8 & Gao-Guenie (H5), 1x \\
\hline 9 & ES (Tonschiefer), 1x \\
\hline 10 & ES (Tonschiefer), $1 \mathrm{x}$ \\
\hline 11 & NWA 869 (L5), 1x \\
\hline 12 & NWA 869 (L5), 1x \\
\hline 13 & Sayh al Uhaymir 001 (L4-5), 1x \\
\hline 14 & Sayh al Uhaymir 001 (L4-5), 1x \\
\hline 15 & Murchison-A (CM2), 1x \\
\hline 16 & Murchison-A (CM2), 1x \\
\hline 17 & Murchison-B (CM2), 1x \\
\hline 18 & Murchison-B (CM2), 1x \\
\hline
\end{tabular}

\begin{tabular}{|c|c|}
\hline Nr. & Probe \\
\hline 19 & Murchison-C (CM2), 1x \\
\hline 20 & Murchison-C (CM2), 1x \\
\hline 21 & Allende-A (CV3), 1x \\
\hline 22 & Allende-A (CV3), 1x \\
\hline 23 & Allende-B (CV3), 1x \\
\hline 24 & Allende-B (CV3), 1x \\
\hline 25 & Allende-C (CV3), 1x \\
\hline 26 & Allende-C (CV3), 1x \\
\hline 27 & Bilanga (DIO), 1x \\
\hline 28 & Bilanga (DIO), $1 x$ \\
\hline 29 & Pultusk-A (H5), 1x \\
\hline 30 & Pultusk-A (H5), 1x \\
\hline 31 & Pultusk-B (H5), 1x \\
\hline 32 & Pultusk-B (H5), 1x \\
\hline 33 & Mokoia (CV3), 1x \\
\hline 34 & Mokoia (CV3), 1x \\
\hline \multicolumn{2}{|c|}{ Schliff 3} \\
\hline 1 & Alais (CI1), 1x \\
\hline 2 & Alais (CI1), 2x \\
\hline 3 & Ivuna (CI1), 1x \\
\hline 4 & Ivuna (CI1), 2x \\
\hline 5 & Ivuna (CI1), 2x \\
\hline 6 & Orgueil-B (CI1), 1x \\
\hline 7 & Orgueil-B (CI1), 2x \\
\hline 8 & Orgueil-C (CI1), 1x \\
\hline 9 & Orgueil-C (CI1), 2x \\
\hline 10 & Orgueil-C (CI1), 2x \\
\hline 11 & Orgueil-D (CI1), 1x \\
\hline 12 & Cold Bokkeveld (CM2), 1x \\
\hline 13 & Cold Bokkeveld (CM2), 1x \\
\hline 14 & Cold Bokkeveld (CM2), 2x \\
\hline 15 & Mighei (CM2), 1x \\
\hline 16 & Mighei (CM2), 1x \\
\hline 17 & Mighei (CM2), 2x \\
\hline 18 & Murchison-E (CM2), 1x \\
\hline 19 & Murchison-E (CM2), 1x \\
\hline 20 & Murchison-E (CM2), 2x \\
\hline 21 & Murray (CM2), 1x \\
\hline 22 & Murray (CM2), 1x \\
\hline 23 & Murray (CM2), 2x \\
\hline 24 & Allende-E (CV3), 2x \\
\hline 25 & Allende-E (CV3), 1x \\
\hline 26 & Allende-E (CV3), 2x \\
\hline 27 & Allende-M (CV3), 1x \\
\hline 28 & Allende-M (CV3), 2x \\
\hline 29 & Allende-S (CV3), 1x \\
\hline 30 & Allende-S (CV3), 2x \\
\hline 31 & Allende-S (CV3), 3x \\
\hline 32 & Allende-S (CV3), 4x \\
\hline 33 & Vigarano (CV3), 1x \\
\hline 34 & Vigarano (CV3), 1x \\
\hline 35 & Vigarano (CV3), 2x \\
\hline 36 & Karoonda-B (CK4), 1x \\
\hline 37 & Karoonda-B (CK4), 1x \\
\hline 38 & Karoonda-B (CK4), 2x \\
\hline
\end{tabular}


Tabelle A.7 (Fortsetzung)

\begin{tabular}{|c|c|}
\hline \multicolumn{2}{|c|}{ Nr. $\quad$ Probe } \\
\hline \multicolumn{2}{|c|}{ Schliff 4} \\
\hline 1 & GZG.GSP.13450 (Lherzolith), 1x \\
\hline 2 & GZG.GSP.13450 (Lherzolith), $2 \mathrm{x}$ \\
\hline 3 & De-1 (Peridotit), 1x \\
\hline 4 & De-1 (Peridotit), 2x \\
\hline 5 & De-2 (Peridotit), 1x \\
\hline 6 & De-2 (Peridotit), $2 \mathrm{x}$ \\
\hline 7 & De-3 (Peridotit), 1x \\
\hline 8 & De-3 (Peridotit), $2 \mathrm{x}$ \\
\hline 9 & Zm (Peridotit), 1x \\
\hline 10 & Zm (Peridotit), $2 \mathrm{x}$ \\
\hline 11 & SC-1 (Peridotit), 1x \\
\hline 12 & SC-1 (Peridotit), 2x \\
\hline 13 & SC-2 (Peridotit), $1 \mathrm{x}$ \\
\hline 14 & SC-2 (Peridotit), 2x \\
\hline 15 & Ba-1 (Peridotit), 1x \\
\hline 16 & Ba-1 (Peridotit), 2x \\
\hline 17 & Ba-2 (Peridotit), 1x \\
\hline 18 & Ba-2 (Peridotit), $2 \mathrm{x}$ \\
\hline 19 & PCC-1 (Peridotit), $1 \mathrm{x}$ \\
\hline 20 & PCC-1 (Peridotit), 2x \\
\hline 21 & JP-1 (Peridotit), 1x \\
\hline 22 & JP-1 (Peridotit), $2 \mathrm{x}$ \\
\hline 23 & DTS-1 (Dunit), 1x \\
\hline 24 & DTS-1 (Dunit), 2x \\
\hline 25 & NIM-D (Dunit), $1 \mathrm{x}$ \\
\hline 26 & NIM-D (Dunit), $2 \mathrm{x}$ \\
\hline 27 & NIM-P (Pyroxenit), 1x \\
\hline 28 & NIM-P (Pyroxenit), $2 \mathrm{x}$ \\
\hline 29 & UB-N (Serpentinit), $1 \mathrm{x}$ \\
\hline 30 & UB-N (Serpentinit), $2 \mathrm{x}$ \\
\hline 31 & Orgueil-B (CI1), 1x \\
\hline 32 & Orgueil-B (CI1), 2x \\
\hline \multicolumn{2}{|c|}{ Schliff 5} \\
\hline 1 & Chainpur-B (LL3), 1x \\
\hline 2 & Chainpur-B (LL3), 2x \\
\hline 3 & Saint-Séverin (LL6), 1x \\
\hline 4 & Saint-Séverin (LL6), 2x \\
\hline 5 & Bruderheim (L6), 1x \\
\hline 6 & Bruderheim (L6), $2 \mathrm{x}$ \\
\hline 7 & El Hammami (H5), 1x \\
\hline 8 & El Hammami (H5), 2x \\
\hline 9 & Thuathe $(\mathrm{H} 4 / 5), 1 \mathrm{x}$ \\
\hline 10 & Thuathe $(\mathrm{H} 4 / 5), 2 \mathrm{x}$ \\
\hline 11 & Thuathe $(\mathrm{H} 4 / 5), 3 \mathrm{x}$ \\
\hline 12 & Thuathe $(\mathrm{H} 4 / 5), 4 \mathrm{x}$ \\
\hline 13 & Abee $(\mathrm{EH} 4), 1 \mathrm{x}$ \\
\hline 14 & Abee (EH4), 2x \\
\hline 15 & Indarch (EH4), 1x \\
\hline 16 & Indarch (EH4), 2x \\
\hline 17 & St. Mark's (EH5), 1x \\
\hline 18 & St. Mark's (EH5), 2x \\
\hline 19 & NWA 753 (R3-6), 1x \\
\hline 20 & NWA 753 (R3-6), 2x \\
\hline 21 & Dhofar 182 (EUC), 1x \\
\hline
\end{tabular}

\begin{tabular}{ll}
\hline Nr. & Probe \\
\hline 22 & Dhofar 182 (EUC), 2x \\
23 & Juvinas (EUC), 1x \\
24 & Juvinas (EUC), 2x \\
25 & Millbillillie-B (EUC), 1x \\
26 & Millbillillie-B (EUC), 2x \\
27 & NWA 3359 (EUC), 1x \\
28 & NWA 3359 (EUC), 2x \\
29 & Dhofar 911 (Mond), 1x \\
30 & Dhofar 911 (Mond), 2x \\
31 & Kalahari 008 (Mond), 1x \\
32 & Kalahari 008 (Mond), 2x \\
33 & Kalahari 009 (Mond), 1x \\
34 & Kalahari 009 (Mond), 2x \\
35 & NWA 4864 (Mars), 1x \\
36 & NWA 4864 (Mars), 2x \\
37 & SAU 051 (Mars), 1x \\
38 & SAU 051 (Mars), 2x \\
39 & Orgueil-B (CI1), 1x \\
Schliff 6 \\
1 & El-1 (Serpentinit), 1x \\
2 & El-1 (Serpentinit), 2x \\
3 & El-3 (Gabbro), 1x \\
4 & El-3 (Gabbro), 2x \\
5 & 24803 (Komatiit), 1x \\
6 & 24803 (Komatiit), 2x \\
7 & 26575 (Komatiit), 1x \\
8 & 26575 (Komatiit), 2x \\
9 & 26581 (Komatiit), 1x \\
10 & 26581 (Komatiit), 2x \\
11 & Za-28a (Komatiit), 1x \\
12 & Za-28a (Komatiit), 2x \\
13 & ZA-29a (Komatiit), 1x \\
14 & ZA-29a (Komatiit), 2x \\
15 & Za-27 (Peridotit), 1x \\
16 & Za-27 (Peridotit), 2x \\
17 & LP1 (OIB), 1x \\
18 & LP1 (OIB), 2x \\
19 & LP4 (OIB), 1x \\
20 & LP4 (OIB), 2x \\
21 & SC-3 (Basalt), 1x \\
22 & SC-3 (Basalt), 2x \\
23 & Pan-02-047 (Granit), 1x \\
24 & Pan-02-047 (Granit), 2x \\
25 & MOL (Tektit), 1x \\
26 & MOL (Tektit), 2x \\
27 & KOH (Tektit), 1x \\
28 & KOH (Tektit), 2x \\
29 & MUO (Tektit), 1x \\
30 & MUO (Tektit), 2x \\
33 & QUA (Tektit), 1x \\
35 & El-2 (Radiolarit), 1x \\
36 & Kalkstein + SiO),, $1 \mathrm{x}$ \\
37 & Orgueil-B (CI1), 1x \\
\hline &
\end{tabular}


Tabelle A.7 (Fortsetzung)

\begin{tabular}{|c|c|c|c|}
\hline \multicolumn{2}{|c|}{ Nr. Probe } & $\mathrm{Nr}$ & Probe \\
\hline \multicolumn{2}{|c|}{ Schliff 7} & 39 & Gao-Guenie-F (H5), 2x \\
\hline 1 & 138 DS-1 (MORB) & 40 & Orgueil-B (CI1) \\
\hline 2 & 138 DS-1 (MORB) & \multicolumn{2}{|c|}{ Schliff A } \\
\hline 3 & 155 DS-1 (MORB) & 1 & Lherzolith, $2 \mathrm{x}$ \\
\hline 4 & 155 DS-1 (MORB) & 2 & Lherzolith, $2 \mathrm{x}$ \\
\hline 5 & 139 DS-2 (MORB) & 3 & Lherzolith, $2 \mathrm{x}$ \\
\hline 6 & 139 DS-2 (MORB) & 4 & Lherzolith \\
\hline 7 & 157 DS-3 (MORB) & 5 & Lherzolith $+\mathrm{Fe}, \mathrm{Ni}$, Co-Metall, $2 \mathrm{x}$ \\
\hline 8 & 157 DS-3 (MORB) & 6 & Lherzolith $+\mathrm{Fe}, \mathrm{Ni}$, Co-Metall, $2 \mathrm{x}$ \\
\hline 9 & 137 DS-4 (MORB) & 7 & Lherzolith $+\mathrm{Fe}$, Ni, Co-Metall, $2 \mathrm{x}$ \\
\hline 10 & 137 DS-4 (MORB) & 8 & Lherzolith $+\mathrm{Fe}, \mathrm{Ni}$, Co-Metall, $2 \mathrm{x}$ \\
\hline 11 & GZG.GSP.12569 (Obsidian) & 9 & Lherzolith $+\mathrm{Fe}$, Ni, Co-Metall, $2 \mathrm{x}$ \\
\hline 12 & GZG.GSP.12569 (Obsidian) & 10 & Olivin, $2 \mathrm{x}$ \\
\hline 13 & GZG.GSP.12569 (Obsidian), 1x & 11 & Olivin, $2 \mathrm{x}$ \\
\hline 14 & GZG.GSP.12569 (Obsidian), 2x & 12 & Olivin, $2 \mathrm{x}$ \\
\hline 15 & Orgueil-B (CI1) & 13 & Lherzolith $+\mathrm{Fe}$, Ni, Co-Metall, $2 \mathrm{x}$ \\
\hline \multicolumn{2}{|c|}{ Schliff 8} & 14 & Lherzolith $+\mathrm{Fe}, \mathrm{Ni}$, Co-Metall, $2 \mathrm{x}$ \\
\hline 1 & Peridotit $+\mathrm{SiO}_{2}, \mathrm{Fe}_{2} \mathrm{O}_{3}, \mathrm{NiO}$ & 15 & Allende-A (CV3), 2x \\
\hline 2 & Peridotit $+\mathrm{SiO}_{2}, \mathrm{Fe}_{2} \mathrm{O}_{3}, \mathrm{NiO}$ & 16 & Allende-A (CV3), 1x \\
\hline 3 & Peridotit $+\mathrm{SiO}_{2}, \mathrm{Fe}_{2} \mathrm{O}_{3}, \mathrm{NiO}$ & 17 & Allende-A (CV3), 1x \\
\hline 4 & Peridotit $+\mathrm{SiO}_{2}, \mathrm{Fe}_{2} \mathrm{O}_{3}, \mathrm{NiO}$ & 18 & Hammond Downs (H4), 1x \\
\hline 5 & Peridotit $+\mathrm{SiO}_{2}, \mathrm{Fe}_{2} \mathrm{O}_{3}, \mathrm{NiO}$ & 19 & Hammond Downs (H4), 2x \\
\hline 6 & Peridotit $+\mathrm{SiO}_{2}, \mathrm{Fe}_{2} \mathrm{O}_{3}, \mathrm{NiO}$ & 20 & Richardton (H5), 1x \\
\hline 7 & Peridotit $+\mathrm{Fe}_{2} \mathrm{O}_{3}, \mathrm{NiO}$ & 21 & Richardton (H5), 2x \\
\hline 8 & Peridotit $+\mathrm{Fe}_{2} \mathrm{O}_{3}, \mathrm{NiO}$ & 22 & Forest City (H5), 1x \\
\hline 9 & Peridotit $+\mathrm{Fe}_{2} \mathrm{O}_{3}, \mathrm{NiO}$ & 23 & Forest City (H5), 2x \\
\hline 10 & Peridotit $+\mathrm{Fe}_{2} \mathrm{O}_{3}, \mathrm{NiO}$ & 24 & Pultusk-A (H5), 1x \\
\hline 11 & Peridotit $+\mathrm{Fe}_{2} \mathrm{O}_{3}, \mathrm{NiO}$ & 25 & Pultusk-A (H5), 2x \\
\hline 12 & Peridotit $+\mathrm{Fe}_{2} \mathrm{O}_{3}, \mathrm{NiO}$ & 26 & Jilin (H5), 1x \\
\hline 13 & Peridotit $+\mathrm{SiO}_{2}$ & 27 & Jilin (H5), 2x \\
\hline 14 & Peridotit $+\mathrm{SiO}_{2}$ & 28 & Hvittis (EL6), 1x \\
\hline 15 & $\mathrm{SiO}_{2}$ & 29 & Hvittis (EL6), 2x \\
\hline 16 & $\mathrm{SiO}_{2}$ & 30 & Zavid (L6), 1x \\
\hline 17 & $\mathrm{Fe}_{2} \mathrm{O}_{3}$ & 31 & Zavid (L6), 2x \\
\hline 18 & $\mathrm{Fe}_{2} \mathrm{O}_{3}$ & 32 & Orgueil-A (CI1), $2 \mathrm{x}$ \\
\hline 19 & $\mathrm{Fe}_{2} \mathrm{O}_{3}+\mathrm{NiO}$ & \multicolumn{2}{|c|}{ Schliff B } \\
\hline 20 & $\mathrm{Fe}_{2} \mathrm{O}_{3}+\mathrm{NiO}$ & 1 & Ijopega $(\mathrm{H} 6), 1 \mathrm{x}$ \\
\hline 21 & Gao-Guenie, 30 min gemahlen, $1 x$ & 2 & Ijopega (H6), 2x \\
\hline 22 & Gao-Guenie, 60 min gemahlen, $1 x$ & 3 & Chainpur (LL3), 1x \\
\hline 23 & Gao-Guenie, 60 min gemahlen, $2 \mathrm{x}$ & 4 & Chainpur (LL3), 2x \\
\hline 24 & Gao-Guenie, 90 min gemahlen, $1 x$ & 5 & Chainpur (LL3) \\
\hline 25 & Gao-Guenie, 90 min gemahlen, 2x & 6 & Adrian (H4), 1x \\
\hline 26 & Gao-Guenie, 120 min gemahlen, $1 \mathrm{x}$ & 7 & Adrian (H4), 2x \\
\hline 27 & Gao-Guenie, 120 min gemahlen, $2 x$ & 8 & Mokoia (CV3), $1 \mathrm{x}$ \\
\hline 28 & Gao-Guenie-A (H5), 1x & 9 & Mokoia (CV3), 2x \\
\hline 29 & Gao-Guenie-A (H5), 2x & 10 & Pillistfer (EL6), 1x \\
\hline 30 & Gao-Guenie-B (H5), 1x & 11 & Pillistfer (EL6), 2x \\
\hline 31 & Gao-Guenie-B (H5), 2x & 12 & St. Michel (L6), 1x \\
\hline 32 & Gao-Guenie-C (H5), 1x & 13 & St. Michel (L6), 2x \\
\hline 33 & Gao-Guenie-C (H5), 2x & 14 & Karoonda-A (CK4), 1x \\
\hline 34 & Gao-Guenie-D (H5), 1x & 15 & Karoonda-A (CK4), 2x \\
\hline 35 & Gao-Guenie-D (H5), 2x & 16 & Murchison-D (CM2), 1x \\
\hline 36 & Gao-Guenie-E (H5), 1x & 17 & Murchison-D (CM2), 2x \\
\hline 37 & Gao-Guenie-E (H5), 2x & 18 & Lake Labyrinth (LL6), 1x \\
\hline 38 & Gao-Guenie-F (H5), 1x & 19 & Lake Labyrinth (LL6), 2x \\
\hline
\end{tabular}


Tabelle A.7 (Fortsetzung)

\begin{tabular}{|c|c|c|c|}
\hline & Probe & $\mathrm{Nr}$. & Probe \\
\hline 20 & Allende-B (CV3), 1x & 9 & Ijopega (H6) \\
\hline 21 & Allende-B (CV3), 2x & 10 & Ijopega (H6) \\
\hline 22 & Allende-B (CV3), 2x & 11 & Zavid (L6) \\
\hline 23 & Orgueil (CI1), 1x & 12 & Zavid (L6) \\
\hline 24 & Orgueil (CI1), 1x & 13 & St. Michel (L6) \\
\hline 25 & Orgueil (CI1), 2x & 14 & St. Michel (L6) \\
\hline \multicolumn{2}{|c|}{ Schliff C } & 15 & Hvittis (EL6) \\
\hline 1 & Mount Tazerzait (L5), 1x & 16 & Hvittis (EL6) \\
\hline 2 & Mount Tazerzait (L5), 2x & 17 & Lherzolith \\
\hline 3 & Axtell (CV3), 1x & 18 & Lherzolith \\
\hline 4 & Axtell (CV3), 2x & 19 & Orgueil-A (CI1) \\
\hline 5 & Djoumine (H5-6), 1x & 20 & Orgueil-A (CI1) \\
\hline 6 & Djoumine (H5-6), 2x & 21 & Orgueil-A (CI1) \\
\hline 7 & Eagle (EL6), 1x & 22 & Orgueil-A (CI1) \\
\hline 8 & Eagle (EL6), 2x & 23 & Orgueil-A (CI1) \\
\hline 9 & NWA 3003 (CO3), 1x & \multicolumn{2}{|c|}{ Schliff S } \\
\hline 10 & NWA 3003 (CO3), 2x & 1 & Quarz \\
\hline 11 & Camel Donga (EUC), 1x & 2 & Quarz, 1x \\
\hline 12 & Camel Donga (EUC), $2 \mathrm{x}$ & 3 & Quarz, $2 \mathrm{x}$ \\
\hline 13 & Dar al Gani 300 (H3-6), 1x & 4 & Quarz, 3x \\
\hline 14 & Dar al Gani 300 (H3-6), 2x & 5 & GSP-2 (Granodiorit) \\
\hline 15 & Padvarninkai (EUC), 1x & 6 & GSP-2 (Granodiorit), 1x \\
\hline 16 & Padvarninkai (EUC), 2x & 7 & GSP-2 (Granodiorit), 2x \\
\hline 17 & Bencubbin (CB), 1x & 8 & GSP-2 (Granodiorit), 3x \\
\hline 18 & Bencubbin $(\mathrm{CB}), 2 \mathrm{x}$ & 9 & BCR-2 (Basalt) \\
\hline 19 & Millbillillie-A (EUC), 1x & 10 & BCR-2 (Basalt), 1x \\
\hline 20 & Millbillillie-A (EUC), 2x & 11 & BCR-2 (Basalt), $2 \mathrm{x}$ \\
\hline 21 & Dar al Gani 298 (LL4), 1x & 12 & BCR-2 (Basalt), 3x \\
\hline 22 & Dar al Gani 298 (LL4), 2x & 13 & BIR-1 (Basalt) \\
\hline 23 & Atlanta (EL6), 1x & 14 & BIR-1 (Basalt), 1x \\
\hline 24 & Atlanta (EL6), 2x & 15 & BIR-1 (Basalt), 2x \\
\hline 25 & Homestead (L5), 1x & 16 & BIR-1 (Basalt), 3x \\
\hline 26 & Homestead (L5), 2x & 17 & RGM-2 (Rhyolith) \\
\hline 27 & Sahara 97072 (EH3), 1x & 18 & RGM-2 (Rhyolith), 1x \\
\hline 28 & Orgueil-E (CI1), >3x & 19 & RGM-2 (Rhyolith), 2x \\
\hline 29 & Orgueil-E (CI1), 2x & 20 & RGM-2 (Rhyolith), 3x \\
\hline 30 & Orgueil-E (CI1), 1x & 21 & BHVO-2 (Basalt) \\
\hline 31 & Orgueil-E (CI1), 2x & 22 & BHVO-2 (Basalt), 1x \\
\hline 32 & Orgueil-A (CI1), 1x & 23 & BHVO-2 (Basalt), 2x \\
\hline 33 & Orgueil-A (CI1), 2x & 24 & BHVO-2 (Basalt), 3x \\
\hline 34 & NWA 1457 (WIN), 1x & 25 & W-2a (Diabas) \\
\hline 35 & NWA 1457 (WIN), 2x & 26 & W-2a (Diabas), 1x \\
\hline 36 & Mount Egerton (AUB), 1x & 27 & W-2a (Diabas), 2x \\
\hline 37 & Mount Egerton (AUB), 2x & 28 & W-2a (Diabas), 3x \\
\hline 38 & Allende-D (CV3) & 29 & $\mathrm{QC}$ \\
\hline 39 & Allende-D (CV3) & 30 & $\mathrm{QC}, 1 \mathrm{x}$ \\
\hline \multicolumn{2}{|c|}{ Schliff D } & 31 & $\mathrm{QC}, 2 \mathrm{x}$ \\
\hline 1 & Hammond Downs (H4) & 32 & $\mathrm{QC}, 3 \mathrm{x}$ \\
\hline 2 & Hammond Downs (H4) & 33 & AGV-2 (Andesit) \\
\hline 3 & Richardton (H5) & 34 & AGV-2 (Andesit), 1x \\
\hline 4 & Richardton (H5) & 35 & AGV-2 (Andesit), 2x \\
\hline 5 & Adrian (H4) & 36 & AGV-2 (Andesit), 3x \\
\hline 6 & Adrian (H4) & & \\
\hline 7 & Forest City (H5) & & \\
\hline 8 & Forest City (H5) & & \\
\hline
\end{tabular}





\section{Literaturverzeichnis}

Alexander, C. M. O. (2005). Re-examining the role of chondrules in producing the elemental fractionations in chondrites. Meteoritics \& Planetary Science, 40(7):943-965.

Alexander, C. M. O., Grossman, J. N., Ebel, D. S., und Ciesla, F. J. (2008). The Formation Conditions of Chondrules and Chondrites. Science, 320(5883):16171619.

Allègre, C., Manhès, G., und Lewin, É. (2001). Chemical composition of the Earth and the volatility control on planetary genetics. Earth and Planetary Science Letters, 185(1-2):49-69.

Amelin, Y., Kaltenbach, A., Iizuka, T., Stirling, C. H., Ireland, T. R., Petaev, M., und Jacobsen, S. B. (2010). U-Pb chronology of the Solar System's oldest solids with variable ${ }^{238} \mathrm{U} /{ }^{235} \mathrm{U}$. Earth and Planetary Science Letters, 300(3-4):343-350.

Anders, E. und Grevesse, N. (1989). Abundances of the elements: Meteoritic and solar. Geochimica et Cosmochimica Acta, 53(1):197-214.

Asphaug, E., Jutzi, M., und Movshovitz, N. (2011). Chondrule formation during planetesimal accretion. Earth and Planetary Science Letters, 308(3-4):369-379.

Barrat, J. A., Zanda, B., Moynier, F., Bollinger, C., Liorzou, C., und Bayon, G. (2012). Geochemistry of CI chondrites: Major and trace elements, and $\mathrm{Cu}$ and Zn Isotopes. Geochimica et Cosmochimica Acta, 83:79-92.

Bau, M. (1996). Controls on the fractionation of isovalent trace elements in magmatic and aqueous systems: evidence from $\mathrm{Y} / \mathrm{Ho}, \mathrm{Zr} / \mathrm{Hf}$, and lanthanide tetrad effect. Contributions to Mineralogy and Petrology, 123(3):323-333.

Bischoff, A. (2001). Meteorite classification and the definition of new chondrite classes as a result of successful meteorite search in hot and cold deserts. Planetary and Space Science, 49(8):769-776.

Bischoff, A. und Keil, K. (1984). Al-rich objects in ordinary chondrites: Related origin of carbonaceous and ordinary chondrites and their constituents. Geochimica et Cosmochimica Acta, 48(4):693-709. 
Bizzarro, M., Baker, J. A., und Haack, H. (2004). Mg isotope evidence for contemporaneous formation of chondrules and refractory inclusions. Nature, 431(7006):275278.

Bland, P. A., Alard, O., Benedix, G. K., Kearsley, A. T., Menzies, O. N., Watt, L. E., und Rogers, N. W. (2005). Volatile fractionation in the early solar system and chondrule/matrix complementarity. Proceedings of the National Academy of Sciences of the United States of America, 102(39):13755-13760.

Bonal, L., Quirico, E., Bourot-Denise, M., und Montagnac, G. (2006). Determination of the petrologic type of CV3 chondrites by Raman spectroscopy of included organic matter. Geochimica et Cosmochimica Acta, 70(7):1849-1863.

Borisov, A., Pack, A., Kropf, A., und Palme, H. (2008). Partitioning of Na between olivine and melt: An experimental study with application to the formation of meteoritic $\mathrm{Na}_{2} \mathrm{O}$-rich chondrule glass and refractory forsterite grains. Geochimica et Cosmochimica Acta, 72(22):5558-5573.

Boss, A. P. und Durisen, R. H. (2005). Chondrule-forming Shock Fronts in the Solar Nebula: A Possible Unified Scenario for Planet and Chondrite Formation. The Astrophysical Journal Letters, 621:L137-L140.

Bouvier, A., Vervoort, J. D., und Patchett, P. J. (2008). The Lu-Hf and Sm-Nd isotopic composition of CHUR: Constraints from unequilibrated chondrites and implications for the bulk composition of terrestrial planets. Earth and Planetary Science Letters, 273(1-2):48-57.

Bouvier, A. und Wadhwa, M. (2010). The age of the Solar System redefined by the oldest $\mathrm{Pb}-\mathrm{Pb}$ age of a meteoritic inclusion. Nature Geoscience, 3(9):637-641.

Boynton, W. V. (1975). Fractionation in the solar nebula: condensation of yttrium and the rare earth elements. Geochimica et Cosmochimica Acta, 39(5):569-584.

Boynton, W. V. (1984). Cosmochemistry of the Rare Earth Elements: Meteorite studies. In: Henderson, P. (Hrsg.), Rare Earth Element Geochemistry, Developments in Geochemistry, Band 2, Seiten 90-114. Elsevier, New York.

Boynton, W. V. (1989). Cosmochemistry of the rare earth elements: Condensation and evaporation processes. In: Lipin, B. R. und McKay, G. A. (Hrsg.), Geochemistry and mineralogy of rare earth elements, Reviews in Mineralogy, Seiten 1-24. Mineralogical Society of America, Washington, DC.

Boynton, W. V., Frazier, R. M., und MacDougall, J. D. (1980). Identification of an Ultra-Refractory Component in the Murchison Meteorite. In: Lunar and Planetary Institute Science Conference Abstracts, Band 11, Seiten 103-105. 
Burkhardt, C., Kleine, T., Bourdon, B., Palme, H., Zipfel, J., Friedrich, J. M., und Ebel, D. S. (2008). Hf-W mineral isochron for Ca,Al-rich inclusions: Age of the solar system and the timing of core formation in planetesimals. Geochimica et Cosmochimica Acta, 72(24):6177-6197.

Busemann, H., Young, A. F., Alexander, C. M. O., Hoppe, P., Mukhopadhyay, S., und Nittler, L. R. (2006). Interstellar Chemistry Recorded in Organic Matter from Primitive Meteorites. Science, 312(5774):727-730.

Caro, G., Bourdon, B., Halliday, A. N., und Quitte, G. (2008). Super-chondritic $\mathrm{Sm} / \mathrm{Nd}$ ratios in Mars, the Earth and the Moon. Nature, 452(7185):336-339.

Chambers, J. E. (2004). Planetary accretion in the inner Solar System. Earth and Planetary Science Letters, 223(3-4):241-252.

Ciesla, F. J. (2007). Outward Transport of High-Temperature Materials Around the Midplane of the Solar Nebula. Science, 318(5850):613-615.

Clayton, R. N., Mayeda, T. K., und Rubin, A. E. (1984). Oxygen isotopic compositions of enstatite chondrites and aubrites. Journal of Geophysical Research: Solid Earth, 89(S01):C245-C249.

Connelly, J. N., Bizzarro, M., Krot, A. N., Nordlund, A., Wielandt, D., und Ivanova, M. A. (2012). The Absolute Chronology and Thermal Processing of Solids in the Solar Protoplanetary Disk. Science, 338(6107):651-655.

Crozaz, G., Floss, C., und Wadhwa, M. (2003). Chemical alteration and REE mobilization in meteorites from hot and cold deserts. Geochimica et Cosmochimica Acta, 67(24):4727-4741.

Crozaz, G. und Wadhwa, M. (2001). The terrestrial alteration of Saharan Shergottites Dar al Gani 476 and 489: A case study of weathering in a hot desert environment. Geochimica et Cosmochimica Acta, 65(6):971-977.

Cuzzi, J. N., Davis, S. S., und Dobrovolskis, A. R. (2003). Blowing in the wind. II. Creation and redistribution of refractory inclusions in a turbulent protoplanetary nebula. Icarus, 166(2):385-402.

Davis, A. M. (1991). Ultrarefractory inclusions and the nature of the group II REE fractionation (abstract). Meteoritics, 26:330.

Desch, S. J. und Connolly, H. C. (2002). A model of the thermal processing of particles in solar nebula shocks: Application to the cooling rates of chondrules. Meteoritics \&f Planetary Science, 37(2):183-207. 
Dulski, P. (2001). Reference Materials for Geochemical Studies: New Analytical Data by ICP-MS and Critical Discussion of Reference Values. Geostandards Newsletter, 25(1):87-125.

El Goresy, A., Zinner, E., Matsunami, S., Palme, H., Spettel, B., Lin, Y., und Nazarov, M. (2002). Efremovka 101.1: A CAI with ultrarefractory REE patterns and enormous enrichments of Sc, Zr, and Y in Fassaite and Perovskite. Geochimica et Cosmochimica Acta, 66(8):1459-1491.

Evensen, N. M., Hamilton, P. J., und O'Nions, R. K. (1978). Rare-earth abundances in chondritic meteorites. Geochimica et Cosmochimica Acta, 42(8):1199-1212.

Fahey, A. J., Zinner, E., Kurat, G., und Kracher, A. (1994). Hibonite-hercynite inclusion HH-1 from the Lancé (CO3) meteorite: The history of an ultrarefractory CAI. Geochimica et Cosmochimica Acta, 58(21):4779-4793.

Frank, D., Zolensky, M., Martinez, J., Mikouchi, T., Ohsumi, K., Hagiya, K., Satake, W., Le, L., Ross, D., und Peslier, A. (2011). A CAI in the Ivuna CI1 Chondrite. In: Lunar and Planetary Institute Science Conference Abstracts, Band 42, Seite 2785 .

Gao, S., Liu, X., Yuan, H., Hattendorf, B., Günther, D., Chen, L., und Hu, S. (2002). Determination of Forty Two Major and Trace Elements in USGS and NIST SRM Glasses by Laser Ablation-Inductively Coupled Plasma-Mass Spectrometry. Geostandards Newsletter, 26(2):181-196.

Greenwood, R. C., Franchi, I. A., Kearsley, A. T., und Alard, O. (2010). The relationship between CK and CV chondrites. Geochimica et Cosmochimica Acta, 74(5):1684-1705.

Grevesse, N., Asplund, M., und Sauval, A. J. (2007). The Solar Chemical Composition. Space Science Reviews, 130(1-4):105-114.

Grossman, L. und Ganapathy, R. (1976a). Trace elements in the Allende meteorite-I. Coarse-grained, Ca-rich inclusions. Geochimica et Cosmochimica Acta, 40(3):331344 .

Grossman, L. und Ganapathy, R. (1976b). Trace elements in the Allende meteoriteII. Fine-grained, Ca-rich inclusions. Geochimica et Cosmochimica Acta, 40(8):967977.

Grossman, L., Ganapathy, R., Methot, R. L., und Davis, A. M. (1979). Trace elements in the Allende meteorite-IV. Amoeboid olivine aggregates. Geochimica et Cosmochimica Acta, 43(6):817-829. 
Hewins, R. H., Connolly Jr., H. C., Lofgren, G. E., und Libourel, G. (2005). Experimental Constraints on Chondrule Formation. In: Krot, A. N., Scott, E. R. D., und Reipurth, B. (Hrsg.), Chondrites and the Protoplanetary Disk, Band 341 von Astronomical Society of the Pacific Conference Series, Seiten 286-316.

Hezel, D. C. und Palme, H. (2008). Constraints for chondrule formation from $\mathrm{Ca}-\mathrm{Al}$ distribution in carbonaceous chondrites. Earth and Planetary Science Letters, 265(3-4):716-725.

Hezel, D. C. und Palme, H. (2010). The chemical relationship between chondrules and matrix and the chondrule matrix complementarity. Earth and Planetary Science Letters, 294(1-2):85-93.

Hezel, D. C., Russell, S. S., Ross, A. J., und Kearsley, A. T. (2008). Modal abundances of CAIs: Implications for bulk chondrite element abundances and fractionations. Meteoritics \& Planetary Science, 43(11):1879-1894.

Hood, L. L., Ciesla, F. J., Artemieva, N. A., Marzari, F., und Weidenschilling, S. J. (2009). Nebular shock waves generated by planetesimals passing through Jovian resonances: Possible sites for chondrule formation. Meteoritics 85 Planetary Science, 44(3):327-342.

Huang, F., Li, S., Dong, F., Li, Q., Chen, F., Wang, Y., und Yang, W. (2007). Recycling of deeply subducted continental crust in the Dabie Mountains, central China. Lithos, 96(1-2):151-169.

Huang, S., Farkaš, J., Yu, G., Petaev, M. I., und Jacobsen, S. B. (2012). Calcium isotopic ratios and rare earth element abundances in refractory inclusions from the Allende CV3 chondrite. Geochimica et Cosmochimica Acta, 77:252-265.

Hutchison, R. (2004). Meteorites: A petrologic, chemical, and isotopic synthesis. Cambridge University Press.

Ireland, T. R. (1990). Presolar isotopic and chemical signatures in hibonite-bearing refractory inclusions from the Murchison carbonaceous chondrite. Geochimica et Cosmochimica Acta, 54(11):3219-3237.

Ireland, T. R., Fahey, A. J., und Zinner, E. K. (1988). Trace-element abundances in hibonites from the Murchison carbonaceous chondrite: Constraints on hightemperature processes in the solar nebula. Geochimica et Cosmochimica Acta, 52(12):2841-2854.

Jacquet, E., Alard, O., und Gounelle, M. (2012). Chondrule trace element geochemistry at the mineral scale. Meteoritics \& Planetary Science, 47(11):1695-1714. 
Jarosewich, E. (1990). Chemical analyses of meteorites: A compilation of stony and iron meteorite analyses. Meteoritics, 25(4):323-337.

Jarosewich, E., Clarke Jr., R. S., und Barrows, J. N. (1987). The Allende Meteorite Reference Sample. Smithsonian Contributions to the Earth Sciences, 27.

Javoy, M. (1995). The integral enstatite chondrite model of the Earth. Geophysical Research Letters, 22(16):2219-2222.

Javoy, M., Kaminski, E., Guyot, F., Andrault, D., Sanloup, C., Moreira, M., Labrosse, S., Jambon, A., Agrinier, P., Davaille, A., und Jaupart, C. (2010). The chemical composition of the Earth: Enstatite chondrite models. Earth and Planetary Science Letters, 293(3-4):259-268.

Jenner, F. E. und O'Neill, H. St. C. (2012a). Analysis of 60 elements in 616 ocean floor basaltic glasses. Geochemistry, Geophysics, Geosystems, 13(2).

Jenner, F. E. und O'Neill, H. St. C. (2012b). Major and trace analysis of basaltic glasses by laser-ablation ICP-MS. Geochemistry, Geophysics, Geosystems, 13(3).

Jochum, K. P., Stolz, A. J., und McOrist, G. (2000). Niobium and tantalum in carbonaceous chondrites: Constraints on the solar system and primitive mantle niobium/tantalum, zirconium/niobium, and niobium/uranium ratio. Meteoritics Ef Planetary Science, 35(2):229-235.

Jochum, K. P., Weis, U., Stoll, B., Kuzmin, D., Yang, Q., Raczek, I., Jacob, D. E., Stracke, A., Birbaum, K., Frick, D. A., et al. (2011). Determination of Reference Values for NIST SRM 610-617 Glasses Following ISO Guidelines. Geostandards and Geoanalytical Research, 35(4):397-429.

Jones, R. H., Grossman, J. N., und Rubin, A. E. (2005). Chemical, Mineralogical and Isotopic Properties of Chondrules: Clues to Their Origin. In: Krot, A. N., Scott, E. R. D., und Reipurth, B. (Hrsg.), Chondrites and the Protoplanetary Disk, Band 341 von Astronomical Society of the Pacific Conference Series, Seiten 251-285.

Jones, R. H. und Norman, M. D. (2008). Trace Elements in Aluminum-rich Chondrules from the Mokoia CV Chondrite. In: Lunar and Planetary Institute Science Conference Abstracts, Band 39, Seite 2051.

Kallemeyn, G. W., Boynton, W. V., Willis, J., und Wasson, J. T. (1978). Formation of the Bencubbin polymict meteoritic breccia. Geochimica et Cosmochimica Acta, 42(5):507-515. 
Kallemeyn, G. W. und Wasson, J. T. (1979). Refractory element fractionations among carbonaceous chondrite groups. Nature, 282(5741):827-829.

Kallemeyn, G. W. und Wasson, J. T. (1981). The compositional classification of chondrites-I. The carbonaceous chondrite groups. Geochimica et Cosmochimica Acta, 45(7):1217-1230.

King, E. A., Schonfeld, E., Richardson, K. A., und Eldridge, J. S. (1969). Meteorite Fail at Pueblito de Allende, Chihuahua, Mexico: Preliminary Information. Science, 163(3870):928-929.

Klerner, S. und Palme, H. (1999). Origin of Chondrules and Matrix in Carbonaceous Chondrites. In: Lunar and Planetary Institute Science Conference Abstracts, Band 30, Seite 1272.

König, S., Münker, C., Schuth, S., und Garbe-Schönberg, D. (2008). Mobility of tungsten in subduction zones. Earth and Planetary Science Letters, 274(1-2):8292.

Kornacki, A. S. und Fegley, B. J. (1986). The abundance and relative volatility of refractory trace elements in Allende Ca,Al-rich inclusions: implications for chemical and physical processes in the solar nebula. Earth and Planetary Science Letters, 79(3-4):217-234.

Krot, A. N., Amelin, Y., Bland, P., Ciesla, F. J., Connelly, J., Davis, A. M., Huss, G. R., Hutcheon, I. D., Makide, K., Nagashima, K., et al. (2009). Origin and chronology of chondritic components: A review. Geochimica et Cosmochimica Acta, 73(17):4963-4997.

Krot, A. N., Keil, K., Goodrich, C. A., Scott, E. R. D., und Weisberg, M. K. (2003). Classification of Meteorites. In: Davis, A. M., Holland, H. D., und Turekian, K. K. (Hrsg.), Treatise on Geochemistry: Meteorites, Comets and Planets, Band 1, Seiten 83-128. Elsevier.

Lahaye, Y., Lambert, D., und Walters, S. (1997). Ultraviolet Laser Sampling and High Resolution Inductively Coupled Plasma-Mass Spectrometry of NIST and BCR-2G Glass Reference Materials. Geostandards Newsletter, 21(2):205-214.

Larimer, J. W. und Anders, E. (1970). Chemical fractionations in meteorites-III. Major element fractionations in chondrites. Geochimica et Cosmochimica Acta, $34(3): 367-387$.

Liu, Y.-G. und Schmitt, R. A. (1988). The Relative Nebular Locations for the Formation of Parental Components in the CV3 Chondrites Kaba, Mokoia and 
Allende. In: Lunar and Planetary Institute Science Conference Abstracts, Band 19, Seite 684 .

Lodders, K. (2003). Solar System Abundances and Condensation Temperatures of the Elements. The Astrophysical Journal, 591:1220-1247.

Lodders, K., Palme, H., und Gail, H. P. (2009). Abundances of the elements in the solar system. In: Trümper, J. E. (Hrsg.), Landolt-Börnstein - Group VI Astronomy and Astrophysics, Band 4B, Kapitel 4.4, Seiten 560-598. SpringerVerlag.

MacPherson, G. J. und Davis, A. M. (1994). Refractory inclusions in the prototypical CM chondrite, Mighei. Geochimica et Cosmochimica Acta, 58(24):5599-5625.

MacPherson, G. J., Davis, A. M., und Grossman, J. N. (1989). Refractory Inclusions in the Unique Chondrite ALH85085. LPI Contributions, 712:143.

MacPherson, G. J. und Huss, G. R. (2005). Petrogenesis of Al-rich chondrules: Evidence from bulk compositions and phase equilibria. Geochimica et Cosmochimica Acta, 69(12):3099-3127.

MacPherson, G. J., Kita, N. T., Ushikubo, T., Bullock, E. S., und Davis, A. M. (2012). Well-resolved variations in the formation ages for $\mathrm{Ca}-\mathrm{Al}$-rich inclusions in the early Solar System. Earth and Planetary Science Letters, 331-332:43-54.

Mao, X.-Y., Ward, B. J., Grossman, L., und MacPherson, G. J. (1990). Chemical compositions of refractory inclusions from the Vigarano and Leoville carbonaceous chondrites. Geochimica et Cosmochimica Acta, 54(7):2121-2132.

Martin, P. M. und Mason, B. (1974). Major and trace elements in the Allende meteorite. Nature, 249(5455):333-334.

Marty, B., Chaussidon, M., Wiens, R. C., Jurewicz, A. J. G., und Burnett, D. S. (2011). A ${ }^{15}$ N-Poor Isotopic Composition for the Solar System As Shown by Genesis Solar Wind Samples. Science, 332(6037):1533-1536.

Mason, B. und Taylor, S. R. (1982). Inclusions in the Allende Meteorite. Smithsonian Contributions to the Earth Sciences, 25:1-30.

Masuda, A., Nakamura, N., und Tanaka, T. (1973). Fine structures of mutually normalized rare-earth patterns of chondrites. Geochimica et Cosmochimica Acta, $37(2): 239-248$.

McSween, H. Y. (1977). Petrographic variations among carbonaceous chondrites of the Vigarano type. Geochimica et Cosmochimica Acta, 41(12):1777-1790. 
McSween Jr., H. Y. (1977). Chemical and petrographic constraints on the origin of chondrules and inclusions in carbonaceous chondrites. Geochimica et Cosmochimica Acta, 41(12):1843-1860.

Minami, E. (1935). Gehalte an seltenen Erden in europäischen und japanischen Tonschiefern. Nachr. Ges. Wiss. Göttingen, 1(14):155-170.

Misawa, K. und Fujita, T. (1994). A relict refractory inclusion in a ferromagnesian chondrule from the Allende meteorite. Nature, 368(6473):723-726.

Misawa, K. und Nakamura, N. (1988a). Demonstration of REE fractionation among individual chondrules from the Allende (CV3) chondrite. Geochimica et Cosmochimica Acta, 52(6):1699-1710.

Misawa, K. und Nakamura, N. (1988b). Highly fractionated rare-earth elements in ferromagnesian chondrules from the Felix (CO3) meteorite. Nature, 334(6177):4750 .

Misawa, K. und Nakamura, N. (1988c). Rare earth elements in chondrules from the Felix (CO3) chondrite: Comparison with Allende (CV) chondrules. Antarctic Meteorite Research, 1:215-223.

Monecke, T., Kempe, U., Monecke, J., Sala, M., und Wolf, D. (2002). Tetrad effect in rare earth element distribution patterns: A method of quantification with application to rock and mineral samples from granite-related rare metal deposits. Geochimica et Cosmochimica Acta, 66(7):1185-1196.

Morlok, A., Bischoff, A., Stephan, T., Floss, C., Zinner, E., und Jessberger, E. K. (2006). Brecciation and chemical heterogeneities of CI chondrites. Geochimica et Cosmochimica Acta, 70(21):5371-5394.

Münker, C. (2010). A high field strength element perspective on early lunar differentiation. Geochimica et Cosmochimica Acta, 74(24):7340-7361.

Münker, C., Pfänder, J. A., Weyer, S., Büchl, A., Kleine, T., und Mezger, K. (2003). Evolution of Planetary Cores and the Earth-Moon System from Nb/Ta Systematics. Science, 301(5629):84-87.

Münker, C., Stracke, A., Bendel, V., Palme, H., und Pack, A. (2012). Is the bulk Earth Nb/Ta chondritic? Mineralogical Magazine, 76(6):2148.

Münker, C., Weyer, S., Scherer, E., und Mezger, K. (2001). Separation of high field strength elements (Nb, Ta, Zr, Hf) and Lu from rock samples for MC-ICPMS measurements. Geochemistry, Geophysics, Geosystems, 2(12). 
Nagashima, K., Krot, A. N., und Yurimoto, H. (2004). Stardust silicates from primitive meteorites. Nature, 428(6986):921-924.

Nakamura, N. (1974). Determination of REE, Ba, Fe, Mg, Na and K in carbonaceous and ordinary chondrites. Geochimica et Cosmochimica Acta, 38(5):757-775.

Nakamura, N. und Masuda, A. (1973). Chondrites with peculiar rare-earth patterns. Earth and Planetary Science Letters, 19(4):429-437.

Pack, A., Kremer, K., Albrecht, N., Simon, K., und Kronz, A. (2010). Description of an aerodynamic levitation apparatus with applications in Earth sciences. Geochemical Transactions, 11(1):1-16.

Pack, A., Russell, S. S., Shelley, J. M. G., und Van Zuilen, M. (2007). Geo- and cosmochemistry of the twin elements yttrium and holmium. Geochimica et Cosmochimica Acta, 71(18):4592-4608.

Pack, A., Shelley, J. M. G., und Palme, H. (2004). Chondrules with Peculiar REE Patterns: Implications for Solar Nebular Condensation at High C/O. Science, 303(5660):997-1000.

Palme, H. und O’Neill, H. S. C. (2003). Cosmochemical Estimates of Mantle Composition. In: Holland, H. D. und Turekian, K. K. (Hrsg.), Treatise on Geochemistry: The Mantel and Core, Band 2, Seiten 1-38. Elsevier.

Palme, H., Wlotzka, F., Nagel, K., und El Goresy, A. (1982). An ultra-refractory inclusion from the Ornans carbonaceous chondrite. Earth and Planetary Science Letters, 61(1):1-12.

Patzer, A., Hezel, D. C., Bendel, V., und Pack, A. (2012). Chondritic ingredients: I. Usual suspects and some oddballs in the Leoville CV3 meteorite. Meteoritics $\&$ Planetary Science, 47(1):142-157.

Patzer, A., Pack, A., und Gerdes, A. (2010). Zirconium and hafnium in meteorites. Meteoritics \& Planetary Science, 45(7):1136-1151.

Paulick, H., Münker, C., und Schuth, S. (2010). The influence of small-scale mantle heterogeneities on Mid-Ocean Ridge volcanism: Evidence from the southern MidAtlantic Ridge ( $7^{\circ} 30^{\prime} \mathrm{S}$ to $\left.11^{\circ} 30^{\prime} \mathrm{S}\right)$ and Ascension Island. Earth and Planetary Science Letters, 296(3-4):299-310.

Pin, C. und Zalduegui, J. F. S. (1997). Sequential separation of light rare-earth elements, thorium and uranium by miniaturized extraction chromatography: Application to isotopic analyses of silicate rocks. Analytica Chimica Acta, 339(12):79-89. 
Pourmand, A., Dauphas, N., und Ireland, T. J. (2012). A novel extraction chromatography and MC-ICP-MS technique for rapid analysis of REE, Sc and Y: Revising CI-chondrite and Post-Archean Australian Shale (PAAS) abundances. Chemical Geology, 291:38-54.

Rubin, A. E. (2010). Physical properties of chondrules in different chondrite groups: Implications for multiple melting events in dusty environments. Geochimica et Cosmochimica Acta, 74(16):4807-4828.

Rubin, A. E. (2011). Origin of the differences in refractory-lithophile-element abundances among chondrite groups. Icarus, 213(2):547-558.

Rubin, A. E. und Bottke, W. F. (2009). On the origin of shocked and unshocked $\mathrm{CM}$ clasts in H-chondrite regolith breccias. Meteoritics $\&$ Planetary Science, 44(5):701-724.

Russell, C. T., Raymond, C. A., Coradini, A., McSween, H. Y., Zuber, M. T., Nathues, A., De Sanctis, M. C., Jaumann, R., Konopliv, A. S., Preusker, F., et al. (2012). Dawn at Vesta: Testing the Protoplanetary Paradigm. Science, 336(6082):684-686.

Russell, S. S., Krot, A. N., Huss, G. R., Keil, K., Itoh, S., Yurimoto, H., und MacPherson, G. J. (2005). The Genetic Relationship between Refractory Inclusions and Chondrules. In: Krot, A. N., Scott, E. R. D., und Reipurth, B. (Hrsg.), Chondrites and the Protoplanetary Disk, Band 341 von Astronomical Society of the Pacific Conference Series, Seiten 317-350.

Sanders, I. S. und Scott, E. R. D. (2012). The origin of chondrules and chondrites: Debris from low-velocity impacts between molten planetesimals? Meteoritics $\mathscr{6}$ Planetary Science, 47(12):2170-2192.

Scherer, P. und Schultz, L. (2000). Noble gas record, collisional history, and pairing of CV, CO, CK, and other carbonaceous chondrites. Meteoritics $\&$ Planetary Science, 35(1):145-153.

Schulze, H., Bischoff, A., Palme, H., Spettel, B., Dreibus, G., und Otto, J. (1994). Mineralogy and chemistry of Rumuruti: The first meteorite fall of the new $\mathrm{R}$ chondrite group. Meteoritics, 29(2):275-286.

Scott, E. R. D. (2007). Chondrites and the Protoplanetary Disk. Annual Review of Earth and Planetary Sciences, 35:577-620.

Scott, E. R. D. und Krot, A. N. (2003). Chondrites and their Components. In: Davis, A. M., Holland, H. D., und Turekian, K. K. (Hrsg.), Treatise on Geochemistry: Meteorites, Comets and Planets, Band 1, Seiten 143-200. Elsevier. 
Scott, E. R. D. und Krot, A. N. (2005). Thermal Processing of Silicate Dust in the Solar Nebula: Clues from Primitive Chondrite Matrices. The Astrophysical Journal, 623:571-578.

Sears, D. W., Kallemeyn, G. W., und Wasson, J. T. (1982). The compositional classification of chondrites: II The enstatite chondrite groups. Geochimica et Cosmochimica Acta, 46(4):597-608.

Shannon, R. D. (1976). Revised effective ionic radii and systematic studies of interatomic distances in halides and chalcogenides. Acta Crystallographica Section A, 32(5):751-767.

Shu, F. H., Shang, H., und Lee, T. (1996). Toward an astrophysical theory of chondrites. Science, 271:1545-1552.

Simon, S. B., Davis, A. M., und Grossman, L. (1996). A unique ultrarefractory inclusion from the Murchison meteorite. Meteoritics $\& 5$ Planetary Science, 31(1):106-115.

Stracke, A., Palme, H., Gellissen, M., Münker, C., Kleine, T., Birbaum, K., Günther, D., Bourdon, B., und Zipfel, J. (2012). Refractory element fractionation in the Allende meteorite: Implications for solar nebula condensation and the chondritic composition of planetary bodies. Geochimica et Cosmochimica Acta, 85:114-141.

Strnad, L., Mihaljevic, M., und Sebek, O. (2005). Laser Ablation and Solution ICP-MS Determination of Rare Earth Elements in USGS BIR-1G, BHVO-2G and BCR-2G Glass Reference Materials. Geostandards and Geoanalytical Research, 29(3):303-314.

Sylvester, P. J. (2008). LA-(MC)-ICP-MS Trends in 2006 and 2007 with Particular Emphasis on Measurement Uncertainties. Geostandards and Geoanalytical Research, 32(4):469-488.

Sylvester, P. J., Simon, S. B., und Grossman, L. (1993). Refractory inclusions from the Leoville, Efremovka, and Vigarano C3V chondrites: Major element differences between types $\mathrm{A}$ and $\mathrm{B}$, and extraordinary refractory siderophile element compositions. Geochimica et Cosmochimica Acta, 57(15):3763-3784.

Tomeoka, K. und Buseck, P. R. (1988). Matrix mineralogy of the Orgueil CI carbonaceous chondrite. Geochimica et Cosmochimica Acta, 52(6):1627-1640.

Van Schmus, W. R. und Wood, J. A. (1967). A chemical-petrologic classification for the chondritic meteorites. Geochimica et Cosmochimica Acta, 31(5):747-765. 
Wakaki, S., Itoh, S., Tanaka, T., und Yurimoto, H. (2013). Petrology, trace element abundances and oxygen isotopic compositions of a compound CAI-chondrule object from Allende. Geochimica et Cosmochimica Acta, 102:261-279.

Warren, P. H. (2011). Stable-isotopic anomalies and the accretionary assemblage of the Earth and Mars: A subordinate role for carbonaceous chondrites. Earth and Planetary Science Letters, 311(1-2):93-100.

Wasson, J. T. (2013). Vesta and extensively melted asteroids: Why HED meteorites are probably not from Vesta. Earth and Planetary Science Letters, 381:138-146.

Wegner, W., Wörner, G., Harmon, R. S., und Jicha, B. R. (2011). Magmatic history and evolution of the Central American Land Bridge in Panama since Cretaceous times. Geological Society of America Bulletin, 123(3-4):703-724.

Weisberg, M. K., McCoy, T. J., und Krot, A. N. (2006). Systematics and Evaluation of Meteorite Classification. In: Lauretta, D. S. und McSween, H. Y. (Hrsg.), Meteorites and the Early Solar System, Band 2, Seiten 19-52. Univ. of Arizona Press.

Weyer, S., Münker, C., Rehkämper, M., und Mezger, K. (2002). Determination of ultra-low $\mathrm{Nb}$, Ta, Zr and Hf concentrations and the chondritic $\mathrm{Zr} / \mathrm{Hf}$ and $\mathrm{Nb} / \mathrm{Ta}$ ratios by isotope dilution analyses with multiple collector ICP-MS. Chemical Geology, 187(3-4):295-313.

Willbold, M. und Jochum, K. P. (2005). Multi-Element Isotope Dilution Sector Field ICP-MS: A Precise Technique for the Analysis of Geological Materials and its Application to Geological Reference Materials. Geostandards and Geoanalytical Research, 29(1):63-82.

Wolf, D. und Palme, H. (2001). The solar system abundances of phosphorus and titanium and the nebular volatility of phosphorus. Meteoritics \& Planetary Science, 36(4):559-571.

Wurm, G. und Haack, H. (2009). Outward transport of CAIs during FU-Orionis events. Meteoritics \& Planetary Science, 44(5):689-699.

Zolensky, M., Barrett, R., und Browning, L. (1993). Mineralogy and composition of matrix and chondrule rims in carbonaceous chondrites. Geochimica et Cosmochimica Acta, 57(13):3123-3148.

Zolensky, M. E., Weisberg, M. K., Buchanan, P. C., und Mittlefehldt, D. W. (1996). Mineralogy of carbonaceous chondrite clasts in HED achondrites and the Moon. Meteoritics \& Planetary Science, 31(4):518-537. 
Zolensky, M. E., Zega, T. J., Yano, H., Wirick, S., Westphal, A. J., Weisberg, M. K., Weber, I., Warren, J. L., Velbel, M. A., Tsuchiyama, A., et al. (2006). Mineralogy and Petrology of Comet 81P/Wild 2 Nucleus Samples. Science, 314(5806):1735-1739. 


\section{Publikationen}

\section{Zeitschriftenartikel}

- In Vorbereitung:

Bendel, V., Pack, A., Patzer, A., O’Neill, H.St.C., Jenner, F.E. und Münker, C. „Chemical dichotomy of inner solar system bodies - implications from heavy rare earth element variations".

- Bendel, V., Ueltzhöffer, K.J., Freitag, J., Kipfstuhl, S., Kuhs, W.F., Garbe, C.S. und Faria, S.H. „High-resolution variations in size, number and arrangement of air bubbles in the EPICA DML (Antarctica) ice core", Journal of Glaciology 59(217), 972-980 (2013).

- Patzer, A., Hezel, D.C., Bendel, V. und Pack, A. „Chondritic ingredients: I. Usual suspects and some oddballs in the Leoville CV3 meteorite", Meteoritics and Planetary Science 47, 142-157 (2012).

- Ueltzhöffer, K.J., Bendel, V., Freitag, J., Kipfstuhl, S., Wagenbach, D., Faria, S.H. und Garbe, C.S. „Distribution of air bubbles in the EDML and EDC (Antarctica) ice cores, using a new method of automatic image analysis", Journal of Glaciology 56(196), 339-348 (2010).

- Bendel, V. und Schmidt, B.C. „Raman spectroscopic characterisation of disordered alkali feldspars along the join $\mathrm{KAlSi}_{3} \mathrm{O}_{8}-\mathrm{NaAlSi}_{3} \mathrm{O}_{8}$ : application to natural sanidine and anorthoclase", European Journal of Mineralogy 20(6), 1055-1065 (2008).

\section{Konferenzbeiträge}

- Bendel, V., Pack, A.*, Münker, C., Jenner, F.E. und O’Neill, H.St.C. „Heavy REE fractionation in chondrites, achondrites and planets", Paneth Kolloquium, Nördlingen (2013).

- Bendel, V., Pack, A. und O'Neill, H.St.C. „Rare earth elements in CI1-chondrites and planetary samples", 43rd Lunar and Planetary Science Conference, The Woodlands, Texas (2012). 
- Bendel, V.*, Pack, A., O’Neill, H.St.C., Jenner, F.E. und Münker, C. „Volatility-controlled rare earth element fractionation in the early Solar System“, 75th Annual Meteoritical Society Meeting, Cairns (2012).

- Bendel, V.*, Pack, A., O’Neill, H.St.C., Jenner, F.E. und Münker, C. „Planetary-scale volatility-controlled fractionation of the rare earth elements in the early Solar System", European Mineralogical Conference, Frankfurt (2012).

- Bendel, V.*, Pack, A., O’Neill, H.St.C., Jenner, F.E. und Münker, C. „Volatility-controlled rare earth element fractionation - meteorites and Earth", Paneth Kolloquium, Nördlingen (2012).

- Münker, C.*, Stracke, A., Bendel, V., Palme, H. und Pack, A. „Is the bulk Earth Nb/Ta chondritic?", Goldschmidt Conference, Montreal (2012).

- Bendel, V.*, Patzer, A., Pack, A., Hezel, D.C. und Münker, C. „Rare earth elements in bulk chondrites and chondrite components", 42nd Lunar and Planetary Science Conference, The Woodlands, Texas (2011).

- Patzer, A.*, Hezel. D.C., Bendel, V., und Pack, A. „The Leoville CV3 chondrite revisited: prime material for the study of refractory trace elements", 42nd Lunar and Planetary Science Conference, The Woodlands, Texas (2011).

- Bendel, V.*, Patzer, A., Pack, A., Allen, C. und Münker, C. „Rare earth element abundances in bulk chondrites and earth", 88th Annual Meeting of the German Mineralogical Society (DMG), Münster (2010).

- Bendel, V.*, Patzer, A., Pack, A., Allen, C. und Münker, C. „Rare earth elements as indicator for volatility controlled fractionation processes on planetary scale", Paneth Kolloquium, Nördlingen (2010).

* Präsentierender Autor 


\section{Danksagung}

Die vorliegende Doktorarbeit wurde am Geowissenschaftlichen Zentrum der Universität Göttingen in der Abteilung Isotopengeologie bei Prof. Dr. Andreas Pack angefertigt und von der Deutschen Forschungsgemeinschaft durch das Projekt PA 909/7-1/2 innerhalb des Schwerpunktprogrammes 1385 "The first 10 million years of the solar system - a planetary materials approach" gefördert.

Ich möchte mich an dieser Stelle bei allen bedanken, die zum Erfolg der Arbeit beigetragen haben. Prof. Dr. Andreas Pack danke ich für die engagierte Betreuung dieses spannenden Dissertationsprojektes. Das gemeinsame Diskutieren der Ergebnisse hat sehr zum Gelingen der Arbeit beigetragen. Prof. Dr. Bent T. Hansen danke ich herzlich für die freundliche Übernahme des Koreferates.

Das Projekt profitierte sehr von der großzügigen Bereitstellung vielfältiger Proben. Hierfür danke ich A. Bischoff, T. McCoy, L. Welzenbach, M. Shelley, B. Zanda, R. Schönberg, A. Gehler, J. Zipfel, K. Bermingham, M. Reich, G. Hartmann, K. H. Wedepohl, B. Schmidt, W. Wegner, H.-P. Schertl, H. Paulick und C. Münker.

Weiterhin möchte ich Prof. Dr. Carsten Münker für die freundliche Betreuung während der Aufenthalte in Bonn sowie die Durchführung der MC-ICPMS-Messungen danken. Auch die intensive Unterstützung durch Prof. Dr. Hugh O'Neill und Dr. Charlotte Allen bei den LA-ICPMS-Messungen an der Australian National University Canberra war sehr hilfreich. Bei Dr. Andrea Patzer möchte ich mich für die Durchführung einiger Messungen und die sehr angenehme Zusammenarbeit bedanken.

Für die Durchführung und Vorbereitung der Röntgenfluoreszenzanalysen möchte ich Dr. Gerald Hartmann und Angelika Reitz meinen Dank aussprechen. Ein herzliches Dankeschön geht außerdem an Dr. Andreas Kronz und Gabriele Mengel für die Hilfestellung bei den Mikrosondenmessungen sowie an Fabian Hartmann und Nina Albrecht für die Unterstützung bei der Probenpräparation. Auch allen anderen Kollegen bin ich für die sehr angenehme Arbeitsatmosphäre dankbar. Die gemeinsame Doktorandenzeit mit Sukanya Sen Gupta, Magdalena Hofmann und Alexander Gehler wird mir in guter Erinnerung bleiben.

Sehr wertvoll war für mich die ununterbrochene moralische Unterstützung durch meine lieben Freunde, Eltern und Geschwister. Ganz besonders danke ich meinem Freund Jon-Olaf Krisponeit. Sein gründliches Korrekturlesen der Arbeit, die tatkräftige Unterstützung und ausgezeichnete Beratung bei zahlreichen Computerfragen waren äußerst hilfreich. Seine beständige Liebe und Aufmunterung halfen mir sehr, diese Arbeit zu vollenden. Für all die schönen Momente und Abwechslungen während dieser Zeit bin ich ihm zu größtem Dank verpflichtet. 